Supplement of Atmos. Chem. Phys., 19, 4075-4091, 2019

https://doi.org/10.5194/acp-19-4075-2019-supplement

(c) Author(s) 2019. This work is distributed under

the Creative Commons Attribution 4.0 License.

(c) (1)

Supplement of

\title{
Mechanistic and kinetics investigations of oligomer formation from Criegee intermediate reactions with hydroxyalkyl hydroperoxides
}

\section{Long Chen et al.}

Correspondence to: Yu Huang (huangyu@ieecas.cn) and Junji Cao (cao@loess.llqg.ac.cn)

The copyright of individual parts of the supplement might differ from the CC BY 4.0 License. 


\section{Contents:}

Table S1 Y/X (Y = M06-2X, CCSD(T), X = 6-311+G(2df,2p), def2-TZVP) calculated energy barrier $\left(\Delta E_{a}^{\#}, \Delta G_{a}^{\#}\right)$ for the addition reactions of carbonyl oxides with HHPs based on the M06-2X/6-311+G(2df,2p) optimized geometries $\left(\mathrm{kcal} \cdot \mathrm{mol}^{-1}\right.$ )

Table S2 The partition function of every vibrational mode involved in the complex IM1a

Table S3 The partition function of every vibrational mode involved in the complex IM2a

Table S4 AIM properties at the bond critical points for the forming bonds B1-B4

Table S5 Rate coefficients $\left(\mathrm{cm}^{3}\right.$ molecule $\left.{ }^{-1} \mathrm{~s}^{-1}\right)$ of $\mathrm{CH}_{2} \mathrm{OO}$ reactions with $\mathrm{P} 1 \mathrm{a}, \mathrm{P} 1 \mathrm{~b}$, P1c and P1d computed at different temperatures

Table S6 Rate coefficients $\left(\mathrm{cm}^{3}\right.$ molecule $\mathrm{s}^{-1}$ ) of anti- $\mathrm{CH}_{3} \mathrm{CHOO}$ reactions with $\mathrm{P} 3 \mathrm{a}$, $\mathrm{P} 3 \mathrm{~b}, \mathrm{P} 3 \mathrm{c}$ and $\mathrm{P} 3 \mathrm{~d}$ computed at different temperatures

Table S7 Rate coefficients $\left(\mathrm{cm}^{3}\right.$ molecule $\left.\mathrm{s}^{-1}\right)$ of $s y n-\mathrm{CH}_{3} \mathrm{CHOO}$ reactions with $\mathrm{P} 5 \mathrm{a}$, P5b, P5c and P5d computed at different temperatures

Table S8 Rate coefficients $\left(\mathrm{cm}^{3}\right.$ molecule $\left.\mathrm{e}^{-1} \mathrm{~s}^{-1}\right)$ of $\left(\mathrm{CH}_{3}\right)_{2} \mathrm{CHOO}$ reactions with $\mathrm{P} 7 \mathrm{a}$, P7b, P7c and P7d computed at different temperatures

Table S9 Rate coefficients $\left(\mathrm{cm}^{3}\right.$ molecule $\left.{ }^{-1} \mathrm{~s}^{-1}\right)$ of elementary reactions R1a and R9 Figure S1. PES ( $\Delta G$ and $\Delta E$ (italic) ) for the reaction of $\mathrm{CH}_{2} \mathrm{OO}$ with $\mathrm{HO}-\mathrm{CH}_{2} \mathrm{OO}-\mathrm{H}$ $\left(\mathrm{Pb}_{1}\right)$ calculated at the M06-2X/def2-TZVP//M06-2X/6-311+G(2df,2p) level of theory Figure S2 PES ( $\Delta G$ and $\Delta E$ (italic)) for the reaction of $\mathrm{HO}-\mathrm{C}\left(\mathrm{CH}_{3}\right) \mathrm{HOO}-\mathrm{H}$ with anti-(a) and syn- $\mathrm{CH}_{3} \mathrm{CHOO}$ (b) calculated at the M06-2X/def2-TZVP//M06-2X/6-311 $+\mathrm{G}(2 \mathrm{df}, 2 \mathrm{p})$ level of theory

Figure S3 PES $(\Delta G$ and $\Delta E$ (italic) $)$ for the reaction of $\left(\mathrm{CH}_{3}\right)_{2} \mathrm{COO}$ with HO-C $\left(\mathrm{CH}_{3}\right)_{2} \mathrm{OO}-\mathrm{H}\left(\mathrm{Pb}_{3}\right)$ calculated at the M06-2X/def2-TZVP//M06-2X/6-311+ $\mathrm{G}(2 \mathrm{df}, 2 \mathrm{p})$ level of theory

Figure S4. Optimized geometries, z-matrices and vibrational frequencies of all stationary points in the reaction of $2 \mathrm{CH}_{2} \mathrm{OO}$ with $\mathrm{HO}-\mathrm{CH}_{2} \mathrm{OO}-\mathrm{H}$ at the M06-2X/6-311+G(2df,2p) level of theory 
Figure S5. PES ( $\Delta G$ and $\Delta E$ (italic)) for the reaction of $\mathrm{HO}-\mathrm{CH}_{2} \mathrm{OO}-\mathrm{H}\left(\mathrm{Pa}_{1}\right)$ with $\mathrm{OH}$ computed at the M06-2X/def2-TZVP//M06-2X/6-311+G(2df,2p) level of theory

Figure S6. Optimized geometries, z-matrices and vibrational frequencies of all stationary points in the reaction of 2 anti- $\mathrm{CH}_{3} \mathrm{CHOO}$ with $\mathrm{HO}-\mathrm{CH}\left(\mathrm{CH}_{3}\right) \mathrm{OO}-\mathrm{H}\left(\mathrm{Pa}_{2}\right.$ and $\left.\mathrm{Pb}_{2}\right)$ at the M06-2X/6-311+G(2df,2p) level of theory

Figure S7. Optimized geometries, z-matrices and vibrational frequencies of all stationary points in the reaction of 2 syn- $\mathrm{CH}_{3} \mathrm{CHOO}$ with $\mathrm{HO}-\mathrm{CH}\left(\mathrm{CH}_{3}\right) \mathrm{OO}-\mathrm{H}\left(\mathrm{Pa}_{2}\right.$ and $\left.\mathrm{Pb}_{2^{\prime}}\right)$ at the M06-2X/6-311+G(2df,2p) level of theory

Figure S8. Optimized geometries, z-matrices and vibrational frequencies of all stationary points in the reaction of $\left(\mathrm{CH}_{3}\right)_{2} \mathrm{COO}$ with $\mathrm{HO}-\mathrm{C}\left(\mathrm{CH}_{3}\right)_{2} \mathrm{OO}-\mathrm{H}$ at the M06-2X/6-311+G(2df,2p) level of theory

Figure S9. PES $(\Delta G$ and $\Delta E$ (italic) $)$ of distinct SCI reactions with $\mathrm{HO}-\mathrm{CH}\left(\mathrm{CH}_{3}\right) \mathrm{OO}-\mathrm{H}\left(\mathrm{Pa}_{2}\right)$ calculated at the M06-2X/def2-TZVP//M06-2X/6-311+ G(2df,2p) level of theory

Figure S10. PES $(\Delta G$ and $\Delta E$ (italic)) of distinct SCI reactions with $\mathrm{HO}-\mathrm{CH}\left(\mathrm{CH}_{3}\right) \mathrm{OO}-\mathrm{H}\left(\mathrm{Pa}_{2}\right)$ calculated at the M06-2X/def2-TZVP//M06-2X/6-311+ $\mathrm{G}(2 \mathrm{df}, 2 \mathrm{p})$ level of theory

Figure S11. PES $(\Delta G$ and $\Delta E$ (italic)) of distinct SCI reactions with $\mathrm{HO}-\mathrm{C}\left(\mathrm{CH}_{3}\right)_{2} \mathrm{OO}-\mathrm{H}\left(\mathrm{Pa}_{3}\right)$ calculated at the M06-2X/def2-TZVP//M06-2X/6-311+ $\mathrm{G}(2 \mathrm{df}, 2 \mathrm{p})$ level of theory 
Table S1 Y/X ( $\mathrm{Y}=\mathrm{M} 06-2 \mathrm{X}, \mathrm{CCSD}(\mathrm{T}), \mathrm{X}=6-311+\mathrm{G}(2 \mathrm{df}, 2 \mathrm{p})$, def2-TZVP) calculated energy barrier $\left(\Delta E_{a}^{\#}, \Delta G_{a}^{\#}\right)$ for the addition reactions of carbonyl oxides with HHPs based on the M06-2X/6-311+G(2df,2p) optimized geometries $\left(\mathrm{kcal} \cdot \mathrm{mol}^{-1}\right)$

\begin{tabular}{|c|c|c|c|c|}
\hline \multirow{2}{*}{ Reactions } & \multicolumn{2}{|c|}{$\operatorname{CCSD}(\mathrm{T}) / 6-311+\mathrm{G}(2 \mathrm{df}, 2 \mathrm{p})$} & \multicolumn{2}{|c|}{ M06-2X/def2-TZVP } \\
\hline & $\Delta E_{a}^{\#}$ & $\Delta G_{a}^{\#}$ & $\Delta E_{a}^{\#}$ & $\Delta G_{a}^{\#}$ \\
\hline R1a & $9.4^{\mathrm{a}} ; 7.9^{\mathrm{b}}$ & $10.1^{\mathrm{a}} ; 8.6^{\mathrm{b}}$ & $8.0^{\mathrm{a}} ; 7.6^{\mathrm{b}}$ & $8.8^{\mathrm{a}} ; 8.4^{\mathrm{b}}$ \\
\hline $\mathrm{R} 1 \mathrm{~b}$ & $13.0^{\mathrm{a}} ; 11.6^{\mathrm{b}}$ & $13.3^{\mathrm{a}} ; 11.9^{\mathrm{b}}$ & $11.9^{\mathrm{a}} ; 11.4^{\mathrm{b}}$ & $12.2^{\mathrm{a}} ; 11.7^{\mathrm{b}}$ \\
\hline $\mathrm{R} 1 \mathrm{c}$ & $8.1^{\mathrm{a}} ; 6.7^{\mathrm{b}}$ & $9.2^{\mathrm{a}} ; 7.8^{\mathrm{b}}$ & $6.9^{\mathrm{a}} ; 6.4^{\mathrm{b}}$ & $7.9^{\mathrm{a}} ; 7.4^{\mathrm{b}}$ \\
\hline R1d & $8.6^{\mathrm{a}} ; 7.6^{\mathrm{b}}$ & $10.2^{\mathrm{a}} ; 9.2^{\mathrm{b}}$ & $7.0^{\mathrm{a}} ; 6.7^{\mathrm{b}}$ & $8.7^{\mathrm{a}} ; 8.4^{\mathrm{b}}$ \\
\hline R3a & $6.6^{\mathrm{a}} ; 4.9^{\mathrm{b}}$ & $7.1^{\mathrm{a}} ; 5.4^{\mathrm{b}}$ & $5.8^{\mathrm{a}} ; 4.4^{\mathrm{b}}$ & $6.2^{\mathrm{a}} ; 5.8^{\mathrm{b}}$ \\
\hline $\mathrm{R} 3 \mathrm{~b}$ & $8.4^{\mathrm{a}} ; 7.3^{\mathrm{b}}$ & $9.7^{\mathrm{a}} ; 8.6^{\mathrm{b}}$ & $7.3^{\mathrm{a}} ; 6.8^{\mathrm{b}}$ & $8.8^{\mathrm{a}} ; 8.3^{\mathrm{b}}$ \\
\hline $\mathrm{R} 3 \mathrm{c}$ & $6.5^{\mathrm{a}} ; 4.8^{\mathrm{b}}$ & $7.3^{\mathrm{a}} ; 5.6^{\mathrm{b}}$ & $5.8^{\mathrm{a}} ; 5.4^{\mathrm{b}}$ & $6.6^{\mathrm{a}} ; 6.2^{\mathrm{b}}$ \\
\hline R3d & $8.4^{\mathrm{a}} ; 6.8^{\mathrm{b}}$ & $9.7^{\mathrm{a}} ; 8.1^{\mathrm{b}}$ & $7.3^{\mathrm{a}} ; 6.8^{\mathrm{b}}$ & $8.6^{\mathrm{a}} ; 8.1^{\mathrm{b}}$ \\
\hline R5a & $12.3^{\mathrm{a}} ; 10.9^{\mathrm{b}}$ & $13.2^{\mathrm{a}} ; 11.8^{\mathrm{b}}$ & $11.5^{\mathrm{a}} ; 11.1^{\mathrm{b}}$ & $12.5^{\mathrm{a}} ; 12.1^{\mathrm{b}}$ \\
\hline $\mathrm{R} 5 \mathrm{~b}$ & $11.0^{\mathrm{a}} ; 9.4^{\mathrm{b}}$ & $12.4^{\mathrm{a}} ; 10.8^{\mathrm{b}}$ & $10.6^{\mathrm{a}} ; 10.1^{\mathrm{b}}$ & $12.0^{\mathrm{a}} ; 11.5^{\mathrm{b}}$ \\
\hline $\mathrm{R} 5 \mathrm{c}$ & $12.0^{\mathrm{a}} ; 10.6^{\mathrm{b}}$ & $13.3^{\mathrm{a}} ; 11.9^{\mathrm{b}}$ & $11.3^{\mathrm{a}} ; 10.9^{\mathrm{b}}$ & $12.5^{\mathrm{a}} ; 12.1^{\mathrm{b}}$ \\
\hline R5d & $12.2^{\mathrm{a}} ; 11.0^{\mathrm{b}}$ & $13.8^{\mathrm{a}} ; 12.6^{\mathrm{b}}$ & $11.4^{\mathrm{a}} ; 11.0^{\mathrm{b}}$ & $13.0^{\mathrm{a}} ; 12.6^{\mathrm{b}}$ \\
\hline
\end{tabular}

$\mathrm{a}$ and $\mathrm{b}$ represent without and with considering the BSSE correction 
According to the principle of statistical thermodynamics, the entropy can be described as eqn (1)

$$
S=\frac{H-G}{T}
$$

where $\mathrm{H}$ is the enthalpy; $\mathrm{G}$ is the free energy; $\mathrm{T}$ is the temperature in Kelvin. The relationship between the entropy and the partition function can be written as eqn (2)

$$
\mathrm{S}=k \ln \frac{q^{\mathrm{N}}}{N !}+N k T\left(\frac{\partial \ln q}{\partial T}\right)_{V, N}
$$

where $k$ is the Boltzmann constant; $q$ is the partition function, it mainly includes translation $\left(q_{\text {trans }}\right)$, vibration $\left(q_{v i b}\right)$, external rotation $\left(q_{r o t}\right)$, electronic $\left(q_{e l e}\right)$ and torsional $\left(q_{t o r}\right)$ partition functions ( $\left.q=q_{t r a n s} q_{v i b} q_{r o t} q_{e l e} q_{t o r}\right)$ (Mendes, et al., 2014). Among these partition functions, the $q_{v i b}$ plays an important role in determining the entropic factors. Thus, the $q_{v i b}$ is used to evaluate the contribution of vibrational mode to the entropic factor. The $q_{v i b}$ can be described as eqn (3)

$$
q_{\mathrm{vib}}=\exp \left(-\frac{1}{2} \frac{h v}{k T}\right) \times\left(\frac{1}{1-e^{-h v / k T}}\right)
$$


Table S2 The partition function of every vibrational mode involved in the complex IM1a

\begin{tabular}{|c|c|c|c|}
\hline $\mathrm{N}$ & $v$ & $q_{\mathrm{vib}}$ & percent $(\%)$ \\
\hline 1 & 51.49 & 4.03 & 30.43 \\
\hline 2 & 123.66 & 1.66 & 12.52 \\
\hline 3 & 149.16 & 1.37 & 10.31 \\
\hline 4 & 158.76 & 1.28 & 9.66 \\
\hline 5 & 199.39 & 1.00 & 7.58 \\
\hline 6 & 242.53 & 0.81 & 6.12 \\
\hline 7 & 257.19 & 0.76 & 5.74 \\
\hline 8 & 422.78 & 0.42 & 3.15 \\
\hline 9 & 501.75 & 0.33 & 2.48 \\
\hline 10 & 538.04 & 0.30 & 2.24 \\
\hline 11 & 642.00 & 0.22 & 1.69 \\
\hline 12 & 723.92 & 0.18 & 1.37 \\
\hline 13 & 858.58 & 0.13 & 0.97 \\
\hline 14 & 896.89 & 0.12 & 0.89 \\
\hline 15 & 958.09 & 0.10 & 0.76 \\
\hline 16 & 1070.70 & 0.08 & 0.58 \\
\hline 17 & 1093.27 & 0.07 & 0.55 \\
\hline 18 & 1112.93 & 0.07 & 0.52 \\
\hline 19 & 1134.12 & 0.07 & 0.50 \\
\hline 20 & 1270.31 & 0.05 & 0.36 \\
\hline 21 & 1300.08 & 0.04 & 0.33 \\
\hline 22 & 1397.45 & 0.03 & 0.26 \\
\hline 23 & 1429.61 & 0.03 & 0.24 \\
\hline 24 & 1456.77 & 0.03 & 0.23 \\
\hline 25 & 1506.87 & 0.03 & 0.20 \\
\hline 26 & 1588.46 & 0.02 & 0.17 \\
\hline 27 & 1674.72 & 0.02 & 0.13 \\
\hline 28 & 3088.99 & 0.00 & 0.00 \\
\hline 29 & 3148.98 & 0.00 & 0.00 \\
\hline 30 & 3162.08 & 0.00 & 0.00 \\
\hline 31 & 3162.65 & 0.00 & 0.00 \\
\hline 32 & 3305.08 & 0.00 & 0.00 \\
\hline 33 & 3868.49 & 0.00 & 0.00 \\
\hline
\end{tabular}


Table S3 The partition function of every vibrational mode involved in the complex IM2a

\begin{tabular}{|c|c|c|c|}
\hline $\mathrm{N}$ & $v$ & $q_{\text {vib }}$ & percent $(\%)$ \\
\hline 1 & 45.57 & 4.56 & 19.74 \\
\hline 2 & 66.77 & 3.10 & 13.44 \\
\hline 3 & 93.88 & 2.20 & 9.52 \\
\hline 4 & 102.82 & 2.00 & 8.68 \\
\hline 5 & 122.46 & 1.67 & 7.25 \\
\hline 6 & 144.25 & 1.41 & 6.12 \\
\hline 7 & 151.51 & 1.34 & 5.82 \\
\hline 8 & 186.88 & 1.08 & 4.66 \\
\hline 9 & 236.15 & 0.84 & 3.62 \\
\hline 10 & 242.41 & 0.81 & 3.52 \\
\hline 11 & 324.58 & 0.58 & 2.51 \\
\hline 12 & 383.38 & 0.47 & 2.05 \\
\hline 13 & 460.65 & 0.37 & 1.61 \\
\hline 14 & 475.56 & 0.35 & 1.54 \\
\hline 15 & 546.86 & 0.29 & 1.25 \\
\hline 16 & 589.00 & 0.26 & 1.12 \\
\hline 17 & 676.46 & 0.20 & 0.89 \\
\hline 18 & 704.48 & 0.19 & 0.82 \\
\hline 19 & 751.93 & 0.17 & 0.73 \\
\hline 20 & 889.93 & 0.12 & 0.52 \\
\hline 21 & 904.59 & 0.12 & 0.50 \\
\hline 22 & 957.33 & 0.10 & 0.44 \\
\hline 23 & 1053.66 & 0.08 & 0.35 \\
\hline 24 & 1082.87 & 0.07 & 0.33 \\
\hline 25 & 1103.67 & 0.07 & 0.31 \\
\hline 26 & 1123.39 & 0.07 & 0.29 \\
\hline 27 & 1129.87 & 0.07 & 0.29 \\
\hline 28 & 1139.95 & 0.06 & 0.28 \\
\hline 29 & 1188.07 & 0.06 & 0.25 \\
\hline 30 & 1271.30 & 0.05 & 0.20 \\
\hline 31 & 1306.97 & 0.04 & 0.19 \\
\hline 32 & 1353.81 & 0.04 & 0.17 \\
\hline 33 & 1383.99 & 0.04 & 0.16 \\
\hline 34 & 1415.10 & 0.03 & 0.14 \\
\hline 35 & 1438.67 & 0.03 & 0.14 \\
\hline 36 & 1455.62 & 0.03 & 0.13 \\
\hline 37 & 1457.70 & 0.03 & 0.13 \\
\hline 38 & 1501.08 & 0.03 & 0.12 \\
\hline 39 & 1569.37 & 0.02 & 0.10 \\
\hline 40 & 1663.59 & 0.02 & 0.08 \\
\hline 41 & 3104.57 & 0.00 & 0.00 \\
\hline 42 & 3113.88 & 0.00 & 0.00 \\
\hline
\end{tabular}




\begin{tabular}{lccl}
\hline 43 & 3140.47 & 0.00 & 0.00 \\
44 & 3173.49 & 0.00 & 0.00 \\
45 & 3187.95 & 0.00 & 0.00 \\
46 & 3286.9 & 0.00 & 0.00 \\
47 & 3436.06 & 0.00 & 0.00 \\
48 & 3910.66 & 0.00 & 0.00 \\
\hline
\end{tabular}



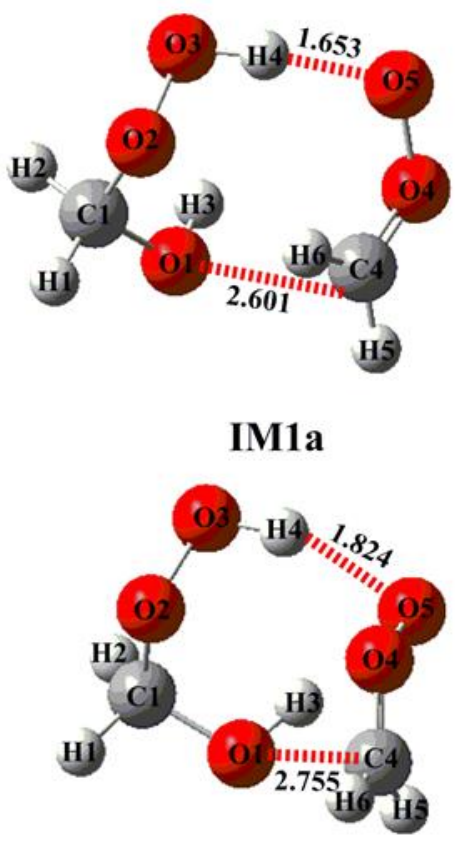

IM1b

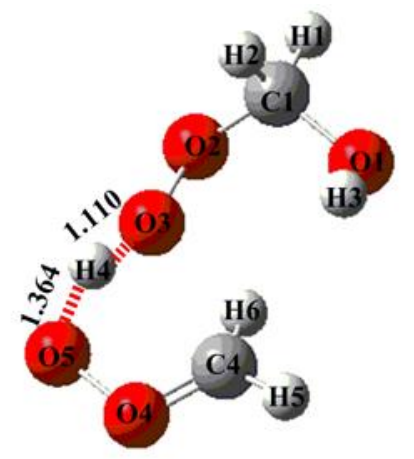

TS1a

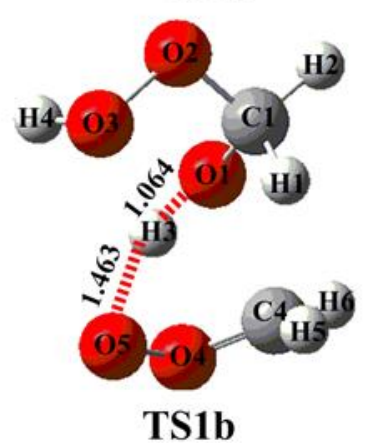

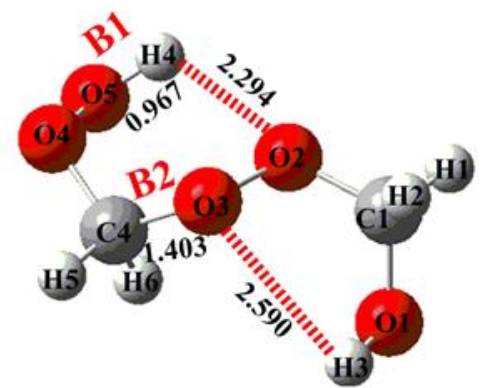

P1a

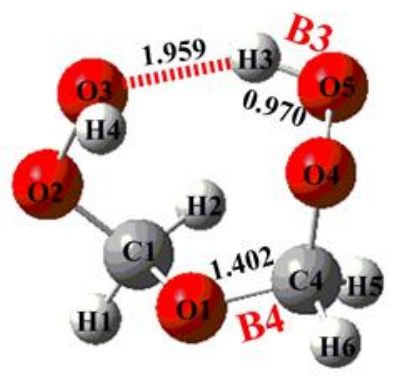

P1b

Table S4 AIM properties at the bond critical points for the forming bonds B1-B4

\begin{tabular}{cccccc}
\hline Bond & $\rho\left(\mathrm{e} / \AA^{3}\right)$ & $\nabla^{2}\left(\mathrm{e} / \AA^{3}\right)$ & Eigenvalue 1 & Eigenvalue 2 & Eigenvalue 3 \\
\hline B1(O5-H4) & 0.370 & -2.776 & -1.9316 & -1.8598 & 1.0148 \\
B2(O3-C4) & 0.271 & -0.559 & -0.5734 & -0.5112 & 0.5258 \\
B3(O5-H3) & 0.364 & -2.760 & -1.945 & -1.8743 & 1.0588 \\
B4(O1-C4) & 0.269 & -0.565 & -0.5734 & -0.5054 & 0.5139 \\
\hline
\end{tabular}


Table S5 Rate coefficients $\left(\mathrm{cm}^{3}\right.$ molecule $\left.\mathrm{s}^{-1} \mathrm{~s}^{-1}\right)$ of $\mathrm{CH}_{2} \mathrm{OO}$ reactions with $\mathrm{P} 1 \mathrm{a}, \mathrm{P} 1 \mathrm{~b}, \mathrm{P} 1 \mathrm{c}$ and $\mathrm{P} 1 \mathrm{~d}$ computed at different temperatures

\begin{tabular}{lllll}
\hline $\mathrm{T} / \mathrm{K}$ & $k_{(\mathrm{CH} 2 \mathrm{OO}+\mathrm{Pla})}$ & $k_{(\mathrm{CH} 2 \mathrm{OO}+\mathrm{P} 1 \mathrm{~b})}$ & $k_{(\mathrm{CH} 2 \mathrm{OO}+\mathrm{Plc})}$ & $k_{(\mathrm{CH} 2 \mathrm{OO}+\mathrm{Pld})}$ \\
\hline 273 & $5.1 \times 10^{-12}$ & $3.5 \times 10^{-12}$ & $8.2 \times 10^{-13}$ & $4.8 \times 10^{-11}$ \\
280 & $4.0 \times 10^{-12}$ & $2.9 \times 10^{-12}$ & $7.1 \times 10^{-13}$ & $3.7 \times 10^{-11}$ \\
298 & $2.3 \times 10^{-12}$ & $1.9 \times 10^{-12}$ & $5.2 \times 10^{-13}$ & $1.9 \times 10^{-11}$ \\
300 & $2.2 \times 10^{-12}$ & $1.8 \times 10^{-12}$ & $5.0 \times 10^{-13}$ & $1.8 \times 10^{-11}$ \\
320 & $1.3 \times 10^{-12}$ & $1.2 \times 10^{-12}$ & $3.7 \times 10^{-13}$ & $1.0 \times 10^{-11}$ \\
340 & $8.3 \times 10^{-13}$ & $8.5 \times 10^{-13}$ & $2.8 \times 10^{-13}$ & $5.9 \times 10^{-12}$ \\
360 & $5.6 \times 10^{-13}$ & $6.3 \times 10^{-13}$ & $2.3 \times 10^{-13}$ & $3.8 \times 10^{-12}$ \\
380 & $4.0 \times 10^{-13}$ & $4.9 \times 10^{-13}$ & $1.9 \times 10^{-13}$ & $2.5 \times 10^{-12}$ \\
400 & $3.0 \times 10^{-13}$ & $3.9 \times 10^{-13}$ & $1.6 \times 10^{-13}$ & $1.8 \times 10^{-12}$ \\
\hline
\end{tabular}


Table S6 Rate coefficients $\left(\mathrm{cm}^{3}\right.$ molecule $\left.\mathrm{s}^{-1} \mathrm{~s}^{-1}\right)$ of anti- $\mathrm{CH}_{3} \mathrm{CHOO}$ reactions with $\mathrm{P} 3 \mathrm{a}, \mathrm{P} 3 \mathrm{~b}, \mathrm{P} 3 \mathrm{c}$ and $\mathrm{P} 3 \mathrm{~d}$ computed at different temperatures

\begin{tabular}{ccccc}
\hline $\mathrm{T} / \mathrm{K}$ & $k_{(\text {anti+P3a) }}$ & $k_{(\text {anti+P3b) }}$ & $k_{(\text {anti+P3c) }}$ & $k_{(\text {anti+P3d) }}$ \\
\hline 273 & $3.5 \times 10^{-9}$ & $2.3 \times 10^{-9}$ & $1.7 \times 10^{-7}$ & $2.1 \times 10^{-9}$ \\
280 & $2.3 \times 10^{-9}$ & $1.4 \times 10^{-9}$ & $1.0 \times 10^{-7}$ & $1.4 \times 10^{-9}$ \\
298 & $8.8 \times 10^{-10}$ & $5.8 \times 10^{-10}$ & $2.9 \times 10^{-8}$ & $5.9 \times 10^{-10}$ \\
300 & $8.0 \times 10^{-10}$ & $5.3 \times 10^{-10}$ & $2.6 \times 10^{-8}$ & $5.4 \times 10^{-10}$ \\
320 & $3.2 \times 10^{-10}$ & $2.3 \times 10^{-10}$ & $7.9 \times 10^{-9}$ & $2.3 \times 10^{-10}$ \\
340 & $1.4 \times 10^{-10}$ & $1.1 \times 10^{-10}$ & $2.8 \times 10^{-9}$ & $1.1 \times 10^{-10}$ \\
360 & $7.1 \times 10^{-11}$ & $5.8 \times 10^{-11}$ & $1.1 \times 10^{-9}$ & $5.9 \times 10^{-11}$ \\
380 & $3.8 \times 10^{-11}$ & $3.3 \times 10^{-11}$ & $4.9 \times 10^{-10}$ & $3.4 \times 10^{-11}$ \\
400 & $2.2 \times 10^{-11}$ & $2.0 \times 10^{-11}$ & $2.4 \times 10^{-10}$ & $2.0 \times 10^{-11}$ \\
\hline
\end{tabular}


Table S7 Rate coefficients $\left(\mathrm{cm}^{3}\right.$ molecule $\left.{ }^{-1} \mathrm{~s}^{-1}\right)$ of $s y n-\mathrm{CH}_{3} \mathrm{CHOO}$ reactions with P5a, P5b, P5c and P5d computed at different temperatures

\begin{tabular}{ccccc}
\hline $\mathrm{T} / \mathrm{K}$ & $k_{(\mathrm{syn}+\mathrm{P} 5 \mathrm{a})}$ & $k_{(\mathrm{syn}+\mathrm{P} 5 \mathrm{~b})}$ & $k_{(\mathrm{syn}+\mathrm{P} 5 \mathrm{c})}$ & $k_{(\mathrm{syn}+\mathrm{P} 5 \mathrm{~d})}$ \\
\hline 273 & $2.1 \times 10^{-11}$ & $1.7 \times 10^{-13}$ & $2.1 \times 10^{-11}$ & $2.4 \times 10^{-13}$ \\
280 & $1.5 \times 10^{-11}$ & $1.4 \times 10^{-13}$ & $1.5 \times 10^{-11}$ & $2.0 \times 10^{-13}$ \\
298 & $7.5 \times 10^{-12}$ & $1.0 \times 10^{-13}$ & $7.6 \times 10^{-12}$ & $1.4 \times 10^{-13}$ \\
300 & $6.9 \times 10^{-12}$ & $9.7 \times 10^{-14}$ & $7.0 \times 10^{-12}$ & $1.4 \times 10^{-13}$ \\
320 & $3.5 \times 10^{-12}$ & $6.9 \times 10^{-14}$ & $3.6 \times 10^{-12}$ & $9.6 \times 10^{-14}$ \\
340 & $1.9 \times 10^{-12}$ & $5.2 \times 10^{-14}$ & $2.0 \times 10^{-12}$ & $7.1 \times 10^{-14}$ \\
360 & $1.1 \times 10^{-12}$ & $4.0 \times 10^{-14}$ & $1.2 \times 10^{-12}$ & $5.5 \times 10^{-14}$ \\
380 & $7.1 \times 10^{-13}$ & $3.0 \times 10^{-14}$ & $7.3 \times 10^{-13}$ & $4.4 \times 10^{-14}$ \\
400 & $4.7 \times 10^{-13}$ & $2.6 \times 10^{-14}$ & $4.9 \times 10^{-13}$ & $3.6 \times 10^{-14}$ \\
\hline
\end{tabular}


Table S8 Rate coefficients $\left(\mathrm{cm}^{3}\right.$ molecule $\left.\mathrm{s}^{-1}\right)$ of $\left(\mathrm{CH}_{3}\right)_{2} \mathrm{CHOO}$ reactions with $\mathrm{P} 7 \mathrm{a}, \mathrm{P} 7 \mathrm{~b}, \mathrm{P} 7 \mathrm{c}$ and P7d computed at different temperatures

\begin{tabular}{ccccc}
\hline $\mathrm{T} / \mathrm{K}$ & $k_{((\mathrm{CH} 3) 2 \mathrm{CHOO}+\mathrm{P7a})}$ & $k_{((\mathrm{CH} 3) 2 \mathrm{CHOO}+\mathrm{P} 7 \mathrm{~b})}$ & $k_{((\mathrm{CH} 3) 2 \mathrm{CHOO}+\mathrm{P7c})}$ & $k_{((\mathrm{CH} 3) 2 \mathrm{CHOO}+\mathrm{P} 7 \mathrm{~d})}$ \\
\hline 273 & $7.8 \times 10^{-14}$ & $9.1 \times 10^{-14}$ & $1.8 \times 10^{-12}$ & $4.5 \times 10^{-13}$ \\
280 & $6.8 \times 10^{-14}$ & $8.2 \times 10^{-14}$ & $1.5 \times 10^{-12}$ & $3.9 \times 10^{-13}$ \\
298 & $5.0 \times 10^{-14}$ & $6.6 \times 10^{-14}$ & $1.0 \times 10^{-12}$ & $2.8 \times 10^{-13}$ \\
300 & $4.8 \times 10^{-14}$ & $6.5 \times 10^{-14}$ & $9.9 \times 10^{-13}$ & $2.7 \times 10^{-13}$ \\
320 & $3.6 \times 10^{-14}$ & $5.3 \times 10^{-14}$ & $6.9 \times 10^{-13}$ & $1.9 \times 10^{-13}$ \\
340 & $2.8 \times 10^{-14}$ & $4.5 \times 10^{-14}$ & $5.1 \times 10^{-13}$ & $1.5 \times 10^{-13}$ \\
360 & $2.2 \times 10^{-14}$ & $3.9 \times 10^{-14}$ & $3.9 \times 10^{-13}$ & $1.2 \times 10^{-13}$ \\
380 & $1.9 \times 10^{-14}$ & $3.5 \times 10^{-14}$ & $3.1 \times 10^{-13}$ & $9.6 \times 10^{-14}$ \\
400 & $1.6 \times 10^{-14}$ & $3.1 \times 10^{-14}$ & $2.5 \times 10^{-13}$ & $8.0 \times 10^{-14}$ \\
\hline
\end{tabular}


Table S9 Rate coefficients $\left(\mathrm{cm}^{3}\right.$ molecule $\left.\mathrm{s}^{-1}\right)$ of elementary reactions R1a and R9

\begin{tabular}{ccccc}
\hline $\mathrm{T}$ & $k_{\mathrm{TST}(\mathrm{R} 1 \mathrm{a})}$ & $k_{\mathrm{CVTST}(\mathrm{R} 1 \mathrm{a})}$ & $k_{\mathrm{TST}(\mathrm{R} 9)}$ & $k_{\mathrm{CVTST}(\mathrm{R} 9)}$ \\
\hline 273 & $1.2 \times 10^{-11}$ & $3.5 \times 10^{-11}$ & $5.5 \times 10^{-9}$ & $1.3 \times 10^{-8}$ \\
280 & $9.4 \times 10^{-12}$ & $2.6 \times 10^{-11}$ & $3.7 \times 10^{-9}$ & $8.5 \times 10^{-9}$ \\
298 & $5.4 \times 10^{-12}$ & $1.4 \times 10^{-11}$ & $1.5 \times 10^{-9}$ & $3.2 \times 10^{-9}$ \\
300 & $5.1 \times 10^{-12}$ & $1.3 \times 10^{-11}$ & $1.3 \times 10^{-9}$ & $2.9 \times 10^{-9}$ \\
320 & $3.0 \times 10^{-12}$ & $7.2 \times 10^{-12}$ & $5.5 \times 10^{-10}$ & $1.1 \times 10^{-9}$ \\
340 & $1.9 \times 10^{-12}$ & $4.2 \times 10^{-12}$ & $2.6 \times 10^{-10}$ & $5.0 \times 10^{-10}$ \\
360 & $1.2 \times 10^{-12}$ & $2.7 \times 10^{-12}$ & $1.4 \times 10^{-10}$ & $2.4 \times 10^{-10}$ \\
380 & $8.6 \times 10^{-13}$ & $1.8 \times 10^{-12}$ & $7.1 \times 10^{-11}$ & $1.3 \times 10^{-10}$ \\
400 & $6.3 \times 10^{-13}$ & $1.2 \times 10^{-12}$ & $4.1 \times 10^{-11}$ & $7.3 \times 10^{-11}$ \\
\hline
\end{tabular}




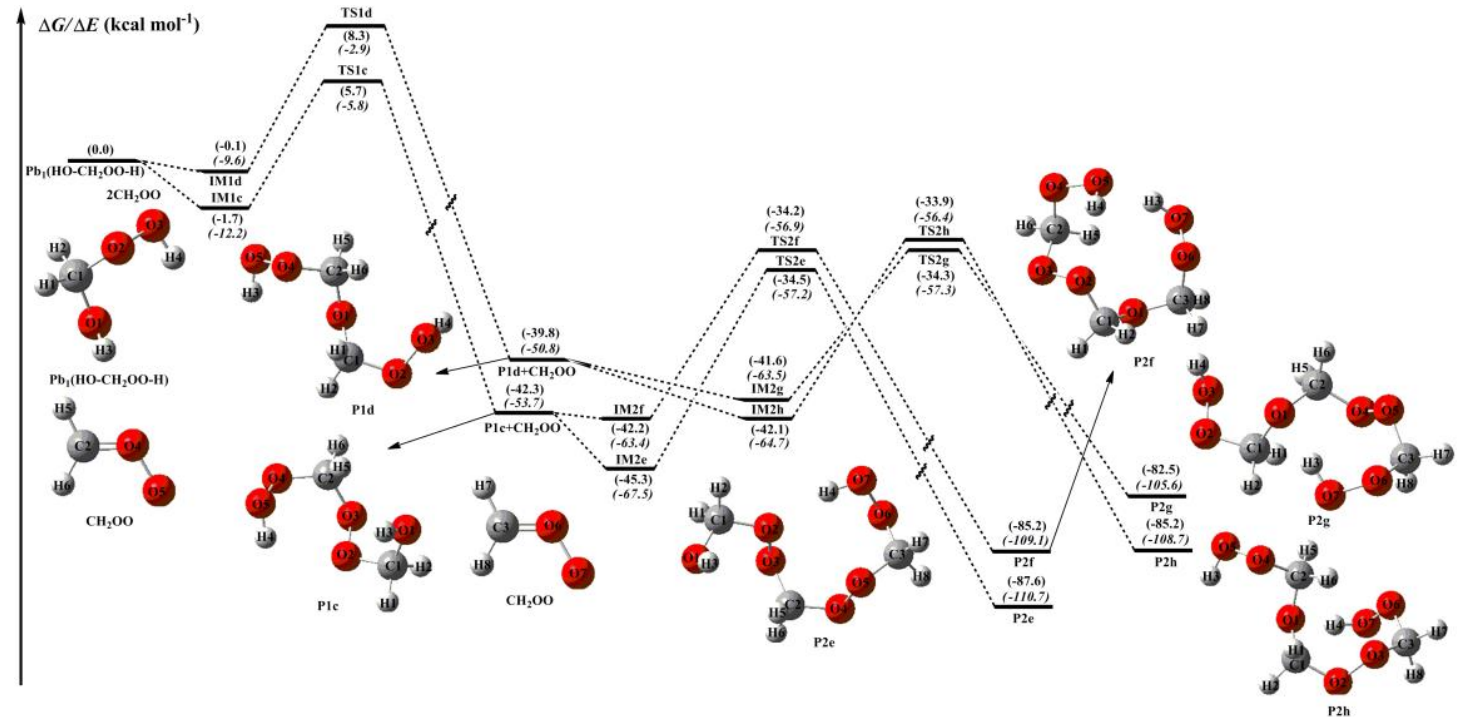

Figure S1. PES $\left(\Delta G\right.$ and $\Delta E$ (italic)) for the reaction of $\mathrm{CH}_{2} \mathrm{OO}$ with $\mathrm{HO}-\mathrm{CH}_{2} \mathrm{OO}-\mathrm{H}\left(\mathrm{Pb}_{1}\right)$ calculated at the M06-2X/def2-TZVP//M06-2X/6-311+G(2df,2p) level of theory 

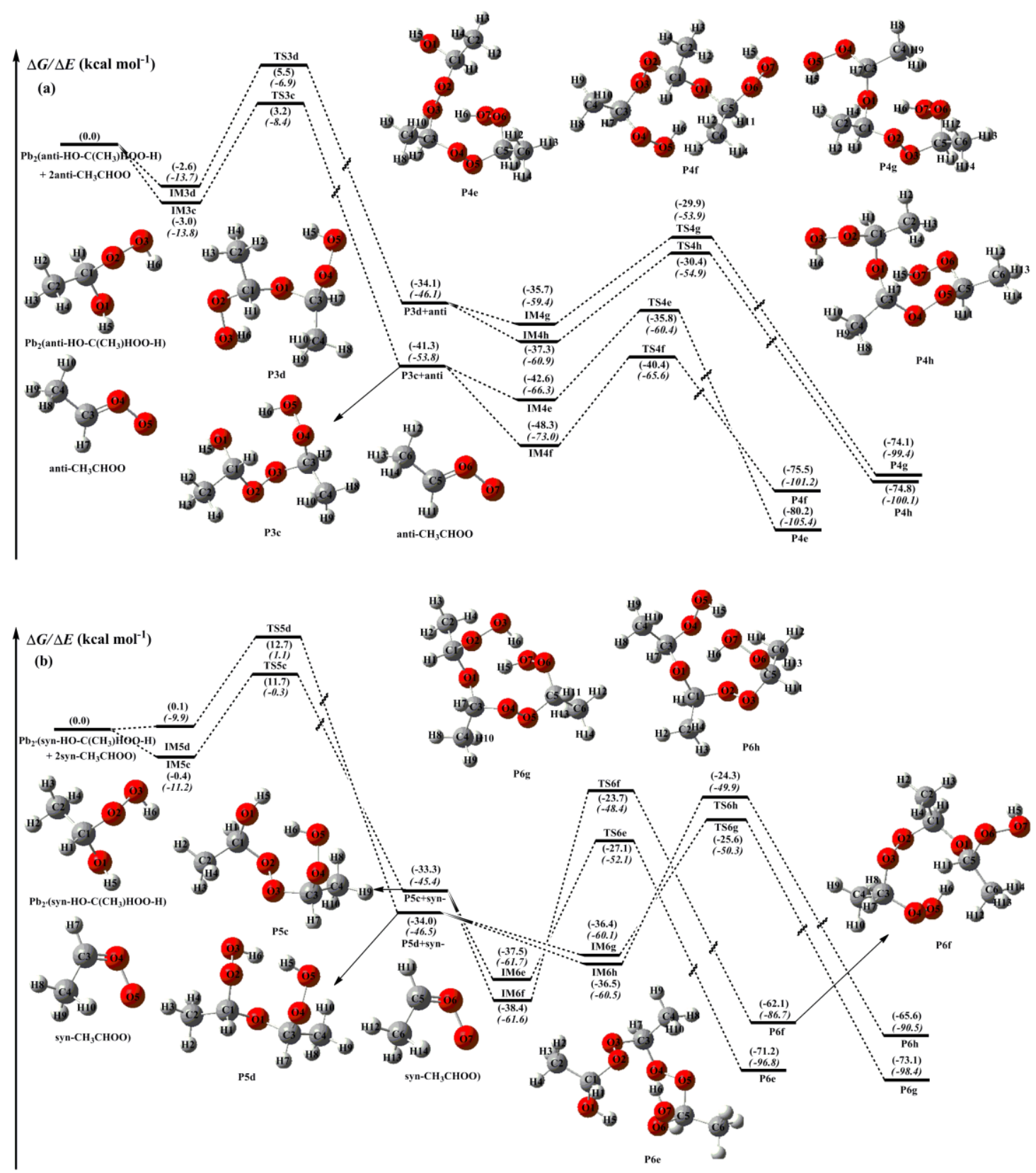

Figure S2 PES ( $\Delta G$ and $\Delta E$ (italic)) for the reaction of $\mathrm{HO}-\mathrm{C}\left(\mathrm{CH}_{3}\right) \mathrm{HOO}-\mathrm{H}$ with anti-(a) and syn- $\mathrm{CH}_{3} \mathrm{CHOO}(\mathrm{b})$ calculated at the M06-2X/def2-TZVP//M06-2X/6-311+G(2df,2p) level of theory 


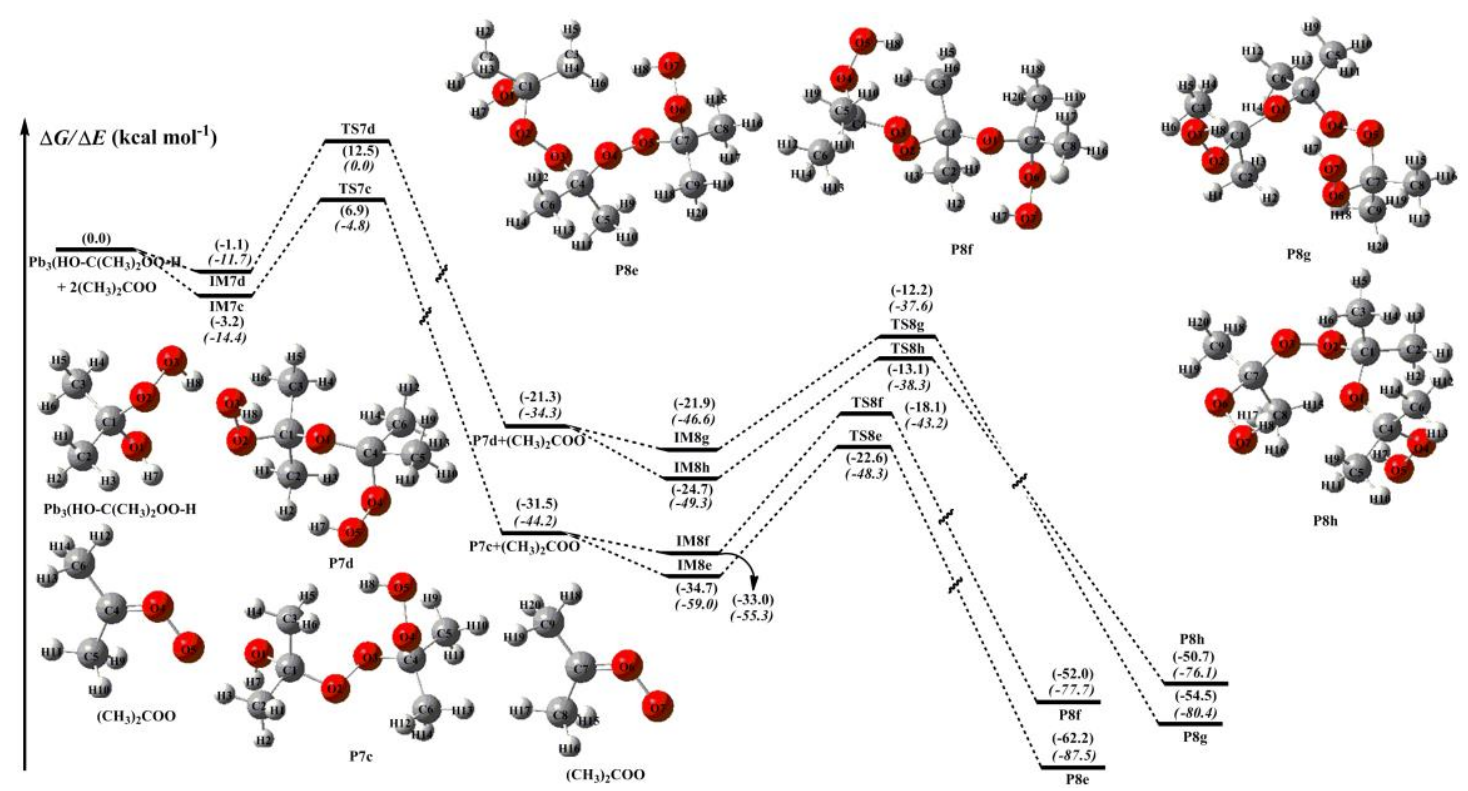

Figure S3 PES ( $\Delta G$ and $\Delta E$ (italic) ) for the reaction of $\left(\mathrm{CH}_{3}\right)_{2} \mathrm{COO}$ with $\mathrm{HO}-\mathrm{C}\left(\mathrm{CH}_{3}\right)_{2} \mathrm{OO}-\mathrm{H}\left(\mathrm{Pb}_{3}\right)$ calculated at the M06-2X/def2-TZVP//M06-2X/6-311+G(2df,2p) level of theory 


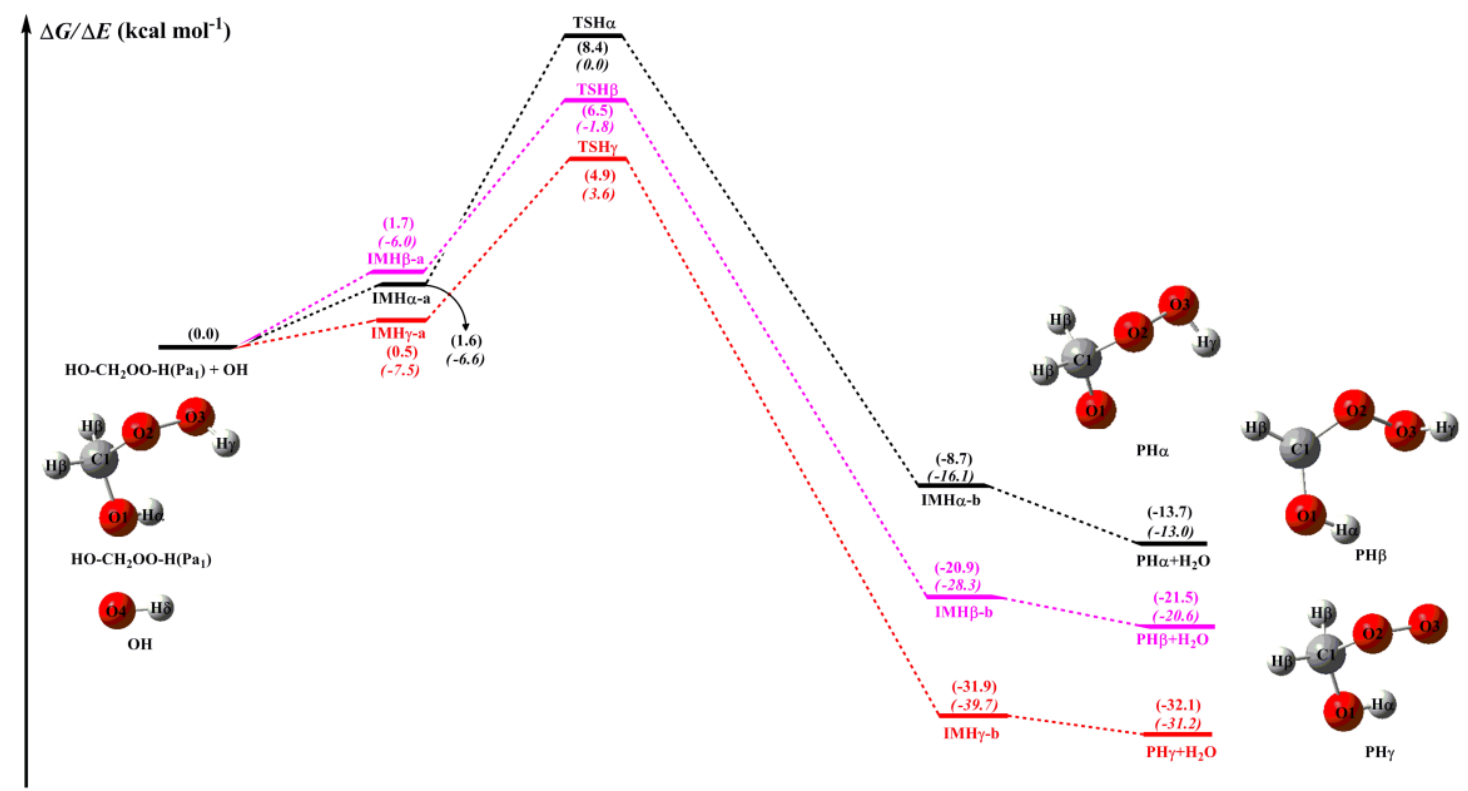

Figure S5. PES ( $\Delta G$ and $\Delta E$ (italic)) for the reaction of $\mathrm{HO}-\mathrm{CH}_{2} \mathrm{OO}-\mathrm{H}\left(\mathrm{Pa}_{1}\right)$ with $\mathrm{OH}$ computed at the M06-2X/def2-TZVP//M06-2X/6-311+G(2df,2p) level of theory 


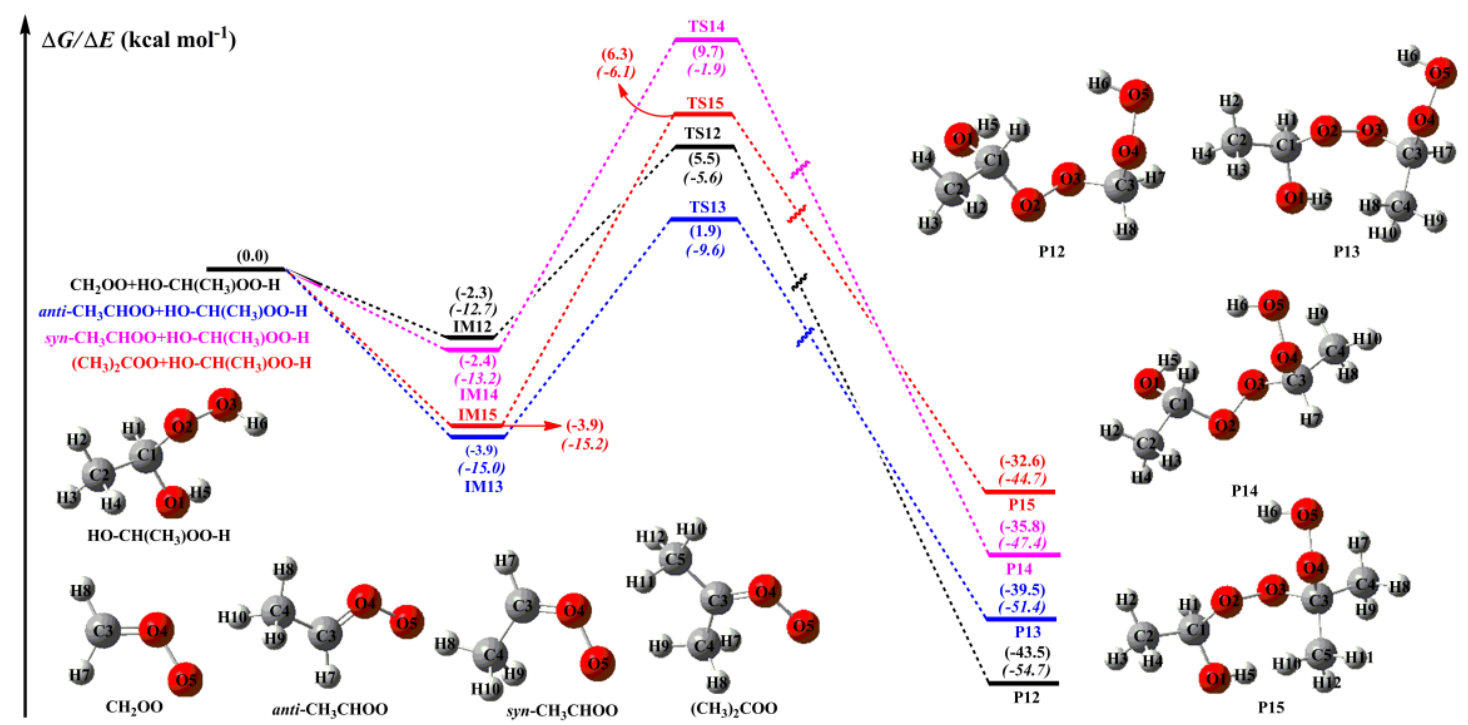

Figure S9. PES ( $\Delta G$ and $\Delta E$ (italic)) of distinct SCI reactions with $\mathrm{HO}-\mathrm{CH}\left(\mathrm{CH}_{3}\right) \mathrm{OO}-\mathrm{H}\left(\mathrm{Pa}_{2}\right)$ calculated at the M06-2X/def2-TZVP//M06-2X/6-311+G(2df,2p) level of theory 


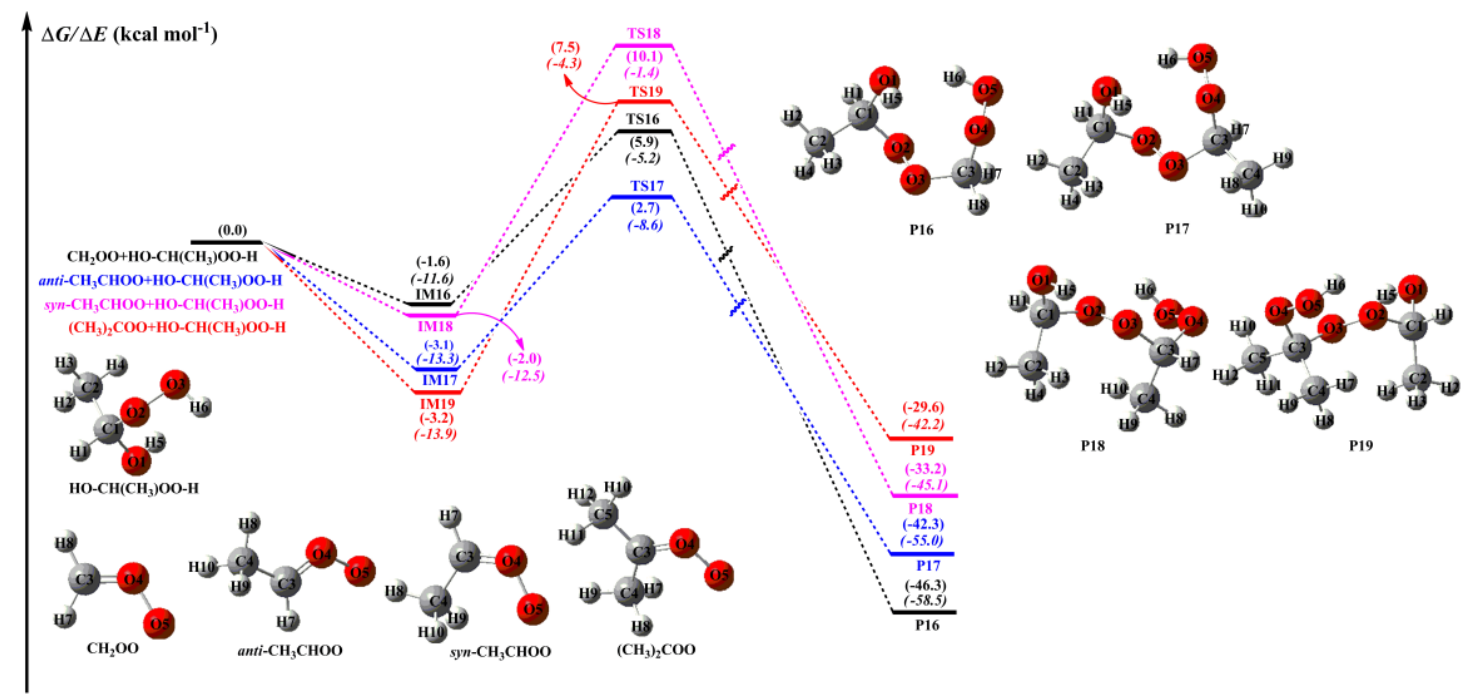

Figure S10. PES $\left(\Delta G\right.$ and $\Delta E$ (italic)) of distinct SCI reactions with $\mathrm{HO}-\mathrm{CH}\left(\mathrm{CH}_{3}\right) \mathrm{OO}-\mathrm{H}\left(\mathrm{Pa}_{2}\right)$ calculated at the M06-2X/def2-TZVP//M06-2X/6-311+G(2df,2p) level of theory 


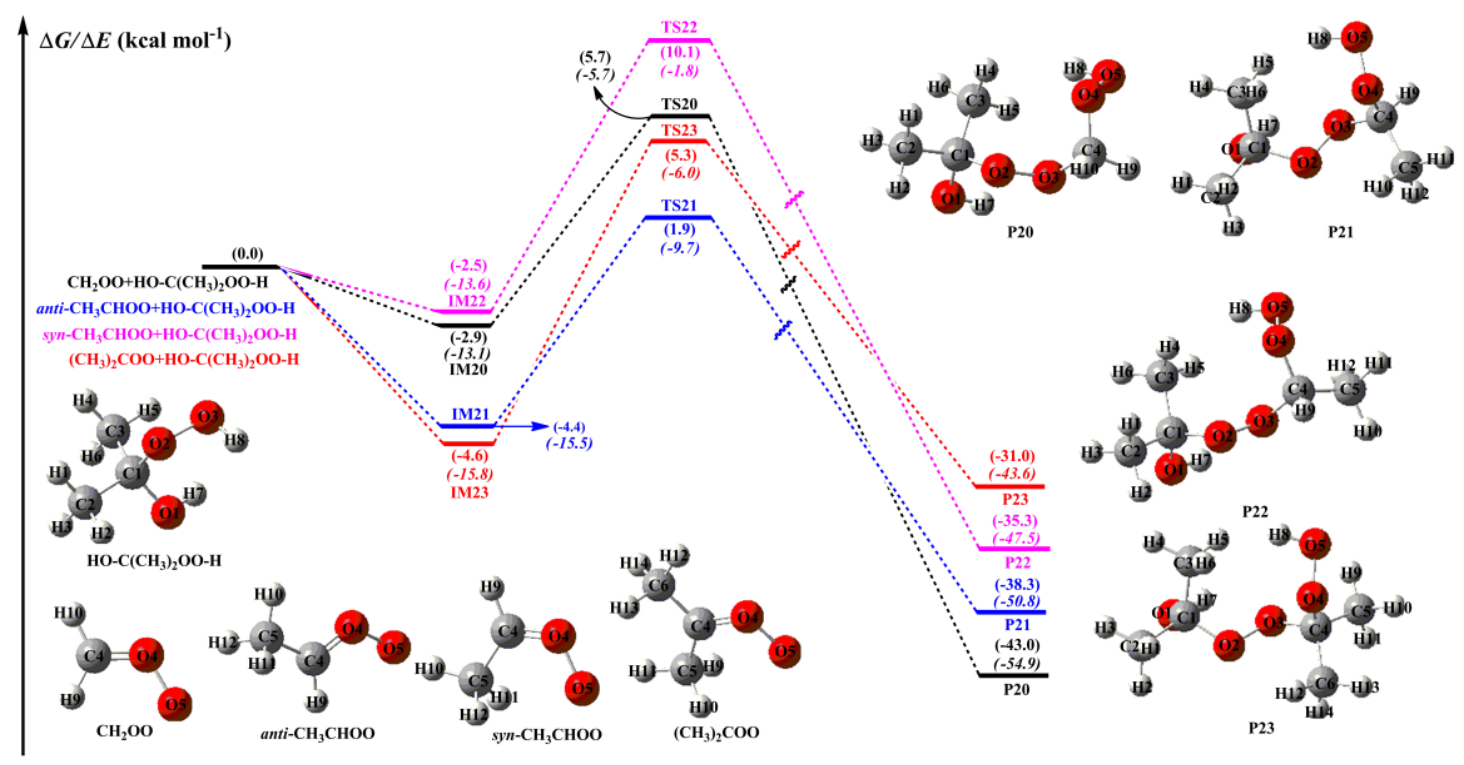

Figure S11. PES $\left(\Delta G\right.$ and $\Delta E$ (italic)) of distinct SCI reactions with $\mathrm{HO}-\mathrm{C}\left(\mathrm{CH}_{3}\right)_{2} \mathrm{OO}-\mathrm{H}\left(\mathrm{Pa}_{3}\right)$ calculated at the M06-2X/def2-TZVP//M06-2X/6-311+G(2df,2p) level of theory 
Figure S4. Optimized geometries, z-matrices and vibrational frequencies of all stationary points in the reaction of $2 \mathrm{CH}_{2} \mathrm{OO}$ with $\mathrm{HO}-\mathrm{CH}_{2} \mathrm{OO}-\mathrm{H}$ at the M06-2X/6-311+G(2df,2p) level of theory

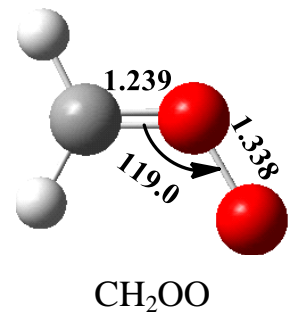

$\mathrm{C}$

O $11 \quad$ B1

$\mathrm{O} 2 \mathrm{~B} 2$

$\mathrm{H} \quad 11 \quad \mathrm{~B} 3$

$\mathrm{H}$

$1 \quad \mathrm{~B} 4$

B2 1

B3 2

A1

B4 2

A2 $3 \quad$ D1 0

A3 $3 \quad$ D2 0

Variables:

$\begin{array}{lr}\text { B1 } & 1.23895372 \\ \text { B2 } & 1.33773725 \\ \text { B3 } & 1.08411000 \\ \text { B4 } & 1.08104852 \\ \text { A1 } & 119.01084624 \\ \text { A2 } & 118.71143342 \\ \text { A3 } & 115.75496765 \\ \text { D1 } & 0.00000000 \\ \text { D2 } & 180.00000000\end{array}$

Harmonic Vibrational Frequencies $\left(\mathbf{c m}^{-1}\right)$ :

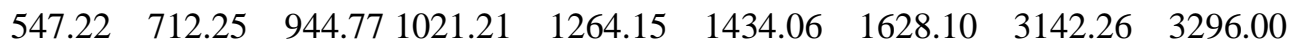

$\begin{array}{llll}\text { Rotational constants }(\boldsymbol{B} / \mathbf{G H Z}): & 81.8651497 & 12.6910280 & 10.9876788\end{array}$

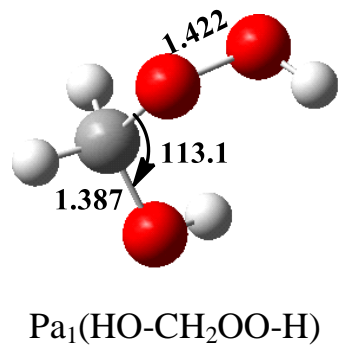

$\mathrm{C}$

O 11

$\mathrm{O} \quad 2 \quad \mathrm{~B} 2$

$\mathrm{H} \quad 1$

$\mathrm{H} \quad 1$

B3 2

A2

B4 2

O 1

B5 2

A

A4

B6 2

A5 1

B7 1

A6 2

D1 0

$\mathrm{H} 3$

6

1.40359950

B1

1.42184472

B2 


$\begin{array}{lr}\text { B3 } & 1.08832012 \\ \text { B4 } & 1.09298865 \\ \text { B5 } & 1.38702567 \\ \text { B6 } & 0.96387803 \\ \text { B7 } & 0.95983640 \\ \text { A1 } & 106.22681004 \\ \text { A2 } & 103.84544465 \\ \text { A3 } & 108.76259188 \\ \text { A4 } & 113.09492724 \\ \text { A5 } & 101.24544362 \\ \text { A6 } & 108.59940983 \\ \text { D1 } & -174.17439700 \\ \text { D2 } & -55.77995767 \\ \text { D3 } & 68.78269175 \\ \text { D4 } & -116.34697036 \\ \text { D5 } & -78.72946929\end{array}$

Harmonic Vibrational Frequencies $\left(\mathbf{c m}^{-\mathbf{1}}\right)$ :

$\begin{array}{llllllllll}187.55 & 261.78 & 396.49 & 476.16 & 636.54 & 947.37 & 1066.60 & 1100.18 & 1138.48 & 1294.22\end{array}$ $\begin{array}{llllllll}1395.95 & 1421.31 & 1430.83 & 1501.76 & 3073.24 & 3162.27 & 3850.45 & 3903.89\end{array}$

Rotational constants (B/GHZ): $17.3837324 \quad 6.0872099 \quad 5.0358587$

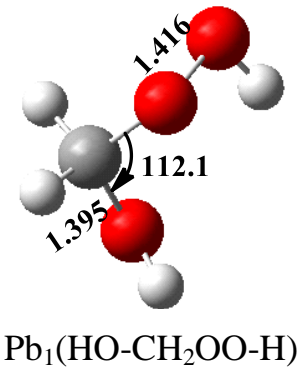

$\mathrm{C}$

$\mathrm{O} \quad 1 \quad \mathrm{~B} 1$

$\begin{array}{lllll}\mathrm{O} & 2 & \mathrm{~B} 2 & 1 & \mathrm{~A} 1\end{array}$

$\begin{array}{llllllll}\mathrm{H} & 1 & \mathrm{~B} 3 & 2 & \mathrm{~A} 2 & 3 & \mathrm{D} 1 & 0\end{array}$

$\begin{array}{llllllll}\mathrm{H} & 1 & \mathrm{~B} 4 & 2 & \mathrm{~A} 3 & 3 & \mathrm{D} 2 & 0\end{array}$

$\begin{array}{llllllll}\mathrm{O} & 1 & \mathrm{~B} 5 & 2 & \mathrm{~A} 4 & 3 & \text { D3 } & 0\end{array}$

$\begin{array}{llllllll}\mathrm{H} & 3 & \mathrm{~B} 6 & 2 & \mathrm{~A} 5 & 1 & \mathrm{D} 4 & 0\end{array}$

$\begin{array}{llllllll}\mathrm{H} & 6 & \mathrm{~B} 7 & 1 & \mathrm{~A} 6 & 2 & \text { D5 } & 0\end{array}$

Variables:

$\begin{array}{lr}\text { B1 } & 1.39846990 \\ \text { B2 } & 1.41549630 \\ \text { B3 } & 1.09221672 \\ \text { B4 } & 1.08851868 \\ \text { B5 } & 1.39462498 \\ \text { B6 } & 0.96568050 \\ \text { B7 } & 0.96059450 \\ \text { A1 } & 107.51359566\end{array}$




$\begin{array}{lr}\text { A2 } & 103.96617277 \\ \text { A3 } & 110.05328386 \\ \text { A4 } & 112.05221718 \\ \text { A5 } & 102.42638690 \\ \text { A6 } & 109.14726274 \\ \text { D1 } & -168.56099031 \\ \text { D2 } & -49.08547865 \\ \text { D3 } & 69.49009664 \\ \text { D4 } & -75.33464713 \\ \text { D5 } & 70.40550778\end{array}$

Harmonic Vibrational Frequencies $\left(\mathbf{c m}^{-1}\right)$ :

$\begin{array}{llllllllll}185.98 & 350.68 & 427.05 & 458.09 & 649.20 & 961.31 & 1059.11 & 1114.93 & 1125.96 & 1292.58\end{array}$ $\begin{array}{llllllll}1382.71 & 1430.87 & 1444.34 & 1504.42 & 3085.79 & 3154.05 & 3828.21 & 3893.48\end{array}$

Rotational constants (B/GHZ): $17.7484920 \quad 5.9989349 \quad 5.0693236$

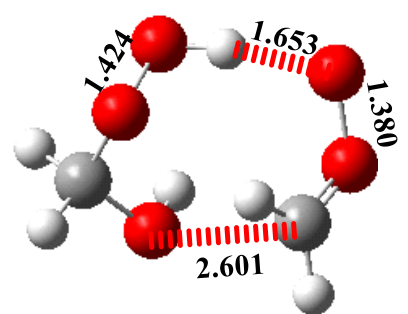

IM1a
$\mathrm{C}$

$\mathrm{H} \quad \mathrm{B} \quad \mathrm{B} 1$

$\mathrm{H} \quad 1 \quad \mathrm{~B} 2 \quad 2$

$\begin{array}{llll}\mathrm{O} & 1 & \mathrm{~B} 3 & 3\end{array}$

$\begin{array}{llll}\mathrm{O} & 1 & \mathrm{~B} 4 & 4\end{array}$

$\mathrm{H} \quad 4 \quad$ B5 1

C 5

H 7

$\mathrm{H} \quad 7$

$\mathrm{O}$

$\mathrm{O}$

O 1

$\mathrm{H} \quad 12$

Variables:

B1

B2

B3

B4

B5

B6

B7

B8

B9

$\begin{array}{ll}1 & 1.09193463 \\ 2 & 1.08849176 \\ 3 & 2.24430673 \\ 4 & 1.39051082 \\ 5 & 0.99633873 \\ 6 & 3.06560927 \\ 7 & 1.08259664 \\ 8 & 1.08495294 \\ 9 & 2.22859774\end{array}$

A1

A2

A3 2

D1 0

A4 5

D2 0

A5 4

D3 0

D4 0

A6 1

D5 0

A7 1

D6 0

A8 1

D7 0

$\begin{array}{llll}\text { A9 } & 1 & \text { D8 } & 0\end{array}$

$\begin{array}{llll}\text { A10 } & 4 & \text { D9 } & 0\end{array}$

A11 5 


$\begin{array}{lr}\text { B10 } & 1.23265549 \\ \text { B11 } & 1.40521222 \\ \text { B12 } & 0.96272306 \\ \text { A1 } & 111.28650697 \\ \text { A2 } & 142.88476256 \\ \text { A3 } & 37.63600756 \\ \text { A4 } & 114.28220506 \\ \text { A5 } & 87.19146587 \\ \text { A6 } & 128.81480221 \\ \text { A7 } & 44.14002611 \\ \text { A8 } & 71.65730745 \\ \text { A9 } & 97.34905266 \\ \text { A10 } & 112.10771126 \\ \text { A11 } & 107.25214062 \\ \text { D1 } & 113.20365830 \\ \text { D2 } & -129.89551688 \\ \text { D3 } & 75.73345389 \\ \text { D4 } & -89.79001655 \\ \text { D5 } & -17.33812910 \\ \text { D6 } & -120.38255440 \\ \text { D7 } & 136.53226895 \\ \text { D8 } & 114.73764822 \\ \text { D9 } & 68.63114204 \\ \text { D10 } & -75.96210566\end{array}$

Harmonic Vibrational Frequencies $\left(\mathbf{c m}^{-1}\right)$ :

$\begin{array}{lccccccccr}51.49 & 123.65 & 149.15 & 158.76 & 199.39 & 242.52 & 257.19 & 422.77 & 501.74 & 538.03 \\ 641.99 & 723.92 & 858.57 & 896.89 & 958.08 & 1070.70 & 1093.26 & 1112.92 & 1134.11 & 1270.31 \\ 1300.07 & 1397.45 & 1429.61 & 1456.76 & 1506.87 & 1588.45 & 1674.71 & 3088.99 & 3148.97 \\ 3162.07 & 3162.64 & 3305.07 & 3868.49 & & & & & \end{array}$

Rotational constants (B/GHZ): $3.7514986 \quad 1.8833579 \quad 1.4348672$

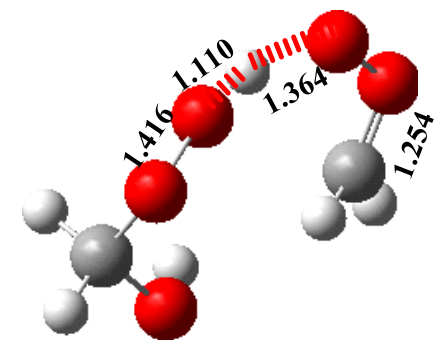

TS1a

$\mathrm{C}$

$\mathrm{H} \quad 1 \quad$ B1

$\mathrm{H} \quad 1 \quad$ B2

$\begin{array}{lll}\mathrm{O} & 1 & \mathrm{~B} 3\end{array}$

$\mathrm{O} \quad 1 \quad \mathrm{~B} 4$

$\mathrm{H} \quad 4$

A

$\begin{array}{llllll}\text { B3 } & 3 & \text { A2 } & 2 & \text { D1 } & 0\end{array}$

$\begin{array}{llllll}\text { B4 } & 4 & \text { A3 } & 2 & \text { D2 } & 0\end{array}$

$\begin{array}{llllll}\text { B5 } & 1 & \text { A4 } & 5 & \text { D3 } & 0\end{array}$ 


$\begin{array}{rrrrrrrr}\text { C } & 4 & \text { B6 } & 1 & \text { A5 } & 5 & \text { D4 } & 0 \\ \text { H } & 7 & \text { B7 } & 4 & \text { A6 } & 1 & \text { D5 } & 0 \\ \text { H } & 7 & \text { B8 } & 4 & \text { A7 } & 1 & \text { D6 } & 0 \\ \text { O } & 7 & \text { B9 } & 4 & \text { A8 } & 1 & \text { D7 } & 0 \\ \text { O } & 7 & \text { B10 } & 4 & \text { A9 } & 1 & \text { D8 } & 0 \\ \text { O } & 1 & \text { B11 } & 5 & \text { A10 } & 4 & \text { D9 } & 0 \\ \text { H } & 12 & \text { B12 } & 1 & \text { A11 } & 5 & \text { D10 } & 0\end{array}$

Variables:

$\begin{array}{ll}\text { B1 } & 1.09236135 \\ \text { B2 } & 1.08804886 \\ \text { B3 } & 2.24424874 \\ \text { B4 } & 1.39811878 \\ \text { B5 } & 1.10965849 \\ \text { B6 } & 2.11376185 \\ \text { B7 } & 1.08564528 \\ \text { B8 } & 1.08422517 \\ \text { B9 } & 2.17270169 \\ \text { B10 } & 1.25371695 \\ \text { B11 } & 1.39546227 \\ \text { B12 } & 0.95934668\end{array}$

A1 111.33252841

A2 141.56828263

A3 37.39819679

A4 $\quad 138.86224365$

A5 106.32634151

A6 112.74521149

A7 $\quad 79.64310141$

A8 $\quad 68.13881085$

A9 $\quad 91.69184623$

A10 112.30561165

A11 $\quad 108.39573179$

D1 $\quad 116.64933959$

D2 $\quad-125.38714617$

D3 $\quad 5.28588587$

D4 86.71869259

D5 $\quad 70.46269736$

D6 $\quad-49.76277905$

D7 $\quad-140.38093414$

D8 $\quad-171.03190235$

D9 $\quad 64.04282937$

D10 -88.61169385

Harmonic Vibrational Frequencies $\left(\mathbf{c m}^{-1}\right)$ :

$\begin{array}{llllllllll}590.85 i & 61.86 & 109.90 & 155.04 & 258.66 & 330.27 & 385.33 & 433.96 & 466.27 & 556.61\end{array}$ $\begin{array}{llllllllll}651.70 & 842.43 & 908.64 & 957.59 & 1057.32 & 1094.16 & 1115.57 & 1135.39 & 1210.01 & 1259.37\end{array}$ 


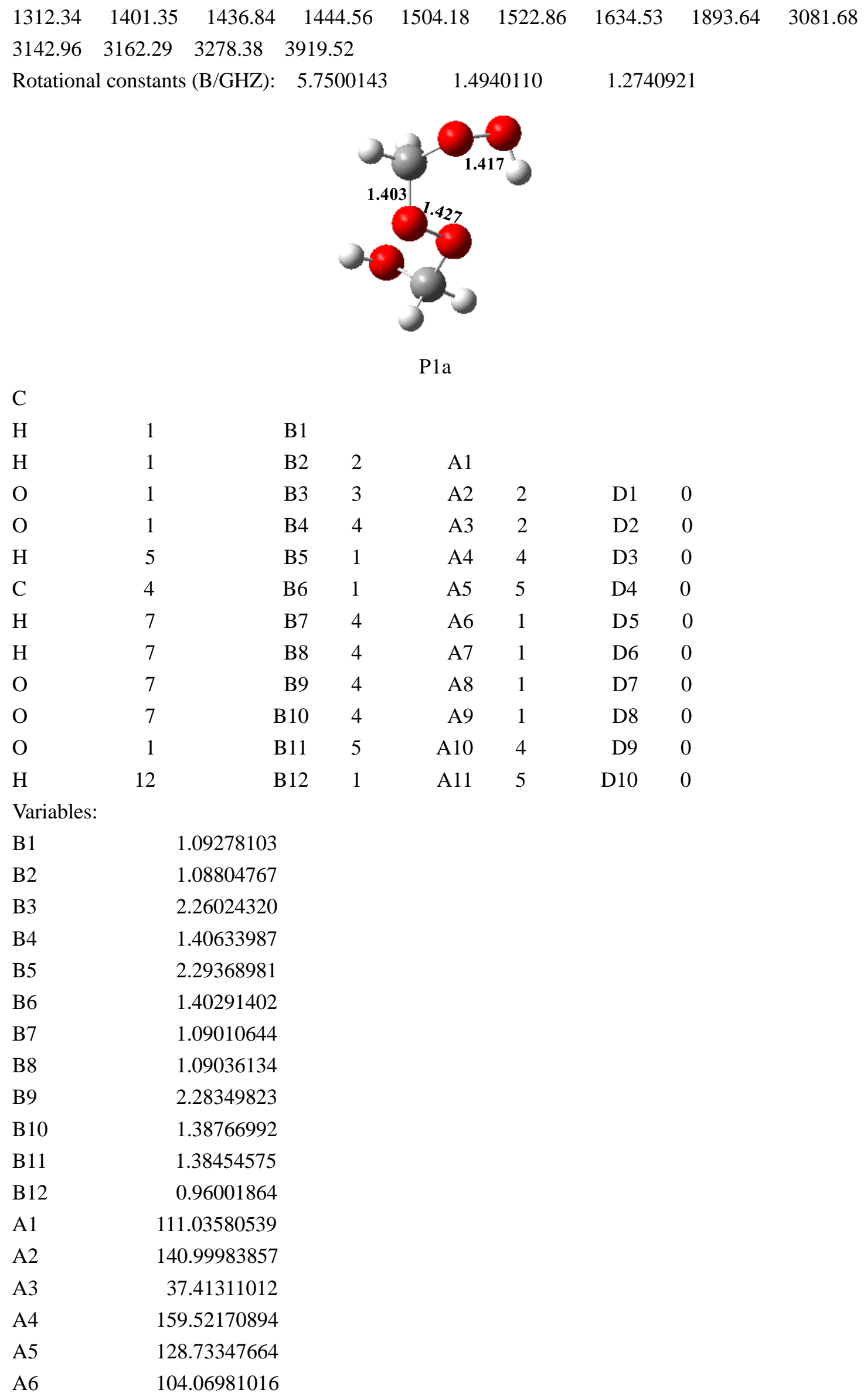




$\begin{array}{lr}\text { A7 } & 110.34401285 \\ \text { A8 } & 106.81981294 \\ \text { A9 } & 112.99544627 \\ \text { A10 } & 112.90571768 \\ \text { A11 } & 108.81709236 \\ \text { D1 } & 110.30671017 \\ \text { D2 } & -128.62298419 \\ \text { D3 } & -163.71292412 \\ \text { D4 } & 64.16608871 \\ \text { D5 } & 136.46268868 \\ \text { D6 } & 14.81755872 \\ \text { D7 } & -71.43436757 \\ \text { D8 } & -109.16769572 \\ \text { D9 } & 69.36407861 \\ \text { D10 } & -78.57478123\end{array}$

Harmonic Vibrational Frequencies $\left(\mathbf{c m}^{-\mathbf{1}}\right)$ :

$\begin{array}{lllllllllll}30.18 & 72.61 & 157.09 & 181.77 & 305.50 & 376.67 & 418.82 & 421.71 & 490.98 & 610.56 & 680.47\end{array}$ $\begin{array}{lllllllll}892.84 & 966.13 & 1053.48 & 1067.22 & 1116.56 & 1140.12 & 1142.95 & 1185.22 & 1305.98\end{array}$ $\begin{array}{lllllllll}1339.30 & 1398.02 & 1410.36 & 1432.97 & 1457.82 & 1458.26 & 1504.03 & 3083.47 & 3102.60\end{array}$ $\begin{array}{llll}3160.70 & 3174.77 \quad 3802.76 & 3909.20\end{array}$

Rotational constants (B/GHZ): $\quad 5.9322654 \quad 1.4514673 \quad 1.3429018$

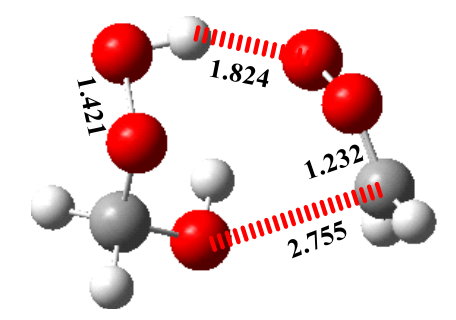

IM1b

$\mathrm{C}$

$\begin{array}{lll}\mathrm{O} & 1 & \mathrm{~B} 1\end{array}$

$\begin{array}{lllll}\mathrm{H} & 2 & \mathrm{~B} 2 & 1 & \mathrm{~A} 1\end{array}$

$\begin{array}{llllllll}\mathrm{O} & 1 & \mathrm{~B} 3 & 2 & \mathrm{~A} 2 & 3 & \mathrm{D} 1 & 0\end{array}$

$\begin{array}{llllllll}\mathrm{C} & 2 & \mathrm{~B} 4 & 1 & \mathrm{~A} 3 & 4 & \mathrm{D} 2 & 0\end{array}$

$\begin{array}{llllllll}\mathrm{H} & 5 & \mathrm{~B} 5 & 2 & \mathrm{~A} 4 & 1 & \mathrm{D} 3 & 0\end{array}$

$\begin{array}{llllllll}\mathrm{H} & 5 & \mathrm{~B} 6 & 2 & \mathrm{~A} 5 & 1 & \mathrm{D} 4 & 0\end{array}$

$\begin{array}{llllllll}\mathrm{O} & 5 & \mathrm{~B} 7 & 2 & \mathrm{~A} 6 & 1 & \mathrm{D} 5 & 0\end{array}$

$\begin{array}{llllllll}\mathrm{O} & 5 & \mathrm{~B} 8 & 2 & \mathrm{~A} 7 & 1 & \mathrm{D} 6 & 0\end{array}$

$\begin{array}{lllllllll}\mathrm{H} & 1 & \mathrm{~B} 9 & 2 & \mathrm{~A} 8 & 5 & \mathrm{D} 7 & 0\end{array}$

$\begin{array}{llllllll}\mathrm{H} & 1 & \mathrm{~B} 10 & 2 & \mathrm{~A} 9 & 5 & \mathrm{D} 8 & 0\end{array}$

$\begin{array}{llllllll}\mathrm{O} & 1 & \mathrm{~B} 11 & 2 & \mathrm{~A} 10 & 5 & \mathrm{D} 9 & 0\end{array}$

$\begin{array}{llllllll}\mathrm{H} & 4 & \mathrm{~B} 12 & 1 & \mathrm{~A} 11 & 12 & \mathrm{D} 10 & 0\end{array}$

Variables:

$\begin{array}{ll}\text { B1 } & 1.40080832 \\ \text { B2 } & 0.96726628\end{array}$ 


$\begin{array}{lr}\text { B3 } & 2.26933462 \\ \text { B4 } & 2.75470408 \\ \text { B5 } & 1.08334781 \\ \text { B6 } & 1.08276277 \\ \text { B7 } & 2.22141378 \\ \text { B8 } & 1.23165769 \\ \text { B9 } & 1.08880954 \\ \text { B10 } & 1.09185771 \\ \text { B11 } & 1.39521073 \\ \text { B12 } & 0.97956338 \\ \text { A1 } & 108.07810054 \\ \text { A2 } & 101.44725504 \\ \text { A3 } & 116.14951018 \\ \text { A4 } & 121.41226346 \\ \text { A5 } & 63.56643339 \\ \text { A6 } & 68.95898494 \\ \text { A7 } & 87.04598340 \\ \text { A8 } & 107.15652046 \\ \text { A9 } & 111.17484923 \\ \text { A10 } & 112.63307909 \\ \text { A11 } & 101.66754461 \\ \text { D1 } & -22.98049505 \\ \text { D2 } & 65.04627094 \\ \text { D3 } & 59.89222066 \\ \text { D4 } & 176.04280736 \\ \text { D5 } & -87.05510272 \\ \text { D6 } & -58.36146674 \\ \text { D7 } & -87.09989462 \\ \text { D8 } & 151.03444773 \\ \text { D9 } & 28.36800286 \\ \text { D10 } & 94.81429795 \\ \text { Har }\end{array}$

Harmonic Vibrational Frequencies $\left(\mathbf{c m}^{-\mathbf{1}}\right)$ :

$\begin{array}{lllllllllll}72.04 & 86.72 & 139.83 & 162.23 & 177.47 & 249.40 & 270.96 & 438.36 & 528.28 & 549.83 & 627.73\end{array}$ $\begin{array}{llllllllll}698.19 & 718.23 & 906.21 & 946.33 & 1090.21 & 1099.12 & 1110.60 & 1133.31 & 1263.78 & 1302.17\end{array}$ $\begin{array}{lllllllll}1394.48 & 1439.43 & 1457.30 & 1502.53 & 1542.61 & 1681.85 & 3079.78 & 3152.18 & 3153.95\end{array}$ $3299.66 \quad 3522.35 \quad 3791.16$

Rotational constants (B/GHZ): $3.6364140 \quad 1.9053619 \quad 1.5431218$

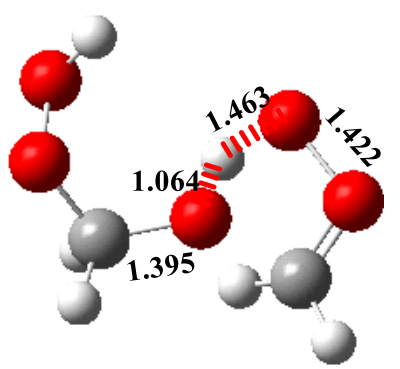


TS1b

$\mathrm{C}$

$\begin{array}{lll}\mathrm{O} & 1 & \mathrm{~B} 1\end{array}$

H 2

O 1

C 2

$\mathrm{H} \quad 5$

$\mathrm{H} \quad 5$

O 5

O 5

$\mathrm{H} \quad 1$

$\mathrm{H} \quad 1$

O 1

$\mathrm{H} \quad 4$

Variables:

B1

B2

B3

B4

B5

B6

B7

B8

B9

B10

B11

B12

A1

A2

A3

A4

A5

A6

A7

A8

A9

A10

A11

D1

D2

D3

D4

D5

D6
1.39505172

1.06436688

2.25738999

2.02056504

1.08479080

1.08427018

2.16375976

1.25550533

1.08948659

1.09600897

1.39429682

0.96524204

109.89749303

96.63285190

119.36262780

106.95259056

87.46712512

71.09182158

92.70873641

109.15338965

111.01751086

111.65375567

108.13664266

$-33.80470151$

$-117.52849662$

$-100.45382300$

22.17662678

108.65489249

141.36310093
B2

B3 2

B4 1

B5 2

B6 2

B7 2

B8 2

B9 2

B10 2

B11 2

B12 1
A1

$\begin{array}{llll}\mathrm{A} 2 & 3 & \mathrm{D} 1 & 0\end{array}$

A3 4

D2 0

A4 1

D3 0

A5 1

D4 0

A6 1

D5 0

A7 1

D6 0

A8 5

D7 0

$\begin{array}{llll}\text { A9 } & 5 & \text { D8 } & 0\end{array}$

$\begin{array}{llll}\mathrm{A} 10 & 5 & \mathrm{D} 9 & 0\end{array}$

A11 12

D10 0 


$\begin{array}{lr}\text { D7 } & 92.44996439 \\ \text { D8 } & -30.73008333 \\ \text { D9 } & -152.57144045 \\ \text { D10 } & 85.99718652\end{array}$

Harmonic Vibrational Frequencies $\left(\mathbf{c m}^{-1}\right)$ :

$\begin{array}{lllllllllll}415.22 i & 40.20 & 99.91 & 148.49 & 205.00 & 314.15 & 349.23 & 431.37 & 437.34 & 543.07 & 653.19\end{array}$ $\begin{array}{lllllllll}860.79 & 906.53 & 964.29 & 1081.17 & 1109.99 & 1138.65 & 1204.54 & 1237.56 & 1252.56\end{array}$ $\begin{array}{lllllllll}1345.51 & 1423.49 & 1427.10 & 1438.53 & 1497.31 & 1537.87 & 1648.60 & 2188.55 & 3048.32\end{array}$ $\begin{array}{llll}3150.56 & 3154.89 & 3280.64 & 3823.52\end{array}$

Rotational constants (B/GHZ): $5.2607094 \quad 1.4842848 \quad 1.2265723$

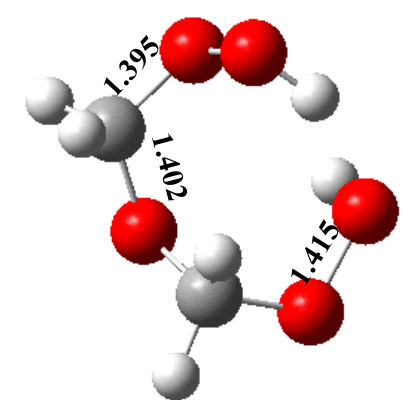

$\mathrm{P} 1 \mathrm{~b}$
$\mathrm{C}$

$\begin{array}{lll}\mathrm{O} & \mathrm{B} 1\end{array}$

$\begin{array}{lll}\mathrm{H} & 1 & \mathrm{~B} 2\end{array}$

$\mathrm{O} \quad 1$

C 2

$\mathrm{H} 5$

$\mathrm{H} \quad 5$

O 5

O 5

$\mathrm{H} \quad 1$

$\mathrm{H} \quad 1$

O 1

$\mathrm{H} \quad 4$

Variables:

B1

1.39646466

B2

2.81289310

B3

2.28646347

B4

1.40195246

B5

1.08773954

B6

1.09184508

B7

2.29107602

B8

1.39505286

B9

1.09030466

B10

1.09296995

B11

1.39952417
B2 2

B3 2

B4 1

B5 2

B6 2

B7 2

B8 2

B9 2

B10 2

B11 2

B12 1

$\begin{array}{rrrr}\text { A1 } & & & \\ \text { A2 } & 3 & \text { D1 } & 0 \\ \text { A3 } & 4 & \text { D2 } & 0 \\ \text { A4 } & 1 & \text { D3 } & 0 \\ \text { A5 } & 1 & \text { D4 } & 0 \\ \text { A6 } & 1 & \text { D5 } & 0 \\ \text { A7 } & 1 & \text { D6 } & 0 \\ \text { A8 } & 5 & \text { D7 } & 0 \\ \text { A9 } & 5 & \text { D8 } & 0 \\ \text { A10 } & 5 & \text { D9 } & 0 \\ \text { A11 } & 2 & \text { D10 } & 0\end{array}$




$\begin{array}{lr}\text { B12 } & 0.96841304 \\ \text { A1 } & 84.04500359 \\ \text { A2 } & 93.33213382 \\ \text { A3 } & 117.88717196 \\ \text { A4 } & 105.66638951 \\ \text { A5 } & 111.18657932 \\ \text { A6 } & 108.78967345 \\ \text { A7 } & 114.09161968 \\ \text { A8 } & 109.64046157 \\ \text { A9 } & 111.00065620 \\ \text { A10 } & 110.84454877 \\ \text { A11 } & 95.79808701 \\ \text { D1 } & -42.74310231 \\ \text { D2 } & -83.79203073 \\ \text { D3 } & -168.60139383 \\ \text { D4 } & -47.40725336 \\ \text { D5 } & 38.07853217 \\ \text { D6 } & 76.32887374 \\ \text { D7 } & 131.10525667 \\ \text { D8 } & 7.65714895 \\ \text { D9 } & -116.00092950 \\ \text { D10 } & -18.80481243\end{array}$

Harmonic Vibrational Frequencies $\left(\mathrm{cm}^{-1}\right)$ :

$\begin{array}{lccccccccr}135.00 & 145.58 & 202.53 & 213.91 & 346.24 & 421.73 & 442.75 & 481.31 & 592.03 & 611.01 \\ 640.47 & 947.94 & 955.88 & 1007.76 & 1102.38 & 1118.74 & 1131.21 & 1192.18 & 1221.83 \\ 1326.90 & 1342.21 & 1419.16 & 1434.41 & 1445.01 & 1482.50 & 1487.94 & 1522.29 & 3081.27 \\ 3094.55 & 3146.10 & 3170.45 & 3727.57 & 3794.55 & & & & \\ \text { Rotational constants (B/GHZ): } 3.8977194 & 2.2929822 & 1.6941060 & & \end{array}$

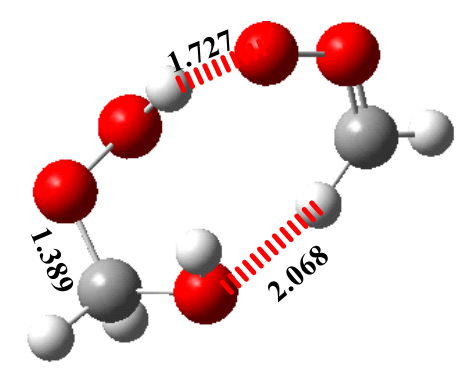

IM1c

$\mathrm{C}$

$\begin{array}{lll}\mathrm{H} & 1 & \mathrm{~B} 1\end{array}$

$\begin{array}{lllll}\mathrm{H} & 1 & \mathrm{~B} 2 & 2 & \mathrm{~A} 1\end{array}$

O $11 \quad$ B3 2

$\begin{array}{lll}\mathrm{O} & 1 & \mathrm{~B} 4\end{array}$

$\begin{array}{llll}\mathrm{H} & 4 & \mathrm{~B} 5 & 1\end{array}$

A2 $3 \quad$ D1 0

$\begin{array}{llll}\mathrm{C} & 4 & \mathrm{~B} 6 & 1\end{array}$

A3 $2 \quad$ D2 0

A4 $5 \quad$ D3 0

$\begin{array}{ll}\mathrm{H} & 7\end{array}$

B7 4

A5 5

D4 0

A6 $1 \quad$ D5 0 


$\begin{array}{rrrrrrrr}\mathrm{H} & 7 & \mathrm{~B} 8 & 4 & \text { A7 } & 1 & \text { D6 } & 0 \\ \mathrm{O} & 7 & \text { B9 } & 4 & \text { A8 } & 1 & \text { D7 } & 0 \\ \mathrm{O} & 7 & \text { B10 } & 4 & \text { A9 } & 1 & \text { D8 } & 0 \\ \text { O } & 1 & \text { B11 } & 5 & \text { A10 } & 4 & \text { D9 } & 0 \\ \text { H } & 12 & \text { B12 } & 1 & \text { A11 } & 5 & \text { D10 } & 0\end{array}$

Variables:

B1 1.09046364

B2 1.09261770

B3 2.27498814

B4 1.38916094

B5 0.98761059

B6 3.04672330

B7 1.08393484

B8 1.08944632

B9 2.23124355

B10 1.23599820

B11 1.40212287

B12 0.96429357

A1 $\quad 111.05797735$

A2 87.45762310

A3 36.32914297

A4 $\quad 104.58806432$

A5 91.90056559

A6 133.50361129

A7 $\quad 59.98514632$

A8 $\quad 58.71049427$

A9 79.87433376

A10 112.68359912

A11 $\quad 108.27238062$

D1 $\quad-143.15021687$

D2 $\quad-131.88141217$

D3 $\quad 88.16754998$

D4 $\quad 143.38332110$

D5 $\quad 117.96489328$

D6 5.28488483

D7 $\quad-100.38932397$

D8 $\quad-127.52170069$

D9 $\quad 66.46706035$

D10 44.27403697

Harmonic Vibrational Frequencies $\left(\mathbf{c m}^{-\mathbf{1}}\right)$ :

$\begin{array}{lllllllllll}77.37 & 87.29 & 118.68 & 143.76 & 174.83 & 226.15 & 270.08 & 412.86 & 446.90 & 551.99 & 631.82\end{array}$ $\begin{array}{llllllllll}703.53 & 770.05 & 899.27 & 963.03 & 1075.11 & 1125.66 & 1127.41 & 1138.57 & 1290.24 & 1292.41\end{array}$ $\begin{array}{lllllllll}1368.81 & 1445.95 & 1465.16 & 1514.58 & 1592.94 & 1656.63 & 3078.32 & 3106.21 & 3137.37\end{array}$ $3262.65 \quad 3333.76 \quad 3847.00$ 


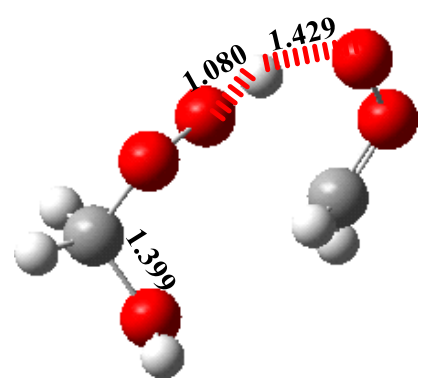

TS1c

C

$\begin{array}{lll}\mathrm{H} & 1 & \mathrm{~B} 1\end{array}$

$\begin{array}{lll}\mathrm{H} & 1 & \mathrm{~B} 2\end{array}$

O 1

O 1

B3 2

A1

$\mathrm{H} \quad 4$

C 4

$\begin{array}{ll}\mathrm{H} & 7\end{array}$

$\mathrm{H} \quad 7$

O 7

O 7

O 1

$\mathrm{H} \quad 12$

B4 4

A2

A3 2

D1 0

B5 1

A4 5

D2 0

A5 5

D3 0

B6 1

D4 0

B7 4

A6 1

D5 0

B8 4

A7 1

D6 0

B9 4

A8 1

D7 0

B10 4

A9 1

D8 0

B11 5

$\begin{array}{llll}\text { A10 } & 4 & \text { D9 } & 0\end{array}$

Variables:

B1

1.08834267

B2

1.09098647

B3

2.24850821

B4

1.39542161

B5

1.07990772

B6

2.10510054

B7

1.08477369

B8

1.08304653

B9

2.17191319

B10

1.25228579

B11

1.39940584

B12

0.96008114

A1

111.34228110

A2

82.25871571

A3

37.18494329

A4

137.39356271

A5

102.38445807

A6

110.23472364

A7

81.62634578

A8

69.10655673 


$\begin{array}{lr}\text { A9 } & 91.25481400 \\ \text { A10 } & 112.69680826 \\ \text { A11 } & 109.11408821 \\ \text { D1 } & -140.35342444 \\ \text { D2 } & -137.77810136 \\ \text { D3 } & 23.43473810 \\ \text { D4 } & 102.14409738 \\ \text { D5 } & 69.08255577 \\ \text { D6 } & -51.96070192 \\ \text { D7 } & -140.37025526 \\ \text { D8 } & -172.14953376 \\ \text { D9 } & 73.90957895 \\ \text { D10 } & 78.04491034\end{array}$

Harmonic Vibrational Frequencies $\left(\mathrm{cm}^{-\mathbf{1}}\right)$ :

$\begin{array}{lrllllllrr}444.43 i & 85.01 & 121.97 & 150.19 & 224.38 & 316.38 & 391.24 & 412.83 & 473.35 & 561.73 \\ 640.54 & 837.64 & 902.44 & 964.66 & 1056.78 & 1104.75 & 1122.39 & 1144.23 & 1230.55 & 1261.66 \\ 1313.57 & 1388.98 & 1435.68 & 1442.39 & 1502.48 & 1545.25 & 1648.19 & 2075.05 & 3100.75 \\ 3150.53 & 3172.59 & 3284.86 & 3900.85 & & & & & \\ \text { Rotational constants (B/GHZ): } & 5.4134777 & 1.5210588 & 1.2867685 & \end{array}$

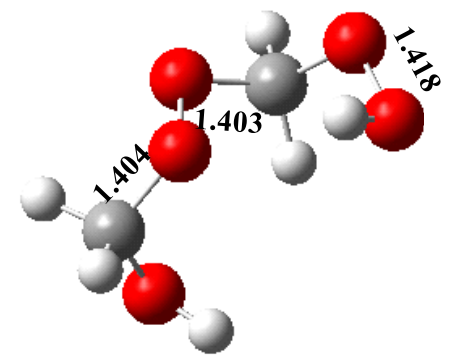

P1c

C

$\begin{array}{lll}\mathrm{H} & 1 & \mathrm{~B} 1\end{array}$

$\begin{array}{llll}\mathrm{H} & 1 & \mathrm{~B} 2 & 2\end{array}$

O 1

B3 2

A1

O 1

B4 4

A2 $3 \quad$ D1 0

$\mathrm{H} \quad 5$

B5 1

A3 2

D2 0

A4 $4 \quad$ D3 0

C 4

B6 1

A5 5

D4 0

$\mathrm{H} \quad 7$

B7 4

A6 1

D5 0

$\mathrm{H} \quad 7$

B8 4

A7 1

D6 0

$\begin{array}{ll}\mathrm{O} & 7\end{array}$

B9 4

A8 1

D7 0

O 7

B10 4

$\begin{array}{llll}\text { A9 } & 1 & \text { D8 } & 0\end{array}$

O 1

B11 5

$\begin{array}{llll}\text { A10 } & 4 & \text { D9 } & 0\end{array}$

$\mathrm{H} \quad 12$

B12 1

A11 5

D10 0

Variables:

$\begin{array}{ll}\text { B1 } & 1.08799697 \\ \text { B2 } & 1.09266730 \\ \text { B3 } & 2.28275845\end{array}$




$\begin{array}{lr}\text { B4 } & 1.40439172 \\ \text { B5 } & 2.30963075 \\ \text { B6 } & 1.40301252 \\ \text { B7 } & 1.08999704 \\ \text { B8 } & 1.08884085 \\ \text { B9 } & 2.2838931 \\ \text { B10 } & 1.38942698 \\ \text { B11 } & 1.38890401 \\ \text { B12 } & 0.96086739 \\ \text { A1 } & 111.14188870 \\ \text { A2 } & 83.33957356 \\ \text { A3 } & 36.42924134 \\ \text { A4 } & 142.55329882 \\ \text { A5 } & 116.44916198 \\ \text { A6 } & 103.75434043 \\ \text { A7 } & 110.81234223 \\ \text { A8 } & 106.60253194 \\ \text { A9 } & 113.29761144 \\ \text { A10 } & 113.18409069 \\ \text { A11 } & 109.34093879 \\ \text { D1 } & -139.20137611 \\ \text { D2 } & -136.55054616 \\ \text { D3 } & -123.05508909 \\ \text { D4 } & 85.73972105 \\ \text { D5 } & 135.82759445 \\ \text { D6 } & 14.74523235 \\ \text { D7 } & -72.22722885 \\ \text { D8 } & -109.92615292 \\ \text { D9 } & 72.80077979 \\ \text { D10 } & 67.55024409 \\ \text { Har } & \end{array}$

Harmonic Vibrational Frequencies $\left(\mathbf{c m}^{-1}\right)$ :

$\begin{array}{llllllllll}52.34 & 122.81 & 165.98 & 193.47 & 360.42 & 393.57 & 413.65 & 461.69 & 494.73 & 637.42\end{array}$ $\begin{array}{lllllllll}669.12 & 895.05 & 962.81 & 1055.36 & 1071.58 & 1112.53 & 1132.52 & 1139.34 & 1187.24\end{array}$ $\begin{array}{lllllllll}1304.97 & 1329.91 & 1386.94 & 1406.08 & 1443.87 & 1458.11 & 1468.76 & 1511.42 & 3084.92\end{array}$ $\begin{array}{lllll}3113.13 & 3176.99 & 3185.22 & 3806.52 & 3893.02\end{array}$

Rotational constants (B/GHZ): $4.8837919 \quad 1.6280646 \quad 1.5251948$

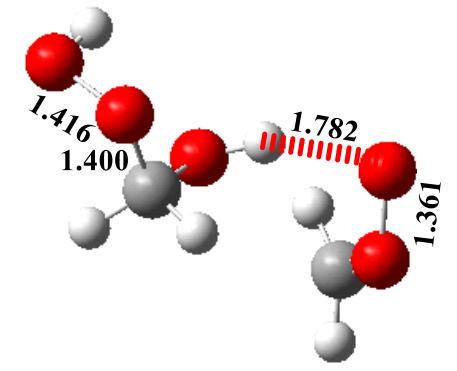

IM1d 
$\mathrm{C}$

$\begin{array}{lll}\mathrm{O} & 1 & \mathrm{~B} 1\end{array}$

$\begin{array}{lllll}\mathrm{H} & 2 & \mathrm{~B} 2 & 1 & \mathrm{~A} 1\end{array}$

$\begin{array}{llll}\mathrm{O} & 1 & \mathrm{~B} 3 & 2\end{array}$

$\begin{array}{llll}\mathrm{C} & 2 & \mathrm{~B} 4 & 1\end{array}$

$\begin{array}{llll}\mathrm{H} & 5 & \mathrm{~B} 5 & 2\end{array}$

$\begin{array}{llll}\mathrm{H} & 5 & \mathrm{~B} 6 & 2\end{array}$

$\begin{array}{llll}\mathrm{O} & 5 & \mathrm{~B} 7 & 2\end{array}$

$\begin{array}{llll}\mathrm{O} & 5 & \mathrm{~B} 8 & 2\end{array}$

$\begin{array}{llll}\mathrm{H} & 1 & \mathrm{~B} 9 & 2\end{array}$

$\begin{array}{llll}\mathrm{H} & 1 & \mathrm{~B} 10 & 2\end{array}$

O 1

$\mathrm{H} \quad 4$

B11 2

B12 1

$\begin{array}{rrrr}\text { A2 } & 3 & \text { D1 } & 0 \\ \text { A3 } & 4 & \text { D2 } & 0 \\ \text { A4 } & 1 & \text { D3 } & 0 \\ \text { A5 } & 1 & \text { D4 } & 0 \\ \text { A6 } & 1 & \text { D5 } & 0 \\ \text { A7 } & 1 & \text { D6 } & 0 \\ \text { A8 } & 8 & \text { D7 } & 0 \\ \text { A9 } & 8 & \text { D8 } & 0 \\ \text { A10 } & 8 & \text { D9 } & 0 \\ \text { A11 } & 2 & \text { D10 } & 0\end{array}$

Variables:

B1 1.39184722

B2 0.97951118

B3 2.26831849

B4 2.84383962

B5 1.08289919

B6 1.08392000

B7 2.22421909

B8 1.23490771

B9 1.08994860

B10 1.09316960

B11 1.39949743

B12 0.96615439

A1 108.66118511

A2 95.15492618

A3 121.49458385

A4 $\quad 128.59537979$

A5 $\quad 61.57653409$

A6 62.57822124

A7 $\quad 82.22777023$

A8 $\quad 107.72780280$

A9 112.18565229

A10 $\quad 112.03065997$

A11 91.43188118

D1 $\quad 112.04097538$

D2 $\quad-176.68359091$

D3 $\quad 53.99856023$

D4 $\quad 167.57297018$

D5 $\quad-90.32950518$

D6 $\quad-62.59597667$

D7 $\quad-141.59020116$ 
D8

$-18.33374995$

D9

97.82144144

D10

$-11.86208360$

Harmonic Vibrational Frequencies $\left(\mathrm{cm}^{-1}\right)$ :

$\begin{array}{lllllllllll}34.20 & 59.12 & 96.11 & 105.16 & 176.74 & 202.51 & 263.42 & 349.72 & 443.45 & 545.48 & 640.52\end{array}$ $\begin{array}{llllllllll}697.12 & 843.16 & 915.62 & 960.73 & 1079.52 & 1109.59 & 1116.80 & 1136.09 & 1266.48 & 1323.65\end{array}$ $\begin{array}{lllllllll}1429.27 & 1440.47 & 1446.10 & 1473.38 & 1502.30 & 1656.34 & 3082.10 & 3146.58 & 3147.18\end{array}$ $3293.83 \quad 3467.77 \quad 3809.79$

Rotational constants (B/GHZ):

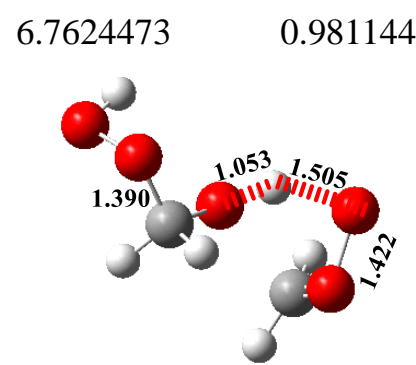

TS1d

C

$\begin{array}{lll}\mathrm{O} & 1 & \mathrm{~B} 1\end{array}$

$\begin{array}{llll}\mathrm{H} & 2 & \mathrm{~B} 2 & 1\end{array}$

O 1

B3 2

A1

C 2

B4 1

B5 2

B6 2

B7 2

B8 2

B9 2

B10 2

B11 2

B12 1

A2

A3 4

D1 0

A4 1

D2 0

A5

D3 0

D4 0

A6 1 D5 0

O 5

A 7

$\begin{array}{ll}\mathrm{H} & 1 \\ & 1\end{array}$

O 1

$\mathrm{H} \quad 4$

A8

D6 0

$\begin{array}{lll}5 & \text { D7 } 0\end{array}$

A9 $5 \quad$ D8 0

A10 5 D9 0

$\begin{array}{llll}\text { A11 } 12 & \text { D10 }\end{array}$

Variables:

$\begin{array}{lr}\text { B1 } & 1.40541536 \\ \text { B2 } & 1.05306931 \\ \text { B3 } & 2.26335509 \\ \text { B4 } & 2.01062737 \\ \text { B5 } & 1.08597113 \\ \text { B6 } & 1.08260109 \\ \text { B7 } & 2.16703780 \\ \text { B8 } & 1.25738886 \\ \text { B9 } & 1.09046133 \\ \text { B10 } & 1.09313474 \\ \text { B11 } & 1.38997707 \\ \text { B12 } & 0.96551861 \\ \text { A1 } & 108.04626998 \\ \text { A2 } & 95.81237725\end{array}$




\begin{tabular}{lr} 
A3 & 112.24458528 \\
A4 & 108.32341448 \\
A5 & 84.09262558 \\
A6 & 71.82185776 \\
A7 & 94.93969707 \\
A8 & 107.98183471 \\
A9 & 111.10058481 \\
A10 & 111.01747364 \\
A11 & 97.83507199 \\
D1 & 112.85465019 \\
D2 & -165.16194270 \\
D3 & 52.12346906 \\
D4 & 173.29716560 \\
D5 & -98.63749406 \\
D6 & -67.23448226 \\
D7 & -78.65069600 \\
D8 & 44.70191553 \\
D9 & 160.43655525 \\
D10 & 100.07184294 \\
\hline
\end{tabular}

Harmonic Vibrational Frequencies $\left(\mathbf{c m}^{-1}\right)$ :

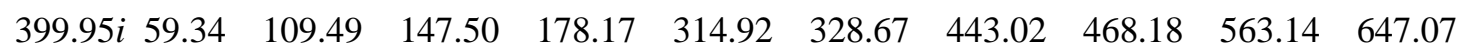
$\begin{array}{lllllllll}857.27 & 913.34 & 970.10 & 1055.70 & 1132.49 & 1139.96 & 1208.96 & 1233.28 & 1281.29\end{array}$ $\begin{array}{lllllllll}1342.36 & 1417.61 & 1432.86 & 1447.95 & 1500.20 & 1534.71 & 1607.27 & 2318.31 & 3084.38\end{array}$ $3148.33 \quad 3151.10 \quad 3278.92 \quad 3822.07$

Rotational constants (B/GHZ): $\quad 6.6335969 \quad 1.2418419 \quad 1.1818513$

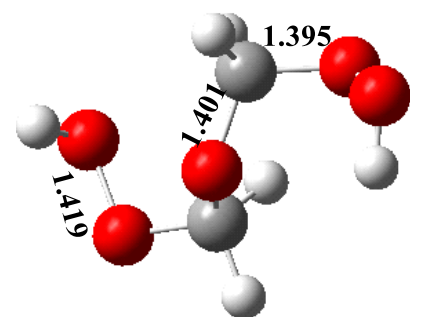

P1d

$\mathrm{C}$

O 11

$\begin{array}{llll}\mathrm{H} & 2 & \mathrm{~B} 2 & 1\end{array}$

$\begin{array}{llll}\mathrm{O} & 1 & \mathrm{~B} 3 & 2\end{array}$

C 2

B4 1

A1

$\mathrm{H} \quad 5$

B5 2

B6 2

$\begin{array}{llll}\mathrm{A} 2 & 3 & \mathrm{D} 1 & 0\end{array}$

A3 $4 \quad$ D2 0

$\mathrm{H} \quad 5$

A4 $1 \quad$ D3 0

O 5

B7 2

A5 1

D4 0

O 5

B8 2

A6 $1 \quad$ D5 0

$\mathrm{H} \quad 1$

B9 2

A7 $1 \quad$ D6 0

$\mathrm{H} \quad 1$

O 1

B10 2

$\begin{array}{llll}\mathrm{A} 8 & 5 & \mathrm{D} 7 & 0\end{array}$

B11 2

A9 5

D8 0

$\mathrm{A} 10 \quad 5 \quad \mathrm{D} 9 \quad 0$ 


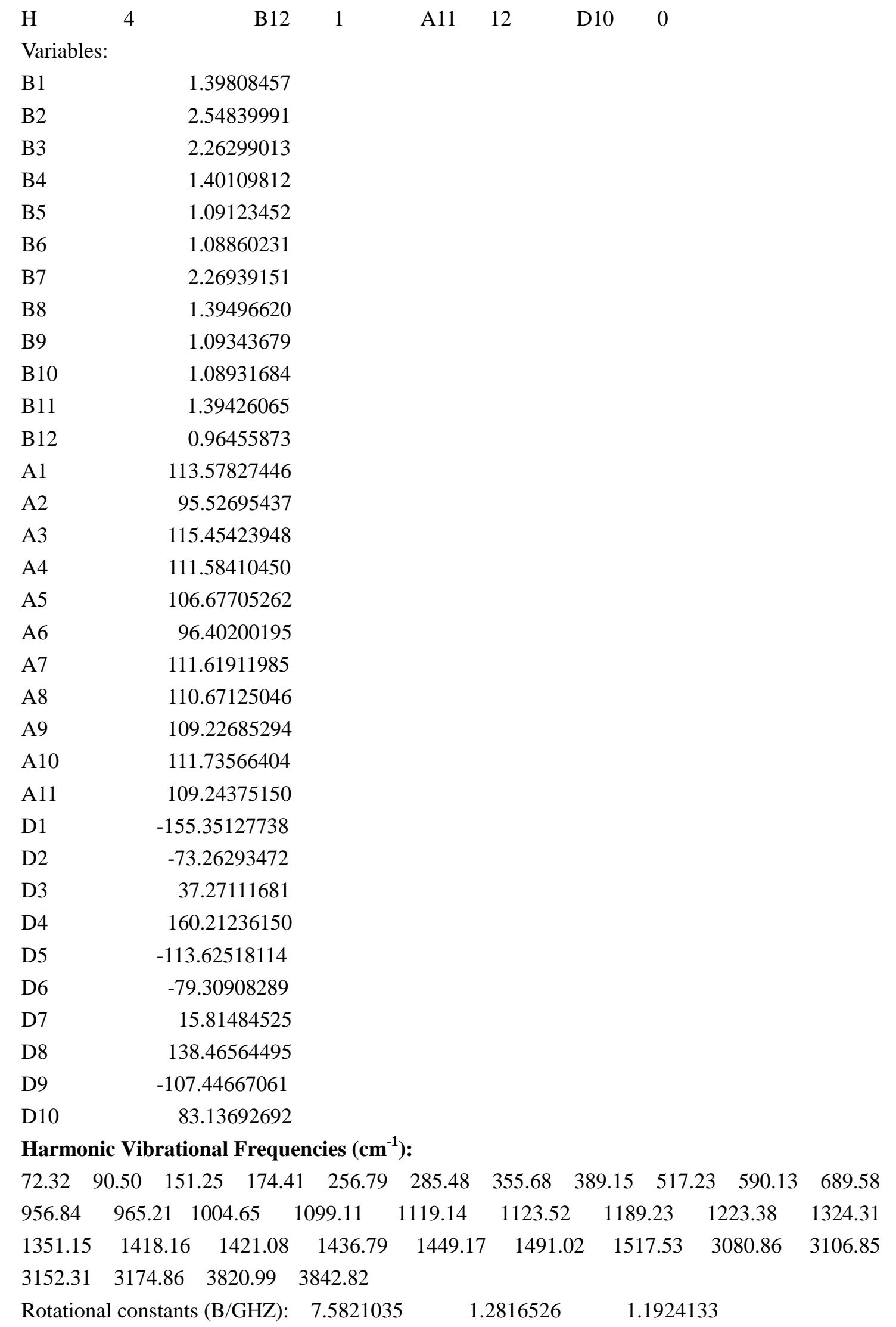




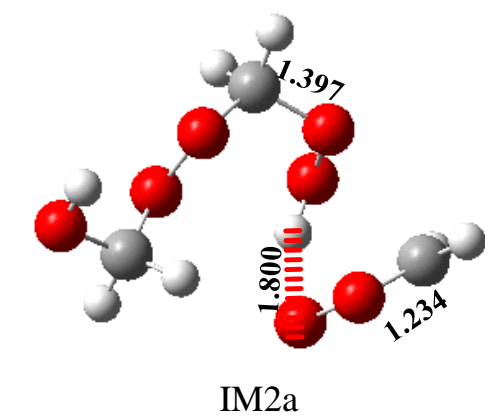

C $\begin{array}{lll}\mathrm{H} & 1 & \mathrm{~B} 1\end{array}$

$\mathrm{H} \quad 1$

O 1

O 1

$\mathrm{H} \quad 4$

C 5

$\begin{array}{ll}\mathrm{H} & 7\end{array}$

$\begin{array}{ll}\mathrm{H} & 7\end{array}$

O 7

O 7

O 1

O 12

C $\quad 13$

$\mathrm{H} \quad 14$

$\mathrm{H} \quad 14$

O 14

$\begin{array}{ll}\mathrm{H} & 17\end{array}$

B2

B2 2

A1

B3 2

B4 4

$\begin{array}{lll}3 & \text { D1 } & 0\end{array}$

B5 1

A3 $2 \quad$ D2 0

A4 $5 \quad$ D3 0

B6 1

A5 $4 \quad$ D4 0

B7 5

A6 $1 \quad$ D5 0

B8 5

A7 $1 \quad$ D6 0

$\begin{array}{llllll}\text { B9 } & 5 & \text { A8 } & 1 & \text { D7 } & 0\end{array}$

B10 5

A9 $1 \quad$ D8 0

B11 5

$\begin{array}{llll}\text { A10 } & 4 & \text { D9 } & 0\end{array}$

B12 1

$\begin{array}{llll}\text { A11 } 5 & \text { D10 } & 0\end{array}$

B13 12

A12 11 D11 0

B14 13

A13 $12 \quad$ D12 0

B15 13

$\begin{array}{llll}\text { A14 } 12 & \text { D13 } & 0\end{array}$

B16 13

A15 $12 \quad$ D14 0

B17 14

A16 13

D15 0

Variables:

B1

B2

B3

B4

B5

B6

B7

B8

B9

B10

B11

B12

B13

B14

B15

B16

B17
1.08988363

1.08990783

2.30167009

1.39674415

0.98469338

2.70891340

1.08284091

1.08512776

2.22979926

1.23359936

1.39017378

1.43268519

1.40341318

1.08778468

1.09010598

1.38936274

0.96003195 


$\begin{array}{lr}\text { A1 } & 112.56515164 \\ \text { A2 } & 80.07049835 \\ \text { A3 } & 35.55734957 \\ \text { A4 } & 98.64788909 \\ \text { A5 } & 137.48661170 \\ \text { A6 } & 111.68529288 \\ \text { A7 } & 68.55344125 \\ \text { A8 } & 76.93420325 \\ \text { A9 } & 90.54297570 \\ \text { A10 } & 113.76157523 \\ \text { A11 } & 106.63788571 \\ \text { A12 } & 105.35826636 \\ \text { A13 } & 103.89028096 \\ \text { A14 } & 108.71124010 \\ \text { A15 } & 112.73237897 \\ \text { A16 } & 108.74926881 \\ \text { D1 } & -137.47081525 \\ \text { D2 } & -145.80060400 \\ \text { D3 } & 97.56904113 \\ \text { D4 } & -107.63461386 \\ \text { D5 } & -83.38171650 \\ \text { D6 } & 156.31654248 \\ \text { D7 } & 64.37397360 \\ \text { D8 } & 34.37027424 \\ \text { D9 } & 88.30041855 \\ \text { D10 } & -80.05073945 \\ \text { D11 } & 161.56374166 \\ \text { D12 } & -170.05631440 \\ \text { D13 } & -52.25066845 \\ \text { D14 } & 73.23464199 \\ \text { D15 } & -72.71154424 \\ \text { Harn }\end{array}$

Harmonic Vibrational Frequencies $\left(\mathbf{c m}^{-1}\right)$ :

$\begin{array}{lllllllllll}45.57 & 66.77 & 93.87 & 102.81 & 122.46 & 144.25 & 151.50 & 186.87 & 236.15 & 242.40 & 324.57\end{array}$ $\begin{array}{llllllllll}383.37 & 460.65 & 475.55 & 546.85 & 588.99 & 676.46 & 704.47 & 751.92 & 889.93 & 904.59\end{array}$ $\begin{array}{lllllllll}957.32 & 1053.65 & 1082.87 & 1103.67 & 1123.38 & 1129.87 & 1139.95 & 1188.07 & 1271.30\end{array}$ $\begin{array}{llllllllll}1306.97 & 1353.81 & 1383.99 & 1415.10 & 1438.66 & 1455.62 & 1457.70 & 1501.07 & 1569.37\end{array}$ $\begin{array}{lllllllll}1663.59 & 3104.56 & 3113.87 & 3140.47 & 3173.48 & 3187.95 & 3286.92 & 3436.05 & 3910.66\end{array}$

Rotational constants (B/GHZ): $1.6892434 \quad 0.9067425 \quad 0.7015963$ 


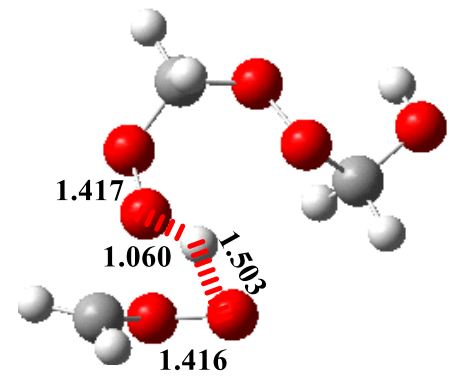

TS2a

C

$\begin{array}{lll}\mathrm{H} & 1 & \mathrm{~B} 1\end{array}$

$\mathrm{H} \quad 1$

O 1

O 1

$\mathrm{H} \quad 4$

C 4

$\mathrm{H} \quad 7$

$\begin{array}{ll}\mathrm{H} & 7\end{array}$

O 7

$\begin{array}{ll}\mathrm{O} & 7\end{array}$

O 1

O 12

C 13

$\mathrm{H} \quad 14$

$\mathrm{H} \quad 14$

O 14

H 17

Variables:

B

B2

B3

B4

B5

B6

B7

B8

B9

B10

B11

B12

B13

B14

B15

B16

1.09048353

1.08946064

2.29390121

1.40053475

1.05987870

2.03446157

1.08499052

1.08348969

2.16595257

1.25473572

1.38695811

1.43058879

1.40977475

1.08774904

1.08984162

1.38682739

$$
\text { B2 }
$$

B3 3

B4 4

B5 1

B6 1

B7 4

B8 4

B9 4

B10 4

B11 5

B12 1

B13 12

B14 13

B15 13

B16 13

B17 14
A1

$\begin{array}{llll}\mathrm{A} 2 & 2 & \mathrm{D} 1 & 0\end{array}$

A3 $2 \quad$ D2 0

A4 $5 \quad$ D3 0

A5 $5 \quad$ D4 0

A6 1 D5 0

A7 $1 \quad$ D6 0

$\begin{array}{llll}\text { A8 } & 1 & \text { D7 } & 0\end{array}$

$\begin{array}{llll}\text { A9 } & 1 & \text { D8 } & 0\end{array}$

$\begin{array}{llll}\mathrm{A} 10 & 4 & \mathrm{D} 9 & 0\end{array}$

$\begin{array}{llll}\mathrm{A} 11 & 5 & \mathrm{D} 10 & 0\end{array}$

A12 1 D11 0

$\begin{array}{llll}\text { A13 } 12 & \text { D12 } & 0\end{array}$

$\begin{array}{llll}\text { A14 } 12 & \text { D13 } & 0\end{array}$

$\begin{array}{llll}\text { A15 } 12 & \text { D14 } & 0\end{array}$

A16 $13 \quad$ D15 0 


$\begin{array}{lr}\text { B17 } & 0.96001843 \\ \text { A1 } & 112.45099433 \\ \text { A2 } & 137.04083125 \\ \text { A3 } & 35.74938867 \\ \text { A4 } & 97.18922543 \\ \text { A5 } & 136.15498864 \\ \text { A6 } & 107.47965602 \\ \text { A7 } & 83.65696172 \\ \text { A8 } & 71.50085949 \\ \text { A9 } & 94.31535569 \\ \text { A10 } & 113.63871609 \\ \text { A11 } & 106.45848008 \\ \text { A12 } & 105.59804582 \\ \text { A13 } & 103.75707173 \\ \text { A14 } & 108.66367202 \\ \text { A15 } & 112.40634252 \\ \text { A16 } & 108.85458353 \\ \text { D1 } & 99.12083365 \\ \text { D2 } & -146.59130157 \\ \text { D3 } & 104.09980232 \\ \text { D4 } & 26.29211804 \\ \text { D5 } & -66.74191041 \\ \text { D6 } & 171.57560754 \\ \text { D7 } & 83.52045154 \\ \text { D8 } & 52.15373696 \\ \text { D9 } & 89.49960538 \\ \text { D10 } & -74.85799528 \\ \text { D11 } & 147.67673854 \\ \text { D12 } & -170.95609238 \\ \text { D13 } & -53.29097995 \\ \text { D14 } & 72.26569892 \\ \text { D15 } & -74.65608515 \\ \text { Hart }\end{array}$

Harmonic Vibrational Frequencies $\left(\mathbf{c m}^{-1}\right)$ :

$\begin{array}{lccccccccccr}422.04 i & 45.71 & 79.71 & 99.69 & 105.97 & 140.19 & 165.91 & 176.37 & 321.38 & 337.62 & 374.91 \\ 442.37 & 466.90 & 480.64 & 579.15 & 606.29 & 680.92 & 842.96 & 891.11 & 913.74 & 965.11 \\ 1053.02 & 1068.77 & 1102.60 & 1135.64 & 1142.15 & 1172.02 & 1190.47 & 1239.94 & 1264.83 \\ 1307.38 & 1347.52 & 1393.62 & 1410.33 & 1433.68 & 1443.30 & 1458.72 & 1491.80 & 1574.24 \\ 1616.94 & 2254.04 & 3107.48 & 3113.55 & 3166.94 & 3176.08 & 3188.73 & 3297.14 & 3905.15 \\ \text { Rotational constants (B/GHZ): } & 1.9471020 & 0.8831406 & 0.6636227 & & \end{array}$




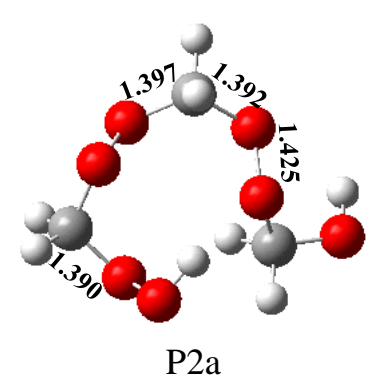

C

$\begin{array}{lll}\mathrm{H} & 1 & \mathrm{~B} 1\end{array}$

$\begin{array}{lllll}\mathrm{H} & 1 & \mathrm{~B} 2 & 2 & \mathrm{~A} 1\end{array}$

O 1

O 1

B3 3

$\begin{array}{llll}\text { A2 } 2 & \text { D1 } & 0\end{array}$

B4 4

A3 2

D2 0

B5 1

A4 5

D3 0

B6 1

A5 5

D4 0

B7 4

A6 1

D5 0

B8 4

A7 1

D6 0

B9 4

A8 1

D7 0

B10 4

A9 1

D8 0

B11 5

A10 4

D9 0

B12 1

A11 5

D10 0

B13 12

A12 1

D11 0

B14 13

A13 12

D12 0

B15 13

A14 12

D13 0

B16 13

A15 12

D14 0

B17 14

A16 13

D15 0

Variables:

B1

B2

B3

B4

B5

B6

B7

B8

B9

B10

B11

B12

B13

B14

B15

B16

B17

A1
1.08996953

1.08989693

2.29105378

1.39705916

2.53724197

1.39570642

1.08997228

1.09061376

2.26051329

1.38984608

1.39157329

1.42458804

1.41623303

1.08836733

1.09049357

1.38234993

0.96044175

111.81227637 


$\begin{array}{lr}\text { A2 } & 132.26587240 \\ \text { A3 } & 36.26474406 \\ \text { A4 } & 85.91659127 \\ \text { A5 } & 130.53995581 \\ \text { A6 } & 111.06955137 \\ \text { A7 } & 103.92225613 \\ \text { A8 } & 95.25773354 \\ \text { A9 } & 113.06503292 \\ \text { A10 } & 113.67872899 \\ \text { A11 } & 108.24810586 \\ \text { A12 } & 105.48195397 \\ \text { A13 } & 104.09420845 \\ \text { A14 } & 108.10670957 \\ \text { A15 } & 112.31296775 \\ \text { A16 } & 109.03401662 \\ \text { D1 } & 89.07606744 \\ \text { D2 } & -161.48051552 \\ \text { D3 } & 124.26494635 \\ \text { D4 } & 59.52514124 \\ \text { D5 } & -74.12610961 \\ \text { D6 } & 164.48719366 \\ \text { D7 } & 77.48674924 \\ \text { D8 } & 44.19866703 \\ \text { D9 } & 105.98976860 \\ \text { D10 } & -72.88027127 \\ \text { D11 } & 135.66147153 \\ \text { D12 } & -171.39482513 \\ \text { D13 } & -53.42255826 \\ \text { D14 } & 71.65226008 \\ \text { D15 } & -73.31751266 \\ \text { Har } & \end{array}$

Harmonic Vibrational Frequencies $\left(\mathrm{cm}^{-1}\right)$ :

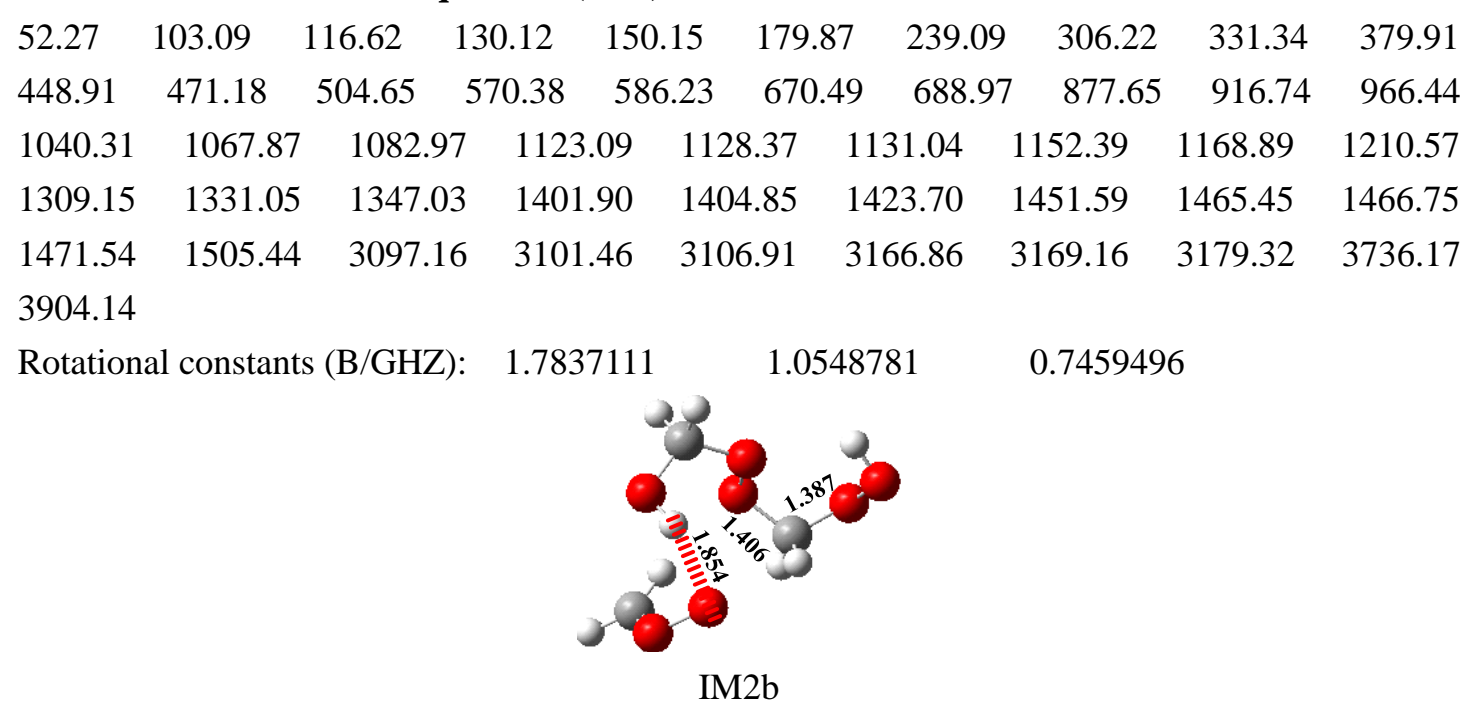


C

\begin{tabular}{|c|c|c|c|c|c|c|c|}
\hline $\mathrm{O}$ & 1 & B1 & & & & & \\
\hline $\mathrm{H}$ & 2 & B2 & 1 & $\mathrm{~A} 1$ & & & \\
\hline $\mathrm{O}$ & 1 & B3 & 2 & A2 & 3 & D1 & 0 \\
\hline $\mathrm{C}$ & 2 & B4 & 1 & A3 & 4 & D2 & 0 \\
\hline $\mathrm{H}$ & 5 & B5 & 2 & A4 & 1 & D3 & 0 \\
\hline $\mathrm{H}$ & 5 & B6 & 2 & A5 & 1 & D4 & 0 \\
\hline $\mathrm{O}$ & 5 & B7 & 2 & A6 & 1 & D5 & 0 \\
\hline $\mathrm{O}$ & 5 & B8 & 2 & A7 & 1 & D6 & 0 \\
\hline $\mathrm{H}$ & 1 & B9 & 2 & A8 & 8 & D7 & 0 \\
\hline $\mathrm{H}$ & 1 & B10 & 2 & A9 & 8 & D8 & 0 \\
\hline $\mathrm{O}$ & 1 & B11 & 2 & A10 & 8 & D9 & 0 \\
\hline $\mathrm{C}$ & 4 & B 12 & 1 & A11 & 2 & D10 & 0 \\
\hline $\mathrm{H}$ & 13 & B13 & 4 & A12 & 1 & D11 & 0 \\
\hline $\mathrm{H}$ & 13 & B14 & 4 & A13 & 1 & D12 & 0 \\
\hline $\mathrm{O}$ & 13 & B15 & 4 & A14 & 1 & D13 & 0 \\
\hline $\mathrm{O}$ & 16 & B16 & 13 & A15 & 4 & D14 & 0 \\
\hline $\mathrm{H}$ & 17 & B17 & 16 & A16 & 13 & D15 & 0 \\
\hline
\end{tabular}

Variables:

$\begin{array}{lr}\text { B1 } & 1.38110497 \\ \text { B2 } & 0.97806555 \\ \text { B3 } & 2.28717162 \\ \text { B4 } & 2.78408003 \\ \text { B5 } & 1.08347963 \\ \text { B6 } & 1.08586218 \\ \text { B7 } & 2.22647943 \\ \text { B8 } & 1.23496196 \\ \text { B9 } & 1.09420375 \\ \text { B10 } & 1.09089084 \\ \text { B11 } & 1.40561815 \\ \text { B12 } & 1.40632027 \\ \text { B13 } & 1.09093937 \\ \text { B14 } & 1.09061095 \\ \text { B15 } & 1.38743459 \\ \text { B16 } & 1.41689926 \\ \text { B17 } & 0.96679943 \\ \text { A1 } & 108.87790588 \\ \text { A2 } & 95.07927923 \\ \text { A3 } & 127.87407982 \\ \text { A4 } & 126.66948896 \\ \text { A5 } & 61.21356138 \\ \text { A6 } & 64.36724709 \\ \text { A7 } & 83.59146399 \\ \text { A8 } & 112.88898577\end{array}$




$\begin{array}{lr}\text { A9 } & 107.99969684 \\ \text { A10 } & 113.03863037 \\ \text { A11 } & 136.61980845 \\ \text { A12 } & 103.98919999 \\ \text { A13 } & 109.97048831 \\ \text { A14 } & 112.74021862 \\ \text { A15 } & 109.14749481 \\ \text { A16 } & 102.46600373 \\ \text { D1 } & 71.61729278 \\ \text { D2 } & -8.18908864 \\ \text { D3 } & -121.73394953 \\ \text { D4 } & -7.34620069 \\ \text { D5 } & 93.51373958 \\ \text { D6 } & 121.57231875 \\ \text { D7 } & -100.67987837 \\ \text { D8 } & 136.65223571 \\ \text { D9 } & 14.50151620 \\ \text { D10 } & -83.50487238 \\ \text { D11 } & 149.52685355 \\ \text { D12 } & 28.42751256 \\ \text { D13 } & -96.41461274 \\ \text { D14 } & 88.46210761 \\ \text { D15 } & -77.34408045 \\ \text { Har }\end{array}$

Harmonic Vibrational Frequencies $\left(\mathbf{c m}^{-\mathbf{1}}\right)$ :

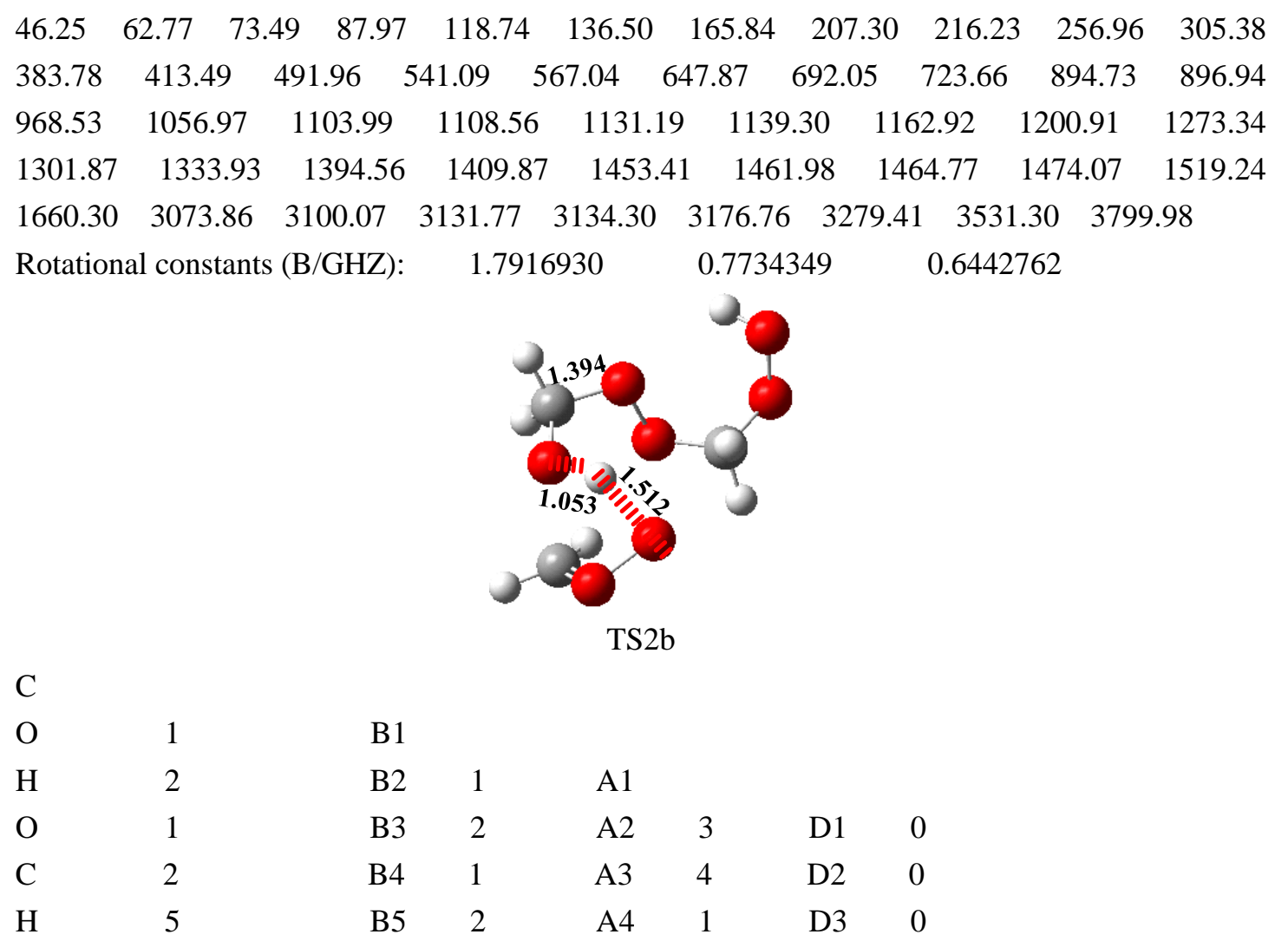




$\begin{array}{rrrrrrrr}\mathrm{H} & 5 & \text { B6 } & 2 & \text { A5 } & 1 & \text { D4 } & 0 \\ \mathrm{O} & 5 & \text { B7 } & 2 & \text { A6 } & 1 & \text { D5 } & 0 \\ \text { O } & 5 & \text { B8 } & 2 & \text { A7 } & 1 & \text { D6 } & 0 \\ \text { H } & 1 & \text { B9 } & 2 & \text { A8 } & 5 & \text { D7 } & 0 \\ \mathrm{H} & 1 & \text { B10 } & 2 & \text { A9 } & 5 & \text { D8 } & 0 \\ \text { O } & 1 & \text { B11 } & 2 & \text { A10 } & 5 & \text { D9 } & 0 \\ \text { C } & 4 & \text { B12 } & 1 & \text { A11 } & 12 & \text { D10 } & 0 \\ \text { H } & 13 & \text { B13 } & 4 & \text { A12 } & 1 & \text { D11 } & 0 \\ \text { H } & 13 & \text { B14 } & 4 & \text { A13 } & 1 & \text { D12 } & 0 \\ \text { O } & 13 & \text { B15 } & 4 & \text { A14 } & 1 & \text { D13 } & 0 \\ \text { O } & 16 & \text { B16 } & 13 & \text { A15 } & 4 & \text { D14 } & 0 \\ \text { H } & 17 & \text { B17 } & 16 & \text { A16 } & 13 & \text { D15 } & 0\end{array}$

Variables:

\begin{tabular}{lr} 
B1 & 1.39502528 \\
B2 & 1.05260297 \\
B3 & 2.26047176 \\
B4 & 2.02403623 \\
B5 & 1.08523997 \\
B6 & 1.08433626 \\
B7 & 2.17140994 \\
B8 & 1.25568657 \\
B9 & 1.09110598 \\
B10 & 1.09445435 \\
B11 & 1.39416675 \\
B12 & 1.40833772 \\
B13 & 1.08909028 \\
B14 & 1.08795784 \\
B15 & 1.38725212 \\
B16 & 1.41708408 \\
B17 & 0.96615157 \\
A1 & 111.61493178 \\
A2 & 98.01644964 \\
A3 & 120.02520637 \\
A4 & 107.27681762 \\
A5 & 86.34704641 \\
A6 & 71.51306729 \\
A7 & 92.61939092 \\
A8 & 109.79557098 \\
A9 & 109.58878956 \\
A10 & 113.02497097 \\
A11 & 133.75223406 \\
A12 & 104.63837476 \\
A13 & 110.14185079 \\
A14 & 112.32775449 \\
& \\
\hline
\end{tabular}




$\begin{array}{lr}\text { A15 } & 108.59744787 \\ \text { A16 } & 102.58741540 \\ \text { D1 } & 38.00190645 \\ \text { D2 } & -49.25705322 \\ \text { D3 } & -99.28023230 \\ \text { D4 } & 23.43900737 \\ \text { D5 } & 110.64015518 \\ \text { D6 } & 143.17008711 \\ \text { D7 } & 159.17335504 \\ \text { D8 } & 37.63768563 \\ \text { D9 } & -85.02396625 \\ \text { D10 } & 53.65158443 \\ \text { D11 } & 132.50697958 \\ \text { D12 } & 11.82954061 \\ \text { D13 } & -112.95957016 \\ \text { D14 } & 83.05278903 \\ \text { D15 } & -82.68278521\end{array}$

Harmonic Vibrational Frequencies $\left(\mathbf{c m}^{-1}\right)$ :

$\begin{array}{lllllllllll}406.95 i & 55.00 & 60.59 & 89.20 & 136.11 & 142.34 & 169.06 & 196.89 & 274.70 & 313.18 & 361.41\end{array}$ $\begin{array}{llllllllll}429.25 & 439.30 & 484.52 & 545.20 & 613.54 & 686.26 & 859.81 & 890.21 & 911.14 & 963.21\end{array}$ $\begin{array}{lllllllll}1031.81 & 1064.85 & 1115.81 & 1129.07 & 1134.76 & 1163.12 & 1193.48 & 1214.41 & 1247.77\end{array}$ $\begin{array}{lllllllll}1312.87 & 1340.34 & 1405.96 & 1421.04 & 1434.67 & 1444.79 & 1449.77 & 1483.22 & 1513.70\end{array}$ $\begin{array}{lllllllll}1625.15 & 2336.79 & 3068.43 & 3116.20 & 3135.60 & 3142.64 & 3188.30 & 3271.80 & 3813.88\end{array}$ Rotational constants (B/GHZ): $\quad 2.2651765 \quad 0.7597666 \quad 0.6430710$

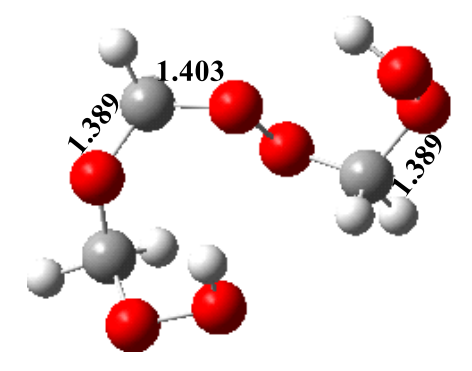

$\mathrm{P} 2 \mathrm{~b}$

$\mathrm{C}$

$\begin{array}{lll}\mathrm{O} & 1 & \mathrm{~B} 1\end{array}$

$\begin{array}{lllll}\mathrm{H} & 2 & \mathrm{~B} 2 & 1 & \mathrm{~A} 1\end{array}$

$\begin{array}{lllll}\mathrm{O} & 1 & \mathrm{~B} 3 & 2 & \mathrm{~A} 2\end{array}$

C 2

$\mathrm{H} \quad 5$

$\mathrm{H} \quad 5$

O 5

$\mathrm{O} \quad 5$

$\mathrm{H} \quad 1$

B4 1

A2 $3 \quad$ D1 0

$\mathrm{H} \quad 1$

O 1

B5 2

B6 2

A4 $1 \quad$ D3 0

$\begin{array}{llllll}\text { B7 } & 2 & \text { A6 } & 1 & \text { D5 } & 0\end{array}$

$\begin{array}{llllll}\text { B8 } & 2 & \text { A7 } & 1 & \text { D6 } & 0\end{array}$

$\begin{array}{llllll}\text { B9 } & 2 & \text { A8 } & 5 & \text { D7 } & 0\end{array}$

$\begin{array}{llllll}\mathrm{B} 10 & 2 & \mathrm{~A} 9 & 5 & \mathrm{D} 8 & 0\end{array}$

$\begin{array}{llllll}\mathrm{B} 11 & 2 & \mathrm{~A} 10 & 5 & \mathrm{D} 9 & 0\end{array}$ 


$\begin{array}{rrrrrrrr}\mathrm{C} & 4 & \text { B12 } & 1 & \text { A11 } & 2 & \text { D10 } & 0 \\ \mathrm{H} & 13 & \text { B13 } & 4 & \text { A12 } & 1 & \text { D11 } & 0 \\ \mathrm{H} & 13 & \text { B14 } & 4 & \text { A13 } & 1 & \text { D12 } & 0 \\ \mathrm{O} & 13 & \text { B15 } & 4 & \text { A14 } & 1 & \text { D13 } & 0 \\ \mathrm{O} & 16 & \text { B16 } & 13 & \text { A15 } & 4 & \text { D14 } & 0 \\ \mathrm{H} & 17 & \text { B17 } & 16 & \text { A16 } & 13 & \text { D15 } & 0\end{array}$

Variables:

$\begin{array}{ll}\text { B1 } & 1.38923007 \\ \text { B2 } & 2.47710069 \\ \text { B3 } & 2.27535158 \\ \text { B4 } & 1.40812992 \\ \text { B5 } & 1.09024509 \\ \text { B6 } & 1.09152910 \\ \text { B7 } & 2.26689874 \\ \text { B8 } & 1.38976120 \\ \text { B9 } & 1.08773067 \\ \text { B10 } & 1.09222482 \\ \text { B11 } & 1.40308826 \\ \text { B12 } & 1.39938622 \\ \text { B13 } & 1.08943437 \\ \text { B14 } & 1.08897042 \\ \text { B15 } & 1.38947138 \\ \text { B16 } & 1.41735556 \\ \text { B17 } & 0.96627625\end{array}$

A1 100.74337231

A2 109.37831232

A3 118.07175381

A4 109.29106202

A5 110.59965632

A6 93.87139699

A7 $\quad 109.94625415$

A8 106.21614541

A9 112.00249249

A10 114.00410880

A11 141.02106713

A12 103.73756378

A13 110.84141369

A14 113.02432109

A15 108.86687448

A16 102.36095130

D1 40.71739136

D2 $\quad-38.28209208$

D3 $\quad-123.98237274$

D4 -0.09671928 


$\begin{array}{lr}\text { D5 } & 89.21285794 \\ \text { D6 } & 122.74711748 \\ \text { D7 } & 166.79996842 \\ \text { D8 } & 45.32337066 \\ \text { D9 } & -78.12030609 \\ \text { D10 } & -77.69334427 \\ \text { D11 } & 156.21249938 \\ \text { D12 } & 35.52046663 \\ \text { D13 } & -88.93935348 \\ \text { D14 } & 88.20021650 \\ \text { D15 } & -81.57055734\end{array}$

Harmonic Vibrational Frequencies $\left(\mathbf{c m}^{-\mathbf{1}}\right)$ :

$\begin{array}{lllllllllll}49.63 & 85.41 & 100.29 & 114.16 & 143.49 & 170.55 & 196.92 & 300.77 & 339.79 & 373.56 & 385.00\end{array}$ $\begin{array}{llllllllll}422.92 & 430.48 & 491.87 & 577.64 & 620.92 & 683.71 & 886.83 & 964.30 & 967.28 & 994.18\end{array}$ $\begin{array}{lllllllll}1071.88 & 1111.61 & 1118.78 & 1135.66 & 1138.19 & 1171.59 & 1203.77 & 1226.50 & 1324.92\end{array}$ $\begin{array}{lllllllll}1335.18 & 1354.65 & 1399.72 & 1429.53 & 1444.13 & 1447.95 & 1454.31 & 1459.82 & 1485.46\end{array}$ $\begin{array}{lllllllll}1505.45 & 3096.88 & 3104.02 & 3115.79 & 3167.24 & 3172.80 & 3184.82 & 3802.56 & 3804.43\end{array}$ Rotational constants (B/GHZ): $\quad 2.3684565 \quad 0.7647451 \quad 0.6638408$

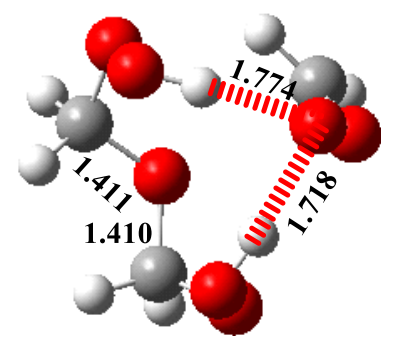

IM2c

$\mathrm{C}$

$\begin{array}{lll}\mathrm{H} & 1 & \mathrm{~B} 1\end{array}$

$\mathrm{H}-1-\mathrm{B} 2$

O 1

O 1

$\mathrm{H} \quad 4$

C 5

$\begin{array}{ll}\mathrm{H} & 7\end{array}$

$\begin{array}{ll}\mathrm{H} & 7\end{array}$

O 7

O 7

O 1

C $\quad 12$

B2 2

A1

B3 2 A2

A2 3

D1 0

B4 4

A3 3

D2 0

B5 1

A4 5

D3 0

B6 1

A5 4

D4 0

B7 $5 \quad$ A6 1

D5 0

$\begin{array}{llll}\text { B8 } & 5 & \text { A7 } & 1\end{array}$

D6 0

$\begin{array}{llll}\text { B9 } & 5 & \text { A8 } & 1\end{array}$

D7 0

B10 5

A9 1

D8 0

B11 5

A10 4

D9 0

B12 1

A11 5

D10 0

$\begin{array}{ll}\mathrm{H} & 13\end{array}$

B13 12

A12 1

D11 0

$\mathrm{H} \quad 13$

B14 12

A13 1

D12 0

O 13

B15 12

A14 1

D13 0

O 16

B16 13

A15 12

D14 0

$\begin{array}{ll}\mathrm{H} & 17\end{array}$

B17 16

A16 13

D15 0 


\begin{tabular}{|c|c|}
\hline Varia & \\
\hline B1 & 1.09173357 \\
\hline B2 & 1.09176734 \\
\hline B3 & 2.27577887 \\
\hline B4 & 1.38540952 \\
\hline B5 & 0.98225148 \\
\hline B6 & 3.15396968 \\
\hline B7 & 1.08351633 \\
\hline B8 & 1.08593208 \\
\hline B9 & 2.22471154 \\
\hline B10 & 1.23092006 \\
\hline B11 & 1.41043256 \\
\hline B12 & 1.41127256 \\
\hline B13 & 1.09272220 \\
\hline B14 & 1.09064191 \\
\hline B15 & 1.38086711 \\
\hline B16 & 1.41872472 \\
\hline B17 & 0.97937323 \\
\hline $\mathrm{A} 1$ & 111.75486058 \\
\hline $\mathrm{A} 2$ & 138.05627343 \\
\hline A3 & 36.17564227 \\
\hline A4 & 100.35720007 \\
\hline A5 & 89.14156252 \\
\hline A6 & 130.84903723 \\
\hline A7 & 30.92016001 \\
\hline A8 & 75.05781953 \\
\hline A9 & 104.97079052 \\
\hline A 10 & 110.13473721 \\
\hline A11 & 118.80943987 \\
\hline A 12 & 109.99615222 \\
\hline A13 & 108.88105126 \\
\hline A14 & 111.48489040 \\
\hline A 15 & 107.93570473 \\
\hline A 16 & 101.34831941 \\
\hline D1 & -102.98030066 \\
\hline D2 & 141.56318281 \\
\hline D3 & -97.21480171 \\
\hline D4 & 92.81035561 \\
\hline D5 & 54.77784378 \\
\hline D6 & 145.67746133 \\
\hline D7 & -105.07658079 \\
\hline D8 & -89.91177852 \\
\hline D9 & -81.03102241 \\
\hline D10 & 137.28754439 \\
\hline
\end{tabular}




$\begin{array}{lr}\text { D11 } & -3.10865002 \\ \text { D12 } & 119.26575569 \\ \text { D13 } & -125.94687678 \\ \text { D14 } & 78.42619470 \\ \text { D15 } & -103.17900909\end{array}$

Harmonic Vibrational Frequencies $\left(\mathbf{c m}^{-1}\right)$ :

$\begin{array}{lllllllllll}58.24 & 74.27 & 98.25 & 124.05 & 144.64 & 150.67 & 186.06 & 198.66 & 257.24 & 274.00 & 356.48\end{array}$ $\begin{array}{llllllllll}431.97 & 446.67 & 551.94 & 555.92 & 574.44 & 651.59 & 724.28 & 756.86 & 908.71 & 956.01\end{array}$ $\begin{array}{lllllllll}964.84 & 1009.82 & 1094.74 & 1130.53 & 1149.53 & 1154.97 & 1182.88 & 1217.23 & 1271.00\end{array}$ $\begin{array}{lllllllll}1326.64 & 1347.04 & 1423.57 & 1459.49 & 1465.41 & 1490.68 & 1515.50 & 1550.13 & 1598.10\end{array}$ $\begin{array}{lllllllll}1692.66 & 3074.86 & 3083.55 & 3140.80 & 3149.45 & 3149.98 & 3295.73 & 3449.88 & 3522.12\end{array}$ Rotational constants (B/GHZ): $1.3332535 \quad 1.2296427 \quad 0.9436559$

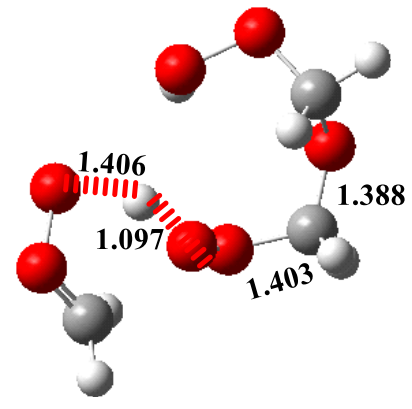

$\mathrm{TS} 2 \mathrm{c}$

$\mathrm{C}$

$\begin{array}{lll}\mathrm{H} & 1 & \mathrm{~B} 1\end{array}$

$\mathrm{H} \quad 1$

O 1

O 1

$\mathrm{H} \quad 4$

C 4

H 7

$\begin{array}{ll}\mathrm{H} & 7\end{array}$

O 7

O 7

O 1

C $\quad 12$

$\mathrm{H} \quad 13$

$\mathrm{H} \quad 13$

O 13

O 16

$\mathrm{H} \quad 17$

B2

B3 $2 \quad$ A2

B4 4

A3 3

D1 0

B5 1

B6

A4 5

D3 0

B7

A5 5

D4 0

$4 \quad$ A6 11 D5 0

$\begin{array}{llllll}\text { B8 } & 4 & \text { A7 } & 1 & \text { D6 } & 0\end{array}$

$\begin{array}{llllll}\text { B9 } & 4 & \text { A8 } & 1 & \text { D7 } & 0\end{array}$

$\begin{array}{llllll}\text { B10 } & 4 & \text { A9 } & 1 & \text { D8 } & 0\end{array}$

$\begin{array}{llllll}\text { B11 } & 5 & \text { A10 } & 4 & \text { D9 } & 0\end{array}$

$\begin{array}{llllll}\mathrm{B} 12 & 1 & \mathrm{~A} 11 & 5 & \mathrm{D} 10 & 0\end{array}$

$\begin{array}{llllll}\mathrm{B} 13 & 12 & \mathrm{~A} 12 & 1 & \mathrm{D} 11 & 0\end{array}$

$\begin{array}{llllll}\mathrm{B} 14 & 12 & \mathrm{~A} 13 & 1 & \mathrm{D} 12 & 0\end{array}$

$\begin{array}{llllll}\text { B15 } & 12 & \text { A14 } & 1 & \text { D13 } & 0\end{array}$

$\begin{array}{llllll}\text { B16 } & 13 & \text { A15 } & 12 & \text { D14 } & 0\end{array}$

Variables:

$\begin{array}{ll}\text { B1 } & 1.08822105 \\ \text { B2 } & 1.09248724 \\ \text { B3 } & 2.29205468 \\ \text { B4 } & 1.40275015\end{array}$




\begin{tabular}{|c|c|}
\hline B5 & 1.09651837 \\
\hline B6 & 2.07146171 \\
\hline B7 & 1.08539676 \\
\hline B8 & 1.08431856 \\
\hline B9 & 2.17197829 \\
\hline B10 & 1.25381589 \\
\hline B11 & 1.38807186 \\
\hline B12 & 1.40660899 \\
\hline B13 & 1.09237305 \\
\hline B14 & 1.09030052 \\
\hline B15 & 1.38788552 \\
\hline B16 & 1.41449609 \\
\hline B17 & 0.96710312 \\
\hline A1 & 111.33825263 \\
\hline $\mathrm{A} 2$ & 135.75219725 \\
\hline A3 & 35.99138020 \\
\hline A4 & 120.17714399 \\
\hline A5 & 132.92302060 \\
\hline A6 & 110.26438685 \\
\hline A7 & 82.04308471 \\
\hline A 8 & 69.22682265 \\
\hline A9 & 92.55809505 \\
\hline A 10 & 114.19942473 \\
\hline A11 & 117.95478561 \\
\hline A12 & 110.52380147 \\
\hline A13 & 108.98011506 \\
\hline A14 & 110.67815682 \\
\hline A15 & 108.02146988 \\
\hline A16 & 102.57729529 \\
\hline D1 & -95.33460589 \\
\hline D2 & 146.11137347 \\
\hline D3 & -67.86854107 \\
\hline D4 & 29.49492823 \\
\hline D5 & 91.33413052 \\
\hline D6 & -29.85607405 \\
\hline D7 & -119.42611990 \\
\hline D8 & -150.28906792 \\
\hline D9 & -90.27296692 \\
\hline D10 & 78.75076545 \\
\hline D11 & 2.46988242 \\
\hline D12 & 125.91857628 \\
\hline D13 & -120.56991234 \\
\hline D14 & 71.90661523 \\
\hline D15 & -90.05706114 \\
\hline
\end{tabular}


Harmonic Vibrational Frequencies $\left(\mathbf{c m}^{-1}\right)$ :

$\begin{array}{lccccccccccr}521.14 i & 60.42 & 73.14 & 96.87 & 121.27 & 154.30 & 180.40 & 205.45 & 337.52 & 362.68 & 383.27 \\ 388.08 & 441.97 & 468.13 & 559.95 & 597.94 & 628.83 & 846.51 & 908.05 & 942.68 & 965.92 \\ 1002.96 & 1072.84 & 1118.98 & 1142.01 & 1153.50 & 1192.73 & 1217.33 & 1224.76 & 1251.51 \\ 1341.83 & 1350.29 & 1415.05 & 1431.90 & 1435.19 & 1450.90 & 1486.56 & 1512.98 & 1557.08 \\ 1640.72 & 1973.12 & 3080.76 & 3088.40 & 3146.38 & 3150.59 & 3160.74 & 3279.20 & 3799.25 \\ \text { Rotational constants (B/GHZ): } & 2.1368669 & 0.8386239 & 0.6464839 & & \end{array}$

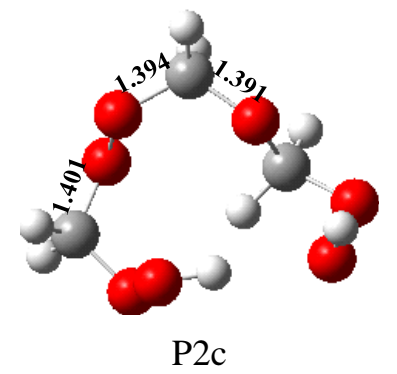

C

$\begin{array}{lll}\mathrm{H} & 1 & \mathrm{~B} 1\end{array}$

$\begin{array}{lll}\mathrm{H} & 1 & \mathrm{~B} 2\end{array}$

O 1

O 1

$\mathrm{H} \quad 5$

C 4

$\begin{array}{ll}\mathrm{H} & 7\end{array}$

$\mathrm{H} \quad 7$

$\begin{array}{ll}\mathrm{O} & 7\end{array}$

$\begin{array}{ll}\mathrm{O} & 7\end{array}$

O 1

C 12

$\mathrm{H} \quad 13$

$\mathrm{H} \quad 13$

O 13

O 16

H 17

Variables:

B

1.08804196

B2

1.09491762

B3

2.27492032

B4

1.39372009

B5

3.20695464

B6 1.40061720

B7 1.08973201

B8 1.08965214

B9 2.27878583

B10 1.38871558

B11

1.39135346 


\begin{tabular}{|c|c|}
\hline B12 & 1.40718095 \\
\hline B13 & 1.09130927 \\
\hline B14 & 1.09398138 \\
\hline B15 & 1.38804938 \\
\hline B16 & 1.41944571 \\
\hline B17 & 0.96616097 \\
\hline $\mathrm{A} 1$ & 111.00826776 \\
\hline $\mathrm{A} 2$ & 138.51001701 \\
\hline A3 & 36.87277193 \\
\hline $\mathrm{A} 4$ & 89.16382476 \\
\hline A5 & 132.45947809 \\
\hline A6 & 104.70141827 \\
\hline A7 & 109.70028309 \\
\hline A8 & 106.31788028 \\
\hline A9 & 114.41532465 \\
\hline A10 & 114.13728410 \\
\hline A11 & 116.16639715 \\
\hline A12 & 111.27663138 \\
\hline A13 & 111.15030493 \\
\hline A14 & 107.75516983 \\
\hline A 15 & 107.11471579 \\
\hline A16 & 101.55343398 \\
\hline D1 & -102.06238759 \\
\hline D2 & 138.34038038 \\
\hline D3 & 76.73333567 \\
\hline D4 & -56.63472133 \\
\hline D5 & -173.80604446 \\
\hline D6 & 65.36888885 \\
\hline D7 & -21.90081340 \\
\hline D8 & -59.73989428 \\
\hline D9 & -80.86100177 \\
\hline D10 & 86.34647865 \\
\hline D11 & -63.10900622 \\
\hline D12 & 61.11192440 \\
\hline D13 & 175.41256076 \\
\hline D14 & 68.09959073 \\
\hline D15 & -97.62181004 \\
\hline
\end{tabular}

Harmonic Vibrational Frequencies $\left(\mathrm{cm}^{-1}\right)$ :

$\begin{array}{lllllllllll}56.64 & 99.70 & 115.04 & 128.67 & 157.70 & 175.85 & 189.49 & 292.64 & 353.62 & 360.84 & 396.61\end{array}$ $\begin{array}{llllllllll}446.88 & 461.30 & 497.57 & 556.01 & 631.36 & 686.12 & 887.38 & 962.60 & 968.62 & 1020.98\end{array}$ $\begin{array}{lllllllll}1077.96 & 1130.34 & 1133.83 & 1141.82 & 1157.78 & 1174.32 & 1181.70 & 1223.52 & 1302.07\end{array}$ $\begin{array}{lllllllll}1342.74 & 1361.21 & 1413.93 & 1421.96 & 1429.11 & 1450.06 & 1457.67 & 1491.60 & 1507.42\end{array}$ $\begin{array}{lllllllll}1538.72 & 3063.26 & 3080.40 & 3102.23 & 3146.16 & 3161.26 & 3173.43 & 3742.99 & 3805.38\end{array}$ Rotational constants (B/GHZ): $1.7171591 \quad 1.0738626 \quad 0.7770133$ 


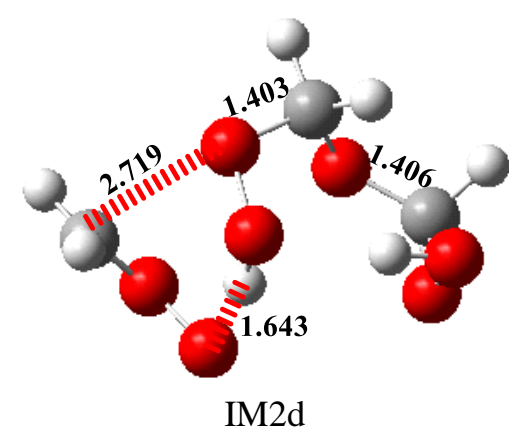

$\begin{array}{lr}\mathrm{C} & \\ \mathrm{H} & 1 \\ \mathrm{H} & 1 \\ \mathrm{O} & 1 \\ \mathrm{O} & 1 \\ \mathrm{H} & 4 \\ \mathrm{C} & 5 \\ \mathrm{H} & 7 \\ \mathrm{H} & 7 \\ \mathrm{O} & 7 \\ \mathrm{O} & 7 \\ \mathrm{O} & 1 \\ \mathrm{C} & 12 \\ \mathrm{H} & 13 \\ \mathrm{H} & 13 \\ \mathrm{O} & 13 \\ \mathrm{O} & 16 \\ \mathrm{H} & 17\end{array}$

Variables:

$\begin{array}{ll}\text { B1 } & 1.09230618 \\ \text { B2 } & 1.09152965 \\ \text { B3 } & 2.30712600 \\ \text { B4 } & 1.40339209 \\ \text { B5 } & 1.00358637 \\ \text { B6 } & 2.71928638 \\ \text { B7 } & 1.08298022 \\ \text { B8 } & 1.08478245 \\ \text { B9 } & 2.22786991 \\ \text { B10 } & 1.23283725 \\ \text { B11 } & 1.38988740 \\ \text { B12 } & 1.40618075 \\ \text { B13 } & 1.08735793 \\ \text { B14 } & 1.09272513 \\ \text { B15 } & 1.38978946 \\ \text { B16 } & 1.42037272 \\ \text { B17 } & 0.97170844\end{array}$




$\begin{array}{lr}\text { A1 } & 111.15122046 \\ \text { A2 } & 138.03696929 \\ \text { A3 } & 35.45614286 \\ \text { A4 } & 98.02714931 \\ \text { A5 } & 121.92372641 \\ \text { A6 } & 112.60041067 \\ \text { A7 } & 66.63663805 \\ \text { A8 } & 77.10976933 \\ \text { A9 } & 91.91971324 \\ \text { A10 } & 111.17745592 \\ \text { A11 } & 118.06891513 \\ \text { A12 } & 105.26935443 \\ \text { A13 } & 110.48948435 \\ \text { A14 } & 115.10760989 \\ \text { A15 } & 108.93009905 \\ \text { A16 } & 100.92143105 \\ \text { D1 } & 113.57810685 \\ \text { D2 } & -129.02111946 \\ \text { D3 } & 99.26769621 \\ \text { D4 } & -102.83902346 \\ \text { D5 } & -74.47040132 \\ \text { D6 } & 166.14673737 \\ \text { D7 } & 73.76952847 \\ \text { D8 } & 44.28779259 \\ \text { D9 } & 68.38655223 \\ \text { D10 } & -114.07100982 \\ \text { D11 } & -172.75006226 \\ \text { D12 } & -52.05805084 \\ \text { D13 } & 71.97089060 \\ \text { D14 } & -86.61494647 \\ \text { D15 } & 98.61402746 \\ \text { Hart }\end{array}$

Harmonic Vibrational Frequencies $\left(\mathbf{c m}^{-1}\right)$ :

$\begin{array}{lllllllllll}51.63 & 67.67 & 84.47 & 130.39 & 147.39 & 173.10 & 189.60 & 212.21 & 243.54 & 283.78 & 351.96\end{array}$ $\begin{array}{llllllllll}431.17 & 457.85 & 550.78 & 605.23 & 609.59 & 650.22 & 711.33 & 876.70 & 903.01 & 943.94\end{array}$ $\begin{array}{lllllllll}952.56 & 1006.74 & 1101.57 & 1105.39 & 1119.94 & 1139.49 & 1194.68 & 1220.63 & 1267.55\end{array}$ $\begin{array}{llllllllll}1329.67 & 1348.73 & 1423.54 & 1442.74 & 1454.52 & 1483.95 & 1489.03 & 1509.51 & 1638.73\end{array}$ $\begin{array}{lllllllll}1667.96 & 3011.00 & 3078.93 & 3085.14 & 3142.96 & 3145.11 & 3165.71 & 3289.07 & 3683.63\end{array}$ Rotational constants (B/GHZ): $1.9076250 \quad 0.9078872 \quad 0.8500031$ 


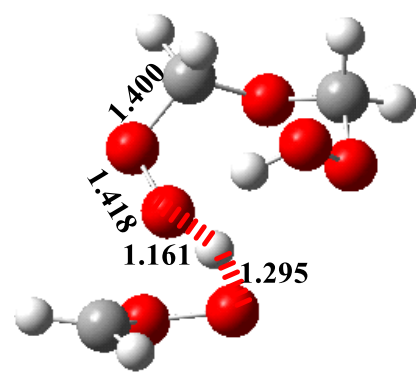

TS2d

C $\begin{array}{lll}\mathrm{H} & 1 & \mathrm{~B} 1\end{array}$

$\mathrm{H} \quad 1$

O 1

O 1

$\mathrm{H} \quad 4$

C 4

$\begin{array}{ll}\mathrm{H} & 7\end{array}$

$\begin{array}{ll}\mathrm{H} & 7\end{array}$

O 7

O 7

O 1

C 12

$\mathrm{H} \quad 13$

$\mathrm{H} \quad 13$

O 13

O 16

$\mathrm{H} \quad 17$

Variables:

B1

B2

B3

B4

B5

B6

B7

B8

B9

B10

B11

B12

B13

B14

B15

B16

B17
B2 2

B3 3

B4 4

B5 1

B6 1

B7 4

B8 4

B9 4

B10 4

B11 5

B12 1

B13 12

B14 12

B15 12

B16 13

B17 16
A1

A2 $2 \quad$ D1 0

A3 $2 \quad$ D2 0

A4 $5 \quad$ D3 0

A5 $5 \quad$ D4 0

A6 $1 \quad$ D5 0

A7 $1 \quad$ D6 0

$\begin{array}{llll}\text { A8 } & 1 & \text { D7 } & 0\end{array}$

$\begin{array}{llll}\text { A9 } & 1 & \text { D8 } & 0\end{array}$

$\begin{array}{llll}\mathrm{A} 10 & 4 & \mathrm{D} 9 & 0\end{array}$

$\begin{array}{llll}\text { A11 } 5 & \text { D10 } & 0\end{array}$

$\begin{array}{llll}\text { A12 } & 1 & \text { D11 } & 0\end{array}$

A13 $1 \quad$ D12 0

$\begin{array}{llll}\text { A14 } 1 & \text { D13 } & 0\end{array}$

$\begin{array}{llll}\text { A15 } 12 & \text { D14 } & 0\end{array}$

$\begin{array}{llll}\text { A16 } 13 & \text { D15 }\end{array}$
1.09367104

1.09074277

2.29192599

1.40042830

1.16093597

2.14527910

1.08508067

1.08212318

2.16523921

1.24990183

1.39167318

1.40093186

1.08757378

1.09277147

1.39383116

1.42031374

0.97225980 


$\begin{array}{lr}\text { A1 } & 111.15228335 \\ \text { A2 } & 138.52218970 \\ \text { A3 } & 35.85178712 \\ \text { A4 } & 97.59744781 \\ \text { A5 } & 132.88810683 \\ \text { A6 } & 111.47931341 \\ \text { A7 } & 80.02282469 \\ \text { A8 } & 67.24828457 \\ \text { A9 } & 90.61177313 \\ \text { A10 } & 111.56428225 \\ \text { A11 } & 118.11874004 \\ \text { A12 } & 105.58182353 \\ \text { A13 } & 110.98005521 \\ \text { A14 } & 114.59734224 \\ \text { A15 } & 108.91138900 \\ \text { A16 } & 101.19496613 \\ \text { D1 } & 116.61836747 \\ \text { D2 } & -126.35933158 \\ \text { D3 } & 102.13141964 \\ \text { D4 } & 31.03457895 \\ \text { D5 } & -68.81143216 \\ \text { D6 } & 169.93317752 \\ \text { D7 } & 80.23864756 \\ \text { D8 } & 49.30639345 \\ \text { D9 } & 64.69441313 \\ \text { D10 } & -115.06128116 \\ \text { D11 } & -168.87398847 \\ \text { D12 } & -47.77997472 \\ \text { D13 } & 76.06955785 \\ \text { D14 } & -86.88702127 \\ \text { D15 } & 94.21529419 \\ \text { Har } & \end{array}$

Harmonic Vibrational Frequencies $\left(\mathbf{c m}^{-1}\right)$ :

$\begin{array}{lllllllllll}842.60 i & 45.38 & 75.99 & 94.81 & 137.87 & 157.71 & 201.42 & 218.92 & 309.76 & 376.68 & 412.21\end{array}$ $\begin{array}{llllllllll}454.76 & 468.16 & 571.62 & 608.44 & 615.84 & 657.87 & 791.37 & 804.93 & 915.84 & 946.09\end{array}$ $\begin{array}{lllllllll}971.18 & 1010.57 & 1107.48 & 1128.39 & 1138.23 & 1162.38 & 1195.79 & 1220.25 & 1250.91\end{array}$ $\begin{array}{lllllllll}1329.64 & 1344.08 & 1421.76 & 1437.82 & 1445.38 & 1485.06 & 1487.53 & 1516.13 & 1566.53\end{array}$ $\begin{array}{lllllllll}1634.61 & 1774.95 & 3076.33 & 3083.61 & 3151.78 & 3156.58 & 3168.93 & 3290.32 & 3656.04\end{array}$ Rotational constants (B/GHZ): $2.0039339 \quad 0.8782998 \quad 0.8104643$ 


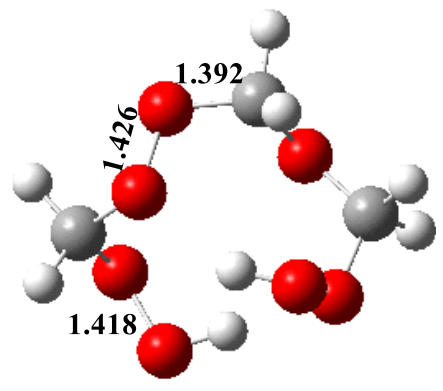

P2d

C

$\mathrm{H} \quad 1$

H 1

O 1

O 1

H 4

C 4

H $\quad 7$

H 7

O 7

O 7

O 1

C 12

H 13

$\mathrm{H} \quad 13$

O 13

O 16

H 17

Variables:

B1

B2

B3

B4

B5

B6

B7

B8

B9

B10

B11

B12

B13

B14

B15

B16
B1

B2

B3 3

B4 4

B5 1

B6 1

B7 4

B8 4

B9 4

B10 4

B11 5

B12 1

B13 12

B14 12

B15 12

B16 13

B17 16

A1

$\begin{array}{llll}\text { A2 } 2 & \text { D1 } & 0\end{array}$

A3 2

D2 0

A4 5

D3 0

A5 5

D4 0

A6 1

D5 0

A7 1

D6 0

$\begin{array}{llll}\text { A8 } & 1 & \text { D7 } & 0\end{array}$

A9 $1 \quad$ D8 0

$\begin{array}{llll}\text { A10 } 4 & \text { D9 } & 0\end{array}$

A11 $5 \quad$ D10 0

A12 $1 \quad$ D11 0

A13 11 D12 0

A14 $1 \quad$ D13 0

A15 $12 \quad$ D14 0

A16 $13 \quad$ D15 0

1.09309772

1.09100771

2.29414924

1.39192073

2.83987031

1.40870539

1.08905637

1.09062584

2.25155136

1.37965101

1.39878983

1.39203121

1.08803263

1.09196967

1.40090693

1.41817956 


$\begin{array}{lr}\text { B17 } & 0.97146288 \\ \text { A1 } & 111.11151288 \\ \text { A2 } & 137.48993681 \\ \text { A3 } & 35.98118791 \\ \text { A4 } & 88.65888437 \\ \text { A5 } & 128.38693063 \\ \text { A6 } & 109.70296427 \\ \text { A7 } & 104.03733893 \\ \text { A8 } & 98.58895242 \\ \text { A9 } & 113.42065813 \\ \text { A10 } & 111.69210369 \\ \text { A11 } & 118.28325766 \\ \text { A12 } & 106.85412716 \\ \text { A13 } & 111.87883549 \\ \text { A14 } & 113.12884958 \\ \text { A15 } & 108.55256572 \\ \text { A16 } & 102.48700391 \\ \text { D1 } & 108.77249315 \\ \text { D2 } & -133.69594214 \\ \text { D3 } & 123.79490551 \\ \text { D4 } & 61.26578478 \\ \text { D5 } & -72.84257660 \\ \text { D6 } & 166.41785372 \\ \text { D7 } & 80.90048485 \\ \text { D8 } & 45.49097179 \\ \text { D9 } & 73.82769244 \\ \text { D10 } & -126.40161905 \\ \text { D11 } & -157.64639852 \\ \text { D12 } & -35.23447306 \\ \text { D13 } & 87.88033664 \\ \text { D14 } & -81.82169669 \\ \text { D15 } & 91.20787985 \\ \text { Hart }\end{array}$

Harmonic Vibrational Frequencies $\left(\mathbf{c m}^{-1}\right)$ :

$\begin{array}{lllllllllll}67.95 & 70.34 & 92.00 & 145.56 & 185.43 & 210.22 & 242.88 & 275.79 & 377.58 & 400.76 & 462.28\end{array}$ $\begin{array}{llllllllll}504.46 & 516.17 & 588.53 & 619.04 & 650.40 & 679.53 & 893.44 & 947.19 & 969.44 & 1012.39\end{array}$ $\begin{array}{lllllllll}1058.51 & 1101.68 & 1124.39 & 1139.97 & 1144.57 & 1173.58 & 1210.46 & 1231.14 & 1327.15\end{array}$ $\begin{array}{llllllllll}1346.08 & 1350.55 & 1409.66 & 1428.29 & 1451.21 & 1459.64 & 1466.44 & 1492.92 & 1495.42\end{array}$ $\begin{array}{lllllllll}1513.94 & 3076.60 & 3091.99 & 3102.62 & 3143.70 & 3172.18 & 3174.95 & 3698.15 & 3727.35\end{array}$

Rotational constants (B/GHZ): $1.6405933 \quad 1.2080624 \quad 0.9120953$ 


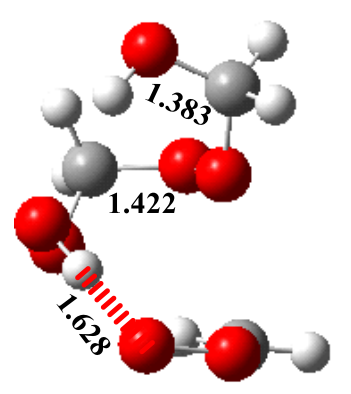

IM2e

C

H 1

H 1

O 1

O 1

$\mathrm{H} \quad 4$

C 5

H 7

H 7

$\begin{array}{ll}\mathrm{O} & 7\end{array}$

O 7

O 1

O 12

C 13

$\begin{array}{ll}\mathrm{H} & 14\end{array}$

$\mathrm{H} \quad 14$

O 14

$\mathrm{H} \quad 17$

Variables:

B

B2

B3

B4

B5

B6

B7

B8

B9

B10

B11

B12

B13

B14

B15

B16
B1

B2

B3 2

B4 4

B5 1

B6 1

B7 5

B8 5

B9 5

B10 5

B11 5

B12 1

B13 12

B14 13

B15 13

B16 13

B17 14
A1

A2 $3 \quad$ D1 0

A3 $2 \quad$ D2 0

A4 $5 \quad$ D3 0

A5 $4 \quad$ D4 0

A6 $1 \quad$ D5 0

A7 $1 \quad$ D6 0

$\begin{array}{llll}\text { A8 } & 1 & \text { D7 } & 0\end{array}$

$\begin{array}{llll}\text { A9 } & 1 & \text { D8 } & 0\end{array}$

$\mathrm{A} 10 \quad 4 \quad \mathrm{D} 9 \quad 0$

A11 $5 \quad$ D10 0

A12 11 D11 0

A13 12 D12 0

A14 $12 \quad$ D13 0

A15 $12 \quad$ D14 0

A16 13 D15 0

1.08612208

1.09029262

2.27923749

1.38262711

1.00102219

3.17989086

1.08308732

1.08639653

2.22949630

1.23244809

1.42234880

1.41793320

1.41067028

1.08917389

1.09452666

1.38261190 


$\begin{array}{lr}\text { B17 } & 0.96679914 \\ \text { A1 } & 112.18094293 \\ \text { A2 } & 79.08160700 \\ \text { A3 } & 36.37876894 \\ \text { A4 } & 117.41065646 \\ \text { A5 } & 101.43461274 \\ \text { A6 } & 137.57409026 \\ \text { A7 } & 26.55513924 \\ \text { A8 } & 70.76204339 \\ \text { A9 } & 101.84737662 \\ \text { A10 } & 113.35923781 \\ \text { A11 } & 109.99771538 \\ \text { A12 } & 111.11237852 \\ \text { A13 } & 110.02178604 \\ \text { A14 } & 102.06270164 \\ \text { A15 } & 113.07241891 \\ \text { A16 } & 108.62181219 \\ \text { D1 } & -138.30405178 \\ \text { D2 } & -149.10446672 \\ \text { D3 } & 69.50390516 \\ \text { D4 } & -92.93423693 \\ \text { D5 } & -28.78859466 \\ \text { D6 } & -101.16008569 \\ \text { D7 } & 134.04256453 \\ \text { D8 } & 122.82912041 \\ \text { D9 } & 92.65887420 \\ \text { D10 } & -53.96454365 \\ \text { D11 } & -89.53289303 \\ \text { D12 } & -49.16824222 \\ \text { D13 } & -167.23418630 \\ \text { D14 } & 71.18242593 \\ \text { D15 } & 46.07669395 \\ \text { Hart }\end{array}$

Harmonic Vibrational Frequencies $\left(\mathbf{c m}^{-1}\right)$ :

$\begin{array}{lllllllllll}18.74 & 62.73 & 93.56 & 112.18 & 134.59 & 152.20 & 200.02 & 225.26 & 248.99 & 267.68 & 350.47\end{array}$ $\begin{array}{llllllllll}384.50 & 482.22 & 509.90 & 544.98 & 633.06 & 668.30 & 726.94 & 873.25 & 889.63 & 910.48\end{array}$ $\begin{array}{lllllllll}963.42 & 1022.69 & 1072.75 & 1115.25 & 1135.50 & 1151.35 & 1160.45 & 1183.86 & 1271.41\end{array}$ $\begin{array}{lllllllll}1293.62 & 1324.70 & 1378.24 & 1418.58 & 1448.98 & 1460.48 & 1495.79 & 1506.11 & 1619.70\end{array}$ $\begin{array}{lllllllll}1677.30 & 3066.91 & 3075.16 & 3110.59 & 3144.53 & 3145.62 & 3189.36 & 3288.77 & 3800.66\end{array}$ Rotational constants (B/GHZ): $\quad 1.5962138 \quad 0.9800565 \quad 0.8857907$ 


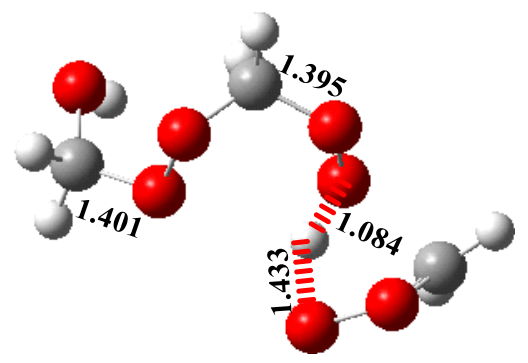

TS2e

C

$\mathrm{H} \quad 1$

$\mathrm{H} \quad 1$

O 1

O 1

$\mathrm{H} \quad 4$

C 4

H 7

H 7

O 7

O 7

O 1

O 12

C 13

$\mathrm{H} \quad 14$

H 14

O 14

$\begin{array}{ll}\mathrm{H} & 17\end{array}$

Variables:

B1

B2

B3

B4

B5

B6

B7

B8

B9

B10

B11

B12

B13

B14

B15

B16

B17
B1

B2 2

B3 2

B4 4

B5 1

B6 1

B7 4

B8 4

B9 4

B10 4

B11 5

B12 1

B13 12

B14 13

B15 13

B16 13

B17 14

A1

A2 $3 \quad$ D1 0

A3 $2 \quad$ D2 0

A4 $5 \quad$ D3 0

A5 $5 \quad$ D4 0

A6 $1 \quad$ D5 0

A7 $1 \quad$ D6 0

$\begin{array}{llll}\text { A8 } & 1 & \text { D7 } & 0\end{array}$

$\begin{array}{llll}\text { A9 } & 1 & \text { D8 } & 0\end{array}$

$\begin{array}{llll}\mathrm{A} 10 & 4 & \mathrm{D} 9 & 0\end{array}$

$\begin{array}{llll}\text { A11 } 5 & \text { D10 } & 0\end{array}$

A12 1 D11 0

A13 $12 \quad$ D12 0

$\begin{array}{llll}\text { A14 } 12 & \text { D13 } & 0\end{array}$

A15 $12 \quad$ D14 0

A16 $13 \quad$ D15 0

1.08923401

1.09009800

2.28173243

1.39493380

1.08394794

2.06772845

1.08527351

1.08303179

2.16473001

1.25271213

1.39370527

1.42174647

1.40069839

1.08821486

1.09222745

1.39235043

0.96045827 


$\begin{array}{lr}\text { A1 } & 112.44183552 \\ \text { A2 } & 83.04375779 \\ \text { A3 } & 36.13688195 \\ \text { A4 } & 98.38106256 \\ \text { A5 } & 135.75998694 \\ \text { A6 } & 108.70818668 \\ \text { A7 } & 82.38067310 \\ \text { A8 } & 69.97320235 \\ \text { A9 } & 93.28957040 \\ \text { A10 } & 113.71568944 \\ \text { A11 } & 108.95746625 \\ \text { A12 } & 107.17241240 \\ \text { A13 } & 109.72907776 \\ \text { A14 } & 103.35368248 \\ \text { A15 } & 113.17076732 \\ \text { A16 } & 109.03910104 \\ \text { D1 } & -140.71295350 \\ \text { D2 } & -139.43368109 \\ \text { D3 } & 102.47258006 \\ \text { D4 } & 26.25380766 \\ \text { D5 } & -66.01218646 \\ \text { D6 } & 172.56940833 \\ \text { D7 } & 83.91203637 \\ \text { D8 } & 52.86941997 \\ \text { D9 } & 81.49511747 \\ \text { D10 } & -80.05930208 \\ \text { D11 } & -112.03080042 \\ \text { D12 } & -45.86700062 \\ \text { D13 } & -164.49220104 \\ \text { D14 } & 73.41567897 \\ \text { D15 } & 69.34137392 \\ \text { Darn }\end{array}$

Harmonic Vibrational Frequencies $\left(\mathbf{c m}^{-\mathbf{1}}\right)$ :

$\begin{array}{lllllllllll}479.67 i & 42.58 & 49.40 & 70.78 & 89.93 & 144.75 & 159.03 & 194.15 & 329.38 & 370.29 & 402.61\end{array}$ $\begin{array}{llllllllll}442.37 & 462.08 & 476.78 & 582.04 & 632.67 & 671.20 & 817.87 & 896.24 & 904.96 & 955.69\end{array}$ $\begin{array}{lllllllll}1060.75 & 1069.80 & 1101.00 & 1122.26 & 1138.93 & 1156.21 & 1183.89 & 1232.02 & 1300.08\end{array}$ $\begin{array}{lllllllll}1301.41 & 1332.66 & 1384.12 & 1408.33 & 1437.32 & 1440.60 & 1457.24 & 1500.86 & 1584.36\end{array}$ $\begin{array}{lllllllll}1623.66 & 2047.92 & 3083.89 & 3105.96 & 3150.92 & 3165.02 & 3174.98 & 3281.72 & 3896.13\end{array}$

Rotational constants (B/GHZ): $\quad 2.5144665 \quad 0.6930923 \quad 0.6237335$ 


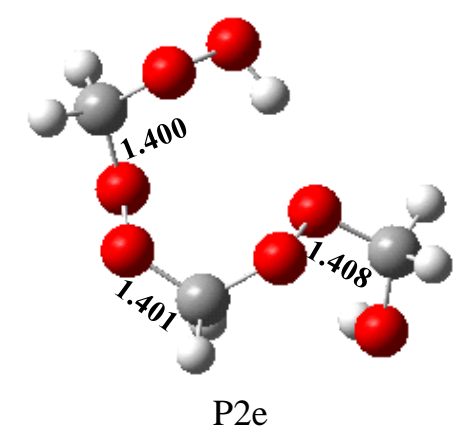

C

$\mathrm{H} \quad 1$

$\mathrm{H} \quad 1$

O 1

O 1

$\mathrm{H} \quad 4$

C 4

H 7

$\mathrm{H} \quad 7$

O 7

O 7

O 1

O 12

C 13

$\mathrm{H} \quad 14$

$\mathrm{H} \quad 14$

O 14

H $\quad 17$

B1

B2 2 A1

B3 2 A2

B4 4

A3 2

D1 0

$\begin{array}{llllll}\text { B5 } & 1 & \text { A4 } & 5 & \text { D3 } & 0\end{array}$

$\begin{array}{llllll}\text { B6 } & 1 & \text { A5 } & 5 & \text { D4 } & 0\end{array}$

$\begin{array}{llllll}\text { B7 } & 4 & \text { A6 } & 1 & \text { D5 } & 0\end{array}$

$\begin{array}{llllll}\text { B8 } & 4 & \text { A7 } & 1 & \text { D6 } & 0\end{array}$

$\begin{array}{llllll}\text { B9 } & 4 & \text { A8 } & 1 & \text { D7 } & 0\end{array}$

$\begin{array}{llllll}\mathrm{B} 10 & 4 & \mathrm{~A} 9 & 1 & \mathrm{D} 8 & 0\end{array}$

$\begin{array}{llllll}\mathrm{B} 11 & 5 & 10 & 4 & \mathrm{D} 9 & 0\end{array}$

$\begin{array}{llllll}\mathrm{B} 12 & 1 & 11 & 5 & \mathrm{D} 10 & 0\end{array}$

$\begin{array}{llllll}\mathrm{B} 13 & 12 & 12 & 1 & \mathrm{D} 11 & 0\end{array}$

$\begin{array}{llllll}\text { B14 } & 13 & 13 & 12 & \text { D12 } & 0\end{array}$

$\begin{array}{llllll}\text { B15 } & 13 & 14 & 12 & \text { D13 } & 0\end{array}$

$\begin{array}{llllll}\text { B16 } & 13 & 15 & 12 & \text { D14 } & 0\end{array}$

$\begin{array}{llllll}\text { B17 } & 14 & 16 & 13 & \text { D15 } & 0\end{array}$

Variables:

B2 1.08968958

B3 2.27065662

B4 1.38754669

B5 2.68713858

B6 1.40044393

B7 1.09038160

B8 1.09068247

B9 2.26491757

B10 1.38426644

B11 1.40114368

B12 1.42057882

B13 1.40828970

B14 1.08765346

B15 1.09240083

B16 1.38617180

B17 0.96060309 


$\begin{array}{lr}\text { A1 } & 112.12362214 \\ \text { A2 } & 84.39851150 \\ \text { A3 } & 36.81331179 \\ \text { A4 } & 84.78505923 \\ \text { A5 } & 129.06210279 \\ \text { A6 } & 110.43738593 \\ \text { A7 } & 103.88268553 \\ \text { A8 } & 98.04488206 \\ \text { A9 } & 113.56000497 \\ \text { A10 } & 113.96981209 \\ \text { A11 } & 109.54417534 \\ \text { A12 } & 107.31305993 \\ \text { A13 } & 109.40632468 \\ \text { A14 } & 103.37934023 \\ \text { A15 } & 112.66415694 \\ \text { A16 } & 109.45194888 \\ \text { D1 } & -142.25081628 \\ \text { D2 } & -137.16686151 \\ \text { D3 } & 121.12189566 \\ \text { D4 } & 60.31391196 \\ \text { D5 } & -77.88693348 \\ \text { D6 } & 161.13639535 \\ \text { D7 } & 74.62735218 \\ \text { D8 } & 40.09496279 \\ \text { D9 } & 78.28285708 \\ \text { D10 } & -90.68241610 \\ \text { D11 } & -112.85526833 \\ \text { D12 } & -46.22804825 \\ \text { D13 } & -164.83120013 \\ \text { D14 } & 72.94418472 \\ \text { D15 } & 70.84294455 \\ \text { Harn }\end{array}$

Harmonic Vibrational Frequencies $\left(\mathbf{c m}^{-1}\right)$ :

$\begin{array}{lllllllllll}41.45 & 56.84 & 89.21 & 107.18 & 148.65 & 190.21 & 238.15 & 273.49 & 340.14 & 414.07 & 428.68\end{array}$ $\begin{array}{llllllllll}471.14 & 502.64 & 527.73 & 626.92 & 658.35 & 686.63 & 882.30 & 918.49 & 965.75 & 1045.85\end{array}$ $\begin{array}{lllllllll}1060.95 & 1079.08 & 1113.56 & 1134.80 & 1136.36 & 1141.42 & 1164.76 & 1213.13 & 1300.46\end{array}$ $\begin{array}{lllllllll}1324.56 & 1347.10 & 1392.02 & 1400.41 & 1422.61 & 1438.11 & 1457.42 & 1466.31 & 1501.72\end{array}$ $\begin{array}{lllllllll}1505.05 & 3091.26 & 3098.08 & 3108.12 & 3166.03 & 3167.91 & 3175.88 & 3737.06 & 3894.23\end{array}$

Rotational constants (B/GHZ): $\quad 2.1188528 \quad 0.8569316 \quad 0.6925481$ 


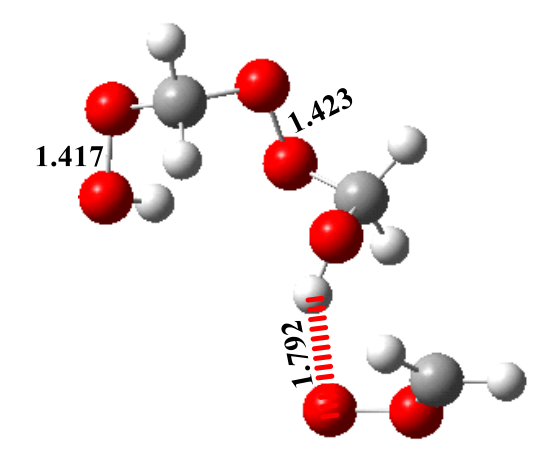

IM2f

C

$\mathrm{O}$

$\mathrm{H}$

$\mathrm{O}$

C

$\mathrm{H}$

$\mathrm{H}$

$\mathrm{O}$

$\mathrm{O}$

$\mathrm{H}$

$\mathrm{H}$

$\mathrm{O}$

C

$\mathrm{H}$

$\mathrm{H}$

$\mathrm{O}$

$\mathrm{O}$

$\mathrm{H}$

Variables:

B1

B2

B3

B4

B5

B6

B7

B8

B9

B10

B11

B12

B13

B14

B15
B1

B2 1

B3 2

B4 1

B5 2

B6 2

B7 2

B8 2

B9 2

B10 2

B11 2

B12 1

B13 4

B14 4

B15 4

B16 13

B17 16
A1

A2 3

A3 4

A4 1

A5 1

A6 1

A7 1

A8 8

A9 8

A10 8

A11 2

A12 1

A13 1

A14 1

A15 4

A16 13
D1 0

D2 0

D3 0

D4 0

D5 0

D6 0

D7 0

D8 0

D9 0

D10 0

D11 0

D12 0

D13 0

D14 0

D15 0

1.38541949

0.97881337

2.27986516

2.85195410

1.08281979

1.08406020

2.22515895

1.23488140

1.08920564

1.09371930

1.40676994

1.40377379

1.08892979

1.09022278

1.38916304 


$\begin{array}{lr}\text { B16 } & 1.41743589 \\ \text { B17 } & 0.96689024 \\ \text { A1 } & 107.88364174 \\ \text { A2 } & 98.97126201 \\ \text { A3 } & 118.60526939 \\ \text { A4 } & 128.04137336 \\ \text { A5 } & 62.88419809 \\ \text { A6 } & 62.39075969 \\ \text { A7 } & 81.48096122 \\ \text { A8 } & 108.19585792 \\ \text { A9 } & 112.10662447 \\ \text { A10 } & 113.11187194 \\ \text { A11 } & 116.58836494 \\ \text { A12 } & 110.74221169 \\ \text { A13 } & 103.60174462 \\ \text { A14 } & 113.06229427 \\ \text { A15 } & 109.01415233 \\ \text { A16 } & 102.44190489 \\ \text { D1 } & 114.44477801 \\ \text { D2 } & -175.34010402 \\ \text { D3 } & 58.01265176 \\ \text { D4 } & 172.45032314 \\ \text { D5 } & -86.01791353 \\ \text { D6 } & -57.85909124 \\ \text { D7 } & -139.81605346 \\ \text { D8 } & -16.56835866 \\ \text { D9 } & 99.65813261 \\ \text { D10 } & -31.88923729 \\ \text { D11 } & 15.33568935 \\ \text { D12 } & -77.33818027 \\ \text { D13 } & -109.06072058 \\ \text { D14 } & \text { D15 }\end{array}$

Harmonic Vibrational Frequencies $\left(\mathbf{c m}^{-1}\right)$ :

$\begin{array}{lllllllllll}32.45 & 38.20 & 60.54 & 83.33 & 94.99 & 133.96 & 165.32 & 190.61 & 197.23 & 251.06 & 363.22\end{array}$ $\begin{array}{llllllllll}396.34 & 401.69 & 494.97 & 542.92 & 630.74 & 664.85 & 705.89 & 794.45 & 894.60 & 912.69\end{array}$ $\begin{array}{lllllllll}966.80 & 1050.80 & 1082.92 & 1110.22 & 1113.80 & 1134.71 & 1142.36 & 1188.63 & 1268.30\end{array}$ $\begin{array}{lllllllll}1326.70 & 1335.88 & 1407.30 & 1434.12 & 1450.33 & 1463.64 & 1467.83 & 1480.08 & 1498.30\end{array}$ $\begin{array}{lllllllll}1658.51 & 3082.16 & 3112.34 & 3152.53 & 3154.83 & 3183.50 & 3301.60 & 3487.41 & 3822.53\end{array}$

Rotational constants (B/GHZ): $\quad 2.4734210 \quad 0.5244542 \quad 0.4772716$ 


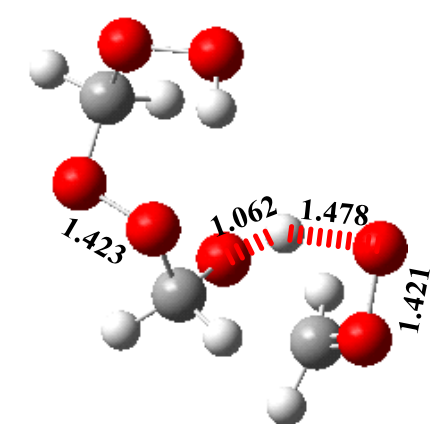

TS2f

$\mathrm{C}$

$\begin{array}{lll}\mathrm{O} & 1 & \mathrm{~B} 1\end{array}$

$\mathrm{H} 2$

O 1

C 2

$\mathrm{H} \quad 5$

$\mathrm{H} \quad 5$

O 5

O 5

$\mathrm{H} \quad 1$

$\mathrm{H} \quad 1$

O 1

C 4

$\mathrm{H} \quad 13$

$\mathrm{H} \quad 13$

O 13

O 16

$\mathrm{H} \quad 17$

Variables:

B1

B2

B3

B4

B5

B6

B7

B8

B9

B10

B11

B12

B13

B14

B15

B16
1.39961428

1.06230827

2.27469756

2.02225615

1.08600759

1.08273072

2.16672676

1.25760257

1.08954590

1.09438224

1.39494950

1.40641657

1.08920709

1.09012719

1.38691368

1.41721953
B2

B3 2

B4 1

B5 2

B6 2

B7 2

B8 2

B9 2

B10 2

B11 2

B12 1

B13 4

B14 4

B15 4

B16 13

B17 16

A1

$\begin{array}{llll}\text { A2 } & 3 & \text { D1 } & 0\end{array}$

A3 4 D2 0

A4 $1 \quad$ D3 0

A5 $1 \quad$ D4 0

A6 $1 \quad$ D5 0

A7 $1 \quad$ D6 0

$\begin{array}{llll}\mathrm{A} 8 & 5 & \mathrm{D} 7 & 0\end{array}$

$\begin{array}{llll}\text { A9 } & 5 & \text { D8 } & 0\end{array}$

A10 $5 \quad$ D9 0

$\begin{array}{llll}\text { A11 } 12 & \text { D10 } & 0\end{array}$

A12 11 D11 0

A13 $1 \quad$ D12 0

A14 11 D13 0

A15 4 D14 0

A16 $13 \quad$ D15 0 


$\begin{array}{lr}\text { B17 } & 0.96665240 \\ \text { A1 } & 106.91873974 \\ \text { A2 } & 98.51220404 \\ \text { A3 } & 112.18553269 \\ \text { A4 } & 108.73508948 \\ \text { A5 } & 83.74583601 \\ \text { A6 } & 71.26994541 \\ \text { A7 } & 94.45826679 \\ \text { A8 } & 108.35761304 \\ \text { A9 } & 111.22899059 \\ \text { A10 } & 111.73604657 \\ \text { A11 } & 116.89660233 \\ \text { A12 } & 110.84006237 \\ \text { A13 } & 103.28386193 \\ \text { A14 } & 113.12151268 \\ \text { A15 } & 109.00330423 \\ \text { A16 } & 102.55150867 \\ \text { D1 } & 102.71963239 \\ \text { D2 } & -176.8299113 \\ \text { D3 } & 54.15560690 \\ \text { D4 } & 175.35081731 \\ \text { D5 } & -96.47401378 \\ \text { D6 } & -65.12487889 \\ \text { D7 } & -91.09240334 \\ \text { D8 } & 32.22709201 \\ \text { D9 } & 147.70420891 \\ \text { D10 } & 84.27230204 \\ \text { D11 } & 17.90426513 \\ \text { D12 } & 138.88468532 \\ \text { D13 } & -106.44822035 \\ \text { D14 } & 90.22906792 \\ \text { D15 } & -81.53657170 \\ \text { Harti }\end{array}$

Harmonic Vibrational Frequencies $\left(\mathbf{c m}^{-1}\right)$ :

$\begin{array}{lllllllllll}420.24 i & 24.74 & 53.34 & 92.84 & 109.60 & 150.44 & 169.51 & 196.96 & 328.73 & 363.63 & 395.24\end{array}$ $\begin{array}{llllllllll}402.67 & 468.24 & 500.11 & 569.79 & 640.57 & 667.15 & 850.24 & 898.34 & 910.17 & 967.97\end{array}$ $\begin{array}{lllllllll}1059.22 & 1069.61 & 1125.31 & 1128.51 & 1143.47 & 1191.06 & 1201.95 & 1227.86 & 1287.64\end{array}$ $\begin{array}{llllllllll}1332.36 & 1356.94 & 1412.18 & 1420.50 & 1448.55 & 1457.98 & 1476.54 & 1499.78 & 1547.63\end{array}$ $\begin{array}{lllllllll}1610.13 & 2187.80 & 3076.48 & 3116.86 & 3146.00 & 3148.12 & 3188.00 & 3276.65 & 3824.95\end{array}$

Rotational constants (B/GHZ): $\quad 2.3942415 \quad 0.6652077 \quad 0.5803588$ 


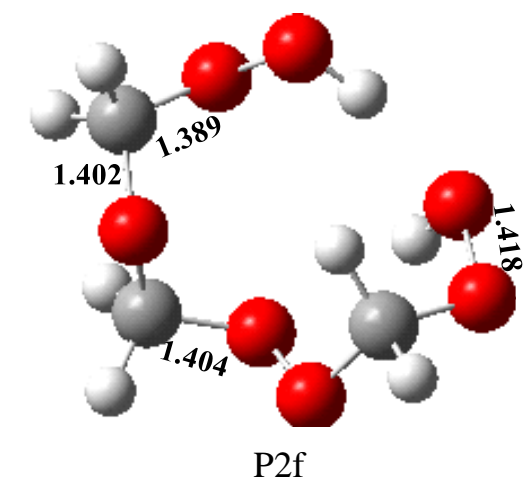

C

$\begin{array}{lll}\mathrm{O} & 1 & \mathrm{~B} 1\end{array}$

$\mathrm{H} \quad 2$

B2

B3 2

A1

O 1

C 2

$\mathrm{H} \quad 5$

B4 1

A2 3

D1 0

B5 2

A3 4

D2 0

$\mathrm{H} \quad 5$

B6 2

A4 1

D3 0

O 5

B7 2

A5 1

D4 0

O 5

B8 2

A6 1

D5 0

$\mathrm{H} \quad 1$

$\mathrm{H} \quad 1$

B9 2

A7 1

D6 0

B10 2

A8 5

D7 0

O 1

C 4

$\mathrm{H} \quad 13$

B11 2

A9 5

D8 0

B12 1

A10 5

D9 0

B13 4

A11 2

D10 0

$\mathrm{H} \quad 13$

O 13

O 16

B14 4

A12 1

D11 0

B15 4

A13 1

D12 0

A14 1

D13 0

B16 13

A15 4

D14 0

H 17

B17 16

A16 13

D15 0

Variables:

B1

1.39016151

B2

3.17128950

B3

2.28378685

1.40213902

B4

1.09381529

B6

1.08795931

2.26618927

B7

1.38905157

B9

1.08838158

B10

1.09364724

B11

1.40427350

B12

1.40134802

B13

1.08979752

B14

1.08978026

B15

1.39478933

B16

1.41782831 


$\begin{array}{lr}\text { B17 } & 0.96837901 \\ \text { A1 } & 99.81199139 \\ \text { A2 } & 100.36555267 \\ \text { A3 } & 115.28611557 \\ \text { A4 } & 110.45574588 \\ \text { A5 } & 106.18580949 \\ \text { A6 } & 101.73168033 \\ \text { A7 } & 114.36884630 \\ \text { A8 } & 107.66155829 \\ \text { A9 } & 111.69357720 \\ \text { A10 } & 113.26034996 \\ \text { A11 } & 114.69693545 \\ \text { A12 } & 110.72534321 \\ \text { A13 } & 104.18113993 \\ \text { A14 } & 112.90563740 \\ \text { A15 } & 109.43100524 \\ \text { A16 } & 103.06092293 \\ \text { D1 } & 59.59624301 \\ \text { D2 } & 125.58457954 \\ \text { D3 } & 51.64532794 \\ \text { D4 } & 172.78300804 \\ \text { D5 } & -101.57273673 \\ \text { D6 } & -65.09533819 \\ \text { D7 } & -149.81749699 \\ \text { D8 } & -26.93769359 \\ \text { D9 } & 89.86669200 \\ \text { D10 } & -28.03390935 \\ \text { D11 } & 15.68529921 \\ \text { D12 } & 136.88246327 \\ \text { D13 } & -109.46425857 \\ \text { D14 } & 83.52303734 \\ \text { D15 } & -75.21026115 \\ \text { Hart }\end{array}$

Harmonic Vibrational Frequencies $\left(\mathbf{c m}^{-1}\right)$ :

$\begin{array}{lllllllllll}52.91 & 95.56 & 111.93 & 132.16 & 178.78 & 197.26 & 236.35 & 319.29 & 371.98 & 402.47 & 446.61\end{array}$ $\begin{array}{llllllllll}486.16 & 519.34 & 582.49 & 613.86 & 667.11 & 685.08 & 895.34 & 956.86 & 957.99 & 994.35\end{array}$ $\begin{array}{lllllllll}1055.88 & 1106.03 & 1111.17 & 1126.66 & 1136.57 & 1182.62 & 1199.50 & 1224.93 & 1329.27\end{array}$ $\begin{array}{llllllllll}1338.13 & 1362.85 & 1413.36 & 1427.77 & 1444.14 & 1449.49 & 1470.95 & 1484.88 & 1505.65\end{array}$ $\begin{array}{lllllllll}1518.67 & 3082.24 & 3088.08 & 3108.05 & 3161.03 & 3166.16 & 3178.87 & 3667.48 & 3767.38\end{array}$ Rotational constants (B/GHZ): $1.7576020 \quad 1.1421941 \quad 0.8117293$ 


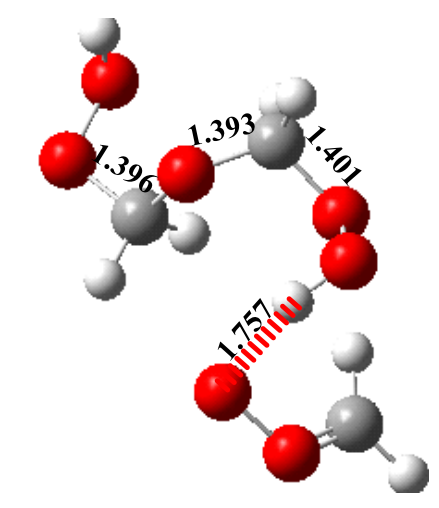

IM2g

C

$\begin{array}{lll}\mathrm{H} & 1 & \mathrm{~B} 1\end{array}$

$\mathrm{H} \quad 1$

O 1

O 1

H 4

C 4

$\begin{array}{ll}\mathrm{H} & 7\end{array}$

$\begin{array}{ll}\mathrm{H} & 7\end{array}$

$\begin{array}{ll}\text { O } & 7\end{array}$

$\begin{array}{ll}\mathrm{O} & 7\end{array}$

O 1

C 12

$\mathrm{H} \quad 13$

$\mathrm{H} \quad 13$

O 13

O 16

$\begin{array}{ll}\mathrm{H} & 17\end{array}$

Variables:

B1

B2

B3

B4

B5

B6

B7

B8

B9

B10

B11

B12

B13

B14
B2 2

A1

A2 $2 \quad$ D1 0

A3 $3 \quad$ D2 0

A4 $5 \quad$ D3 0

A5 $5 \quad$ D4 0

A6 $1 \quad$ D5 0

A7 $1 \quad$ D6 0

A8 $1 \quad$ D7 0

$\begin{array}{llllll}\mathrm{B} 10 & 4 & \mathrm{~A} 9 & 1 & \mathrm{D} 8 & 0\end{array}$

$\begin{array}{llllll}\mathrm{B} 11 & 5 & \mathrm{~A} 10 & 4 & \mathrm{D} 9 & 0\end{array}$

$\begin{array}{llllll}\mathrm{B} 12 & 1 & \mathrm{~A} 11 & 5 & \mathrm{D} 10 & 0\end{array}$

$\begin{array}{llllll}\mathrm{B} 13 & 12 & \mathrm{~A} 12 & 1 & \mathrm{D} 11 & 0\end{array}$

$\begin{array}{llllll}\mathrm{B} 14 & 12 & \mathrm{~A} 13 & 1 & \mathrm{D} 12 & 0\end{array}$

$\begin{array}{llllll}\text { B15 } & 12 & \text { A14 } & 1 & \text { D13 } & 0\end{array}$

$\begin{array}{llllll}\mathrm{B} 16 & 13 & \mathrm{~A} 15 & 12 & \mathrm{D} 14 & 0\end{array}$

$\begin{array}{llllll}\mathrm{B} 17 & 16 & \mathrm{~A} 16 & 13 & \mathrm{D} 15 & 0\end{array}$

1.09251099

1.08857672

2.28184846

1.40104938

0.98793151

2.91142732

1.08333958

1.08666208

2.22617563

1.23529145

1.39336495

1.39873873

1.09338349

1.08793991 


$\begin{array}{lr}\text { B15 } & 1.39594118 \\ \text { B16 } & 1.41932363 \\ \text { B17 } & 0.96444280 \\ \text { A1 } & 112.29161584 \\ \text { A2 } & 86.49480522 \\ \text { A3 } & 36.19947374 \\ \text { A4 } & 93.62676194 \\ \text { A5 } & 109.19439623 \\ \text { A6 } & 133.30938701 \\ \text { A7 } & 56.12162122 \\ \text { A8 } & 61.14500383 \\ \text { A9 } & 83.55764158 \\ \text { A10 } & 112.33947202 \\ \text { A11 } & 115.72248522 \\ \text { A12 } & 110.53858517 \\ \text { A13 } & 109.19011473 \\ \text { A14 } & 111.43558064 \\ \text { A15 } & 107.29833333 \\ \text { A16 } & 101.47327251 \\ \text { D1 } & 143.24263789 \\ \text { D2 } & 131.30999379 \\ \text { D3 } & -104.43033882 \\ \text { D4 } & -41.64727776 \\ \text { D5 } & 136.41637140 \\ \text { D6 } & 27.59241056 \\ \text { D7 } & -79.45326753 \\ \text { D8 } & -104.94110326 \\ \text { D9 } & -65.49269616 \\ \text { D10 } & -71.37026394 \\ \text { D11 } & 14.73887242 \\ \text { D12 } & -108.24045279 \\ \text { D13 } & \text { D14 } \\ \text { D15 } & \text { 103761 } \\ \text { D } 13990991\end{array}$

Harmonic Vibrational Frequencies $\left(\mathbf{c m}^{-1}\right)$ :

$\begin{array}{llllllllllll}46.21 & 66.36 & 76.76 & 99.71 & 117.70 & 127.51 & 164.59 & 192.81 & 207.05 & 265.81 & 296.07\end{array}$ $\begin{array}{llllllllll}349.87 & 398.31 & 491.96 & 546.01 & 598.84 & 691.64 & 702.03 & 843.86 & 908.97 & 956.44\end{array}$ $\begin{array}{lllllllll}959.98 & 1013.02 & 1098.00 & 1102.94 & 1114.39 & 1126.36 & 1196.16 & 1221.22 & 1281.06\end{array}$ $\begin{array}{llllllllll}1322.84 & 1349.48 & 1412.90 & 1415.48 & 1443.48 & 1459.35 & 1491.24 & 1516.57 & 1537.98\end{array}$ $\begin{array}{lllllllll}1659.80 & 3072.75 & 3094.81 & 3134.12 & 3165.17 & 3182.78 & 3282.19 & 3355.77 & 3832.79\end{array}$ Rotational constants (B/GHZ): $2.0751858 \quad 0.6898281 \quad 0.5863434$ 


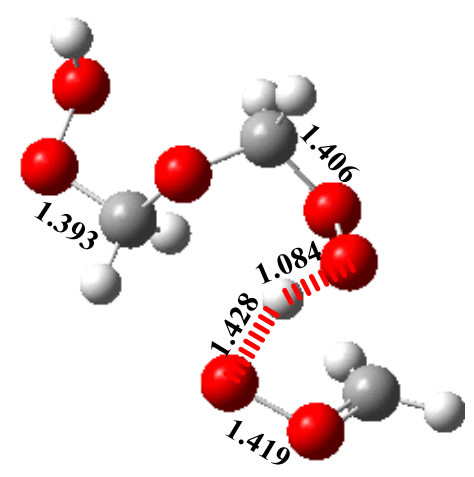

TS2g

C

$\begin{array}{lll}\mathrm{H} & 1 & \mathrm{~B} 1\end{array}$

$\mathrm{H} \quad 1$

O 1

O 1

$\mathrm{H} \quad 4$

$\begin{array}{ll}\text { C } & 4\end{array}$

$\begin{array}{ll}\mathrm{H} & 7\end{array}$

$\mathrm{H} \quad 7$

O 7

O $\quad 7$

O 1

C 12

$\mathrm{H} \quad 13$

$\mathrm{H} \quad 13$

O 13

O 16

$\begin{array}{ll}\mathrm{H} & 17\end{array}$

Variables:

B1

1.09255349

B2

1.08880208

B3

2.27735311

B4

1.40560362

B5 1.08429991

B6

2.05063373

B7

1.08531656

B8

1.08428220

B9

2.16784408

B10

1.25646182

B11

1.38989073

B12

1.40270194

B13

1.09298237

B14

1.08874137

B15

1.39321724

A1

A2 $2 \quad$ D1 0

A3 $3 \quad$ D2 0

A4 $5 \quad$ D3 0

A5 $5 \quad$ D4 0

A6 $1 \quad$ D5 0

A7 $1 \quad$ D6 0

$\begin{array}{llll}\text { A8 } & 1 & \text { D7 } & 0\end{array}$

$\begin{array}{llll}\text { A9 } & 1 & \text { D8 } & 0\end{array}$

A10 $4 \quad$ D9 0

$\begin{array}{llll}\text { A11 } 5 & \text { D10 } & 0\end{array}$

$\begin{array}{llll}\mathrm{A} 12 & 1 & \mathrm{D} 11 & 0\end{array}$

A13 $1 \quad$ D12 0

$\begin{array}{llll}\mathrm{A} 14 & 1 & \mathrm{D} 13 & 0\end{array}$

$\begin{array}{llll}\text { A15 } 12 & \text { D14 } & 0\end{array}$

A16 $13 \quad$ D15 0 


$\begin{array}{lr}\text { B16 } & 1.41990570 \\ \text { B17 } & 0.96444818 \\ \text { A1 } & 112.21428794 \\ \text { A2 } & 88.41870957 \\ \text { A3 } & 36.37581507 \\ \text { A4 } & 89.14953315 \\ \text { A5 } & 130.25346852 \\ \text { A6 } & 109.45917960 \\ \text { A7 } & 82.71542406 \\ \text { A8 } & 70.10523631 \\ \text { A9 } & 93.41373454 \\ \text { A10 } & 111.99920686 \\ \text { A11 } & 115.90753998 \\ \text { A12 } & 110.53177004 \\ \text { A13 } & 108.89039209 \\ \text { A14 } & 111.26312212 \\ \text { A15 } & 107.10448192 \\ \text { A16 } & 101.37931161 \\ \text { D1 } & 144.48717432 \\ \text { D2 } & 128.17118389 \\ \text { D3 } & -112.24729833 \\ \text { D4 } & -43.28307859 \\ \text { D5 } & 134.06370574 \\ \text { D6 } & 12.64959652 \\ \text { D7 } & -76.21104489 \\ \text { D8 } & -107.37611105 \\ \text { D9 } & -61.53055399 \\ \text { D10 } & -69.57946020 \\ \text { D11 } & 13.25499114 \\ \text { D12 } & 135.36579299 \\ \text { D13 } & -109.67520965 \\ \text { D14 } & 68.56946770 \\ \text { D15 } & \text { - } \\ \text { Dar } & \end{array}$

Harmonic Vibrational Frequencies $\left(\mathrm{cm}^{-1}\right)$ :

$\begin{array}{llllllllllll}480.25 i & 48.26 & 64.12 & 72.78 & 115.01 & 156.90 & 178.67 & 219.92 & 297.00 & 334.03 & 382.58\end{array}$ $\begin{array}{llllllllll}399.30 & 444.36 & 474.42 & 558.72 & 597.44 & 702.54 & 851.96 & 910.73 & 956.36 & 967.34\end{array}$ $\begin{array}{lllllllll}1004.76 & 1081.33 & 1109.81 & 1129.81 & 1168.14 & 1194.16 & 1218.94 & 1228.05 & 1263.09\end{array}$ $\begin{array}{lllllllll}1319.98 & 1344.81 & 1413.67 & 1420.53 & 1430.76 & 1445.13 & 1494.17 & 1515.07 & 1579.79\end{array}$ $\begin{array}{lllllllll}1669.50 & 2011.72 & 3080.15 & 3091.69 & 3144.56 & 3158.77 & 3162.57 & 3275.66 & 3850.65\end{array}$ Rotational constants (B/GHZ): $2.5857978 \quad 0.6776697 \quad 0.5836400$ 


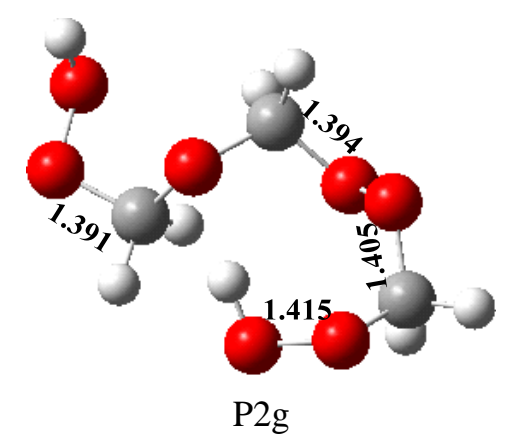

C $\begin{array}{lll}\mathrm{H} & 1 & \mathrm{~B} 1\end{array}$

$\mathrm{H} \quad 1$

O 1

O 1

$\begin{array}{ll}\mathrm{H} & 4\end{array}$

C 4

$\begin{array}{ll}\mathrm{H} & 7\end{array}$

$\begin{array}{ll}\mathrm{H} & 7\end{array}$

$\begin{array}{ll}\mathrm{O} & 7\end{array}$

$\begin{array}{ll}\mathrm{O} & 7\end{array}$

O 1

C 12

$\mathrm{H} \quad 13$

$\mathrm{H} \quad 13$

O 13

O 16

$\begin{array}{ll}\mathrm{H} & 17\end{array}$

Variables:

B1

B2

1.09103800

1.08796265

B3

2.27623751

B4

1.39395661

B5

2.62642987

B6

1.40546038

B7

1.08987311

1.08960326

B8

2.27070349

B10

1.38779544

B11

1.40283305

B12

1.40603069

B13

1.09270609

B14

1.08985450

B15

1.39054820

B16

1.41922653

B17

0.96456561 


$\begin{array}{lr}\text { A1 } & 111.79194762 \\ \text { A2 } & 80.67936969 \\ \text { A3 } & 36.74951676 \\ \text { A4 } & 69.59693642 \\ \text { A5 } & 130.07016792 \\ \text { A6 } & 106.32062282 \\ \text { A7 } & 109.22904256 \\ \text { A8 } & 99.01915591 \\ \text { A9 } & 112.71971506 \\ \text { A10 } & 112.98726579 \\ \text { A11 } & 115.04877759 \\ \text { A12 } & 110.35423705 \\ \text { A13 } & 109.40362864 \\ \text { A14 } & 111.30136070 \\ \text { A15 } & 107.01317549 \\ \text { A16 } & 101.50851226 \\ \text { D1 } & 137.94182976 \\ \text { D2 } & 143.37539711 \\ \text { D3 } & -105.16687005 \\ \text { D4 } & -64.81225627 \\ \text { D5 } & 176.29465784 \\ \text { D6 } & 54.66300917 \\ \text { D7 } & -34.81203912 \\ \text { D8 } & -69.92150218 \\ \text { D9 } & -80.59422487 \\ \text { D10 } & -77.50311667 \\ \text { D11 } & 13.94032389 \\ \text { D12 } & 135.98580146 \\ \text { D13 } & -109.32325411 \\ \text { D14 } & 68.70910568 \\ \text { D15 } & -108.04090264 \\ \text { Har } & \end{array}$

Harmonic Vibrational Frequencies $\left(\mathbf{c m}^{-1}\right)$ :

$\begin{array}{lllllllllll}55.26 & 67.33 & 95.53 & 115.19 & 145.26 & 172.37 & 197.67 & 265.66 & 319.69 & 391.55 & 393.86\end{array}$ $\begin{array}{llllllllll}445.40 & 473.18 & 516.78 & 578.66 & 627.55 & 682.22 & 897.91 & 957.76 & 971.25 & 996.07\end{array}$ $\begin{array}{lllllllll}1064.82 & 1098.79 & 1127.59 & 1132.20 & 1139.79 & 1170.32 & 1185.30 & 1228.99 & 1321.87\end{array}$ $\begin{array}{lllllllll}1336.31 & 1362.46 & 1403.43 & 1422.23 & 1423.36 & 1449.01 & 1464.02 & 1490.39 & 1508.87\end{array}$ $\begin{array}{lllllllll}1523.76 & 3082.99 & 3101.23 & 3105.61 & 3154.56 & 3170.14 & 3170.53 & 3740.03 & 3846.15\end{array}$

Rotational constants (B/GHZ): $2.6948311 \quad 0.7063897 \quad 0.5917402$ 


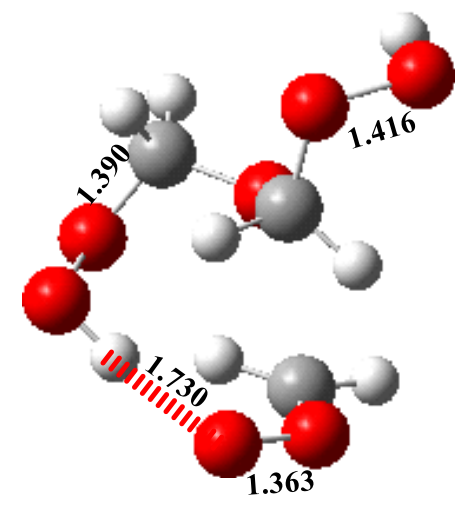

IM2h

C $\begin{array}{lll}\mathrm{H} & 1 & \mathrm{~B} 1\end{array}$

O 1

O 1

$\mathrm{H} \quad 3$

C 4

O 6

O 6

$\mathrm{H} \quad 6$

O 1

C $\quad 10$

$\mathrm{H} \quad 11$

O 11

O 13

$\mathrm{H} \quad 14$

$\mathrm{H} \quad 6$

$\mathrm{H} \quad 1$

$\mathrm{H} \quad 11$

$$
\text { B2 }
$$

B3 3

A1

B4 1

A2 2

D1 0

A3 4

D2 0

B5 1

A4 3

D3 0

B6 4

A5 1

D4 0

B7 4

A6 1

D5 0

B8 4

A7 1

D6 0

B9 4

A8 3

D7 0

B10 1

A9 4

D8 0

B11 10

A10 1

D9 0

B12 10

A11 1

D10 0

B13 11

A12 10

D11 0

B14 13

A13 11

D12 0

B15 4

A14 1

D13 0

B16 4

A15 3

D14 0

B17 10

A16 1

D15 0

Variables:

B1

B2

B3

B4

B5

B6

B7

B8

B9

B10

B11

B12

B13

B14
1.08971566

2.27253275

1.39010865

0.98769047

3.15440970

2.22974974

1.23417325

1.08321969

1.40755424

1.40860821

1.09000645

1.39385093

1.41648418

0.96491137 


$\begin{array}{lr}\text { B15 } & 1.08567381 \\ \text { B16 } & 1.09194504 \\ \text { B17 } & 1.08792016 \\ \text { A1 } & 139.89223970 \\ \text { A2 } & 36.43624281 \\ \text { A3 } & 99.55580024 \\ \text { A4 } & 89.68900009 \\ \text { A5 } & 73.26297427 \\ \text { A6 } & 103.02761207 \\ \text { A7 } & 134.13522847 \\ \text { A8 } & 111.47502245 \\ \text { A9 } & 115.76480717 \\ \text { A10 } & 111.52522327 \\ \text { A11 } & 111.23720722 \\ \text { A12 } & 107.81788764 \\ \text { A13 } & 102.44573130 \\ \text { A14 } & 29.50851920 \\ \text { A15 } & 110.39820795 \\ \text { A16 } & 106.82828904 \\ \text { D1 } & -19.06547212 \\ \text { D2 } & 97.63418694 \\ \text { D3 } & -93.77028877 \\ \text { D4 } & 100.08621750 \\ \text { D5 } & 86.63980538 \\ \text { D6 } & -59.75685416 \\ \text { D7 } & 75.91639981 \\ \text { D8 } & -47.28301404 \\ \text { D9 } & 160.41736294 \\ \text { D10 } & -78.72373717 \\ \text { D11 } & 8.63424103 \\ \text { D12 } & \text { D13 } \\ \text { D14 } & \text { D15 }\end{array}$

Harmonic Vibrational Frequencies $\left(\mathbf{c m}^{-1}\right)$ :

$\begin{array}{lllllllllll}63.01 & 96.56 & 101.18 & 112.73 & 135.00 & 150.20 & 172.78 & 189.46 & 238.90 & 249.70 & 259.90\end{array}$ $\begin{array}{llllllllll}342.47 & 419.74 & 517.21 & 559.22 & 591.11 & 689.18 & 703.57 & 759.75 & 917.57 & 956.05\end{array}$ $\begin{array}{lllllllll}964.42 & 985.14 & 1086.25 & 1095.61 & 1118.11 & 1140.32 & 1178.22 & 1222.63 & 1276.04\end{array}$ $\begin{array}{lllllllll}1324.77 & 1351.96 & 1418.18 & 1429.22 & 1443.23 & 1462.70 & 1482.44 & 1507.39 & 1606.93\end{array}$ $\begin{array}{lllllllll}1669.06 & 3089.67 & 3114.87 & 3141.22 & 3156.10 & 3184.69 & 3288.70 & 3334.45 & 3825.83\end{array}$ Rotational constants (B/GHZ): $1.5653054 \quad 0.8960914 \quad 0.7090610$ 


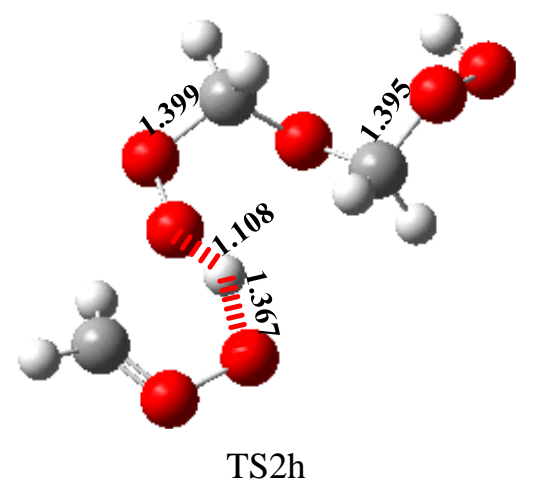

C

$\begin{array}{lll}\mathrm{H} & 1 & \mathrm{~B} 1\end{array}$

O 1

B2

B3 3

A1

O 1

B4 1

$\begin{array}{llll}\mathrm{A} 2 & 2 & \mathrm{D} 1 & 0\end{array}$

$\mathrm{H} \quad 3$

B5 1

A3 4

D2 0

B6 3

A4 4

D3 0

O 6

O 6

$\mathrm{H} \quad 6$

O 1

C $\quad 10$

B7 3

A5 1

D4 0

A6 1

D5 0

B8 3

D6 0

$\begin{array}{llllll}\text { B9 } & 4 & \text { A8 } & 3 & \text { D7 } & 0\end{array}$

$\mathrm{H} \quad 11$

B10 1

A9 4

D8 0

B11 10

A10 1

D9 0

B12 10

A11 1

D10 0

B13 11

A12 10

D11 0

B14 13

A13 11

D12 0

B15 3

A14 1

D13 0

B16 10

A15 11

D14 0

B17 10

A16 1

D15 0

Variables:

$\begin{array}{ll}\text { B1 } & 1.08918921 \\ \text { B2 } & 2.26991947 \\ \text { B3 } & 1.39919125 \\ \text { B4 } & 1.10798769 \\ \text { B5 } & 2.08121138 \\ \text { B6 } & 2.16695998 \\ \text { B7 } & 1.25553177 \\ \text { B8 } & 1.08509206 \\ \text { B9 } & 1.39446402 \\ \text { B10 } & 1.40449342 \\ \text { B11 } & 1.09180998 \\ \text { B12 } & 1.39458612 \\ \text { B13 } & 1.41596698 \\ \text { B14 } & 0.96581822 \\ \text { B15 } & 1.08455134 \\ \text { B16 } & 1.09333226\end{array}$




$\begin{array}{lr}\text { B17 } & 1.08792582 \\ \text { A1 } & 139.97834002 \\ \text { A2 } & 36.53355088 \\ \text { A3 } & 92.52524319 \\ \text { A4 } & 131.57776929 \\ \text { A5 } & 68.93566771 \\ \text { A6 } & 92.09186719 \\ \text { A7 } & 110.47761309 \\ \text { A8 } & 111.40216306 \\ \text { A9 } & 116.18639986 \\ \text { A10 } & 111.90979077 \\ \text { A11 } & 111.29608539 \\ \text { A12 } & 107.67231763 \\ \text { A13 } & 102.55390087 \\ \text { A14 } & 81.75860943 \\ \text { A15 } & 111.00467033 \\ \text { A16 } & 106.57033144 \\ \text { D1 } & -9.45173549 \\ \text { D2 } & 110.86891332 \\ \text { D3 } & 43.13314291 \\ \text { D4 } & 81.24394911 \\ \text { D5 } & 112.60440441 \\ \text { D6 } & -129.13157205 \\ \text { D7 } & 68.99287148 \\ \text { D8 } & -104.42811431 \\ \text { D9 } & 35.45960935 \\ \text { D10 } & -80.98099262 \\ \text { D11 } & -70.40695770 \\ \text { D12 } & 78.26282618 \\ \text { D13 } & -7.74798712 \\ \text { D14 } & 18.60760720 \\ \text { D15 } & 158.05968250 \\ \text { Har } & \end{array}$

Harmonic Vibrational Frequencies $\left(\mathbf{c m}^{-1}\right)$ :

$\begin{array}{lllllllllll}549.49 i & 33.55 & 67.89 & 76.07 & 94.02 & 136.29 & 160.73 & 195.71 & 270.92 & 351.39 & 370.04\end{array}$ $\begin{array}{llllllllll}408.02 & 449.10 & 510.08 & 551.76 & 598.56 & 688.47 & 842.37 & 912.08 & 960.19 & 964.27\end{array}$ $\begin{array}{lllllllll}1000.43 & 1073.52 & 1110.24 & 1120.12 & 1154.09 & 1189.09 & 1219.79 & 1233.45 & 1299.58\end{array}$ $\begin{array}{lllllllll}1326.35 & 1354.13 & 1414.09 & 1428.17 & 1444.30 & 1456.05 & 1488.19 & 1506.86 & 1514.47\end{array}$ $\begin{array}{lllllllll}1636.06 & 1931.93 & 3075.48 & 3101.07 & 3149.11 & 3150.66 & 3175.01 & 3282.00 & 3801.91\end{array}$ Rotational constants (B/GHZ): $2.6170536 \quad 0.6374371 \quad 0.5516667$ 


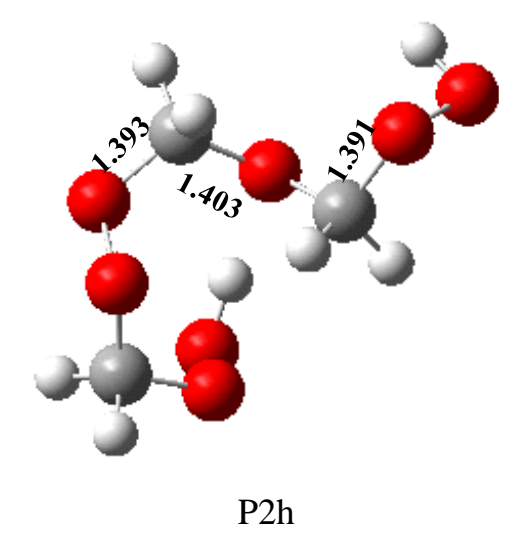

C

$\begin{array}{rr}\mathrm{H} & 1 \\ \mathrm{O} & 1 \\ \mathrm{O} & 1 \\ \mathrm{H} & 4 \\ \mathrm{C} & 3 \\ \mathrm{O} & 6 \\ \mathrm{O} & 6 \\ \mathrm{H} & 6 \\ \mathrm{O} & 1 \\ \mathrm{C} & 10 \\ \mathrm{H} & 11 \\ \mathrm{O} & 11 \\ \mathrm{O} & 13 \\ \mathrm{H} & 14 \\ \mathrm{H} & 6 \\ \mathrm{H} & 1 \\ \mathrm{H} & 11\end{array}$

B1

B2 2

A1

B3 3

$\begin{array}{llll}\mathrm{A} 2 & 2 & \mathrm{D} 1 & 0\end{array}$

B4 1

A3 $3 \quad$ D2 0

B5 1

A4 4 D3 0

B6 3

A5 $1 \quad$ D4 0

B7 3

$\begin{array}{llll}\text { A6 } & 1 & \text { D5 } & 0\end{array}$

B8 3

A7 $1 \quad$ D6 0

B9 4

A8 3

D7 0

B10 1

$\begin{array}{llll}\text { A9 } & 4 & \text { D8 } & 0\end{array}$

$\begin{array}{llllll}\mathrm{B} 11 & 10 & \mathrm{~A} 10 & 1 & \mathrm{D} 9 & 0\end{array}$

B12 10

$\begin{array}{llll}\text { A11 } & 1 & \text { D10 } & 0\end{array}$

B13 11

A12 $10 \quad$ D11 0

B14 13

A13 11

D12 0

B15 3

A14 1

D13 0

B16 4

A15 3

D14 0

B17 10

A16 1

D15 0

Variables:

B1

B2

B3

B4

B5

B6

B7

B8

B9

B10

B11

B12

B13

B14

B15
1.08877532

2.26930949

1.39311289

2.60325504

1.40328883

2.29327111

1.39002715

1.09013041

1.40308882

1.41270503

1.08960069

1.39064916

1.41592648

0.96542230

1.08910671 


$\begin{array}{lr}\text { B16 } & 1.09235409 \\ \text { B17 } & 1.08800381 \\ \text { A1 } & 139.83750564 \\ \text { A2 } & 36.90786676 \\ \text { A3 } & 79.00641048 \\ \text { A4 } & 122.95847928 \\ \text { A5 } & 110.44440725 \\ \text { A6 } & 113.70385650 \\ \text { A7 } & 103.51745521 \\ \text { A8 } & 111.84987135 \\ \text { A9 } & 115.80114981 \\ \text { A10 } & 111.29150182 \\ \text { A11 } & 111.00868536 \\ \text { A12 } & 107.75688776 \\ \text { A13 } & 102.58653419 \\ \text { A14 } & 110.46809897 \\ \text { A15 } & 110.60726161 \\ \text { A16 } & 106.54763988 \\ \text { D1 } & -16.83301383 \\ \text { D2 } & -86.30584833 \\ \text { D3 } & 75.00811407 \\ \text { D4 } & 3.82125513 \\ \text { D5 } & 42.23051816 \\ \text { D6 } & 156.99521408 \\ \text { D7 } & 74.16713403 \\ \text { D8 } & -102.87408219 \\ \text { D9 } & 35.50686411 \\ \text { D10 } & -81.40705961 \\ \text { D11 } & -71.64962251 \\ \text { D12 } & 84.40368265 \\ \text { D13 } & -82.27628607 \\ \text { D14 } & 157.60846268 \\ \text { D15 } & \text {. }\end{array}$

Harmonic Vibrational Frequencies $\left(\mathrm{cm}^{-1}\right)$ :

$\begin{array}{lllllllllll}62.61 & 95.37 & 96.61 & 137.04 & 165.34 & 183.54 & 223.39 & 271.71 & 321.30 & 344.66 & 438.00\end{array}$ $\begin{array}{llllllllll}485.19 & 515.12 & 548.16 & 601.37 & 641.25 & 691.64 & 891.03 & 963.61 & 965.40 & 981.57\end{array}$ $\begin{array}{lllllllll}1063.41 & 1092.97 & 1125.06 & 1130.25 & 1136.19 & 1168.25 & 1198.38 & 1224.40 & 1320.88\end{array}$ $\begin{array}{lllllllll}1342.85 & 1355.74 & 1408.88 & 1422.13 & 1434.65 & 1445.05 & 1470.08 & 1484.39 & 1494.07\end{array}$ $\begin{array}{lllllllll}1511.90 & 3087.34 & 3114.50 & 3118.45 & 3158.17 & 3184.10 & 3186.59 & 3701.23 & 3814.21\end{array}$

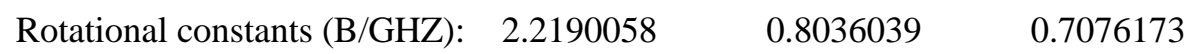


Figure S6. Optimized geometries, z-matrices and vibrational frequencies of all stationary points in the reaction of 2anti- $\mathrm{CH}_{3} \mathrm{CHOO}$ with $\mathrm{HO}-\mathrm{CH}\left(\mathrm{CH}_{3}\right) \mathrm{OO}-\mathrm{H}\left(\mathrm{Pa}_{2}\right.$ and $\left.\mathrm{Pb}_{2}\right)$ at the M06-2X/6-311+G(2df,2p) level of theory

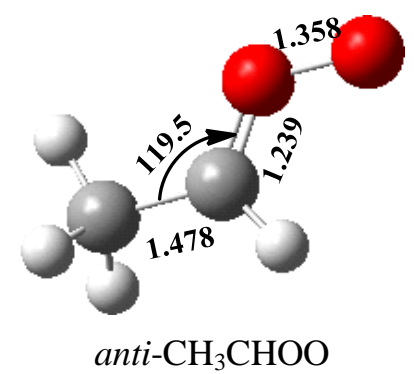

$\mathrm{C}$

C $\quad 1 \quad$ B1

$\begin{array}{lllll}\mathrm{O} & 2 & \mathrm{~B} 2 & 1 & \mathrm{~A} 1\end{array}$

$\begin{array}{llllllll}\mathrm{O} & 3 & \mathrm{~B} 3 & 2 & \mathrm{~A} 2 & 1 & \mathrm{D} 1 & 0\end{array}$

$\begin{array}{lllllllll}\mathrm{H} & 2 & \mathrm{~B} 4 & 1 & \mathrm{~A} 3 & 3 & \mathrm{D} 2 & 0\end{array}$

$\begin{array}{llllllll}\mathrm{H} & 1 & \mathrm{~B} 5 & 2 & \mathrm{~A} 4 & 3 & \mathrm{D} 3 & 0\end{array}$

$\begin{array}{llllllll}\mathrm{H} & 1 & \mathrm{~B} 6 & 2 & \mathrm{~A} 5 & 3 & \mathrm{D} 4 & 0\end{array}$

$\begin{array}{llllllll}\mathrm{H} & 1 & \mathrm{~B} 7 & 2 & \mathrm{~A} 6 & 3 & \mathrm{D} 5 & 0\end{array}$

Variables:

B1 1.47773142

B2 1.23946346

B3 $\quad 1.35750070$

B4 $\quad 1.08771168$

B5 1.08635238

B6 1.09128861

B7 1.09128861

A1 $\quad 119.45495955$

A2 118.49336247

A3 $\quad 124.16595089$

A4 110.51284798

A5 109.76373093

A6 $\quad 109.76373093$

D1 $\quad 180.00000000$

D2 180.00000000

D3 0.00000000

D4 $\quad 121.23944242$

D5 $\quad-121.23944242$

Harmonic Vibrational Frequencies $\left(\mathbf{c m}^{-1}\right)$ :

$\begin{array}{llllllllll}123.60 & 267.33 & 326.90 & 569.53 & 885.58 & 907.63 & 994.50 & 1094.19 & 1168.65 & 1361.95\end{array}$ $\begin{array}{llllllll}1421.37 & 1464.01 & 1466.30 & 1669.61 & 3041.35 & 3100.89 & 3154.85 & 3185.97\end{array}$

Rotational constants (B/GHZ): $50.3397406 \quad 4.4856969 \quad 4.2257340$ 


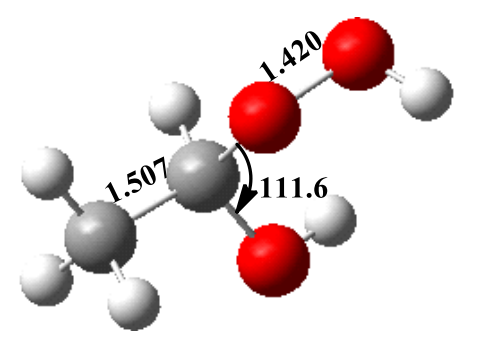

$\mathrm{Pa} 2$ (anti-HO-C( $\left.\left(\mathrm{CH}_{3}\right) \mathrm{HOO}-\mathrm{H}\right)$

C

$\begin{array}{lll}\mathrm{O} & 1 & \mathrm{~B} 1\end{array}$

O 2

$\mathrm{H} \quad 1$

B2 1

B3 2

A1

O 1

B4 2

A2 $3 \quad$ D1 0

$\mathrm{H} \quad 3$

B5 2

A3 $3 \quad$ D2 0

$\mathrm{H} \quad 5$

A4 1 D3 0

C 1

B6 1

A5 $2 \quad$ D4 0

$\mathrm{H} \quad 8$

B7 5

A6 2 D5 0

$\mathrm{H} \quad 8$

B8 1

A7 5 D6 0

$\mathrm{H} \quad 8$

B9 1

A8 $5 \quad \mathrm{D} 7 \quad 0$

Variables:

B1

B10 1

A9 $5 \quad$ D8 0

B2

1.41569532

B3

1.42017416

B4

1.09482603

1.39030971

B5

0.96410626

B6

0.96025509

B7

1.50671757

B8

1.08714989

B9

1.08735218

B10

1.08861151

A1

106.67614608

A2

107.36440783

A3

111.57568824

A4

101.25233335

A5

108.50688635

A6

109.19015556

A7

110.32004034

A8

109.28533909

A9

109.19173123

D1

$-56.73123655$

D2

64.50648454

D3

$-115.03145039$

D4

$-73.83304950$

D5

$-116.37221789$ 
D6

176.51039554

D7

$-63.03391591$

D8

56.09516575

Harmonic Vibrational Frequencies $\left(\mathbf{c m}^{-1}\right)$ :

$\begin{array}{lccccccccr}168.88 & 206.99 & 270.98 & 301.52 & 386.96 & 487.11 & 542.40 & 621.29 & 881.39 & 944.49 \\ 1050.37 & 1085.29 & 1149.31 & 1197.29 & 1287.22 & 1382.43 & 1405.19 & 1423.97 & 1474.84 \\ 1490.31 & 1497.81 & 3062.41 & 3076.94 & 3159.39 & 3166.55 & 3841.24 & 3901.81 & \\ \text { Rotational constants (B/GHZ): } & 8.9648260 & 4.0384667 & 3.0258813 & \end{array}$

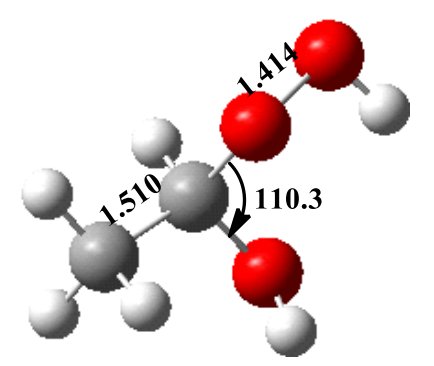

$\mathrm{Pb} 2\left(\right.$ anti-HO-C$\left.\left(\mathrm{CH}_{3}\right) \mathrm{HOO}-\mathrm{H}\right)$

$\mathrm{C}$

$\begin{array}{lll}\mathrm{O} & 1 & \mathrm{~B} 1\end{array}$

$\begin{array}{lll}\mathrm{O} & 2 & \mathrm{~B} 2\end{array}$

$\mathrm{H} \quad 1$

A1

O 1

B3 2

A2

B4 2

A3 3

D1 0

$\mathrm{H} \quad 3$

B5 2

A4 1

D2 0

$\mathrm{H} \quad 5$

B6 1

D3 0

C 1

A5

B7 5

D4 0

$\mathrm{H} \quad 8$

B8 1

A6 2 D5 0

$\mathrm{H} \quad 8$

A7 5

D6 0

B9 1

A8 $5 \quad$ D7 0

H 8

B10 1

A9 5

D8 0

Variables:

$\begin{array}{lr}\text { B1 } & 1.40563851 \\ \text { B2 } & 1.41436283 \\ \text { B3 } & 1.09042144 \\ \text { B4 } & 1.40172694 \\ \text { B5 } & 0.96713594 \\ \text { B6 } & 0.96232772 \\ \text { B7 } & 1.51031333 \\ \text { B8 } & 1.08742974 \\ \text { B9 } & 1.08760410 \\ \text { B10 } & 1.09109605 \\ \text { A1 } & 107.90919012 \\ \text { A2 } & 108.92681699 \\ \text { A3 } & 110.31500838 \\ \text { A4 } & 102.43358095 \\ \text { A5 } & 108.67336606 \\ \text { A6 } & 113.23886840\end{array}$




$\begin{array}{lc}\text { A7 } & 110.39831349 \\ \text { A8 } & 109.43760934 \\ \text { A9 } & 109.91873676 \\ \text { D1 } & -49.35090905 \\ \text { D2 } & 66.01742464 \\ \text { D3 } & -69.81772721 \\ \text { D4 } & 73.46672198 \\ \text { D5 } & -118.58772121 \\ \text { D6 } & -178.27029831 \\ \text { D7 } & -57.81897386 \\ \text { D8 } & 62.08270738\end{array}$

\section{Harmonic Vibrational Frequencies $\left(\mathrm{cm}^{-\mathbf{1}}\right)$ :}

$\begin{array}{llllllllll}168.12 & 232.23 & 288.62 & 363.46 & 403.13 & 437.44 & 552.43 & 615.43 & 884.89 & 938.21\end{array}$ $\begin{array}{lllllllll}1058.32 & 1081.91 & 1145.96 & 1190.98 & 1305.12 & 1375.74 & 1414.66 & 1446.95 & 1450.02\end{array}$ $\begin{array}{llllllll}1492.38 & 1500.00 & 3064.20 & 3122.16 & 3147.46 & 3168.68 & 3791.89 & 3868.85\end{array}$

Rotational constants (B/GHZ): $8.7844581 \quad 4.0553836 \quad 3.0361122$

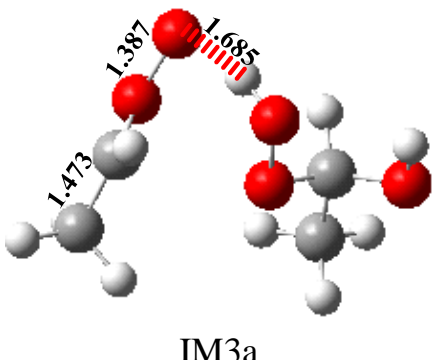

$\mathrm{C}$

C 11

$\begin{array}{lllll}\mathrm{H} & 2 & \mathrm{~B} 2 & 1 & \mathrm{~A} 1\end{array}$

$\mathrm{O} \quad 2$

$\mathrm{O} \quad 2$

B3 $1 \quad$ A2

B4 $1 \quad$ A3

A2 $3 \quad$ D1 0

$\mathrm{H} \quad 4$

B5 2

4

D2 0

C 5

B6 2

A4 1

D3 0

C 7

B7 5

A5 1

D4 0

$\mathrm{H} \quad 8$

B8 7

A6 2

D5 0

H 8

B9 7

A7 5

D6 0

H 8

A8

D7 0

O 7

$\begin{array}{ll}\text { O } & 7\end{array}$

B10 7

A9 5

D8 0

$\begin{array}{llllll}\mathrm{B} 11 & 5 & \mathrm{~A} 10 & 2 & \mathrm{D} 9 & 0\end{array}$

$\mathrm{H} \quad 7$

B12 5

A11 2

D10 0

$\mathrm{H} \quad 1$

$\mathrm{H} \quad 1$

B13 5

A12 2

D11 0

B14 2

A13 5

D12 0

B15 2

A14 5

D13 0

$\mathrm{H} \quad 1$

O 2

B16 2

A15 5

D14 0

$\mathrm{H} \quad 18$

B17 1

A16 5

D15 0

B18 2

A17 1

D16 0

Variables:

B1 


$\begin{array}{lr}\text { B2 } & 1.09588134 \\ \text { B3 } & 2.28606161 \\ \text { B4 } & 1.41705760 \\ \text { B5 } & 0.99701842 \\ \text { B6 } & 2.59897264 \\ \text { B7 } & 1.47301445 \\ \text { B8 } & 1.08601017 \\ \text { B9 } & 1.09244501 \\ \text { B10 } & 1.09033213 \\ \text { B11 } & 2.23959042 \\ \text { B12 } & 1.23746317 \\ \text { B13 } & 1.08743103 \\ \text { B14 } & 1.08744946 \\ \text { B15 } & 1.08897381 \\ \text { B16 } & 1.08887468 \\ \text { B17 } & 1.38882283 \\ \text { B18 } & 0.96279214 \\ \text { A1 } & 112.13754806 \\ \text { A2 } & 142.64497767 \\ \text { A3 } & 106.41150456 \\ \text { A4 } & 95.72684391 \\ \text { A5 } & 125.61685596 \\ \text { A6 } & 108.65017375 \\ \text { A7 } & 111.10967367 \\ \text { D8 } 8 & 107.60088200 \\ \text { D10 } & 109.81576832 \\ \text { A9 } & 75.56088665 \\ \text { A10 } & 88.94494959 \\ \text { A11 } & 71.51387444 \\ \text { A12 } & 109.20222614 \\ \text { A13 } & 109.39308447 \\ \text { A14 } & 110.34489610 \\ \text { A15 } & 109.16737299 \\ \text { A16 } & 107.36515794 \\ \text { A17 } & 111.71784539 \\ \text { D1 } & \end{array}$




$\begin{array}{lr}\text { D11 } & -151.68081177 \\ \text { D12 } & -178.87559666 \\ \text { D13 } & -59.80323837 \\ \text { D14 } & 61.32949895 \\ \text { D15 } & 119.67301473 \\ \text { D16 } & -174.10957451\end{array}$

Harmonic Vibrational Frequencies $\left(\mathbf{c m}^{-\mathbf{1}}\right)$ :

$\begin{array}{lccccccccccr}47.71 & 59.00 & 76.97 & 107.97 & 188.52 & 192.14 & 220.40 & 246.89 & 252.67 & 298.71 & 308.41 \\ 360.37 & 383.00 & 440.77 & 550.52 & 568.35 & 626.28 & 874.46 & 886.13 & 896.09 & 933.03 \\ 946.12 & 997.64 & 1045.06 & 1083.50 & 1122.51 & 1158.81 & 1184.54 & 1203.62 & 1292.90 \\ 1382.97 & 1395.65 & 1408.10 & 1431.47 & 1468.30 & 1475.05 & 1477.58 & 1493.81 & 1496.03 \\ 1593.99 & 1694.84 & 3061.88 & 3066.98 & 3067.59 & 3134.37 & 3147.02 & 3158.88 & 3167.83 \\ 3174.55 & 3199.28 & 3864.38 & & & & & & \\ \text { Rotational constants (B/GHZ): } & 2.2080409 & 1.0023933 & 0.8428133 & & \end{array}$

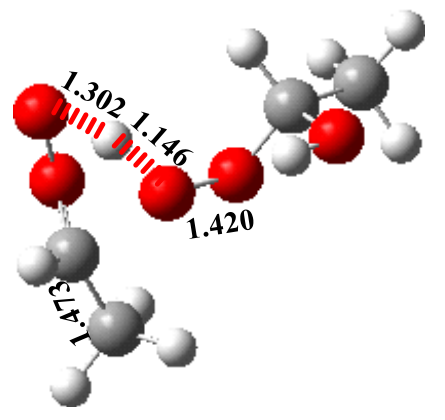

TS3a

$\mathrm{C}$

$\begin{array}{lll}\mathrm{C} & 1 & \mathrm{~B} 1\end{array}$

$\begin{array}{lllll}\mathrm{H} & 2 & \mathrm{~B} 2 & 1 & \mathrm{~A} 1\end{array}$

$\begin{array}{llllllll}\mathrm{O} & 2 & \mathrm{~B} 3 & 1 & \mathrm{~A} 2 & 3 & \mathrm{D} 1 & 0\end{array}$

$\begin{array}{llllllll}\mathrm{O} & 2 & \mathrm{~B} 4 & 1 & \mathrm{~A} 3 & 4 & \mathrm{D} 2 & 0\end{array}$

$\begin{array}{llllllll}\mathrm{H} & 4 & \mathrm{~B} 5 & 2 & \mathrm{~A} 4 & 1 & \mathrm{D} 3 & 0\end{array}$

$\begin{array}{llllllll}\mathrm{C} & 4 & \mathrm{~B} 6 & 2 & \mathrm{~A} 5 & 1 & \mathrm{D} 4 & 0\end{array}$

$\begin{array}{llllllll}\mathrm{C} & 7 & \mathrm{~B} 7 & 4 & \mathrm{~A} 6 & 2 & \mathrm{D} 5 & 0\end{array}$

$\begin{array}{llllllll}\mathrm{H} & 8 & \mathrm{~B} 8 & 7 & \mathrm{~A} 7 & 4 & \text { D6 } & 0\end{array}$

$\begin{array}{llllllll}\mathrm{H} & 8 & \mathrm{~B} 9 & 7 & \mathrm{~A} 8 & 4 & \mathrm{D} 7 & 0\end{array}$

$\begin{array}{llllllll}\mathrm{H} & 8 & \mathrm{~B} 10 & 7 & \mathrm{~A} 9 & 4 & \mathrm{D} 8 & 0\end{array}$

$\begin{array}{llllllll}\mathrm{O} & 7 & \mathrm{~B} 11 & 4 & \mathrm{~A} 10 & 2 & \mathrm{D} 9 & 0\end{array}$

$\begin{array}{llllllll}\mathrm{O} & 7 & \mathrm{~B} 12 & 4 & \mathrm{~A} 11 & 2 & \mathrm{D} 10 & 0\end{array}$

$\begin{array}{lllllllll}\mathrm{H} & 7 & \mathrm{~B} 13 & 4 & \mathrm{~A} 12 & 2 & \mathrm{D} 11 & 0\end{array}$

$\begin{array}{llllllll}\mathrm{H} & 1 & \mathrm{~B} 14 & 2 & \mathrm{~A} 13 & 5 & \mathrm{D} 12 & 0\end{array}$

$\begin{array}{llllllll}\mathrm{H} & 1 & \mathrm{~B} 15 & 2 & \mathrm{~A} 14 & 5 & \mathrm{D} 13 & 0\end{array}$

$\begin{array}{llllllll}\mathrm{H} & 1 & \mathrm{~B} 16 & 2 & \mathrm{~A} 15 & 5 & \mathrm{D} 14 & 0\end{array}$

$\begin{array}{llllllll}\mathrm{O} & 2 & \mathrm{~B} 17 & 1 & \mathrm{~A} 16 & 5 & \mathrm{D} 15 & 0\end{array}$

$\begin{array}{llllllll}\mathrm{H} & 18 & \mathrm{~B} 18 & 2 & \mathrm{~A} 17 & 1 & \mathrm{D} 16 & 0\end{array}$

Variables:

B1 


\begin{tabular}{|c|c|}
\hline B2 & 1.09680033 \\
\hline B3 & 2.27537540 \\
\hline B4 & 1.41267018 \\
\hline B5 & 1.14629694 \\
\hline B6 & 2.09266996 \\
\hline B7 & 1.47314801 \\
\hline B8 & 1.08562748 \\
\hline B9 & 1.09139659 \\
\hline B10 & 1.08954279 \\
\hline B11 & 2.17830566 \\
\hline B12 & 1.26509406 \\
\hline B13 & 1.08442645 \\
\hline B14 & 1.08740960 \\
\hline B15 & 1.08872873 \\
\hline B16 & 1.08728928 \\
\hline B17 & 1.39196119 \\
\hline B18 & 0.96206416 \\
\hline $\mathrm{A} 1$ & 112.12615210 \\
\hline $\mathrm{A} 2$ & 142.99993630 \\
\hline A3 & 106.45456874 \\
\hline A4 & 98.52823318 \\
\hline A5 & 132.39413035 \\
\hline A6 & 109.42652762 \\
\hline A7 & 110.69685909 \\
\hline A8 & 106.94235463 \\
\hline A9 & 110.14408341 \\
\hline A10 & 67.73088327 \\
\hline A11 & 91.56398322 \\
\hline A 12 & 81.72079728 \\
\hline A13 & 109.16316794 \\
\hline A14 & 109.19415616 \\
\hline A15 & 110.32371413 \\
\hline A16 & 109.05867000 \\
\hline A 17 & 107.56277655 \\
\hline D1 & 113.41450101 \\
\hline D2 & 3.72670173 \\
\hline D3 & -95.07909793 \\
\hline D4 & -21.63742703 \\
\hline D5 & 68.83295965 \\
\hline D6 & -98.50512974 \\
\hline D7 & 20.53512345 \\
\hline D8 & 138.04082086 \\
\hline D9 & -82.48237143 \\
\hline D10 & -52.02823463 \\
\hline
\end{tabular}




$\begin{array}{lr}\text { D11 } & -169.94234885 \\ \text { D12 } & -179.76790553 \\ \text { D13 } & -60.81273213 \\ \text { D14 } & 59.88635127 \\ \text { D15 } & 120.02083831 \\ \text { D16 } & -175.20892712\end{array}$

Harmonic Vibrational Frequencies $\left(\mathbf{c m}^{-\mathbf{1}}\right)$ :

$\begin{array}{lccccccccccr}760.59 i & 31.44 & 71.46 & 83.50 & 133.16 & 182.72 & 211.84 & 228.31 & 312.04 & 328.67 & 370.32 \\ 395.31 & 450.26 & 515.55 & 551.06 & 574.16 & 630.10 & 848.54 & 890.14 & 892.66 & 945.48 \\ 978.12 & 1004.62 & 1068.06 & 1093.53 & 1143.18 & 1167.27 & 1181.59 & 1204.03 & 1289.46 \\ 1362.14 & 1394.15 & 1399.75 & 1420.52 & 1455.39 & 1470.77 & 1471.41 & 1490.72 & 1496.52 \\ 1577.39 & 1620.43 & 1793.12 & 3050.67 & 3069.61 & 3072.46 & 3143.83 & 3152.24 & 3159.82 \\ 3180.57 & 3213.23 & 3879.88 & & & & & & \\ \text { Rotational constants (B/GHZ): } & 2.6545881 & 0.8829012 & 0.8283969 & & \end{array}$

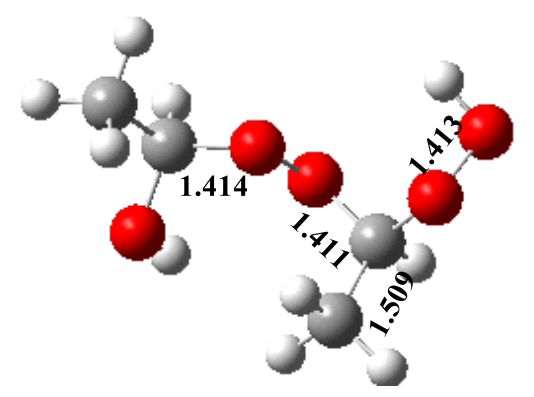

P3a

C

$\begin{array}{lll}\text { C } & 1 & \text { B1 }\end{array}$

$\mathrm{H} 2 \mathrm{~B}$

O 2

O 2

$\mathrm{H} \quad 4$

$\begin{array}{ll}\mathrm{C} & 4\end{array}$

$\begin{array}{ll}\text { C } & 7\end{array}$

$\mathrm{H} \quad 8$

$\mathrm{H} \quad 8$

$\mathrm{H} \quad 8$

$\begin{array}{ll}\mathrm{O} & 7\end{array}$

O 7

$\mathrm{H} \quad 7$

$\mathrm{H} \quad 1$

$\mathrm{H} \quad 1$

$\mathrm{H} \quad 1$

O 2

$\mathrm{H} \quad 18$

$1-A 1$

B3 1

A

B4 1

A2 3

D1 0

B5 2

A3 4

D2 0

B6 2

A4 1

D3 0

B7

A5 1

D4 0

B8 7

A6 2

D5 0

B9 7

A7 4

D6 0

B10 7

A8 4

D7 0

$\begin{array}{llll}\text { A } 9 & 4 & \text { D8 } & 0\end{array}$

$\begin{array}{llllll}\text { B11 } & 4 & \text { A10 } & 2 & \text { D9 } & 0\end{array}$

$\begin{array}{llllll}\mathrm{B} 12 & 4 & \mathrm{~A} 11 & 2 & \mathrm{D} 10 & 0\end{array}$

$\begin{array}{llllll}\mathrm{B} 13 & 4 & \mathrm{~A} 12 & 2 & \mathrm{D} 11 & 0\end{array}$

$\begin{array}{llllll}\mathrm{B} 14 & 2 & \mathrm{~A} 13 & 5 & \mathrm{D} 12 & 0\end{array}$

$\begin{array}{llllll}\mathrm{B} 15 & 2 & \mathrm{~A} 14 & 5 & \mathrm{D} 13 & 0\end{array}$

$\begin{array}{llllll}\mathrm{B} 16 & 2 & \mathrm{~A} 15 & 5 & \mathrm{D} 14 & 0\end{array}$

$\begin{array}{llllll}\text { B17 } & 1 & \text { A16 } & 5 & \text { D15 } & 0\end{array}$

Variables:

B1 


\begin{tabular}{|c|c|}
\hline B2 & 1.09491115 \\
\hline B3 & 2.26630708 \\
\hline B4 & 1.41372421 \\
\hline B5 & 2.49099596 \\
\hline B6 & 1.41142786 \\
\hline B7 & 1.50946176 \\
\hline B8 & 1.08627522 \\
\hline B9 & 1.08763812 \\
\hline B10 & 1.08753086 \\
\hline B11 & 2.27669741 \\
\hline B12 & 1.39592198 \\
\hline B13 & 1.09294165 \\
\hline B14 & 1.08715034 \\
\hline B15 & 1.08859529 \\
\hline B16 & 1.08717461 \\
\hline B17 & 1.39223791 \\
\hline B18 & 0.96066653 \\
\hline A1 & 112.35980412 \\
\hline $\mathrm{A} 2$ & 142.83491688 \\
\hline $\mathrm{A} 3$ & 105.73052198 \\
\hline A 4 & 122.40293209 \\
\hline A5 & 132.66947382 \\
\hline A6 & 114.52002117 \\
\hline A7 & 110.28259467 \\
\hline A8 & 109.60337723 \\
\hline A9 & 109.20949822 \\
\hline A 10 & 92.04386608 \\
\hline A11 & 110.87381857 \\
\hline A12 & 101.75024035 \\
\hline A13 & 109.08803930 \\
\hline A14 & 109.25921967 \\
\hline A15 & 110.45558069 \\
\hline A16 & 109.00843256 \\
\hline A17 & 108.35450949 \\
\hline D1 & 112.61435024 \\
\hline D2 & 4.50037556 \\
\hline D3 & -35.39188089 \\
\hline D4 & 66.19824516 \\
\hline D5 & 22.75594823 \\
\hline D6 & -66.60412420 \\
\hline D7 & 53.23355628 \\
\hline D8 & 173.03325184 \\
\hline D9 & -129.53463826 \\
\hline D10 & -97.90640667 \\
\hline
\end{tabular}




$\begin{array}{lr}\text { D11 } & 145.30971230 \\ \text { D12 } & 178.82165302 \\ \text { D13 } & -62.11988246 \\ \text { D14 } & 58.37736945 \\ \text { D15 } & 120.19231452 \\ \text { D16 } & 171.69769825\end{array}$

Harmonic Vibrational Frequencies $\left(\mathbf{c m}^{-1}\right)$ :

$\begin{array}{lccccccccccr}49.07 & 72.08 & 109.32 & 159.58 & 199.78 & 211.56 & 247.01 & 273.05 & 321.64 & 363.27 & 376.25 \\ 390.49 & 430.45 & 520.05 & 586.09 & 615.27 & 698.51 & 879.77 & 897.41 & 933.68 & 949.05 \\ 1013.58 & 1062.37 & 1086.76 & 1137.70 & 1150.45 & 1157.58 & 1188.85 & 1220.13 & 1291.81 \\ 1365.80 & 1391.02 & 1400.64 & 1408.40 & 1429.49 & 1441.78 & 1476.11 & 1488.89 & 1492.48 \\ 1495.08 & 1498.79 & 3066.69 & 3073.40 & 3085.37 & 3111.40 & 3153.93 & 3168.12 & 3168.81 \\ 3174.59 & 3822.63 & 3892.80 & & & & & & \end{array}$

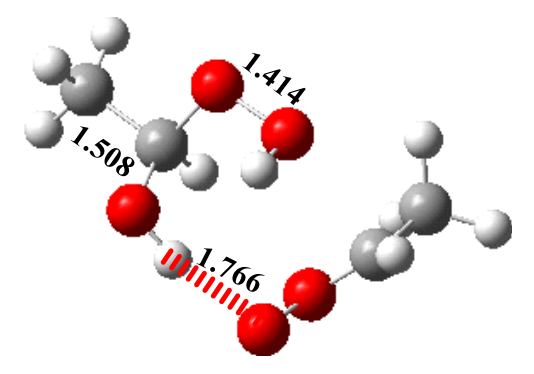

IM3b

C

$\begin{array}{lll}\mathrm{C} & 1 & \mathrm{~B} 1\end{array}$

$\begin{array}{lll}\mathrm{H} & 2 & \mathrm{~B} 2\end{array}$

$\mathrm{H} \quad 2$

$\mathrm{H} \quad 2$

O 1

$\mathrm{H} \quad 6$

O 1

C $\quad 8$

C 9

$\mathrm{H} \quad 10$

$\mathrm{H} \quad 10$

$$
\text { B3 } 1 \quad \text { A2 }
$$

A1

$\begin{array}{llllll}\text { B4 } & 1 & \text { A3 } & 3 & \text { D2 } & 0\end{array}$

$\begin{array}{llllll}\text { B5 } & 2 & \text { A4 } & 3 & \text { D3 } & 0\end{array}$

$\begin{array}{llllll}\mathrm{B} 6 & 1 & \mathrm{~A} 5 & 2 & \mathrm{D} 4 & 0\end{array}$

$\begin{array}{llllll}\text { B7 } & 6 & \text { A6 } & 2 & \text { D5 } & 0\end{array}$

$\begin{array}{llllll}\text { B8 } & 1 & \text { A7 } & 6 & \text { D6 } & 0\end{array}$

$\begin{array}{llllll}\text { B9 } & 8 & \text { A8 } & 1 & \text { D7 } & 0\end{array}$

$\mathrm{H} \quad 10$

O 9

O 9

$\mathrm{H} \quad 9$

$\mathrm{H} \quad 1$

O $\quad 8$

$\mathrm{H} \quad 8$

$\begin{array}{llllll}\text { B10 } & 9 & \text { A9 } & 8 & \text { D8 } & 0\end{array}$

$\begin{array}{llllll}\text { B11 } & 9 & \text { A10 } & 8 & \text { D9 } & 0\end{array}$

$\begin{array}{llllll}\mathrm{B} 12 & 9 & \mathrm{~A} 11 & 8 & \mathrm{D} 10 & 0\end{array}$

$\begin{array}{llllll}\text { B13 } & 8 & \text { A12 } & 1 & \text { D11 } & 0\end{array}$

$\begin{array}{llllll}\text { B14 } & 8 & \text { A13 } & 1 & \text { D12 } & 0\end{array}$

$\begin{array}{llllll}\text { B15 } & 8 & \text { A14 } & 1 & \text { D13 } & 0\end{array}$

$\begin{array}{llllll}\text { B16 } & 6 & \text { A15 } & 2 & \text { D14 } & 0\end{array}$

$\begin{array}{llllll}\text { B17 } & 1 & \text { A16 } & 6 & \text { D15 } & 0\end{array}$

Variables:

B1

B2

1.08742419 


\begin{tabular}{|c|c|}
\hline B3 & 1.08849329 \\
\hline B4 & 1.08801695 \\
\hline B5 & 1.37963044 \\
\hline B6 & 0.98140154 \\
\hline B7 & 2.29812352 \\
\hline B8 & 2.61762296 \\
\hline B9 & 1.47212864 \\
\hline B10 & 1.09291525 \\
\hline B11 & 1.09006863 \\
\hline B12 & 1.08583169 \\
\hline B13 & 2.22848650 \\
\hline B14 & 1.23644418 \\
\hline B15 & 1.08641135 \\
\hline B16 & 1.09605670 \\
\hline B17 & 1.41388068 \\
\hline B18 & 0.96988235 \\
\hline A1 & 109.04063971 \\
\hline $\mathrm{A} 2$ & 109.00976048 \\
\hline A3 & 110.83837920 \\
\hline A4 & 110.69646544 \\
\hline A5 & 109.56835659 \\
\hline A6 & 90.06949017 \\
\hline A7 & 98.04100798 \\
\hline A8 & 105.48106580 \\
\hline A9 & 107.23490174 \\
\hline A 10 & 110.05995475 \\
\hline A11 & 111.12688858 \\
\hline A12 & 78.37575326 \\
\hline A13 & 92.09357087 \\
\hline A14 & 71.68440492 \\
\hline A15 & 110.77549308 \\
\hline A16 & 36.32329653 \\
\hline A17 & 87.12518764 \\
\hline D1 & 118.80009668 \\
\hline D2 & -120.64392270 \\
\hline D3 & -64.02594521 \\
\hline D4 & 152.51063018 \\
\hline D5 & 145.58714926 \\
\hline D6 & 86.49384873 \\
\hline D7 & 93.51104707 \\
\hline D8 & 14.97659590 \\
\hline D9 & 131.71522148 \\
\hline D10 & -105.36166400 \\
\hline D11 & -59.57857943 \\
\hline
\end{tabular}




$\begin{array}{lr}\text { D12 } & -28.25144516 \\ \text { D13 } & -146.03504076 \\ \text { D14 } & -125.02492738 \\ \text { D15 } & -127.16654047 \\ \text { D16 } & -12.27407397\end{array}$

Harmonic Vibrational Frequencies $\left(\mathbf{c m}^{-\mathbf{1}}\right)$ :

$\begin{array}{lllllllllll}48.80 & 58.14 & 107.77 & 120.57 & 132.66 & 187.07 & 190.50 & 232.68 & 247.56 & 298.83 & 328.29\end{array}$ $\begin{array}{llllllllll}339.80 & 395.23 & 468.03 & 548.30 & 568.34 & 625.06 & 835.37 & 875.57 & 877.41 & 905.48\end{array}$ $\begin{array}{lllllllll}946.51 & 992.40 & 1058.26 & 1117.03 & 1122.63 & 1146.30 & 1179.94 & 1203.32 & 1366.89\end{array}$ $\begin{array}{lllllllll}1373.09 & 1393.82 & 1410.21 & 1420.47 & 1442.30 & 1462.87 & 1473.30 & 1489.60 & 1499.05\end{array}$ $\begin{array}{lllllllll}1527.16 & 1703.80 & 3052.21 & 3055.92 & 3075.25 & 3122.17 & 3157.34 & 3163.19 & 3172.25\end{array}$ $3207.48 \quad 3417.56 \quad 3748.50$

Rotational constants (B/GHZ): $2.2886834 \quad 0.9674864 \quad 0.8347377$

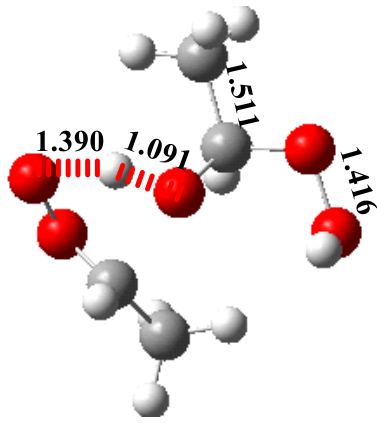

TS3b

$\mathrm{C}$

C $11 \quad$ B1

$\mathrm{H} \quad 2 \quad \mathrm{~B} 2$

$\mathrm{H} \quad 2$

B3 1

A1

$\mathrm{H} \quad 2$

O 1

B4 1

B5 2

B6 1

$\begin{array}{llll}\mathrm{A} 2 & 3 & \mathrm{D} 1 & 0\end{array}$

A3 3

D2 0

$\mathrm{H} \quad 6$

A4 5

D3 0

O 1

C 6

B7 6

A5 2

D4 0

C 9

B

A6 2

D5 0

$\mathrm{H} \quad 10$

B

A7 2

D6 0

B9 6

A8 1

D7 0

$\mathrm{H} \quad 10$

B10 9

A9 6

D8 0

$\mathrm{H} \quad 10$

B11 9

A10 6

D9 0

O 9

B12 9

A11 6

D10 0

B13 6

A12 1

D11 0

O 9

B14 6

A13 1

D12 0

H 9

B15 6

A14 1

D13 0

$\mathrm{H} \quad 1$

B16 6

A15 9

D14 0

O 1

B17 6

A16 9

D15 0

$\mathrm{H}$

B18 1

A17 6

D16 0

Variables:

B1

1.51062631

B2

1.08894606 


\begin{tabular}{|c|c|}
\hline B3 & 1.08900550 \\
\hline B4 & 1.08757170 \\
\hline B5 & 1.39929354 \\
\hline B6 & 1.09113654 \\
\hline B7 & 2.28023194 \\
\hline B8 & 2.03408399 \\
\hline B9 & 1.47661477 \\
\hline B10 & 1.09235790 \\
\hline B11 & 1.08957650 \\
\hline B12 & 1.08662645 \\
\hline B13 & 2.18171319 \\
\hline B14 & 1.26979616 \\
\hline B15 & 1.08415993 \\
\hline B16 & 1.09872965 \\
\hline B17 & 1.40485157 \\
\hline B18 & 0.96555941 \\
\hline $\mathrm{A} 1$ & 109.01941750 \\
\hline $\mathrm{A} 2$ & 109.38592021 \\
\hline A3 & 110.27797062 \\
\hline A4 & 112.40756686 \\
\hline A5 & 110.46802222 \\
\hline A6 & 89.71843740 \\
\hline A7 & 113.07122217 \\
\hline A8 & 110.52064325 \\
\hline A9 & 108.01940160 \\
\hline A10 & 109.72632567 \\
\hline A11 & 110.76039368 \\
\hline A12 & 69.24310544 \\
\hline A13 & 93.06772810 \\
\hline A14 & 82.65874760 \\
\hline A 15 & 109.66074057 \\
\hline A16 & 108.73198273 \\
\hline A17 & 99.79921535 \\
\hline D1 & 119.39230675 \\
\hline D2 & -120.28745656 \\
\hline D3 & 178.77025123 \\
\hline D4 & 36.93816828 \\
\hline D5 & 148.69453395 \\
\hline D6 & 118.44094878 \\
\hline D7 & 50.01032463 \\
\hline D8 & 19.26879146 \\
\hline D9 & 137.11342074 \\
\hline D10 & -100.69445777 \\
\hline D11 & -102.08684848 \\
\hline
\end{tabular}




$\begin{array}{lr}\text { D12 } & -71.55593788 \\ \text { D13 } & 171.06876081 \\ \text { D14 } & -6.04388695 \\ \text { D15 } & -124.25596382 \\ \text { D16 } & -27.43712274\end{array}$

Harmonic Vibrational Frequencies $\left(\mathbf{c m}^{-1}\right)$ :

$\begin{array}{lllllllllll}474.45 i & 62.09 & 97.56 & 123.53 & 165.09 & 185.11 & 208.12 & 248.10 & 295.94 & 314.49 & 362.82\end{array}$ $\begin{array}{llllllllll}393.60 & 431.70 & 507.10 & 542.37 & 560.69 & 632.44 & 865.08 & 894.08 & 942.11 & 986.23\end{array}$ $\begin{array}{lllllllll}996.69 & 1050.51 & 1094.51 & 1138.22 & 1177.78 & 1182.24 & 1194.41 & 1249.18 & 1348.53\end{array}$ $\begin{array}{llllllllll}1358.39 & 1403.00 & 1415.93 & 1423.96 & 1433.14 & 1464.67 & 1476.49 & 1481.10 & 1493.14\end{array}$ $\begin{array}{llllllllll}1513.21 & 1609.62 & 1970.27 & 3037.07 & 3059.73 & 3063.19 & 3131.25 & 3145.94 & 3155.89\end{array}$ $3167.76 \quad 3204.51 \quad 3816.84$

Rotational constants (B/GHZ): $\quad 2.1197962 \quad 1.2237817 \quad 0.8718358$

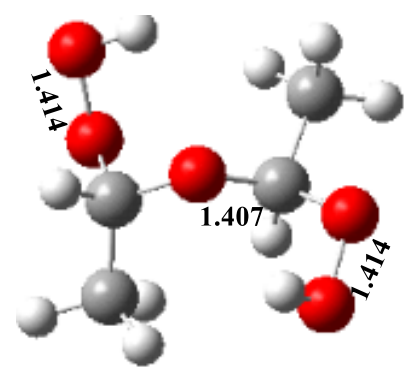

$\mathrm{P} 3 \mathrm{~b}$

$\mathrm{C}$

$\begin{array}{lll}\mathrm{C} & 1 & \mathrm{~B} 1\end{array}$

$\mathrm{H} 2$

$\mathrm{H} 2$

B2 $1 \quad$ A1

$\mathrm{H} \quad 2$

B3 1 A2

B4 $1 \quad$ A3

B5 2 A4 5

B6 $1 \quad$ A5 2

$\begin{array}{llll}\text { B7 } & 6 & \text { A6 } & 2\end{array}$

$\begin{array}{llll}\text { B8 } & 1 & \text { A7 } & 2\end{array}$

B9 $6 \quad$ A8 1

$\begin{array}{llrl}\mathrm{B} 10 & 9 & \mathrm{~A} 9 & 6 \\ \mathrm{~B} 11 & 9 & \mathrm{~A} 10 & 6\end{array}$

D1 0

O 1

$\mathrm{H} \quad 6$

O 1

C 6

C 9

$\mathrm{H} \quad 10$

$\mathrm{H} \quad 10$

B12 9

A11 6

D2 0

$\mathrm{H} \quad 10$

B13 6

A12 1

D3 0

O 9

B14 6

A13 1

D4 0

O 9

B15 6

A14 1

D5 0

$\mathrm{H} \quad 9$

B16 6

A15 9

D6 0

$\mathrm{H} \quad 1$

O 1

B17 6

A16 9

D7 0

$\mathrm{H} \quad 8$

B18 1

A17 18

D8 0

D9 0

D10 0

D11 0

D12 0

D13 0

D14 0

D15 0

Variables:

B1 1.51068400

B2 1.08948177

B3

1.08964319 


\begin{tabular}{|c|c|}
\hline B4 & 1.08797851 \\
\hline B5 & 1.41354738 \\
\hline B6 & 2.37964189 \\
\hline B7 & 2.27279279 \\
\hline B8 & 1.40730535 \\
\hline B9 & 1.51599776 \\
\hline B10 & 1.08810642 \\
\hline B11 & 1.08719265 \\
\hline B12 & 1.08776051 \\
\hline B13 & 2.28350987 \\
\hline B14 & 1.40455768 \\
\hline B15 & 1.09017820 \\
\hline B16 & 1.09117736 \\
\hline B17 & 1.39742619 \\
\hline B18 & 0.96582125 \\
\hline $\mathrm{A} 1$ & 109.25678648 \\
\hline $\mathrm{A} 2$ & 109.40120634 \\
\hline A3 & 110.35780723 \\
\hline A4 & 111.50523270 \\
\hline A5 & 102.66767236 \\
\hline A6 & 90.90151083 \\
\hline A7 & 117.72303081 \\
\hline A8 & 115.65532016 \\
\hline A9 & 109.64118902 \\
\hline A10 & 108.94687840 \\
\hline A11 & 111.64286441 \\
\hline A12 & 93.46293253 \\
\hline A13 & 110.14539866 \\
\hline A14 & 104.02643067 \\
\hline A 15 & 110.19153149 \\
\hline A16 & 107.38733033 \\
\hline A17 & 96.83812200 \\
\hline D1 & 120.45693880 \\
\hline D2 & -119.55490049 \\
\hline D3 & 174.17223229 \\
\hline D4 & 20.89587741 \\
\hline D5 & 148.84115021 \\
\hline D6 & 101.40900703 \\
\hline D7 & 53.91118029 \\
\hline D8 & 41.20979940 \\
\hline D9 & 161.02335061 \\
\hline D10 & -79.76057145 \\
\hline D11 & -99.20642815 \\
\hline D12 & -66.41172449 \\
\hline
\end{tabular}




$\begin{array}{lc}\text { D13 } & 176.82418888 \\ \text { D14 } & -23.86872240 \\ \text { D15 } & -142.63706892 \\ \text { D16 } & 100.55168646\end{array}$

Harmonic Vibrational Frequencies $\left(\mathbf{c m}^{-1}\right)$ :

$\begin{array}{lllllllllll}41.22 & 77.44 & 148.20 & 170.40 & 195.42 & 221.03 & 259.35 & 281.52 & 297.98 & 339.47 & 352.64\end{array}$ $\begin{array}{llllllllll}375.25 & 438.01 & 517.45 & 554.97 & 586.72 & 695.19 & 855.83 & 896.05 & 925.73 & 970.53\end{array}$ $\begin{array}{lllllllll}1055.61 & 1058.87 & 1107.49 & 1133.25 & 1149.10 & 1186.69 & 1194.34 & 1226.73 & 1357.54\end{array}$ $\begin{array}{lllllllll}1368.53 & 1406.03 & 1413.35 & 1429.66 & 1437.03 & 1440.74 & 1455.38 & 1487.56 & 1500.81\end{array}$ $\begin{array}{lllllllll}1501.82 & 1512.39 & 3065.42 & 3070.69 & 3119.97 & 3130.97 & 3146.88 & 3154.98 & 3158.09\end{array}$ $3165.83 \quad 3777.32 \quad 3812.77$

Rotational constants (B/GHZ): $\quad 2.4247505 \quad 1.1625174 \quad 0.8900905$

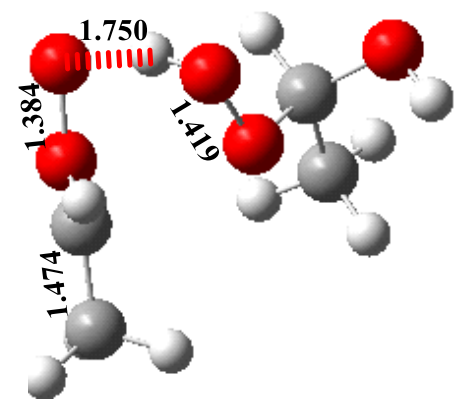

IM3c

$\mathrm{C}$

C $1 \quad$ B1

$\mathrm{H} \quad 2$

O 2

B2

O 2

B3 1

A1

$\mathrm{H} 4$

B4 1

A2

D1 0

A3 4

D2 0

C 5

A4 1

D3 0

B5 2

A5 1

D4 0

C 7

B6 2

A6 2

D5 0

$\mathrm{H} \quad 8$

B7 5

A7 5

D6 0

H 8

B8 7

A8 5

D7 0

$\mathrm{H} \quad 8$

A9 5

D8 0

O 7

B10 7

A10 2

D9 0

O 7

B11 5

A11 2

D10 0

H 7

B12 5

A12 2

D11 0

H 1

B13 5

A13 5

D12 0

$\mathrm{H} \quad 1$

B14 2

A14 5

D13 0

H 1

B15 2

A15 5

D14 0

O 2

B16 2

A16 5

D15 0

H 18

B18 2

A17 1

D16 0

Variables:

B1

1.51557311

B2

1.09090764

B3

2.31387011 


\begin{tabular}{|c|c|}
\hline B4 & 1.41340867 \\
\hline B5 & 0.98968588 \\
\hline B6 & 2.60636525 \\
\hline B7 & 1.47374587 \\
\hline B8 & 1.08615479 \\
\hline B9 & 1.09252696 \\
\hline B10 & 1.09015192 \\
\hline B11 & 2.23980231 \\
\hline B12 & 1.23764064 \\
\hline B13 & 1.08733254 \\
\hline B14 & 1.08731829 \\
\hline B15 & 1.09160500 \\
\hline B16 & 1.08941931 \\
\hline B17 & 1.38994353 \\
\hline B18 & 0.96183438 \\
\hline $\mathrm{A} 1$ & 111.96190063 \\
\hline $\mathrm{A} 2$ & 138.90040289 \\
\hline A3 & 104.91744742 \\
\hline A4 & 92.69338531 \\
\hline A5 & 121.93956092 \\
\hline A6 & 109.44285781 \\
\hline A7 & 111.06296548 \\
\hline A8 & 107.65100903 \\
\hline A9 & 109.98393190 \\
\hline A 10 & 74.67227951 \\
\hline A11 & 88.29793044 \\
\hline A 12 & 71.25950956 \\
\hline A13 & 109.22096192 \\
\hline A14 & 110.01436840 \\
\hline A 15 & 110.62038368 \\
\hline A16 & 113.16459695 \\
\hline A 17 & 108.59782348 \\
\hline D1 & 106.22672556 \\
\hline D2 & 11.69280182 \\
\hline D3 & -83.81560938 \\
\hline D4 & -61.93995909 \\
\hline D5 & 90.52522798 \\
\hline D6 & -103.62338377 \\
\hline D7 & 16.90104495 \\
\hline D8 & 133.70127963 \\
\hline D9 & -60.86894526 \\
\hline D10 & -30.02052062 \\
\hline D11 & -149.99250764 \\
\hline D12 & -176.52125053 \\
\hline
\end{tabular}




$\begin{array}{lr}\text { D13 } & -57.18926337 \\ \text { D14 } & 63.51990960 \\ \text { D15 } & 122.03227877 \\ \text { D16 } & -63.69917379\end{array}$

Harmonic Vibrational Frequencies $\left(\mathbf{c m}^{-\mathbf{1}}\right)$ :

$\begin{array}{lccccccccccc}46.89 & 58.09 & 63.24 & 106.00 & 166.90 & 189.04 & 222.61 & 227.77 & 236.13 & 287.39 & 299.82 \\ 324.06 & 361.28 & 393.61 & 550.62 & 568.92 & 594.12 & 842.02 & 875.55 & 887.66 & 912.13 \\ 941.21 & 997.12 & 1057.44 & 1083.89 & 1115.13 & 1130.17 & 1182.18 & 1193.48 & 1316.56 \\ 1375.59 & 1385.72 & 1408.93 & 1434.10 & 1435.32 & 1466.98 & 1472.85 & 1487.90 & 1498.18 \\ 1573.86 & 1692.68 & 3046.56 & 3060.89 & 3118.41 & 3128.51 & 3136.50 & 3149.22 & 3171.22 \\ 3204.53 & 3310.30 & 3872.96 & & & & & & \\ \text { Rotational constants (B/GHZ): } & 2.1902236 & 1.0176992 & 0.8757031 & \end{array}$

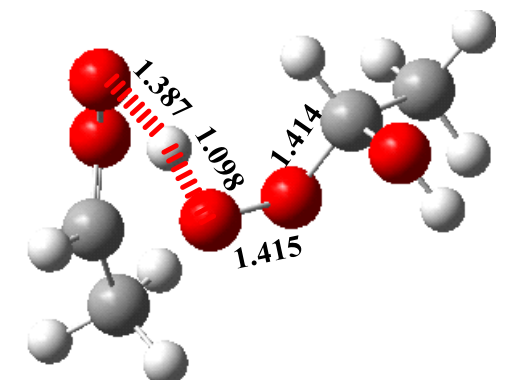

$\mathrm{TS} 3 \mathrm{c}$

C

$\begin{array}{lll}\text { C } & 1 & \text { B1 }\end{array}$

$\mathrm{H} \quad 2$

O 2

O 2

$\mathrm{H} \quad 4$

C 4

C $\quad 7$

$\mathrm{H} \quad 8$

$\mathrm{H} \quad 8$

$\mathrm{H} \quad 8$

O 7

O 7

$\mathrm{H} \quad 7$

$\mathrm{H} \quad 1$

B2

B2 1

A1

B3 1

A2 $3 \quad$ D1 0

B4 1

A3 4

D2 0

B5 2

A4 1

D3 0

B6 2

$\begin{array}{llll}\text { A5 } & 1 & \text { D4 } & 0\end{array}$

B7 4

A6 2

D5 0

B8 7

A7 4

D6 0

B9 7

A8 4

D7 0

B10 7

A9 4

D8 0

B11 4

$\begin{array}{llll}\text { A10 } 2 & \text { D9 } & 0\end{array}$

B12 4

A11 2

D10 0

B13 4

A12 2 D11 0

B14 2

A13 5

D12 0

$\mathrm{H} \quad 1$

B15 2

A14 5

D13 0

B16 2

A15 5

D14 0

O 2

B17 1

A16 5

D15 0

$\mathrm{H} \quad 18$

B18 2

A17 1

D16 0

Variables:

$\begin{array}{ll}\text { B1 } & 1.51347450 \\ \text { B2 } & 1.09200831 \\ \text { B3 } & 2.30796299\end{array}$




\begin{tabular}{|c|c|}
\hline B4 & 1.41401246 \\
\hline B5 & 1.09820114 \\
\hline B6 & 2.06523848 \\
\hline B7 & 1.47530888 \\
\hline $\mathrm{B} 8$ & 1.08551818 \\
\hline B9 & 1.08953515 \\
\hline B 10 & 1.09072505 \\
\hline B11 & 2.17903111 \\
\hline B12 & 1.26492397 \\
\hline B13 & 1.08522293 \\
\hline B14 & 1.08734268 \\
\hline B 15 & 1.09118181 \\
\hline B16 & 1.08761227 \\
\hline B17 & 1.39016122 \\
\hline B18 & 0.96193845 \\
\hline $\mathrm{A} 1$ & 111.85102583 \\
\hline A2 & 139.56881374 \\
\hline $\mathrm{A} 3$ & 104.89303342 \\
\hline A4 & 85.19227706 \\
\hline A5 & 124.01680680 \\
\hline A6 & 108.94402735 \\
\hline A7 & 110.66712727 \\
\hline A8 & 107.80724483 \\
\hline A9 & 109.55842511 \\
\hline A 10 & 69.09863294 \\
\hline A11 & 92.40710665 \\
\hline A12 & 82.87124982 \\
\hline A13 & 108.99644049 \\
\hline A14 & 109.88902269 \\
\hline A15 & 110.65551456 \\
\hline A16 & 113.35950288 \\
\hline A17 & 108.71714304 \\
\hline D1 & 109.52610156 \\
\hline D2 & 8.45140619 \\
\hline D3 & -106.39983305 \\
\hline D4 & -40.49880834 \\
\hline D5 & 84.82122756 \\
\hline D6 & -88.75849014 \\
\hline D7 & 31.51701080 \\
\hline D8 & 148.95966522 \\
\hline D9 & -67.22279330 \\
\hline D10 & -36.38631620 \\
\hline D11 & -154.04165291 \\
\hline D12 & -178.59743440 \\
\hline
\end{tabular}




$\begin{array}{lr}\text { D13 } & -59.28127759 \\ \text { D14 } & 60.97819578 \\ \text { D15 } & 122.33459091 \\ \text { D16 } & -57.91138606\end{array}$

Harmonic Vibrational Frequencies $\left(\mathbf{c m}^{-\mathbf{1}}\right)$ :

$\begin{array}{lccccccccccr}478.26 i & 35.65 & 73.12 & 80.16 & 123.78 & 176.55 & 219.32 & 246.76 & 309.23 & 321.92 & 343.85 \\ 378.31 & 437.87 & 520.74 & 563.75 & 578.05 & 603.56 & 885.27 & 893.39 & 941.11 & 974.45 \\ 999.95 & 1040.67 & 1089.99 & 1114.60 & 1131.25 & 1182.55 & 1194.46 & 1277.56 & 1318.88 \\ 1365.43 & 1389.78 & 1415.91 & 1421.19 & 1447.23 & 1453.36 & 1474.75 & 1489.68 & 1502.94 \\ 1611.34 & 1628.35 & 1887.50 & 3065.65 & 3067.16 & 3122.48 & 3141.54 & 3148.00 & 3163.51 \\ 3183.30 & 3207.58 & 3875.75 & & & & & & & \\ \text { Rotational constants (B/GHZ): } & 2.6131001 & 0.9239361 & 0.8711024 & \end{array}$

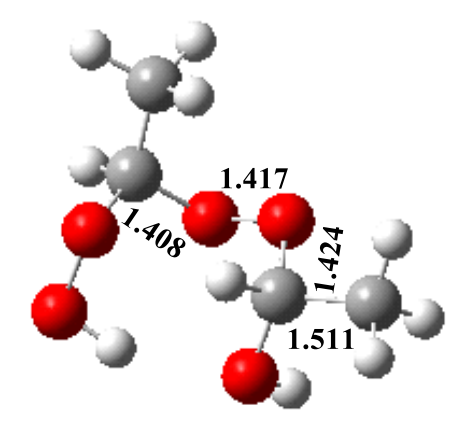

$\mathrm{P} 3 \mathrm{c}$

$\mathrm{C}$

C $1 \quad$ B1

$\begin{array}{lllll}\mathrm{H} & 2 & \mathrm{~B} 2 & 1 & \mathrm{~A} 1\end{array}$

$\begin{array}{llllllll}\mathrm{O} & 2 & \mathrm{~B} 3 & 1 & \mathrm{~A} 2 & 3 & \mathrm{D} 1 & 0\end{array}$

$\begin{array}{llllllll}\mathrm{O} & 4 & \mathrm{~B} 4 & 2 & \mathrm{~A} 3 & 1 & \mathrm{D} 2 & 0\end{array}$

$\begin{array}{llllllll}\mathrm{H} & 4 & \mathrm{~B} 5 & 2 & \mathrm{~A} 4 & 1 & \mathrm{D} 3 & 0\end{array}$

$\begin{array}{llllllll}\mathrm{C} & 4 & \mathrm{~B} 6 & 2 & \mathrm{~A} 5 & 1 & \mathrm{D} 4 & 0\end{array}$

$\begin{array}{llllllll}\mathrm{C} & 7 & \mathrm{~B} 7 & 4 & \mathrm{~A} 6 & 2 & \mathrm{D} 5 & 0\end{array}$

$\begin{array}{llllllll}\mathrm{H} & 8 & \mathrm{~B} 8 & 7 & \mathrm{~A} 7 & 4 & \mathrm{D} 6 & 0\end{array}$

$\begin{array}{llllllll}\mathrm{H} & 8 & \mathrm{~B} 9 & 7 & \mathrm{~A} 8 & 4 & \mathrm{D} 7 & 0\end{array}$

$\begin{array}{llllllll}\mathrm{H} & 8 & \mathrm{~B} 10 & 7 & \mathrm{~A} 9 & 4 & \mathrm{D} 8 & 0\end{array}$

$\begin{array}{llllllll}\mathrm{O} & 7 & \mathrm{~B} 11 & 4 & \mathrm{~A} 10 & 2 & \mathrm{D} 9 & 0\end{array}$

$\begin{array}{llllllll}\mathrm{O} & 7 & \mathrm{~B} 12 & 4 & \mathrm{~A} 11 & 2 & \mathrm{D} 10 & 0\end{array}$

$\begin{array}{llllllll}\mathrm{H} & 7 & \mathrm{~B} 13 & 4 & \mathrm{~A} 12 & 2 & \mathrm{D} 11 & 0\end{array}$

$\begin{array}{llllllll}\mathrm{H} & 1 & \mathrm{~B} 14 & 2 & \mathrm{~A} 13 & 5 & \mathrm{D} 12 & 0\end{array}$

$\begin{array}{llllllll}\mathrm{H} & 1 & \mathrm{~B} 15 & 2 & \mathrm{~A} 14 & 5 & \mathrm{D} 13 & 0\end{array}$

$\begin{array}{llllllll}\mathrm{H} & 1 & \mathrm{~B} 16 & 2 & \mathrm{~A} 15 & 5 & \mathrm{D} 14 & 0\end{array}$

$\begin{array}{llllllll}\mathrm{O} & 2 & \mathrm{~B} 17 & 1 & \mathrm{~A} 16 & 5 & \mathrm{D} 15 & 0\end{array}$

$\begin{array}{llllllll}\mathrm{H} & 18 & \mathrm{~B} 18 & 2 & \mathrm{~A} 17 & 1 & \mathrm{D} 16 & 0\end{array}$

Variables:

$\begin{array}{ll}\text { B1 } & 1.51072575 \\ \text { B2 } & 1.08912457 \\ \text { B3 } & 2.34851031\end{array}$




\begin{tabular}{|c|c|}
\hline B4 & 1.41662705 \\
\hline B5 & 2.47427596 \\
\hline B6 & 1.40844697 \\
\hline B7 & 1.50877995 \\
\hline B8 & 1.08741022 \\
\hline B9 & 1.08743051 \\
\hline B10 & 1.08713084 \\
\hline B11 & 2.28344763 \\
\hline B12 & 1.40021873 \\
\hline B13 & 1.09293893 \\
\hline B14 & 1.08761040 \\
\hline B15 & 1.09021355 \\
\hline B16 & 1.08828438 \\
\hline B17 & 1.39633155 \\
\hline B18 & 0.96351575 \\
\hline $\mathrm{A} 1$ & 112.06604004 \\
\hline $\mathrm{A} 2$ & 136.97320321 \\
\hline A3 & 34.35559065 \\
\hline A4 & 67.54573384 \\
\hline A5 & 104.34354920 \\
\hline A6 & 114.34998474 \\
\hline A7 & 110.40288894 \\
\hline A8 & 109.28093071 \\
\hline A9 & 109.43187243 \\
\hline A 10 & 91.77378084 \\
\hline A11 & 111.29316642 \\
\hline A 12 & 101.58133542 \\
\hline A13 & 109.18061569 \\
\hline A14 & 109.87176982 \\
\hline A 15 & 110.44422465 \\
\hline A16 & 113.51786416 \\
\hline A 17 & 109.01895802 \\
\hline D1 & 131.45671080 \\
\hline D2 & -21.11341616 \\
\hline D3 & 162.04968288 \\
\hline D4 & -129.31785414 \\
\hline D5 & 81.85744072 \\
\hline D6 & -69.76624023 \\
\hline D7 & 50.06738650 \\
\hline D8 & 170.16532842 \\
\hline D9 & -70.31944503 \\
\hline D10 & -39.24070240 \\
\hline D11 & -155.76145142 \\
\hline D12 & 178.47100275 \\
\hline
\end{tabular}




$\begin{array}{lr}\text { D13 } & -61.52582127 \\ \text { D14 } & 58.33366633 \\ \text { D15 } & 121.66821320 \\ \text { D16 } & -62.48549906\end{array}$

Harmonic Vibrational Frequencies $\left(\mathbf{c m}^{-1}\right)$ :

$\begin{array}{lcccccccccc}59.06 & 100.54 & 144.23 & 172.05 & 219.95 & 239.94 & 261.82 & 280.99 & 318.08 & 338.27 \\ 408.94 & 425.65 & 520.26 & 538.81 & 578.60 & 621.30 & 724.66 & 853.62 & 885.79 & 920.87 \\ 935.39 & 1016.58 & 1060.61 & 1085.22 & 1126.05 & 1138.01 & 1150.73 & 1193.99 & 1210.27 \\ 1305.75 & 1358.68 & 1375.10 & 1396.83 & 1420.48 & 1424.34 & 1461.46 & 1483.00 & 1489.09 \\ 1495.83 & 1501.89 & 1517.25 & 3067.49 & 3069.59 & 3104.35 & 3130.63 & 3152.01 & 3153.17 \\ 3158.98 & 3168.66 & 3704.64 & 3852.07 & & & & & & \end{array}$

$\begin{array}{llll}\text { Rotational constants (B/GHZ): } \quad 2.3376001 & 1.2905174 & 1.0017937\end{array}$

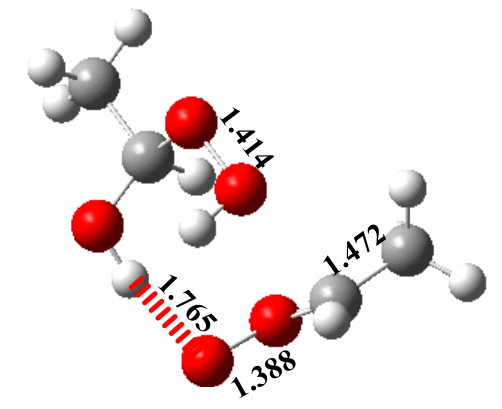

IM3d

$\mathrm{C}$

$\begin{array}{lll}\mathrm{C} & 1 & \mathrm{~B} 1\end{array}$

$\mathrm{H} \quad 2$

$\mathrm{H} \quad 2$

B2 1

A1

$\mathrm{H} 2$

B3 1

A2

B4 1

A3 3

D1 0

B5 2

A4 5

D2 0

O 1

H 6

B6 1

A5 2

D3 0

O 1

B7 6

A6 2

D4 0

C 8

B8 1

A7 6

D5 0

C 9

B9 8

D6 0

$\mathrm{H} \quad 10$

B10 9

A8 1

D7 0

$\mathrm{H} \quad 10$

B11 9

A9 8

D8 0

$\mathrm{H} \quad 10$

A10 8

D9 0

B12 9

A11 8

D10 0

O 9

B13 8

A12 1

D11 0

O 9

B14 8

A13 1

D12 0

$\mathrm{H} \quad 9$

B15 8

A14 1

D13 0

B16 6

A15 2

D14 0

$\begin{array}{ll}\text { O } & 8\end{array}$

B17 1

A16 6

D15 0

$\mathrm{H} \quad 8$

B18 1

A17 6

D16 0

Variables:

$\begin{array}{ll}\text { B1 } & 1.50826493 \\ \text { B2 } & 1.08849529 \\ \text { B3 } & 1.08801670\end{array}$




\begin{tabular}{|c|c|}
\hline B4 & 1.08742205 \\
\hline B5 & 1.37962600 \\
\hline B6 & 0.98142278 \\
\hline B7 & 2.29806678 \\
\hline B8 & 2.61776907 \\
\hline B9 & 1.47212942 \\
\hline B10 & 1.09290962 \\
\hline B11 & 1.09006994 \\
\hline B12 & 1.08583184 \\
\hline B13 & 2.22846638 \\
\hline B14 & 1.23643882 \\
\hline B15 & 1.08641314 \\
\hline B16 & 1.09605777 \\
\hline B17 & 1.41388495 \\
\hline B18 & 0.96988115 \\
\hline $\mathrm{A} 1$ & 109.01036072 \\
\hline $\mathrm{A} 2$ & 110.84148501 \\
\hline A3 & 109.03763998 \\
\hline A4 & 110.69434922 \\
\hline A5 & 109.57607280 \\
\hline A6 & 90.05983673 \\
\hline A7 & 98.01723396 \\
\hline A8 & 105.47799746 \\
\hline A9 & 107.23694494 \\
\hline A 10 & 110.05720912 \\
\hline A11 & 111.12794525 \\
\hline A 12 & 78.37768584 \\
\hline A13 & 92.09396020 \\
\hline A14 & 71.68952638 \\
\hline A 15 & 110.77581185 \\
\hline A16 & 36.32281308 \\
\hline A 17 & 87.13287244 \\
\hline D1 & 120.55712250 \\
\hline D2 & -118.79854602 \\
\hline D3 & -64.00008235 \\
\hline D4 & 152.51486167 \\
\hline D5 & 145.58311616 \\
\hline D6 & 86.50072674 \\
\hline D7 & 93.48177167 \\
\hline D8 & 14.99025232 \\
\hline D9 & 131.72828005 \\
\hline D10 & -105.35056108 \\
\hline D11 & -59.60987445 \\
\hline D12 & -28.28139720 \\
\hline
\end{tabular}




$\begin{array}{ll}\text { D13 } & -146.06327171 \\ \text { D14 } & -125.01900609 \\ \text { D15 } & -127.18120992 \\ \text { D16 } & -12.30393477\end{array}$

Harmonic Vibrational Frequencies $\left(\mathbf{c m}^{-\mathbf{1}}\right)$ :

$\begin{array}{lcccccccccc}48.70 & 58.15 & 107.79 & 120.47 & 132.63 & 187.12 & 190.52 & 232.69 & 247.40 & 298.78 & 328.26 \\ 339.79 & 395.24 & 467.75 & 548.30 & 568.33 & 625.09 & 835.47 & 875.63 & 877.40 & 905.42 \\ 946.53 & 992.40 & 1058.25 & 1116.94 & 1122.66 & 1146.33 & 1179.94 & 1203.35 & 1366.90 \\ 1373.10 & 1393.85 & 1410.20 & 1420.48 & 1442.26 & 1462.87 & 1473.29 & 1489.60 & 1499.05 \\ 1527.18 & 1703.83 & 3052.27 & 3055.85 & 3075.24 & 3122.20 & 3157.33 & 3163.17 & 3172.24 \\ 3207.37 & 3417.10 & 3748.46 & & & & & & \end{array}$

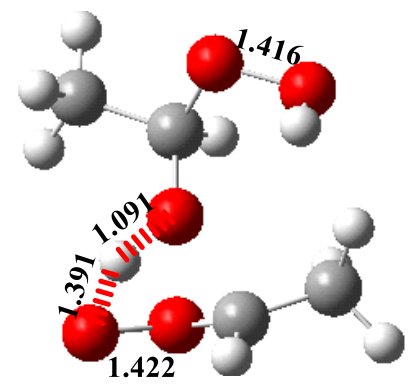

TS3d

$\mathrm{C}$

$\begin{array}{lll}\mathrm{C} & 1 & \mathrm{~B} 1\end{array}$

$\mathrm{H}-2$

$\begin{array}{lllll}\mathrm{H} & 2 & \mathrm{~B} 3 & 1 & \mathrm{~A} 2\end{array}$

$\mathrm{H} \quad 2$

O 1

$\mathrm{H} \quad 6$

O 1

C 6

C 9

$\mathrm{H} \quad 10$

$\mathrm{H} \quad 10$

$\mathrm{H} \quad 10$

B4 1

A3 3

D1 0

B5 2

A4 4

D2 0

B6 1

D3 0

B7 6

A5 2

D4 0

B8 1

A6 2

D5 0

A7 2

D6 0

$\begin{array}{llll}\text { B9 } & 6 & \text { A8 } & 1\end{array}$

D7 0

$\begin{array}{llllll}\mathrm{B} 10 & 9 & \mathrm{~A} 9 & 6 & \mathrm{D} 8 & 0\end{array}$

O 9

B11 9

A10 6

D9 0

B12 9 A11 6

D10 0

B13 6

A12 1

D11 0

O 9

B14 6

A13 1

D12 0

$\mathrm{H} \quad 9$

B15 6

A14 1

D13 0

B16 6

A15 9

D14 0

O 1

B17 6

A16 9

D15 0

$\begin{array}{ll}\mathrm{H} & 8\end{array}$

B18 1

A17 6

D16 0

Variables:

$\begin{array}{ll}\text { B1 } & 1.51062721 \\ \text { B2 } & 1.08899963 \\ \text { B3 } & 1.08754863\end{array}$




\begin{tabular}{|c|c|}
\hline B4 & 1.08890640 \\
\hline B5 & 1.39944841 \\
\hline B6 & 1.09076945 \\
\hline B7 & 2.28016169 \\
\hline B8 & 2.03285956 \\
\hline B9 & 1.47667443 \\
\hline B10 & 1.09231438 \\
\hline B11 & 1.08958067 \\
\hline B12 & 1.08662402 \\
\hline B13 & 2.18183027 \\
\hline B14 & 1.26986233 \\
\hline B15 & 1.08417695 \\
\hline B16 & 1.09869541 \\
\hline B17 & 1.40479016 \\
\hline B18 & 0.96552862 \\
\hline $\mathrm{A} 1$ & 109.39921212 \\
\hline $\mathrm{A} 2$ & 110.26379595 \\
\hline A3 & 109.01878635 \\
\hline A4 & 112.46332959 \\
\hline A5 & 110.52821037 \\
\hline A6 & 89.74982896 \\
\hline A7 & 113.12856475 \\
\hline A8 & 110.51338887 \\
\hline A9 & 108.02666454 \\
\hline A 10 & 109.72007093 \\
\hline A11 & 110.75824359 \\
\hline A 12 & 69.27338968 \\
\hline A13 & 93.10712764 \\
\hline A14 & 82.67386623 \\
\hline A 15 & 109.64796687 \\
\hline A16 & 108.70777143 \\
\hline A17 & 99.96845148 \\
\hline D1 & 120.32575407 \\
\hline D2 & -119.39763046 \\
\hline D3 & 179.37812485 \\
\hline D4 & 36.79687479 \\
\hline D5 & 148.73522994 \\
\hline D6 & 118.34880438 \\
\hline D7 & 50.11453130 \\
\hline D8 & 19.40476679 \\
\hline D9 & 137.27097159 \\
\hline D10 & -100.55672073 \\
\hline D11 & -101.98145046 \\
\hline D12 & -71.46640659 \\
\hline
\end{tabular}




$\begin{array}{lr}\text { D13 } & 171.15901035 \\ \text { D14 } & -6.15783029 \\ \text { D15 } & -124.34519710 \\ \text { D16 } & -27.57342043\end{array}$

Harmonic Vibrational Frequencies $\left(\mathbf{c m}^{-1}\right)$ :

$\begin{array}{lllllllllll}473.45 i & 62.49 & 98.03 & 123.37 & 165.61 & 185.49 & 208.87 & 248.37 & 296.01 & 314.43 & 362.94\end{array}$ $\begin{array}{llllllllll}394.65 & 431.58 & 507.10 & 542.48 & 560.59 & 632.45 & 865.10 & 894.24 & 941.62 & 986.56\end{array}$ $\begin{array}{lllllllll}996.59 & 1050.51 & 1094.91 & 1137.71 & 1177.82 & 1182.67 & 1194.81 & 1248.99 & 1348.35\end{array}$ $\begin{array}{llllllllll}1358.43 & 1403.00 & 1415.77 & 1424.17 & 1433.04 & 1464.64 & 1476.06 & 1481.07 & 1493.79\end{array}$ $\begin{array}{llllllllll}1513.03 & 1609.43 & 1973.17 & 3037.43 & 3059.86 & 3062.85 & 3131.43 & 3146.14 & 3154.55\end{array}$ $3167.57 \quad 3204.15 \quad 3816.88$

Rotational constants (B/GHZ): $\quad 2.1199984 \quad 1.2235984 \quad 0.8716425$

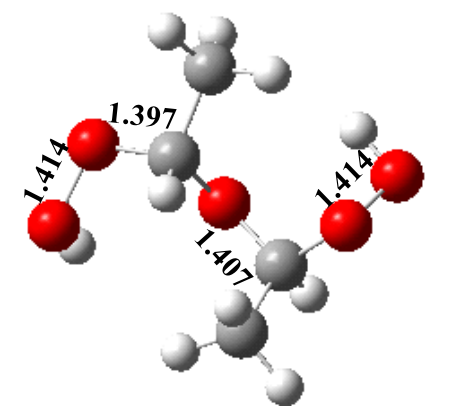

P3d

$\mathrm{C}$

C $\quad 1 \quad$ B1

$\begin{array}{lllll}\mathrm{H} & 2 & \mathrm{~B} 2 & 1 & \mathrm{~A} 1\end{array}$

$\begin{array}{llllllll}\mathrm{H} & 2 & \mathrm{~B} 3 & 1 & \mathrm{~A} 2 & 3 & \mathrm{D} 1 & 0\end{array}$

$\begin{array}{llllllll}\mathrm{H} & 2 & \mathrm{~B} 4 & 1 & \mathrm{~A} 3 & 3 & \mathrm{D} 2 & 0\end{array}$

$\begin{array}{llllllll}\mathrm{O} & 1 & \mathrm{~B} 5 & 2 & \mathrm{~A} 4 & 4 & \mathrm{D} 3 & 0\end{array}$

$\begin{array}{llllllll}\mathrm{H} & 6 & \mathrm{~B} 6 & 1 & \mathrm{~A} 5 & 2 & \mathrm{D} 4 & 0\end{array}$

$\begin{array}{llllllll}\mathrm{O} & 1 & \mathrm{~B} 7 & 6 & \mathrm{~A} 6 & 2 & \mathrm{D} 5 & 0\end{array}$

$\begin{array}{llllllll}\mathrm{C} & 6 & \mathrm{~B} 8 & 1 & \mathrm{~A} 7 & 2 & \mathrm{D} 6 & 0\end{array}$

$\begin{array}{llllllll}\mathrm{C} & 9 & \mathrm{~B} 9 & 6 & \mathrm{~A} 8 & 1 & \mathrm{D} 7 & 0\end{array}$

$\begin{array}{llllllll}\mathrm{H} & 10 & \mathrm{~B} 10 & 9 & \mathrm{~A} 9 & 6 & \mathrm{D} 8 & 0\end{array}$

$\begin{array}{llllllll}\mathrm{H} & 10 & \mathrm{~B} 11 & 9 & \mathrm{~A} 10 & 6 & \mathrm{D} 9 & 0\end{array}$

$\begin{array}{llllllll}\mathrm{H} & 10 & \mathrm{~B} 12 & 9 & \mathrm{~A} 11 & 6 & \mathrm{D} 10 & 0\end{array}$

$\begin{array}{llllllll}\mathrm{O} & 9 & \mathrm{~B} 13 & 6 & \mathrm{~A} 12 & 1 & \mathrm{D} 11 & 0\end{array}$

$\begin{array}{llllllll}\mathrm{O} & 9 & \mathrm{~B} 14 & 6 & \mathrm{~A} 13 & 1 & \mathrm{D} 12 & 0\end{array}$

$\begin{array}{llllllll}\mathrm{H} & 9 & \mathrm{~B} 15 & 6 & \mathrm{~A} 14 & 1 & \mathrm{D} 13 & 0\end{array}$

$\begin{array}{llllllll}\mathrm{H} & 1 & \mathrm{~B} 16 & 6 & \mathrm{~A} 15 & 9 & \mathrm{D} 14 & 0\end{array}$

$\begin{array}{llllllll}\mathrm{O} & 1 & \mathrm{~B} 17 & 6 & \mathrm{~A} 16 & 9 & \mathrm{D} 15 & 0\end{array}$

$\begin{array}{llllllll}\mathrm{H} & 8 & \mathrm{~B} 18 & 1 & \mathrm{~A} 17 & 18 & \mathrm{D} 16 & 0\end{array}$

Variables:

B1 1.51068459

B2 1.08964289

B3 1.08797811 


\begin{tabular}{|c|c|}
\hline B4 & 1.08948149 \\
\hline B5 & 1.41354785 \\
\hline B6 & 2.37962991 \\
\hline B7 & 2.27279198 \\
\hline B8 & 1.40730527 \\
\hline B9 & 1.51599687 \\
\hline B10 & 1.08810672 \\
\hline B11 & 1.08719344 \\
\hline B12 & 1.08776085 \\
\hline B13 & 2.28351177 \\
\hline B14 & 1.40455757 \\
\hline B15 & 1.09017848 \\
\hline B16 & 1.09117794 \\
\hline B17 & 1.39742566 \\
\hline B18 & 0.96582080 \\
\hline $\mathrm{A} 1$ & 109.40116474 \\
\hline $\mathrm{A} 2$ & 110.35773269 \\
\hline A3 & 109.25677114 \\
\hline A4 & 111.50530483 \\
\hline A5 & 102.66783615 \\
\hline A6 & 90.90180133 \\
\hline A7 & 117.72290565 \\
\hline A8 & 115.65533261 \\
\hline A9 & 109.64116860 \\
\hline A 10 & 108.94691120 \\
\hline A11 & 111.64286050 \\
\hline A 12 & 93.46277603 \\
\hline A13 & 110.14548391 \\
\hline A14 & 104.02638792 \\
\hline A 15 & 110.19142788 \\
\hline A16 & 107.38727151 \\
\hline A17 & 96.83861467 \\
\hline D1 & 119.98813338 \\
\hline D2 & -120.45693187 \\
\hline D3 & 174.17320839 \\
\hline D4 & 20.89402614 \\
\hline D5 & 148.84146645 \\
\hline D6 & 101.40736069 \\
\hline D7 & 53.91099463 \\
\hline D8 & 41.20929583 \\
\hline D9 & 161.02283642 \\
\hline D10 & -79.76114649 \\
\hline D11 & -99.20655459 \\
\hline D12 & -66.41199542 \\
\hline
\end{tabular}




$\begin{array}{lr}\text { D13 } & 176.82391288 \\ \text { D14 } & -23.87044330 \\ \text { D15 } & -142.63861301 \\ \text { D16 } & 100.55099936\end{array}$

Harmonic Vibrational Frequencies $\left(\mathbf{c m}^{-1}\right)$ :

$\begin{array}{lccccccccccr}41.22 & 77.45 & 148.20 & 170.39 & 195.42 & 221.03 & 259.36 & 281.53 & 297.99 & 339.48 & 352.64 \\ 375.25 & 438.01 & 517.45 & 554.97 & 586.72 & 695.19 & 855.83 & 896.05 & 925.73 & 970.53 \\ 1055.61 & 1058.87 & 1107.49 & 1133.25 & 1149.10 & 1186.69 & 1194.34 & 1226.73 & 1357.55 \\ 1368.53 & 1406.03 & 1413.35 & 1429.66 & 1437.03 & 1440.74 & 1455.39 & 1487.56 & 1500.81 \\ 1501.82 & 1512.39 & 3065.42 & 3070.69 & 3119.97 & 3130.97 & 3146.88 & 3154.98 & 3158.09 \\ 3165.84 & 3777.32 & 3812.77 & & & & & & \\ \text { Rotational constants (B/GHZ): } & 2.4247574 & 1.1625116 & 0.8900912 & \end{array}$

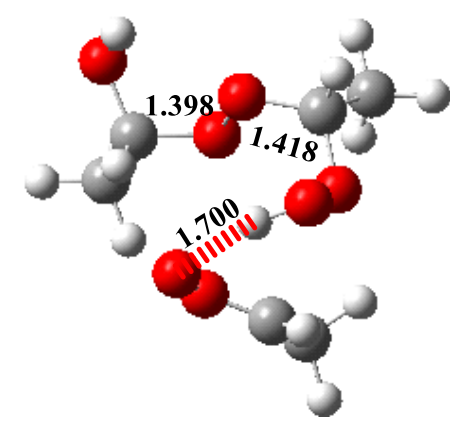

IM4a

C

$\begin{array}{lll}\mathrm{H} & 1 & \mathrm{~B} 1\end{array}$

$\begin{array}{lll}\text { O } & 1 & \text { B2 }\end{array}$

O 1

B2 2

A1

$\mathrm{H} \quad 3$

B3 3

A2

B4 1

A3 4

D1 0

B5 1

A4 3

D2 0

C 4

O 6

B6 4

A5 1

D3 0

O 6

B7 4

A6 1

D4 0

C 1

B8 4

A7 3

D5 0

$\mathrm{H} \quad 9$

B9 1

A8 4

D6 0

$\mathrm{H} \quad 9$

H 9

O 1

B10 1

A9 4

D7 0

B11 1

A10 4

D8 0

C 6

B12 4

A11 3

D9 0

B13 4

A12 1

D10 0

$\mathrm{H} \quad 14$

B14 6

A13 4

D11 0

B15 6

A14 4

D12 0

B16 6

A15 4

D13 0

$\mathrm{H} \quad 14$

H 6

B17 4

A16

D14 0

O 13

B18 1

A17 4

D15 0

B19 13

A18 1

D16 0

C 19

B20 19

A19 13

D17 0

B21 19

A20 13

D18 0

C 20

B22 20

A21 19

D19 0

H 22

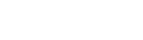




\begin{tabular}{|c|c|c|c|c|c|c|}
\hline $\mathrm{H}$ & 22 & $\mathrm{~B} 23$ & 20 & A22 & 19 & D21 \\
\hline $\mathrm{H}$ & 22 & B24 & 20 & A23 & 19 & D22 \\
\hline $\mathrm{O}$ & 20 & B25 & 19 & A24 & 13 & D23 \\
\hline $\mathrm{H}$ & 26 & B26 & 20 & A25 & 19 & D24 \\
\hline \multicolumn{7}{|c|}{ Variables: } \\
\hline B1 & & 66775 & & & & \\
\hline B2 & & 17669 & & & & \\
\hline B3 & & 04503 & & & & \\
\hline B4 & & 90733 & & & & \\
\hline B5 & & 78378 & & & & \\
\hline B6 & & 68914 & & & & \\
\hline B7 & & 73777 & & & & \\
\hline B8 & & 13066 & & & & \\
\hline B9 & & 29069 & & & & \\
\hline B10 & & 27747 & & & & \\
\hline B11 & & 44854 & & & & \\
\hline B12 & & 45904 & & & & \\
\hline B13 & & 88773 & & & & \\
\hline B14 & & 64633 & & & & \\
\hline B15 & & 02217 & & & & \\
\hline B16 & & 75634 & & & & \\
\hline B17 & & 48199 & & & & \\
\hline B18 & & 68689 & & & & \\
\hline B19 & & 45233 & & & & \\
\hline B20 & & 73645 & & & & \\
\hline B21 & & 12955 & & & & \\
\hline B22 & & 95033 & & & & \\
\hline B23 & & 29256 & & & & \\
\hline B24 & & 09615 & & & & \\
\hline B25 & & 55163 & & & & \\
\hline B26 & & 76856 & & & & \\
\hline A1 & & 97612 & & & & \\
\hline A2 & & 77040 & & & & \\
\hline A3 & & 34786 & & & & \\
\hline A4 & & 60136 & & & & \\
\hline A5 & & 16246 & & & & \\
\hline A6 & & 25061 & & & & \\
\hline A7 & & 01598 & & & & \\
\hline A8 & & 379262 & & & & \\
\hline A9 & & 91113 & & & & \\
\hline A10 & & 362074 & & & & \\
\hline A11 & & 21621 & & & & \\
\hline A12 & & 26548 & & & & \\
\hline A13 & & 73810 & & & & \\
\hline
\end{tabular}




$\begin{array}{lr}\text { A14 } & 111.04562011 \\ \text { A15 } & 107.55418559 \\ \text { A16 } & 70.00450110 \\ \text { A17 } & 108.10333547 \\ \text { A18 } & 104.58906658 \\ \text { A19 } & 107.25223492 \\ \text { A20 } & 105.85349491 \\ \text { A21 } & 110.49336836 \\ \text { A22 } & 108.80333331 \\ \text { A23 } & 109.44885763 \\ \text { A24 } & 110.94573523 \\ \text { A25 } & 108.25525287 \\ \text { D1 } & 138.88980310 \\ \text { D2 } & -94.84525347 \\ \text { D3 } & 107.30526467 \\ \text { D4 } & -73.69143791 \\ \text { D5 } & -43.11851795 \\ \text { D6 } & 165.71105953 \\ \text { D7 } & -66.18987301 \\ \text { D8 } & 54.46006409 \\ \text { D9 } & 173.65981488 \\ \text { D10 } & -69.07274747 \\ \text { D11 } & 78.53534425 \\ \text { D12 } & 133.82235279 \\ \text { D13 } & -103.42640012 \\ \text { D14 } & 17.06617301 \\ \text { D15 } & -161.12785937 \\ \text { D16 } & -71.30904863 \\ \text { D17 } & 164.53806241 \\ \text { D18 } & -56.00117457 \\ \text { D19 } & -176.63502519 \\ \text { D20 } & 61.63454919 \\ \text { D21 } & -59.73308936 \\ \text { D22 } & \text { D23 } \\ \text { D24 } & -46179980 \\ \text { Hart } & \end{array}$

Harmonic Vibrational Frequencies $\left(\mathbf{c m}^{-1}\right)$ :

$\begin{array}{lllllllllll}40.01 & 53.06 & 73.16 & 81.06 & 97.16 & 111.48 & 120.06 & 141.56 & 158.72 & 206.97 & 219.15\end{array}$ $\begin{array}{llllllllll}229.12 & 233.63 & 254.40 & 287.07 & 304.46 & 327.77 & 353.49 & 386.89 & 411.05 & 424.79\end{array}$ $\begin{array}{llllllllll}520.43 & 567.14 & 579.64 & 601.10 & 699.97 & 823.22 & 878.22 & 886.27 & 901.62 & 905.09\end{array}$ $\begin{array}{lllllllll}937.09 & 954.15 & 994.24 & 1006.60 & 1060.33 & 1088.76 & 1111.31 & 1143.82 & 1150.60\end{array}$ $\begin{array}{lllllllll}1161.98 & 1182.34 & 1185.69 & 1213.59 & 1297.07 & 1346.72 & 1373.68 & 1393.34 & 1398.23\end{array}$ $\begin{array}{lllllllll}1422.26 & 1423.32 & 1423.89 & 1463.61 & 1468.81 & 1483.71 & 1485.21 & 1496.59 & 1497.81\end{array}$ $\begin{array}{lllllllll}1500.44 & 1617.78 & 1695.77 & 3055.82 & 3077.49 & 3081.33 & 3114.26 & 3117.08 & 3127.61\end{array}$ 


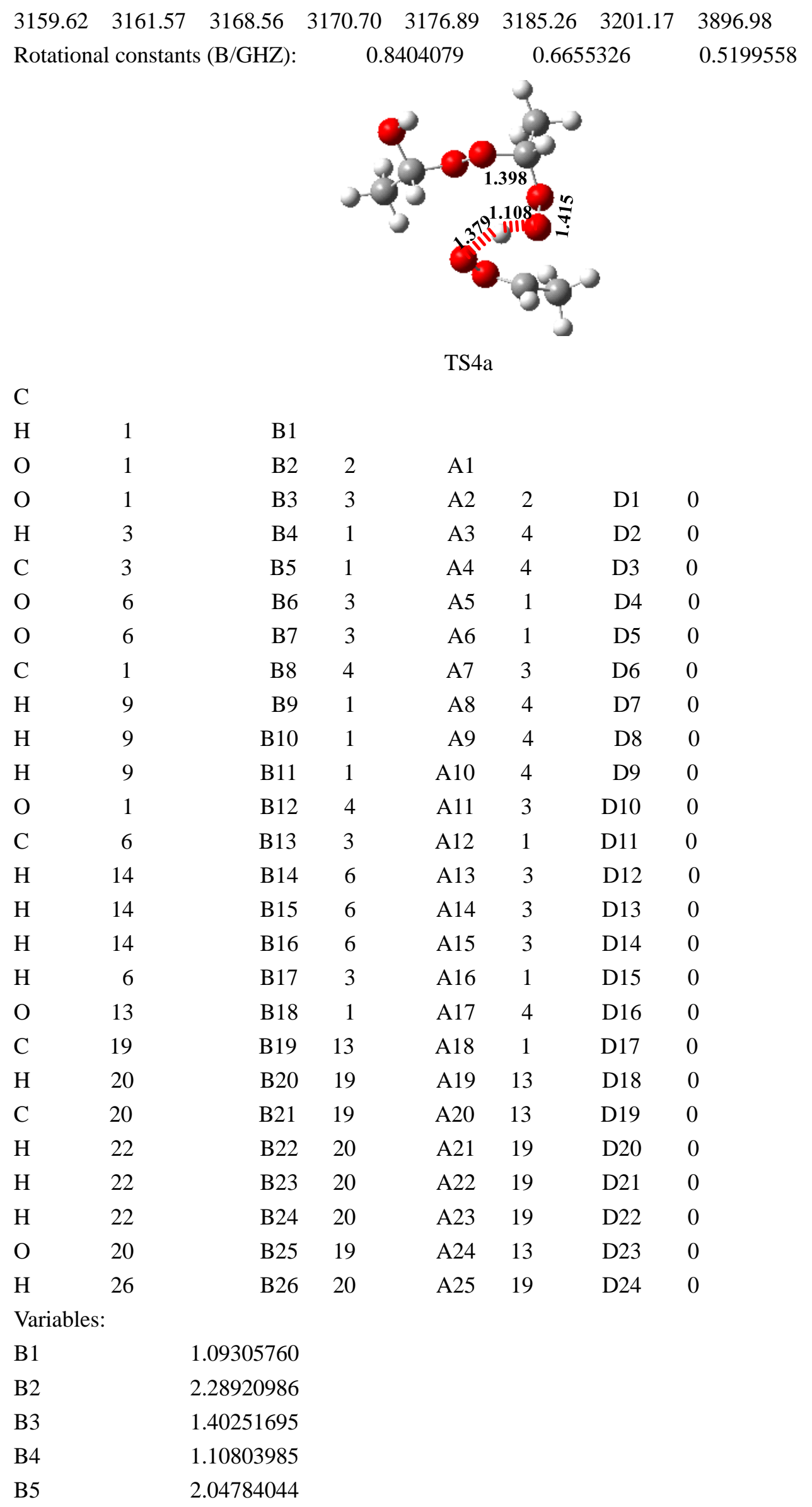




\begin{tabular}{|c|c|}
\hline B6 & 2.17345685 \\
\hline B7 & 1.26616672 \\
\hline B8 & 1.51146059 \\
\hline B9 & 1.08750242 \\
\hline B10 & 1.08663416 \\
\hline B11 & 1.08755991 \\
\hline B12 & 1.39787808 \\
\hline B13 & 1.47523420 \\
\hline B14 & 1.09110229 \\
\hline B15 & 1.08542504 \\
\hline B16 & 1.08914207 \\
\hline B17 & 1.08509114 \\
\hline B18 & 1.43087617 \\
\hline B19 & 1.41171451 \\
\hline B20 & 1.09090000 \\
\hline B21 & 1.50573353 \\
\hline B22 & 1.08727855 \\
\hline B23 & 1.08732745 \\
\hline B24 & 1.08906741 \\
\hline B25 & 1.39718939 \\
\hline B26 & 0.96040756 \\
\hline $\mathrm{A} 1$ & 85.63299725 \\
\hline $\mathrm{A} 2$ & 35.82719462 \\
\hline A3 & 93.38569115 \\
\hline A4 & 129.97126223 \\
\hline A5 & 69.35897017 \\
\hline A6 & 92.91593901 \\
\hline A7 & 106.11341755 \\
\hline A8 & 109.66420352 \\
\hline A9 & 110.07153147 \\
\hline A10 & 109.04012241 \\
\hline A11 & 111.66210862 \\
\hline A12 & 108.87267572 \\
\hline A13 & 109.18338956 \\
\hline A14 & 110.66379939 \\
\hline A15 & 108.07029571 \\
\hline A16 & 83.20141216 \\
\hline A17 & 107.48035991 \\
\hline A18 & 105.16912477 \\
\hline A19 & 107.44743296 \\
\hline A20 & 105.67402457 \\
\hline A 21 & 109.47506435 \\
\hline A 22 & 109.04108824 \\
\hline A 23 & 109.72218512 \\
\hline
\end{tabular}




$\begin{array}{lr}\text { A24 } & 111.13681846 \\ \text { A25 } & 108.29576429 \\ \text { D1 } & 132.93866792 \\ \text { D2 } & -110.93651103 \\ \text { D3 } & -38.54409198 \\ \text { D4 } & -79.73361701 \\ \text { D5 } & -48.76383637 \\ \text { D6 } & 173.15024269 \\ \text { D7 } & -66.20545521 \\ \text { D8 } & 54.35860537 \\ \text { D9 } & 173.88149122 \\ \text { D10 } & -61.89888204 \\ \text { D11 } & 72.67064407 \\ \text { D12 } & 151.80761150 \\ \text { D13 } & -86.20023555 \\ \text { D14 } & 34.36895698 \\ \text { D15 } & -166.17766104 \\ \text { D16 } & -65.53116459 \\ \text { D17 } & 169.22210117 \\ \text { D18 } & -48.74501194 \\ \text { D19 } & -168.27994875 \\ \text { D20 } & 62.00204995 \\ \text { D21 } & -178.13719277 \\ \text { D22 } & -58.92423512 \\ \text { D23 } & 73.30128810 \\ \text { D24 } & -67.80762676 \\ \text { Har } & \end{array}$

Harmonic Vibrational Frequencies $\left(\mathbf{c m}^{-1}\right)$ :

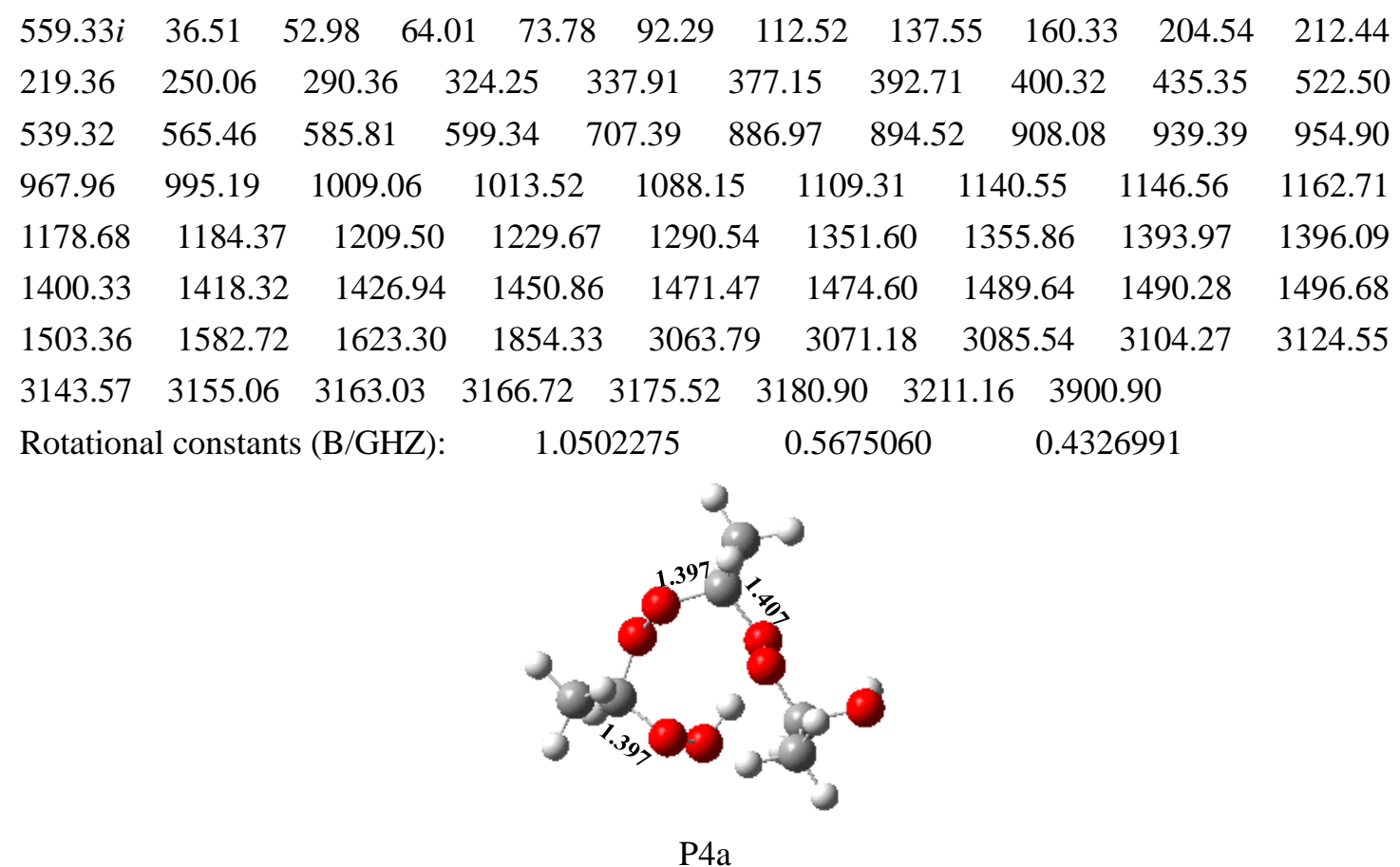


C

$\mathrm{H} \quad 1$

O 1

O 1

$\mathrm{H} \quad 3$

C 3

O 6

O 6

C 1

$\mathrm{H} \quad 9$

$\mathrm{H} \quad 9$

$\mathrm{H} \quad 9$

O 1

C 6

$\mathrm{H} \quad 14$

H 14

$\mathrm{H} \quad 14$

H 6

O 13

C 19

H 20

C 20

$\mathrm{H} \quad 22$

$\mathrm{H} \quad 22$

$\mathrm{H} \quad 22$

O 20

$\mathrm{H} \quad 26$

Variables:

B

B2

B3

B4

B5

B6

B7

B8

B9

B10

B11

B12

B13

B14

B15

B16
B1

B2 $2 \quad$ A1

B3 $3 \quad$ A2

B4

B5

B6 3

B7 3

B8 4

B9

B10 1

B11

B12 4

B13 3

B14 6

B15 6

B16 6

B17 3

B18 1

B19 13

B20 19

B21 19

B22 20

B23 20

B24 20

B25 19

B26 20

D1 0

A3 $4 \quad$ D2 0

A4 4 D3 0

A5 $1 \quad$ D4 0

A6 1 D5 0

A7 $3 \quad$ D6 0

$\begin{array}{llll}\text { A8 } & 4 & \text { D7 } & 0\end{array}$

$\begin{array}{llll}\text { A9 } & 4 & \text { D8 } & 0\end{array}$

$\begin{array}{llll}\mathrm{A} 10 & 4 & \mathrm{D} 9 & 0\end{array}$

A11 $3 \quad$ D10 0

A12 $1 \quad$ D11 0

A13 $3 \quad$ D12 0

A14 $3 \quad$ D13 0

A15 $3 \quad$ D14 0

A16 1 D15 0

A17 4 D16 0

$\begin{array}{llll}\text { A18 } 1 & \text { D17 } & 0\end{array}$

A19 $13 \quad$ D18 0

$\begin{array}{llll}\text { A20 } & 13 & \text { D19 } & 0\end{array}$

A21 $19 \quad$ D20 0

A22 $19 \quad$ D21 0

$\begin{array}{llll}\text { A23 } 19 & \text { D22 } & 0\end{array}$

A24 $13 \quad$ D23 0

A25 $19 \quad$ D24 0

1.09236410

2.27624819

1.39659979

2.53268836

1.40991757

2.27741737

1.39746599

1.51130089

1.08708862

1.08635936

1.08748501

1.40742215

1.50939067

1.08730608

1.08713452

1.08753335 


\begin{tabular}{|c|c|}
\hline B17 & 1.09267857 \\
\hline B18 & 1.43043745 \\
\hline B19 & 1.41230303 \\
\hline $\mathrm{B} 20$ & 1.09291560 \\
\hline B21 & 1.50683556 \\
\hline $\mathrm{B} 22$ & 1.08719759 \\
\hline B23 & 1.08714733 \\
\hline B24 & 1.08876305 \\
\hline $\mathrm{B} 25$ & 1.39432692 \\
\hline B26 & 0.96039074 \\
\hline A1 & 81.97205284 \\
\hline $\mathrm{A} 2$ & 36.53396844 \\
\hline A3 & 73.83803625 \\
\hline A4 & 124.25240040 \\
\hline A5 & 92.91926410 \\
\hline A6 & 112.00617768 \\
\hline A7 & 106.50856878 \\
\hline A8 & 109.32301756 \\
\hline A9 & 110.29129115 \\
\hline A10 & 109.15510741 \\
\hline A11 & 112.03526591 \\
\hline A12 & 114.00690044 \\
\hline A13 & 109.42351105 \\
\hline A14 & 109.88740119 \\
\hline A15 & 109.38988789 \\
\hline A16 & 101.40730278 \\
\hline A17 & 107.01788247 \\
\hline A18 & 106.01005338 \\
\hline A19 & 107.85648477 \\
\hline A20 & 105.39320408 \\
\hline A21 & 110.06078305 \\
\hline A22 & 108.96569767 \\
\hline A23 & 109.40964461 \\
\hline A24 & 111.41360556 \\
\hline A 25 & 108.94946055 \\
\hline D1 & 139.64237489 \\
\hline D2 & -133.95408747 \\
\hline D3 & -75.35413053 \\
\hline D4 & -67.27321830 \\
\hline D5 & -35.62985652 \\
\hline D6 & 165.17342551 \\
\hline D7 & -68.52740365 \\
\hline D8 & 51.76353862 \\
\hline D9 & 171.50680838 \\
\hline
\end{tabular}




$\begin{array}{lr}\text { D10 } & -70.06800310 \\ \text { D11 } & 85.39489019 \\ \text { D12 } & 169.35294427 \\ \text { D13 } & -70.52489663 \\ \text { D14 } & 49.38585820 \\ \text { D15 } & -152.40756194 \\ \text { D16 } & -67.71068351 \\ \text { D17 } & 174.96989933 \\ \text { D18 } & -51.93411078 \\ \text { D19 } & -171.76797334 \\ \text { D20 } & 60.37926654 \\ \text { D21 } & -179.45991700 \\ \text { D22 } & -60.41974203 \\ \text { D23 } & 70.29652453 \\ \text { D24 } & -68.56422670\end{array}$

Harmonic Vibrational Frequencies $\left(\mathbf{c m}^{-\mathbf{1}}\right)$ :

$\begin{array}{lccccccccccr}34.03 & 41.01 & 73.16 & 99.64 & 128.17 & 138.78 & 157.80 & 173.79 & 186.74 & 214.15 & 219.67 \\ 266.07 & 287.73 & 307.95 & 314.55 & 353.82 & 383.09 & 413.96 & 431.68 & 503.31 & 519.55 \\ 569.26 & 587.19 & 594.39 & 697.53 & 699.28 & 875.92 & 891.23 & 908.87 & 929.83 & 939.46 \\ 953.30 & 1006.23 & 1020.91 & 1059.14 & 1083.26 & 134.11 & 1141.67 & 1147.59 & 1150.92 \\ 1156.53 & 1179.82 & 1201.96 & 1230.09 & 1285.22 & 1357.07 & 1364.07 & 1393.53 & 1396.18 \\ 1401.87 & 1410.27 & 1420.22 & 1425.92 & 1460.73 & 1469.39 & 1483.24 & 1484.42 & 1490.30 \\ 1495.12 & 1497.34 & 1498.17 & 3073.94 & 3077.01 & 3077.77 & 3108.15 & 3110.02 & 3112.27 \\ 3158.13 & 3159.82 & 3159.91 & 3163.38 & 3167.54 & 3168.97 & 3765.41 & 3901.87 & \\ \text { Rotational constants (B/GHZ): } & 0.9989220 & 0.6205029 & 0.4912401 & \end{array}$

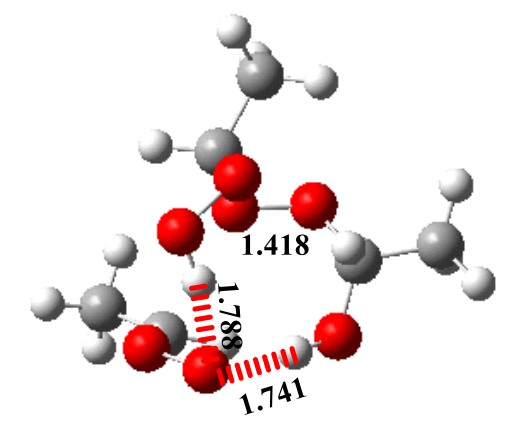

IM4b

$\mathrm{C}$

$\begin{array}{lll}\mathrm{C} & 1 & \mathrm{~B} 1\end{array}$

$\begin{array}{lllll}\mathrm{H} & 2 & \mathrm{~B} 2 & 1 & \mathrm{~A} 1\end{array}$

$\begin{array}{llllllll}\mathrm{H} & 2 & \mathrm{~B} 3 & 1 & \mathrm{~A} 2 & 3 & \mathrm{D} 1 & 0\end{array}$

$\begin{array}{llllllll}\mathrm{H} & 2 & \mathrm{~B} 4 & 1 & \mathrm{~A} 3 & 3 & \mathrm{D} 2 & 0\end{array}$

$\begin{array}{llllllll}\mathrm{O} & 1 & \mathrm{~B} 5 & 2 & \mathrm{~A} 4 & 3 & \mathrm{D} 3 & 0\end{array}$

$\begin{array}{llllllll}\mathrm{H} & 6 & \mathrm{~B} 6 & 1 & \mathrm{~A} 5 & 2 & \mathrm{D} 4 & 0\end{array}$

$\begin{array}{llllllll}\mathrm{C} & 6 & \mathrm{~B} 7 & 1 & \mathrm{~A} 6 & 2 & \text { D5 } & 0\end{array}$

$\begin{array}{llllllll}\mathrm{C} & 8 & \mathrm{~B} 8 & 6 & \mathrm{~A} 7 & 1 & \text { D6 } & 0\end{array}$

$\begin{array}{llllllll}\mathrm{H} & 9 & \mathrm{~B} 9 & 8 & \mathrm{~A} 8 & 6 & \mathrm{D} 7 & 0\end{array}$ 


\begin{tabular}{|c|c|c|c|c|c|c|}
\hline $\mathrm{H}$ & 9 & B10 & 8 & A9 & 6 & D8 \\
\hline $\mathrm{H}$ & 9 & B11 & 8 & A 10 & 6 & D9 \\
\hline $\mathrm{O}$ & 8 & B12 & 6 & A11 & 1 & D10 \\
\hline $\mathrm{O}$ & 8 & B13 & 6 & A12 & 1 & D11 \\
\hline $\mathrm{H}$ & 8 & B14 & 6 & A13 & 1 & D12 \\
\hline $\mathrm{H}$ & 1 & B15 & 6 & A14 & 2 & D13 \\
\hline $\mathrm{O}$ & 1 & B16 & 6 & A15 & 2 & D14 \\
\hline $\mathrm{O}$ & 17 & B17 & 1 & A16 & 6 & D15 \\
\hline $\mathrm{C}$ & 18 & B18 & 17 & A17 & 1 & D16 \\
\hline $\mathrm{H}$ & 19 & B19 & 18 & A18 & 17 & D17 \\
\hline $\mathrm{C}$ & 19 & B20 & 18 & A19 & 17 & D18 \\
\hline $\mathrm{H}$ & 21 & B21 & 19 & A20 & 18 & D19 \\
\hline $\mathrm{H}$ & 21 & B22 & 19 & A 21 & 18 & D20 \\
\hline $\mathrm{H}$ & 21 & B23 & 19 & A22 & 18 & D21 \\
\hline $\mathrm{O}$ & 19 & B24 & 18 & $\mathrm{~A} 23$ & 17 & D22 \\
\hline $\mathrm{O}$ & 25 & B25 & 19 & A24 & 18 & D23 \\
\hline $\mathrm{H}$ & 26 & B26 & 25 & A25 & 19 & D24 \\
\hline
\end{tabular}

Variables:

$\begin{array}{ll}\text { B1 } & 1.50971389 \\ \text { B2 } & 1.08731480 \\ \text { B3 } & 1.08893475 \\ \text { B4 } & 1.08783924 \\ \text { B5 } & 1.38126481 \\ \text { B6 } & 0.97997535 \\ \text { B7 } & 3.09683344 \\ \text { B8 } & 1.47208233 \\ \text { B9 } & 1.09199636 \\ \text { B10 } & 1.09067128 \\ \text { B11 } & 1.08573996 \\ \text { B12 } & 2.24965673 \\ \text { B13 } & 1.23877899 \\ \text { B14 } & 1.08821958 \\ \text { B15 } & 1.09209117 \\ \text { B16 } & 1.43363029 \\ \text { B17 } & 1.41761745 \\ \text { B18 } & 1.42376575 \\ \text { B19 } & 1.09248423 \\ \text { B20 } & 1.50972754 \\ \text { B21 } & 1.08705435 \\ \text { B22 } & 1.08725755 \\ \text { B23 } & 1.08726875 \\ \text { B24 } & 1.39136939 \\ \text { B25 } & 1.41639088 \\ \text { B26 } & 0.98337025\end{array}$




\begin{tabular}{|c|c|}
\hline A1 & 108.87248638 \\
\hline $\mathrm{A} 2$ & 109.22580512 \\
\hline A3 & 110.92826347 \\
\hline A4 & 109.98380516 \\
\hline A5 & 109.27330830 \\
\hline A6 & 106.93379592 \\
\hline A7 & 147.20662058 \\
\hline A8 & 107.31325578 \\
\hline A9 & 109.51363687 \\
\hline A10 & 111.32287060 \\
\hline A11 & 58.22785283 \\
\hline A 12 & 90.90545366 \\
\hline A13 & 30.82054598 \\
\hline A14 & 110.98798739 \\
\hline A15 & 111.64623190 \\
\hline A16 & 110.19204826 \\
\hline A17 & 111.15543258 \\
\hline A18 & 102.30121766 \\
\hline A19 & 113.42440636 \\
\hline A20 & 108.83008232 \\
\hline A 21 & 110.27952279 \\
\hline A22 & 109.93569481 \\
\hline A23 & 111.80295282 \\
\hline A24 & 108.74772470 \\
\hline A 25 & 104.21966745 \\
\hline D1 & 118.73839395 \\
\hline D2 & -120.52078093 \\
\hline D3 & -61.95233780 \\
\hline D4 & 157.13852095 \\
\hline D5 & -146.89835259 \\
\hline D6 & 64.65969904 \\
\hline D7 & -31.85145049 \\
\hline D8 & 84.36506363 \\
\hline D9 & -152.84950939 \\
\hline D10 & -98.63323274 \\
\hline D11 & -91.23153019 \\
\hline D12 & 114.31367953 \\
\hline D13 & -125.10161198 \\
\hline D14 & 114.78552957 \\
\hline D15 & 66.55979873 \\
\hline D16 & 94.70615654 \\
\hline D17 & 159.76604016 \\
\hline D18 & 37.74099439 \\
\hline D19 & 166.25762108 \\
\hline
\end{tabular}




$\begin{array}{lr}\text { D20 } & -74.17596929 \\ \text { D21 } & 45.81783677 \\ \text { D22 } & -83.84925776 \\ \text { D23 } & -75.23575748 \\ \text { D24 } & 87.03251440\end{array}$

Harmonic Vibrational Frequencies $\left(\mathbf{c m}^{-\mathbf{1}}\right)$ :

$\begin{array}{llllllllllll}34.30 & 63.36 & 78.34 & 99.69 & 118.45 & 121.17 & 129.17 & 149.64 & 179.36 & 211.79 & 219.88 \\ 235.22 & 240.87 & 273.44 & 303.47 & 317.64 & 333.39 & 362.58 & 397.46 & 426.64 & 530.64 \\ 566.74 & 578.08 & 621.37 & 707.45 & 780.95 & 852.16 & 875.47 & 887.20 & 899.36 & 915.60 \\ 925.75 & 955.09 & 1009.29 & 1014.82 & 1062.08 & 1108.48 & 1119.68 & 1128.41 & 1140.11 \\ 1160.06 & 1187.17 & 1197.20 & 1206.04 & 1356.42 & 1361.35 & 1388.17 & 1393.31 & 1402.31 \\ 1410.99 & 1425.34 & 1444.34 & 1465.44 & 1467.50 & 1485.38 & 1490.86 & 1493.45 & 1496.01 \\ 1508.31 & 1530.24 & 1697.23 & 3057.56 & 3071.21 & 3078.63 & 3099.53 & 3101.43 & 3122.74 \\ 3151.85 & 3162.00 & 3165.71 & 3166.38 & 3173.89 & 3197.03 & 3404.80 & 3455.08 & \\ \text { Rotational constants (B/GHZ): } & 0.8262655 & 0.6476380 & 0.5240421 & \end{array}$

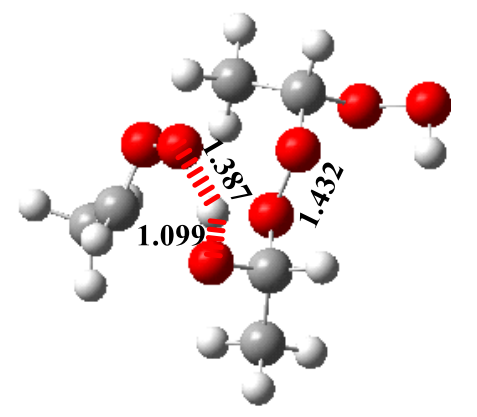

TS4b

C

$\begin{array}{lll}\mathrm{C} & 1 & \mathrm{~B} 1\end{array}$

$\begin{array}{lllll}\mathrm{H} & 2 & \mathrm{~B} 2 & 1 & \mathrm{~A} 1\end{array}$

$\begin{array}{lllll}\mathrm{H} & 2 & \mathrm{~B} 3 & 1 & \mathrm{~A} 2\end{array}$

$\mathrm{H} \quad 2$

B4 1

$3 \quad \mathrm{D} 1 \quad 0$

$\mathrm{O} \quad 1$

B5 2

A3 $3 \quad$ D2 0

$\mathrm{H} \quad 6$

B6 1

A4 3

D3 0

C 6

B7 1

A5 2

D4 0

C 8

B8 6

A6 2 D5 0

H 9

B9 8

A7 $1 \quad$ D6 0

$\mathrm{H} \quad 9$

H 9

B10 8

$\begin{array}{llll}\text { A8 } & 6 & \text { D7 } & 0\end{array}$

A9 $6 \quad$ D8 0

O 8

B11 8

A10

D9 0

O 8

B12 6

A11 1

D10 0

B13 6

A12 1

D11 0

B14 6

A13 1

D12 0

B15 6

A14 8

D13 0

$\begin{array}{ll}\mathrm{H} & 1 \\ \mathrm{O} & 1\end{array}$

B16 6

A15 8

D14 0

O 17

B17 1

A16 6

D15 0

C 18

B18 17

A17 1

D16 0

H 19

B19 18

A18 17

D17 0 


$\begin{array}{llllllll}\text { C } & 19 & \text { B20 } & 18 & \text { A19 } & 17 & \text { D18 } & 0 \\ \text { H } & 21 & \text { B21 } & 19 & \text { A20 } & 18 & \text { D19 } & 0 \\ \text { H } & 21 & \text { B22 } & 19 & \text { A21 } & 18 & \text { D20 } & 0 \\ \text { H } & 21 & \text { B23 } & 19 & \text { A22 } & 18 & \text { D21 } & 0 \\ \text { O } & 19 & \text { B24 } & 18 & \text { A23 } & 17 & \text { D22 } & 0 \\ \text { O } & 25 & \text { B25 } & 19 & \text { A24 } & 18 & \text { D23 } & 0 \\ \text { H } & 26 & \text { B26 } & 25 & \text { A25 } & 19 & \text { D24 } & 0\end{array}$

Variables:

1.51135490

B2 1.08719356

B3 1.08941491

B4 1.08750585

B5 1.39206112

B6 1.09904681

B7 2.01655519

B8 1.47946920

B9 1.08702995

B10 1.09250770

B11 1.08583226

B12 2.17914880

B13 1.26731438

B14 1.08439845

B15 1.09504521

B16 1.40976923

B17 1.43191750

B18 1.40352881

B19 1.09209770

B20 1.51001292

B21 1.08726424

B22 1.08781966

B23 1.08715779

B24 1.39950397

B25 1.41330314

B26 0.96577011

A1 $\quad 109.06279137$

A2 109.86680895

A3 110.57334854

A4 110.01984734

A5 115.37864391

A6 123.21133579

A7 111.90883330

A8 $\quad 109.36933558$

A9 $\quad 108.16701845$

A10 $\quad 110.73345272$ 


\begin{tabular}{|c|c|}
\hline A11 & 69.54778778 \\
\hline A 12 & 94.42132904 \\
\hline A13 & 81.54202377 \\
\hline A14 & 108.78237289 \\
\hline A 15 & 112.68614711 \\
\hline A16 & 105.44692004 \\
\hline A17 & 108.37616026 \\
\hline A18 & 101.67839617 \\
\hline A19 & 114.54676231 \\
\hline A 20 & 109.25889162 \\
\hline A 21 & 110.12328795 \\
\hline A 22 & 108.85927813 \\
\hline A 23 & 111.34170866 \\
\hline A24 & 108.27062600 \\
\hline A 25 & 102.35174224 \\
\hline D1 & 119.22524315 \\
\hline D2 & -120.60214204 \\
\hline D3 & -54.97744044 \\
\hline D4 & 177.55608707 \\
\hline D5 & -93.27045911 \\
\hline D6 & 41.40139413 \\
\hline D7 & 50.14021398 \\
\hline D8 & 167.55375535 \\
\hline D9 & -71.98651333 \\
\hline D10 & -111.41375750 \\
\hline D11 & -81.54589758 \\
\hline D12 & 161.58855492 \\
\hline D13 & 143.79777570 \\
\hline D14 & 24.40159460 \\
\hline D15 & 75.36780610 \\
\hline D16 & 178.03295493 \\
\hline D17 & 173.54672870 \\
\hline D18 & 51.44608850 \\
\hline D19 & 170.35419264 \\
\hline D20 & -70.06144343 \\
\hline D21 & 50.64003125 \\
\hline D22 & -69.44014438 \\
\hline $\mathrm{D} 23$ & -65.30630775 \\
\hline D24 & 79.74807401 \\
\hline
\end{tabular}

Harmonic Vibrational Frequencies $\left(\mathbf{c m}^{-\mathbf{1}}\right)$ :

$\begin{array}{lllllllllll}531.58 i & 51.24 & 56.67 & 67.11 & 88.35 & 112.60 & 140.82 & 162.11 & 175.28 & 206.43 & 221.54\end{array}$ $\begin{array}{llllllllll}236.26 & 254.77 & 258.60 & 285.35 & 316.24 & 342.31 & 354.95 & 399.28 & 421.86 & 480.86\end{array}$ $\begin{array}{llllllllll}527.55 & 571.75 & 582.94 & 617.52 & 703.88 & 869.05 & 890.60 & 899.37 & 927.82 & 943.78\end{array}$ $\begin{array}{lllllllll}951.15 & 1012.66 & 1017.87 & 1061.73 & 1091.11 & 1124.84 & 1144.44 & 1152.31 & 1158.94\end{array}$ 


\begin{tabular}{|c|c|c|c|c|c|c|c|c|c|}
\hline 1181.89 & 1199.36 & 1215.53 & 1238.95 & 1346 & & 1360.55 & 1362. & 1390.77 & 1393.74 \\
\hline 1405.03 & 1414.98 & 1419.91 & 1430.18 & 1446 & & 1454.86 & 1481. & 1490.46 & 1494.72 \\
\hline 1499.94 & 1506.18 & 1615.35 & 1968.74 & 3057 & .14 & 3072.40 & 3072 & 3075.86 & 3115.20 \\
\hline 3138.04 & 3152.74 & 3154.41 & 3168.04 & 3169.91 & 31 & $180.36 \quad 320$ & 9.75 & 3826.63 & \\
\hline \multicolumn{3}{|c|}{ Rotational constants (B/GHZ): } & & 669644 & & 0.5352829 & & 0.4656729 & \\
\hline \multicolumn{9}{|l|}{$\mathrm{C}$} & \\
\hline$C$ & 1 & $\mathrm{~B} 1$ & & & & & & & \\
\hline & & & & & & & & & \\
\hline $\mathrm{H}$ & 2 & B2 & 1 & A1 & & & & & \\
\hline $\mathrm{H}$ & 2 & B3 & 1 & A2 & 3 & D1 & 0 & & \\
\hline $\mathrm{H}$ & 2 & B4 & 1 & A3 & 3 & D2 & 0 & & \\
\hline $\mathrm{O}$ & 1 & B5 & 2 & A4 & 4 & D3 & 0 & & \\
\hline $\mathrm{H}$ & 6 & B6 & 1 & A5 & 2 & D4 & 0 & & \\
\hline $\mathrm{C}$ & 6 & B7 & 1 & A6 & 2 & D5 & 0 & & \\
\hline $\mathrm{C}$ & 8 & B8 & 6 & A7 & 1 & D6 & 0 & & \\
\hline $\mathrm{H}$ & 9 & B9 & 8 & A8 & 6 & D7 & 0 & & \\
\hline $\mathrm{H}$ & 9 & B10 & 8 & A9 & 6 & D8 & 0 & & \\
\hline $\mathrm{H}$ & 9 & B11 & 8 & A10 & 6 & D9 & 0 & & \\
\hline $\mathrm{O}$ & 8 & B12 & 6 & A11 & 1 & D10 & 0 & & \\
\hline $\mathrm{O}$ & 8 & B13 & 6 & A12 & 1 & D11 & 0 & & \\
\hline $\mathrm{H}$ & 8 & B14 & 6 & A13 & 1 & D12 & 0 & & \\
\hline $\mathrm{H}$ & 1 & B15 & 6 & A14 & 8 & D13 & 0 & & \\
\hline $\mathrm{O}$ & 1 & B16 & 6 & A15 & 8 & D14 & 0 & & \\
\hline $\mathrm{O}$ & 17 & B17 & 1 & A16 & 6 & D15 & 0 & & \\
\hline $\mathrm{C}$ & 18 & B18 & 17 & A 17 & 1 & D16 & 0 & & \\
\hline $\mathrm{H}$ & 19 & B19 & 18 & A18 & 17 & D17 & 0 & & \\
\hline $\mathrm{C}$ & 19 & B20 & 18 & A19 & 17 & D18 & 0 & & \\
\hline $\mathrm{H}$ & 21 & B21 & 19 & A 20 & 18 & D19 & 0 & & \\
\hline $\mathrm{H}$ & 21 & B22 & 19 & A21 & 18 & D20 & 0 & & \\
\hline $\mathrm{H}$ & 21 & B23 & 19 & A22 & 18 & D21 & 0 & & \\
\hline $\mathrm{O}$ & 19 & B24 & 18 & A23 & 17 & D22 & 0 & & \\
\hline $\mathrm{O}$ & 25 & B25 & 19 & A24 & 18 & D23 & 0 & & \\
\hline $\mathrm{H}$ & 26 & B26 & 25 & A25 & 19 & D24 & 0 & & \\
\hline \multicolumn{10}{|c|}{ Variables: } \\
\hline \multicolumn{2}{|l|}{ B1 } & .51778472 & & & & & & & \\
\hline \multicolumn{2}{|l|}{ B2 } & .08781576 & & & & & & & \\
\hline
\end{tabular}




\begin{tabular}{|c|c|}
\hline B3 & 1.08592820 \\
\hline B4 & 1.08802912 \\
\hline B5 & 1.40471297 \\
\hline B6 & 2.47347935 \\
\hline B7 & 1.40670352 \\
\hline B8 & 1.51408940 \\
\hline B9 & 1.08763919 \\
\hline B10 & 1.08776992 \\
\hline B11 & 1.08836529 \\
\hline B12 & 2.28963871 \\
\hline B13 & 1.40472425 \\
\hline B14 & 1.09097849 \\
\hline B15 & 1.09229739 \\
\hline B16 & 1.40049727 \\
\hline B17 & 1.43068758 \\
\hline B18 & 1.41373867 \\
\hline B19 & 1.09252189 \\
\hline B20 & 1.50862462 \\
\hline B21 & 1.08718239 \\
\hline B22 & 1.08710720 \\
\hline B23 & 1.08681197 \\
\hline B24 & 1.39576218 \\
\hline B25 & 1.41387598 \\
\hline B26 & 0.96519263 \\
\hline $\mathrm{A} 1$ & 108.81488813 \\
\hline $\mathrm{A} 2$ & 112.01611333 \\
\hline A3 & 109.19802834 \\
\hline A4 & 115.23172648 \\
\hline A5 & 101.13924911 \\
\hline A6 & 122.78553737 \\
\hline A7 & 116.97727072 \\
\hline A8 & 110.17478636 \\
\hline A9 & 108.64193458 \\
\hline A 10 & 110.59049177 \\
\hline A11 & 91.47342435 \\
\hline $\mathrm{A} 12$ & 111.66080708 \\
\hline A 13 & 102.98865479 \\
\hline A14 & 103.23348437 \\
\hline A 15 & 113.45198599 \\
\hline A16 & 106.23972759 \\
\hline A 17 & 107.87736588 \\
\hline A 18 & 101.67656647 \\
\hline A19 & 114.43187866 \\
\hline A20 & 108.91260130 \\
\hline
\end{tabular}




\begin{tabular}{lr} 
A21 & 110.16493996 \\
A22 & 109.14585399 \\
A23 & 110.84622903 \\
A24 & 108.32342169 \\
A25 & 102.30925376 \\
D1 & 120.92997456 \\
D2 & -118.96586348 \\
D3 & 77.28792069 \\
D4 & -160.22610906 \\
D5 & -79.18683181 \\
D6 & 47.19177581 \\
D7 & 51.20582404 \\
D8 & 170.51649771 \\
D9 & -70.50979939 \\
D10 & -103.72892230 \\
D11 & -73.45359851 \\
D12 & 170.07108324 \\
D13 & 160.71757623 \\
D14 & 43.05162316 \\
D15 & 66.40232755 \\
D16 & 179.21301691 \\
D17 & 172.45939587 \\
D18 & 50.22675868 \\
D19 & 169.50672787 \\
D20 & -70.77569657 \\
D21 & 49.15602421 \\
D22 & -70.92089317 \\
D23 & -65.79342151 \\
D24 & 85.65306895 \\
\hline &
\end{tabular}

Harmonic Vibrational Frequencies $\left(\mathrm{cm}^{-1}\right)$ :

$\begin{array}{lccccccccccc}41.73 & 49.93 & 79.89 & 107.21 & 124.79 & 159.28 & 182.13 & 191.62 & 209.26 & 250.57 & 252.74 \\ 266.69 & 292.32 & 309.35 & 321.44 & 335.89 & 352.23 & 413.86 & 432.13 & 487.40 & 515.13 \\ 523.31 & 585.20 & 590.34 & 681.39 & 756.21 & 805.24 & 886.68 & 898.22 & 920.09 & 940.51 \\ 952.40 & 1014.97 & 1060.08 & 1063.71 & 1114.74 & 1133.20 & 1136.44 & 1152.50 & 1157.41 \\ 1170.07 & 1200.42 & 1208.75 & 1232.12 & 1361.67 & 1363.69 & 1377.88 & 1406.84 & 1411.92 \\ 1415.29 & 1426.82 & 1430.16 & 1434.41 & 1449.82 & 1474.24 & 1485.54 & 1487.06 & 1488.74 \\ 1499.51 & 1505.25 & 1516.41 & 3069.67 & 3075.98 & 3077.17 & 3098.27 & 3111.47 & 3118.27 \\ 3149.41 & 3150.71 & 3156.93 & 3159.87 & 3176.11 & 3177.27 & 3761.21 & 3818.68 & \\ \text { Rotational constants (B/GHZ): } & 1.1588304 & 0.5531838 & 0.4628137 & \end{array}$




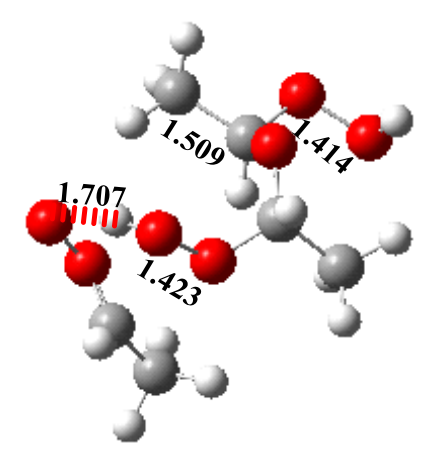

IM4c

C

$\mathrm{H} \quad 1$

O 1

O 1

$\mathrm{H} \quad 3$

C 4

O 6

O 6

C 1

$\mathrm{H} \quad 9$

H 9

H 9

$\begin{array}{ll}\text { O } & 1\end{array}$

C 13

$\mathrm{H} \quad 14$

$\begin{array}{ll}\text { C } & 14\end{array}$

$\mathrm{H} \quad 16$

$\mathrm{H} \quad 16$

$\mathrm{H} \quad 16$

O 14

O 20

$\mathrm{H} \quad 21$

C 6

$\mathrm{H} \quad 23$

$\mathrm{H} \quad 23$

$\mathrm{H} \quad 23$

$\mathrm{H} \quad 6$

Variables:

B1

1.08988603

B2

2.29960509

B3

1.40574902

B4

0.99271921

B5

B6

B1

B2 $2 \quad$ A1

B3 3

A2 2

D1 0

B4 1

A3 4

D2 0

B5 1

A4 3

D3 0

B6 4

A5 1

D4 0

B7 4

A6 1

D5 0

B8 4

A7 3

D6 0

B9 1

A8 4

D7 0

B10 1

A9 4

D8 0

B11 1

A10 4

D9 0

B12 $4 \quad$ A11 3

D10 0

$\begin{array}{llll}\text { B } 13 & 1 & \text { A12 } & 4\end{array}$

D11 0

B14 13

A13 1

D12 0

B15 13

A14 1

D13 0

B16 14

A15 13

D14 0

B17 $14 \quad$ A16 13

D15 0

B18 14

A17 13

D16 0

B19 13

A18 1

D17 0

$\begin{array}{llll}\text { B20 } & 14 & \text { A19 } & 13\end{array}$

D18 0

B21 20

A20 14

D19 0

B22 $4 \quad$ A21 1

D20 0

$\begin{array}{llllll}\text { B23 } & 6 & \text { A22 } & 4 & \text { D21 } & 0\end{array}$

$\begin{array}{llllll}\text { B24 } & 6 & \text { A23 } & 4 & \text { D22 } & 0\end{array}$

$\begin{array}{llllll}\text { B25 } & 6 & \text { A24 } & 4 & \text { D23 } & 0\end{array}$

$\begin{array}{llllll}\text { B26 } & 4 & \text { A25 } & 1 & \text { D24 } & 0\end{array}$

2.23565349 


\begin{tabular}{|c|c|}
\hline B7 & 1.23696252 \\
\hline B8 & 1.51678566 \\
\hline B9 & 1.08834973 \\
\hline B 10 & 1.08832284 \\
\hline B11 & 1.08735019 \\
\hline B12 & 1.40183326 \\
\hline B13 & 1.40807472 \\
\hline B14 & 1.09402576 \\
\hline B15 & 1.50852929 \\
\hline B16 & 1.08871594 \\
\hline B17 & 1.08832217 \\
\hline B 18 & 1.08764312 \\
\hline B19 & 1.40484278 \\
\hline B20 & 1.41402464 \\
\hline B21 & 0.96517079 \\
\hline B22 & 1.47267808 \\
\hline B23 & 1.08607725 \\
\hline B24 & 1.09183282 \\
\hline B 25 & 1.09094578 \\
\hline B26 & 1.08799804 \\
\hline $\mathrm{A} 1$ & 80.59763462 \\
\hline $\mathrm{A} 2$ & 35.88210385 \\
\hline A3 & 118.61058201 \\
\hline A4 & 151.09511942 \\
\hline A5 & 79.63349535 \\
\hline A6 & 90.51805067 \\
\hline A7 & 105.48599470 \\
\hline A8 & 109.24674426 \\
\hline A9 & 111.69896699 \\
\hline A10 & 109.40370252 \\
\hline A11 & 112.48522537 \\
\hline A12 & 118.04993538 \\
\hline A13 & 110.06607374 \\
\hline A14 & 110.46620569 \\
\hline A15 & 108.83260544 \\
\hline A16 & 110.21101903 \\
\hline A17 & 109.74733276 \\
\hline A 18 & 109.34439981 \\
\hline A19 & 108.71266761 \\
\hline A20 & 101.55195751 \\
\hline A21 & 103.98011563 \\
\hline A 22 & 111.09364650 \\
\hline A 23 & 107.78421319 \\
\hline A24 & 109.69459378 \\
\hline
\end{tabular}




$\begin{array}{lr}\text { A25 } & 74.35202657 \\ \text { D1 } & 140.51155807 \\ \text { D2 } & -67.96473918 \\ \text { D3 } & 139.14702820 \\ \text { D4 } & -103.97079536 \\ \text { D5 } & -71.85864678 \\ \text { D6 } & 160.65084845 \\ \text { D7 } & -59.91206576 \\ \text { D8 } & 61.38837581 \\ \text { D9 } & -179.04126659 \\ \text { D10 } & -73.60180211 \\ \text { D11 } & -53.53927270 \\ \text { D12 } & -4.80830856 \\ \text { D13 } & 119.89401887 \\ \text { D14 } & 55.01528347 \\ \text { D15 } & 175.00004187 \\ \text { D16 } & -64.83461936 \\ \text { D17 } & -124.70145506 \\ \text { D18 } & 65.71984230 \\ \text { D19 } & -90.93592897 \\ \text { D20 } & 48.78474884 \\ \text { D21 } & -98.51260590 \\ \text { D22 } & 22.51852363 \\ \text { D23 } & 139.20594197 \\ \text { D24 } & 170.04876827\end{array}$

Harmonic Vibrational Frequencies $\left(\mathrm{cm}^{-1}\right)$ :

$\begin{array}{lllllllllll}26.86 & 47.88 & 69.44 & 80.97 & 110.38 & 120.64 & 147.77 & 166.59 & 181.43 & 206.90 & 225.63\end{array}$ $\begin{array}{llllllllll}227.88 & 253.31 & 266.99 & 269.80 & 308.24 & 318.19 & 338.47 & 349.96 & 362.62 & 434.08\end{array}$ $\begin{array}{lllllllllll}514.98 & 555.47 & 566.95 & 578.60 & 679.20 & 771.20 & 856.49 & 886.99 & 895.78 & 900.65\end{array}$ $\begin{array}{lllllllll}929.94 & 966.81 & 991.27 & 1056.17 & 1062.39 & 1102.75 & 1118.04 & 1137.80 & 1141.70\end{array}$ $\begin{array}{lllllllll}1181.28 & 1187.39 & 1198.96 & 1219.44 & 1354.24 & 1369.97 & 1374.98 & 1408.27 & 1419.08\end{array}$ $\begin{array}{llllllllll}1420.92 & 1425.49 & 1427.21 & 1436.44 & 1467.34 & 1471.01 & 1487.44 & 1497.06 & 1501.07\end{array}$ $\begin{array}{lllllllll}1502.48 & 1614.43 & 1700.01 & 3064.53 & 3069.58 & 3074.40 & 3081.75 & 3123.61 & 3131.67\end{array}$ $\begin{array}{llllllll}3151.31 & 3157.44 & 3158.21 & 3169.27 & 3171.31 & 3191.81 & 3220.42 & 3822.76\end{array}$

Rotational constants (B/GHZ): $\quad 0.9835528 \quad 0.5122121 \quad 0.4796666$

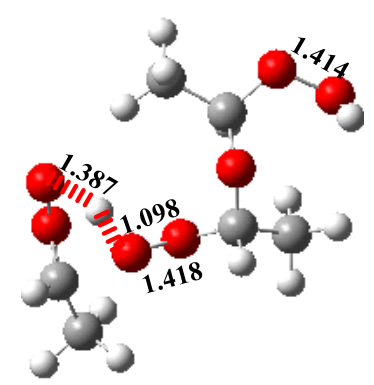

$\mathrm{TS} 4 \mathrm{c}$ 
C

$\begin{array}{lll}\mathrm{H} & 1 & \mathrm{~B} 1\end{array}$

O 1

B1

B2 2

B3 3

A1

O 1

B4 1

A2

A3 4

D1 0

B5 1

A4 4

D2 0

C 3

B6 3

D3 0

B7 3

A5 1

D4 0

O 6

C 1

B8 4

D5 0

A6 1

D6 0

$\begin{array}{llllll}\text { B9 } & 1 & \text { A8 } & 4 & \text { D7 } & 0\end{array}$

$\mathrm{H} \quad 9$

$\mathrm{H} \quad 9$

$\mathrm{H} \quad 9$

O 1

C 13

$\mathrm{H} \quad 14$

C 14

$\mathrm{H} \quad 16$

$\mathrm{H} \quad 16$

B10

B11 1

A9 4

D8 0

B12 4

A10 4

D9 0

B13 1

A11 3

D10 0

A12 $4 \quad$ D11 0

$\begin{array}{llllll}\text { B14 } & 13 & \text { A13 } & 1 & \text { D12 } & 0\end{array}$

B15 13

B16 14

A14 1

D13 0

A15 $13 \quad$ D14 0

$\begin{array}{llllll}\text { B17 } & 14 & \text { A16 } & 13 & \text { D15 } & 0\end{array}$

$\begin{array}{llllll}\mathrm{B} 18 & 14 & \mathrm{~A} 17 & 13 & \mathrm{D} 16 & 0\end{array}$

$\mathrm{H} \quad 16$

O 14

O 20

$\mathrm{H} \quad 21$

C 6

B19 13

A18 1

D17 0

B20 14

A19 13

D18 0

B21 20

A20 14

D19 0

B22 3

A21 1

D20 0

B23 6

A22 3

D21 0

B24 6

A23 3

D22 0

B25 6

A24 3

D23 0

B26 3

A25 1

D24 0

Variables:

$\begin{array}{ll}\text { B1 } & 1.09027824 \\ \text { B2 } & 2.28871652 \\ \text { B3 } & 1.41025512 \\ \text { B4 } & 1.09822617 \\ \text { B5 } & 2.04638788 \\ \text { B6 } & 2.17627638 \\ \text { B7 } & 1.26598624 \\ \text { B8 } & 1.51632880 \\ \text { B9 } & 1.08807711 \\ \text { B10 } & 1.08711601 \\ \text { B11 } & 1.08719138 \\ \text { B12 } & 1.39874708 \\ \text { B13 } & 1.41172244 \\ \text { B14 } & 1.09174360 \\ \text { B15 } & 1.50841540 \\ \text { B16 } & 1.08857215\end{array}$




$\begin{array}{lr}\text { B17 } & 1.08811697 \\ \text { B18 } & 1.08821200 \\ \text { B19 } & 1.40358429 \\ \text { B20 } & 1.41430354 \\ \text { B21 } & 0.96495567 \\ \text { B22 } & 1.47520706 \\ \text { B23 } & 1.08550621 \\ \text { B24 } & 1.08948570 \\ \text { B25 } & 1.09071355 \\ \text { B26 } & 1.08516488 \\ \text { A1 } & 80.90599422 \\ \text { A2 } & 36.09220734 \\ \text { A3 } & 114.02711144 \\ \text { A4 } & 136.71198258 \\ \text { A5 } & 69.20430217 \\ \text { A6 } & 92.89436318 \\ \text { A7 } & 105.52604985 \\ \text { A8 } & 109.04547160 \\ \text { A9 } & 111.99899896 \\ \text { A10 } & 109.37121565 \\ \text { A11 } & 111.87758695 \\ \text { A12 } & 118.22057919 \\ \text { A13 } & 110.18705608 \\ \text { A14 } & 110.66988430 \\ \text { A15 } & 109.24252325 \\ \text { A16 } & 109.98568527 \\ \text { A17 } & 109.61231449 \\ \text { A18 } & 108.91441600 \\ \text { A19 } & 108.48915612 \\ \text { A20 } & 101.53388125 \\ \text { A21 } & 108.92357287 \\ \text { A22 } & 110.71040380 \\ \text { A23 } & 107.8346981515 \\ \text { A24 } & 109.41746432 \\ \text { A25 } & \\ \text { D1 } & \end{array}$




$\begin{array}{lr}\text { D10 } & -72.23568393 \\ \text { D11 } & -59.19140006 \\ \text { D12 } & -5.50395753 \\ \text { D13 } & 118.97155337 \\ \text { D14 } & 52.11950511 \\ \text { D15 } & 172.18648570 \\ \text { D16 } & -68.13675924 \\ \text { D17 } & -125.50807962 \\ \text { D18 } & 66.58663246 \\ \text { D19 } & -93.21220483 \\ \text { D20 } & 45.46956963 \\ \text { D21 } & -89.07354800 \\ \text { D22 } & 31.27686293 \\ \text { D23 } & 148.63928212 \\ \text { D24 } & 166.57886308\end{array}$

Harmonic Vibrational Frequencies $\left(\mathbf{c m}^{-\mathbf{1}}\right)$ :

$\begin{array}{llllllllllll}505.89 i & 23.71 & 45.59 & 70.52 & 95.30 & 104.94 & 119.21 & 126.71 & 154.50 & 180.78 & 225.58 \\ 232.48 & 258.36 & 278.48 & 309.61 & 323.48 & 347.98 & 362.94 & 409.65 & 429.91 & 507.99 \\ 531.23 & 545.34 & 578.05 & 597.41 & 684.76 & 857.11 & 889.45 & 896.51 & 928.00 & 965.62 \\ 975.57 & 999.14 & 1012.81 & 1059.93 & 1097.71 & 1125.38 & 1137.93 & 1141.08 & 1182.45 \\ 1185.69 & 1197.12 & 1219.32 & 1240.41 & 1357.38 & 1363.36 & 1371.92 & 1412.11 & 1414.88 \\ 1421.35 & 1424.08 & 1434.14 & 1440.68 & 1452.31 & 1474.94 & 1484.18 & 1492.48 & 1493.27 \\ 1503.53 & 1577.25 & 1619.99 & 1921.59 & 3067.11 & 3068.84 & 3079.96 & 3118.32 & 3128.47 \\ 3135.79 & 3153.78 & 3158.32 & 3160.93 & 3171.61 & 3185.57 & 3200.64 & 3829.34 & \\ \text { Rotational constants (B/GHZ): } & 1.3716013 & 0.4360083 & 0.3611265 & \end{array}$

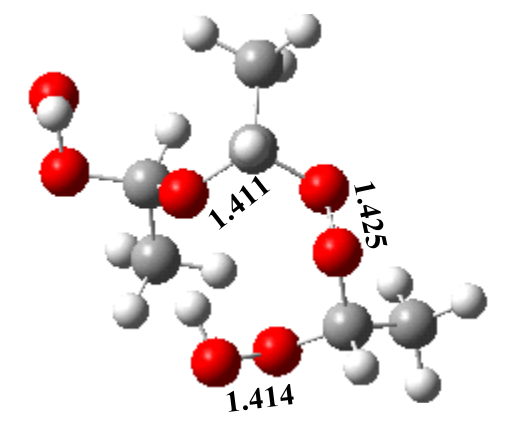

$\mathrm{P} 4 \mathrm{c}$

$\mathrm{C}$

$\begin{array}{lll}\mathrm{H} & 1 & \mathrm{~B} 1\end{array}$

$\begin{array}{lllll}\mathrm{O} & 1 & \mathrm{~B} 2 & 2 & \mathrm{~A} 1\end{array}$

$\begin{array}{llllllll}\mathrm{O} & 1 & \mathrm{~B} 3 & 3 & \mathrm{~A} 2 & 2 & \mathrm{D} 1 & 0\end{array}$

$\begin{array}{llllllll}\mathrm{H} & 3 & \mathrm{~B} 4 & 1 & \mathrm{~A} 3 & 4 & \mathrm{D} 2 & 0\end{array}$

$\begin{array}{lllllllll}\mathrm{C} & 3 & \mathrm{~B} 5 & 1 & \mathrm{~A} 4 & 4 & \mathrm{D} 3 & 0\end{array}$

$\begin{array}{llllllll}\mathrm{O} & 6 & \mathrm{~B} 6 & 3 & \mathrm{~A} 5 & 1 & \mathrm{D} 4 & 0\end{array}$

$\begin{array}{llllllll}\mathrm{O} & 6 & \mathrm{~B} 7 & 3 & \mathrm{~A} 6 & 1 & \mathrm{D} 5 & 0\end{array}$

$\begin{array}{llllllll}\mathrm{C} & 1 & \mathrm{~B} 8 & 4 & \mathrm{~A} 7 & 3 & \text { D6 } & 0\end{array}$

$\begin{array}{llllllll}\mathrm{H} & 9 & \text { B9 } & 1 & \text { A8 } & 4 & \text { D7 } & 0\end{array}$ 


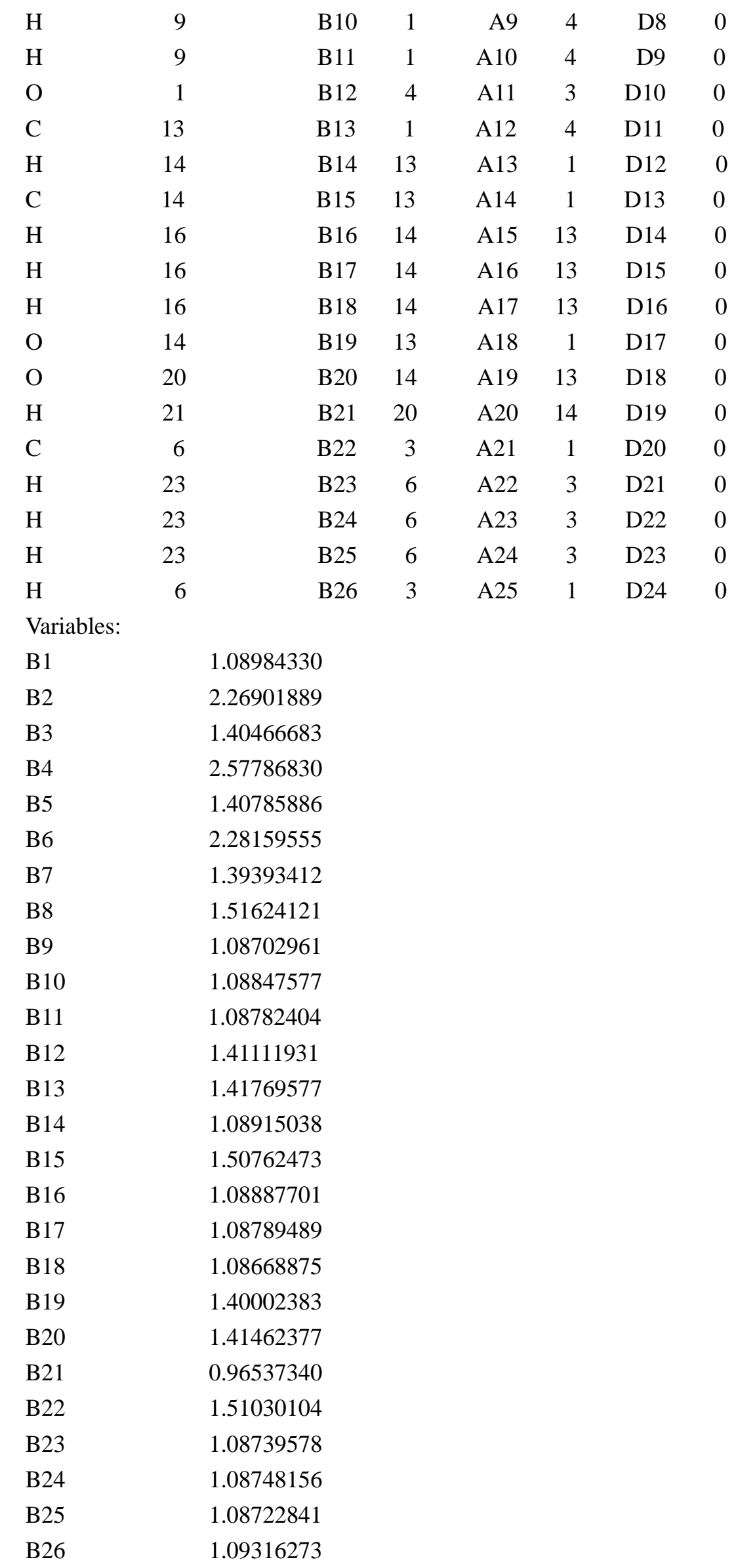




\begin{tabular}{|c|c|}
\hline A1 & 77.72733929 \\
\hline $\mathrm{A} 2$ & 37.01732810 \\
\hline A3 & 70.91714484 \\
\hline A4 & 127.63523441 \\
\hline A5 & 92.45262552 \\
\hline A6 & 112.04838758 \\
\hline A7 & 105.80271809 \\
\hline A8 & 108.13683857 \\
\hline A9 & 111.52423977 \\
\hline A10 & 110.26737557 \\
\hline A11 & 111.49360852 \\
\hline A12 & 120.41187612 \\
\hline A13 & 109.77976998 \\
\hline A14 & 112.21195588 \\
\hline A15 & 110.19990321 \\
\hline A16 & 109.49192381 \\
\hline A17 & 109.28809164 \\
\hline A18 & 107.49928572 \\
\hline A19 & 108.11938108 \\
\hline $\mathrm{A} 20$ & 101.75734298 \\
\hline A21 & 113.77402109 \\
\hline $\mathrm{A} 22$ & 110.03810860 \\
\hline A23 & 109.26792542 \\
\hline A 24 & 109.61596832 \\
\hline A 25 & 101.33262410 \\
\hline D1 & 144.23648516 \\
\hline D2 & -123.54921823 \\
\hline D3 & -71.07298332 \\
\hline D4 & -61.21761047 \\
\hline D5 & -30.20297612 \\
\hline D6 & 155.78733177 \\
\hline D7 & -72.98047146 \\
\hline D8 & 45.53923364 \\
\hline D9 & 167.18252740 \\
\hline D10 & -76.89803133 \\
\hline D11 & -87.46669639 \\
\hline D12 & -12.45243848 \\
\hline D13 & 112.91008497 \\
\hline D14 & 55.11212562 \\
\hline D15 & 175.66756697 \\
\hline D16 & -63.70804031 \\
\hline D17 & -130.74372586 \\
\hline D18 & 66.54326520 \\
\hline D19 & -91.22770080 \\
\hline
\end{tabular}




$\begin{array}{lr}\text { D20 } & 90.69001424 \\ \text { D21 } & -67.45750011 \\ \text { D22 } & 52.32064257 \\ \text { D23 } & 172.35577850 \\ \text { D24 } & -147.60391668\end{array}$

Harmonic Vibrational Frequencies $\left(\mathbf{c m}^{-1}\right)$ :

$\begin{array}{lllllllllll}38.99 & 49.34 & 97.71 & 114.58 & 126.67 & 168.63 & 198.49 & 206.95 & 227.98 & 237.59 & 239.92\end{array}$ $\begin{array}{llllllllll}258.94 & 293.61 & 307.79 & 320.41 & 359.23 & 365.41 & 415.98 & 437.88 & 501.76 & 544.14\end{array}$ $\begin{array}{llllllllll}576.62 & 583.29 & 597.64 & 673.63 & 703.75 & 843.37 & 880.01 & 903.02 & 920.52 & 939.41\end{array}$ $\begin{array}{lllllllll}968.90 & 1011.41 & 1057.48 & 1063.11 & 107.65 & 1137.69 & 1138.75 & 1148.55 & 1153.81\end{array}$ $\begin{array}{lllllllll}1176.98 & 1185.72 & 1216.69 & 1225.97 & 1347.07 & 1360.65 & 1377.84 & 1399.31 & 1411.42\end{array}$ $\begin{array}{lllllllll}1420.10 & 1424.16 & 1426.11 & 1431.22 & 1439.13 & 1477.80 & 1484.31 & 1488.18 & 1493.21\end{array}$ $\begin{array}{llllllllll}1495.22 & 1502.37 & 1513.19 & 3067.59 & 3070.54 & 3078.39 & 3101.13 & 3121.07 & 3132.38\end{array}$ $\begin{array}{llllllll}3150.12 & 3155.15 & 3157.69 & 3162.04 & 3169.34 & 3170.97 & 3697.55 & 3817.60\end{array}$ Rotational constants (B/GHZ): $\quad 1.2463340 \quad 0.5375475 \quad 0.4463976$

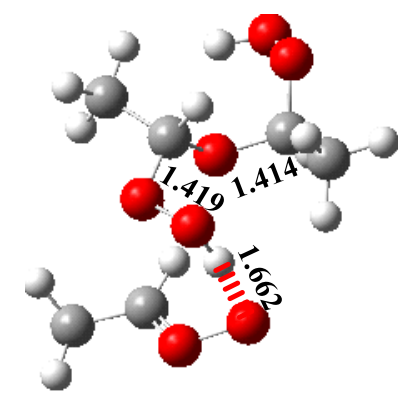

IM4d

$\mathrm{C}$ $\begin{array}{lll}\mathrm{C} & 1 & \mathrm{~B} 1\end{array}$

$\mathrm{H}-2$

2

B2

B3 1

A1

$\mathrm{O} 2$

B4 1

A2

A3 4

D1 0

B5 2

A4 1

D2 0

$\mathrm{H} \quad 4$

B6 2

A5 1

D3 0

C 5

B7 5

A6 2

D4 0

B8 5

A7 2

D5 0

B9 2

A8 5

D6 0

B10 2

A9 5

D7 0

$\mathrm{H} \quad 1$

$\mathrm{H} \quad 1$

B11 2

A10 5

D8 0

O 2

B12 1

A11 5

D9 0

C 13

H 14

B13 2

A12 1

D10 0

B14 13

A13 2

D11 0

C 14

B15 13

A14 2

D12 0

$\mathrm{H} \quad 16$

B16 14

A15 13

D13 0

$\mathrm{H} \quad 16$

B17 14

A16 13

D14 0

$\mathrm{H} \quad 16$

B18 14

A17 13

D15 0

$\begin{array}{ll}\text { O } & 14\end{array}$

B19 13

A18 2

D16 0

D17 0 


$\begin{array}{rrrrrrrr}\mathrm{O} & 20 & \text { B20 } & 14 & \text { A19 } & 13 & \text { D18 } & 0 \\ \mathrm{H} & 21 & \text { B21 } & 20 & \text { A20 } & 14 & \text { D19 } & 0 \\ \mathrm{C} & 7 & \text { B22 } & 5 & \text { A21 } & 2 & \text { D20 } & 0 \\ \mathrm{H} & 23 & \text { B23 } & 7 & \text { A22 } & 5 & \text { D21 } & 0 \\ \mathrm{H} & 23 & \text { B24 } & 7 & \text { A23 } & 5 & \text { D22 } & 0 \\ \mathrm{H} & 23 & \text { B25 } & 7 & \text { A24 } & 5 & \text { D23 } & 0 \\ \mathrm{H} & 7 & \text { B26 } & 5 & \text { A25 } & 2 & \text { D24 } & 0\end{array}$

Variables:

B

1.51253247

B2 1.09002450

B3 2.29688285

B4 1.39852099

B5 0.99644826

B6 2.74829119

B7 2.22986604

B8 1.23683815

B9 1.08935542

B10 1.09031499

B11 1.08731607

B12 1.41489565

B13 1.41448727

B14 1.08994292

B15 1.50987484

B16 1.08773159

B17 1.08794158

B18 1.08712803

B19 1.40430810

B20 1.41537129

B21 0.96648623

B22 1.47326744

B23 1.09019390

B24 1.08576079

B25 1.09240190

B26 1.08835905

A1 111.88991913

A2 138.95182348

A3 $\quad 106.30768723$

A4 103.53883411

A5 109.73275924

A6 77.62401049

A7 $\quad 91.15283378$

A8 109.63517539

A9 $\quad 109.88269228$

A10 $\quad 110.39202284$ 


\begin{tabular}{|c|c|}
\hline A11 & 108.94103835 \\
\hline A12 & 118.60327322 \\
\hline A13 & 104.03648537 \\
\hline A14 & 115.72136362 \\
\hline A15 & 108.70461551 \\
\hline A16 & 108.57754914 \\
\hline A17 & 112.10101312 \\
\hline A18 & 109.46492250 \\
\hline A19 & 108.20292526 \\
\hline A20 & 102.78595340 \\
\hline A 21 & 107.29128280 \\
\hline A 22 & 109.75145875 \\
\hline A 23 & 111.23376050 \\
\hline A24 & 107.55495231 \\
\hline A 25 & 69.93050512 \\
\hline D1 & 101.38891279 \\
\hline D2 & 17.73649325 \\
\hline D3 & 123.01717701 \\
\hline D4 & 93.78809210 \\
\hline D5 & 94.76092423 \\
\hline D6 & 126.50630702 \\
\hline D7 & 176.23490727 \\
\hline D8 & -64.06667414 \\
\hline D9 & 56.58135390 \\
\hline D10 & 118.30925829 \\
\hline D11 & 124.58816952 \\
\hline D12 & 179.89091595 \\
\hline D13 & 57.34317368 \\
\hline D14 & 56.96251331 \\
\hline D15 & 176.06997882 \\
\hline D16 & -63.47468766 \\
\hline D17 & -63.75198366 \\
\hline D18 & -68.41260880 \\
\hline D19 & 69.82063930 \\
\hline D20 & -112.30689726 \\
\hline $\mathrm{D} 21$ & 131.42236342 \\
\hline D22 & -105.67348616 \\
\hline $\mathrm{D} 23$ & 14.68929608 \\
\hline D24 & 8.13720535 \\
\hline
\end{tabular}

Harmonic Vibrational Frequencies $\left(\mathbf{c m}^{-\mathbf{1}}\right)$ :

$\begin{array}{lllllllllll}46.24 & 50.10 & 56.61 & 76.90 & 95.88 & 98.87 & 125.17 & 152.35 & 179.88 & 202.20 & 217.58\end{array}$ $\begin{array}{llllllllll}229.88 & 245.57 & 250.86 & 284.25 & 306.58 & 322.23 & 334.90 & 342.76 & 363.56 & 427.36\end{array}$ $\begin{array}{llllllllll}510.02 & 555.42 & 563.09 & 587.73 & 687.32 & 850.17 & 878.51 & 893.83 & 898.88 & 902.56\end{array}$ $\begin{array}{lllllllll}919.71 & 961.52 & 991.62 & 1056.08 & 1061.90 & 1098.41 & 1132.72 & 1138.87 & 1151.45\end{array}$ 


$\begin{array}{lcccccccc}1174.93 & 1179.51 & 1190.61 & 1216.20 & 1359.30 & 1371.17 & 1377.21 & 1401.20 & 1411.40 \\ 1424.99 & 1425.73 & 1432.41 & 1437.06 & 1462.95 & 1469.99 & 1484.65 & 1490.50 & 1495.87 \\ 1500.05 & 1607.85 & 1704.48 & 3056.99 & 3059.51 & 3071.95 & 3119.05 & 3126.94 & 3130.21 \\ 3143.91 & 3148.45 & 3151.70 & 3155.33 & 3168.28 & 3177.58 & 3210.21 & 3794.31 & \\ \text { Rotational constants (B/GHZ): } & 1.0778706 & 0.4825751 & 0.4350330 & \end{array}$

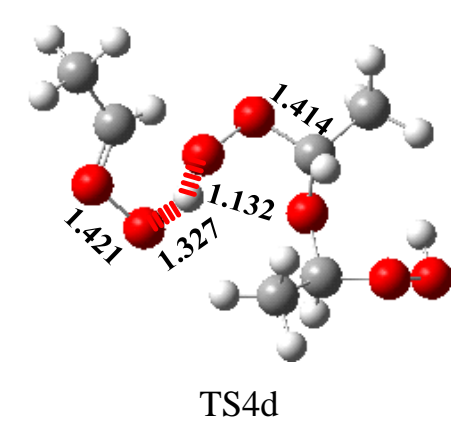

C

$\begin{array}{lll}\text { C } & 1 & \text { B1 }\end{array}$

$\mathrm{H} 2$

O 2

O 2

$\mathrm{H} \quad 4$

C 4

$\begin{array}{ll}\mathrm{O} & 7\end{array}$

$\begin{array}{ll}\text { O } & 7\end{array}$

$\mathrm{H} \quad 1$

$\begin{array}{ll}\mathrm{H} & 1\end{array}$

$\mathrm{H} \quad 1$

O 2

C 13

$\mathrm{H} \quad 14$

C 14

$\mathrm{H} \quad 16$

$\mathrm{H} \quad 16$

$\mathrm{H} \quad 16$

O 14

O 20

$\mathrm{H} \quad 21$

$\begin{array}{ll}\text { C } & 7\end{array}$

$\mathrm{H} \quad 23$

$\mathrm{H} \quad 23$

$\mathrm{H} \quad 23$

$\begin{array}{ll}\mathrm{H} & 7\end{array}$

B2 1

A1

B3 1

A2

B4 1

A3 4

D1 0

B5 2

A4 1

D2 0

B6 2

A5 1

D3 0

B7 4

A6 2

D4 0

B8 4

A7 2

D5 0

B9 2

A8 5

D6 0

B10 2

A9 5

D7 0

B11 2

A10 5

D8 0

B12 1

A11 5

D9 0

B13 2

A12 1

D10 0

B14 13

A13 2

D11 0

B15 13

A14 2

D12 0

B16 14

A15 13

D13 0

B17 14

A16 13

D14 0

B18 14

A17 13

D15 0

B19 13

A18 2

D16 0

B20 14

A19 13

D17 0

B21 20

A20 14

D18 0

B22 4

A21 2

D19 0

B23 7

A22 4

D20 0

B24 7

A23 4

D21 0

B25 7

A24 4

D22 0

B26 4

A25 2

D23 0

Variables:

B1

1.51113226

B2

1.09106878

B3

2.27658486 


\begin{tabular}{|c|c|}
\hline B4 & 1.40233705 \\
\hline B5 & 1.13174835 \\
\hline B6 & 2.08423609 \\
\hline B7 & 2.17793278 \\
\hline B8 & 1.26478990 \\
\hline B9 & 1.08968353 \\
\hline B10 & 1.08898223 \\
\hline B11 & 1.08781630 \\
\hline B12 & 1.40784866 \\
\hline B13 & 1.41240667 \\
\hline B14 & 1.08985931 \\
\hline B15 & 1.51177144 \\
\hline B16 & 1.08852348 \\
\hline B17 & 1.08793894 \\
\hline B18 & 1.08733343 \\
\hline B19 & 1.40501003 \\
\hline B20 & 1.41641966 \\
\hline B21 & 0.96759122 \\
\hline B22 & 1.47420366 \\
\hline B23 & 1.09118828 \\
\hline B24 & 1.08563478 \\
\hline B25 & 1.08953256 \\
\hline B26 & 1.08624902 \\
\hline A1 & 111.63612894 \\
\hline $\mathrm{A} 2$ & 141.95490742 \\
\hline A3 & 106.14071454 \\
\hline A4 & 93.95388048 \\
\hline A5 & 132.15204701 \\
\hline A6 & 68.05247230 \\
\hline A7 & 91.20213651 \\
\hline A8 & 109.17762042 \\
\hline A9 & 109.59923202 \\
\hline A10 & 110.55980123 \\
\hline A11 & 110.39588466 \\
\hline A12 & 117.79714561 \\
\hline A 13 & 104.06505743 \\
\hline A14 & 115.61481159 \\
\hline A 15 & 108.97579474 \\
\hline A16 & 108.63870620 \\
\hline A17 & 112.33333223 \\
\hline A 18 & 109.52176804 \\
\hline A19 & 108.08814577 \\
\hline A20 & 102.86249331 \\
\hline A21 & 110.66802606 \\
\hline
\end{tabular}




$\begin{array}{lr}\text { A22 } & 109.12435515 \\ \text { A23 } & 111.19523436 \\ \text { A24 } & 107.75276587 \\ \text { A25 } & 81.54746671 \\ \text { D1 } & 111.74224517 \\ \text { D2 } & 7.07506803 \\ \text { D3 } & 121.71009461 \\ \text { D4 } & 52.17605770 \\ \text { D5 } & 84.19564459 \\ \text { D6 } & 115.47119995 \\ \text { D7 } & 173.73856526 \\ \text { D8 } & -66.16895990 \\ \text { D9 } & 54.61408812 \\ \text { D10 } & 117.87262483 \\ \text { D11 } & 117.44944932 \\ \text { D12 } & -177.30987383 \\ \text { D13 } & 60.35047191 \\ \text { D14 } & 58.06845447 \\ \text { D15 } & 176.86817215 \\ \text { D16 } & -62.78615214 \\ \text { D17 } & -60.81570501 \\ \text { D18 } & -68.03420334 \\ \text { D19 } & 63.03925458 \\ \text { D20 } & -123.14457865 \\ \text { D21 } & 150.53793960 \\ \text { D22 } & -87.43396628 \\ \text { D23 } & 33.68050923 \\ \text { D24 } & -2.69392539 \\ \text { Hart }\end{array}$

Harmonic Vibrational Frequencies $\left(\mathrm{cm}^{-1}\right)$ :

$\begin{array}{lllllllllll}658.18 i & 34.26 & 53.14 & 67.91 & 91.27 & 100.51 & 116.33 & 142.82 & 183.09 & 203.80 & 234.78\end{array}$

$\begin{array}{llllllllll}246.53 & 271.59 & 295.63 & 303.11 & 318.58 & 349.06 & 356.13 & 399.91 & 454.44 & 481.10\end{array}$

$\begin{array}{llllllllll}520.59 & 546.07 & 571.12 & 595.63 & 694.41 & 853.80 & 889.06 & 898.99 & 914.10 & 934.14\end{array}$

$\begin{array}{lllllllll}966.75 & 993.98 & 1003.37 & 1054.82 & 1089.70 & 1108.44 & 1139.34 & 1148.39 & 1174.69\end{array}$

$\begin{array}{lllllllll}1188.52 & 1190.54 & 1200.18 & 1222.32 & 1362.93 & 1370.67 & 1372.81 & 1404.02 & 1416.11\end{array}$

$\begin{array}{llllllllll}1420.49 & 1431.78 & 1439.54 & 1447.59 & 1457.11 & 1470.61 & 1481.12 & 1493.25 & 1496.95\end{array}$

$\begin{array}{lllllllll}1503.39 & 1523.09 & 1628.03 & 1877.96 & 3060.64 & 3065.30 & 3080.68 & 3128.24 & 3134.71\end{array}$

$\begin{array}{llllllll}3135.09 & 3140.36 & 3154.80 & 3161.93 & 3171.42 & 3172.85 & 3207.05 & 3772.62\end{array}$

Rotational constants (B/GHZ): $1.4526259 \quad 0.4180410 \quad 0.3586741$ 


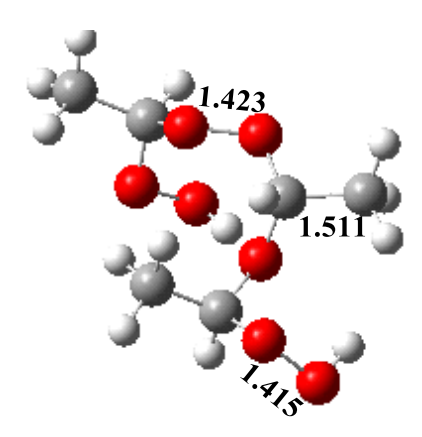

P4d

C

C 1

$\mathrm{H} \quad 2$

O 2

O 2

H 5

C 4

O 7

O 7

H 1

H 1

H 1

O 2

C 13

$\mathrm{H} \quad 14$

C 14

$\mathrm{H} \quad 16$

H 16

$\mathrm{H} \quad 16$

O 14

O 20

$\mathrm{H} \quad 21$

$\begin{array}{ll}\text { C } & 7\end{array}$

$\mathrm{H} \quad 23$

$\mathrm{H} \quad 23$

H 23

H 7

Variables:

B1

B2

B3

B4

B5

B6

B7
B1

B2

B3 1

B4 1

B5 2

B6 2

B7 4

B8 4

B9 2

B10 2

B11 2

B12 1

B13 2

B14 13

B15 13

B16 14

B17 14

B18 14

B19 13

B20 14

B21 20

B22 4

B23 7

B24 7

B25 7

B26 4
A1

A2 $3 \quad$ D1 0

A3 $4 \quad$ D2 0

A4 $1 \quad$ D3 0

$\begin{array}{llll}\text { A5 } & 1 & \text { D4 } & 0\end{array}$

A6 2 D5 0

A7 $2 \quad$ D6 0

$\begin{array}{llll}\mathrm{A} 8 & 5 & \mathrm{D} 7 & 0\end{array}$

$\begin{array}{llll}\text { A9 } & 5 & \text { D8 } & 0\end{array}$

$\begin{array}{llll}\mathrm{A} 10 & 5 & \mathrm{D} 9 & 0\end{array}$

A11 $5 \quad$ D10 0

A12 $1 \quad$ D11 0

A13 2 D12 0

A14 $2 \quad$ D13 0

A15 $13 \quad$ D14 0

A16 $13 \quad$ D15 0

A17 $13 \quad$ D16 0

$\begin{array}{llll}\text { A18 } 2 & \text { D17 } & 0\end{array}$

A19 $13 \quad$ D18 0

$\begin{array}{llll}\text { A20 } & 14 & \text { D19 } & 0\end{array}$

$\begin{array}{llll}\mathrm{A} 21 & 2 & \mathrm{D} 20 & 0\end{array}$

A22 $4 \quad$ D21 0

A23 4 D22 0

A24 $4 \quad$ D23 0

$\begin{array}{llll}\text { A25 } 2 & \text { D24 }\end{array}$
1.51094944

1.09015830

2.27654943

1.39738897

2.54668381

1.41228959

2.30392621 


\begin{tabular}{|c|c|}
\hline B8 & 1.39719820 \\
\hline B9 & 1.08950085 \\
\hline B 10 & 1.08899092 \\
\hline B11 & 1.08733998 \\
\hline B12 & 1.41604970 \\
\hline B13 & 1.41912046 \\
\hline B14 & 1.08996381 \\
\hline B15 & 1.51061946 \\
\hline B16 & 1.08705922 \\
\hline B17 & 1.08785878 \\
\hline B18 & 1.08732663 \\
\hline B19 & 1.40062225 \\
\hline B20 & 1.41525997 \\
\hline B21 & 0.96692423 \\
\hline B22 & 1.51145596 \\
\hline B23 & 1.08649179 \\
\hline B24 & 1.08925210 \\
\hline B25 & 1.08660228 \\
\hline B26 & 1.09053277 \\
\hline A1 & 111.76271615 \\
\hline $\mathrm{A} 2$ & 141.07524901 \\
\hline A3 & 106.04710991 \\
\hline A4 & 80.88701993 \\
\hline A5 & 126.64552408 \\
\hline A6 & 106.65300592 \\
\hline A7 & 112.60661551 \\
\hline A8 & 109.22702497 \\
\hline A9 & 109.76470852 \\
\hline A 10 & 110.37936152 \\
\hline A11 & 109.89697371 \\
\hline A12 & 117.97222871 \\
\hline A13 & 103.93309889 \\
\hline A 14 & 115.32744007 \\
\hline A15 & 108.59286405 \\
\hline A16 & 108.51369423 \\
\hline A 17 & 112.24104066 \\
\hline A 18 & 109.27287591 \\
\hline A19 & 108.28282811 \\
\hline A20 & 102.97433461 \\
\hline A21 & 105.24732763 \\
\hline $\mathrm{A} 22$ & 109.32522277 \\
\hline A23 & 109.74420507 \\
\hline A24 & 109.85553198 \\
\hline A25 & 108.93765872 \\
\hline
\end{tabular}




\begin{tabular}{lr} 
D1 & 106.29855659 \\
D2 & 13.10482566 \\
D3 & 108.64749281 \\
D4 & 91.71323222 \\
D5 & 7.07985745 \\
D6 & 44.10716519 \\
D7 & 174.98865182 \\
D8 & -64.79816109 \\
D9 & 55.79812871 \\
D10 & 118.14925333 \\
D11 & 118.57633844 \\
D12 & -178.98341258 \\
D13 & 58.66364651 \\
D14 & 55.14080836 \\
D15 & 174.45668484 \\
D16 & -65.38053141 \\
D17 & -62.41151261 \\
D18 & -69.46737861 \\
D19 & 69.92706513 \\
D20 & 160.22718749 \\
D21 & 179.51000388 \\
D22 & -61.01317969 \\
D23 & 58.91156449 \\
D24 & -77.06902110 \\
\hline
\end{tabular}

Harmonic Vibrational Frequencies $\left(\mathrm{cm}^{-1}\right)$ :

$\begin{array}{lccccccccccr}51.54 & 58.85 & 77.21 & 107.58 & 122.34 & 156.01 & 175.18 & 192.17 & 212.40 & 221.95 & 244.44 \\ 283.87 & 297.75 & 318.23 & 332.10 & 355.46 & 360.60 & 427.50 & 441.82 & 500.85 & 529.92 \\ 571.69 & 584.68 & 610.26 & 636.21 & 696.37 & 852.43 & 893.01 & 904.24 & 918.44 & 937.49 \\ 969.12 & 1057.25 & 1059.03 & 1066.99 & 1098.69 & 1120.87 & 1137.89 & 1151.68 & 1163.47 \\ 1178.74 & 1191.97 & 1203.33 & 1234.61 & 1359.86 & 1365.73 & 1369.53 & 1392.65 & 1405.65 \\ 1416.24 & 1419.73 & 1425.14 & 1436.79 & 1443.90 & 1486.34 & 1490.24 & 1492.75 & 1496.06 \\ 1498.24 & 1501.99 & 1511.04 & 3060.09 & 3070.00 & 3086.39 & 3124.23 & 3125.44 & 3133.10 \\ 3144.02 & 3152.30 & 3154.25 & 3165.6 & 3166.24 & 3185.01 & 3685.28 & 3790.98 & \\ \text { Rotational constants (B/GHZ): } & 1.2530048 & 0.5100976 & 0.4622789 & \end{array}$

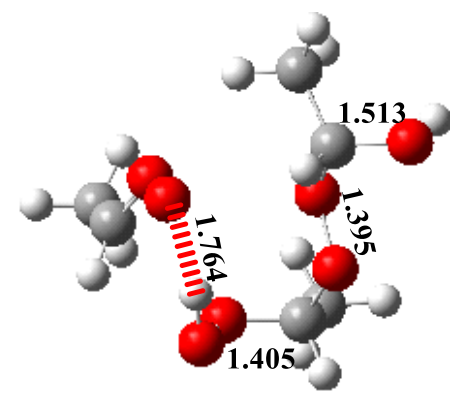

IM4e

C 


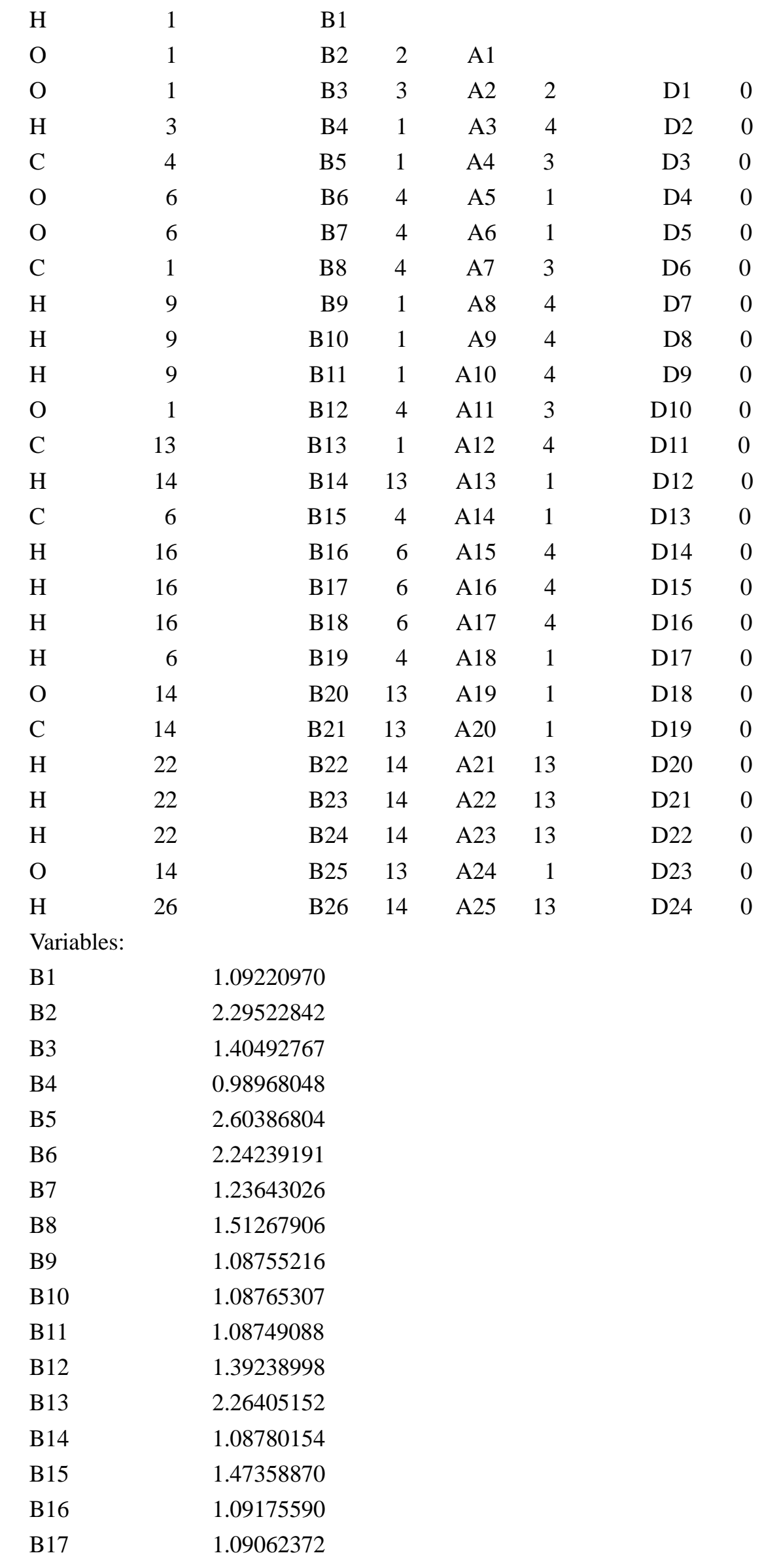




\begin{tabular}{|c|c|}
\hline B18 & 1.0859693 \\
\hline B19 & 1.08739056 \\
\hline B20 & 1.41289320 \\
\hline B21 & 1.51261133 \\
\hline B22 & 1.08756319 \\
\hline B23 & 1.09198260 \\
\hline B24 & 1.08590923 \\
\hline B25 & 1.39458926 \\
\hline B26 & 0.96144134 \\
\hline A1 & 84.80415733 \\
\hline A2 & 35.79607281 \\
\hline A3 & 100.70188354 \\
\hline A4 & 138.46932268 \\
\hline A5 & 78.38797973 \\
\hline A6 & 91.02059383 \\
\hline A7 & 106.13661164 \\
\hline A8 & 109.75126776 \\
\hline A9 & 110.22653164 \\
\hline A10 & 109.08141611 \\
\hline A11 & 112.45242789 \\
\hline A12 & 143.17951957 \\
\hline A13 & 82.72109841 \\
\hline A14 & 106.34304468 \\
\hline A15 & 107.64947562 \\
\hline A16 & 109.85753602 \\
\hline A17 & 110.99531500 \\
\hline A18 & 71.58198198 \\
\hline A19 & 37.45795410 \\
\hline A20 & 141.75176202 \\
\hline A21 & 108.63260471 \\
\hline A 22 & 110.13101323 \\
\hline A23 & 110.39435097 \\
\hline A24 & 94.52819455 \\
\hline A 25 & 108.59816838 \\
\hline D1 & 133.29298164 \\
\hline D2 & -94.01674098 \\
\hline D3 & 110.84337941 \\
\hline D4 & -68.24642324 \\
\hline D5 & -37.30557012 \\
\hline D6 & 171.89735998 \\
\hline D7 & -64.47075845 \\
\hline D8 & 56.30053450 \\
\hline D9 & 175.68729752 \\
\hline D10 & -62.51185308 \\
\hline
\end{tabular}




$\begin{array}{lr}\text { D11 } & -53.01133099 \\ \text { D12 } & 109.15363614 \\ \text { D13 } & 83.63928741 \\ \text { D14 } & 19.68054073 \\ \text { D15 } & 136.47363364 \\ \text { D16 } & -100.95991226 \\ \text { D17 } & -156.09248675 \\ \text { D18 } & -24.89949059 \\ \text { D19 } & -7.52021913 \\ \text { D20 } & 173.72722224 \\ \text { D21 } & -67.15204267 \\ \text { D22 } & 53.93885205 \\ \text { D23 } & -144.58242849 \\ \text { D24 } & 97.63127663\end{array}$

Harmonic Vibrational Frequencies $\left(\mathbf{c m}^{-1}\right)$ :

$\begin{array}{lllllllllll}39.92 & 44.71 & 64.48 & 86.35 & 103.00 & 109.50 & 134.31 & 145.57 & 159.46 & 185.56 & 213.79\end{array}$ $\begin{array}{llllllllll}227.63 & 246.56 & 261.29 & 288.25 & 306.93 & 326.54 & 353.49 & 364.48 & 398.51 & 418.50\end{array}$ $\begin{array}{llllllllll}535.81 & 567.10 & 573.87 & 586.58 & 695.12 & 779.21 & 878.66 & 890.36 & 907.02 & 909.86\end{array}$ $\begin{array}{lllllllll}943.81 & 952.88 & 995.63 & 1014.45 & 1060.92 & 1091.25 & 1120.88 & 1133.85 & 1141.58\end{array}$ $\begin{array}{lllllllll}1165.51 & 1179.72 & 1183.03 & 1216.26 & 1312.53 & 1353.84 & 1377.60 & 1380.26 & 1398.46\end{array}$ $\begin{array}{llllllllll}1408.99 & 1419.00 & 1429.73 & 1437.59 & 1466.76 & 1467.73 & 1487.03 & 1489.41 & 1494.97\end{array}$ $\begin{array}{llllllllll}1502.73 & 1572.51 & 1701.18 & 3054.81 & 3063.12 & 3069.32 & 3110.08 & 3118.40 & 3138.93\end{array}$ $\begin{array}{llllllll}3152.00 & 3156.53 & 3165.05 & 3174.40 & 3182.76 & 3202.08 & 3328.47 & 3882.56\end{array}$ Rotational constants (B/GHZ): $0.8894646 \quad 0.6295060 \quad 0.4899185$

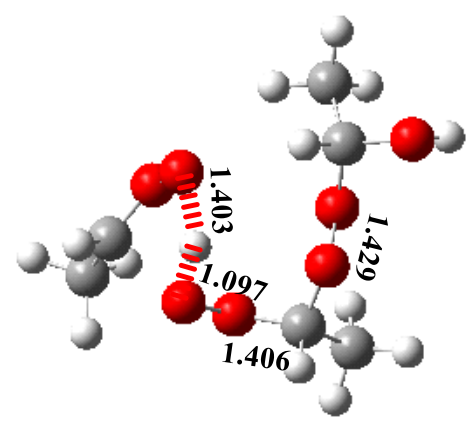

TS4e

$\mathrm{C}$ $\mathrm{H} \quad \mathrm{B} 1$

$\begin{array}{lll}\mathrm{O} & 1 & \mathrm{~B} 2\end{array}$

O 11 B3

$\begin{array}{lll}\mathrm{H} & 3 & \mathrm{~B} 4\end{array}$

C 3

O 6

O 6

C 1

$\mathrm{H} \quad 9$

$\mathrm{H} \quad 9$

B5 1

B6 3

B7 3

B8 4

B9 1

B10 1

$\begin{array}{llll}\text { A1 } & & & \\ \text { A2 } & 2 & \text { D1 } & 0 \\ \text { A3 } & 4 & \text { D2 } & 0 \\ \text { A4 } & 4 & \text { D3 } & 0 \\ \text { A5 } & 1 & \text { D4 } & 0 \\ \text { A6 } & 1 & \text { D5 } & 0 \\ \text { A7 } & 3 & \text { D6 } & 0 \\ \text { A8 } & 4 & \text { D7 } & 0 \\ \text { A9 } & 4 & \text { D8 } & 0\end{array}$




$\begin{array}{rrrrrrrr}\text { H } & 9 & \text { B11 } & 1 & \text { A10 } & 4 & \text { D9 } & 0 \\ \text { O } & 1 & \text { B12 } & 4 & \text { A11 } & 3 & \text { D10 } & 0 \\ \text { C } & 13 & \text { B13 } & 1 & \text { A12 } & 4 & \text { D11 } & 0 \\ \text { H } & 14 & \text { B14 } & 13 & \text { A13 } & 1 & \text { D12 } & 0 \\ \mathrm{C} & 6 & \text { B15 } & 3 & \text { A14 } & 1 & \text { D13 } & 0 \\ \mathrm{H} & 16 & \text { B16 } & 6 & \text { A15 } & 3 & \text { D14 } & 0 \\ \text { H } & 16 & \text { B17 } & 6 & \text { A16 } & 3 & \text { D15 } & 0 \\ \text { H } & 16 & \text { B18 } & 6 & \text { A17 } & 3 & \text { D16 } & 0 \\ \text { H } & 6 & \text { B19 } & 3 & \text { A18 } & 1 & \text { D17 } & 0 \\ \text { O } & 14 & \text { B20 } & 13 & \text { A19 } & 1 & \text { D18 } & 0 \\ \text { C } & 14 & \text { B21 } & 13 & \text { A20 } & 1 & \text { D19 } & 0 \\ \text { H } & 22 & \text { B22 } & 14 & \text { A21 } & 13 & \text { D20 } & 0 \\ \text { H } & 22 & \text { B23 } & 14 & \text { A22 } & 13 & \text { D21 } & 0 \\ \text { H } & 22 & \text { B24 } & 14 & \text { A23 } & 13 & \text { D22 } & 0 \\ \text { O } & 14 & \text { B25 } & 13 & \text { A24 } & 1 & \text { D23 } & 0 \\ \text { H } & 26 & \text { B26 } & 14 & \text { A25 } & 13 & \text { D24 } & 0\end{array}$

Variables:

$\begin{array}{ll}\text { B1 } & 1.09287817 \\ \text { B2 } & 2.28984621 \\ \text { B3 } & 1.40557360 \\ \text { B4 } & 1.09706182 \\ \text { B5 } & 2.03945879 \\ \text { B6 } & 2.17472036 \\ \text { B7 } & 1.26620599 \\ \text { B8 } & 1.51188526 \\ \text { B9 } & 1.08752614 \\ \text { B10 } & 1.08679533 \\ \text { B11 } & 1.08759252 \\ \text { B12 } & 1.39467985 \\ \text { B13 } & 2.27531668 \\ \text { B14 } & 1.08693985 \\ \text { B15 } & 1.47569140 \\ \text { B16 } & 1.08894021 \\ \text { B17 } & 1.09114703 \\ \text { B18 } & 1.08548074 \\ \text { B19 } & 1.08522402 \\ \text { B20 } & 1.41011336 \\ \text { B21 } & 1.51234598 \\ \text { B22 } & 1.08764745 \\ \text { B23 } & 1.09183501 \\ \text { B24 } & 1.08729959 \\ \text { B25 } & 1.39587460 \\ \text { B26 } & 0.96135497 \\ \text { A1 } & 85.78814742\end{array}$




\begin{tabular}{|c|c|}
\hline $\mathrm{A} 2$ & 35.83488413 \\
\hline A3 & 93.25440023 \\
\hline A4 & 130.66482395 \\
\hline A5 & 69.73718130 \\
\hline A6 & 93.18145956 \\
\hline A7 & 105.91197458 \\
\hline A8 & 109.75788025 \\
\hline A9 & 110.10829823 \\
\hline A10 & 108.89311895 \\
\hline A11 & 111.94457018 \\
\hline A12 & 142.25882840 \\
\hline A13 & 80.47355313 \\
\hline A14 & 108.67338487 \\
\hline A 15 & 108.19714714 \\
\hline A16 & 109.13172173 \\
\hline A 17 & 110.61292724 \\
\hline A18 & 83.45229567 \\
\hline A19 & 37.02660812 \\
\hline A20 & 139.24754252 \\
\hline A21 & 109.03075853 \\
\hline A22 & 110.26547268 \\
\hline A23 & 109.63113971 \\
\hline A24 & 98.43684239 \\
\hline A25 & 108.51228439 \\
\hline D1 & 132.48197594 \\
\hline D2 & -110.57073829 \\
\hline D3 & -37.65025627 \\
\hline D4 & -79.42378884 \\
\hline D5 & -48.40421172 \\
\hline D6 & 173.36292452 \\
\hline D7 & -66.01404221 \\
\hline D8 & 54.53530879 \\
\hline D9 & 174.17923200 \\
\hline D10 & -61.40562578 \\
\hline D11 & -51.77350181 \\
\hline D12 & 118.01818308 \\
\hline D13 & 73.09059040 \\
\hline D14 & 35.64322942 \\
\hline D15 & 153.16928567 \\
\hline D16 & -84.99802503 \\
\hline D17 & -165.72255577 \\
\hline D18 & -21.91971176 \\
\hline D19 & 5.66370608 \\
\hline D20 & 166.79290348 \\
\hline
\end{tabular}




$\begin{array}{lr}\text { D21 } & -73.65364693 \\ \text { D22 } & 46.83753809 \\ \text { D23 } & -136.53357630 \\ \text { D24 } & 99.05969059\end{array}$

Harmonic Vibrational Frequencies $\left(\mathbf{c m}^{-\mathbf{1}}\right)$ :

$\begin{array}{lccccccccccr}489.06 i & 40.44 & 59.52 & 75.62 & 87.92 & 107.34 & 117.83 & 135.76 & 162.56 & 198.51 & 221.59 \\ 232.93 & 250.26 & 291.33 & 323.63 & 336.67 & 359.75 & 388.50 & 397.95 & 434.76 & 535.10 \\ 541.59 & 562.83 & 578.34 & 589.35 & 701.28 & 885.68 & 895.65 & 904.67 & 939.83 & 952.53 \\ 970.13 & 1005.56 & 1015.37 & 1030.64 & 1090.97 & 1122.61 & 1131.64 & 1139.28 & 1165.45 \\ 1176.71 & 1184.40 & 1213.08 & 1250.71 & 1312.30 & 1354.10 & 1358.59 & 1379.70 & 1395.35 \\ 1411.60 & 1418.77 & 1424.46 & 1441.80 & 1453.64 & 1476.96 & 1489.37 & 1490.48 & 1496.87 \\ 1501.40 & 1583.38 & 1620.74 & 1932.60 & 3059.40 & 3065.43 & 3082.76 & 3107.42 & 3137.78 \\ 3144.80 & 3165.00 & 3165.41 & 3171.25 & 3175.56 & 3181.77 & 3208.82 & 3881.16 & \\ \text { Rotational constants (B/GHZ): } & 1.0432275 & 0.5769855 & 0.4340642 & \end{array}$

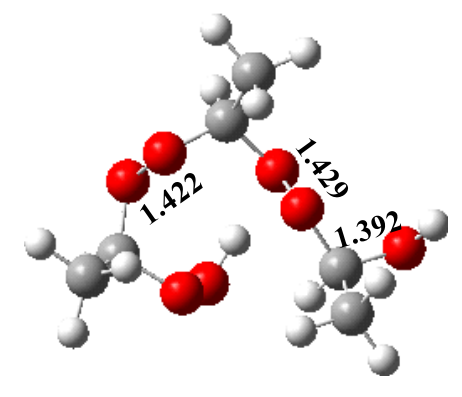

$\mathrm{P} 4 \mathrm{e}$

C

$\begin{array}{lll}\mathrm{H} & 1 & \mathrm{~B} 1\end{array}$

$\begin{array}{lll}\mathrm{O} & 1 & \mathrm{~B} 2\end{array}$

O 1

2 A1

$\mathrm{H} \quad 3$

C 3

O 6

O 6

C 1

H 9

$\mathrm{H} \quad 9$

H 9

O 1

C 13

$\mathrm{H} \quad 14$

C 6

$\mathrm{H} \quad 16$

$\mathrm{H} \quad 16$

$\mathrm{H} \quad 16$

$\mathrm{H} \quad 6$

O 14

C $\quad 14$

B3 3

A2 $2 \quad$ D1 0

B4 1

A3 4 D2 0

B5 1

A4 4

D3 0

B6 3

A5 1

D4 0

B7 3

A6 1

D5 0

B8 4

A7 3

D6 0

B9 1

A8 4

D7 0

B10 1

A9 4

D8 0

B11 1

A10 4

D9 0

B12 4

A11 3

D10 0

B13 1

A12 4

D11 0

B14 13

A13 1

D12 0

B15 3

A14 1

D13 0

B16 6

A15 3

D14 0

B17 6

A16 3

D15 0

B18 6

A17 3

D16 0

B19 3

A18 1

D17 0

B20 13

A19 1

D18 0

B21 13

A20 1

D19 0 


$\begin{array}{rrrrrrrr}\mathrm{H} & 22 & \text { B22 } & 14 & \text { A21 } & 13 & \text { D20 } & 0 \\ \mathrm{H} & 22 & \text { B23 } & 14 & \text { A22 } & 13 & \text { D21 } & 0 \\ \mathrm{H} & 22 & \text { B24 } & 14 & \text { A23 } & 13 & \text { D22 } & 0 \\ \mathrm{O} & 14 & \text { B25 } & 13 & \text { A24 } & 1 & \text { D23 } & 0 \\ \mathrm{H} & 26 & \text { B26 } & 14 & \text { A25 } & 13 & \text { D24 } & 0\end{array}$

Variables:

B1 1.09222826

B2 2.27651153

B3 1.39821153

B4 2.58358522

B5 1.40850208

B6 2.27818159

B7 1.39674139

B8 1.51185906

B9 $\quad 1.08708121$

B10 1.08651710

B11 1.08749742

B12 1.40525614

B13 2.29008779

B14 1.08829230

B15 1.50974902

B16 1.08757006

B17 1.08728053

B18 1.08713004

B19 1.09260914

B20 1.41134882

B21 1.51360235

B22 1.08740044

B23 1.09128742

B24 1.08755477

B25 1.39234294

B26 0.96173596

A1 $\quad 81.51555155$

A2 36.54811300

A3 73.08520154

A4 124.02662811

A5 93.38832111

A6 112.00121963

A7 106.39444339

A8 $\quad 109.36735568$

A9 $\quad 110.32432812$

A10 $\quad 109.09850615$

A11 112.49288735

A12 141.80734940 


\begin{tabular}{|c|c|}
\hline A13 & 84.15830724 \\
\hline A14 & 113.94244596 \\
\hline A 15 & 109.34093690 \\
\hline A16 & 109.47031533 \\
\hline A17 & 109.94902728 \\
\hline A18 & 101.51441331 \\
\hline A19 & 36.52675526 \\
\hline A20 & 139.93761546 \\
\hline A21 & 108.88744131 \\
\hline A22 & 110.11495768 \\
\hline A23 & 110.34682790 \\
\hline A24 & 95.30311123 \\
\hline A25 & 108.77755867 \\
\hline D1 & 140.38445983 \\
\hline D2 & -132.83480775 \\
\hline D3 & -75.49781690 \\
\hline D4 & -66.24791196 \\
\hline D5 & -34.32472818 \\
\hline D6 & 163.94405201 \\
\hline D7 & -67.59865416 \\
\hline D8 & 52.61630911 \\
\hline D9 & 172.52545124 \\
\hline D10 & -70.99255703 \\
\hline D11 & -56.94674703 \\
\hline D12 & 115.19566810 \\
\hline D13 & 86.69489040 \\
\hline D14 & 49.91622666 \\
\hline D15 & 169.84382793 \\
\hline D16 & -69.93059696 \\
\hline D17 & -151.19334435 \\
\hline D18 & -19.26833600 \\
\hline D19 & -2.02393493 \\
\hline D20 & 172.09428491 \\
\hline D21 & -68.41730073 \\
\hline D22 & 51.90940033 \\
\hline D23 & -139.05182444 \\
\hline D24 & 97.12413633 \\
\hline
\end{tabular}

Harmonic Vibrational Frequencies $\left(\mathbf{c m}^{-1}\right)$ :

$\begin{array}{lccccccccccr}42.09 & 68.53 & 93.40 & 107.55 & 132.52 & 150.09 & 166.92 & 180.41 & 189.71 & 220.77 & 243.40 \\ 270.07 & 291.99 & 311.49 & 320.40 & 345.96 & 386.94 & 414.69 & 433.18 & 533.50 & 557.53 \\ 578.06 & 587.16 & 593.24 & 692.01 & 700.93 & 877.34 & 890.18 & 909.80 & 932.85 & 940.13 \\ 951.42 & 1009.12 & 1024.02 & 1060.61 & 1096.60 & 1133.22 & 1138.14 & 1142.74 & 1149.59 \\ 1157.83 & 1179.40 & 1203.40 & 1229.40 & 1316.79 & 1357.83 & 1359.68 & 1389.02 & 1394.15 \\ 1402.54 & 1416.11 & 1420.75 & 1426.22 & 1451.53 & 1467.46 & 1485.13 & 1486.12 & 1488.14\end{array}$




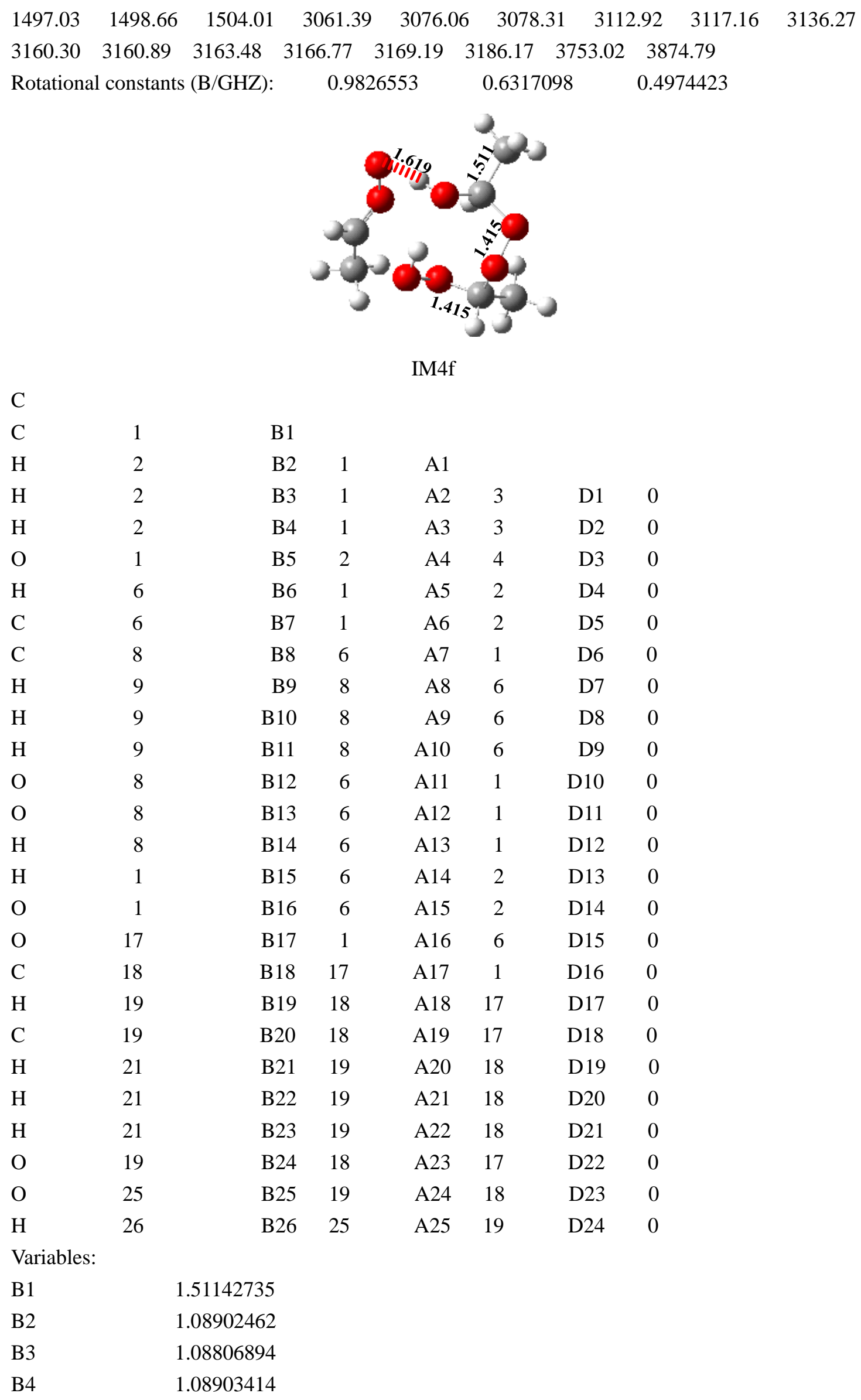




\begin{tabular}{|c|c|}
\hline B5 & 1.39157906 \\
\hline B6 & 0.99932699 \\
\hline B7 & 3.63082891 \\
\hline $\mathrm{B} 8$ & 1.47165878 \\
\hline B9 & 1.08541214 \\
\hline B 10 & 1.08953440 \\
\hline B11 & 1.09204001 \\
\hline B12 & 2.23559074 \\
\hline B13 & 1.23714932 \\
\hline B14 & 1.08665629 \\
\hline B15 & 1.09617765 \\
\hline B 16 & 1.42586452 \\
\hline B17 & 1.41487055 \\
\hline B 18 & 1.39574176 \\
\hline B19 & 1.09301048 \\
\hline B20 & 1.50855606 \\
\hline B21 & 1.08757429 \\
\hline B22 & 1.08718741 \\
\hline B 23 & 1.08736669 \\
\hline B24 & 1.41519190 \\
\hline B 25 & 1.41829273 \\
\hline B26 & 0.98625205 \\
\hline A1 & 108.51579916 \\
\hline $\mathrm{A} 2$ & 109.20006379 \\
\hline A3 & 110.81700105 \\
\hline A4 & 112.79616090 \\
\hline A5 & 106.28892428 \\
\hline A6 & 100.58445595 \\
\hline A7 & 130.53689279 \\
\hline A8 & 110.89301447 \\
\hline A9 & 108.16262133 \\
\hline A10 & 108.80824986 \\
\hline A11 & 45.40064927 \\
\hline A12 & 55.07362937 \\
\hline A13 & 84.67564192 \\
\hline A14 & 109.54434259 \\
\hline A15 & 109.90201139 \\
\hline A16 & 112.39555480 \\
\hline A17 & 112.97288203 \\
\hline A18 & 102.11736495 \\
\hline A19 & 115.19437005 \\
\hline A 20 & 109.68120044 \\
\hline A 21 & 110.60862035 \\
\hline A22 & 108.95371781 \\
\hline
\end{tabular}




$\begin{array}{lr}\text { A23 } & 111.03437554 \\ \text { A24 } & 108.02178903 \\ \text { A25 } & 100.63840339 \\ \text { D1 } & 119.29107274 \\ \text { D2 } & -119.78178423 \\ \text { D3 } & 55.88095261 \\ \text { D4 } & 82.63220104 \\ \text { D5 } & 127.93721337 \\ \text { D6 } & 48.01327880 \\ \text { D7 } & -61.27863246 \\ \text { D8 } & 59.64803091 \\ \text { D9 } & 176.96640049 \\ \text { D10 } & -96.66967907 \\ \text { D11 } & -53.48852664 \\ \text { D12 } & 178.42627704 \\ \text { D13 } & -124.04732900 \\ \text { D14 } & 115.99935918 \\ \text { D15 } & 41.15508527 \\ \text { D16 } & 77.60846712 \\ \text { D17 } & 165.96188924 \\ \text { D18 } & 42.86467920 \\ \text { D19 } & 170.91543307 \\ \text { D20 } & -68.41380216 \\ \text { D21 } & 51.08170309 \\ \text { D22 } & -78.26159118 \\ \text { D23 } & -55.63033381 \\ \text { D24 } & 72.36844891 \\ \text { Dar } & \end{array}$

Harmonic Vibrational Frequencies $\left(\mathbf{c m}^{-1}\right)$ :

$\begin{array}{lllllllllll}46.64 & 58.48 & 75.27 & 111.99 & 128.59 & 132.86 & 155.21 & 179.66 & 189.75 & 200.58 & 232.47\end{array}$ $\begin{array}{llllllllll}242.90 & 265.60 & 281.77 & 285.45 & 330.10 & 333.95 & 340.15 & 409.07 & 432.80 & 519.62\end{array}$ $\begin{array}{llllllllll}561.97 & 579.23 & 615.67 & 718.30 & 740.14 & 851.70 & 885.17 & 886.67 & 895.66 & 920.56\end{array}$ $\begin{array}{lllllllll}944.67 & 987.72 & 1017.42 & 1036.55 & 1055.32 & 1110.40 & 1120.95 & 1131.33 & 1153.68\end{array}$ $\begin{array}{lllllllll}1158.30 & 1182.71 & 1194.11 & 1217.91 & 1356.87 & 1368.33 & 1370.48 & 1394.52 & 1406.34\end{array}$ $\begin{array}{lllllllll}1421.74 & 1425.38 & 1430.64 & 1462.18 & 1468.44 & 1473.89 & 1486.93 & 1487.48 & 1492.13\end{array}$ $\begin{array}{lllllllll}1514.43 & 1562.63 & 1699.11 & 3043.35 & 3060.48 & 3060.98 & 3070.94 & 3075.28 & 3098.86\end{array}$ $\begin{array}{llllllll}3130.07 & 3142.56 & 3154.30 & 3156.83 & 3163.14 & 3183.95 & 3218.16 & 3446.80\end{array}$ $\begin{array}{llll}\text { Rotational constants (B/GHZ): } & 0.9429473 & 0.6563090 & 0.4629794\end{array}$

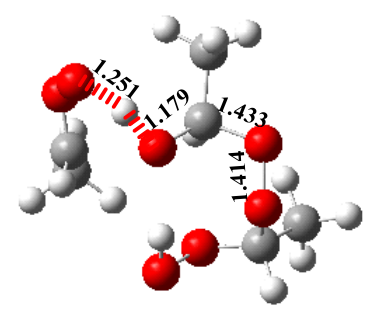

TS4f 
C

$\begin{array}{lll}\mathrm{C} & 1 & \mathrm{~B} 1\end{array}$

$\mathrm{H} \quad 2$

H 2

B2

B3 1

A1

$\mathrm{H} 2$

B4 1

$\begin{array}{llll}\mathrm{A} 2 & 3 & \mathrm{D} 1 & 0\end{array}$

B5 2

A3 4 D2 0

O 1

$\mathrm{H} \quad 6$

C 6

C 8

$\mathrm{H} \quad 9$

$\mathrm{H} \quad 9$

$\mathrm{H} \quad 9$

O 8

O 8

$\mathrm{H} \quad 8$

$\mathrm{H} \quad 1$

O 1

O 17

C 18

$\mathrm{H} \quad 19$

C 19

$\mathrm{H} \quad 21$

$\mathrm{H} \quad 21$

$\mathrm{H} \quad 21$

O 19

O 25

B6 1

A4 5

D3 0

B7 1

A5 2

D4 0

B8 6

A6 2 D5 0

B9 8

A7 $1 \quad$ D6 0

B10 8

A8 $6 \quad$ D7 0

A9 $6 \quad$ D8 0

B11 8

$\begin{array}{llll}\mathrm{A} 10 & 6 & \mathrm{D} 9 & 0\end{array}$

$\begin{array}{llllll}\mathrm{B} 12 & 6 & \mathrm{~A} 11 & 1 & \mathrm{D} 10 & 0\end{array}$

$\begin{array}{llllll}\mathrm{B} 13 & 6 & \mathrm{~A} 12 & 1 & \mathrm{D} 11 & 0\end{array}$

$\begin{array}{llllll}\mathrm{B} 14 & 6 & \mathrm{~A} 13 & 1 & \mathrm{D} 12 & 0\end{array}$

$\begin{array}{llllll}\mathrm{B} 15 & 6 & \mathrm{~A} 14 & 8 & \mathrm{D} 13 & 0\end{array}$

$\begin{array}{llllll}\text { B16 } & 6 & \text { A15 } & 8 & \text { D14 } & 0\end{array}$

$\begin{array}{llllll}\mathrm{B} 17 & 1 & \mathrm{~A} 16 & 6 & \mathrm{D} 15 & 0\end{array}$

$\begin{array}{llllll}\mathrm{B} 18 & 17 & \mathrm{~A} 17 & 1 & \mathrm{D} 16 & 0\end{array}$

$\begin{array}{llllll}\text { B19 } & 18 & \text { A18 } & 17 & \text { D17 } & 0\end{array}$

$\begin{array}{llllll}\text { B20 } & 18 & \text { A19 } & 17 & \text { D18 } & 0\end{array}$

$\begin{array}{llllll}\text { B21 } & 19 & \text { A20 } & 18 & \text { D19 } & 0\end{array}$

$\begin{array}{llllll}\text { B22 } & 19 & \text { A21 } & 18 & \text { D20 } & 0\end{array}$

$\begin{array}{llllll}\mathrm{B} 23 & 19 & \mathrm{~A} 22 & 18 & \mathrm{D} 21 & 0\end{array}$

$\begin{array}{llllll}\text { B24 } & 18 & \text { A23 } & 17 & \text { D22 } & 0\end{array}$

$\begin{array}{llllll}\text { B25 } & 19 & \text { A24 } & 18 & \text { D23 } & 0\end{array}$

$\mathrm{H} \quad 26$

B26 25

A25 19

D24 0

Variables:

$\begin{array}{ll}\text { B1 } & 1.51365507 \\ \text { B2 } & 1.08893647 \\ \text { B3 } & 1.08896139 \\ \text { B4 } & 1.08838192 \\ \text { B5 } & 1.38264951 \\ \text { B6 } & 1.17902118 \\ \text { B7 } & 2.12575719 \\ \text { B8 } & 1.46990104 \\ \text { B9 } & 1.08618478 \\ \text { B10 } & 1.09244701 \\ \text { B11 } & 1.09108271 \\ \text { B12 } & 2.18394190 \\ \text { B13 } & 1.26772206 \\ \text { B14 } & 1.08355815 \\ \text { B15 } & 1.09765692 \\ \text { B16 } & 1.43252556\end{array}$




$\begin{array}{lr}\text { B17 } & 1.41412955 \\ \text { B18 } & 1.40065094 \\ \text { B19 } & 1.09262656 \\ \text { B20 } & 1.50801058 \\ \text { B21 } & 1.08733642 \\ \text { B22 } & 1.08729733 \\ \text { B23 } & 1.08736733 \\ \text { B24 } & 1.41152028 \\ \text { B25 } & 1.41701412 \\ \text { B26 } & 0.97706823 \\ \text { A1 } & 109.73065520 \\ \text { A2 } & 109.45946259 \\ \text { A3 } & 110.14338221 \\ \text { A4 } & 113.87010037 \\ \text { A5 } & 114.58950205 \\ \text { A6 } & 113.94533734 \\ \text { A7 } & 113.13789462 \\ \text { A8 } & 111.19291612 \\ \text { A9 } & 107.74688520 \\ \text { A10 } & 108.89562246 \\ \text { A11 } & 66.46636284 \\ \text { A12 } & 90.90422750 \\ \text { A13 } & 79.44991169 \\ \text { A14 } & 109.13746509 \\ \text { A15 } & 111.11071122 \\ \text { A16 } & 112.34644084 \\ \text { A17 } & 113.01384575 \\ \text { A18 } & 102.05854810 \\ \text { A19 } & 115.13675530 \\ \text { A20 } & 109.51387763 \\ \text { A21 } & 110.77664955 \\ \text { A22 } & 108.99627783 \\ \text { A23 } & 110.79277701 \\ \text { A24 } & 107.99136507 \\ \text { A25 } & 100.47296530 \\ \text { D1 } & \\ \text { D2 } & 175.095298877 \\ \text { D } & \end{array}$




$\begin{array}{lr}\text { D10 } & -107.21991061 \\ \text { D11 } & -77.36344476 \\ \text { D12 } & 164.45529515 \\ \text { D13 } & -35.52151663 \\ \text { D14 } & -154.30952033 \\ \text { D15 } & 40.04359979 \\ \text { D16 } & 76.59132876 \\ \text { D17 } & 165.01345565 \\ \text { D18 } & 42.10947386 \\ \text { D19 } & 171.15938536 \\ \text { D20 } & -68.28513438 \\ \text { D21 } & 51.24615609 \\ \text { D22 } & -79.39084813 \\ \text { D23 } & -57.60381880 \\ \text { D24 } & 69.74750755\end{array}$

Harmonic Vibrational Frequencies $\left(\mathbf{c m}^{-1}\right)$ :

$\begin{array}{llllllllllll}995.34 i & 33.90 & 71.61 & 78.57 & 128.47 & 132.22 & 149.54 & 155.72 & 174.59 & 192.72 & 227.03 \\ 260.11 & 260.30 & 290.14 & 318.24 & 321.19 & 370.28 & 382.64 & 434.49 & 492.09 & 509.78 \\ 563.99 & 586.63 & 608.79 & 630.80 & 728.83 & 761.41 & 850.33 & 881.16 & 890.60 & 923.53 \\ 934.88 & 976.35 & 1006.11 & 1019.45 & 1056.55 & 1113.25 & 1122.71 & 1136.68 & 1150.27 \\ 1164.77 & 1188.48 & 1198.36 & 1229.54 & 1351.18 & 1363.21 & 1385.37 & 1398.71 & 1415.23 \\ 1417.05 & 1425.46 & 1431.88 & 1449.39 & 1464.00 & 1481.18 & 1486.44 & 1491.97 & 1497.30 \\ 1505.45 & 1547.62 & 1608.30 & 1801.72 & 3039.05 & 3055.49 & 3062.43 & 3078.44 & 3113.02 \\ 3122.62 & 3146.08 & 3151.87 & 3159.32 & 3167.40 & 3169.86 & 3223.17 & 3620.99 & \\ \text { Rotational constants (B/GHZ): } & 1.1699816 & 0.5715202 & 0.4631327 & \end{array}$

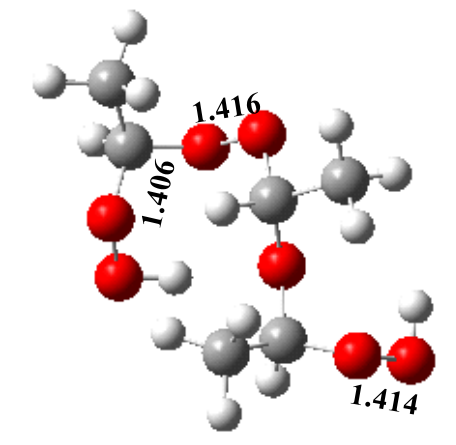

P4f

C

$\begin{array}{llllllll}\mathrm{C} & 1 & \text { B1 } & & & & & \\ \mathrm{H} & 2 & \text { B2 } & 1 & \text { A1 } & & & \\ \mathrm{H} & 2 & \text { B3 } & 1 & \text { A2 } & 3 & \text { D1 } & 0 \\ \mathrm{H} & 2 & \text { B4 } & 1 & \text { A3 } & 4 & \text { D2 } & 0 \\ \text { O } & 1 & \text { B5 } & 2 & \text { A4 } & 3 & \text { D3 } & 0 \\ \text { H } & 6 & \text { B6 } & 1 & \text { A5 } & 2 & \text { D4 } & 0 \\ \text { C } & 6 & \text { B7 } & 1 & \text { A6 } & 2 & \text { D5 } & 0 \\ \text { C } & 8 & \text { B8 } & 6 & \text { A7 } & 1 & \text { D6 } & 0\end{array}$




\begin{tabular}{|c|c|c|c|c|c|c|}
\hline $\mathrm{H}$ & 9 & B9 & 8 & A8 & 6 & D7 \\
\hline $\mathrm{H}$ & 9 & B10 & 8 & A9 & 6 & D8 \\
\hline $\mathrm{H}$ & 9 & B11 & 8 & A10 & 6 & D9 \\
\hline $\mathrm{O}$ & 8 & B12 & 6 & A11 & 1 & D10 \\
\hline $\mathrm{O}$ & 8 & B13 & 6 & A12 & 1 & D11 \\
\hline $\mathrm{H}$ & 8 & B14 & 6 & A13 & 1 & D12 \\
\hline $\mathrm{H}$ & 1 & B15 & 6 & A14 & 8 & D13 \\
\hline $\mathrm{O}$ & 1 & B16 & 6 & A15 & 8 & D14 \\
\hline $\mathrm{O}$ & 17 & B17 & 1 & A16 & 6 & D15 \\
\hline $\mathrm{C}$ & 18 & B18 & 17 & A17 & 1 & D16 \\
\hline $\mathrm{H}$ & 19 & B19 & 18 & A18 & 17 & D17 \\
\hline $\mathrm{C}$ & 19 & B20 & 18 & A19 & 17 & D18 \\
\hline $\mathrm{H}$ & 21 & B21 & 19 & A20 & 18 & D19 \\
\hline $\mathrm{H}$ & 21 & B22 & 19 & A21 & 18 & D20 \\
\hline $\mathrm{H}$ & 21 & B23 & 19 & A22 & 18 & D21 \\
\hline $\mathrm{O}$ & 19 & B24 & 18 & A23 & 17 & D22 \\
\hline $\mathrm{O}$ & 25 & B25 & 19 & A24 & 18 & D23 \\
\hline $\mathrm{H}$ & 26 & B26 & 25 & A25 & 19 & D24 \\
\hline \multicolumn{7}{|c|}{ Variables: } \\
\hline B1 & & 1.51209329 & & & & \\
\hline B2 & & 1.08702362 & & & & \\
\hline B3 & & 1.08936431 & & & & \\
\hline B4 & & 1.08860446 & & & & \\
\hline B5 & & 1.41079284 & & & & \\
\hline B6 & & 2.40152825 & & & & \\
\hline B7 & & 1.41516231 & & & & \\
\hline B8 & & 1.51326773 & & & & \\
\hline B9 & & 1.08913324 & & & & \\
\hline B 10 & & 1.08876286 & & & & \\
\hline B11 & & 1.08708082 & & & & \\
\hline B 12 & & 2.28041386 & & & & \\
\hline B13 & & 1.40185680 & & & & \\
\hline B14 & & 1.09078294 & & & & \\
\hline B15 & & 1.09085298 & & & & \\
\hline B16 & & 1.41180654 & & & & \\
\hline B17 & & 1.41601692 & & & & \\
\hline B18 & & 1.40671252 & & & & \\
\hline B19 & & 1.09268617 & & & & \\
\hline B20 & & 1.50796295 & & & & \\
\hline B21 & & 1.08711592 & & & & \\
\hline B22 & & 1.08746755 & & & & \\
\hline B23 & & 1.08745735 & & & & \\
\hline B24 & & 1.40616304 & & & & \\
\hline B25 & & 1.41555893 & & & & \\
\hline
\end{tabular}




\begin{tabular}{|c|c|}
\hline B26 & 0.97244740 \\
\hline A1 & 110.12699829 \\
\hline $\mathrm{A} 2$ & 109.54181221 \\
\hline A3 & 109.59387595 \\
\hline A4 & 113.93573669 \\
\hline A5 & 113.08703645 \\
\hline A6 & 118.08306445 \\
\hline A7 & 115.68186609 \\
\hline A8 & 111.30620261 \\
\hline A9 & 109.76804649 \\
\hline A10 & 108.47783422 \\
\hline A11 & 92.93225597 \\
\hline A12 & 110.25670321 \\
\hline A13 & 103.95229737 \\
\hline A14 & 108.98840206 \\
\hline A15 & 107.69508869 \\
\hline A16 & 112.79307307 \\
\hline A17 & 112.86930290 \\
\hline A18 & 101.82889840 \\
\hline A19 & 114.95355349 \\
\hline A 20 & 109.32013123 \\
\hline A 21 & 111.00989883 \\
\hline A22 & 109.09627925 \\
\hline A 23 & 110.49975008 \\
\hline A24 & 107.85556957 \\
\hline A 25 & 101.43617805 \\
\hline D1 & 120.15427817 \\
\hline D2 & 119.42683613 \\
\hline D3 & -65.65157791 \\
\hline D4 & -12.74369651 \\
\hline D5 & 73.28305853 \\
\hline D6 & 40.38429918 \\
\hline D7 & -72.65656306 \\
\hline D8 & 49.02804660 \\
\hline D9 & 168.41097603 \\
\hline D10 & -112.81316013 \\
\hline D11 & -80.34736991 \\
\hline D12 & 163.32827421 \\
\hline D13 & -52.37617768 \\
\hline D14 & -171.63370871 \\
\hline D15 & 47.61972724 \\
\hline D16 & 74.85989322 \\
\hline D17 & 164.51230186 \\
\hline D18 & 41.93096863 \\
\hline
\end{tabular}




$\begin{array}{lr}\text { D19 } & 171.03441459 \\ \text { D20 } & -68.63997699 \\ \text { D21 } & 51.20616491 \\ \text { D22 } & -79.72665617 \\ \text { D23 } & -59.74850479 \\ \text { D24 } & 69.33987951\end{array}$

Harmonic Vibrational Frequencies $\left(\mathbf{c m}^{-\mathbf{1}}\right)$ :

$\begin{array}{lllllllllll}53.08 & 63.95 & 99.85 & 113.61 & 149.88 & 168.19 & 191.36 & 203.75 & 222.57 & 240.90 & 260.34\end{array}$ $\begin{array}{llllllllll}273.07 & 296.15 & 307.88 & 331.69 & 364.54 & 380.49 & 405.36 & 428.16 & 509.60 & 534.12\end{array}$ $\begin{array}{llllllllll}568.88 & 588.88 & 598.83 & 690.09 & 728.85 & 841.79 & 861.93 & 902.19 & 914.12 & 936.07\end{array}$ $\begin{array}{lllllllll}955.70 & 1021.13 & 1058.94 & 1064.19 & 1110.22 & 1135.26 & 1142.62 & 1146.95 & 1149.84\end{array}$ $\begin{array}{llllllllll}1182.21 & 1193.96 & 1206.25 & 1212.48 & 1362.03 & 1382.77 & 1390.85 & 1402.27 & 1419.54\end{array}$ $\begin{array}{lllllllll}1425.90 & 1429.31 & 1438.58 & 1449.75 & 1453.26 & 1485.97 & 1487.36 & 1490.25 & 1493.04\end{array}$ $\begin{array}{lllllllll}1502.05 & 1504.75 & 1511.54 & 3062.33 & 3064.11 & 3079.71 & 3112.62 & 3121.96 & 3128.35\end{array}$ $\begin{array}{llllllll}3145.48 & 3148.78 & 3157.13 & 3161.22 & 3161.80 & 3167.27 & 3703.12 & 3785.57\end{array}$ $\begin{array}{llll}\text { Rotational constants (B/GHZ): } & 1.3096654 & 0.5273363 & 0.4555924\end{array}$

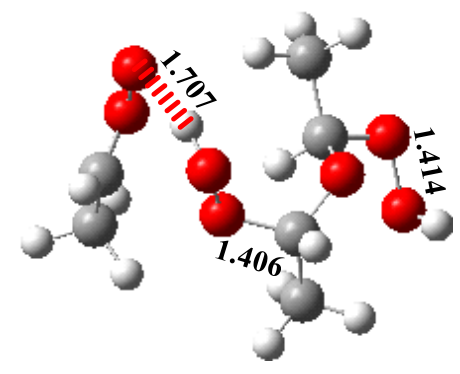

IM4g

$\mathrm{C}$

$\begin{array}{lll}\mathrm{H} & 1 & \mathrm{~B}\end{array}$

$\begin{array}{lll}\mathrm{O} & 1 & \mathrm{~B} 2\end{array}$

O 1

B2 2

A1

$\mathrm{H} \quad 3$

B3 3

A2

B4 1

A3 4

D1 0

B5 1

A4 3

D2 0

C 4

B6 4

A5 1

D3 0

O 6

B7 4

A6

D4 0

C 1

B8 4

A7 3

D5 0

$\mathrm{H} \quad 9$

B9 1

A8 4

D6 0

$\mathrm{H} \quad 9$

$\mathrm{H} \quad 9$

B10 1

A9 4

D7 0

B11 1

A10 4

D8 0

O 1

C 13

B12 4

A11 3

D9 0

B13 1

D10 0

A12 $4 \quad$ D11 0

B14 13

A13 1

D12 0

B15 13

A14 1

D13 0

B16 14

A15 13

D14 0

B17 14

A16 13

D15 0

B18 14

A17 13

D16 0

B19 13

A18 1

D17 0 


\begin{tabular}{|c|c|c|c|c|c|c|}
\hline $\mathrm{O}$ & 20 & B20 & 14 & A19 & 13 & D18 \\
\hline $\mathrm{H}$ & 21 & B21 & 20 & A20 & 14 & D19 \\
\hline $\mathrm{C}$ & 6 & B22 & 4 & $\mathrm{~A} 21$ & 1 & D20 \\
\hline $\mathrm{H}$ & 23 & B23 & 6 & A22 & 4 & D21 \\
\hline $\mathrm{H}$ & 23 & B24 & 6 & A23 & 4 & D22 \\
\hline $\mathrm{H}$ & 23 & B25 & 6 & A24 & 4 & D23 \\
\hline $\mathrm{H}$ & 6 & B26 & 4 & $\mathrm{~A} 25$ & 1 & D24 \\
\hline \multicolumn{7}{|c|}{ Variables: } \\
\hline B1 & \multicolumn{2}{|c|}{1.08989225} & & & & \\
\hline B2 & \multicolumn{2}{|c|}{2.29949457} & & & & \\
\hline B3 & \multicolumn{2}{|c|}{1.40567969} & & & & \\
\hline B4 & \multicolumn{2}{|c|}{0.99262132} & & & & \\
\hline B5 & \multicolumn{2}{|c|}{2.59813611} & & & & \\
\hline B6 & \multicolumn{2}{|c|}{2.23571782} & & & & \\
\hline B7 & \multicolumn{2}{|c|}{1.23692891} & & & & \\
\hline B8 & \multicolumn{2}{|c|}{1.51678502} & & & & \\
\hline B9 & \multicolumn{2}{|c|}{1.08836639} & & & & \\
\hline B10 & \multicolumn{2}{|c|}{1.08832020} & & & & \\
\hline B11 & \multicolumn{2}{|c|}{1.08734580} & & & & \\
\hline B12 & \multicolumn{2}{|c|}{1.40181228} & & & & \\
\hline B13 & \multicolumn{2}{|c|}{1.40798287} & & & & \\
\hline B14 & \multicolumn{2}{|c|}{1.09401405} & & & & \\
\hline B15 & \multicolumn{2}{|c|}{1.50854856} & & & & \\
\hline B16 & \multicolumn{2}{|c|}{1.08870052} & & & & \\
\hline B17 & \multicolumn{2}{|c|}{1.08832000} & & & & \\
\hline B18 & \multicolumn{2}{|c|}{1.08765414} & & & & \\
\hline B19 & \multicolumn{2}{|c|}{1.40485863} & & & & \\
\hline B20 & \multicolumn{2}{|c|}{1.41407393} & & & & \\
\hline B21 & \multicolumn{2}{|c|}{0.96516271} & & & & \\
\hline B22 & \multicolumn{2}{|c|}{1.47265702} & & & & \\
\hline B23 & \multicolumn{2}{|c|}{1.08607334} & & & & \\
\hline B24 & & & & & & \\
\hline B25 & & & & & & \\
\hline B26 & & & & & & \\
\hline $\mathrm{A} 1$ & & & & & & \\
\hline $\mathrm{A} 2$ & & & & & & \\
\hline $\mathrm{A} 3$ & & & & & & \\
\hline A4 & & & & & & \\
\hline A5 & & & & & & \\
\hline A6 & & & & & & \\
\hline A7 & & & & & & \\
\hline A8 & & & & & & \\
\hline A9 & & & & & & \\
\hline A10 & & & & & & \\
\hline
\end{tabular}




\begin{tabular}{|c|c|}
\hline A11 & 112.48098249 \\
\hline A 12 & 118.04986420 \\
\hline A13 & 110.07398802 \\
\hline A14 & 110.47563080 \\
\hline A 15 & 108.84796777 \\
\hline A16 & 110.20108242 \\
\hline A17 & 109.74513674 \\
\hline A18 & 109.34341255 \\
\hline A19 & 108.70507464 \\
\hline A 20 & 101.55028858 \\
\hline A 21 & 103.94932138 \\
\hline A 22 & 111.10089095 \\
\hline A 23 & 107.76094252 \\
\hline A24 & 109.70541927 \\
\hline A 25 & 74.38022597 \\
\hline D1 & 140.48415744 \\
\hline D2 & -67.95223672 \\
\hline D3 & 139.09194859 \\
\hline D4 & -103.96836887 \\
\hline D5 & -71.84875693 \\
\hline D6 & 160.68689674 \\
\hline D7 & -59.89457908 \\
\hline D8 & 61.40228711 \\
\hline D9 & -179.01705674 \\
\hline D10 & -73.56617720 \\
\hline D11 & -53.53552790 \\
\hline D12 & -4.81315748 \\
\hline D13 & 119.88252934 \\
\hline D14 & 55.06037640 \\
\hline D15 & 175.04927881 \\
\hline D16 & -64.78934850 \\
\hline D17 & -124.70321124 \\
\hline D18 & 65.76088256 \\
\hline D19 & -91.03747684 \\
\hline D20 & 48.79600857 \\
\hline D21 & -98.59107351 \\
\hline D22 & 22.40915402 \\
\hline $\mathrm{D} 23$ & 139.09464990 \\
\hline D24 & 170.06310544 \\
\hline
\end{tabular}

Harmonic Vibrational Frequencies $\left(\mathbf{c m}^{-\mathbf{1}}\right)$ :

$\begin{array}{lllllllllll}26.88 & 47.86 & 69.56 & 80.85 & 110.38 & 120.40 & 147.65 & 165.06 & 181.25 & 206.91 & 225.35\end{array}$ $\begin{array}{llllllllll}227.02 & 253.22 & 266.58 & 269.18 & 308.21 & 318.01 & 338.28 & 349.85 & 362.42 & 433.95\end{array}$

$\begin{array}{llllllllll}514.93 & 555.45 & 566.86 & 578.57 & 679.25 & 770.53 & 856.48 & 886.80 & 895.74 & 900.49\end{array}$ $\begin{array}{lllllllll}929.91 & 966.82 & 991.20 & 1056.06 & 1062.27 & 1102.88 & 1117.93 & 1137.76 & 1141.71\end{array}$ 


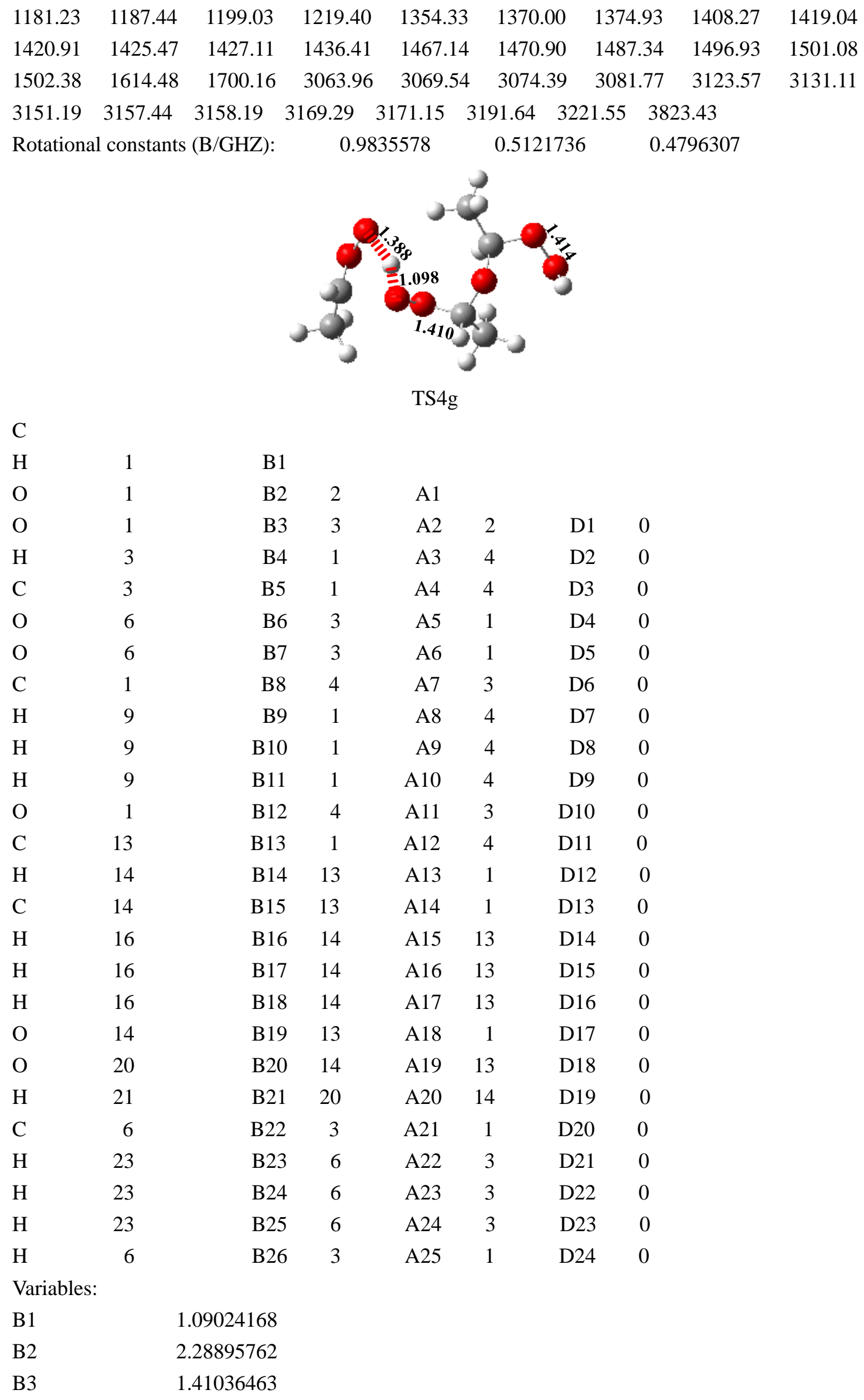




\begin{tabular}{|c|c|}
\hline B4 & 1.09788771 \\
\hline B5 & 2.04590628 \\
\hline B6 & 2.17626749 \\
\hline B7 & 1.26601066 \\
\hline B8 & 1.51634779 \\
\hline B9 & 1.08807732 \\
\hline B10 & 1.08712510 \\
\hline B11 & 1.08713639 \\
\hline B12 & 1.39874285 \\
\hline B13 & 1.41175945 \\
\hline B14 & 1.09175802 \\
\hline B15 & 1.50838974 \\
\hline B16 & 1.08860773 \\
\hline B17 & 1.08812782 \\
\hline B18 & 1.08822610 \\
\hline B19 & 1.40363916 \\
\hline B20 & 1.41429717 \\
\hline B21 & 0.96493845 \\
\hline B22 & 1.47520435 \\
\hline B23 & 1.08554143 \\
\hline B24 & 1.08937415 \\
\hline B25 & 1.09082228 \\
\hline B26 & 1.08516786 \\
\hline $\mathrm{A} 1$ & 80.81186551 \\
\hline $\mathrm{A} 2$ & 36.08311069 \\
\hline A3 & 113.60147481 \\
\hline A4 & 136.81139745 \\
\hline A5 & 69.22649495 \\
\hline A6 & 92.90675000 \\
\hline A7 & 105.52008523 \\
\hline A8 & 109.01350230 \\
\hline A9 & 112.01805121 \\
\hline A10 & 109.39082940 \\
\hline A11 & 111.86800981 \\
\hline A12 & 118.25116172 \\
\hline A 13 & 110.18233351 \\
\hline A14 & 110.67852053 \\
\hline A 15 & 109.22976709 \\
\hline A16 & 109.97113199 \\
\hline A17 & 109.64815535 \\
\hline A 18 & 108.91990231 \\
\hline A19 & 108.50032237 \\
\hline A20 & 101.52803244 \\
\hline A21 & 108.93864492 \\
\hline
\end{tabular}




$\begin{array}{lr}\text { A22 } & 110.71040096 \\ \text { A23 } & 107.91061737 \\ \text { A24 } & 109.36440160 \\ \text { A25 } & 83.07607491 \\ \text { D1 } & 139.90143922 \\ \text { D2 } & -81.88035803 \\ \text { D3 } & 10.77072312 \\ \text { D4 } & -106.22674341 \\ \text { D5 } & -75.43454796 \\ \text { D6 } & 161.17789773 \\ \text { D7 } & -59.98461846 \\ \text { D8 } & 60.72283575 \\ \text { D9 } & -179.12579189 \\ \text { D10 } & -72.45228860 \\ \text { D11 } & -59.45402485 \\ \text { D12 } & -5.40239008 \\ \text { D13 } & 119.09214963 \\ \text { D14 } & 52.04153254 \\ \text { D15 } & 172.07606159 \\ \text { D16 } & -68.19957370 \\ \text { D17 } & -125.39754087 \\ \text { D18 } & 66.58579608 \\ \text { D19 } & -93.31387562 \\ \text { D20 } & 46.03432020 \\ \text { D21 } & -88.34484221 \\ \text { D22 } & 32.09125297 \\ \text { D23 } & 149.45874943 \\ \text { D24 } & 167.14488114 \\ \text { Hart }\end{array}$

Harmonic Vibrational Frequencies $\left(\mathbf{c m}^{-1}\right)$ :

$\begin{array}{lcccccccccc}503.922 i & 20.12 & 43.73 & 73.92 & 94.65 & 105.22 & 119.78 & 133.34 & 154.39 & 180.26 & 226.15 \\ 232.55 & 257.79 & 278.22 & 309.91 & 323.95 & 347.47 & 361.81 & 409.47 & 430.05 & 508.14 \\ 531.33 & 545.78 & 578.04 & 597.42 & 684.40 & 856.79 & 889.56 & 896.69 & 927.68 & 965.44 \\ 975.37 & 999.85 & 1013.00 & 1059.76 & 1097.61 & 1125.54 & 1137.48 & 1140.87 & 1182.45 \\ 1186.16 & 197.15 & 1219.16 & 1240.43 & 1356.64 & 1363.54 & 1372.31 & 1412.04 & 1415.84 \\ 1421.22 & 1424.22 & 1434.24 & 1440.30 & 1452.83 & 1475.81 & 1484.11 & 1491.45 & 1493.32 \\ 1503.86 & 1576.19 & 1620.02 & 1926.55 & 3066.94 & 3068.49 & 3079.93 & 3117.73 & 3129.08 \\ 3135.18 & 3153.16 & 3158.12 & 3160.98 & 3172.13 & 3187.20 & 3200.51 & 3828.64 & \\ \text { Rotational constants (B/GHZ): } & 1.3699825 & 0.4366109 & 0.3615594 & \end{array}$




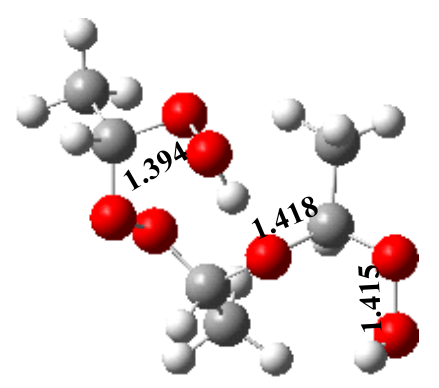

$\mathrm{P} 4 \mathrm{~g}$

C

$\begin{array}{lll}\mathrm{H} & 1 & \mathrm{~B} 1\end{array}$

O 1

O 1

$\mathrm{H} \quad 3$

C 3

O 6

O 6

C 1

$\begin{array}{ll}\mathrm{H} & 9\end{array}$

$\mathrm{H} \quad 9$

H 9

$\begin{array}{ll}\mathrm{O} & 1\end{array}$

C 13

$\begin{array}{ll}\mathrm{H} & 14\end{array}$

C 14

$\mathrm{H} \quad 16$

$\mathrm{H} \quad 16$

$\mathrm{H} \quad 16$

O 14

O 20

$\mathrm{H} \quad 21$

C 6

$\mathrm{H} \quad 23$

$\mathrm{H} \quad 23$

$\mathrm{H} \quad 23$

$\mathrm{H} \quad 6$

B2 2

A1

B3 3

A2 2

D1 0

B4 1

A3 4

D2 0

B5 1

A4 4

D3 0

B6 3

A5 1

D4 0

B7 3

A6 1

D5 0

B8 4

A7 3

D6 0

$\begin{array}{llllll}\text { B9 } & 1 & \text { A8 } & 4 & \text { D7 } & 0\end{array}$

$\begin{array}{llllll}\text { B10 } & 1 & \text { A9 } & 4 & \text { D8 } & 0\end{array}$

$\begin{array}{llllll}\text { B11 } & 1 & \text { A10 } & 4 & \text { D9 } & 0\end{array}$

$\begin{array}{llllll}\mathrm{B} 12 & 4 & \mathrm{~A} 11 & 3 & \mathrm{D} 10 & 0\end{array}$

$\begin{array}{llllll}\text { B13 } & 1 & \text { A12 } & 4 & \text { D11 } & 0\end{array}$

B14 13

A13 1

D12 0

B15 13

A14 1

D13 0

B16 14

A15 13

D14 0

B17 14

A16 13

D15 0

B18 14

A17 13

D16 0

B19 13

A18 1

D17 0

B20 14

A19 13

D18 0

B21 20

A20 14

D19 0

B22 3

A21 1

D20 0

B23 6

A22 3

D21 0

B24 6

A23 3

D22 0

B25 6

A24 3

D23 0

B26 3

A25 1

D24 0

Variables:

$\begin{array}{ll}\text { B1 } & 1.08985158 \\ \text { B2 } & 2.26901670 \\ \text { B3 } & 1.40474999 \\ \text { B4 } & 2.57754897 \\ \text { B5 } & 1.40775324 \\ \text { B6 } & 2.28166469 \\ \text { B7 } & 1.39405356 \\ \text { B8 } & 1.51622451\end{array}$




\begin{tabular}{|c|c|}
\hline B9 & 1.08703337 \\
\hline B10 & 1.08847495 \\
\hline B11 & 1.08782495 \\
\hline B12 & 1.41099061 \\
\hline B13 & 1.41776867 \\
\hline B14 & 1.08913605 \\
\hline B15 & 1.50759213 \\
\hline B16 & 1.08886732 \\
\hline B17 & 1.08789395 \\
\hline B18 & 1.08670431 \\
\hline B19 & 1.39997740 \\
\hline $\mathrm{B} 20$ & 1.41458143 \\
\hline B21 & 0.96536910 \\
\hline $\mathrm{B} 22$ & 1.51030382 \\
\hline B23 & 1.08739045 \\
\hline B24 & 1.08748243 \\
\hline $\mathrm{B} 25$ & 1.08722838 \\
\hline B26 & 1.09315776 \\
\hline $\mathrm{A} 1$ & 77.73690322 \\
\hline $\mathrm{A} 2$ & 37.01854144 \\
\hline $\mathrm{A} 3$ & 70.93072060 \\
\hline A4 & 127.64112143 \\
\hline A5 & 92.44993805 \\
\hline A6 & 112.04876561 \\
\hline A7 & 105.79741176 \\
\hline A8 & 108.13077247 \\
\hline A9 & 111.52895514 \\
\hline A10 & 110.26747150 \\
\hline A11 & 111.48521801 \\
\hline A12 & 120.42062766 \\
\hline A13 & 109.77510499 \\
\hline A14 & 112.19994183 \\
\hline A15 & 110.20172979 \\
\hline A16 & 109.49566984 \\
\hline A17 & 109.28395458 \\
\hline A18 & 107.50243326 \\
\hline A19 & 108.11526033 \\
\hline $\mathrm{A} 20$ & 101.75680472 \\
\hline A21 & 113.78133568 \\
\hline A22 & 110.03731035 \\
\hline A23 & 109.26952836 \\
\hline A24 & 109.61529064 \\
\hline A25 & 101.33808511 \\
\hline D1 & 144.19978524 \\
\hline
\end{tabular}




\begin{tabular}{lr} 
D2 & -123.56769645 \\
D3 & -71.06947094 \\
D4 & -61.23705574 \\
D5 & -30.22554036 \\
D6 & 155.81622840 \\
D7 & -72.91438515 \\
D8 & 45.60455364 \\
D9 & 167.25210733 \\
D10 & -76.86288701 \\
D11 & -87.53615682 \\
D12 & -12.41258604 \\
D13 & 112.94598345 \\
D14 & 55.06824669 \\
D15 & 175.63356396 \\
D16 & -63.74585689 \\
D17 & -130.70264127 \\
D18 & 66.56541665 \\
D19 & -91.27047117 \\
D20 & 90.66866401 \\
D21 & -67.47485429 \\
D22 & 52.30525140 \\
D23 & 172.34006859 \\
D24 & -147.61887767 \\
\hline Har
\end{tabular}

Harmonic Vibrational Frequencies $\left(\mathbf{c m}^{-1}\right)$ :

$\begin{array}{lllllllllll}38.96 & 49.34 & 97.66 & 114.64 & 126.70 & 168.55 & 198.58 & 206.77 & 228.61 & 238.70 & 240.12\end{array}$ $\begin{array}{llllllllll}258.89 & 293.56 & 307.63 & 320.37 & 359.13 & 365.42 & 416.00 & 437.85 & 501.75 & 544.14\end{array}$ $\begin{array}{llllllllll}576.62 & 583.29 & 597.60 & 673.59 & 703.72 & 843.35 & 879.99 & 902.95 & 920.48 & 939.40\end{array}$ $\begin{array}{lllllllll}968.94 & 1011.39 & 1057.58 & 1063.09 & 1107.62 & 1137.69 & 1138.86 & 1148.62 & 1153.73\end{array}$ $\begin{array}{llllllllll}1177.02 & 1185.76 & 1216.75 & 1225.90 & 1347.01 & 1360.70 & 1377.87 & 1399.36 & 1411.39\end{array}$ $\begin{array}{lllllllll}1420.10 & 1424.16 & 1426.10 & 1431.23 & 1439.13 & 1478.17 & 1484.28 & 1488.17 & 1493.15\end{array}$ $\begin{array}{llllllllll}1495.30 & 1502.33 & 1513.27 & 3067.54 & 3070.50 & 3078.37 & 3101.23 & 3120.98 & 3132.44\end{array}$ $\begin{array}{llllllll}3150.14 & 3155.17 & 3157.68 & 3161.96 & 3169.10 & 3170.95 & 3697.82 & 3817.66\end{array}$ $\begin{array}{llll}\text { Rotational constants (B/GHZ): } & 1.2463103 & 0.5375417 & 0.4463805\end{array}$

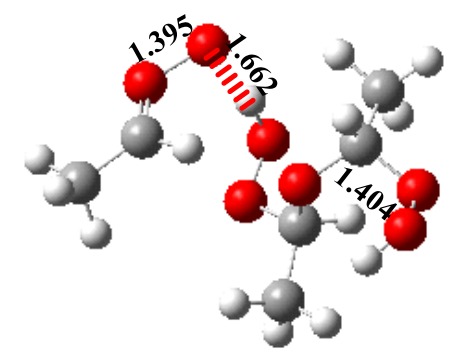

IM4h

$\mathrm{C}$ C 11

$\begin{array}{lllll}\mathrm{H} & 2 & \mathrm{~B} 2 & 1 & \mathrm{~A} 1\end{array}$ 


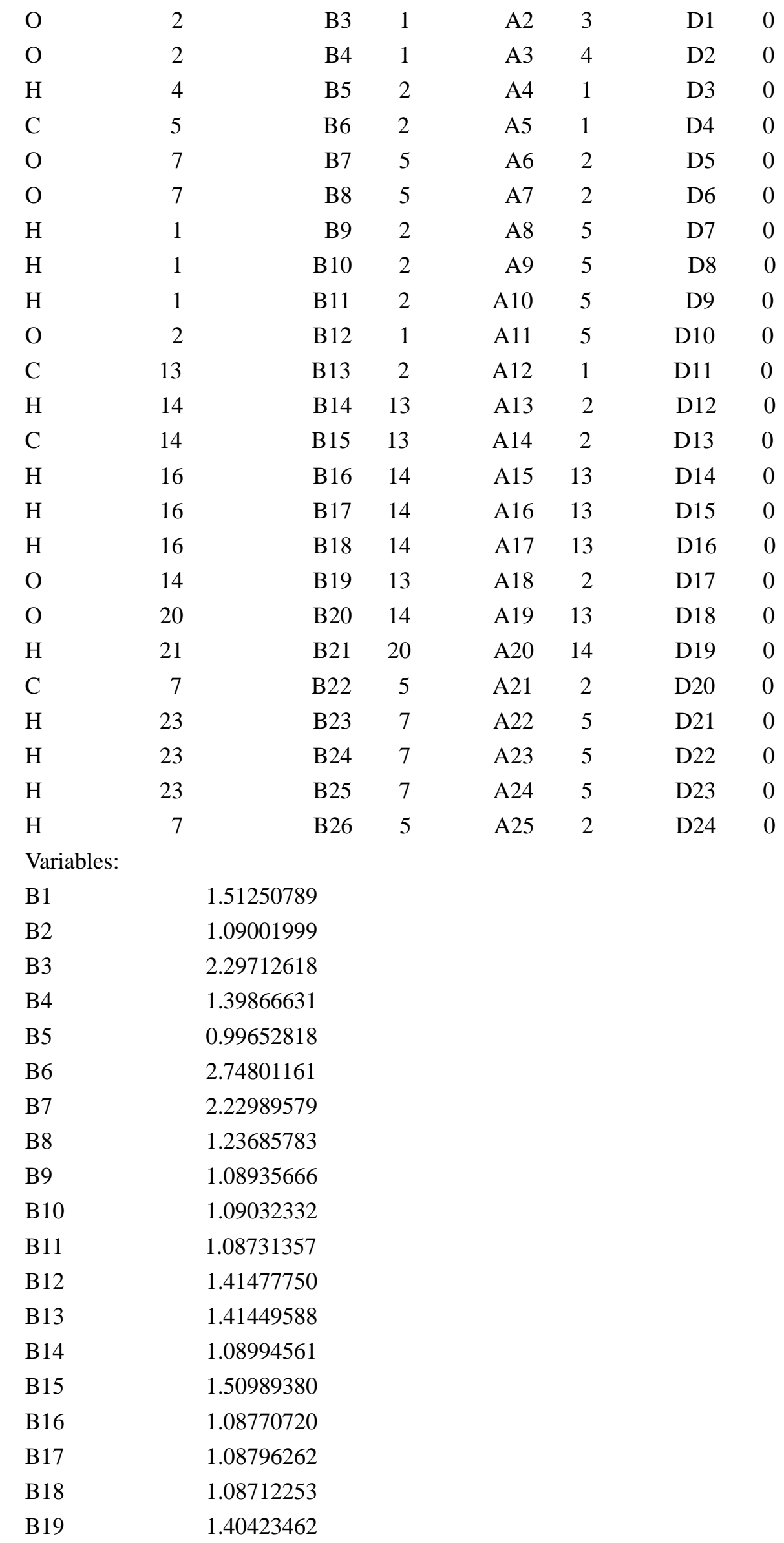




\begin{tabular}{|c|c|}
\hline B20 & 1.41536296 \\
\hline B21 & 0.96648727 \\
\hline B22 & 1.47323096 \\
\hline B23 & 1.09019255 \\
\hline B24 & 1.08575402 \\
\hline B25 & 1.09242137 \\
\hline B26 & 1.08834197 \\
\hline A1 & 111.88944299 \\
\hline A2 & 138.93223725 \\
\hline A3 & 106.30611569 \\
\hline A4 & 103.49678822 \\
\hline A5 & 109.75053148 \\
\hline A6 & 77.61586504 \\
\hline A7 & 91.11819032 \\
\hline A8 & 109.63423583 \\
\hline A9 & 109.88430873 \\
\hline A10 & 110.39255344 \\
\hline A11 & 108.94581132 \\
\hline A12 & 118.60381164 \\
\hline A13 & 104.03554679 \\
\hline A14 & 115.72288898 \\
\hline A15 & 108.70340980 \\
\hline A16 & 108.57019001 \\
\hline A17 & 112.10507134 \\
\hline A18 & 109.46456417 \\
\hline A19 & 108.20535467 \\
\hline $\mathrm{A} 20$ & 102.78717962 \\
\hline A21 & 107.25728258 \\
\hline A22 & 109.76336014 \\
\hline A23 & 111.23967914 \\
\hline A24 & 107.53490663 \\
\hline A25 & 69.99282901 \\
\hline D1 & 101.34523386 \\
\hline D2 & 17.76350071 \\
\hline D3 & 123.13241061 \\
\hline D4 & 93.82388050 \\
\hline D5 & 94.71329763 \\
\hline D6 & 126.47027223 \\
\hline D7 & 176.29898494 \\
\hline D8 & -63.99975027 \\
\hline D9 & 56.64375032 \\
\hline D10 & 118.30787089 \\
\hline D11 & 124.60109674 \\
\hline D12 & 179.87460504 \\
\hline
\end{tabular}




$\begin{array}{lr}\text { D13 } & 57.33552640 \\ \text { D14 } & 56.93716557 \\ \text { D15 } & 176.03712331 \\ \text { D16 } & -63.52097297 \\ \text { D17 } & -63.76356444 \\ \text { D18 } & -68.39924327 \\ \text { D19 } & 69.84639321 \\ \text { D20 } & -112.35819504 \\ \text { D21 } & 131.32852575 \\ \text { D22 } & -105.74074788 \\ \text { D23 } & 14.60561958 \\ \text { D24 } & 8.10266926\end{array}$

Harmonic Vibrational Frequencies $\left(\mathrm{cm}^{-1}\right)$ :

$\begin{array}{lcccccccccc}46.25 & 50.01 & 56.54 & 76.82 & 95.96 & 98.86 & 125.72 & 152.66 & 179.97 & 202.50 & 217.67 \\ 230.05 & 245.84 & 251.13 & 284.15 & 306.65 & 322.30 & 334.87 & 342.71 & 363.60 & 427.32 \\ 510.02 & 555.35 & 563.05 & 587.70 & 687.29 & 850.24 & 878.90 & 893.98 & 899.20 & 902.86 \\ 919.79 & 961.60 & 991.76 & 1056.10 & 1061.86 & 1098.54 & 1132.94 & 1138.80 & 1151.44 \\ 1175.01 & 1179.56 & 1190.51 & 1216.18 & 1359.20 & 1371.18 & 1377.15 & 1401.16 & 1411.40 \\ 1424.98 & 1425.76 & 1432.40 & 1437.10 & 1463.00 & 1470.06 & 1484.65 & 1490.53 & 1495.90 \\ 1500.07 & 1607.87 & 1704.35 & 3057.05 & 3059.56 & 3071.87 & 3119.07 & 3127.06 & 3130.20 \\ 3142.75 & 3147.99 & 3151.74 & 3154.92 & 3168.41 & 3177.72 & 3210.09 & 3794.26 & \\ \text { Rotational constants (B/GHZ): } & 1.0780273 & 0.4826535 & 0.4350768 & \end{array}$

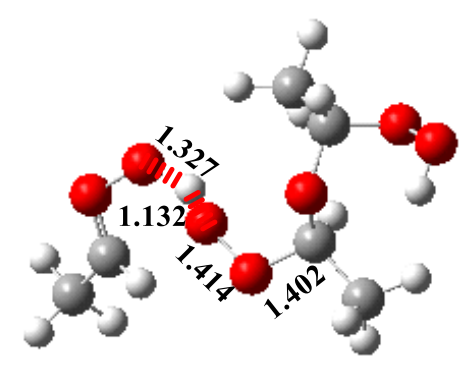

TS4h

C $\begin{array}{lll}\mathrm{C} & 1 & \mathrm{~B} 1\end{array}$

$\begin{array}{lll}\mathrm{H} & 2 & \mathrm{~B} 2\end{array}$

$\begin{array}{lll}\mathrm{O} & 2 & \mathrm{~B} 3\end{array}$

O 2

$\mathrm{H} \quad 4$

C 4

O 7

O 7

$\mathrm{H} \quad 1$

$\mathrm{H} \quad 1$

$\mathrm{H} \quad 1$

O 2

C 13

$\begin{array}{cl}\text { B2 } & 1 \\ \text { B3 } & 1 \\ \text { B4 } & 1 \\ \text { B5 } & 2 \\ \text { B6 } & 2 \\ \text { B7 } & 4 \\ \text { B8 } & 4 \\ \text { B9 } & 2 \\ \text { B10 } & 2 \\ \text { B11 } & 2 \\ \text { B12 } & 1 \\ \text { B13 } & 2\end{array}$

A1

A2 $3 \quad$ D1 0

A3 $4 \quad$ D2 0

A4 $1 \quad$ D3 0

A5 $1 \quad$ D4 0

A6 2 D5 0

A7 $2 \quad$ D6 0

$\begin{array}{llll}\text { A8 } & 5 & \text { D7 } & 0\end{array}$

A9 $5 \quad$ D8 0

$\begin{array}{llll}\mathrm{A} 10 & 5 & \mathrm{D} 9 & 0\end{array}$

A11 $5 \quad$ D10 0

A12 $1 \quad$ D11 0




\begin{tabular}{|c|c|c|c|c|c|c|}
\hline $\mathrm{H}$ & 14 & B14 & 13 & A13 & 2 & D12 \\
\hline $\mathrm{C}$ & 14 & B15 & 13 & A14 & 2 & D13 \\
\hline $\mathrm{H}$ & 16 & B16 & 14 & A15 & 13 & D14 \\
\hline $\mathrm{H}$ & 16 & B17 & 14 & A16 & 13 & D15 \\
\hline $\mathrm{H}$ & 16 & B18 & 14 & A17 & 13 & D16 \\
\hline $\mathrm{O}$ & 14 & B19 & 13 & A18 & 2 & D17 \\
\hline $\mathrm{O}$ & 20 & $\mathrm{~B} 20$ & 14 & A19 & 13 & D18 \\
\hline $\mathrm{H}$ & 21 & B21 & 20 & A20 & 14 & D19 \\
\hline $\mathrm{C}$ & 7 & $\mathrm{~B} 22$ & 4 & $\mathrm{~A} 21$ & 2 & D20 \\
\hline $\mathrm{H}$ & 23 & B23 & 7 & $\mathrm{~A} 22$ & 4 & D21 \\
\hline $\mathrm{H}$ & 23 & B24 & 7 & A23 & 4 & D22 \\
\hline $\mathrm{H}$ & 23 & B25 & 7 & A24 & 4 & D23 \\
\hline $\mathrm{H}$ & 7 & B26 & 4 & A25 & 2 & D24 \\
\hline \multicolumn{7}{|c|}{ Variables: } \\
\hline B1 & \multicolumn{2}{|c|}{1.51111735} & & & & \\
\hline B2 & \multicolumn{2}{|c|}{1.09105838} & & & & \\
\hline B3 & \multicolumn{2}{|c|}{2.27645529} & & & & \\
\hline B4 & \multicolumn{2}{|c|}{1.40230789} & & & & \\
\hline B5 & \multicolumn{2}{|c|}{1.13180531} & & & & \\
\hline B6 & \multicolumn{2}{|c|}{2.08422088} & & & & \\
\hline B7 & \multicolumn{2}{|c|}{2.17784018} & & & & \\
\hline B8 & \multicolumn{2}{|c|}{1.26479641} & & & & \\
\hline B9 & \multicolumn{2}{|c|}{1.08968677} & & & & \\
\hline B 10 & \multicolumn{2}{|c|}{1.08898829} & & & & \\
\hline B11 & \multicolumn{2}{|c|}{1.08781564} & & & & \\
\hline B12 & \multicolumn{2}{|c|}{1.40787769} & & & & \\
\hline B13 & \multicolumn{2}{|c|}{1.41238814} & & & & \\
\hline B14 & \multicolumn{2}{|c|}{1.08986656} & & & & \\
\hline B 15 & \multicolumn{2}{|c|}{1.51177989} & & & & \\
\hline B16 & \multicolumn{2}{|c|}{1.08853822} & & & & \\
\hline B 17 & \multicolumn{2}{|c|}{1.08793419} & & & & \\
\hline B18 & \multicolumn{2}{|c|}{1.08733194} & & & & \\
\hline B19 & \multicolumn{2}{|c|}{1.40499994} & & & & \\
\hline B20 & \multicolumn{2}{|c|}{1.41642665} & & & & \\
\hline B21 & \multicolumn{2}{|c|}{0.96760269} & & & & \\
\hline B22 & \multicolumn{2}{|c|}{1.47420472} & & & & \\
\hline B23 & \multicolumn{2}{|c|}{1.09120637} & & & & \\
\hline B24 & & 402 & & & & \\
\hline B25 & & 528 & & & & \\
\hline B26 & & 575 & & & & \\
\hline A1 & & 401 & & & & \\
\hline $\mathrm{A} 2$ & & 623 & & & & \\
\hline A 3 & & 372 & & & & \\
\hline A4 & & 395 & & & & \\
\hline
\end{tabular}




\begin{tabular}{|c|c|}
\hline A5 & 132.17568156 \\
\hline A6 & 68.05685016 \\
\hline A7 & 91.20462764 \\
\hline A8 & 109.17059399 \\
\hline A9 & 109.60210841 \\
\hline A10 & 110.56148587 \\
\hline A11 & 110.40403911 \\
\hline A 12 & 117.79727901 \\
\hline A13 & 104.06236731 \\
\hline A14 & 115.60328882 \\
\hline A15 & 108.96741211 \\
\hline A 16 & 108.64539910 \\
\hline A17 & 112.33605555 \\
\hline A18 & 109.52344320 \\
\hline A19 & 108.08797307 \\
\hline A20 & 102.86301599 \\
\hline A21 & 110.65725518 \\
\hline A22 & 109.11616926 \\
\hline A23 & 111.19756840 \\
\hline A24 & 107.76019999 \\
\hline A25 & 81.56356643 \\
\hline D1 & 111.70436240 \\
\hline D2 & 7.11425937 \\
\hline D3 & 121.82731959 \\
\hline D4 & 52.31045191 \\
\hline D5 & 84.13396097 \\
\hline D6 & 115.41825100 \\
\hline D7 & 173.81278815 \\
\hline D8 & -66.09662555 \\
\hline D9 & 54.69059127 \\
\hline D10 & 117.86410146 \\
\hline D11 & 117.35404347 \\
\hline D12 & -177.30242789 \\
\hline D13 & 60.36650278 \\
\hline D14 & 58.15305529 \\
\hline D15 & 176.95982726 \\
\hline D16 & -62.69092568 \\
\hline D17 & -60.80584079 \\
\hline D18 & -68.03694900 \\
\hline D19 & 62.95635100 \\
\hline D20 & -123.19444407 \\
\hline D21 & 150.71284912 \\
\hline D22 & -87.27216538 \\
\hline D23 & 33.85671228 \\
\hline
\end{tabular}


D24

$-2.74069095$

Harmonic Vibrational Frequencies $\left(\mathbf{c m}^{-1}\right)$ :

$\begin{array}{lllllllllllr}658.86 i & 34.38 & 53.42 & 68.01 & 91.99 & 100.45 & 118.06 & 144.55 & 183.18 & 204.13 & 234.89 \\ 246.46 & 270.97 & 295.62 & 302.86 & 318.42 & 348.45 & 356.20 & 399.73 & 454.30 & 481.18 \\ 520.58 & 546.09 & 571.06 & 595.56 & 694.41 & 853.80 & 889.11 & 898.93 & 913.93 & 934.00 \\ 966.68 & 993.85 & 1003.35 & 1054.79 & 1089.64 & 1108.40 & 1139.31 & 1148.40 & 1174.66 \\ 1188.50 & 1190.56 & 1200.10 & 1222.32 & 1362.94 & 1370.62 & 1372.83 & 1403.97 & 1416.06 \\ 1420.50 & 1431.74 & 1439.41 & 1447.78 & 1457.24 & 1470.75 & 1480.86 & 1493.27 & 1497.12 \\ 1503.46 & 1522.55 & 1627.98 & 1877.87 & 3060.70 & 3065.53 & 3080.58 & 3128.27 & 3134.93 \\ 3135.07 & 3140.42 & 3154.89 & 3161.79 & 3171.35 & 3173.14 & 3207.03 & 3772.52 & \\ \text { Rotational constants (B/GHZ): } & 1.4526870 & 0.4180504 & 0.3586739 & \end{array}$

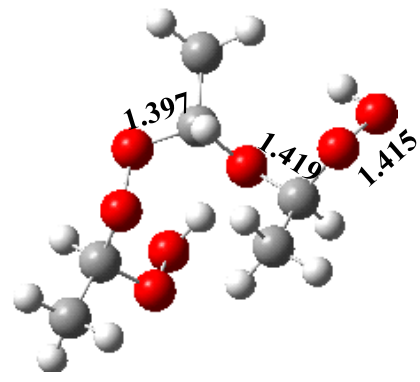

P4h

C

$\begin{array}{lll}\text { C } & 1 & \text { B1 }\end{array}$

$\begin{array}{lll}\mathrm{H} & 2 & \mathrm{~B} 2\end{array}$

O 2

O 2

$\mathrm{H} \quad 5$

C 4

O 7

O 7

$\mathrm{H} \quad 1$

$\mathrm{H} \quad 1$

$\mathrm{H} \quad 1$

O 2

C 13

$\mathrm{H} \quad 14$

C $\quad 14$

$\mathrm{H} \quad 16$

$\mathrm{H} \quad 16$

$\mathrm{H} \quad 16$

O 14

O 20

$\mathrm{H} \quad 21$

$\begin{array}{ll}\text { C } & 7\end{array}$

H 23

$\begin{array}{rrrrrr}\text { B2 } & 1 & \text { A1 } & & & \\ \text { B3 } & 1 & \text { A2 } & 3 & \text { D1 } & 0 \\ \text { B4 } & 1 & \text { A3 } & 4 & \text { D2 } & 0 \\ \text { B5 } & 2 & \text { A4 } & 1 & \text { D3 } & 0 \\ \text { B6 } & 2 & \text { A5 } & 1 & \text { D4 } & 0 \\ \text { B7 } & 4 & \text { A6 } & 2 & \text { D5 } & 0 \\ \text { B8 } & 4 & \text { A7 } & 2 & \text { D6 } & 0 \\ \text { B9 } & 2 & \text { A8 } & 5 & \text { D7 } & 0 \\ \text { B10 } & 2 & \text { A9 } & 5 & \text { D8 } & 0 \\ \text { B11 } & 2 & \text { A10 } & 5 & \text { D9 } & 0 \\ \text { B12 } & 1 & \text { A11 } & 5 & \text { D10 } & 0 \\ \text { B13 } & 2 & \text { A12 } & 1 & \text { D11 } & 0 \\ \text { B14 } & 13 & \text { A13 } & 2 & \text { D12 } & 0 \\ \text { B15 } & 13 & \text { A14 } & 2 & \text { D13 } & 0 \\ \text { B16 } & 14 & \text { A15 } & 13 & \text { D14 } & 0 \\ \text { B17 } & 14 & \text { A16 } & 13 & \text { D15 } & 0 \\ \text { B18 } & 14 & \text { A17 } & 13 & \text { D16 } & 0 \\ \text { B19 } & 13 & \text { A18 } & 2 & \text { D17 } & 0 \\ \text { B20 } & 14 & \text { A19 } & 13 & \text { D18 } & 0 \\ \text { B21 } & 20 & \text { A20 } & 14 & \text { D19 } & 0 \\ \text { B22 } & 4 & \text { A21 } & 2 & \text { D20 } & 0 \\ \text { B23 } & 7 & \text { A22 } & 4 & \text { D21 } & 0 \\ & & & & & \end{array}$




$\begin{array}{rrrrrrrr}\mathrm{H} & 23 & \text { B24 } & 7 & \text { A23 } & 4 & \text { D22 } & 0 \\ \mathrm{H} & 23 & \text { B25 } & 7 & \text { A24 } & 4 & \text { D23 } & 0 \\ \mathrm{H} & 7 & \text { B26 } & 4 & \text { A25 } & 2 & \text { D24 } & 0\end{array}$

Variables:

B1

1.51095093

B2

1.09015900

B3

2.27655151

B4

1.39738860

B5

2.54666883

B6

1.41228831

B7

2.30393211

B8

1.39720376

B9

1.08950125

B10

1.08899058

B11

1.08734011

B12

1.41604750

B13

1.41912472

B14

1.08996315

B15

1.51062057

B16

1.08705881

B17

1.08785832

B18

1.08732574

B19

1.40062011

B20

1.41526075

B21

0.96692404

B22

1.51145397

B23

1.08649080

B24

1.08925296

B25

1.08660209

B26

1.09053221

A1

111.76215365

A2

141.07429025

A3

106.04628949

A4

80.88838164

A5

126.64507046

A6

106.65403001

A7

112.60652492

A8

109.22682241

A9

109.76470979

A10 $\quad 110.37934085$

A11 109.89654146

A12 117.97250882

A13 103.93314659

A14

115.32787692 


$\begin{array}{lr}\text { A15 } & 108.59267295 \\ \text { A16 } & 108.51370622 \\ \text { A17 } & 112.24114827 \\ \text { A18 } & 109.27263751 \\ \text { A19 } & 108.28301023 \\ \text { A20 } & 102.97455620 \\ \text { A21 } & 105.2475337 \\ \text { A22 } & 109.32485505 \\ \text { A23 } & 109.74427763 \\ \text { A24 } & 109.85547163 \\ \text { A25 } & 108.93751579 \\ \text { D1 } & 106.29807388 \\ \text { D2 } & 13.10504939 \\ \text { D3 } & 108.64607982 \\ \text { D4 } & 91.71238897 \\ \text { D5 } & 7.07974137 \\ \text { D6 } & 44.10721597 \\ \text { D7 } & 174.98671073 \\ \text { D8 } & -64.80013914 \\ \text { D9 } & 55.79629527 \\ \text { D10 } & 118.14995971 \\ \text { D11 } & 118.57792920 \\ \text { D12 } & -178.97992775 \\ \text { D13 } & 58.66729935 \\ \text { D14 } & 55.14354213 \\ \text { D15 } & 174.45927415 \\ \text { D16 } & -65.37740815 \\ \text { D17 } & -62.40829344 \\ \text { D18 } & -69.46719065 \\ \text { D19 } & 69.92683651 \\ \text { D20 } & 160.22743689 \\ \text { D21 } & 179.50505644 \\ \text { D22 } & -61.01838330 \\ \text { D23 } & 58.90648117 \\ \text { D24 } & -77.06864978 \\ \text { D } & \end{array}$

Harmonic Vibrational Frequencies $\left(\mathbf{c m}^{-\mathbf{1}}\right)$ :

$\begin{array}{lllllllllll}51.54 & 58.86 & 77.19 & 107.58 & 122.34 & 156.01 & 175.19 & 192.17 & 212.38 & 221.94 & 244.43\end{array}$ $\begin{array}{llllllllll}283.91 & 297.76 & 318.22 & 332.10 & 355.45 & 360.58 & 427.50 & 441.83 & 500.85 & 529.92\end{array}$ $\begin{array}{llllllllll}571.69 & 584.68 & 610.26 & 636.22 & 696.37 & 852.43 & 893.01 & 904.25 & 918.44 & 937.49\end{array}$ $\begin{array}{lllllllll}969.12 & 1057.26 & 1059.03 & 1066.99 & 1098.69 & 1120.87 & 1137.89 & 1151.68 & 1163.47\end{array}$ $\begin{array}{lllllllll}1178.74 & 1191.97 & 1203.33 & 1234.61 & 1359.86 & 1365.73 & 1369.54 & 1392.65 & 1405.65\end{array}$ $\begin{array}{lllllllll}1416.24 & 1419.72 & 1425.14 & 1436.79 & 1443.90 & 1486.34 & 1490.24 & 1492.75 & 1496.07\end{array}$ $\begin{array}{llllllllll}1498.25 & 1502.00 & 1511.03 & 3060.10 & 3070.00 & 3086.40 & 3124.22 & 3125.45 & 3133.10\end{array}$ $\begin{array}{llllllll}3144.02 & 3152.30 & 3154.25 & 3165.67 & 3166.25 & 3185.02 & 3685.27 & 3791.00\end{array}$ 
Rotational constants (B/GHZ):

1.2530071

0.5100953

0.4622752 
Figure S7. Optimized geometries, z-matrices and vibrational frequencies of all stationary points in the reaction of 2 syn- $\mathrm{CH}_{3} \mathrm{CHOO}$ with $\mathrm{HO}-\mathrm{CH}\left(\mathrm{CH}_{3}\right) \mathrm{OO}-\mathrm{H}\left(\mathrm{Pa}_{2}\right.$ and $\left.\mathrm{Pb}_{2}\right)$ at the M06-2X/6-311+G(2df,2p) level of theory

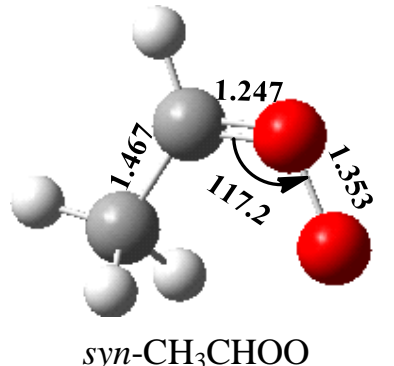

$\mathrm{C}$

$\begin{array}{lll}\mathrm{O} & 1 & \mathrm{~B} 1\end{array}$

$\mathrm{O} 2 \mathrm{~B} 2$

$\mathrm{H} \quad 1 \quad \mathrm{~B} 3$

C 11 B

$\mathrm{H} \quad 5$

$\mathrm{H} \quad 5$

B5

$\mathrm{H} \quad 5$

B3 2

A1

B4 2

A2 3

D1 0

A3 3

D2 0

B5 1

A4 2

D3 0

B6 1

A5 2

D4 0

Variables:

B1

1.24673463

B2

1.35257838

B3

1.08417638

B4

1.46657543

B5

1.08452020

B6

1.09346997

B7

1.09346997

A1

117.18473613

A2

113.50906167

A3

121.22148887

A4

111.67299194

A5

108.36774689

A6

108.36774689

D1

180.00000000

D2

0.00000000

D3

180.00000000

D4

$-56.62427783$

D5

56.62427783

Harmonic Vibrational Frequencies $\left(\mathrm{cm}^{-1}\right)$ :

$\begin{array}{llllllllll}180.02 & 313.30 & 470.29 & 682.06 & 784.99 & 938.95 & 995.40 & 1065.23 & 1108.04 & 1360.12\end{array}$ $\begin{array}{llllllll}1409.89 & 1435.62 & 1462.37 & 1644.74 & 3035.96 & 3082.86 & 3172.74 & 3221.03\end{array}$

Rotational constants (B/GHZ): $\quad 17.9036510 \quad 7.2529111 \quad 5.3265305$ 


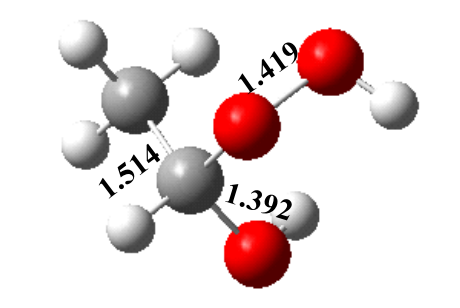

$\mathrm{Pa}_{2}$ (syn-HO-CH$\left(\mathrm{CH}_{3}\right) \mathrm{OO}-\mathrm{H}$ )

$\mathrm{C}$

$\begin{array}{lll}\text { O } & 1 & \text { B1 }\end{array}$

$\mathrm{O} 2 \mathrm{~B} 2$

$\mathrm{H} \quad 1 \quad \mathrm{~B} 3$

O 1

$\mathrm{H} \quad 3$

B 4

A2 $3 \quad$ D1 0

A3 $3 \quad$ D2 0

$\mathrm{H} \quad 5$

B5

A4 $1 \quad$ D3 0

C 1

B6

A5 2 D4 0

$\begin{array}{ll}\mathrm{H} & 8\end{array}$

$\mathrm{H} \quad 8$

$\mathrm{H} \quad 8$

B

B8

A6 2 D5 0

A7 5 D6 0

$\begin{array}{llllll}\text { B9 } & 1 & \text { A8 } & 5 & \text { D7 } & 0\end{array}$

Variables:

B1

1.41574412

B2

1.41909669

B3

1.09067286

B4

1.39184386

B5

0.96378500

B6

0.96073234

B7

1.51389468

B8

1.08824849

B9

1.08773853

B10

1.08994559

A1 $\quad 107.86168065$

A2 101.74558425

A3 111.37946066

A4 101.19029547

A5 108.64817138

A6 112.66192372

A7 $\quad 109.67570337$

A8 $\quad 110.13170367$

A9 $\quad 110.09828549$

D1 $\quad 177.82956009$

D2 $\quad 64.09238847$

D3 $\quad-105.11788339$

D4 $\quad-86.73514620$

D5 $\quad 126.56282225$

D6 $\quad 59.47979574$ 
D7

179.80552806

D8

$-60.73368006$

Harmonic Vibrational Frequencies $\left(\mathbf{c m}^{-1}\right)$ :

$\begin{array}{llllllllll}150.83 & 237.33 & 243.85 & 312.28 & 420.17 & 438.32 & 507.63 & 696.96 & 860.78 & 957.79\end{array}$ $\begin{array}{lllllllll}1012.49 & 1090.91 & 1133.80 & 1175.40 & 1314.13 & 1389.46 & 1412.85 & 1418.37 & 1438.19\end{array}$ $\begin{array}{llllllll}1493.54 & 1503.52 & 3067.74 & 3128.13 & 3149.52 & 3154.52 & 3842.07 & 3889.62\end{array}$

Rotational constants (B/GHZ): $\quad 6.9706500 \quad 4.5813473 \quad 3.6600767$

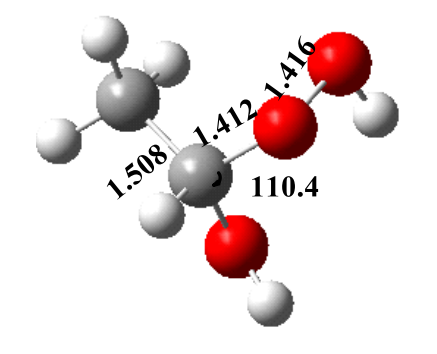

$\mathrm{P}_{\mathrm{b} 2}$ (syn-HO-CH$\left.\left(\mathrm{CH}_{3}\right) \mathrm{OO}-\mathrm{H}\right)$

$\mathrm{C}$

O $\quad 1 \quad$ B1

$\begin{array}{llll}\mathrm{O} & 2 & \mathrm{~B} 2 & 1\end{array}$

$\begin{array}{llll}\mathrm{H} & 1 & \mathrm{~B} 3 & 2\end{array}$

$\begin{array}{llll}\mathrm{O} & 1 & \mathrm{~B} 4 & 2\end{array}$

A1

$\mathrm{H} \quad 3$

B5 2

$\begin{array}{llll}\mathrm{A} 2 & 3 & \mathrm{D} 1 & 0\end{array}$

$\mathrm{H} \quad 5$

B6 1

A $3-3=$ D2 0

C 1

B7 5

A4 1 D3 0

$\mathrm{H} \quad 8$

$\mathrm{H} \quad 8$

B8 1

B9 1

$\begin{array}{llll}\mathrm{A} 5 & 2 & \mathrm{D} 4 & 0\end{array}$

H $\quad 8$

B10 1

A6 2 D5 0

A7 5 D6 0

$\begin{array}{llll}\mathrm{A} 8 & 5 & \mathrm{D} 7 & 0\end{array}$

Variables:

1.41195863

B2

1.41583897

B3

1.09421397

B4

1.40063180

B5

0.96614133

B6

0.96082394

B7

1.50834430

B8

1.08789377

B9

1.08768717

B10

1.08657519

A1 $\quad 109.03213737$

A2 $\quad 101.56077691$

A3 110.37617334

A4 $\quad 102.39067420$

A5 109.02869039

A6 $\quad 108.36044040$

A7 $\quad 109.33838888$ 


$\begin{array}{lr}\text { A8 } & 109.65383915 \\ \text { A9 } & 110.04773295 \\ \text { D1 } & -175.01638651 \\ \text { D2 } & 66.80673155 \\ \text { D3 } & -73.86249428 \\ \text { D4 } & 67.10948874 \\ \text { D5 } & 124.99768807 \\ \text { D6 } & 65.51240659 \\ \text { D7 } & -174.39368180 \\ \text { D8 } & -54.24919950\end{array}$

Harmonic Vibrational Frequencies $\left(\mathbf{c m}^{-\mathbf{1}}\right)$ :

$\begin{array}{llllllllll}156.41 & 244.75 & 281.39 & 328.73 & 432.26 & 440.40 & 512.59 & 711.49 & 867.12 & 958.92\end{array}$ $\begin{array}{lllllllll}1014.22 & 1099.27 & 1144.99 & 1166.60 & 1293.62 & 1392.09 & 1403.03 & 1432.91 & 1463.23\end{array}$ $\begin{array}{llllllll}1493.99 & 1499.42 & 3085.44 & 3090.77 & 3164.63 & 3180.81 & 3816.10 & 3899.46\end{array}$

$\begin{array}{llll}\text { Rotational constants (B/GHZ): } & 6.9585956 & 4.6233182 & 3.6586659\end{array}$

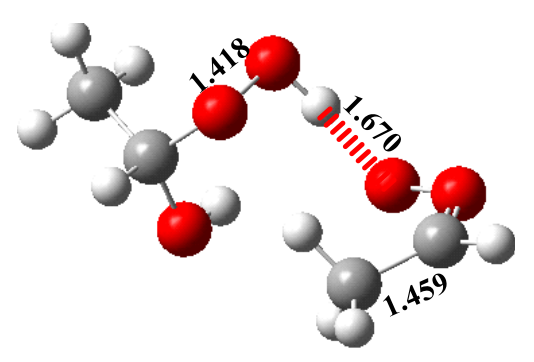

IM5a

$\mathrm{C}$

$\begin{array}{lll}\mathrm{C} & 1 & \mathrm{~B} 1\end{array}$

$\begin{array}{lllll}\mathrm{H} & 2 & \mathrm{~B} 2 & 1 & \mathrm{~A} 1\end{array}$

$\mathrm{O} 2$

$\mathrm{O} \quad 2$

B3 1

A2 $3 \quad$ D1 0

$\mathrm{H} \quad 4$

B4 1

A3 $4 \quad$ D2 0

C $\quad 5$

$\begin{array}{ll}\mathrm{C} & 7\end{array}$

B5 2

A4 $1 \quad$ D3 0

$\begin{array}{llllll}\text { B6 } & 2 & \text { A5 } & 1 & \text { D4 } & 0\end{array}$

$\mathrm{H} \quad 8$

B7 5

A6 2

D5 0

B8 7

A7 5

D6 0

$\mathrm{H} \quad 8$

$\mathrm{H} \quad 8$

B9 7

A8 5

D7 0

B10 7

A9 5

D8 0

$\begin{array}{ll}\mathrm{O} & 7\end{array}$

$\begin{array}{ll}\text { O } & 7\end{array}$

B11 5

A10 2

D9 0

B12 5

A11 2

D10 0

$\begin{array}{ll}\mathrm{H} & 7\end{array}$

H 1

H 1

B13 5

A12 2

D11 0

B14 2

A13 5

D12 0

B15 2

A14 5

D13 0

$\mathrm{H} \quad 1$

B16 2

A15 5

D14 0

O 2

B17 1

A16 5

D15 0

H 18

B18 2

A17 1

D16 0

Variables:

B1 


\begin{tabular}{|c|c|}
\hline B2 & 1.09148326 \\
\hline B3 & 2.30175361 \\
\hline B4 & 1.41316732 \\
\hline B5 & 0.99123294 \\
\hline B6 & 3.80798980 \\
\hline B7 & 1.45937289 \\
\hline B8 & 1.09838575 \\
\hline B9 & 1.09231742 \\
\hline B10 & 1.08423902 \\
\hline B11 & 2.22991873 \\
\hline B12 & 1.24547630 \\
\hline B13 & 1.08586593 \\
\hline B14 & 1.08811022 \\
\hline B15 & 1.08832284 \\
\hline B16 & 1.08794594 \\
\hline B17 & 1.39816762 \\
\hline B18 & 0.96353069 \\
\hline A1 & 111.24205607 \\
\hline $\mathrm{A} 2$ & 90.16727392 \\
\hline A3 & 112.49873523 \\
\hline A4 & 101.63697870 \\
\hline A5 & 115.38994230 \\
\hline A6 & 58.08195389 \\
\hline A7 & 107.93333155 \\
\hline A8 & 109.21460269 \\
\hline A9 & 111.35907755 \\
\hline A 10 & 58.55894465 \\
\hline A11 & 81.34839804 \\
\hline A12 & 136.26835627 \\
\hline A13 & 109.13831307 \\
\hline A14 & 110.09310841 \\
\hline A 15 & 110.30830528 \\
\hline A 16 & 112.66177549 \\
\hline A17 & 108.75473046 \\
\hline D1 & -142.94101934 \\
\hline D2 & 28.61034137 \\
\hline D3 & -133.14507035 \\
\hline D4 & -142.77404916 \\
\hline D5 & -42.27148165 \\
\hline D6 & -11.40171370 \\
\hline D7 & 101.07931270 \\
\hline D8 & -133.95521395 \\
\hline D9 & 69.48216489 \\
\hline D10 & 95.63674146 \\
\hline
\end{tabular}




$\begin{array}{lr}\text { D11 } & -150.77017919 \\ \text { D12 } & -172.55267086 \\ \text { D13 } & -52.35149091 \\ \text { D14 } & 67.44394802 \\ \text { D15 } & -126.86212449 \\ \text { D16 } & 59.30536832\end{array}$

Harmonic Vibrational Frequencies (cm-1):

$\begin{array}{lccccccccccr}37.05 & 58.37 & 65.97 & 94.23 & 124.20 & 157.77 & 224.83 & 245.64 & 273.30 & 316.45 & 333.64 \\ 381.92 & 431.45 & 474.78 & 527.19 & 677.38 & 682.12 & 789.85 & 860.29 & 916.91 & 929.88 \\ 953.93 & 1005.27 & 1016.97 & 1083.84 & 1094.75 & 1119.42 & 1147.76 & 1165.11 & 1311.70 \\ 1361.67 & 1387.61 & 1411.96 & 1416.26 & 1426.39 & 1445.94 & 1471.70 & 1489.72 & 1501.46 \\ 1547.10 & 1667.87 & 3018.98 & 3073.84 & 3092.66 & 3108.59 & 3153.43 & 3164.72 & 3179.49 \\ 3207.88 & 3237.73 & 3857.81 & & & & & & & \\ \text { Rotational constants (B/GHZ): } & 2.8536693 & 0.8515420 & 0.7880124 & \end{array}$

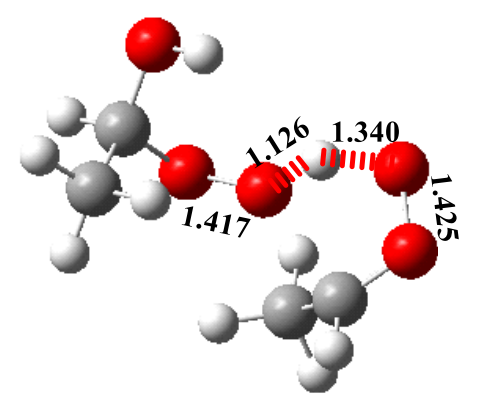

TS5a

$\mathrm{C}$

$\begin{array}{lll}\text { C } & 1 & \text { B } 1\end{array}$

$\begin{array}{lllll}\mathrm{H} & 2 & \mathrm{~B} 2 & 1 & \mathrm{~A} 1\end{array}$

$\begin{array}{llllllll}\mathrm{O} & 2 & \mathrm{~B} 3 & 1 & \mathrm{~A} 2 & 3 & \mathrm{D} 1 & 0\end{array}$

$\begin{array}{llllllll}\mathrm{O} & 4 & \mathrm{~B} 4 & 2 & \mathrm{~A} 3 & 1 & \mathrm{D} 2 & 0\end{array}$

$\begin{array}{llllllll}\mathrm{H} & 4 & \mathrm{~B} 5 & 2 & \mathrm{~A} 4 & 1 & \mathrm{D} 3 & 0\end{array}$

$\begin{array}{llllllll}\mathrm{C} & 4 & \mathrm{~B} 6 & 2 & \mathrm{~A} 5 & 1 & \mathrm{D} 4 & 0\end{array}$

$\begin{array}{llllllll}\mathrm{C} & 7 & \mathrm{~B} 7 & 4 & \mathrm{~A} 6 & 2 & \mathrm{D} 5 & 0\end{array}$

$\begin{array}{llllllll}\mathrm{H} & 8 & \mathrm{~B} 8 & 7 & \mathrm{~A} 7 & 4 & \mathrm{D} 6 & 0\end{array}$

$\begin{array}{llllllll}\mathrm{H} & 8 & \text { B9 } & 7 & \text { A8 } & 4 & \text { D7 } & 0\end{array}$

$\begin{array}{llllllll}\mathrm{H} & 8 & \mathrm{~B} 10 & 7 & \text { A9 } & 4 & \text { D8 } & 0\end{array}$

$\begin{array}{llllllll}\mathrm{O} & 7 & \mathrm{~B} 11 & 4 & \mathrm{~A} 10 & 2 & \mathrm{D} 9 & 0\end{array}$

$\begin{array}{llllllll}\mathrm{O} & 7 & \mathrm{~B} 12 & 4 & \mathrm{~A} 11 & 2 & \mathrm{D} 10 & 0\end{array}$

$\begin{array}{llllllll}\mathrm{H} & 7 & \mathrm{~B} 13 & 4 & \mathrm{~A} 12 & 2 & \mathrm{D} 11 & 0\end{array}$

$\begin{array}{llllllll}\mathrm{H} & 1 & \mathrm{~B} 14 & 2 & \mathrm{~A} 13 & 5 & \mathrm{D} 12 & 0\end{array}$

$\begin{array}{llllllll}\mathrm{H} & 1 & \mathrm{~B} 15 & 2 & \mathrm{~A} 14 & 5 & \mathrm{D} 13 & 0\end{array}$

$\begin{array}{llllllll}\mathrm{H} & 1 & \mathrm{~B} 16 & 2 & \mathrm{~A} 15 & 5 & \mathrm{D} 14 & 0\end{array}$

$\begin{array}{llllllll}\mathrm{O} & 2 & \mathrm{~B} 17 & 1 & \mathrm{~A} 16 & 5 & \mathrm{D} 15 & 0\end{array}$

$\begin{array}{llllllll}\mathrm{H} & 18 & \mathrm{~B} 18 & 2 & \mathrm{~A} 17 & 1 & \mathrm{D} 16 & 0\end{array}$

Variables:

B1 


\begin{tabular}{|c|c|}
\hline B2 & 1.09054669 \\
\hline B3 & 2.27837592 \\
\hline B4 & 1.41668873 \\
\hline B5 & 1.12561008 \\
\hline B6 & 2.07261490 \\
\hline B7 & 1.47689215 \\
\hline B8 & 1.08713872 \\
\hline B9 & 1.08289876 \\
\hline B10 & 1.09450647 \\
\hline B11 & 2.20608522 \\
\hline B12 & 1.26994802 \\
\hline B13 & 1.08831658 \\
\hline B14 & 1.08790940 \\
\hline B15 & 1.08892423 \\
\hline B16 & 1.08891949 \\
\hline B17 & 1.38838475 \\
\hline B18 & 0.96229392 \\
\hline $\mathrm{A} 1$ & 111.44734145 \\
\hline $\mathrm{A} 2$ & 92.23926399 \\
\hline A3 & 36.53493936 \\
\hline A4 & 122.80651359 \\
\hline A5 & 144.16589608 \\
\hline A6 & 95.43679993 \\
\hline A7 & 109.64992009 \\
\hline A8 & 111.71216658 \\
\hline A9 & 105.85070803 \\
\hline A 10 & 67.66216296 \\
\hline A11 & 87.96345251 \\
\hline A12 & 106.13635289 \\
\hline A13 & 109.25803934 \\
\hline A14 & 110.35958281 \\
\hline A15 & 110.26166795 \\
\hline A16 & 112.60970163 \\
\hline A 17 & 108.07480576 \\
\hline D1 & -145.43878710 \\
\hline D2 & 124.80535965 \\
\hline D3 & -169.32563500 \\
\hline D4 & 80.18369833 \\
\hline D5 & 35.95190351 \\
\hline D6 & -58.12652476 \\
\hline D7 & 64.78356427 \\
\hline D8 & -174.84686220 \\
\hline D9 & 129.32399617 \\
\hline D10 & 161.74682279 \\
\hline
\end{tabular}




$\begin{array}{lr}\text { D11 } & -86.07921082 \\ \text { D12 } & -174.06269633 \\ \text { D13 } & -54.08384737 \\ \text { D14 } & 66.28167159 \\ \text { D15 } & -126.42300420 \\ \text { D16 } & 58.44358289\end{array}$

Harmonic Vibrational Frequencies $\left(\mathbf{c m}^{\mathbf{- 1}}\right)$ :

$\begin{array}{lllllllllllr}680.11 i & 25.66 & 76.01 & 99.36 & 167.75 & 194.26 & 230.33 & 246.51 & 304.66 & 347.26 & 376.51 \\ 414.12 & 435.22 & 475.71 & 523.20 & 626.52 & 688.16 & 865.24 & 874.07 & 901.31 & 940.69 \\ 962.74 & 972.25 & 1062.79 & 1090.77 & 1119.87 & 1161.84 & 1176.19 & 1186.50 & 1322.92 \\ 1370.91 & 1380.75 & 1403.54 & 1410.28 & 1417.86 & 1432.11 & 1456.53 & 1479.99 & 1493.63 \\ 1497.45 & 1623.39 & 1942.40 & 3053.61 & 3060.51 & 3123.18 & 3142.77 & 3145.28 & 3149.84 \\ 3189.91 & 3208.62 & 3874.81 & & & & & & & \\ \text { Rotational constants (B/GHZ): } & 2.9518200 & 0.8863593 & 0.7997626 & \end{array}$

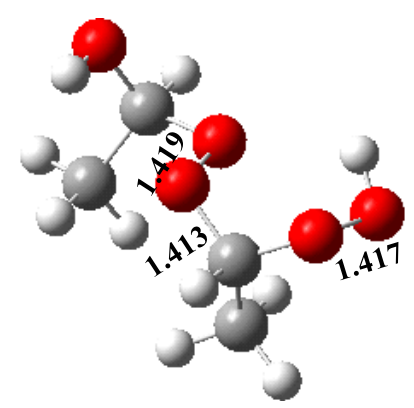

P5a

$\mathrm{C}$

$\begin{array}{lll}\text { C } & 1 & \text { B1 }\end{array}$

$\begin{array}{lllll}\mathrm{H} & 2 & \mathrm{~B} 2 & 1 & \mathrm{~A} 1\end{array}$

O 2

O 2

B3 1

A2

B4 1

A2 $3 \quad$ D1 0

$\mathrm{H} \quad 5$

C 4

$\begin{array}{ll}\mathrm{C} & 7\end{array}$

B5 2

A3 4 D2 0

B6 2

A4 $1 \quad$ D3 0

$\mathrm{H} \quad 8$

B7 4

A5 1 D4 0

$\begin{array}{ll}\mathrm{H} & 8\end{array}$

$\mathrm{H} \quad 8$

B8 7

A7 4 D6 0

B9 7

A8 4

D7 0

$\begin{array}{ll}\mathrm{O} & 7\end{array}$

$\begin{array}{ll}\text { O } & 7\end{array}$

B10 7

A9 4

D8 0

B11 4

A10 2

D9 0

B12 4

A11 2 D10 0

$\begin{array}{ll}\mathrm{H} & 7\end{array}$

B13 4

$\mathrm{A} 122 \mathrm{D} 11 \quad 0$

$\mathrm{H} \quad 1$

$\mathrm{H} \quad 1$

B14 2

A13 5 D12 0

B15 2

A14 5

D13 0

$\mathrm{H} \quad 1$

B16 2

A15 5

D14 0

O 2

B17 1

A16 5 D15 0

$\mathrm{H} \quad 18$

B18 2

A17 1 D16 0

Variables:

B1 


\begin{tabular}{|c|c|}
\hline B2 & 1.09056339 \\
\hline B3 & 2.28628388 \\
\hline B4 & 1.41884962 \\
\hline B5 & 2.25066250 \\
\hline B6 & 1.41284327 \\
\hline B7 & 1.51145540 \\
\hline B8 & 1.08843979 \\
\hline B9 & 1.08488919 \\
\hline B10 & 1.08780553 \\
\hline B11 & 2.31285087 \\
\hline B12 & 1.39840684 \\
\hline B13 & 1.09220839 \\
\hline B14 & 1.08784746 \\
\hline B15 & 1.08874672 \\
\hline B16 & 1.08929913 \\
\hline B17 & 1.38780366 \\
\hline B18 & 0.96218586 \\
\hline A1 & 111.28529884 \\
\hline $\mathrm{A} 2$ & 92.99033146 \\
\hline $\mathrm{A} 3$ & 112.03592930 \\
\hline A 4 & 158.17901614 \\
\hline A5 & 135.07293481 \\
\hline A6 & 113.60579390 \\
\hline A7 & 109.13872625 \\
\hline A8 & 111.03113365 \\
\hline A9 & 109.00303414 \\
\hline A 10 & 103.56744328 \\
\hline A11 & 111.32715224 \\
\hline A12 & 101.80247766 \\
\hline A13 & 109.06595637 \\
\hline A14 & 110.34933562 \\
\hline A15 & 110.50671992 \\
\hline A16 & 112.88962011 \\
\hline A17 & 108.49872867 \\
\hline D1 & -145.81289978 \\
\hline D2 & 32.38257590 \\
\hline D3 & 134.93127510 \\
\hline D4 & 70.46048513 \\
\hline D5 & -27.99804965 \\
\hline D6 & -60.00951979 \\
\hline D7 & 60.83649868 \\
\hline D8 & -179.32046621 \\
\hline D9 & 66.67034906 \\
\hline D10 & 102.56205577 \\
\hline
\end{tabular}




$\begin{array}{lr}\text { D11 } & -148.39983846 \\ \text { D12 } & -173.69098816 \\ \text { D13 } & -53.74968015 \\ \text { D14 } & 66.76242380 \\ \text { D15 } & -126.70718453 \\ \text { D16 } & 58.88801737\end{array}$

Harmonic Vibrational Frequencies $\left(\mathbf{c m}^{-\mathbf{1}}\right)$ :

$\begin{array}{lllllllllll}44.92 & 81.36 & 108.30 & 146.16 & 218.75 & 230.92 & 252.03 & 263.54 & 329.38 & 350.08 & 378.65\end{array}$ $\begin{array}{llllllllll}416.19 & 469.20 & 528.08 & 538.54 & 663.89 & 747.49 & 860.07 & 875.81 & 931.69 & 954.92\end{array}$ $\begin{array}{lllllllll}997.17 & 1019.91 & 1088.20 & 1121.91 & 1138.50 & 1169.16 & 1178.87 & 1189.76 & 1322.30\end{array}$ $\begin{array}{lllllllll}1356.58 & 1374.51 & 1396.83 & 1415.38 & 1421.64 & 1427.66 & 1461.50 & 1483.73 & 1489.26\end{array}$ $\begin{array}{lllllllll}1499.26 & 1505.88 & 3063.50 & 3081.79 & 3121.51 & 3132.98 & 3145.54 & 3153.32 & 3155.21\end{array}$ $3186.18 \quad 3790.70 \quad 3878.79$

Rotational constants (B/GHZ): $\quad 2.9580302 \quad 1.0025194 \quad 0.9026314$

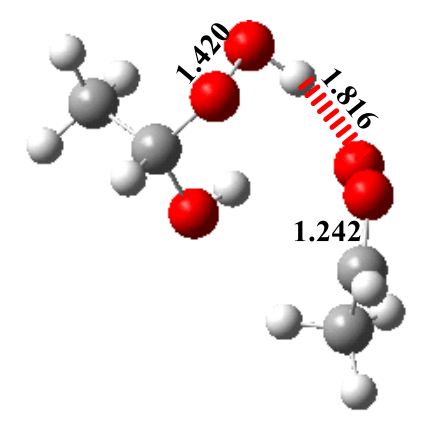

IM5b

$\mathrm{C}$

$\begin{array}{lll}\mathrm{C} & 1 & \mathrm{~B} 1\end{array}$

$\begin{array}{lllll}\mathrm{H} & 2 & \mathrm{~B} 2 & 1 & \mathrm{~A} 1\end{array}$

$\mathrm{H}-2-\mathrm{B} 3-1$

$\mathrm{H} 2$

B3 1

B4 1

A2 $3 \quad$ D1 0

O 1

B5 2

A3 3

D2 0

A4 $5 \quad$ D3 0

$\mathrm{H} \quad 6$

B6 1

A5 $2 \quad$ D4 0

O 1

B7 6

A6 2 D5 0

C 6

B8 1

A7 2 D6 0

C 9

B9 6

$\begin{array}{llll}\text { A8 } & 1 & \text { D7 } & 0\end{array}$

$\mathrm{H} \quad 10$

B10 9

$\begin{array}{llll}\text { A9 } & 6 & \text { D8 } & 0\end{array}$

$\mathrm{H} \quad 10$

B11 9

$\begin{array}{llll}\text { A10 } & 6 & \text { D9 } & 0\end{array}$

$\mathrm{H} \quad 10$

B12 9

$\begin{array}{llll}\text { A11 } & 6 & \text { D10 } & 0\end{array}$

O 9

B13 6

A12 $1 \quad$ D11 0

O 9

B14 6

$\begin{array}{llll}\text { A13 } & 1 & \text { D12 } & 0\end{array}$

$\mathrm{H} \quad 9$

B15 6

$\begin{array}{llll}\text { A14 } 1 & \text { D13 } & 0\end{array}$

$\mathrm{H} \quad 1$

B16 6

A15 2 D14 0

O 1

B17 6

A16 2 D15 0

H 8

B18 1

A17 6

D16 0

Variables:

B1 


$\begin{array}{lr}\text { B2 } & 1.08816420 \\ \text { B3 } & 1.08855431 \\ \text { B4 } & 1.08740114 \\ \text { B5 } & 1.40587702 \\ \text { B6 } & 0.96858792 \\ \text { B7 } & 2.30296672 \\ \text { B8 } & 3.04947260 \\ \text { B9 } & 1.46539209 \\ \text { B10 } & 1.09439214 \\ \text { B11 } & 1.09033465 \\ \text { B12 } & 1.08494745 \\ \text { B13 } & 2.23405779 \\ \text { B14 } & 1.24166790 \\ \text { B15 } & 1.08567814 \\ \text { B16 } & 1.09140892 \\ \text { B17 } & 1.40715244 \\ \text { B18 } & 0.97986526 \\ \text { A1 } & 109.12439437 \\ \text { A2 } & 110.02645515 \\ \text { A3 } & 110.38035038 \\ \text { A4 } & 112.07002288 \\ \text { A5 } & 107.89373027 \\ \text { A6 } & 98.71398587 \\ \text { A7 } & 117.33055010 \\ \text { A8 } & 79.95577098 \\ \text { A9 } & 107.57222984 \\ \text { D10 } & 108.94641969 \\ \text { D10 } & 110.87652254 \\ \text { D1 } & 62.99694955 \\ \text { A12 } & 74.42654949 \\ \text { A13 } & 118.066452015 \\ \text { A14 } & 106.19793603 \\ \text { A15 } & 111.19469227 \\ \text { A16 } & 101.38395754 \\ \text { A17 } & 120.15331873 \\ \text { D1 } & \\ \text { D2 } & -1193435063 \\ \text { D3 } & \end{array}$




$\begin{array}{lr}\text { D11 } & -87.79694349 \\ \text { D12 } & -54.20911843 \\ \text { D13 } & 53.36969103 \\ \text { D14 } & 121.57108270 \\ \text { D15 } & -127.55684725 \\ \text { D16 } & -19.19901843\end{array}$

Harmonic Vibrational Frequencies $\left(\mathbf{c m}^{-1}\right)$ :

$\begin{array}{lllllllllll}55.67 & 67.28 & 107.20 & 113.45 & 145.14 & 153.08 & 211.81 & 228.82 & 274.39 & 326.03 & 338.50\end{array}$ $\begin{array}{llllllllll}418.29 & 438.48 & 477.42 & 531.47 & 672.60 & 689.57 & 731.49 & 793.35 & 864.26 & 921.93\end{array}$ $\begin{array}{lllllllll}952.05 & 995.59 & 1013.11 & 1091.13 & 1109.00 & 1129.00 & 1158.81 & 1163.40 & 1335.27\end{array}$ $\begin{array}{lllllllll}1365.25 & 1384.84 & 1420.63 & 1423.74 & 1428.72 & 1454.75 & 1477.72 & 1493.26 & 1503.73\end{array}$ $\begin{array}{lllllllll}1547.75 & 1686.43 & 3057.42 & 3074.56 & 3109.38 & 3121.07 & 3152.62 & 3166.69 & 3174.82\end{array}$ $3216.31 \quad 3514.40 \quad 3751.46$

Rotational constants (B/GHZ): $\quad 2.6720682 \quad 0.9722782 \quad 0.8326120$

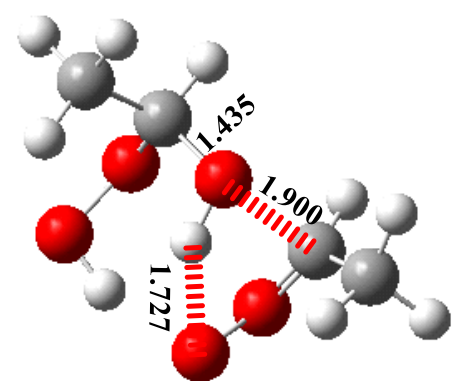

TS5b

$\mathrm{C}$

$\begin{array}{lll}\mathrm{C} & 1 & \mathrm{~B} 1\end{array}$

$\begin{array}{lllll}\mathrm{H} & 2 & \mathrm{~B} 2 & 1 & \mathrm{~A} 1\end{array}$

$\begin{array}{lllll}\mathrm{H} & 2 & \text { B3 } & 1 & \text { A2 }\end{array}$

$\mathrm{H} \quad 2$

O 1

$\mathrm{H} \quad 6$

O 1

C 6

C $\quad 9$

$\mathrm{H} \quad 10$

$\mathrm{H} \quad 10$

$\mathrm{H} \quad 10$

O 9

O 9

$\mathrm{H} \quad 9$

$\mathrm{H} \quad 1$

O 1

$\mathrm{H} \quad 8$

B4 1

A2 $3 \quad$ D1 0

B5 2

A3 4

D2 0

A4 $5 \quad$ D3 0

$\begin{array}{llllll}\text { B6 } & 1 & \text { A5 } & 2 & \text { D4 } & 0\end{array}$

B7 6

A6 2

D5 0

B8 1

A7 2

D6 0

$\begin{array}{llllll}\text { B9 } & 6 & \text { A8 } & 1 & \text { D7 } & 0\end{array}$

Variables:

B1

$\begin{array}{llll}\text { A9 } & 6 & \text { D8 } & 0\end{array}$

$\begin{array}{llllll}\mathrm{B} 11 & 9 & \mathrm{~A} 10 & 6 & \mathrm{D} 9 & 0\end{array}$

$\begin{array}{llllll}\mathrm{B} 12 & 9 & \mathrm{~A} 11 & 6 & \mathrm{D} 10 & 0\end{array}$

$\begin{array}{llllll}\mathrm{B} 13 & 6 & \mathrm{~A} 12 & 1 & \mathrm{D} 11 & 0\end{array}$

$\begin{array}{llllll}\mathrm{B} 14 & 6 & \mathrm{~A} 13 & 1 & \mathrm{D} 12 & 0\end{array}$

$\begin{array}{llllll}\mathrm{B} 15 & 6 & \mathrm{~A} 14 & 1 & \mathrm{D} 13 & 0\end{array}$

$\begin{array}{llllll}\mathrm{B} 16 & 6 & \mathrm{~A} 15 & 9 & \mathrm{D} 14 & 0\end{array}$

$\begin{array}{llllll}\mathrm{B} 17 & 6 & \mathrm{~A} 16 & 9 & \mathrm{D} 15 & 0\end{array}$

$\begin{array}{llllll}\text { B18 } & 1 & \text { A17 } & 18 & \text { D16 } & 0\end{array}$ 


\begin{tabular}{|c|c|}
\hline $\mathrm{B} 2$ & 1.08767122 \\
\hline B3 & 1.08833628 \\
\hline B4 & 1.08686159 \\
\hline B5 & 1.43506519 \\
\hline B6 & 1.00940126 \\
\hline B7 & 2.29073939 \\
\hline B8 & 1.89982191 \\
\hline B9 & 1.48846705 \\
\hline B10 & 1.08583226 \\
\hline B11 & 1.08419963 \\
\hline B12 & 1.09284477 \\
\hline B13 & 2.22018083 \\
\hline B14 & 1.27850834 \\
\hline B15 & 1.08916619 \\
\hline B16 & 1.09082927 \\
\hline B17 & 1.38959239 \\
\hline B18 & 0.98726281 \\
\hline $\mathrm{A} 1$ & 109.60047406 \\
\hline $\mathrm{A} 2$ & 109.04262334 \\
\hline A3 & 110.38705513 \\
\hline A4 & 109.20404196 \\
\hline A5 & 108.91944124 \\
\hline A6 & 96.81216209 \\
\hline A7 & 118.32459590 \\
\hline A 8 & 95.11465779 \\
\hline A9 & 110.34400529 \\
\hline A10 & 111.08844855 \\
\hline A11 & 106.19506527 \\
\hline A 12 & 76.13500039 \\
\hline A13 & 98.44123400 \\
\hline A14 & 103.23745252 \\
\hline A 15 & 106.02667274 \\
\hline A 16 & 111.18135760 \\
\hline A 17 & 98.91528212 \\
\hline D1 & 119.89271416 \\
\hline D2 & 119.39810692 \\
\hline D3 & -59.57754442 \\
\hline D4 & 77.75576884 \\
\hline D5 & -93.38390795 \\
\hline D6 & 171.51722480 \\
\hline D7 & 166.14817545 \\
\hline D8 & -61.52008627 \\
\hline D9 & 62.33010397 \\
\hline D10 & -179.41989651 \\
\hline
\end{tabular}




$\begin{array}{lc}\text { D11 } & -99.65418167 \\ \text { D12 } & -69.38656511 \\ \text { D13 } & 45.57358125 \\ \text { D14 } & -67.73438908 \\ \text { D15 } & 44.21969650 \\ \text { D16 } & 99.60833356\end{array}$

Harmonic Vibrational Frequencies $\left(\mathbf{c m}^{-\mathbf{1}}\right)$ :

$\begin{array}{lccccccccrr}344.99 i & 57.46 & 128.76 & 147.93 & 156.17 & 194.32 & 225.34 & 270.21 & 310.14 & 312.69 \\ 345.68 & 432.33 & 469.21 & 571.75 & 642.86 & 688.91 & 749.96 & 847.11 & 877.92 & 921.99 \\ 937.35 & 979.13 & 995.25 & 1026.34 & 1106.41 & 1128.15 & 1157.78 & 1175.99 & 1193.46 \\ 1342.95 & 1373.28 & 1390.08 & 1401.01 & 1426.99 & 1449.11 & 1465.61 & 1484.77 & 1491.52 \\ 1503.80 & 1565.83 & 1594.73 & 2991.51 & 3059.46 & 3075.60 & 3127.97 & 3148.08 & 3156.99 \\ 3168.61 & 3171.69 & 3197.21 & 3389.68 & & & & & & \\ \text { Rotational constants (B/GHZ): } & 2.5493245 & 1.2339589 & 1.0018028 & \end{array}$

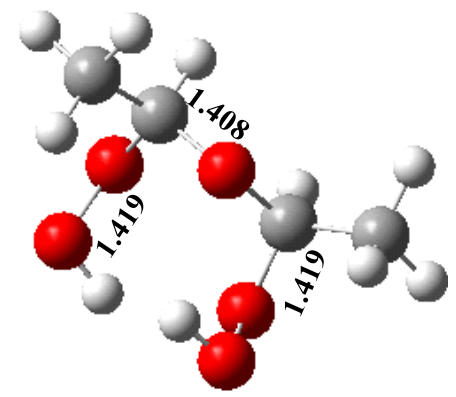

$\mathrm{P} 5 \mathrm{~b}$

$\mathrm{C}$

$\begin{array}{lll}\mathrm{C} & 1 & \mathrm{~B} 1\end{array}$

$\begin{array}{lllll}\mathrm{H} & 2 & \mathrm{~B} 2 & 1 & \mathrm{~A} 1\end{array}$

$\begin{array}{llllllll}\mathrm{H} & 2 & \mathrm{~B} 3 & 1 & \mathrm{~A} 2 & 3 & \mathrm{D} 1 & 0\end{array}$

$\begin{array}{llllllll}\mathrm{H} & 2 & \mathrm{~B} 4 & 1 & \mathrm{~A} 3 & 4 & \mathrm{D} 2 & 0\end{array}$

$\begin{array}{llllllll}\mathrm{O} & 1 & \mathrm{~B} 5 & 2 & \mathrm{~A} 4 & 5 & \mathrm{D} 3 & 0\end{array}$

$\begin{array}{llllllll}\mathrm{H} & 6 & \mathrm{~B} 6 & 1 & \mathrm{~A} 5 & 2 & \mathrm{D} 4 & 0\end{array}$

$\begin{array}{llllllll}\mathrm{O} & 1 & \mathrm{~B} 7 & 6 & \mathrm{~A} 6 & 2 & \mathrm{D} 5 & 0\end{array}$

$\begin{array}{llllllll}\mathrm{C} & 6 & \mathrm{~B} 8 & 1 & \mathrm{~A} 7 & 2 & \text { D6 } & 0\end{array}$

$\begin{array}{llllllll}\text { C } & 9 & \text { B9 } & 6 & \text { A8 } & 1 & \text { D7 } & 0\end{array}$

$\begin{array}{llllllll}\mathrm{H} & 10 & \mathrm{~B} 10 & 9 & \mathrm{~A} 9 & 6 & \mathrm{D} 8 & 0\end{array}$

$\begin{array}{llllllll}\mathrm{H} & 10 & \mathrm{~B} 11 & 9 & \mathrm{~A} 10 & 6 & \mathrm{D} 9 & 0\end{array}$

$\begin{array}{llllllll}\mathrm{H} & 10 & \mathrm{~B} 12 & 9 & \mathrm{~A} 11 & 6 & \mathrm{D} 10 & 0\end{array}$

$\begin{array}{llllllll}\mathrm{O} & 9 & \mathrm{~B} 13 & 6 & \mathrm{~A} 12 & 1 & \mathrm{D} 11 & 0\end{array}$

$\begin{array}{llllllll}\text { O } & 14 & \text { B14 } & 9 & \text { A13 } & 6 & \text { D12 } & 0\end{array}$

$\begin{array}{llllllll}\mathrm{H} & 9 & \mathrm{~B} 15 & 6 & \mathrm{~A} 14 & 1 & \mathrm{D} 13 & 0\end{array}$

$\begin{array}{llllllll}\mathrm{H} & 1 & \mathrm{~B} 16 & 6 & \mathrm{~A} 15 & 9 & \mathrm{D} 14 & 0\end{array}$

$\begin{array}{llllllll}\mathrm{O} & 1 & \mathrm{~B} 17 & 6 & \mathrm{~A} 16 & 9 & \mathrm{D} 15 & 0\end{array}$

$\begin{array}{llllllll}\mathrm{H} & 8 & \mathrm{~B} 18 & 1 & \mathrm{~A} 17 & 18 & \mathrm{D} 16 & 0\end{array}$

Variables:

B1 


\begin{tabular}{|c|c|}
\hline B2 & 1.08778753 \\
\hline B3 & 1.08768481 \\
\hline B4 & 1.08728320 \\
\hline B5 & 1.40840310 \\
\hline B6 & 2.28285434 \\
\hline B7 & 2.30285802 \\
\hline B8 & 1.40006779 \\
\hline B9 & 1.50814338 \\
\hline B10 & 1.08797238 \\
\hline B11 & 1.08677070 \\
\hline B12 & 1.08782637 \\
\hline B13 & 2.30306255 \\
\hline B14 & 1.41735620 \\
\hline B15 & 1.09389402 \\
\hline B16 & 1.09600419 \\
\hline B17 & 1.40394292 \\
\hline B18 & 0.96874445 \\
\hline $\mathrm{A} 1$ & 109.74162108 \\
\hline $\mathrm{A} 2$ & 109.50127609 \\
\hline A3 & 109.64182621 \\
\hline A4 & 107.66570361 \\
\hline A5 & 115.32726405 \\
\hline A6 & 94.01833524 \\
\hline A7 & 116.36640796 \\
\hline A 8 & 108.93409449 \\
\hline A9 & 109.39934458 \\
\hline A10 & 110.04791650 \\
\hline A11 & 109.68170449 \\
\hline A12 & 89.99555915 \\
\hline A13 & 35.72316976 \\
\hline A14 & 111.14750837 \\
\hline A15 & 109.77506437 \\
\hline A 16 & 112.48274600 \\
\hline A 17 & 107.86638874 \\
\hline D1 & 120.26175310 \\
\hline D2 & 119.80250284 \\
\hline D3 & -60.90823506 \\
\hline D4 & 82.51611853 \\
\hline D5 & -94.53347428 \\
\hline D6 & 172.30313558 \\
\hline D7 & 150.81748658 \\
\hline D8 & -64.86327632 \\
\hline D9 & 54.93527223 \\
\hline D10 & 175.08083908 \\
\hline
\end{tabular}




$\begin{array}{lr}\text { D11 } & -116.22201020 \\ \text { D12 } & 124.83687292 \\ \text { D13 } & 26.39322598 \\ \text { D14 } & -65.50294955 \\ \text { D15 } & 46.39340968 \\ \text { D16 } & 81.68075480\end{array}$

Harmonic Vibrational Frequencies $\left(\mathbf{c m}^{\mathbf{- 1}}\right)$ :

$\begin{array}{lccccccccrr}76.27 & 105.35 & 167.37 & 189.29 & 202.66 & 236.72 & 242.56 & 286.41 & 311.10 & 342.85 \\ 374.48 & 439.28 & 466.70 & 586.26 & 600.57 & 692.32 & 726.26 & 848.16 & 878.43 & 935.29 \\ 983.07 & 1006.20 & 1028.22 & 1124.74 & 1136.30 & 1154.32 & 1160.91 & 1178.66 & 1224.77 \\ 1351.76 & 1370.72 & 1387.26 & 1410.41 & 1423.93 & 1434.84 & 1454.29 & 1461.83 & 1489.50 \\ 1490.63 & 1498.00 & 1504.02 & 3071.34 & 3077.39 & 3080.58 & 3096.04 & 3157.73 & 3161.97 \\ 3169.67 & 3171.59 & 3727.75 & 3756.69 & & & & & \\ \text { Rotational constants (B/GHZ): } & 2.4323291 & 1.2895610 & 1.0352789 & \end{array}$

Rotational constants (B/GHZ): $\quad 2.4323291 \quad 1.2895610 \quad 1.0352789$

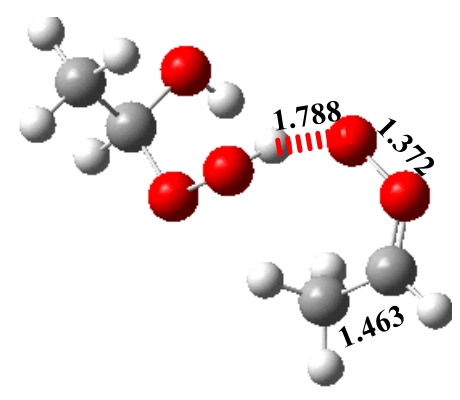

IM5c

$\mathrm{C}$

$\begin{array}{lll}\mathrm{C} & 1 & \mathrm{~B} 1\end{array}$

$\mathrm{H} \quad \mathrm{B} 2$

O 2

$\mathrm{O} \quad 2$

B3 1

A1

A2 3

D1 0

B4 1

A3 4

D2 0

$\mathrm{H} \quad 4$

B5 2

A4 1

D3 0

C 4

B6 2

A5 1

D4 0

$\begin{array}{ll}\text { C } & 7\end{array}$

B7 4

A6 2

D5 0

$\mathrm{H} \quad 8$

B8 7

A7 4

D6 0

$\mathrm{H} \quad 8$

B9 7

A8 4

D7 0

$\mathrm{H} \quad 8$

B10 7

A9 4

D8 0

$\begin{array}{ll}\text { O } & 7\end{array}$

O 7

B11 4

A10 2

D9 0

B12 4

A11 2

D10 0

$\mathrm{H} \quad 7$

B13 4

A12 2

D11 0

B14 2

A13 5

D12 0

$\mathrm{H} \quad 1$

B15 2

A14 5

D13 0

$\mathrm{H} \quad 1$

O 2

B16 2

A15 5

D14 0

$\mathrm{H} \quad 18$

B17 1

A16 5

D15 0

Variables:

B1 


\begin{tabular}{|c|c|}
\hline $\mathrm{B} 2$ & 1.09639410 \\
\hline B3 & 2.31687959 \\
\hline B4 & 1.41600147 \\
\hline B5 & 0.98384803 \\
\hline B6 & 3.39808882 \\
\hline B7 & 1.46273193 \\
\hline B8 & 1.09610288 \\
\hline B9 & 1.09331774 \\
\hline B 10 & 1.08437269 \\
\hline B11 & 2.23400100 \\
\hline B 12 & 1.24437625 \\
\hline B13 & 1.08567670 \\
\hline B14 & 1.08816799 \\
\hline B 15 & 1.08789404 \\
\hline B16 & 1.08693034 \\
\hline B17 & 1.39304527 \\
\hline B 18 & 0.96381004 \\
\hline $\mathrm{A} 1$ & 111.43595735 \\
\hline $\mathrm{A} 2$ & 93.41766811 \\
\hline A3 & 113.11803014 \\
\hline A4 & 90.98570155 \\
\hline A5 & 113.71132546 \\
\hline A6 & 79.08376626 \\
\hline A7 & 108.75057218 \\
\hline A 8 & 108.13753614 \\
\hline A9 & 111.20204608 \\
\hline A10 & 53.02709793 \\
\hline A11 & 67.05417409 \\
\hline A12 & 126.95353408 \\
\hline A13 & 109.38189395 \\
\hline A14 & 109.85834780 \\
\hline A15 & 109.51771127 \\
\hline A16 & 108.30445783 \\
\hline A17 & 109.39959281 \\
\hline D1 & -143.23462844 \\
\hline D2 & 30.13681950 \\
\hline D3 & -124.88766027 \\
\hline D4 & -174.74808810 \\
\hline D5 & -37.22681026 \\
\hline D6 & -8.03032887 \\
\hline D7 & 106.10027942 \\
\hline D8 & -131.02032659 \\
\hline D9 & 60.98354346 \\
\hline D10 & 95.89927513 \\
\hline
\end{tabular}




$\begin{array}{lr}\text { D11 } & -162.37209854 \\ \text { D12 } & -175.98880702 \\ \text { D13 } & -55.84624496 \\ \text { D14 } & 64.48174861 \\ \text { D15 } & -123.70231193 \\ \text { D16 } & 168.11933669\end{array}$

Harmonic Vibrational Frequencies $\left(\mathbf{c m}^{-\mathbf{1}}\right)$ :

$\begin{array}{lllllllllll}50.83 & 63.46 & 77.15 & 103.15 & 129.05 & 158.98 & 214.94 & 231.89 & 263.51 & 315.28 & 329.38\end{array}$ $\begin{array}{llllllllll}426.64 & 437.65 & 471.01 & 501.82 & 678.07 & 696.94 & 782.06 & 800.17 & 864.67 & 927.42\end{array}$ $\begin{array}{lllllllll}963.82 & 1004.18 & 1011.63 & 1097.10 & 1099.24 & 1115.85 & 1149.87 & 1186.30 & 1290.66\end{array}$ $\begin{array}{llllllllll}1372.08 & 1381.25 & 1398.57 & 1421.62 & 1447.41 & 1459.88 & 1475.23 & 1491.99 & 1502.74\end{array}$ $\begin{array}{llllllllll}1561.89 & 1667.47 & 3035.97 & 3059.68 & 3084.79 & 3092.94 & 3163.67 & 3174.73 & 3179.13\end{array}$ $3215.89 \quad 3426.07 \quad 3845.87$

Rotational constants (B/GHZ): $\quad 3.0605179 \quad 0.8101218 \quad 0.7696201$

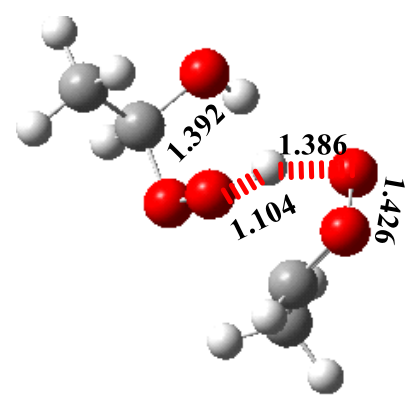

TS5c

$\mathrm{C}$

$\begin{array}{lll}\mathrm{C} & 1 & \mathrm{~B} 1\end{array}$

$\begin{array}{lllll}\mathrm{H} & 2 & \mathrm{~B} 2 & 1 & \mathrm{~A} 1\end{array}$

$\mathrm{O} 2$

O 4

B3 1

A2

B4 2

$\begin{array}{lll}3 & \text { D1 }\end{array}$

$\mathrm{H} \quad 4$

B5 2

A3 $1 \quad$ D2 0

$\begin{array}{ll}\text { C } & 4\end{array}$

B6 2

A4 1

D3 0

$\begin{array}{ll}\text { C } & 7\end{array}$

A5 1 D4 0

$\mathrm{H} \quad 8$

B7 4

A6 2 D5 0

B8 7

A7 4

D6 0

$\begin{array}{ll}\mathrm{H} & 8\end{array}$

B9 7

A8 4

D7 0

$\mathrm{H} \quad 8$

B10 7

A9 4

D8 0

$\begin{array}{ll}\mathrm{O} & 7\end{array}$

$\begin{array}{ll}\text { O } & 7\end{array}$

B11 4

A10 2

D9 0

B12 4

A11 2

D10 0

$\begin{array}{ll}\mathrm{H} & 7\end{array}$

B13 4

A12 2

D11 0

$\mathrm{H} \quad 1$

$\mathrm{H} \quad 1$

B14 2

A13 5

D12 0

B15 2

A14 5

D13 0

$\mathrm{H} \quad 1$

B16 2

A15 5

D14 0

O 2

B17 1

A16 5

D15 0

H 18

B18 2

A17 1

D16 0

Variables:

B1 


\begin{tabular}{|c|c|}
\hline $\mathrm{B} 2$ & 1.09536218 \\
\hline B3 & 2.29975060 \\
\hline B4 & 1.41573133 \\
\hline B5 & 1.10383816 \\
\hline B6 & 2.04182005 \\
\hline B7 & 1.47869391 \\
\hline B8 & 1.08659193 \\
\hline B9 & 1.08231173 \\
\hline $\mathrm{B} 10$ & 1.09463960 \\
\hline B11 & 2.20392514 \\
\hline B12 & 1.27159971 \\
\hline B13 & 1.08812674 \\
\hline B14 & 1.08786440 \\
\hline B15 & 1.08778069 \\
\hline B16 & 1.08691236 \\
\hline B17 & 1.39241511 \\
\hline B18 & 0.96150557 \\
\hline A1 & 111.64134829 \\
\hline $\mathrm{A} 2$ & 92.40717945 \\
\hline A3 & 35.79175608 \\
\hline A4 & 92.85918452 \\
\hline A5 & 143.51127825 \\
\hline A6 & 97.22704675 \\
\hline A7 & 110.17919426 \\
\hline $\mathrm{A} 8$ & 111.58246078 \\
\hline A9 & 105.51840637 \\
\hline A10 & 68.80275494 \\
\hline A11 & 89.29190574 \\
\hline A12 & 104.31135699 \\
\hline A13 & 109.35471748 \\
\hline A14 & 109.85566715 \\
\hline A15 & 109.65486966 \\
\hline A16 & 108.16267677 \\
\hline A17 & 109.63174485 \\
\hline D1 & -143.23270839 \\
\hline D2 & 127.94211881 \\
\hline D3 & -123.05140418 \\
\hline D4 & 167.30574227 \\
\hline D5 & -15.62689755 \\
\hline D6 & -58.34857302 \\
\hline D7 & 65.26106208 \\
\hline D8 & -175.16470345 \\
\hline D9 & 77.46807520 \\
\hline D10 & 109.80801442 \\
\hline
\end{tabular}




$\begin{array}{lr}\text { D11 } & -137.58274081 \\ \text { D12 } & -174.00443044 \\ \text { D13 } & -53.86315879 \\ \text { D14 } & 66.49216464 \\ \text { D15 } & -123.33252797 \\ \text { D16 } & -178.87927080\end{array}$

Harmonic Vibrational Frequencies $\left(\mathbf{c m}^{-\mathbf{1}}\right)$ :

$\begin{array}{lllllllllll}534.18 i & 47.15 & 73.54 & 125.83 & 178.38 & 217.67 & 225.67 & 255.62 & 308.96 & 376.37 & 397.81\end{array}$ $\begin{array}{llllllllll}439.84 & 444.71 & 468.72 & 501.94 & 633.41 & 712.02 & 870.34 & 874.37 & 914.79 & 951.79\end{array}$ $\begin{array}{lllllllll}974.33 & 1000.50 & 1082.00 & 1100.75 & 1136.32 & 1171.52 & 1179.99 & 1212.08 & 1287.92\end{array}$ $\begin{array}{lllllllll}1369.04 & 1378.56 & 1397.39 & 1402.75 & 1447.42 & 1458.63 & 1482.15 & 1489.69 & 1498.27\end{array}$ $\begin{array}{lllllllll}1518.69 & 1645.65 & 1970.29 & 3059.02 & 3073.89 & 3076.61 & 3153.41 & 3157.31 & 3167.05\end{array}$ $3191.87 \quad 3224.45 \quad 3886.57$

Rotational constants (B/GHZ):

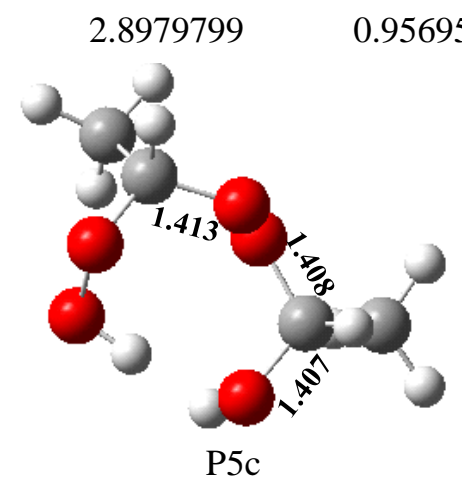

$\begin{array}{rr}\mathrm{C} & \\ \mathrm{C} & 1 \\ \mathrm{H} & 2 \\ \mathrm{O} & 2 \\ \mathrm{O} & 2 \\ \mathrm{H} & 4 \\ \mathrm{C} & 4 \\ \mathrm{C} & 7 \\ \mathrm{H} & 8 \\ \mathrm{H} & 8 \\ \mathrm{H} & 8 \\ \mathrm{O} & 7 \\ \mathrm{O} & 7 \\ \mathrm{H} & 7 \\ \mathrm{H} & 1 \\ \mathrm{H} & 1 \\ \mathrm{H} & 1 \\ \mathrm{O} & 2 \\ \mathrm{H} & 18\end{array}$

Variables:

$\begin{array}{ll}\text { B1 } & 1.50857757 \\ \text { B2 } & 1.09368472\end{array}$




\begin{tabular}{|c|c|}
\hline B3 & 2.30866532 \\
\hline B4 & 1.40778208 \\
\hline B5 & 2.68003297 \\
\hline B6 & 1.41306797 \\
\hline B7 & 1.51039244 \\
\hline B8 & 1.08794823 \\
\hline B9 & 1.08422436 \\
\hline B10 & 1.08770189 \\
\hline B11 & 2.30470002 \\
\hline B12 & 1.40121521 \\
\hline B13 & 1.09243481 \\
\hline B14 & 1.08746877 \\
\hline B15 & 1.08759745 \\
\hline B16 & 1.08660698 \\
\hline B17 & 1.40727618 \\
\hline B18 & 0.96166762 \\
\hline A1 & 111.36324113 \\
\hline A2 & 86.58204024 \\
\hline A3 & 114.01712663 \\
\hline A4 & 68.45133137 \\
\hline A5 & 131.49451198 \\
\hline A6 & 114.17920431 \\
\hline A7 & 109.71354245 \\
\hline A8 & 110.85679343 \\
\hline A9 & 108.31613779 \\
\hline A10 & 98.92869240 \\
\hline A11 & 111.56764351 \\
\hline A12 & 102.55815821 \\
\hline A13 & 109.02775270 \\
\hline A14 & 109.89514691 \\
\hline A15 & 110.01240315 \\
\hline A16 & 108.17484075 \\
\hline A17 & 108.59483488 \\
\hline D1 & -137.22802024 \\
\hline D2 & 23.52891190 \\
\hline D3 & -117.19072410 \\
\hline D4 & -152.96933011 \\
\hline D5 & -66.75103027 \\
\hline D6 & -50.71779327 \\
\hline D7 & 70.01042095 \\
\hline D8 & -170.26000691 \\
\hline D9 & 29.85999435 \\
\hline D10 & 64.17084107 \\
\hline D11 & 172.44874061 \\
\hline
\end{tabular}




$\begin{array}{lr}\text { D12 } & -167.69140661 \\ \text { D13 } & -47.54578710 \\ \text { D14 } & 72.70987565 \\ \text { D15 } & -125.28502675 \\ \text { D16 } & -162.93415657\end{array}$

Harmonic Vibrational Frequencies $\left(\mathbf{c m}^{-1}\right)$ :

$\begin{array}{lcccccccccc}54.01 & 74.71 & 133.53 & 176.48 & 227.06 & 233.18 & 263.08 & 281.60 & 314.58 & 365.98 & 427.85 \\ 465.48 & 499.98 & 510.44 & 595.70 & 677.73 & 736.31 & 853.38 & 875.86 & 933.30 & 953.69 \\ 991.43 & 1015.43 & 1107.55 & 1131.98 & 1145.56 & 1152.98 & 1172.61 & 1191.42 & 1293.40 \\ 1355.93 & 1381.92 & 1400.42 & 1416.56 & 1423.72 & 1454.87 & 1486.25 & 1488.11 & 1496.18 \\ 1503.63 & 1514.04 & 3077.08 & 3082.06 & 3084.78 & 3116.25 & 3158.55 & 3160.74 & 3167.53 \\ 3189.19 & 3734.36 & 3883.31 & & & & & & \\ \text { Rotational constants (B/GHZ): } & 2.5684054 & 1.1939108 & 1.0223523 & \end{array}$

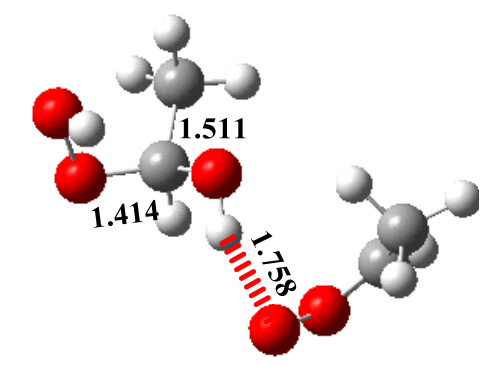

IM5d

$\mathrm{C}$

$\begin{array}{lll}\mathrm{C} & 1 & \mathrm{~B} 1\end{array}$

$\begin{array}{lllll}\mathrm{H} & 2 & \mathrm{~B} 2 & 1 & \mathrm{~A} 1\end{array}$

$\begin{array}{llllllll}\mathrm{H} & 2 & \mathrm{~B} 3 & 1 & \mathrm{~A} 2 & 3 & \mathrm{D} 1 & 0\end{array}$

$\begin{array}{lllllllll}\mathrm{H} & 2 & \mathrm{~B} 4 & 1 & \mathrm{~A} 3 & 3 & \mathrm{D} 2 & 0\end{array}$

$\begin{array}{llllllll}\mathrm{O} & 1 & \mathrm{~B} 5 & 2 & \mathrm{~A} 4 & 5 & \mathrm{D} 3 & 0\end{array}$

$\begin{array}{llllllll}\mathrm{H} & 6 & \mathrm{~B} 6 & 1 & \mathrm{~A} 5 & 2 & \mathrm{D} 4 & 0\end{array}$

$\begin{array}{llllllll}\mathrm{O} & 1 & \mathrm{~B} 7 & 6 & \mathrm{~A} 6 & 2 & \mathrm{D} 5 & 0\end{array}$

$\begin{array}{llllllll}\mathrm{C} & 6 & \mathrm{~B} 8 & 1 & \mathrm{~A} 7 & 2 & \mathrm{D} 6 & 0\end{array}$

$\begin{array}{llllllll}\text { C } & 9 & \text { B9 } & 6 & \text { A8 } & 1 & \text { D7 } & 0\end{array}$

$\begin{array}{llllllll}\mathrm{H} & 10 & \mathrm{~B} 10 & 9 & \mathrm{~A} 9 & 6 & \mathrm{D} 8 & 0\end{array}$

$\begin{array}{llllllll}\mathrm{H} & 10 & \mathrm{~B} 11 & 9 & \mathrm{~A} 10 & 6 & \mathrm{D} 9 & 0\end{array}$

$\begin{array}{llllllll}\mathrm{H} & 10 & \mathrm{~B} 12 & 9 & \mathrm{~A} 11 & 6 & \mathrm{D} 10 & 0\end{array}$

$\begin{array}{llllllll}\mathrm{O} & 9 & \mathrm{~B} 13 & 6 & \mathrm{~A} 12 & 1 & \mathrm{D} 11 & 0\end{array}$

$\begin{array}{llllllll}\mathrm{O} & 9 & \mathrm{~B} 14 & 6 & \mathrm{~A} 13 & 1 & \mathrm{D} 12 & 0\end{array}$

$\begin{array}{llllllll}\mathrm{H} & 9 & \mathrm{~B} 15 & 6 & \mathrm{~A} 14 & 1 & \mathrm{D} 13 & 0\end{array}$

$\begin{array}{llllllll}\mathrm{H} & 1 & \mathrm{~B} 16 & 6 & \mathrm{~A} 15 & 2 & \mathrm{D} 14 & 0\end{array}$

$\begin{array}{llllllll}\mathrm{O} & 1 & \mathrm{~B} 17 & 6 & \mathrm{~A} 16 & 2 & \mathrm{D} 15 & 0\end{array}$

$\begin{array}{llllllll}\text { H } & 8 & \text { B18 } & 1 & \text { A17 } & 6 & \text { D16 } & 0\end{array}$

Variables:

$\begin{array}{ll}\text { B1 } & 1.51110581 \\ \text { B2 } & 1.08880466 \\ \text { B3 } & 1.08792822\end{array}$




\begin{tabular}{|c|c|}
\hline B4 & 1.08716364 \\
\hline B5 & 1.39419412 \\
\hline B6 & 0.98099156 \\
\hline B7 & 2.29771734 \\
\hline B8 & 3.13142443 \\
\hline B9 & 1.46525001 \\
\hline B10 & 1.09519879 \\
\hline B11 & 1.09136916 \\
\hline B12 & 1.08478390 \\
\hline B13 & 2.23310313 \\
\hline B14 & 1.24465627 \\
\hline B15 & 1.08574602 \\
\hline B16 & 1.09559886 \\
\hline B17 & 1.41391391 \\
\hline B18 & 0.96635319 \\
\hline $\mathrm{A} 1$ & 109.62425809 \\
\hline A2 & 110.00252393 \\
\hline A3 & 109.83290599 \\
\hline A4 & 109.23947589 \\
\hline A5 & 108.64080415 \\
\hline A6 & 92.63393522 \\
\hline A7 & 112.12689132 \\
\hline A8 & 78.68153770 \\
\hline A9 & 107.85474841 \\
\hline A 10 & 108.65100472 \\
\hline A11 & 110.96314114 \\
\hline A 12 & 57.05804571 \\
\hline $\mathrm{A} 13$ & 70.17177383 \\
\hline A14 & 123.91662718 \\
\hline A 15 & 110.92207741 \\
\hline A16 & 110.67633843 \\
\hline A17 & 90.08238712 \\
\hline D1 & 120.18396948 \\
\hline D2 & -119.70672523 \\
\hline D3 & -54.87801516 \\
\hline D4 & -157.04056621 \\
\hline D5 & -92.96838461 \\
\hline D6 & -92.80562386 \\
\hline D7 & 167.41527413 \\
\hline D8 & -12.64967925 \\
\hline D9 & 102.35463689 \\
\hline D10 & -133.70230146 \\
\hline D11 & -95.21229285 \\
\hline D12 & -61.02740455 \\
\hline
\end{tabular}




$\begin{array}{rr}\text { D13 } & 43.59511600 \\ \text { D14 } & 123.64523014 \\ \text { D15 } & -124.64069935 \\ \text { D16 } & -12.20718289\end{array}$

Harmonic Vibrational Frequencies $\left(\mathbf{c m}^{-1}\right)$ :

$\begin{array}{lccccccccccc}35.47 & 47.31 & 65.52 & 90.22 & 110.19 & 151.22 & 197.82 & 213.74 & 253.26 & 297.80 & 334.25 \\ 359.84 & 447.27 & 467.37 & 516.77 & 673.20 & 700.56 & 792.14 & 848.65 & 871.53 & 925.93 \\ 963.85 & 995.59 & 1019.21 & 1093.47 & 1119.13 & 1133.63 & 1139.68 & 1174.68 & 1338.96 \\ 1363.83 & 1390.86 & 1407.77 & 1417.28 & 1438.51 & 1448.41 & 1470.64 & 1488.20 & 1492.51 \\ 1498.57 & 1665.13 & 3040.24 & 3072.90 & 3075.16 & 3100.95 & 3148.90 & 3168.72 & 3174.06 \\ 3208.16 & 3431.70 & 3817.63 & & & & & & & \\ \text { Rotational constants (B/GHZ): } & 2.8460478 & 0.7209651 & 0.6841335 & \end{array}$

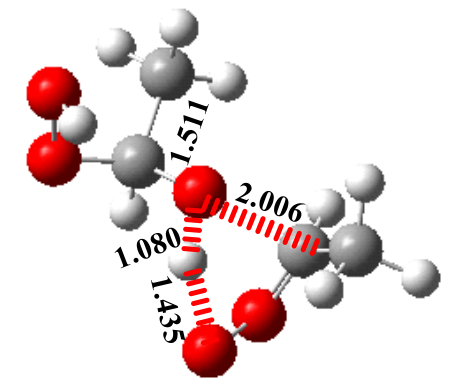

TS5d

$\mathrm{C}$

$\begin{array}{lll}\text { C } & 1 & \text { B1 }\end{array}$

$\begin{array}{lllll}\mathrm{H} & 2 & \mathrm{~B} 2 & 1 & \mathrm{~A} 1\end{array}$

$\begin{array}{lllll}\mathrm{H} & 2 & \mathrm{~B} 3 & 1 & \mathrm{~A} 2\end{array}$

$\begin{array}{lllllllll}\mathrm{H} & 2 & \mathrm{~B} 4 & 1 & \mathrm{~A} 3 & 4 & \mathrm{D} 2 & 0\end{array}$

$\begin{array}{llllllll}\mathrm{O} & 1 & \mathrm{~B} 5 & 2 & \mathrm{~A} 4 & 5 & \mathrm{D} 3 & 0\end{array}$

$\begin{array}{llllllll}\mathrm{H} & 6 & \mathrm{~B} 6 & 1 & \mathrm{~A} 5 & 2 & \mathrm{D} 4 & 0\end{array}$

$\begin{array}{llllllll}\mathrm{O} & 1 & \mathrm{~B} 7 & 6 & \mathrm{~A} 6 & 2 & \mathrm{D} 5 & 0\end{array}$

$\begin{array}{llllllll}\mathrm{C} & 6 & \mathrm{~B} 8 & 1 & \mathrm{~A} 7 & 2 & \mathrm{D} 6 & 0\end{array}$

$\begin{array}{lllllllll}\text { C } & 9 & \text { B9 } & 6 & \text { A8 } & 1 & \text { D7 } & 0\end{array}$

$\begin{array}{llllllll}\mathrm{H} & 10 & \mathrm{~B} 10 & 9 & \mathrm{~A} 9 & 6 & \mathrm{D} 8 & 0\end{array}$

$\begin{array}{llllllll}\mathrm{H} & 10 & \mathrm{~B} 11 & 9 & \mathrm{~A} 10 & 6 & \mathrm{D} 9 & 0\end{array}$

$\begin{array}{llllllll}\mathrm{H} & 10 & \mathrm{~B} 12 & 9 & \mathrm{~A} 11 & 6 & \mathrm{D} 10 & 0\end{array}$

$\begin{array}{llllllll}\mathrm{O} & 9 & \mathrm{~B} 13 & 6 & \mathrm{~A} 12 & 1 & \mathrm{D} 11 & 0\end{array}$

$\begin{array}{llllllll}\mathrm{O} & 9 & \mathrm{~B} 14 & 6 & \mathrm{~A} 13 & 1 & \mathrm{D} 12 & 0\end{array}$

$\begin{array}{llllllll}\mathrm{H} & 9 & \mathrm{~B} 15 & 6 & \mathrm{~A} 14 & 1 & \mathrm{D} 13 & 0\end{array}$

$\begin{array}{llllllll}\mathrm{H} & 1 & \mathrm{~B} 16 & 6 & \mathrm{~A} 15 & 9 & \mathrm{D} 14 & 0\end{array}$

$\begin{array}{llllllll}\mathrm{O} & 1 & \mathrm{~B} 17 & 6 & \mathrm{~A} 16 & 9 & \mathrm{D} 15 & 0\end{array}$

$\begin{array}{llllllll}\text { H } & 8 & \text { B18 } & 1 & \text { A17 } & 18 & \text { D16 } & 0\end{array}$

Variables:

B1 1.51062829

B2 1.08927136

B3 1.08792476 


\begin{tabular}{|c|c|}
\hline B4 & 1.08668999 \\
\hline B5 & 1.40663183 \\
\hline B6 & 1.08041040 \\
\hline B7 & 2.29555555 \\
\hline B8 & 2.00558029 \\
\hline B9 & 1.48340533 \\
\hline B10 & 1.08609956 \\
\hline B11 & 1.08304806 \\
\hline B12 & 1.09392478 \\
\hline B13 & 2.19914624 \\
\hline B14 & 1.27334179 \\
\hline B15 & 1.08929166 \\
\hline B16 & 1.09566361 \\
\hline B17 & 1.40533358 \\
\hline B18 & 0.96521403 \\
\hline $\mathrm{A} 1$ & 110.09934594 \\
\hline $\mathrm{A} 2$ & 109.65725917 \\
\hline A3 & 110.09116743 \\
\hline A4 & 110.02561777 \\
\hline A5 & 107.43747658 \\
\hline A6 & 93.18420134 \\
\hline A7 & 113.31541138 \\
\hline A8 & 93.33469697 \\
\hline A9 & 109.75937091 \\
\hline A 10 & 111.42310478 \\
\hline A11 & 106.17263170 \\
\hline A 12 & 70.22637961 \\
\hline A13 & 92.95364237 \\
\hline A14 & 106.47818263 \\
\hline A 15 & 109.93774865 \\
\hline A16 & 109.37874886 \\
\hline A 17 & 96.59531087 \\
\hline D1 & 119.45139911 \\
\hline D2 & 120.13952923 \\
\hline D3 & -54.34025832 \\
\hline D4 & -163.38270050 \\
\hline D5 & -92.37278002 \\
\hline D6 & -81.45845790 \\
\hline D7 & 167.18365888 \\
\hline D8 & -60.34789337 \\
\hline D9 & 63.21876756 \\
\hline D10 & -177.49954936 \\
\hline D11 & -98.39965615 \\
\hline D12 & -67.68036866 \\
\hline
\end{tabular}




$\begin{array}{lr}\text { D13 } & 46.58430182 \\ \text { D14 } & 42.48242440 \\ \text { D15 } & 153.56172692 \\ \text { D16 } & 101.57303697\end{array}$

Harmonic Vibrational Frequencies $\left(\mathbf{c m}^{-1}\right)$ :

$\begin{array}{lccccccccccr}488.52 i & 43.59 & 96.17 & 100.61 & 138.43 & 155.40 & 211.22 & 248.63 & 284.64 & 295.72 & 339.47 \\ 378.91 & 415.29 & 519.61 & 540.79 & 637.73 & 713.56 & 859.22 & 878.58 & 918.11 & 954.36 \\ 972.51 & 1009.51 & 1095.22 & 1144.96 & 1147.52 & 1155.00 & 1183.60 & 1304.44 & 1363.32 \\ 1377.04 & 1399.35 & 1408.42 & 1425.68 & 1431.37 & 1450.65 & 1480.85 & 1486.90 & 1499.40 \\ 1545.86 & 1608.30 & 2045.48 & 3053.53 & 3070.06 & 3088.81 & 3142.64 & 3144.09 & 3168.99 \\ 3169.92 & 3198.77 & 3823.56 & & & & & & \\ \text { Rotational constants (B/GHZ): } & 2.7039816 & 0.9326103 & 0.8737853 & & \end{array}$

Rotational constants (B/GHZ): $2.7039816 \quad 0.9326103 \quad 0.8737853$

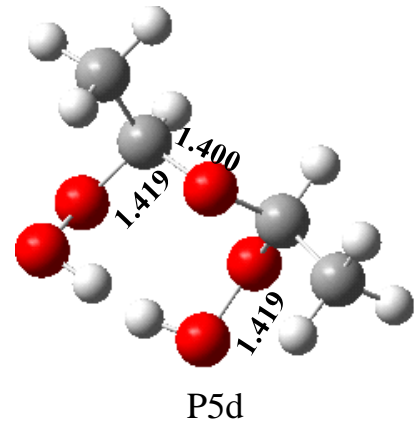

$\mathrm{C}$

C $\quad 1 \quad$ B1

$\begin{array}{lllll}\mathrm{H} & 2 & \mathrm{~B} 2 & 1 & \mathrm{~A} 1\end{array}$

$\mathrm{H} \quad 2$

$\mathrm{H} \quad 2$

B3 1

A2 3

D1 0

B4 1

A3 3

D2 0

O 1

B5 2

A4 5

D3 0

B6 1

A5 2

D4 0

O 1

B7

A6 2

D5 0

C 6

B8 1

D6 0

C 9

$\mathrm{H} \quad 10$

B9 6

A7 2

D7 0

$\mathrm{H} \quad 10$

B10 9

A8 1

D8 0

B11 9

A9 6

D9 0

$\mathrm{H} \quad 10$

B12 9

A10 6

D10 0

O 9

B13 6

A11 6

D11 0

B14 6

A12 1

D12 0

O 9

H $\quad 9$

B15 6

A13 1

D13 0

$\mathrm{H} \quad 1$

B16 6

A14 1

D14 0

B17 1

A15 9

D15 0

H $\quad 8$

B18 1

A16 6

D16 0

Variables:

$\begin{array}{ll}\text { B1 } & 1.50818835 \\ \text { B2 } & 1.08795330 \\ \text { B3 } & 1.08783270 \\ \text { B4 } & 1.08677247\end{array}$




\begin{tabular}{|c|c|}
\hline B5 & 1.40008457 \\
\hline B6 & 2.86300873 \\
\hline B7 & 2.30289480 \\
\hline B8 & 1.40821269 \\
\hline B9 & 1.50739267 \\
\hline B10 & 1.08780228 \\
\hline B11 & 1.08728782 \\
\hline B12 & 1.08766856 \\
\hline B13 & 2.30290612 \\
\hline B14 & 1.40402939 \\
\hline B15 & 1.09604673 \\
\hline B16 & 1.09391248 \\
\hline B17 & 1.41735732 \\
\hline B18 & 0.97034732 \\
\hline A1 & 109.39649757 \\
\hline A 2 & 109.68270261 \\
\hline A3 & 110.04649901 \\
\hline A4 & 108.92328411 \\
\hline A5 & 77.04745371 \\
\hline A6 & 90.02642843 \\
\hline A7 & 116.37608360 \\
\hline A8 & 107.67992309 \\
\hline A9 & 109.74649478 \\
\hline A10 & 109.63823721 \\
\hline A11 & 109.50157912 \\
\hline A12 & 94.02158832 \\
\hline A13 & 112.48916455 \\
\hline A14 & 109.78196710 \\
\hline A15 & 111.14801185 \\
\hline A16 & 35.71978673 \\
\hline A17 & 86.09984124 \\
\hline D1 & 120.06263353 \\
\hline D2 & -119.79683665 \\
\hline D3 & -54.80417520 \\
\hline D4 & 147.77300240 \\
\hline D5 & -92.95118463 \\
\hline D6 & -150.84400215 \\
\hline D7 & -172.28723493 \\
\hline D8 & -59.04007568 \\
\hline D9 & 60.88747044 \\
\hline D10 & -179.30524038 \\
\hline D11 & -77.75466014 \\
\hline D12 & -46.37408592 \\
\hline D13 & 65.51803562 \\
\hline
\end{tabular}




$\begin{array}{lr}\text { D14 } & -26.43530865 \\ \text { D15 } & -124.80010293 \\ \text { D16 } & -7.74887022\end{array}$

Harmonic Vibrational Frequencies $\left(\mathbf{c m}^{-\mathbf{1}}\right)$ :

$\begin{array}{lcccccccccr}76.24 & 105.26 & 166.73 & 189.33 & 202.74 & 236.79 & 242.68 & 286.36 & 311.05 & 342.93 \\ 374.60 & 439.26 & 466.79 & 586.33 & 600.22 & 692.36 & 726.28 & 848.21 & 878.52 & 935.36 \\ 983.00 & 1006.16 & 1028.20 & 1124.95 & 1136.21 & 1154.42 & 1160.83 & 1178.80 & 1224.78 \\ 1351.84 & 1370.90 & 1387.27 & 1410.48 & 1423.88 & 1434.83 & 1454.11 & 1461.76 & 1489.36 \\ 1490.66 & 1498.00 & 1504.03 & 3070.98 & 3077.10 & 3080.67 & 3095.91 & 3157.49 & 3162.09 \\ 3169.41 & 3171.57 & 3728.06 & 3756.89 & & & & & \\ \text { Rotational constants (B/GHZ): } & 2.4325109 & 1.2895706 & 1.0352357 & \end{array}$

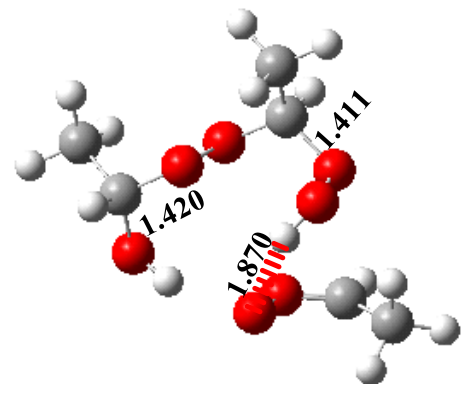

IM6a

\begin{tabular}{|c|c|c|c|c|c|c|}
\hline $\mathrm{H}$ & 1 & B1 & & & & \\
\hline $\mathrm{O}$ & 1 & B2 & 2 & A1 & & \\
\hline $\mathrm{O}$ & 1 & B3 & 3 & A2 & 2 & D1 \\
\hline $\mathrm{H}$ & 3 & B4 & 1 & A3 & 4 & D2 \\
\hline $\mathrm{C}$ & 4 & B5 & 1 & A4 & 3 & D3 \\
\hline $\mathrm{C}$ & 6 & B6 & 4 & A5 & 1 & D4 \\
\hline $\mathrm{H}$ & 7 & B7 & 6 & A6 & 4 & D5 \\
\hline $\mathrm{H}$ & 7 & B8 & 6 & A7 & 4 & D6 \\
\hline $\mathrm{H}$ & 7 & B9 & 6 & A8 & 4 & D7 \\
\hline $\mathrm{O}$ & 6 & B10 & 4 & A9 & 1 & D8 \\
\hline $\mathrm{O}$ & 6 & B11 & 4 & A10 & 1 & D9 \\
\hline $\mathrm{H}$ & 6 & B12 & 4 & A11 & 1 & D10 \\
\hline $\mathrm{C}$ & 1 & B13 & 4 & A12 & 3 & D11 \\
\hline $\mathrm{H}$ & 14 & B14 & 1 & A13 & 4 & D12 \\
\hline $\mathrm{H}$ & 14 & B15 & 1 & A14 & 4 & D13 \\
\hline $\mathrm{H}$ & 14 & B16 & 1 & A15 & 4 & D14 \\
\hline $\mathrm{O}$ & 1 & B17 & 4 & A16 & 3 & D15 \\
\hline $\mathrm{O}$ & 18 & B18 & 1 & A17 & 4 & D16 \\
\hline $\mathrm{C}$ & 19 & B19 & 18 & A18 & 1 & D17 \\
\hline $\mathrm{H}$ & 20 & B20 & 19 & A19 & 18 & D18 \\
\hline $\mathrm{C}$ & 20 & B21 & 19 & A20 & 18 & D19 \\
\hline $\mathrm{H}$ & 22 & B22 & 20 & A21 & 19 & D20 \\
\hline $\mathrm{H}$ & 22 & B23 & 20 & A22 & 19 & $\mathrm{D} 21$ \\
\hline
\end{tabular}




\begin{tabular}{|c|c|c|c|c|c|}
\hline $\mathrm{H}$ & B24 & 20 & A23 & 19 & $\mathrm{D} 22$ \\
\hline $\mathrm{O}$ & B25 & 19 & A24 & 18 & D23 \\
\hline $\mathrm{H}$ & B26 & 20 & A25 & 19 & D24 \\
\hline \multicolumn{6}{|c|}{ Variables: } \\
\hline B1 & 1.09287536 & & & & \\
\hline B2 & 2.32738864 & & & & \\
\hline B3 & 1.41141271 & & & & \\
\hline B4 & 0.98086524 & & & & \\
\hline B5 & 2.87681419 & & & & \\
\hline B6 & 1.46457416 & & & & \\
\hline B7 & 1.09139947 & & & & \\
\hline B8 & 1.08456401 & & & & \\
\hline B9 & 1.09498544 & & & & \\
\hline $\mathrm{B} 10$ & 2.23425260 & & & & \\
\hline B11 & 1.24111813 & & & & \\
\hline B 12 & 1.08577021 & & & & \\
\hline B13 & 1.51181071 & & & & \\
\hline B14 & 1.08387126 & & & & \\
\hline B15 & 1.08802231 & & & & \\
\hline B16 & 1.08818453 & & & & \\
\hline B17 & 1.39787059 & & & & \\
\hline B18 & 1.43399497 & & & & \\
\hline B19 & 1.42019190 & & & & \\
\hline B20 & 1.09807819 & & & & \\
\hline B 21 & 1.51096727 & & & & \\
\hline B22 & 1.08804558 & & & & \\
\hline B23 & 1.08840268 & & & & \\
\hline B24 & 1.08713542 & & & & \\
\hline $\mathrm{B} 25$ & 1.37943248 & & & & \\
\hline B26 & 0.97474228 & & & & \\
\hline A1 & 136.42185312 & & & & \\
\hline A2 & 34.85893841 & & & & \\
\hline $\mathrm{A} 3$ & 97.92337524 & & & & \\
\hline A4 & 124.86729812 & & & & \\
\hline A5 & 87.26356072 & & & & \\
\hline A6 & 108.54494301 & & & & \\
\hline A7 & 110.91374758 & & & & \\
\hline A 8 & 108.04718416 & & & & \\
\hline A9 & 74.73542775 & & & & \\
\hline A10 & 79.91007072 & & & & \\
\hline A11 & 103.86199850 & & & & \\
\hline A 12 & 113.19030673 & & & & \\
\hline A13 & 110.89049875 & & & & \\
\hline A14 & 108.99922854 & & & & \\
\hline
\end{tabular}




$\begin{array}{lr}\text { A15 } & 108.93288371 \\ \text { A16 } & 112.05865525 \\ \text { A17 } & 107.86499372 \\ \text { A18 } & 107.71374457 \\ \text { A19 } & 99.41269395 \\ \text { A20 } & 112.80979003 \\ \text { A21 } & 109.05980856 \\ \text { A22 } & 110.17389826 \\ \text { A23 } & 109.67839216 \\ \text { A24 } & 112.30687072 \\ \text { A25 } & 108.83043346 \\ \text { D1 } & 13.33381438 \\ \text { D2 } & -98.30229382 \\ \text { D3 } & 111.96626526 \\ \text { D4 } & -170.45647803 \\ \text { D5 } & 122.16050601 \\ \text { D6 } & -114.11398966 \\ \text { D7 } & 7.30480567 \\ \text { D8 } & -79.57699612 \\ \text { D9 } & -45.71494835 \\ \text { D10 } & 65.10500140 \\ \text { D11 } & 50.57467422 \\ \text { D12 } & -63.64245638 \\ \text { D13 } & 56.58679910 \\ \text { D14 } & 175.99623126 \\ \text { D15 } & -80.41607682 \\ \text { D16 } & 81.18760018 \\ \text { D17 } & -177.80747878 \\ \text { D18 } & -176.50369968 \\ \text { D19 } & -58.93744991 \\ \text { D20 } & -172.35662132 \\ \text { D21 } & -52.25122026 \\ \text { D22 } & 68.60096661 \\ \text { D23 } & 40.80129617 \\ \text { D24 }\end{array}$

Harmonic Vibrational Frequencies $\left(\mathrm{cm}^{-1}\right)$ :

$\begin{array}{lllllllllll}55.76 & 65.09 & 88.11 & 89.46 & 111.26 & 122.31 & 134.83 & 153.69 & 167.94 & 192.04 & 211.54\end{array}$ $\begin{array}{llllllllll}223.66 & 234.75 & 245.52 & 261.93 & 332.45 & 359.84 & 423.33 & 473.72 & 478.94 & 499.76\end{array}$ $\begin{array}{llllllllll}518.46 & 655.36 & 669.20 & 703.68 & 734.40 & 758.43 & 800.41 & 858.64 & 882.66 & 915.80\end{array}$ $\begin{array}{lllllllll}937.31 & 959.15 & 997.39 & 1003.38 & 1018.55 & 1102.26 & 1109.16 & 1126.71 & 1138.81\end{array}$ $\begin{array}{lllllllll}1145.16 & 1170.31 & 1193.31 & 1214.35 & 1335.74 & 1352.33 & 1370.60 & 1375.72 & 1397.31\end{array}$ $\begin{array}{lllllllll}1405.42 & 1420.06 & 1424.73 & 1455.82 & 1477.81 & 1481.35 & 1483.40 & 1494.21 & 1502.65\end{array}$ $\begin{array}{llllllllll}1504.07 & 1549.50 & 1687.43 & 3042.74 & 3043.87 & 3072.77 & 3079.67 & 3103.79 & 3114.80\end{array}$ $\begin{array}{llllllll}3151.69 & 3154.21 & 3161.86 & 3182.58 & 3186.85 & 3206.47 & 3512.72 & 3606.66\end{array}$ 


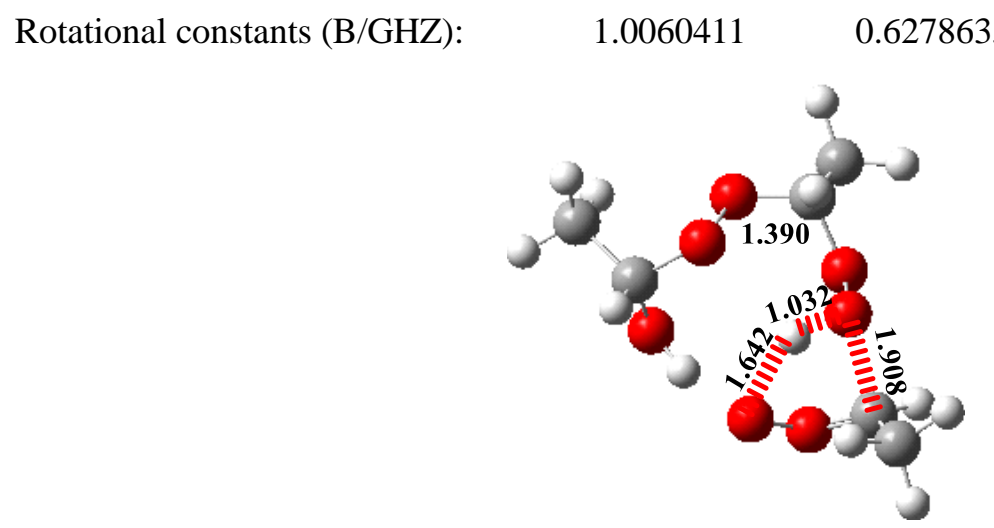

TS6a

C

$\begin{array}{lll}\mathrm{H} & 1 & \mathrm{~B} 1\end{array}$

O 1

O 3

$\mathrm{H} \quad 3$

C 3

C 6

$\begin{array}{ll}\mathrm{H} & 7\end{array}$

$\begin{array}{ll}\mathrm{H} & 7\end{array}$

$\mathrm{H} \quad 7$

O 6

O 6

$\mathrm{H} \quad 6$

C 1

$\mathrm{H} \quad 14$

$\mathrm{H} \quad 14$

$\mathrm{H} \quad 14$

O 1

O 18

C 19

$\mathrm{H} \quad 20$

C 20

$\mathrm{H} \quad 22$

$\mathrm{H} \quad 22$

$\mathrm{H} \quad 22$

O 20

H 26

$$
\text { B2 }
$$

B3 1

A1

B4 1

A2

A3 4

D1 0

B5 1

A4 4

D2 0

B6 3

A5 1

D3 0

$\begin{array}{llll}\text { B7 } & 6 & \text { A6 } & 3\end{array}$

D4 0

B8 6

A7 3

D5 0

B9 6

A8 3

D6 0

B10 3

A9 1

D7 0

B11 3

A10 1

D8 0

B12 3

A11 1

D9 0

B13 4

A12 3

D10 0

$\begin{array}{llll}\text { B14 } & 1 & \text { A13 } & 4\end{array}$

D11 0

B15 1

A14 4

D12 0

B16 1

A15 4

D13 0

B17 4

A16 3

D14 0

B18 1

A17 4

D15 0

B19 18

A18 1

D16 0

B20 19

A19 18

D17 0

B21 19

A20 18

D18 0

B22 20

A21 19

D19 0

B23 20

A22 19

D20 0

B24 20

A23 19

D21 0

B25 19

A24 18

D22 0

B26 20

A25 19

D23 0

D24 0

Variables:

$\begin{array}{ll}\text { B1 } & 1.09183096 \\ \text { B2 } & 2.33197421 \\ \text { B3 } & 1.41685100 \\ \text { B4 } & 1.03225747 \\ \text { B5 } & 1.90829277\end{array}$




\begin{tabular}{|c|c|}
\hline B6 & 1.48722023 \\
\hline B7 & 1.08376033 \\
\hline B8 & 1.09310454 \\
\hline B9 & 1.08613398 \\
\hline B10 & 2.20188774 \\
\hline B11 & 1.27760448 \\
\hline B12 & 1.08826181 \\
\hline B13 & 1.51292417 \\
\hline B14 & 1.08523985 \\
\hline B15 & 1.08802888 \\
\hline B16 & 1.08766105 \\
\hline B17 & 1.39041221 \\
\hline B18 & 1.42921786 \\
\hline B19 & 1.43889664 \\
\hline B20 & 1.09755377 \\
\hline B21 & 1.51013212 \\
\hline B22 & 1.08784261 \\
\hline B23 & 1.08808741 \\
\hline B24 & 1.08750739 \\
\hline B 25 & 1.36919656 \\
\hline B26 & 0.97956998 \\
\hline A1 & 134.14170158 \\
\hline $\mathrm{A} 2$ & 34.74394144 \\
\hline $\mathrm{A} 3$ & 98.12373878 \\
\hline A4 & 138.05780980 \\
\hline A5 & 95.44895921 \\
\hline A6 & 111.48993433 \\
\hline A7 & 106.18624950 \\
\hline A8 & 110.02139260 \\
\hline A9 & 74.91290217 \\
\hline A10 & 96.24918186 \\
\hline A11 & 102.49187922 \\
\hline A12 & 113.36023984 \\
\hline A13 & 111.07409655 \\
\hline A14 & 109.89352536 \\
\hline A15 & 108.21289537 \\
\hline A16 & 111.80764561 \\
\hline A17 & 107.22571632 \\
\hline A18 & 107.31414643 \\
\hline A19 & 99.08168036 \\
\hline A 20 & 111.53136400 \\
\hline A21 & 108.95860392 \\
\hline A 22 & 110.49601309 \\
\hline A23 & 109.52416990 \\
\hline
\end{tabular}




\begin{tabular}{lr} 
A24 & 112.49398268 \\
A25 & 107.80051948 \\
D1 & -27.76538437 \\
D2 & -104.32885156 \\
D3 & -15.01535013 \\
D4 & 173.04130624 \\
D5 & 60.13600344 \\
D6 & 178.57105549 \\
D7 & -63.92713247 \\
D8 & -93.83844414 \\
D9 & -62.23506284 \\
D10 & 52.04833222 \\
D11 & 39.63015828 \\
D12 & -70.87500489 \\
D13 & 50.17841284 \\
D14 & 169.58601900 \\
D15 & -90.53237851 \\
D16 & 67.79116729 \\
D17 & -146.75994374 \\
D18 & -175.83685933 \\
D19 & -58.37616223 \\
D20 & -170.57101551 \\
D21 & -50.24978892 \\
D22 & 70.66379801 \\
D23 & 65.21947214 \\
D24 & 62.95082382 \\
\hline
\end{tabular}

Harmonic Vibrational Frequencies $\left(\mathbf{c m}^{-1}\right)$ :

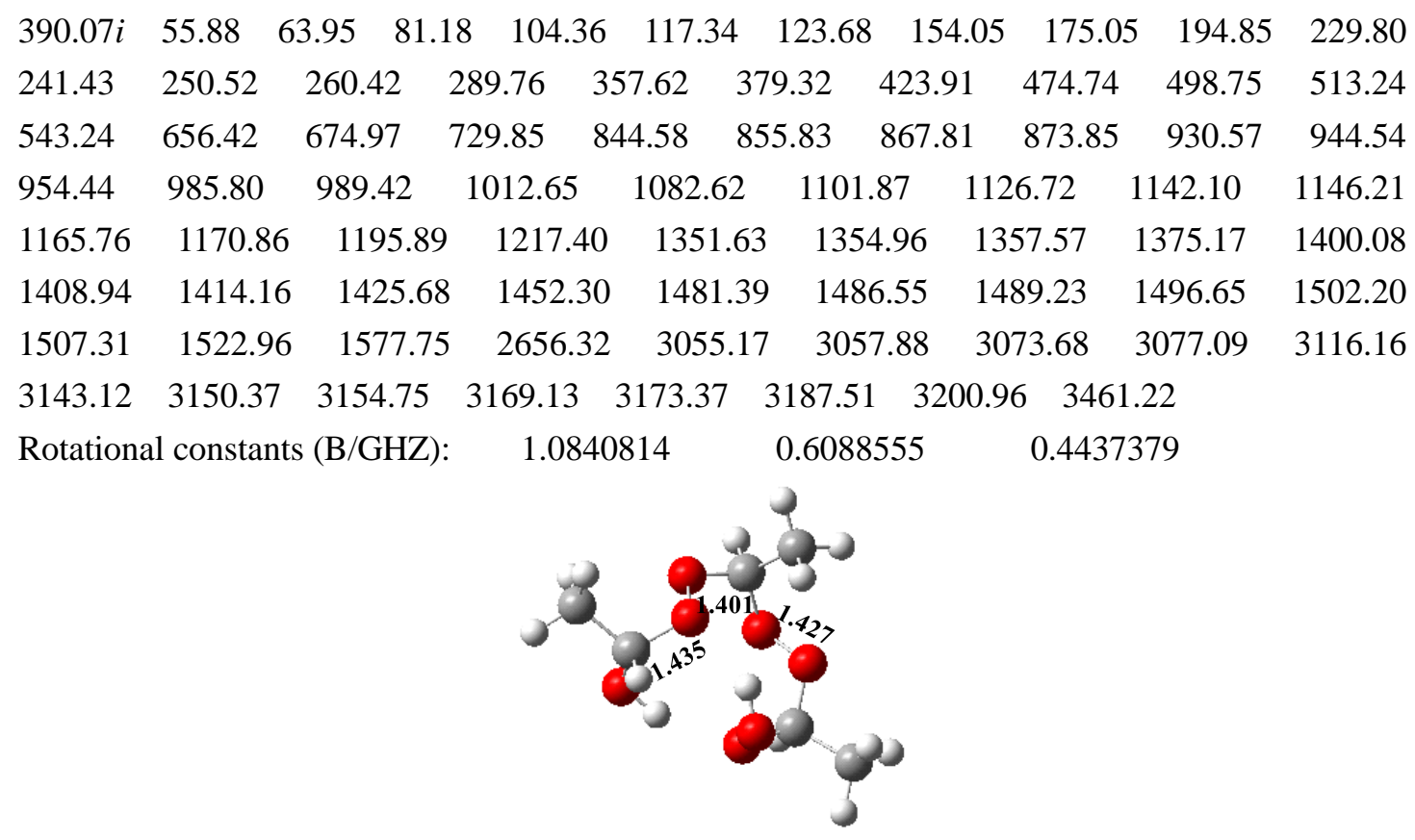

P6a 
C

$\begin{array}{lll}\mathrm{H} & 1\end{array}$

O 1

O 1

$\mathrm{H} \quad 3$

C 3

C 6

$\begin{array}{ll}\mathrm{H} & 7\end{array}$

$\begin{array}{ll}\mathrm{H} & 7\end{array}$

$\begin{array}{ll}\mathrm{H} & 7\end{array}$

O 6

O 6

$\mathrm{H} \quad 6$

C 1

H 14

$\mathrm{H} \quad 14$

$\mathrm{H} \quad 14$

O 1

O 18

C 19

$\mathrm{H} \quad 20$

C 20

$\mathrm{H} \quad 22$

$\mathrm{H} \quad 22$

$\mathrm{H} \quad 22$

O 20

$\mathrm{H} \quad 26$

Variables:

$\begin{array}{ll}\text { B1 } & 1.09232775 \\ \text { B2 } & 2.33227046 \\ \text { B3 } & 1.40996116 \\ \text { B4 } & 2.48304988 \\ \text { B5 } & 1.39609152 \\ \text { B6 } & 1.51052232 \\ \text { B7 } & 1.08672300 \\ \text { B8 } & 1.08747722 \\ \text { B9 } & 1.08717845 \\ \text { B10 } & 2.30016204 \\ \text { B11 } & 1.40984943 \\ \text { B12 } & 1.09180856 \\ \text { B13 } & 1.51263130 \\ \text { B14 } & 1.08574357 \\ \text { B15 } & 1.08745955 \\ \text { B16 } & 1.08735744\end{array}$

B2 $2 \quad$ A1

$\begin{array}{llllll}\text { B } 3 & 3 & \text { A2 } & 2 & \text { D1 } & 0\end{array}$

$\begin{array}{llllll}\text { B4 } & 1 & \text { A3 } & 4 & \text { D2 } & 0\end{array}$

$\begin{array}{llllll}\text { B5 } & 1 & \text { A4 } & 4 & \text { D3 } & 0\end{array}$

$\begin{array}{llllll}\text { B6 } & 3 & \text { A5 } & 1 & \text { D4 } & 0\end{array}$

$\begin{array}{llllll}\text { B7 } & 6 & \text { A6 } & 3 & \text { D5 } & 0\end{array}$

$\begin{array}{llllll}\text { B8 } & 6 & \text { A7 } & 3 & \text { D6 } & 0\end{array}$

$\begin{array}{llllll}\text { B9 } & 6 & \text { A8 } & 3 & \text { D7 } & 0\end{array}$

$\begin{array}{llllll}\mathrm{B} 10 & 3 & \mathrm{~A} 9 & 1 & \mathrm{D} 8 & 0\end{array}$

$\begin{array}{llllll}\text { B11 } & 3 & \text { A10 } & 1 & \text { D9 } & 0\end{array}$

$\begin{array}{llllll}\mathrm{B} 12 & 3 & \mathrm{~A} 11 & 1 & \mathrm{D} 10 & 0\end{array}$

$\begin{array}{llllll}\mathrm{B} 13 & 4 & \mathrm{~A} 12 & 3 & \mathrm{D} 11 & 0\end{array}$

$\begin{array}{llllll}\mathrm{B} 14 & 1 & \mathrm{~A} 13 & 4 & \mathrm{D} 12 & 0\end{array}$

$\begin{array}{llllll}\text { B15 } & 1 & \text { A14 } & 4 & \text { D13 } & 0\end{array}$

$\begin{array}{llllll}\text { B16 } & 1 & \text { A15 } & 4 & \text { D14 } & 0\end{array}$

$\begin{array}{llllll}\mathrm{B} 17 & 4 & \mathrm{~A} 16 & 3 & \mathrm{D} 15 & 0\end{array}$

$\begin{array}{llllll}\mathrm{B} 18 & 1 & \mathrm{~A} 17 & 4 & \mathrm{D} 16 & 0\end{array}$

$\begin{array}{llllll}\text { B19 } & 18 & \text { A18 } & 1 & \text { D17 } & 0\end{array}$

$\begin{array}{llllll}\text { B20 } & 19 & \text { A19 } & 18 & \text { D18 } & 0\end{array}$

$\begin{array}{llllll}\text { B21 } & 19 & \text { A20 } & 18 & \text { D19 } & 0\end{array}$

$\begin{array}{llllll}\text { B22 } & 20 & \text { A21 } & 19 & \text { D20 } & 0\end{array}$

$\begin{array}{llllll}\text { B23 } & 20 & \text { A22 } & 19 & \text { D21 } & 0\end{array}$

$\begin{array}{llllll}\text { B24 } & 20 & \text { A23 } & 19 & \text { D22 } & 0\end{array}$

$\begin{array}{llllll}\text { B25 } & 19 & \text { A24 } & 18 & \text { D23 } & 0\end{array}$

$\begin{array}{llllll}\text { B26 } & 20 & \text { A25 } & 19 & \text { D24 } & 0\end{array}$ 


\begin{tabular}{|c|c|}
\hline B17 & 1.40129954 \\
\hline B18 & 1.42405746 \\
\hline B19 & 1.43518760 \\
\hline $\mathrm{B} 20$ & 1.09686422 \\
\hline B21 & 1.50851575 \\
\hline $\mathrm{B} 22$ & 1.08777840 \\
\hline B23 & 1.08793578 \\
\hline B24 & 1.08716011 \\
\hline $\mathrm{B} 25$ & 1.37686178 \\
\hline B26 & 0.96634165 \\
\hline A1 & 130.89861590 \\
\hline $\mathrm{A} 2$ & 34.96018747 \\
\hline A3 & 84.70741739 \\
\hline A4 & 133.32536480 \\
\hline A5 & 106.48227027 \\
\hline A6 & 110.23352883 \\
\hline A7 & 108.88689193 \\
\hline A8 & 109.62562136 \\
\hline A9 & 91.97763633 \\
\hline A10 & 111.25361993 \\
\hline A11 & 110.17852313 \\
\hline A12 & 114.33971172 \\
\hline A13 & 111.11211706 \\
\hline A14 & 110.04900569 \\
\hline A15 & 108.08649059 \\
\hline A16 & 112.07933827 \\
\hline A17 & 109.14848862 \\
\hline A18 & 107.12225815 \\
\hline A19 & 99.98923234 \\
\hline $\mathrm{A} 20$ & 111.88696994 \\
\hline A21 & 109.03752352 \\
\hline A22 & 110.20648783 \\
\hline A23 & 109.59722007 \\
\hline A24 & 112.03825063 \\
\hline A 25 & 108.73180053 \\
\hline D1 & 46.54635227 \\
\hline D2 & -116.51466728 \\
\hline D3 & -50.42379564 \\
\hline D4 & -170.55362763 \\
\hline D5 & 54.59623062 \\
\hline D6 & 174.14923184 \\
\hline D7 & -65.96773505 \\
\hline D8 & -77.56831130 \\
\hline D9 & -46.89186177 \\
\hline
\end{tabular}




$\begin{array}{lr}\text { D10 } & 66.50660692 \\ \text { D11 } & 24.23869591 \\ \text { D12 } & -69.64380208 \\ \text { D13 } & 50.93918457 \\ \text { D14 } & 170.66503171 \\ \text { D15 } & -106.24309267 \\ \text { D16 } & 69.30988564 \\ \text { D17 } & -143.58429472 \\ \text { D18 } & -177.74184681 \\ \text { D19 } & -59.60869062 \\ \text { D20 } & -172.58315682 \\ \text { D21 } & -52.45821765 \\ \text { D22 } & 68.31886924 \\ \text { D23 } & 63.26376140 \\ \text { D24 } & 53.76675720\end{array}$

Harmonic Vibrational Frequencies $\left(\mathbf{c m}^{-1}\right)$ :

$\begin{array}{lllllllllll}58.05 & 73.13 & 100.93 & 117.40 & 140.59 & 158.65 & 188.44 & 201.84 & 225.77 & 239.41 & 245.19\end{array}$ $\begin{array}{llllllllll}260.83 & 278.36 & 311.17 & 347.83 & 400.38 & 428.63 & 469.20 & 493.20 & 521.51 & 552.26\end{array}$ $\begin{array}{lllllllllll}575.16 & 633.04 & 680.30 & 701.42 & 716.79 & 867.54 & 872.22 & 884.62 & 936.70 & 949.90\end{array}$ $\begin{array}{lllllllll}959.39 & 998.67 & 1011.85 & 1032.27 & 1092.68 & 1139.62 & 1141.92 & 1145.93 & 1147.71\end{array}$ $\begin{array}{llllllllll}162.64 & 1190.11 & 1210.18 & 1220.68 & 1331.36 & 1356.20 & 1363.53 & 1374.46 & 1391.45\end{array}$ $\begin{array}{lllllllll}1407.05 & 1414.22 & 1424.19 & 1425.73 & 1478.25 & 1488.79 & 1490.56 & 1491.61 & 1498.05\end{array}$ $\begin{array}{llllllllll}1501.13 & 1502.96 & 1505.86 & 3060.55 & 3079.20 & 3080.12 & 3083.12 & 3115.76 & 3127.66\end{array}$ $\begin{array}{llllllll}3157.22 & 3159.06 & 3165.42 & 3169.59 & 3176.07 & 3178.08 & 3686.91 & 3787.18\end{array}$ Rotational constants (B/GHZ): $\quad 1.1166091 \quad 0.6430181 \quad 0.4836962$

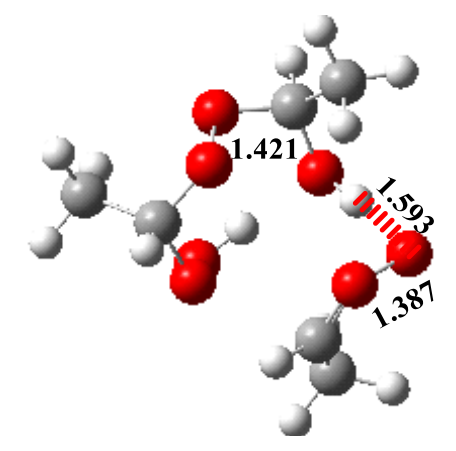

IM6b

$\mathrm{C}$ C $\quad 1 \quad$ B1

$\begin{array}{lllll}\mathrm{H} & 2 & \mathrm{~B} 2 & 1 & \mathrm{~A} 1\end{array}$

$\begin{array}{llllllll}\mathrm{H} & 2 & \mathrm{~B} 3 & 1 & \mathrm{~A} 2 & 3 & \mathrm{D} 1 & 0\end{array}$

$\begin{array}{llllllll}\mathrm{H} & 2 & \mathrm{~B} 4 & 1 & \mathrm{~A} 3 & 3 & \mathrm{D} 2 & 0\end{array}$

$\begin{array}{llllllll}\mathrm{O} & 1 & \mathrm{~B} 5 & 2 & \mathrm{~A} 4 & 5 & \mathrm{D} 3 & 0\end{array}$

$\begin{array}{llllllll}\mathrm{H} & 6 & \mathrm{~B} 6 & 1 & \mathrm{~A} 5 & 2 & \mathrm{D} 4 & 0\end{array}$

$\begin{array}{llllllll}\mathrm{O} & 1 & \mathrm{~B} 7 & 6 & \mathrm{~A} 6 & 2 & \mathrm{D} 5 & 0\end{array}$

$\begin{array}{llllllll}\mathrm{C} & 6 & \mathrm{~B} 8 & 1 & \mathrm{~A} 7 & 2 & \mathrm{D} 6 & 0\end{array}$ 


\begin{tabular}{|c|c|c|c|c|c|c|}
\hline $\mathrm{C}$ & 9 & B9 & 6 & A8 & 1 & D7 \\
\hline $\mathrm{H}$ & 10 & B10 & 9 & A9 & 6 & D8 \\
\hline $\mathrm{H}$ & 10 & B11 & 9 & A10 & 6 & D9 \\
\hline $\mathrm{H}$ & 10 & B12 & 9 & A11 & 6 & D10 \\
\hline $\mathrm{O}$ & 9 & B13 & 6 & A 12 & 1 & D11 \\
\hline $\mathrm{O}$ & 9 & B14 & 6 & A13 & 1 & D12 \\
\hline $\mathrm{H}$ & 1 & B15 & 6 & A14 & 2 & D13 \\
\hline $\mathrm{O}$ & 1 & B16 & 6 & A15 & 2 & D14 \\
\hline $\mathrm{C}$ & 8 & B17 & 1 & A16 & 6 & D15 \\
\hline $\mathrm{H}$ & 18 & B18 & 8 & A17 & 1 & D16 \\
\hline $\mathrm{C}$ & 18 & B19 & 8 & A18 & 1 & D17 \\
\hline $\mathrm{H}$ & 20 & B20 & 18 & A19 & 8 & D18 \\
\hline $\mathrm{H}$ & 20 & B21 & 18 & A20 & 8 & D19 \\
\hline $\mathrm{H}$ & 20 & B22 & 18 & A21 & 8 & D20 \\
\hline $\mathrm{O}$ & 18 & B23 & 8 & A22 & 1 & D21 \\
\hline $\mathrm{O}$ & 24 & B24 & 18 & A23 & 8 & D22 \\
\hline $\mathrm{H}$ & 25 & B25 & 24 & A24 & 18 & D23 \\
\hline $\mathrm{H}$ & 9 & B26 & 6 & A25 & 1 & D24 \\
\hline
\end{tabular}

Variables:

$\begin{array}{ll}\text { B1 } & 1.51317426 \\ \text { B2 } & 1.08840511 \\ \text { B3 } & 1.08804452 \\ \text { B4 } & 1.08735391 \\ \text { B5 } & 1.38992697 \\ \text { B6 } & 1.00260691 \\ \text { B7 } & 2.30936846 \\ \text { B8 } & 3.50119046 \\ \text { B9 } & 1.46537993 \\ \text { B10 } & 1.09108267 \\ \text { B11 } & 1.09242474 \\ \text { B12 } & 1.08427370 \\ \text { B13 } & 2.23403508 \\ \text { B14 } & 1.24319643 \\ \text { B15 } & 1.09320907 \\ \text { B16 } & 1.42107042 \\ \text { B17 } & 1.40370076 \\ \text { B18 } & 1.09357734 \\ \text { B19 } & 1.51040113 \\ \text { B20 } & 1.08784133 \\ \text { B21 } & 1.08794685 \\ \text { B22 } & 1.08379600 \\ \text { B23 } & 1.41124339 \\ \text { B24 } & 1.41677449 \\ \text { B25 } & 0.98427302\end{array}$




\begin{tabular}{|c|c|}
\hline B26 & 1.08478801 \\
\hline A1 & 109.42188671 \\
\hline $\mathrm{A} 2$ & 109.58543282 \\
\hline A3 & 110.50917541 \\
\hline A4 & 112.95366151 \\
\hline A5 & 111.58769451 \\
\hline A6 & 95.86864899 \\
\hline A7 & 117.97673452 \\
\hline A8 & 81.23215186 \\
\hline A9 & 108.90925358 \\
\hline A10 & 108.14001914 \\
\hline A11 & 110.80712111 \\
\hline A12 & 47.68340738 \\
\hline A13 & 62.46799949 \\
\hline A14 & 108.59419711 \\
\hline A15 & 110.73243326 \\
\hline A16 & 125.89146972 \\
\hline A17 & 102.03480640 \\
\hline A18 & 114.05795790 \\
\hline A19 & 109.04087900 \\
\hline A 20 & 108.86854776 \\
\hline A 21 & 110.81228817 \\
\hline A22 & 112.25030447 \\
\hline A 23 & 111.48351364 \\
\hline A24 & 101.35621500 \\
\hline A 25 & 131.26928206 \\
\hline D1 & 120.00218236 \\
\hline D2 & -119.30539364 \\
\hline D3 & -58.76953185 \\
\hline D4 & 16.46870264 \\
\hline D5 & -93.43476402 \\
\hline D6 & 63.78321017 \\
\hline D7 & 168.13713478 \\
\hline D8 & -11.40965599 \\
\hline D9 & 103.86552760 \\
\hline D10 & -132.76709815 \\
\hline D11 & -94.71724775 \\
\hline D12 & -57.05316559 \\
\hline D13 & 123.89810973 \\
\hline D14 & -127.08452562 \\
\hline D15 & -44.86921973 \\
\hline D16 & 156.56772324 \\
\hline D17 & -83.10845370 \\
\hline D18 & -174.38975763 \\
\hline
\end{tabular}




$\begin{array}{lr}\text { D19 } & -54.82769480 \\ \text { D20 } & 65.44514466 \\ \text { D21 } & 47.70671429 \\ \text { D22 } & -83.32124957 \\ \text { D23 } & 79.18160109 \\ \text { D24 } & 40.38019172\end{array}$

Harmonic Vibrational Frequencies $\left(\mathbf{c m}^{-\mathbf{1}}\right)$ :

$\begin{array}{lccccccccccc}31.79 & 45.34 & 71.63 & 91.34 & 119.93 & 132.08 & 140.86 & 152.71 & 167.70 & 211.75 & 217.09 \\ 237.67 & 261.64 & 275.15 & 287.50 & 332.28 & 335.39 & 436.93 & 471.20 & 504.59 & 509.92 \\ 527.62 & 672.74 & 680.10 & 737.88 & 799.94 & 823.86 & 851.51 & 868.41 & 906.46 & 932.23 \\ 954.67 & 991.32 & 1003.98 & 1021.43 & 1046.01 & 1099.33 & 1111.87 & 1120.23 & 1142.33 \\ 1148.34 & 1163.65 & 1194.50 & 1200.62 & 1355.09 & 1358.69 & 1365.28 & 1379.20 & 1409.46 \\ 1413.78 & 1421.14 & 1425.82 & 1444.08 & 1462.90 & 1488.55 & 1490.85 & 1491.21 & 1502.08 \\ 1506.06 & 1549.07 & 1680.30 & 2966.53 & 3060.74 & 3075.03 & 3086.23 & 3100.24 & 3105.57 \\ 3120.26 & 3152.80 & 3160.95 & 3172.54 & 3185.71 & 3195.50 & 3203.23 & 3404.80 & \\ \text { Rotational constants (B/GHZ): } & 0.9289166 & 0.7259506 & 0.5181123 & \end{array}$

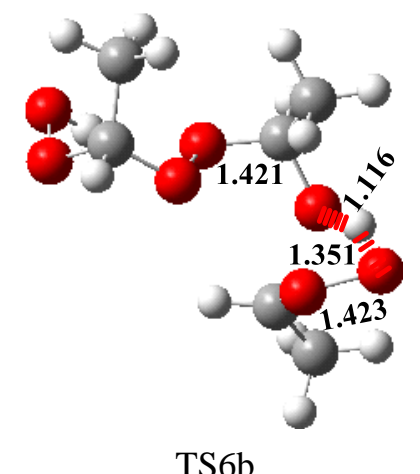

C

$\begin{array}{lll}\mathrm{C} & 1 & \mathrm{~B} 1\end{array}$

$\begin{array}{lll}\mathrm{H} & 2 & \mathrm{~B} 2\end{array}$

$\mathrm{H} \quad 2$

$\mathrm{H} \quad 2$

O 1

$\mathrm{H} \quad 6$

O 1

C 6

C 9

H 10

$\mathrm{H} \quad 10$

$\mathrm{H} \quad 10$

O 9

O 9

$\mathrm{H} \quad 1$

O 1

C 8

$\mathrm{H} \quad 18$

$\begin{array}{ll}\text { B3 } & 1 \\ \text { B4 } & 1 \\ \text { B5 } & 2 \\ \text { B6 } & 1 \\ \text { B7 } & 6 \\ \text { B8 } & 1 \\ \text { B9 } & 6 \\ \text { B10 } & 9 \\ \text { B11 } & 9 \\ \text { B12 } & 9 \\ \text { B13 } & 6 \\ \text { B14 } & 6 \\ \text { B15 } & 6 \\ \text { B16 } & 6 \\ \text { B17 } & 1 \\ \text { B18 } & 8\end{array}$

A1

A2 $3 \quad$ D1 0

A3 $3 \quad$ D2 0

A4 $5 \quad$ D3 0

A5 2 D4 0

A6 2 D5 0

A7 $2 \quad$ D6 0

$\begin{array}{llll}\text { A8 } & 1 & \text { D7 } & 0\end{array}$

A9 $6 \quad$ D8 0

$\begin{array}{llll}\mathrm{A} 10 & 6 & \mathrm{D} 9 & 0\end{array}$

$\begin{array}{llll}\text { A11 } & 6 & \text { D10 } & 0\end{array}$

A12 $1 \quad$ D11 0

A13 $1 \quad$ D12 0

A14 9 D13 0

A15 $9 \quad$ D14 0

A16 $6 \quad$ D15 0

A17 $1 \quad$ D16 0 


$\begin{array}{rrrrrrrr}\mathrm{C} & 18 & \text { B19 } & 8 & \text { A18 } & 1 & \text { D17 } & 0 \\ \mathrm{H} & 20 & \text { B20 } & 18 & \text { A19 } & 8 & \text { D18 } & 0 \\ \mathrm{H} & 20 & \text { B21 } & 18 & \text { A20 } & 8 & \text { D19 } & 0 \\ \mathrm{H} & 20 & \text { B22 } & 18 & \text { A21 } & 8 & \text { D20 } & 0 \\ \mathrm{O} & 18 & \text { B23 } & 8 & \text { A22 } & 1 & \text { D21 } & 0 \\ \mathrm{O} & 24 & \text { B24 } & 18 & \text { A23 } & 8 & \text { D22 } & 0 \\ \mathrm{H} & 25 & \text { B25 } & 24 & \text { A24 } & 18 & \text { D23 } & 0 \\ \mathrm{H} & 9 & \text { B26 } & 6 & \text { A25 } & 1 & \text { D24 } & 0\end{array}$

Variables:

\begin{tabular}{|c|c|}
\hline B1 & 1.51399662 \\
\hline B2 & 1.08895147 \\
\hline B3 & 1.08833497 \\
\hline B4 & 1.08755876 \\
\hline B5 & 1.39235250 \\
\hline B6 & 1.11559127 \\
\hline B7 & 2.32878812 \\
\hline B8 & 2.02765906 \\
\hline B9 & 1.48228714 \\
\hline B10 & 1.08572901 \\
\hline B11 & 1.08276327 \\
\hline B12 & 1.09466229 \\
\hline B13 & 2.20037070 \\
\hline B14 & 1.27565320 \\
\hline B15 & 1.09461200 \\
\hline B16 & 1.42052603 \\
\hline B17 & 1.41330311 \\
\hline B18 & 1.09225917 \\
\hline B19 & 1.51061594 \\
\hline B20 & 1.08781882 \\
\hline B21 & 1.08816878 \\
\hline B22 & 1.08523799 \\
\hline B23 & 1.40006795 \\
\hline B24 & 1.41704229 \\
\hline B25 & 0.96681096 \\
\hline B26 & 1.08727512 \\
\hline $\mathrm{A} 1$ & 108.80132582 \\
\hline A2 & 110.03866314 \\
\hline $\mathrm{A} 3$ & 110.71951449 \\
\hline A4 & 112.87207872 \\
\hline A5 & 113.06415763 \\
\hline A6 & 99.44642741 \\
\hline A7 & 121.08602261 \\
\hline A8 & 91.92531681 \\
\hline A9 & 109.72791230 \\
\hline
\end{tabular}




\begin{tabular}{|c|c|}
\hline A10 & 111.86206078 \\
\hline A11 & 105.72621397 \\
\hline A12 & 68.76500782 \\
\hline A13 & 92.83081702 \\
\hline A14 & 107.86541923 \\
\hline A15 & 110.96154713 \\
\hline A16 & 132.55427674 \\
\hline A17 & 101.65079116 \\
\hline A18 & 113.69161859 \\
\hline A19 & 109.03699467 \\
\hline $\mathrm{A} 20$ & 108.92685577 \\
\hline $\mathrm{A} 21$ & 111.16905132 \\
\hline $\mathrm{A} 22$ & 111.12891098 \\
\hline $\mathrm{A} 23$ & 110.65766449 \\
\hline $\mathrm{A} 24$ & 102.29726169 \\
\hline A25 & 106.95266913 \\
\hline D1 & 119.14264472 \\
\hline D2 & -118.96687882 \\
\hline D3 & -59.29506347 \\
\hline D4 & 3.43441022 \\
\hline D5 & -93.29438901 \\
\hline D6 & 88.69964630 \\
\hline D7 & 159.20467327 \\
\hline D8 & -62.39200400 \\
\hline D9 & 61.39615006 \\
\hline D10 & -179.22084156 \\
\hline D11 & -105.77070651 \\
\hline D12 & -76.20195743 \\
\hline D13 & -148.13872073 \\
\hline D14 & -39.33985431 \\
\hline D15 & -168.56812700 \\
\hline D16 & -151.12819780 \\
\hline D17 & -30.98163922 \\
\hline D18 & 179.32994131 \\
\hline D19 & -61.37434680 \\
\hline D20 & 59.50000732 \\
\hline D21 & 99.63857868 \\
\hline D22 & -86.08386313 \\
\hline D23 & 77.60741106 \\
\hline D24 & 37.93221196 \\
\hline
\end{tabular}

Harmonic Vibrational Frequencies $\left(\mathbf{c m}^{-1}\right)$ :

$\begin{array}{lllllllllll}649.03 i & 34.87 & 38.63 & 78.35 & 89.77 & 108.76 & 134.84 & 150.52 & 202.51 & 219.08 & 225.14\end{array}$ $\begin{array}{llllllllll}241.54 & 250.41 & 269.29 & 310.63 & 337.99 & 369.04 & 385.81 & 441.02 & 462.22 & 507.90\end{array}$ $\begin{array}{llllllllll}527.72 & 541.14 & 631.79 & 681.62 & 745.41 & 850.70 & 858.54 & 871.53 & 921.26 & 931.32\end{array}$ 


$\begin{array}{llllllllll}950.42 & 967.28 & 989.51 & 1020.35 & 1043.65 & 1108.15 & 1127.14 & 1153.28 & 1156.78 \\ 1162.01 & 1172.00 & 1192.23 & 1219.97 & 1353.63 & 1359.10 & 1367.23 & 1376.86 & 1397.56 \\ 1403.48 & 1418.70 & 1422.21 & 1448.00 & 1461.21 & 1479.48 & 1483.75 & 1485.54 & 1494.72 \\ 1499.80 & 1504.78 & 1597.72 & 1880.60 & 3048.09 & 3071.46 & 3076.57 & 3086.11 & 3117.90 \\ 3146.56 & 3151.49 & 3157.05 & 3168.14 & 3183.75 & 3203.26 & 3216.63 & 3802.54 & \\ \text { Rotational constants (B/GHZ): } & 1.3397503 & 0.4587138 & 0.4208758 & \end{array}$

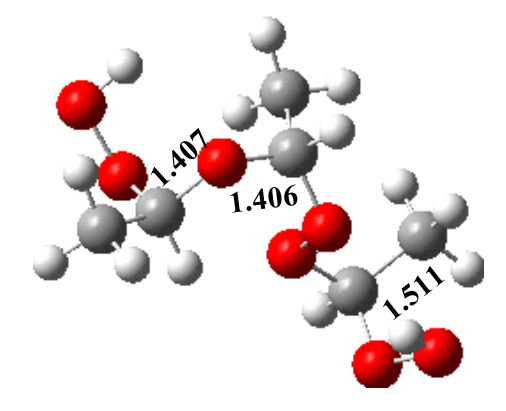

P6b

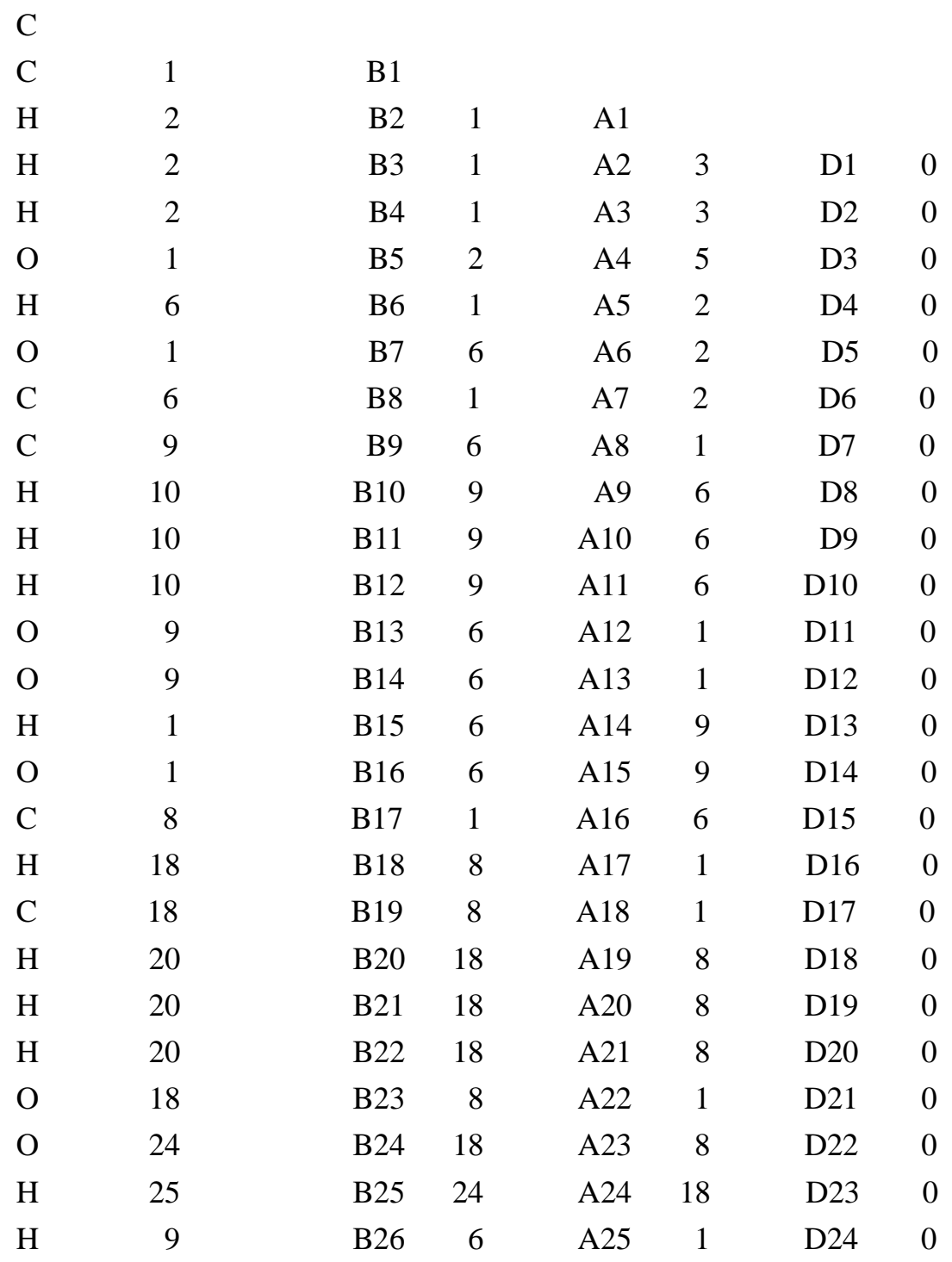

Variables:

B1 


\begin{tabular}{|c|c|}
\hline B2 & 1.08984968 \\
\hline B3 & 1.08885590 \\
\hline B4 & 1.08551591 \\
\hline B5 & 1.40627607 \\
\hline B6 & 2.53388302 \\
\hline B7 & 2.32936067 \\
\hline B8 & 1.40726816 \\
\hline B9 & 1.50880351 \\
\hline B10 & 1.08758529 \\
\hline B11 & 1.08657176 \\
\hline B12 & 1.08792037 \\
\hline B13 & 2.30452359 \\
\hline B14 & 1.41067256 \\
\hline B15 & 1.09121741 \\
\hline B16 & 1.41131701 \\
\hline B17 & 1.41405674 \\
\hline B18 & 1.09201683 \\
\hline B19 & 1.51134044 \\
\hline B20 & 1.08776333 \\
\hline B21 & 1.08829637 \\
\hline B22 & 1.08523736 \\
\hline B23 & 1.39868601 \\
\hline B24 & 1.41689016 \\
\hline B25 & 0.96652364 \\
\hline B26 & 1.09072761 \\
\hline $\mathrm{A} 1$ & 108.25187425 \\
\hline $\mathrm{A} 2$ & 109.55325584 \\
\hline A3 & 111.44597072 \\
\hline A4 & 113.70779610 \\
\hline A5 & 102.43054855 \\
\hline A6 & 106.41497271 \\
\hline A7 & 118.98820491 \\
\hline A8 & 107.45004050 \\
\hline A9 & 109.34452298 \\
\hline A10 & 110.27344214 \\
\hline A11 & 109.39723970 \\
\hline A 12 & 94.42182086 \\
\hline A13 & 111.43212349 \\
\hline A14 & 104.10005531 \\
\hline A15 & 112.27363653 \\
\hline A16 & 133.19787457 \\
\hline A 17 & 101.43222052 \\
\hline A18 & 113.56912065 \\
\hline A19 & 109.08606719 \\
\hline
\end{tabular}




$\begin{array}{lr}\text { A20 } & 109.00075566 \\ \text { A21 } & 111.14940638 \\ \text { A22 } & 111.34154654 \\ \text { A23 } & 110.63066598 \\ \text { A24 } & 102.20651625 \\ \text { A25 } & 110.69356374 \\ \text { D1 } & 117.81243089 \\ \text { D2 } & -120.87017488 \\ \text { D3 } & -61.82055000 \\ \text { D4 } & -0.61021759 \\ \text { D5 } & -93.46094095 \\ \text { D6 } & 78.80582207 \\ \text { D7 } & 165.49149803 \\ \text { D8 } & -65.26258622 \\ \text { D9 } & 54.72699941 \\ \text { D10 } & 174.84159232 \\ \text { D11 } & -101.93468293 \\ \text { D12 } & -69.86832660 \\ \text { D13 } & -161.11388746 \\ \text { D14 } & -51.53678302 \\ \text { D15 } & -157.70696352 \\ \text { D16 } & -153.08035543 \\ \text { D17 } & -32.97466576 \\ \text { D18 } & 177.75671975 \\ \text { D19 } & -63.01711282 \\ \text { D20 } & 57.99206491 \\ \text { D21 } & 97.69601128 \\ \text { D22 } & -86.36157161 \\ \text { D23 } & 81.26654545 \\ \text { D24 } & 42.89362777 \\ \text { Dar } & \end{array}$

Harmonic Vibrational Frequencies $\left(\mathbf{c m}^{-\mathbf{1}}\right)$ :

$\begin{array}{lllllllllll}33.62 & 39.08 & 76.83 & 106.68 & 118.57 & 142.98 & 157.94 & 192.04 & 217.34 & 235.13 & 252.07\end{array}$ $\begin{array}{llllllllll}260.02 & 271.41 & 280.07 & 309.82 & 333.64 & 365.68 & 409.64 & 420.99 & 471.69 & 516.58\end{array}$ $\begin{array}{lllllllllll}533.92 & 569.67 & 694.26 & 715.89 & 750.79 & 832.71 & 859.64 & 884.65 & 928.20 & 938.84\end{array}$ $\begin{array}{lllllllll}965.31 & 999.30 & 1018.52 & 1021.78 & 1109.58 & 1123.29 & 1134.17 & 1154.59 & 1161.22\end{array}$ $\begin{array}{llllllllll}1171.66 & 1189.62 & 1198.40 & 1207.70 & 1355.15 & 1368.38 & 1389.01 & 1391.57 & 1397.95\end{array}$ $\begin{array}{llllllllll}1415.93 & 1419.68 & 1423.57 & 1432.71 & 1441.88 & 1463.52 & 1486.50 & 1487.32 & 1497.82\end{array}$ $\begin{array}{llllllllll}1499.27 & 1505.46 & 1509.63 & 3073.49 & 3078.38 & 3082.97 & 3116.06 & 3121.12 & 3134.99\end{array}$ $\begin{array}{llllllll}3145.00 & 3156.03 & 3159.83 & 3174.81 & 3177.69 & 3181.48 & 3801.67 & 3807.53\end{array}$

$\begin{array}{llll}\text { Rotational constants (B/GHZ): } & 1.4677668 & 0.4281024 & 0.3978915\end{array}$ 


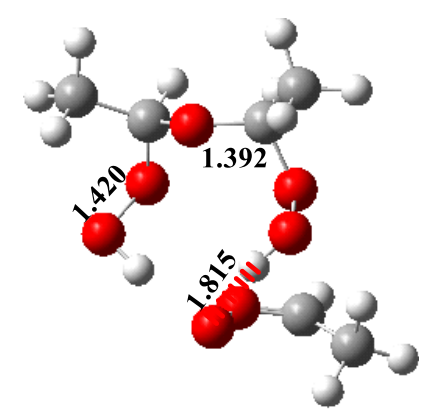

IM6c

C

$\begin{array}{lll}\mathrm{H} & 1 & \mathrm{~B} 1\end{array}$

O 1

O 1

$\mathrm{H} \quad 3$

C 4

C 6

H 7

H $\quad 7$

$\mathrm{H} \quad 7$

O 6

O 6

$\mathrm{H} \quad 6$

C 1

$\mathrm{H} \quad 14$

$\mathrm{H} \quad 14$

$\mathrm{H} \quad 14$

O 1

C 18

H 19

C 19

$\mathrm{H} \quad 21$

$\mathrm{H} \quad 21$

$\mathrm{H} \quad 21$

O 19

O 25

H 26

Variables:

B1

B2

B3

B4

B5

B6

B7

$\begin{array}{ll}\mathrm{B} 1 & 1.09650175 \\ \mathrm{~B} 2 & 2.30179254 \\ \mathrm{~B} 3 & 1.41453735 \\ \mathrm{~B} 4 & 0.97964335 \\ \mathrm{~B} 5 & 2.94886439 \\ \mathrm{~B} 6 & 1.46425930 \\ \mathrm{~B} 7 & 1.09594559\end{array}$

B2

B3 3

B4 1

B5 1

B6 4

B7 6

B8 6

B9 6

B10 4

B11 4

B12 4

B13 4

B14 1

B15 1

B16 1

B17 4

B18 1

B19 18

B20 18

B21 19

B22 19

B23 19

B24 18

B25 19

B26 25
A1

$\begin{array}{llll}\text { A2 } 2 & \text { D1 } & 0\end{array}$

A3 4

D2 0

A4 3

D3 0

A5 1

A6 4

D4 0

D5 0

A7 $4 \quad$ D6 0

$\begin{array}{llll}\text { A8 } & 4 & \text { D7 } & 0\end{array}$

A9 $11 \quad$ D8 0

A10 $1 \quad$ D9 0

A11 $1 \quad$ D10 0

A12 $3 \quad$ D11 0

A13 $4 \quad$ D12 0

A14 $4 \quad$ D13 0

A15 4 D14 0

A16 $3 \quad$ D15 0

A17 $4 \quad$ D16 0

A18 11 D17 0

A19 1 D18 0

$\begin{array}{llll}\text { A20 } & 18 & \text { D19 } & 0\end{array}$

$\begin{array}{llll}\text { A21 } 18 & \text { D20 } & 0\end{array}$

A22 $18 \quad$ D21 0

A23 11 D22 0

$\begin{array}{llll}\text { A24 } & 18 & \text { D23 } & 0\end{array}$

A25 $19 \quad$ D24 0 


\begin{tabular}{|c|c|}
\hline B8 & 1.09065092 \\
\hline B9 & 1.08489577 \\
\hline B 10 & 2.24067000 \\
\hline B11 & 1.24079340 \\
\hline B12 & 1.08600075 \\
\hline B13 & 1.51133505 \\
\hline B14 & 1.08647719 \\
\hline B 15 & 1.08823950 \\
\hline B16 & 1.08801093 \\
\hline B17 & 1.39202231 \\
\hline B18 & 1.40582526 \\
\hline B19 & 1.09666390 \\
\hline B20 & 1.51029084 \\
\hline B21 & 1.08787763 \\
\hline B22 & 1.08787867 \\
\hline B23 & 1.08597582 \\
\hline B24 & 1.40028495 \\
\hline B25 & 1.41993919 \\
\hline B26 & 0.97995523 \\
\hline $\mathrm{A} 1$ & 136.91681269 \\
\hline $\mathrm{A} 2$ & 35.81821623 \\
\hline A3 & 109.04099850 \\
\hline $\mathrm{A} 4$ & 149.12756122 \\
\hline A5 & 87.08318793 \\
\hline A6 & 108.02883607 \\
\hline A7 & 108.85771576 \\
\hline A8 & 110.69713747 \\
\hline A9 & 70.15959267 \\
\hline A 10 & 76.27577065 \\
\hline A11 & 107.84299396 \\
\hline A12 & 112.06685850 \\
\hline A13 & 109.54178283 \\
\hline A14 & 110.01101320 \\
\hline A15 & 109.39349454 \\
\hline A16 & 112.82288332 \\
\hline A17 & 117.23965535 \\
\hline A18 & 109.32499149 \\
\hline A19 & 107.68783209 \\
\hline A20 & 109.38197883 \\
\hline A21 & 109.60946229 \\
\hline A22 & 109.73779621 \\
\hline $\mathrm{A} 23$ & 113.17953961 \\
\hline A24 & 109.35492588 \\
\hline A25 & 101.53680931 \\
\hline
\end{tabular}




\begin{tabular}{lr} 
D1 & 8.84239083 \\
D2 & -80.28448049 \\
D3 & 128.46518964 \\
D4 & -175.73656659 \\
D5 & -2.20194272 \\
D6 & 112.88956557 \\
D7 & -123.19168972 \\
D8 & -83.71249864 \\
D9 & -49.68536053 \\
D10 & 59.55993818 \\
D11 & 54.61077001 \\
D12 & -70.77160758 \\
D13 & 49.39287064 \\
D14 & 169.75200314 \\
D15 & -68.04515670 \\
D16 & -86.02278392 \\
D17 & -36.77210248 \\
D18 & -157.94433911 \\
D19 & 64.92634414 \\
D20 & -174.76620843 \\
D21 & -54.97892285 \\
D22 & 75.81330475 \\
D23 & 75.86746711 \\
D24 & -112.39108021 \\
\hline
\end{tabular}

Harmonic Vibrational Frequencies $\left(\mathbf{c m}^{-1}\right)$ :

$\begin{array}{lccccccccccr}41.59 & 52.23 & 66.76 & 76.77 & 105.50 & 115.07 & 126.87 & 167.32 & 195.36 & 203.01 & 210.89 \\ 219.93 & 225.47 & 278.17 & 307.74 & 325.32 & 355.89 & 363.10 & 411.33 & 469.88 & 473.97 \\ 604.50 & 628.96 & 688.59 & 692.77 & 695.87 & 744.74 & 797.03 & 858.46 & 883.02 & 918.39 \\ 941.61 & 977.79 & 997.95 & 1007.84 & 1033.73 & 1100.23 & 1127.20 & 1129.20 & 1148.75 \\ 1149.72 & 1172.74 & 1184.32 & 1227.72 & 1357.87 & 1371.97 & 1379.03 & 1384.69 & 1420.28 \\ 1422.21 & 1424.75 & 1438.88 & 1448.34 & 1475.82 & 1485.56 & 1487.54 & 1497.98 & 1501.65 \\ 1528.45 & 1560.95 & 1689.23 & 3042.06 & 3056.41 & 3066.13 & 3076.11 & 3080.49 & 3108.03 \\ 3152.38 & 3158.55 & 3173.81 & 3177.62 & 3178.03 & 3201.31 & 3474.02 & 3520.41 & \\ \text { Rotational constants (B/GHZ): } & 1.0047034 & 0.6105839 & 0.4315045 & \end{array}$

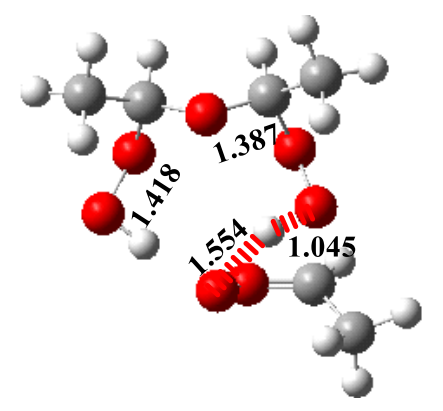

TS6c

C 


\begin{tabular}{|c|c|c|c|c|c|c|c|}
\hline $\mathrm{H}$ & 1 & B1 & & & & & \\
\hline $\mathrm{O}$ & 1 & B2 & 2 & A1 & & & \\
\hline $\mathrm{O}$ & 3 & B3 & 1 & A2 & 2 & D1 & 0 \\
\hline $\mathrm{H}$ & 3 & B4 & 1 & A3 & 4 & D2 & 0 \\
\hline $\mathrm{C}$ & 3 & B5 & 1 & A4 & 4 & D3 & 0 \\
\hline $\mathrm{C}$ & 6 & B6 & 3 & A5 & 1 & D4 & 0 \\
\hline $\mathrm{H}$ & 7 & B7 & 6 & A6 & 3 & D5 & 0 \\
\hline $\mathrm{H}$ & 7 & B8 & 6 & A7 & 3 & D6 & 0 \\
\hline $\mathrm{H}$ & 7 & B9 & 6 & A8 & 3 & D7 & 0 \\
\hline $\mathrm{O}$ & 6 & B10 & 3 & A9 & 1 & D8 & 0 \\
\hline $\mathrm{O}$ & 6 & B11 & 3 & A10 & 1 & D9 & 0 \\
\hline $\mathrm{H}$ & 6 & B12 & 3 & A11 & 1 & D10 & 0 \\
\hline $\mathrm{C}$ & 1 & B13 & 4 & A12 & 3 & D11 & 0 \\
\hline $\mathrm{H}$ & 14 & B14 & 1 & A13 & 4 & D12 & 0 \\
\hline $\mathrm{H}$ & 14 & B15 & 1 & A14 & 4 & D13 & 0 \\
\hline $\mathrm{H}$ & 14 & B16 & 1 & A15 & 4 & D14 & 0 \\
\hline $\mathrm{O}$ & 1 & B17 & 4 & A16 & 3 & D15 & 0 \\
\hline $\mathrm{C}$ & 18 & B18 & 1 & A17 & 4 & D16 & 0 \\
\hline $\mathrm{H}$ & 19 & B19 & 18 & A18 & 1 & D17 & 0 \\
\hline $\mathrm{C}$ & 19 & B20 & 18 & A19 & 1 & D18 & 0 \\
\hline $\mathrm{H}$ & 21 & B21 & 19 & A20 & 18 & D19 & 0 \\
\hline $\mathrm{H}$ & 21 & B22 & 19 & A 21 & 18 & D20 & 0 \\
\hline $\mathrm{H}$ & 21 & B23 & 19 & A22 & 18 & D21 & 0 \\
\hline $\mathrm{O}$ & 19 & B24 & 18 & A 23 & 1 & $\mathrm{D} 22$ & 0 \\
\hline $\mathrm{O}$ & 25 & B25 & 19 & A24 & 18 & $\mathrm{D} 23$ & 0 \\
\hline $\mathrm{H}$ & 26 & B26 & 25 & A25 & 19 & D24 & 0 \\
\hline \multicolumn{7}{|c|}{ Variables: } & \\
\hline B1 & & 1.09560305 & & & & & \\
\hline B2 & & 2.30839318 & & & & & \\
\hline B3 & & 1.41617842 & & & & & \\
\hline B4 & & 1.04539627 & & & & & \\
\hline B5 & & 1.95997817 & & & & & \\
\hline B6 & & 1.48498009 & & & & & \\
\hline B7 & & 1.08606556 & & & & & \\
\hline B8 & & 1.08371614 & & & & & \\
\hline B9 & & 1.09355793 & & & & & \\
\hline B10 & & 2.19649820 & & & & & \\
\hline B11 & & 1.27452426 & & & & & \\
\hline B12 & & 1.08822872 & & & & & \\
\hline B13 & & 1.51094667 & & & & & \\
\hline B14 & & 1.08693765 & & & & & \\
\hline B15 & & 1.08796712 & & & & & \\
\hline B16 & & 1.08769980 & & & & & \\
\hline B17 & & 1.38673477 & & & & & \\
\hline
\end{tabular}




\begin{tabular}{|c|c|}
\hline B 18 & 1.42111594 \\
\hline B19 & 1.09565670 \\
\hline B20 & 1.50956776 \\
\hline B21 & 1.08799222 \\
\hline B22 & 1.08771933 \\
\hline B23 & 1.08578961 \\
\hline B24 & 1.39299349 \\
\hline B 25 & 1.41766508 \\
\hline B26 & 0.98815607 \\
\hline $\mathrm{A} 1$ & 135.54577515 \\
\hline A2 & 35.66324411 \\
\hline A3 & 93.73332142 \\
\hline $\mathrm{A} 4$ & 135.42228298 \\
\hline A5 & 94.27126028 \\
\hline A6 & 109.81765237 \\
\hline A7 & 111.76301824 \\
\hline A8 & 106.13299266 \\
\hline A9 & 72.77118657 \\
\hline A 10 & 94.53080764 \\
\hline A11 & 103.71321433 \\
\hline A 12 & 112.40696130 \\
\hline A13 & 110.08740590 \\
\hline A14 & 110.05435080 \\
\hline A15 & 109.09340442 \\
\hline A16 & 112.12762378 \\
\hline A17 & 116.62498801 \\
\hline A18 & 108.35438876 \\
\hline A19 & 107.43097642 \\
\hline A 20 & 109.43471243 \\
\hline A 21 & 109.43148005 \\
\hline A22 & 109.96117251 \\
\hline A 23 & 112.84731686 \\
\hline A24 & 110.15139079 \\
\hline A 25 & 102.18386441 \\
\hline D1 & -9.83351858 \\
\hline D2 & -109.49752362 \\
\hline D3 & -29.98164606 \\
\hline D4 & -176.60310847 \\
\hline D5 & -64.99738758 \\
\hline D6 & 59.04856676 \\
\hline D7 & 177.69111408 \\
\hline D8 & -83.10280409 \\
\hline D9 & -51.69233105 \\
\hline D10 & 62.24696079 \\
\hline
\end{tabular}




$\begin{array}{lr}\text { D11 } & 54.05361962 \\ \text { D12 } & -72.19055229 \\ \text { D13 } & 48.45181171 \\ \text { D14 } & 168.59807193 \\ \text { D15 } & -69.01443359 \\ \text { D16 } & -83.35116427 \\ \text { D17 } & -34.51863604 \\ \text { D18 } & -155.14899755 \\ \text { D19 } & 66.17774461 \\ \text { D20 } & -173.51433802 \\ \text { D21 } & -54.01061254 \\ \text { D22 } & 78.14497887 \\ \text { D23 } & 80.99908896 \\ \text { D24 } & -98.42616155\end{array}$

Harmonic Vibrational Frequencies $\left(\mathbf{c m}^{-\mathbf{1}}\right)$ :

$\begin{array}{lllllllllllr}403.46 i & 36.31 & 60.01 & 99.28 & 103.33 & 110.63 & 136.01 & 171.60 & 205.98 & 215.67 & 225.41 \\ 246.79 & 272.11 & 294.39 & 324.05 & 342.94 & 361.43 & 390.97 & 409.34 & 470.25 & 529.34 \\ 612.03 & 656.44 & 691.37 & 705.35 & 841.27 & 853.86 & 866.47 & 886.11 & 931.43 & 939.51 \\ 974.81 & 984.53 & 1009.07 & 1024.51 & 1102.46 & 1107.29 & 1136.69 & 1154.76 & 1166.02 \\ 1169.36 & 1189.50 & 1223.64 & 1235.10 & 1361.52 & 1363.17 & 1375.15 & 1385.17 & 1406.35 \\ 1419.49 & 1426.61 & 1440.23 & 1452.28 & 1483.57 & 1484.85 & 1491.81 & 1496.58 & 1500.20 \\ 1531.40 & 1577.95 & 1593.57 & 2419.50 & 3057.41 & 3061.05 & 3073.19 & 3075.05 & 3078.23 \\ 3146.63 & 3155.24 & 3157.98 & 3163.52 & 3176.89 & 3191.06 & 3204.87 & 3313.33 & \\ \text { Rotational constants (B/GHZ): } & 1.0795508 & 0.6078223 & 0.4384923 & \end{array}$

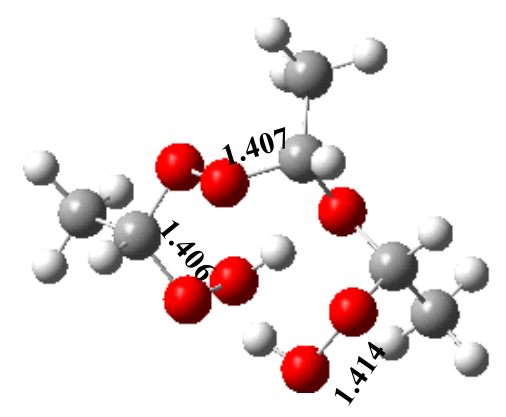

P6c

$\begin{array}{llllllll}\mathrm{C} & & & & & & & \\ \mathrm{H} & 1 & \text { B1 } & & & & & \\ \mathrm{O} & 1 & \text { B2 } & 2 & \text { A1 } & & & \\ \mathrm{O} & 1 & \text { B3 } & 3 & \text { A2 } & 2 & \text { D1 } & 0 \\ \mathrm{H} & 3 & \text { B4 } & 1 & \text { A3 } & 4 & \text { D2 } & 0 \\ \mathrm{C} & 3 & \text { B5 } & 1 & \text { A4 } & 4 & \text { D3 } & 0 \\ \mathrm{C} & 6 & \text { B6 } & 3 & \text { A5 } & 1 & \text { D4 } & 0 \\ \mathrm{H} & 7 & \text { B7 } & 6 & \text { A6 } & 3 & \text { D5 } & 0 \\ \mathrm{H} & 7 & \text { B8 } & 6 & \text { A7 } & 3 & \text { D6 } & 0 \\ \mathrm{H} & 7 & \text { B9 } & 6 & \text { A8 } & 3 & \text { D7 } & 0 \\ \text { O } & 6 & \text { B10 } & 3 & \text { A9 } & 1 & \text { D8 } & 0\end{array}$




\begin{tabular}{|c|c|c|c|c|c|c|}
\hline $\mathrm{O}$ & 6 & B11 & 3 & A 10 & 1 & D9 \\
\hline $\mathrm{H}$ & 6 & $\mathrm{~B} 12$ & 3 & A11 & 1 & D10 \\
\hline $\mathrm{C}$ & 1 & B13 & 4 & A 12 & 3 & D11 \\
\hline $\mathrm{H}$ & 14 & B14 & 1 & A13 & 4 & D12 \\
\hline $\mathrm{H}$ & 14 & B15 & 1 & A14 & 4 & D13 \\
\hline $\mathrm{H}$ & 14 & B16 & 1 & A15 & 4 & D14 \\
\hline $\mathrm{O}$ & 1 & B17 & 4 & A16 & 3 & D15 \\
\hline $\mathrm{C}$ & 18 & B18 & 1 & A17 & 4 & D16 \\
\hline $\mathrm{H}$ & 19 & B19 & 18 & A18 & 1 & D17 \\
\hline $\mathrm{C}$ & 19 & B20 & 18 & A19 & 1 & D18 \\
\hline $\mathrm{H}$ & 21 & B21 & 19 & A20 & 18 & D19 \\
\hline $\mathrm{H}$ & 21 & B22 & 19 & A21 & 18 & D20 \\
\hline $\mathrm{H}$ & 21 & B23 & 19 & A22 & 18 & D21 \\
\hline $\mathrm{O}$ & 19 & B24 & 18 & A23 & 1 & D22 \\
\hline $\mathrm{O}$ & 25 & B25 & 19 & A24 & 18 & D23 \\
\hline $\mathrm{H}$ & 26 & B26 & 25 & A25 & 19 & D24 \\
\hline
\end{tabular}

Variables:

$\begin{array}{lr}\text { B1 } & 1.09273088 \\ \text { B2 } & 2.28740838 \\ \text { B3 } & 1.40696920 \\ \text { B4 } & 2.56874799 \\ \text { B5 } & 1.39918914 \\ \text { B6 } & 1.50921907 \\ \text { B7 } & 1.08730077 \\ \text { B8 } & 1.08662650 \\ \text { B9 } & 1.08742368 \\ \text { B10 } & 2.30678677 \\ \text { B11 } & 1.40628023 \\ \text { B12 } & 1.09307671 \\ \text { B13 } & 1.51063899 \\ \text { B14 } & 1.08670125 \\ \text { B15 } & 1.08794822 \\ \text { B16 } & 1.08811017 \\ \text { B17 } & 1.41036536 \\ \text { B18 } & 1.42028898 \\ \text { B19 } & 1.09502309 \\ \text { B20 } & 1.50767938 \\ \text { B21 } & 1.08785486 \\ \text { B22 } & 1.08768175 \\ \text { B23 } & 1.08721096 \\ \text { B24 } & 1.39569974 \\ \text { B25 } & 1.41445596 \\ \text { B26 } & 0.97305997 \\ \text { A1 } & 137.30810966\end{array}$




\begin{tabular}{|c|c|}
\hline A 2 & 36.43005647 \\
\hline A3 & 70.60121784 \\
\hline A4 & 128.00014020 \\
\hline A5 & 106.30772153 \\
\hline A6 & 109.76475307 \\
\hline A7 & 110.01577235 \\
\hline A8 & 108.85466392 \\
\hline A9 & 92.47933884 \\
\hline A10 & 111.35743826 \\
\hline A11 & 110.12416798 \\
\hline A 12 & 113.18789801 \\
\hline A13 & 110.13348802 \\
\hline A14 & 109.95900869 \\
\hline A15 & 109.11935873 \\
\hline A16 & 110.99215647 \\
\hline A 17 & 115.08242248 \\
\hline A18 & 108.78984752 \\
\hline A19 & 107.84130622 \\
\hline A20 & 109.48121501 \\
\hline A21 & 109.22750324 \\
\hline A22 & 110.34159116 \\
\hline A23 & 111.25869432 \\
\hline A24 & 109.84724950 \\
\hline A25 & 103.57464406 \\
\hline D1 & 14.68520550 \\
\hline D2 & -117.54770524 \\
\hline D3 & -64.69409610 \\
\hline D4 & -153.95981456 \\
\hline D5 & -62.86161353 \\
\hline D6 & 57.80515453 \\
\hline D7 & 177.32837852 \\
\hline D8 & -60.28517621 \\
\hline D9 & -29.65000167 \\
\hline D10 & 83.30823960 \\
\hline D11 & 49.61555489 \\
\hline D12 & -70.91875593 \\
\hline D13 & 49.50076651 \\
\hline D14 & 169.53654836 \\
\hline D15 & -73.45359452 \\
\hline D16 & -100.80677439 \\
\hline D17 & -54.50741795 \\
\hline D18 & -175.96447441 \\
\hline D19 & 63.96254126 \\
\hline D20 & -176.13392732 \\
\hline
\end{tabular}




$\begin{array}{lr}\text { D21 } & -56.63482353 \\ \text { D22 } & 57.34958293 \\ \text { D23 } & 74.46854615 \\ \text { D24 } & -78.10017539\end{array}$

Harmonic Vibrational Frequencies $\left(\mathbf{c m}^{-1}\right)$ :

$\begin{array}{lccccccccccc}58.79 & 61.10 & 88.01 & 114.04 & 127.09 & 186.28 & 190.42 & 202.70 & 213.21 & 228.67 & 243.02 \\ 273.80 & 298.93 & 314.12 & 333.40 & 358.99 & 417.64 & 437.84 & 477.66 & 544.05 & 566.01 \\ 600.03 & 650.14 & 675.04 & 713.43 & 716.85 & 855.66 & 882.27 & 895.10 & 937.04 & 947.40 \\ 985.00 & 1006.64 & 1019.43 & 1030.65 & 1112.93 & 1137.22 & 1148.31 & 1151.46 & 1155.31 \\ 1170.88 & 1174.29 & 1207.08 & 1230.17 & 1358.28 & 1365.23 & 1377.85 & 1393.12 & 1397.83 \\ 1423.20 & 1426.45 & 1431.17 & 1439.14 & 1469.40 & 1486.61 & 1488.37 & 1489.62 & 1498.20 \\ 1500.55 & 1504.32 & 1525.04 & 3070.84 & 3074.94 & 3077.90 & 3085.63 & 3111.48 & 3113.14 \\ 3151.75 & 3157.42 & 3163.48 & 3164.89 & 3166.72 & 3178.23 & 3638.72 & 3670.88 & & \end{array}$

$\begin{array}{llll}\text { Rotational constants (B/GHZ): } & 1.0948628 & 0.6441370 & 0.5157937\end{array}$

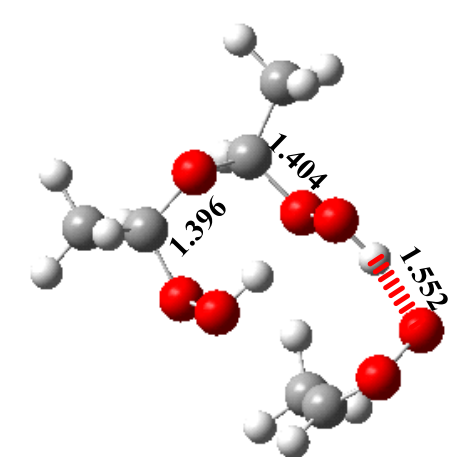

IM6d

C

$\begin{array}{lll}\mathrm{C} & 1 & \mathrm{~B} 1\end{array}$

$\begin{array}{llll}\mathrm{H} & 2 & \mathrm{~B} 2 & 1\end{array}$

$\mathrm{O} 2$

B3 1

A1

$\mathrm{O} 2$

B4 1

$\begin{array}{llll}\mathrm{A} 2 & 3 & \mathrm{D} 1 & 0\end{array}$

$\mathrm{H} \quad 4$

B5 2

A3 4

D2 0

C 4

B6 2

A4 1

D3 0

C $\quad 7$

B7 4

A5 1

D4 0

$\mathrm{H} \quad 8$

B8 7

A6 2

D5 0

H 8

B9 7

A7 4

D6 0

A8 4

D7 0

B10 7

A9 4

D8 0

$\begin{array}{ll}\text { O } & 7\end{array}$

B11 4

A10 2

D9 0

B12 4

A11 2

D10 0

$\begin{array}{ll}\mathrm{H} & 7\end{array}$

B13 4

A12 2

D11 0

B14 2

A13 5

D12 0

B15 2

A14 5

D13 0

B16 2

A15 5

D14 0

B17 1

A16 5

D15 0

C 18

B18 2

A17 1

D16 0

H 19

B19 18

A18 2

D17 0 


$\begin{array}{rrrrrrrr}\mathrm{C} & 19 & \text { B20 } & 18 & \text { A19 } & 2 & \text { D18 } & 0 \\ \mathrm{H} & 21 & \text { B21 } & 19 & \text { A20 } & 18 & \text { D19 } & 0 \\ \mathrm{H} & 21 & \text { B22 } & 19 & \text { A21 } & 18 & \text { D20 } & 0 \\ \mathrm{H} & 21 & \text { B23 } & 19 & \text { A22 } & 18 & \text { D21 } & 0 \\ \mathrm{O} & 19 & \text { B24 } & 18 & \text { A23 } & 2 & \text { D22 } & 0 \\ \text { O } & 25 & \text { B25 } & 19 & \text { A24 } & 18 & \text { D23 } & 0 \\ \text { H } & 26 & \text { B26 } & 25 & \text { A25 } & 19 & \text { D24 } & 0\end{array}$

Variables:

1.50883558

B2 1.09683531

B3 2.30787589

B4 1.41436008

B5 0.98233289

B6 2.71166631

B7 1.46305750

B8 1.08420925

B9 1.09412241

B10 1.09252422

B11 2.23817668

B12 1.24494459

B13 1.08483049

B14 1.08787215

B15 1.08784783

B16 1.08706289

B17 1.39581588

B18 1.40366970

B19 1.09658736

B20 1.50975114

B21 1.08680085

B22 1.08775367

B23 1.08784975

B24 1.40424412

B25 1.43018309

B26 1.01699850

A1 $\quad 112.24477788$

A2 94.37895421

A3 112.70249004

A4 88.95372624

A5 $\quad 131.26610268$

A6 92.24612677

A7 110.80109742

A8 108.54013441

A9 $\quad 108.09481748$

A10 102.01112923 


\begin{tabular}{|c|c|}
\hline A11 & 99.98296411 \\
\hline A 12 & 79.19718589 \\
\hline A13 & 109.72255156 \\
\hline A14 & 109.60287937 \\
\hline A 15 & 109.52339801 \\
\hline A16 & 107.83041070 \\
\hline A17 & 117.68983924 \\
\hline A18 & 110.24835709 \\
\hline A19 & 107.46269876 \\
\hline A 20 & 109.53708665 \\
\hline A 21 & 109.52809085 \\
\hline A 22 & 109.67567938 \\
\hline A 23 & 112.35624463 \\
\hline A24 & 107.45316983 \\
\hline A 25 & 100.67548806 \\
\hline D1 & -145.30840338 \\
\hline D2 & 31.44383795 \\
\hline D3 & -122.87810130 \\
\hline D4 & 157.75549865 \\
\hline D5 & -11.07745960 \\
\hline D6 & -80.03721913 \\
\hline D7 & 41.21230412 \\
\hline D8 & 156.29332843 \\
\hline D9 & 78.64090632 \\
\hline D10 & 113.10301314 \\
\hline D11 & -135.32194067 \\
\hline D12 & -175.06089681 \\
\hline D13 & -54.77773879 \\
\hline D14 & 65.18785358 \\
\hline D15 & -123.80948064 \\
\hline D16 & -170.27215656 \\
\hline D17 & 43.55529549 \\
\hline D18 & 166.07880420 \\
\hline D19 & 57.11286296 \\
\hline D20 & 176.90132728 \\
\hline D21 & -62.78861218 \\
\hline D22 & -69.22357802 \\
\hline $\mathrm{D} 23$ & -60.14993763 \\
\hline D24 & -176.30702421 \\
\hline
\end{tabular}

Harmonic Vibrational Frequencies $\left(\mathbf{c m}^{-\mathbf{1}}\right)$ :

$\begin{array}{lllllllllll}54.05 & 71.85 & 78.02 & 99.69 & 130.53 & 144.54 & 159.61 & 165.45 & 197.67 & 208.30 & 221.24\end{array}$ $\begin{array}{llllllllll}228.95 & 243.26 & 266.73 & 318.41 & 337.96 & 358.29 & 389.73 & 425.93 & 457.69 & 481.80\end{array}$ $\begin{array}{llllllllll}589.03 & 672.99 & 688.47 & 723.52 & 742.18 & 812.34 & 848.75 & 881.26 & 903.86 & 938.19\end{array}$ $\begin{array}{lllllllll}977.43 & 1005.25 & 1008.37 & 1032.37 & 1081.35 & 1113.59 & 1115.33 & 1128.53 & 1145.83\end{array}$ 


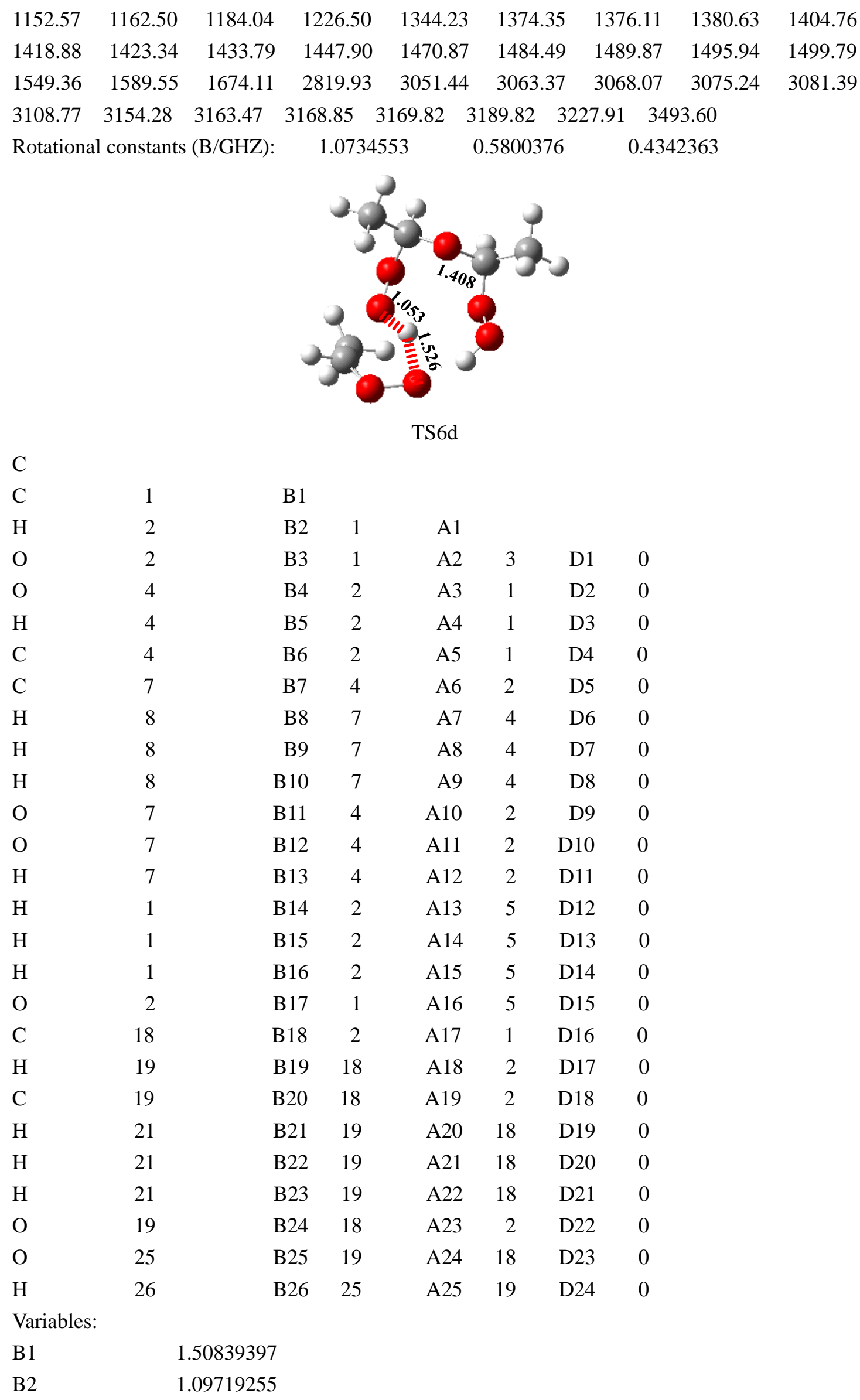




\begin{tabular}{|c|c|}
\hline B3 & 2.28406222 \\
\hline B4 & 1.41130140 \\
\hline B5 & 1.05315022 \\
\hline B6 & 1.92383542 \\
\hline B7 & 1.48290641 \\
\hline B8 & 1.08699841 \\
\hline B9 & 1.08348453 \\
\hline B10 & 1.09338908 \\
\hline B11 & 2.20204570 \\
\hline B12 & 1.27990917 \\
\hline B13 & 1.08788709 \\
\hline B14 & 1.08770950 \\
\hline B15 & 1.08799062 \\
\hline B16 & 1.08720911 \\
\hline B17 & 1.38586199 \\
\hline B18 & 1.40810983 \\
\hline B19 & 1.09588545 \\
\hline B20 & 1.50976011 \\
\hline B21 & 1.08651033 \\
\hline B22 & 1.08776729 \\
\hline B23 & 1.08791112 \\
\hline B24 & 1.40043149 \\
\hline B25 & 1.42707712 \\
\hline B26 & 0.97649954 \\
\hline $\mathrm{A} 1$ & 112.15192248 \\
\hline $\mathrm{A} 2$ & 93.82407211 \\
\hline A3 & 36.58030946 \\
\hline A4 & 110.40718635 \\
\hline A5 & 152.78790389 \\
\hline A6 & 99.02652254 \\
\hline A7 & 109.71624911 \\
\hline A8 & 111.28395758 \\
\hline A9 & 106.24330698 \\
\hline A 10 & 72.36040169 \\
\hline A11 & 91.06677679 \\
\hline $\mathrm{A} 12$ & 102.95371257 \\
\hline A13 & 109.43254337 \\
\hline A14 & 109.89444761 \\
\hline A 15 & 109.53560393 \\
\hline A16 & 108.17515987 \\
\hline A 17 & 116.72666586 \\
\hline A 18 & 109.63170151 \\
\hline A19 & 107.82243457 \\
\hline A20 & 109.57902232 \\
\hline
\end{tabular}




\begin{tabular}{lr} 
A21 & 109.75853446 \\
A22 & 109.46014058 \\
A23 & 112.33310134 \\
A24 & 108.78557965 \\
A25 & 99.76687610 \\
D1 & -144.26126527 \\
D2 & 123.87044182 \\
D3 & -151.13014962 \\
D4 & 98.53689642 \\
D5 & 31.69711412 \\
D6 & -60.71470285 \\
D7 & 62.43724641 \\
D8 & -177.95594559 \\
D9 & 123.41311238 \\
D10 & 156.99532730 \\
D11 & -90.42540012 \\
D12 & -173.41606828 \\
D13 & -53.33632886 \\
D14 & 67.31302039 \\
D15 & -124.66633078 \\
D16 & -176.91329625 \\
D17 & 30.99592584 \\
D18 & 153.16270568 \\
D19 & 54.32265761 \\
D20 & 174.18087020 \\
D21 & -65.40617257 \\
D22 & -81.36602023 \\
D23 & -73.79236609 \\
D24 & 140.04765813 \\
\hline & 9716
\end{tabular}

Harmonic Vibrational Frequencies $\left(\mathbf{c m}^{-1}\right)$ :

$\begin{array}{lllllllllll}391.32 i & 46.77 & 75.30 & 97.28 & 116.64 & 125.59 & 162.72 & 174.58 & 180.83 & 206.66 & 213.96\end{array}$ $\begin{array}{llllllllll}222.93 & 257.32 & 311.80 & 325.55 & 357.85 & 379.03 & 391.70 & 446.35 & 460.05 & 478.57\end{array}$ $\begin{array}{llllllllll}588.94 & 591.70 & 650.00 & 682.88 & 724.33 & 851.98 & 879.79 & 881.21 & 924.93 & 937.48\end{array}$ $\begin{array}{lllllllll}963.35 & 985.64 & 1006.91 & 1024.81 & 1099.87 & 1115.33 & 1145.70 & 1155.65 & 1166.09\end{array}$ $\begin{array}{lllllllll}1169.89 & 1189.12 & 1206.04 & 1231.18 & 1352.75 & 1360.21 & 1374.64 & 1387.08 & 1398.67\end{array}$ $\begin{array}{lllllllll}1415.42 & 1422.97 & 1439.56 & 1445.18 & 1482.74 & 1485.83 & 1487.43 & 1496.09 & 1498.45\end{array}$ $\begin{array}{lllllllll}1503.29 & 531.05 & 1611.38 & 2389.51 & 3061.83 & 3063.08 & 3070.70 & 3073.93 & 3080.34\end{array}$ $\begin{array}{llllllll}3146.43 & 3152.59 & 3161.10 & 3168.71 & 3171.24 & 3188.00 & 3203.11 & 3591.36\end{array}$

Rotational constants (B/GHZ): $\quad 1.0821220 \quad 0.6035619 \quad 0.4460361$ 


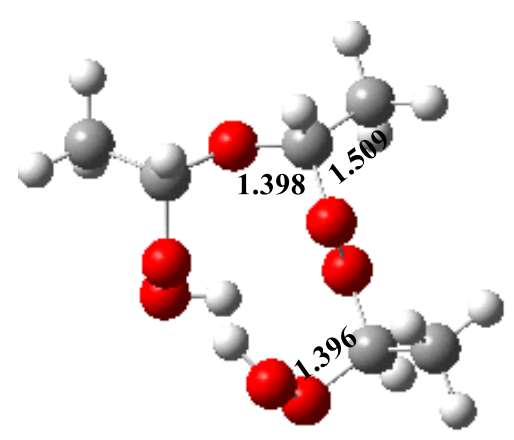

P6d

C

C 1

H 2

O 2

O 2

$\mathrm{H} \quad 5$

C 4

$\begin{array}{ll}\text { C } & 7\end{array}$

H 8

H $\quad 8$

H 8

O 7

O 7

$\begin{array}{ll}\mathrm{H} & 7\end{array}$

H 1

$\mathrm{H} \quad 1$

$\mathrm{H} \quad 1$

O 2

C 18

H 19

C 19

H 21

H 21

$\mathrm{H} \quad 21$

O 19

O 25

H 26

Variables:

B1

B2

B3

B4

B5

B6

B7
B1

B2 $1 \quad$ A1

$\begin{array}{llllll}\text { B3 } & 1 & \text { A2 } & 3 & \text { D1 } & 0\end{array}$

$\begin{array}{llllll}\text { B4 } & 1 & \text { A3 } & 4 & \text { D2 } & 0\end{array}$

$\begin{array}{llllll}\text { B5 } & 2 & \text { A4 } & 1 & \text { D3 } & 0\end{array}$

$\begin{array}{llllll}\text { B6 } & 2 & \text { A5 } & 1 & \text { D4 } & 0\end{array}$

B7 4 A6 2 D5 0

$\begin{array}{llllll}\text { B8 } & 7 & \text { A7 } & 4 & \text { D6 } & 0\end{array}$

$\begin{array}{llllll}\text { B9 } & 7 & \text { A8 } & 4 & \text { D7 } & 0\end{array}$

$\begin{array}{llllll}\mathrm{B} 10 & 7 & \mathrm{~A} 9 & 4 & \mathrm{D} 8 & 0\end{array}$

$\begin{array}{llllll}\mathrm{B} 11 & 4 & \mathrm{~A} 10 & 2 & \mathrm{D} 9 & 0\end{array}$

$\begin{array}{llllll}\mathrm{B} 12 & 4 & \mathrm{~A} 11 & 2 & \mathrm{D} 10 & 0\end{array}$

$\begin{array}{llllll}\mathrm{B} 13 & 4 & \mathrm{~A} 12 & 2 & \mathrm{D} 11 & 0\end{array}$

$\begin{array}{llllll}\mathrm{B} 14 & 2 & \mathrm{~A} 13 & 5 & \mathrm{D} 12 & 0\end{array}$

$\begin{array}{llllll}\mathrm{B} 15 & 2 & \mathrm{~A} 14 & 5 & \mathrm{D} 13 & 0\end{array}$

$\begin{array}{llllll}\mathrm{B} 16 & 2 & \mathrm{~A} 15 & 5 & \mathrm{D} 14 & 0\end{array}$

$\begin{array}{llllll}\text { B17 } & 1 & \text { A16 } & 5 & \text { D15 } & 0\end{array}$

$\begin{array}{llllll}\text { B18 } & 2 & \text { A17 } & 1 & \text { D16 } & 0\end{array}$

$\begin{array}{llllll}\text { B19 } & 18 & \text { A18 } & 2 & \text { D17 } & 0\end{array}$

$\begin{array}{llllll}\text { B20 } & 18 & \text { A19 } & 2 & \text { D18 } & 0\end{array}$

$\begin{array}{llllll}\text { B21 } & 19 & \text { A20 } & 18 & \text { D19 } & 0\end{array}$

$\begin{array}{llllll}\text { B22 } & 19 & \text { A21 } & 18 & \text { D20 } & 0\end{array}$

$\begin{array}{llllll}\mathrm{B} 23 & 19 & \mathrm{~A} 22 & 18 & \mathrm{D} 21 & 0\end{array}$

$\begin{array}{llllll}\text { B24 } & 18 & \text { A23 } & 2 & \text { D22 } & 0\end{array}$

$\begin{array}{llllll}\text { B25 } & 19 & \text { A24 } & 18 & \text { D23 } & 0\end{array}$

$\begin{array}{llllll}\text { B26 } & 25 & \text { A25 } & 19 & \text { D24 } & 0\end{array}$
1.50947108

1.09679183

2.28260436

1.40640036

2.60660857

1.41662598

1.51027904 


$\begin{array}{lr}\text { B8 } & 1.08811475 \\ \text { B9 } & 1.08391221 \\ \text { B10 } & 1.08781694 \\ \text { B11 } & 2.30907153 \\ \text { B12 } & 1.39638624 \\ \text { B13 } & 1.09261305 \\ \text { B14 } & 1.08755105 \\ \text { B15 } & 1.08794282 \\ \text { B16 } & 1.0866964 \\ \text { B17 } & 1.39775918 \\ \text { B18 } & 1.39632479 \\ \text { B19 } & 1.09630606 \\ \text { B20 } & 1.50812598 \\ \text { B21 } & 1.08683182 \\ \text { B22 } & 1.08766693 \\ \text { B23 } & 1.08781224 \\ \text { B24 } & 1.41666974 \\ \text { B25 } & 1.41370485 \\ \text { B26 } & 0.97021768 \\ \text { A1 } & 111.85104021 \\ \text { A2 } & 91.63217525 \\ \text { A3 } & 113.53447511 \\ \text { A4 } & 124.66877705 \\ \text { A23 } & 144.53920220 \\ \text { A2 } & 113.70068633 \\ \text { A6 } & 109.40964512 \\ \text { A7 } & 110.89444772 \\ \text { A8 } & 108.45100280 \\ \text { A9 } & 101.56303140 \\ \text { A10 } & 111.21727278 \\ \text { A11 } & 101.88446815 \\ \text { A12 } & 109.37695782 \\ \text { A13 } & 109.62762129 \\ \text { A14 } & 109.94632273 \\ \text { A15 } & 107.83405836 \\ \text { A17 } & \end{array}$




\begin{tabular}{lr} 
D1 & -143.88141956 \\
D2 & 30.64823415 \\
D3 & -150.57376211 \\
D4 & 127.73533205 \\
D5 & -44.51099162 \\
D6 & -51.91847630 \\
D7 & 69.00163962 \\
D8 & -171.20951740 \\
D9 & 51.08012199 \\
D10 & 86.41666343 \\
D11 & -164.82966596 \\
D12 & -171.32052970 \\
D13 & -51.30914384 \\
D14 & 69.05524015 \\
D15 & -125.24244864 \\
D16 & -175.34928977 \\
D17 & 34.72063409 \\
D18 & 158.32180059 \\
D19 & 57.03748031 \\
D20 & 176.95388041 \\
D21 & -62.75901516 \\
D22 & -77.88235106 \\
D23 & -60.76022825 \\
D24 & 90.02412158 \\
\hline
\end{tabular}

Harmonic Vibrational Frequencies $\left(\mathbf{c m}^{-1}\right)$ :

$\begin{array}{lllllllllll}59.11 & 68.03 & 83.83 & 122.13 & 149.81 & 170.16 & 188.28 & 192.54 & 195.04 & 227.24 & 245.63\end{array}$

$\begin{array}{llllllllll}255.36 & 262.46 & 308.38 & 317.68 & 361.07 & 400.56 & 414.89 & 463.80 & 488.19 & 513.15\end{array}$

$\begin{array}{llllllllll}553.55 & 595.05 & 674.22 & 711.99 & 746.59 & 858.96 & 865.48 & 894.38 & 937.59 & 947.32\end{array}$

$\begin{array}{lllllllll}973.90 & 1001.32 & 1012.66 & 1031.09 & 1126.10 & 1130.99 & 1140.60 & 1153.71 & 1162.99\end{array}$

$\begin{array}{llllllllll}1172.05 & 1190.87 & 1197.35 & 1234.37 & 1353.57 & 1359.54 & 1375.34 & 1380.33 & 1399.09\end{array}$

$\begin{array}{lllllllll}1417.45 & 1421.51 & 1429.78 & 1437.92 & 1483.96 & 1484.80 & 1485.99 & 1489.94 & 1498.94\end{array}$

$\begin{array}{lllllllll}1503.33 & 1507.75 & 1520.96 & 3069.13 & 3070.49 & 3075.54 & 3076.95 & 3082.97 & 3109.12\end{array}$

$\begin{array}{llllllll}3149.32 & 3153.30 & 3157.17 & 3169.02 & 3174.87 & 3194.10 & 3700.96 & 3738.65\end{array}$

$\begin{array}{llll}\text { Rotational constants (B/GHZ): } & 1.1154876 & 0.6590922 & 0.4770579\end{array}$

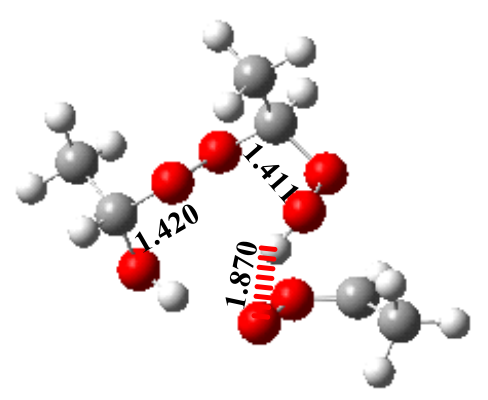

IM6e

$\mathrm{C}$ 


\begin{tabular}{|c|c|c|c|c|c|c|c|}
\hline $\mathrm{H}$ & 1 & B1 & & & & & \\
\hline $\mathrm{O}$ & 1 & B2 & 2 & A1 & & & \\
\hline $\mathrm{O}$ & 1 & B3 & 3 & A2 & 2 & D1 & 0 \\
\hline $\mathrm{O}$ & 1 & B4 & 3 & A3 & 4 & D2 & 0 \\
\hline $\mathrm{H}$ & 4 & B5 & 1 & A4 & 5 & D3 & 0 \\
\hline $\mathrm{C}$ & 5 & B6 & 1 & A5 & 3 & D4 & 0 \\
\hline $\mathrm{C}$ & 7 & B7 & 5 & A6 & 1 & D5 & 0 \\
\hline $\mathrm{H}$ & 8 & B8 & 7 & A7 & 5 & D6 & 0 \\
\hline $\mathrm{H}$ & 8 & B9 & 7 & A8 & 5 & D7 & 0 \\
\hline $\mathrm{H}$ & 8 & B10 & 7 & A9 & 5 & D8 & 0 \\
\hline $\mathrm{O}$ & 7 & B11 & 5 & A10 & 1 & D9 & 0 \\
\hline $\mathrm{O}$ & 7 & B12 & 5 & A11 & 1 & D10 & 0 \\
\hline $\mathrm{C}$ & 1 & B13 & 5 & A12 & 4 & D11 & 0 \\
\hline $\mathrm{H}$ & 14 & B14 & 1 & A13 & 5 & D12 & 0 \\
\hline $\mathrm{H}$ & 14 & B15 & 1 & A14 & 5 & D13 & 0 \\
\hline $\mathrm{H}$ & 14 & B16 & 1 & A15 & 5 & D14 & 0 \\
\hline $\mathrm{O}$ & 1 & B17 & 5 & A16 & 4 & D15 & 0 \\
\hline $\mathrm{C}$ & 3 & B18 & 1 & A17 & 18 & D16 & 0 \\
\hline $\mathrm{H}$ & 19 & B19 & 3 & A18 & 1 & D17 & 0 \\
\hline $\mathrm{C}$ & 19 & B20 & 3 & A19 & 1 & D18 & 0 \\
\hline $\mathrm{H}$ & 21 & B21 & 19 & A20 & 3 & D19 & 0 \\
\hline $\mathrm{H}$ & 21 & B22 & 19 & A 21 & 3 & D20 & 0 \\
\hline $\mathrm{H}$ & 7 & B23 & 5 & A22 & 1 & D21 & 0 \\
\hline $\mathrm{O}$ & 19 & B24 & 3 & A23 & 1 & D22 & 0 \\
\hline $\mathrm{H}$ & 25 & B25 & 19 & A24 & 3 & $\mathrm{D} 23$ & 0 \\
\hline $\mathrm{H}$ & 21 & B26 & 19 & A25 & 3 & D24 & 0 \\
\hline \multicolumn{8}{|c|}{ Variables: } \\
\hline B1 & & 1.09287805 & & & & & \\
\hline B2 & & 2.28915411 & & & & & \\
\hline B3 & & 2.32740991 & & & & & \\
\hline B4 & & 1.41138799 & & & & & \\
\hline B5 & & 0.98085436 & & & & & \\
\hline B6 & & 2.87648149 & & & & & \\
\hline B7 & & 1.46458523 & & & & & \\
\hline B8 & & 1.08457584 & & & & & \\
\hline B9 & & 1.09497181 & & & & & \\
\hline B10 & & 1.09138771 & & & & & \\
\hline B 11 & & 2.23423975 & & & & & \\
\hline B12 & & 1.24111140 & & & & & \\
\hline B 13 & & 1.51181664 & & & & & \\
\hline B14 & & 1.08802348 & & & & & \\
\hline B 15 & & 1.08818186 & & & & & \\
\hline B16 & & 1.08386829 & & & & & \\
\hline B17 & & 1.39787734 & & & & & \\
\hline
\end{tabular}




\begin{tabular}{|c|c|}
\hline B18 & 1.42017120 \\
\hline B19 & 1.09808565 \\
\hline B20 & 1.51097194 \\
\hline B21 & 1.08804482 \\
\hline B22 & 1.08840600 \\
\hline B23 & 1.08576690 \\
\hline B24 & 1.37941385 \\
\hline B25 & 0.97477546 \\
\hline B26 & 1.08713730 \\
\hline $\mathrm{A} 1$ & 138.23943266 \\
\hline $\mathrm{A} 2$ & 76.81222823 \\
\hline $\mathrm{A} 3$ & 102.52179905 \\
\hline A4 & 97.91624670 \\
\hline A5 & 124.87937302 \\
\hline A6 & 87.26993561 \\
\hline A7 & 110.90914727 \\
\hline A8 & 108.04866820 \\
\hline A9 & 108.54877600 \\
\hline A 10 & 74.73980576 \\
\hline A11 & 79.90645347 \\
\hline A 12 & 113.19224751 \\
\hline A13 & 108.99781975 \\
\hline A14 & 108.93335565 \\
\hline A15 & 110.89142838 \\
\hline A16 & 112.05822455 \\
\hline A17 & 143.20635398 \\
\hline A18 & 99.41235488 \\
\hline A19 & 112.81032356 \\
\hline A20 & 109.06092411 \\
\hline A 21 & 110.17331390 \\
\hline A22 & 103.84913444 \\
\hline A23 & 112.30557741 \\
\hline A24 & 108.82945756 \\
\hline A25 & 109.67777597 \\
\hline D1 & -148.72937625 \\
\hline D2 & 23.74068541 \\
\hline D3 & -98.32040905 \\
\hline D4 & 68.66932830 \\
\hline D5 & -170.43838057 \\
\hline D6 & -114.14597933 \\
\hline D7 & 7.26863570 \\
\hline D8 & 122.13040617 \\
\hline D9 & -79.55602163 \\
\hline D10 & -45.69525160 \\
\hline
\end{tabular}




$\begin{array}{lr}\text { D11 } & 50.56670670 \\ \text { D12 } & 56.60101880 \\ \text { D13 } & 176.00920156 \\ \text { D14 } & -63.62836613 \\ \text { D15 } & -80.42537295 \\ \text { D16 } & 3.49026964 \\ \text { D17 } & -178.62272138 \\ \text { D18 } & -61.05797990 \\ \text { D19 } & -172.32074051 \\ \text { D20 } & -52.21439342 \\ \text { D21 } & 65.12697841 \\ \text { D22 } & 62.73877793 \\ \text { D23 } & 40.79450012 \\ \text { D24 } & 68.63581139\end{array}$

Harmonic Vibrational Frequencies $\left(\mathbf{c m}^{-1}\right)$ :

$\begin{array}{lllllllllll}55.79 & 65.10 & 88.11 & 89.50 & 111.28 & 122.32 & 134.82 & 153.70 & 167.93 & 192.02 & 211.17\end{array}$ $\begin{array}{llllllllll}223.37 & 234.73 & 245.50 & 261.91 & 332.51 & 359.85 & 423.37 & 473.69 & 478.96 & 499.79\end{array}$ $\begin{array}{llllllllll}518.47 & 655.38 & 669.19 & 703.62 & 734.43 & 758.68 & 800.42 & 858.65 & 882.68 & 915.94\end{array}$ $\begin{array}{lllllllll}937.31 & 959.16 & 997.41 & 1003.37 & 1018.54 & 1102.23 & 1109.21 & 1126.75 & 1138.82\end{array}$ $\begin{array}{lllllllll}1145.18 & 1170.30 & 1193.31 & 1214.36 & 1335.81 & 1352.34 & 1370.61 & 1375.75 & 1397.35\end{array}$ $\begin{array}{llllllllll}1405.43 & 1420.05 & 1424.76 & 1455.80 & 1477.79 & 1481.39 & 1483.41 & 1494.20 & 1502.68\end{array}$ $\begin{array}{llllllllll}1504.06 & 1549.39 & 1687.48 & 3042.67 & 3043.96 & 3072.79 & 3079.64 & 3103.77 & 3114.91\end{array}$ $\begin{array}{llllllll}3151.69 & 3154.18 & 3161.92 & 3182.46 & 3186.89 & 3206.54 & 3512.81 & 3605.99\end{array}$ Rotational constants (B/GHZ): $\quad 1.0061122 \quad 0.6279030 \quad 0.4466782$

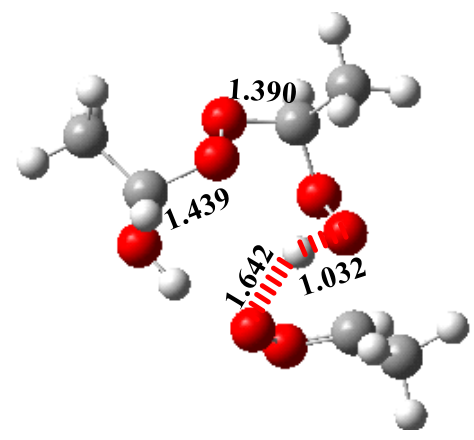

TS6e

$\mathrm{C}$

$\begin{array}{lll}\mathrm{H} & 1 & \mathrm{~B} 1\end{array}$

$\begin{array}{lll}\mathrm{O} & 1 & \mathrm{~B} 2\end{array}$

$\begin{array}{lll}\mathrm{O} & 1 & \mathrm{~B} 3\end{array}$

$\begin{array}{lll}\mathrm{O} & 4 & \mathrm{~B} 4\end{array}$

$\begin{array}{lll}\mathrm{H} & 4 & \text { B5 }\end{array}$

C 4

C $\quad 7$

B6 1

B7 4

B8 7

B9 7

B10 7

A1

A2 $2 \quad$ D1 0

A3 $3 \quad$ D2 0

A4 $5 \quad$ D3 0

A5 $5 \quad$ D4 0

A6 $1 \quad$ D5 0

A7 $4 \quad$ D6 0

$\begin{array}{llllllll}\mathrm{H} & 8 & \text { B9 } & 7 & \text { A8 } & 4 & \text { D7 } & 0\end{array}$

$\begin{array}{ll}\mathrm{H} & 8\end{array}$

A9 4

D8 0 


\begin{tabular}{|c|c|c|c|c|c|c|}
\hline $\mathrm{O}$ & 7 & B11 & 4 & A10 & 1 & D9 \\
\hline $\mathrm{O}$ & 7 & B12 & 4 & A11 & 1 & D10 \\
\hline $\mathrm{C}$ & 1 & B13 & 5 & A 12 & 4 & D11 \\
\hline $\mathrm{H}$ & 14 & B14 & 1 & A13 & 5 & D12 \\
\hline $\mathrm{H}$ & 14 & B15 & 1 & A14 & 5 & D13 \\
\hline $\mathrm{H}$ & 14 & B16 & 1 & A 15 & 5 & D14 \\
\hline $\mathrm{O}$ & 1 & B17 & 5 & A16 & 4 & D15 \\
\hline $\mathrm{C}$ & 3 & B18 & 1 & A 17 & 18 & D16 \\
\hline $\mathrm{H}$ & 19 & B19 & 3 & A18 & 1 & D17 \\
\hline $\mathrm{C}$ & 19 & B20 & 3 & A19 & 1 & D18 \\
\hline $\mathrm{H}$ & 21 & B21 & 19 & A20 & 3 & D19 \\
\hline $\mathrm{H}$ & 21 & B22 & 19 & A 21 & 3 & D20 \\
\hline $\mathrm{H}$ & 7 & B23 & 4 & A22 & 1 & D21 \\
\hline $\mathrm{O}$ & 19 & B24 & 3 & A 23 & 1 & D22 \\
\hline $\mathrm{H}$ & 25 & B25 & 19 & A24 & 3 & D23 \\
\hline $\mathrm{H}$ & 21 & B26 & 19 & A25 & 3 & D24 \\
\hline Varia & & & & & & \\
\hline B1 & \multicolumn{2}{|c|}{1.09183070} & & & & \\
\hline B2 & \multicolumn{2}{|c|}{2.26999537} & & & & \\
\hline B3 & \multicolumn{2}{|c|}{2.33197891} & & & & \\
\hline B4 & \multicolumn{2}{|c|}{1.41685183} & & & & \\
\hline B5 & \multicolumn{2}{|c|}{1.03224466} & & & & \\
\hline B6 & \multicolumn{2}{|c|}{1.90818318} & & & & \\
\hline B7 & \multicolumn{2}{|c|}{1.48722239} & & & & \\
\hline B8 & \multicolumn{2}{|c|}{1.09310614} & & & & \\
\hline B9 & \multicolumn{2}{|c|}{1.08613474} & & & & \\
\hline B10 & \multicolumn{2}{|c|}{1.08375985} & & & & \\
\hline B11 & \multicolumn{2}{|c|}{2.20193964} & & & & \\
\hline B 12 & \multicolumn{2}{|c|}{1.27761968} & & & & \\
\hline B13 & \multicolumn{2}{|c|}{1.51292091} & & & & \\
\hline B14 & \multicolumn{2}{|c|}{1.08802857} & & & & \\
\hline B15 & \multicolumn{2}{|c|}{1.08766103} & & & & \\
\hline B16 & \multicolumn{2}{|c|}{1.08524035} & & & & \\
\hline B17 & \multicolumn{2}{|c|}{1.39039750} & & & & \\
\hline B18 & \multicolumn{2}{|c|}{1.43889897} & & & & \\
\hline B19 & \multicolumn{2}{|c|}{1.09755246} & & & & \\
\hline B20 & \multicolumn{2}{|c|}{1.51013037} & & & & \\
\hline B21 & \multicolumn{2}{|c|}{1.08784287} & & & & \\
\hline B22 & \multicolumn{2}{|c|}{1.08808821} & & & & \\
\hline B23 & \multicolumn{2}{|c|}{1.08826251} & & & & \\
\hline B24 & \multicolumn{2}{|c|}{1.36921798} & & & & \\
\hline B25 & & & & & & \\
\hline B26 & & & & & & \\
\hline $\mathrm{A} 1$ & & 457 & & & & \\
\hline
\end{tabular}




\begin{tabular}{|c|c|}
\hline A2 & 76.01155374 \\
\hline A3 & 34.74418965 \\
\hline A4 & 98.12505653 \\
\hline A5 & 138.06187380 \\
\hline A6 & 95.45304451 \\
\hline A7 & 106.18788181 \\
\hline A8 & 110.02291507 \\
\hline A9 & 111.48452389 \\
\hline A10 & 74.91215455 \\
\hline A11 & 96.25130706 \\
\hline A 12 & 113.36024645 \\
\hline A13 & 109.89326068 \\
\hline A14 & 108.21221162 \\
\hline A15 & 111.07468780 \\
\hline A16 & 111.80704618 \\
\hline A17 & 135.10857019 \\
\hline A18 & 99.08335894 \\
\hline A19 & 111.53133892 \\
\hline A20 & 108.95878301 \\
\hline $\mathrm{A} 21$ & 110.49619002 \\
\hline A22 & 102.49513806 \\
\hline A 23 & 112.49228921 \\
\hline A24 & 107.79815945 \\
\hline A25 & 109.52383848 \\
\hline D1 & -144.82571474 \\
\hline D2 & 121.04252153 \\
\hline D3 & -104.33502524 \\
\hline D4 & -15.01210457 \\
\hline D5 & 173.03637959 \\
\hline D6 & 178.65666916 \\
\hline D7 & -63.84379342 \\
\hline D8 & 60.21832646 \\
\hline D9 & -93.84030288 \\
\hline D10 & -62.24022103 \\
\hline D11 & 39.63074085 \\
\hline D12 & 50.20746535 \\
\hline D13 & 169.61471534 \\
\hline D14 & -70.84640905 \\
\hline D15 & -90.53230189 \\
\hline D16 & 47.86679090 \\
\hline D17 & 157.13107380 \\
\hline D18 & -85.40563862 \\
\hline D19 & -170.56550065 \\
\hline D20 & -50.24430015 \\
\hline
\end{tabular}




$\begin{array}{ll}\text { D21 } & 52.04420862 \\ \text { D22 } & 38.18753161 \\ \text { D23 } & 62.95688675 \\ \text { D24 } & 70.66917103\end{array}$

Harmonic Vibrational Frequencies $\left(\mathbf{c m}^{-\mathbf{1}}\right)$ :

$\begin{array}{lllllllllllr}390.13 i & 55.81 & 63.88 & 80.65 & 104.18 & 117.33 & 123.57 & 154.04 & 174.96 & 195.08 & 229.81 \\ 241.72 & 250.56 & 260.43 & 289.76 & 357.67 & 379.30 & 423.92 & 474.76 & 498.76 & 513.25 \\ 543.20 & 656.37 & 674.97 & 729.89 & 844.59 & 855.83 & 867.82 & 873.86 & 930.52 & 944.55 \\ 954.44 & 985.73 & 989.41 & 1012.64 & 1082.59 & 1101.85 & 1126.71 & 1142.11 & 1146.17 \\ 1165.80 & 1170.90 & 1195.94 & 1217.34 & 1351.64 & 1354.95 & 1357.54 & 1375.16 & 1399.99 \\ 1408.94 & 1414.18 & 1425.70 & 1452.22 & 1481.34 & 1486.59 & 1489.22 & 1496.76 & 1502.19 \\ 1507.28 & 1522.96 & 1577.71 & 2656.54 & 3055.18 & 3057.91 & 3073.70 & 3077.09 & 3116.16 \\ 3143.01 & 3150.37 & 3154.75 & 3169.13 & 3173.39 & 3187.44 & 3200.90 & 3461.20 & \\ \text { Rotational constants (B/GHZ): } & 1.0841080 & 0.6088603 & 0.4437443 & \end{array}$

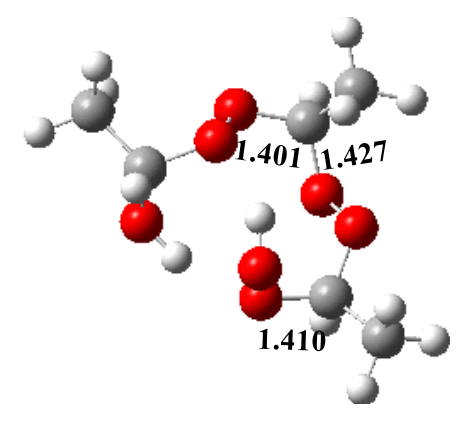

P6e

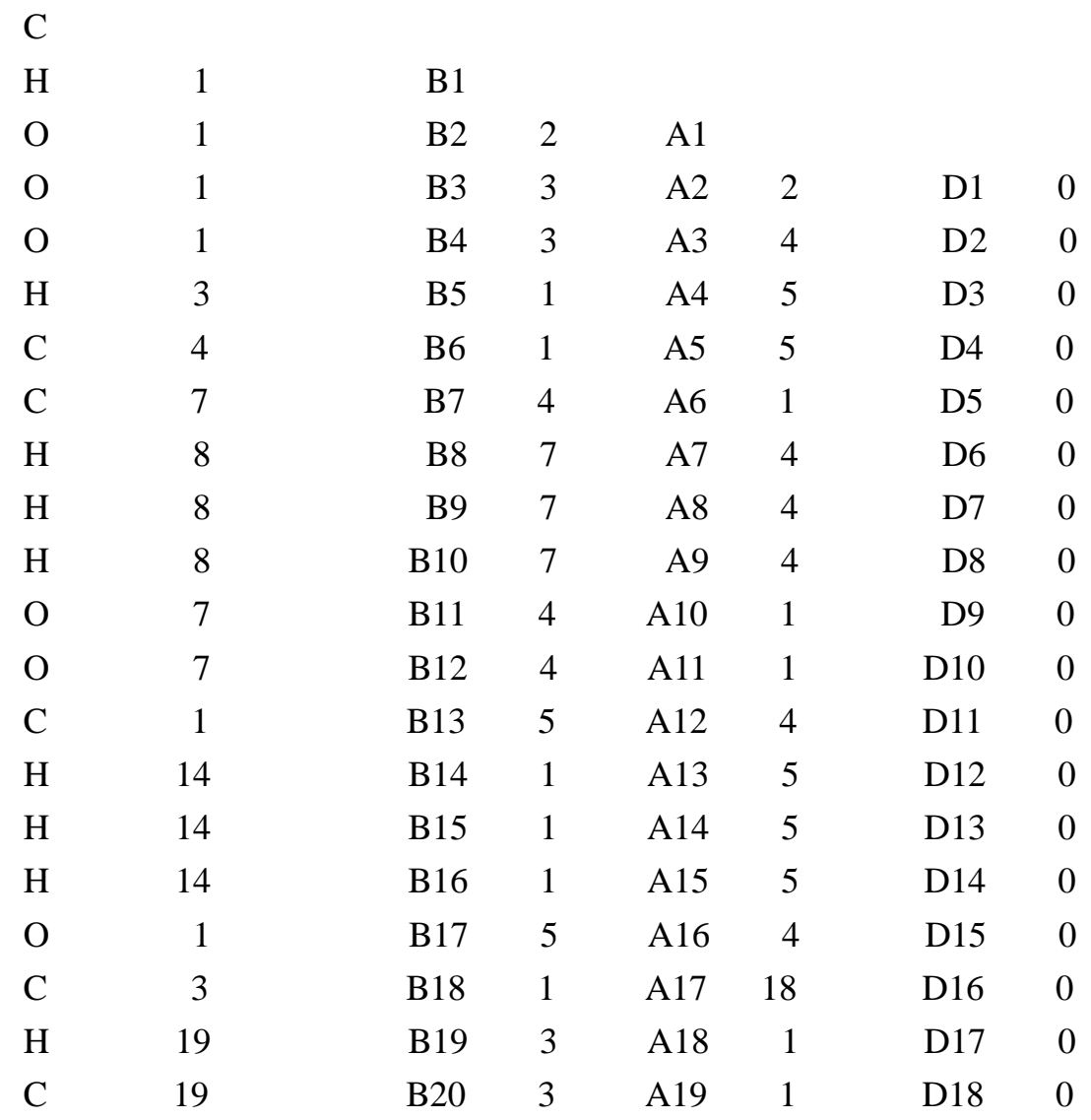




$\begin{array}{rrrrrrrr}\mathrm{H} & 21 & \text { B21 } & 19 & \text { A20 } & 3 & \text { D19 } & 0 \\ \mathrm{H} & 21 & \text { B22 } & 19 & \text { A21 } & 3 & \text { D20 } & 0 \\ \mathrm{H} & 7 & \text { B23 } & 4 & \text { A22 } & 1 & \text { D21 } & 0 \\ \mathrm{O} & 19 & \text { B24 } & 3 & \text { A23 } & 1 & \text { D22 } & 0 \\ \mathrm{H} & 25 & \text { B25 } & 19 & \text { A24 } & 3 & \text { D23 } & 0 \\ \mathrm{H} & 21 & \text { B26 } & 19 & \text { A25 } & 3 & \text { D24 } & 0\end{array}$

Variables:

B

B2

B3

B4

B5

B6

B7

B8

B9

B10

B11

B12

B13

B14

B15

B16

B17

B18

B19

B20

B21

B22

B23

B24

B25

B26

A1

A2

A3

A4

A5

A6

A7

A8

A9

A10

A11
1.09232763

2.30233071

2.33228700

1.40996525

1.97306172

1.39610042

1.51052173

1.08747823

1.08717823

1.08672329

2.30015912

1.40984273

1.51263265

1.08746020

1.08735704

1.08574228

1.40129841

1.43517979

1.09686337

1.50851603

1.08777716

1.08793676

1.09180806

1.37686715

0.96634036

1.08716126

136.74781942

85.72663493

96.53163235

98.49830547

133.32416850

106.48182653

108.88720199

109.62554151

110.23350803

91.97734485

111.25353859 


\begin{tabular}{|c|c|}
\hline A12 & 114.34047917 \\
\hline A13 & 110.04882641 \\
\hline A14 & 108.08655297 \\
\hline A15 & 111.11239609 \\
\hline A16 & 112.07936758 \\
\hline A17 & 133.08536765 \\
\hline A18 & 99.98939102 \\
\hline A19 & 111.88729009 \\
\hline A20 & 109.03755764 \\
\hline $\mathrm{A} 21$ & 110.20663278 \\
\hline A 22 & 110.17779251 \\
\hline A 23 & 112.03845032 \\
\hline A 24 & 108.73204654 \\
\hline A 25 & 109.59702317 \\
\hline D1 & -151.48933084 \\
\hline D2 & 33.30427955 \\
\hline D3 & 44.35298943 \\
\hline D4 & -50.42518604 \\
\hline D5 & -170.55331049 \\
\hline D6 & 174.14782924 \\
\hline D7 & -65.96897981 \\
\hline D8 & 54.59466690 \\
\hline D9 & -77.56696189 \\
\hline D10 & -46.89074661 \\
\hline D11 & 24.23946572 \\
\hline D12 & 50.93900595 \\
\hline D13 & 170.66480832 \\
\hline D14 & -69.64377409 \\
\hline D15 & -106.24356188 \\
\hline D16 & 50.96499844 \\
\hline D17 & 154.39545515 \\
\hline D18 & -87.47126171 \\
\hline D19 & -172.58558718 \\
\hline D20 & -52.46034473 \\
\hline D21 & 66.50759224 \\
\hline D22 & 35.40109965 \\
\hline D23 & 53.76696301 \\
\hline D24 & 68.31648381 \\
\hline
\end{tabular}

Harmonic Vibrational Frequencies $\left(\mathbf{c m}^{-1}\right)$ :

$\begin{array}{lllllllllll}58.04 & 73.13 & 100.93 & 117.40 & 140.57 & 158.65 & 188.44 & 201.84 & 225.77 & 239.40 & 245.18\end{array}$ $\begin{array}{llllllllll}260.82 & 278.30 & 311.16 & 347.81 & 400.38 & 428.62 & 469.19 & 493.20 & 521.50 & 552.25\end{array}$ $\begin{array}{llllllllll}575.13 & 633.00 & 680.29 & 701.42 & 716.79 & 867.54 & 872.22 & 884.62 & 936.70 & 949.90\end{array}$ $\begin{array}{lllllllll}959.39 & 998.67 & 1011.85 & 1032.27 & 1092.68 & 1139.62 & 1141.92 & 1145.92 & 1147.70\end{array}$ $\begin{array}{lllllllll}1162.63 & 1190.10 & 1210.17 & 1220.67 & 1331.35 & 1356.20 & 1363.53 & 1374.46 & 1391.45\end{array}$ 


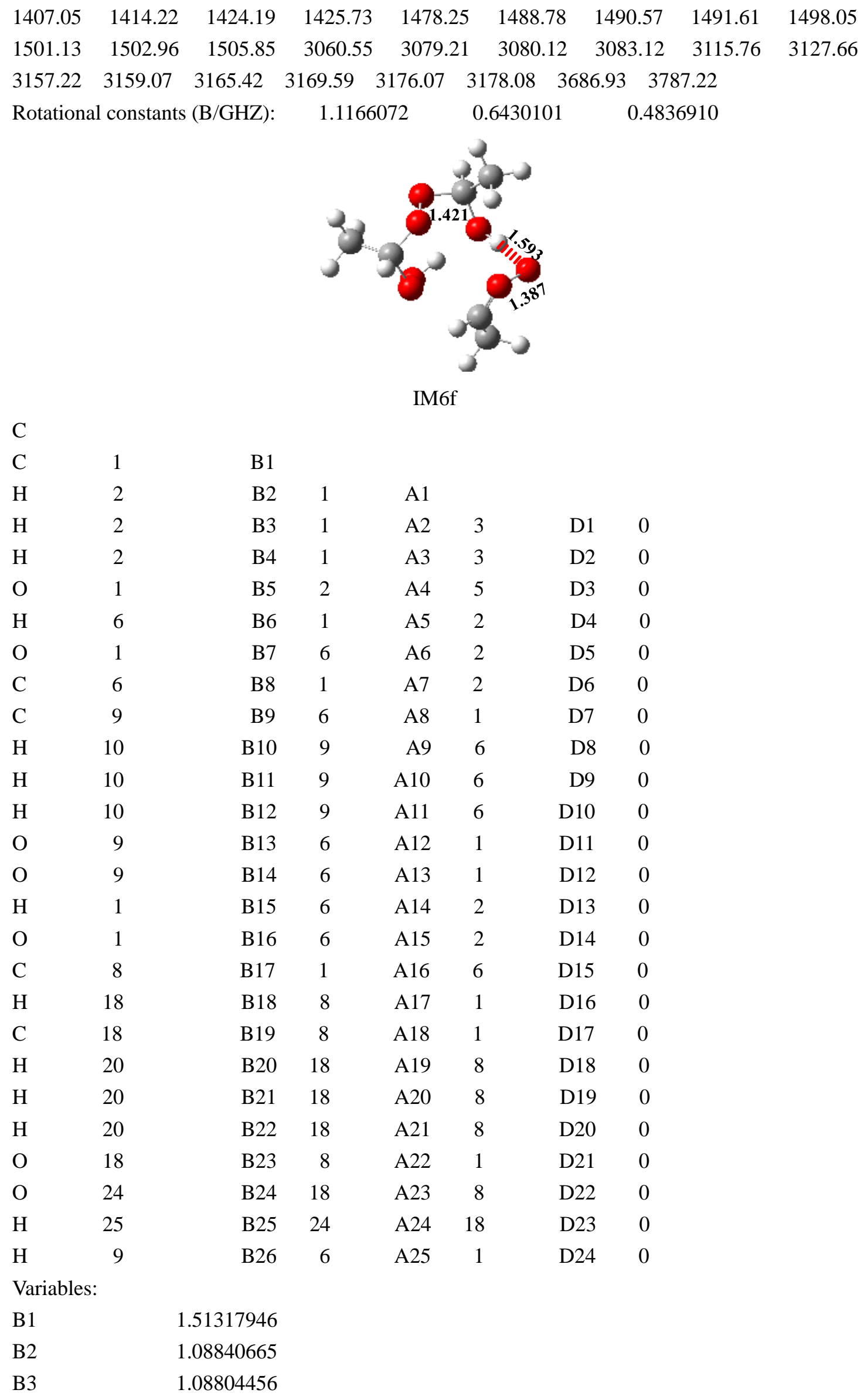




\begin{tabular}{|c|c|}
\hline B4 & 1.08735218 \\
\hline B5 & 1.38994699 \\
\hline B6 & 1.00266894 \\
\hline B7 & 2.30937866 \\
\hline B8 & 3.50139576 \\
\hline B9 & 1.46538382 \\
\hline B10 & 1.08427247 \\
\hline B11 & 1.09107278 \\
\hline B12 & 1.09243110 \\
\hline B13 & 2.23403837 \\
\hline B14 & 1.24319194 \\
\hline B15 & 1.09320928 \\
\hline B16 & 1.42104172 \\
\hline B17 & 1.40372630 \\
\hline B18 & 1.09357910 \\
\hline B19 & 1.51040284 \\
\hline B20 & 1.08794535 \\
\hline B21 & 1.08379688 \\
\hline B22 & 1.08784128 \\
\hline B23 & 1.41121193 \\
\hline B24 & 1.41678031 \\
\hline B25 & 0.98432789 \\
\hline B26 & 1.08479251 \\
\hline A1 & 109.42012466 \\
\hline $\mathrm{A} 2$ & 109.58667469 \\
\hline A3 & 110.50885470 \\
\hline A4 & 112.95115304 \\
\hline A5 & 111.58793350 \\
\hline A6 & 95.87010109 \\
\hline A7 & 117.96084043 \\
\hline A8 & 81.24858666 \\
\hline A9 & 110.80469456 \\
\hline A10 & 108.91235270 \\
\hline A11 & 108.13867789 \\
\hline A12 & 47.67376362 \\
\hline A 13 & 62.45409136 \\
\hline A 14 & 108.59445287 \\
\hline A 15 & 110.73259313 \\
\hline A16 & 125.88854598 \\
\hline A 17 & 102.03442601 \\
\hline A 18 & 114.05630846 \\
\hline A19 & 108.86826981 \\
\hline A20 & 110.81123173 \\
\hline A21 & 109.04144873 \\
\hline
\end{tabular}




$\begin{array}{lr}\text { A22 } & 112.25120391 \\ \text { A23 } & 111.48540253 \\ \text { A24 } & 101.35633255 \\ \text { A25 } & 131.26879988 \\ \text { D1 } & 120.00122761 \\ \text { D2 } & -119.30453605 \\ \text { D3 } & -58.76844478 \\ \text { D4 } & 16.46728057 \\ \text { D5 } & -93.43469368 \\ \text { D6 } & 63.78291555 \\ \text { D7 } & 168.12873958 \\ \text { D8 } & -132.77243000 \\ \text { D9 } & -11.41230641 \\ \text { D10 } & 103.86967944 \\ \text { D11 } & -94.73610220 \\ \text { D12 } & -57.06143004 \\ \text { D13 } & 123.89699981 \\ \text { D14 } & -127.08462855 \\ \text { D15 } & -44.86832325 \\ \text { D16 } & 156.57329203 \\ \text { D17 } & -83.10359704 \\ \text { D18 } & -54.80076772 \\ \text { D19 } & 65.47253030 \\ \text { D20 } & -174.36245236 \\ \text { D21 } & 47.71259707 \\ \text { D22 } & -83.31110651 \\ \text { D23 } & 79.17719125 \\ \text { D24 } & 40.35781894 \\ \text { D } & \end{array}$

Harmonic Vibrational Frequencies (cm-1):

$\begin{array}{lllllllllllr}31.57 & 45.37 & 71.60 & 91.32 & 119.94 & 132.05 & 140.87 & 152.61 & 167.69 & 211.71 & 217.06 \\ 237.67 & 261.70 & 275.17 & 287.55 & 332.27 & 335.39 & 436.93 & 471.19 & 504.60 & 509.93 \\ 527.62 & 672.72 & 680.11 & 737.88 & 800.04 & 824.14 & 851.52 & 868.43 & 906.39 & 932.22 \\ 954.67 & 991.31 & 1003.97 & 1021.41 & 1046.37 & 1099.36 & 1111.88 & 1120.26 & 1142.34 \\ 1148.36 & 1163.63 & 1194.48 & 1200.61 & 1355.07 & 1358.66 & 1365.24 & 1379.19 & 1409.44 \\ 1413.75 & 1421.13 & 1425.81 & 1444.10 & 1462.90 & 1488.54 & 1490.84 & 1491.18 & 1502.12 \\ 1506.04 & 1549.23 & 1680.31 & 2965.70 & 3060.73 & 3075.03 & 3086.22 & 3100.16 & 3105.55 \\ 3120.29 & 3152.80 & 3160.94 & 3172.49 & 3185.69 & 3195.49 & 3203.18 & 3403.81 & \\ \text { Rotational constants (B/GHZ): } & 0.9289128 & 0.7260303 & 0.5181500 & \end{array}$




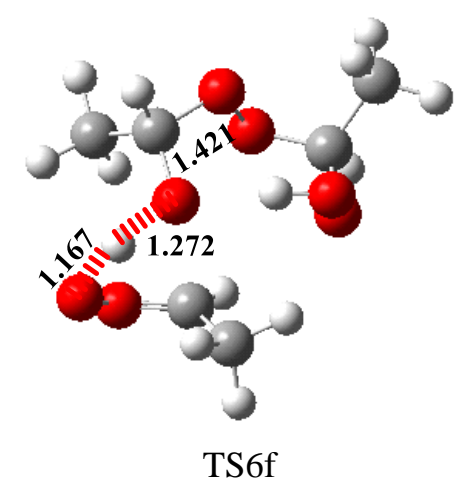

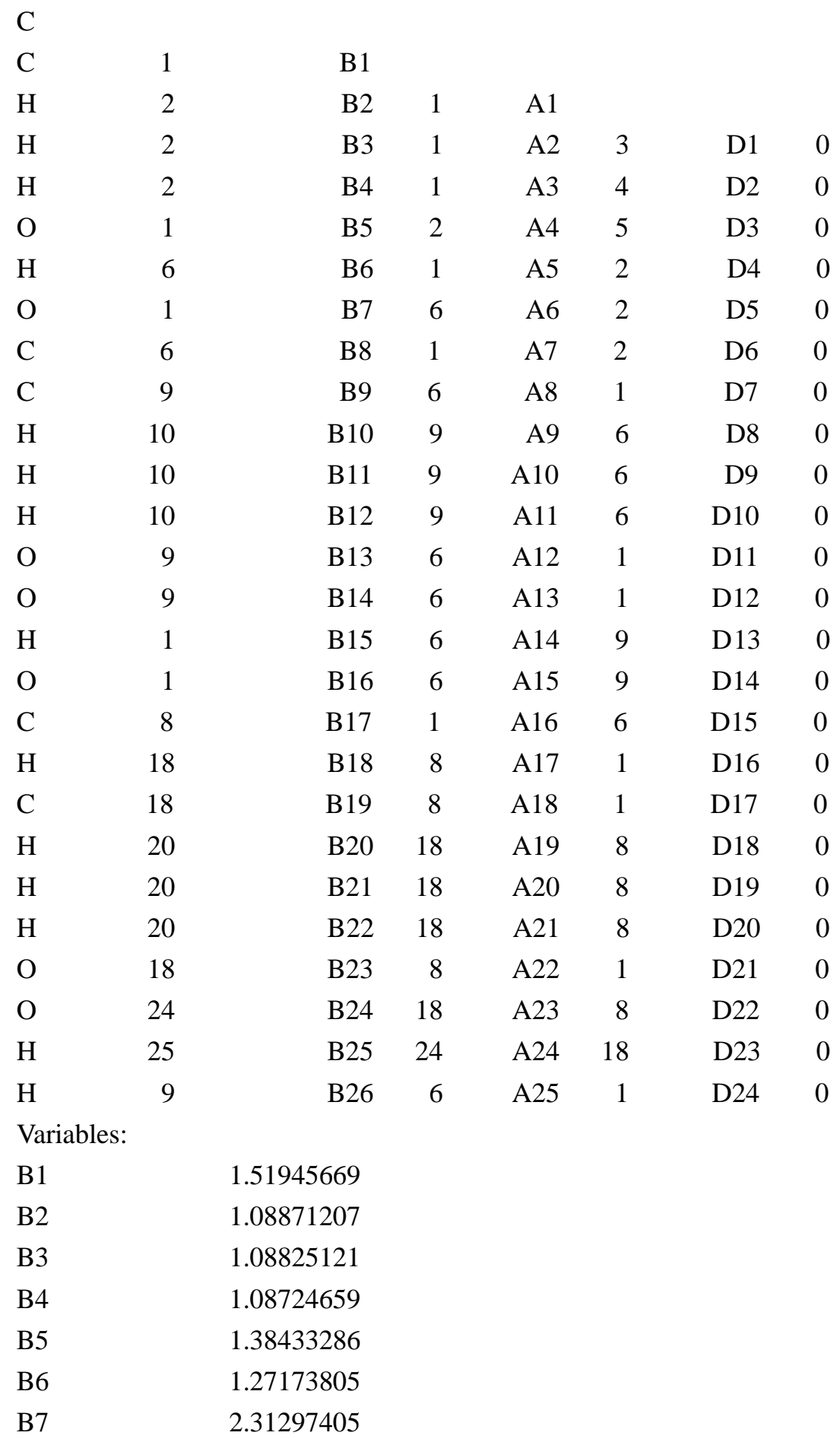




\begin{tabular}{|c|c|}
\hline B8 & 2.23404085 \\
\hline B9 & 1.47195852 \\
\hline B10 & 1.08465653 \\
\hline B11 & 1.08190763 \\
\hline B12 & 1.09769797 \\
\hline B13 & 2.20525129 \\
\hline B14 & 1.26934601 \\
\hline B15 & 1.09796198 \\
\hline B16 & 1.42132035 \\
\hline B17 & 1.40701808 \\
\hline B18 & 1.09332762 \\
\hline B19 & 1.51059492 \\
\hline B20 & 1.08803203 \\
\hline B21 & 1.08383621 \\
\hline B22 & 1.08799779 \\
\hline B23 & 1.40563187 \\
\hline B24 & 1.41980350 \\
\hline B 25 & 0.98322305 \\
\hline B26 & 1.08735213 \\
\hline $\mathrm{A} 1$ & 110.12805894 \\
\hline $\mathrm{A} 2$ & 109.15654378 \\
\hline A3 & 110.84255920 \\
\hline A4 & 114.56556539 \\
\hline A5 & 112.12305452 \\
\hline A6 & 95.26985871 \\
\hline A7 & 122.34605065 \\
\hline A8 & 89.75025650 \\
\hline A9 & 110.18982712 \\
\hline A10 & 112.80553914 \\
\hline A11 & 104.16801365 \\
\hline A 12 & 64.93465324 \\
\hline A 13 & 87.42144854 \\
\hline A14 & 109.66080751 \\
\hline A15 & 110.76579092 \\
\hline A16 & 125.50177285 \\
\hline A 17 & 102.09134642 \\
\hline A18 & 113.77903694 \\
\hline A19 & 108.92196209 \\
\hline A20 & 110.87364085 \\
\hline A21 & 108.99880677 \\
\hline A22 & 111.85415920 \\
\hline A23 & 111.01367568 \\
\hline A24 & 101.28615866 \\
\hline A25 & 110.65362431 \\
\hline
\end{tabular}




$\begin{array}{lr}\text { D1 } & 119.90813905 \\ \text { D2 } & 119.68581317 \\ \text { D3 } & -63.24791476 \\ \text { D4 } & -36.20712732 \\ \text { D5 } & -93.37049852 \\ \text { D6 } & 41.49089783 \\ \text { D7 } & 164.87945943 \\ \text { D8 } & -70.88968758 \\ \text { D9 } & 55.03284894 \\ \text { D10 } & 173.55409655 \\ \text { D11 } & -100.12047293 \\ \text { D12 } & -69.72227892 \\ \text { D13 } & 165.58164355 \\ \text { D14 } & -85.17236538 \\ \text { D15 } & -47.02257099 \\ \text { D16 } & 153.79254788 \\ \text { D17 } & -86.04426095 \\ \text { D18 } & -56.21825952 \\ \text { D19 } & 64.21943886 \\ \text { D20 } & -175.71883353 \\ \text { D21 } & 44.70039168 \\ \text { D22 } & -83.43709024 \\ \text { D23 } & 81.82184920 \\ \text { D24 } & 43.06193513\end{array}$

Harmonic Vibrational Frequencies $\left(\mathbf{c m}^{-1}\right)$ :

$\begin{array}{lccccccccccr}882.91 i & 46.69 & 81.47 & 100.00 & 117.51 & 130.39 & 151.83 & 178.27 & 197.68 & 220.54 & 236.66 \\ 255.13 & 271.32 & 277.10 & 303.64 & 337.33 & 350.07 & 413.46 & 470.57 & 511.11 & 520.60 \\ 527.14 & 636.46 & 671.42 & 733.70 & 745.21 & 823.76 & 854.05 & 855.17 & 876.30 & 928.49 \\ 941.54 & 954.04 & 957.34 & 988.74 & 1018.19 & 1083.42 & 1110.58 & 1138.97 & 1154.51 & 1160.30 \\ 1175.91 & 1193.14 & 1218.87 & 1353.17 & 1360.83 & 1370.43 & 1377.26 & 1397.77 & 1407.20 \\ 1414.33 & 1422.65 & 1429.22 & 1459.43 & 1473.93 & 1484.98 & 1492.23 & 1499.79 & 1502.47 \\ 1540.53 & 1607.43 & 1868.10 & 3026.72 & 3046.64 & 3065.13 & 3091.33 & 3103.64 & 3141.75 \\ 3154.29 & 3155.10 & 3167.30 & 3195.89 & 3204.14 & 3217.29 & 3443.30 & & \end{array}$

Rotational constants (B/GHZ): $\quad 1.2463340 \quad 0.5375475 \quad 0.4463976$

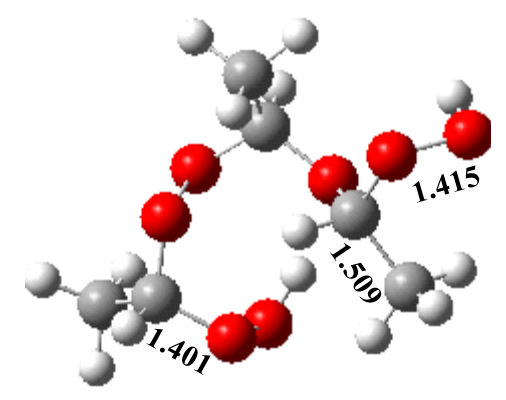

P6f

$\mathrm{C}$ 


\begin{tabular}{|c|c|c|c|c|c|c|c|}
\hline $\mathrm{C}$ & 1 & B1 & & & & & \\
\hline $\mathrm{H}$ & 2 & B2 & 1 & $\mathrm{~A} 1$ & & & \\
\hline $\mathrm{H}$ & 2 & B3 & 1 & A2 & 3 & D1 & 0 \\
\hline $\mathrm{H}$ & 2 & B4 & 1 & A3 & 4 & D2 & 0 \\
\hline $\mathrm{O}$ & 1 & B5 & 2 & A4 & 5 & D3 & 0 \\
\hline $\mathrm{H}$ & 6 & B6 & 1 & A5 & 2 & D4 & 0 \\
\hline $\mathrm{O}$ & 1 & B7 & 6 & A6 & 2 & D5 & 0 \\
\hline $\mathrm{C}$ & 6 & B8 & 1 & A7 & 2 & D6 & 0 \\
\hline $\mathrm{C}$ & 9 & B9 & 6 & A8 & 1 & D7 & 0 \\
\hline $\mathrm{H}$ & 10 & B10 & 9 & A9 & 6 & D8 & 0 \\
\hline $\mathrm{H}$ & 10 & B11 & 9 & A10 & 6 & D9 & 0 \\
\hline $\mathrm{H}$ & 10 & B12 & 9 & A11 & 6 & D10 & 0 \\
\hline $\mathrm{O}$ & 9 & B13 & 6 & A12 & 1 & D11 & 0 \\
\hline $\mathrm{O}$ & 9 & B14 & 6 & A13 & 1 & $\mathrm{D} 12$ & 0 \\
\hline $\mathrm{H}$ & 1 & B15 & 6 & A14 & 9 & D13 & 0 \\
\hline $\mathrm{O}$ & 1 & B16 & 6 & A15 & 9 & D14 & 0 \\
\hline $\mathrm{C}$ & 8 & B 17 & 1 & A 16 & 17 & D15 & 0 \\
\hline $\mathrm{H}$ & 18 & B18 & 8 & A17 & 1 & D16 & 0 \\
\hline $\mathrm{C}$ & 18 & B19 & 8 & A18 & 1 & D17 & 0 \\
\hline $\mathrm{H}$ & 20 & B20 & 18 & A19 & 8 & D18 & 0 \\
\hline $\mathrm{H}$ & 20 & B21 & 18 & A20 & 8 & D19 & 0 \\
\hline $\mathrm{H}$ & 20 & B22 & 18 & A21 & 8 & D20 & 0 \\
\hline $\mathrm{O}$ & 18 & B23 & 8 & A22 & 1 & D21 & 0 \\
\hline $\mathrm{O}$ & 24 & B24 & 18 & A23 & 8 & D22 & 0 \\
\hline $\mathrm{H}$ & 25 & B25 & 24 & A24 & 18 & D23 & 0 \\
\hline $\mathrm{H}$ & 9 & B26 & 6 & A25 & 1 & D24 & 0 \\
\hline
\end{tabular}

Variables:

$\begin{array}{ll}\text { B1 } & 1.51498467 \\ \text { B2 } & 1.08677924 \\ \text { B3 } & 1.08813882 \\ \text { B4 } & 1.08591682 \\ \text { B5 } & 1.41325137 \\ \text { B6 } & 2.54139921 \\ \text { B7 } & 2.29632938 \\ \text { B8 } & 1.41992540 \\ \text { B9 } & 1.50924210 \\ \text { B10 } & 1.08633924 \\ \text { B11 } & 1.08689815 \\ \text { B12 } & 1.08801150 \\ \text { B13 } & 2.30376784 \\ \text { B14 } & 1.40671765 \\ \text { B15 } & 1.09163490 \\ \text { B16 } & 1.40697681 \\ \text { B17 } & 1.41292654\end{array}$




\begin{tabular}{|c|c|}
\hline B 18 & 1.09260695 \\
\hline B19 & 1.51009170 \\
\hline B20 & 1.08780868 \\
\hline B21 & 1.08428702 \\
\hline B22 & 1.08786834 \\
\hline B23 & 1.40133909 \\
\hline B24 & 1.41747588 \\
\hline B 25 & 0.97125762 \\
\hline B26 & 1.09065905 \\
\hline $\mathrm{A} 1$ & 110.44914362 \\
\hline $\mathrm{A} 2$ & 107.61534911 \\
\hline A3 & 112.10535128 \\
\hline $\mathrm{A} 4$ & 116.15703638 \\
\hline A5 & 107.46767446 \\
\hline A6 & 97.83374265 \\
\hline A7 & 120.22645770 \\
\hline A8 & 107.89479500 \\
\hline A9 & 109.90235176 \\
\hline A 10 & 110.14843666 \\
\hline A11 & 108.84722506 \\
\hline A 12 & 94.55060324 \\
\hline A13 & 110.24273645 \\
\hline A14 & 104.95547753 \\
\hline A15 & 110.74457370 \\
\hline A16 & 126.48827666 \\
\hline A17 & 101.59393520 \\
\hline A18 & 113.28349727 \\
\hline A19 & 108.83538156 \\
\hline A 20 & 110.97051647 \\
\hline A 21 & 109.10482545 \\
\hline A22 & 111.74171429 \\
\hline A 23 & 111.31502611 \\
\hline A24 & 101.52659488 \\
\hline A 25 & 110.63759257 \\
\hline D1 & 119.36553687 \\
\hline D2 & 119.50005045 \\
\hline D3 & -60.99070410 \\
\hline D4 & -50.06901703 \\
\hline D5 & -94.65585259 \\
\hline D6 & 31.99955658 \\
\hline D7 & 162.08059276 \\
\hline D8 & -70.76436575 \\
\hline D9 & 50.22145138 \\
\hline D10 & 169.50567890 \\
\hline
\end{tabular}




$\begin{array}{lr}\text { D11 } & -106.66555970 \\ \text { D12 } & -74.04722996 \\ \text { D13 } & 154.12231247 \\ \text { D14 } & -97.66508603 \\ \text { D15 } & 68.78299630 \\ \text { D16 } & 149.83347403 \\ \text { D17 } & -90.17335955 \\ \text { D18 } & -58.82538710 \\ \text { D19 } & 61.67673085 \\ \text { D20 } & -178.31895720 \\ \text { D21 } & 40.68965694 \\ \text { D22 } & -87.70985558 \\ \text { D23 } & 88.47478270 \\ \text { D24 } & 39.13642512\end{array}$

Harmonic Vibrational Frequencies $\left(\mathbf{c m}^{-1}\right)$ :

$\begin{array}{lllllllllll}58.10 & 82.69 & 105.54 & 109.39 & 127.57 & 169.58 & 182.65 & 195.56 & 211.34 & 229.97 & 249.58\end{array}$ $\begin{array}{llllllllll}256.12 & 265.10 & 281.63 & 333.86 & 339.31 & 374.63 & 407.05 & 463.47 & 506.86 & 528.11\end{array}$ $\begin{array}{llllllllll}590.16 & 594.78 & 691.50 & 721.56 & 739.99 & 824.01 & 855.76 & 891.31 & 931.29 & 932.58\end{array}$ $\begin{array}{lllllllll}959.08 & 993.29 & 1016.89 & 1020.75 & 1101.81 & 1128.74 & 1140.24 & 1148.83 & 1157.81\end{array}$ $\begin{array}{llllllllll}1159.86 & 1187.26 & 1195.81 & 1213.31 & 1363.16 & 1368.24 & 1374.19 & 1384.75 & 1388.43\end{array}$ $\begin{array}{llllllllll}1415.10 & 1424.94 & 1428.32 & 1431.03 & 1437.76 & 1478.95 & 1486.30 & 1487.54 & 1494.99\end{array}$ $\begin{array}{llllllllll}1500.56 & 1506.00 & 1507.20 & 3078.88 & 3081.84 & 3082.56 & 3106.83 & 3112.98 & 3143.83\end{array}$ $\begin{array}{llllllll}3157.01 & 3159.00 & 3160.18 & 3174.23 & 3175.68 & 3195.44 & 3700.26 & 3805.64\end{array}$ $\begin{array}{lllll}\text { Rotational constants (B/GHZ): } & 1.3417004 & 0.5635910 & 0.4565588\end{array}$

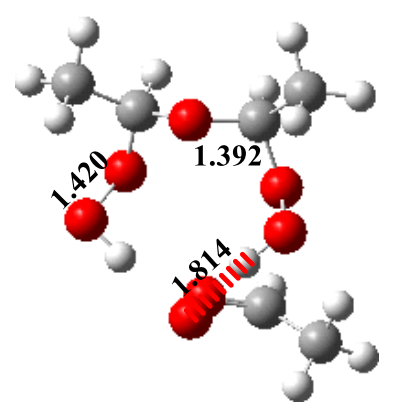

IM6g

$\mathrm{C}$

$\begin{array}{lll}\mathrm{H} & 1 & \mathrm{~B} 1\end{array}$

$\begin{array}{lll}\mathrm{O} & 1 & \mathrm{~B} 2\end{array}$

$\begin{array}{lll}\mathrm{O} & 1 & \mathrm{~B} 3\end{array}$

$\mathrm{H} \quad \mathrm{B} 4 \quad 1$

C 4

B5 1

B6 4

B7 6

B8 6

B9 6

B10 4

A1

A2 $2 \quad$ D1 0

A3 $4 \quad$ D2 0

A4 $3 \quad$ D3 0

A5 $1 \quad$ D4 0

A6 4 D5 0

$\mathrm{H} \quad 7$

A7 4 D6 0

$\mathrm{H} \quad 7$

A8 4

D7 0

O 6

A9 1

D8 0 


$\begin{array}{rr}\mathrm{O} & 6 \\ \mathrm{H} & 6 \\ \mathrm{C} & 1 \\ \mathrm{H} & 14 \\ \mathrm{H} & 14 \\ \mathrm{H} & 14 \\ \mathrm{O} & 1 \\ \mathrm{C} & 18 \\ \mathrm{H} & 19 \\ \mathrm{C} & 19 \\ \mathrm{H} & 21 \\ \mathrm{H} & 21 \\ \mathrm{H} & 21 \\ \mathrm{O} & 19 \\ \mathrm{O} & 25 \\ \mathrm{H} & 26\end{array}$

$\begin{array}{ll}\text { B11 } & 4 \\ \text { B12 } & 4 \\ \text { B13 } & 4 \\ \text { B14 } & 1 \\ \text { B15 } & 1 \\ \text { B16 } & 1 \\ \text { B17 } & 4 \\ \text { B18 } & 1 \\ \text { B19 } & 18 \\ \text { B20 } & 18 \\ \text { B21 } & 19 \\ \text { B22 } & 19 \\ \text { B23 } & 19 \\ \text { B24 } & 18 \\ \text { B25 } & 19 \\ \text { B26 } & 25\end{array}$

$\begin{array}{rrrr}\text { A10 } & 1 & \text { D9 } & 0 \\ \text { A11 } & 1 & \text { D10 } & 0 \\ \text { A12 } & 3 & \text { D11 } & 0 \\ \text { A13 } & 4 & \text { D12 } & 0 \\ \text { A14 } & 4 & \text { D13 } & 0 \\ \text { A15 } & 4 & \text { D14 } & 0 \\ \text { A16 } & 3 & \text { D15 } & 0 \\ \text { A17 } & 4 & \text { D16 } & 0 \\ \text { A18 } & 1 & \text { D17 } & 0 \\ \text { A19 } & 1 & \text { D18 } & 0 \\ \text { A20 } & 18 & \text { D19 } & 0 \\ \text { A21 } & 18 & \text { D20 } & 0 \\ \text { A22 } & 18 & \text { D21 } & 0 \\ \text { A23 } & 1 & \text { D22 } & 0 \\ \text { A24 } & 18 & \text { D23 } & 0 \\ \text { A25 } & 19 & \text { D24 } & 0\end{array}$

Variables:

$\begin{array}{lr}\text { B1 } & 1.09649557 \\ \text { B2 } & 2.30176060 \\ \text { B3 } & 1.41453505 \\ \text { B4 } & 0.97965383 \\ \text { B5 } & 2.94837541 \\ \text { B6 } & 1.46426993 \\ \text { B7 } & 1.09593887 \\ \text { B8 } & 1.09064721 \\ \text { B9 } & 1.08489583 \\ \text { B10 } & 2.24069886 \\ \text { B11 } & 1.24078167 \\ \text { B12 } & 1.08600085 \\ \text { B13 } & 1.51133996 \\ \text { B14 } & 1.08647652 \\ \text { B15 } & 1.08824007 \\ \text { B16 } & 1.08801230 \\ \text { B17 } & 1.39203470 \\ \text { B18 } & 1.40582584 \\ \text { B19 } & 1.09666383 \\ \text { B20 } & 1.51028999 \\ \text { B21 } & 1.08787731 \\ \text { B22 } & 1.08787791 \\ \text { B23 } & 1.08597768 \\ \text { B24 } & 1.40027257 \\ \text { B25 } & 1.41993205 \\ \text { B26 } & 0.97994335 \\ \text { A1 } & 136.91515798 \\ \end{array}$




\begin{tabular}{|c|c|}
\hline A 2 & 35.81860431 \\
\hline A3 & 109.03741938 \\
\hline A4 & 149.11004085 \\
\hline A5 & 87.07937420 \\
\hline A6 & 108.02682625 \\
\hline A7 & 108.86201020 \\
\hline A8 & 110.69569445 \\
\hline A9 & 70.16420026 \\
\hline A10 & 76.28002436 \\
\hline A11 & 107.84233799 \\
\hline A12 & 112.06744738 \\
\hline A13 & 109.54137976 \\
\hline A14 & 110.01138457 \\
\hline A15 & 109.39349606 \\
\hline A16 & 112.82265571 \\
\hline A17 & 117.23992274 \\
\hline A18 & 109.32557199 \\
\hline A19 & 107.68837284 \\
\hline A20 & 109.38144684 \\
\hline A 21 & 109.60996170 \\
\hline A 22 & 109.73842838 \\
\hline A 23 & 113.17843915 \\
\hline A24 & 109.35286247 \\
\hline A 25 & 101.53919946 \\
\hline D1 & 8.84543864 \\
\hline D2 & -80.28135318 \\
\hline D3 & 128.46414001 \\
\hline D4 & -175.75043141 \\
\hline D5 & -2.18580904 \\
\hline D6 & 112.91139840 \\
\hline D7 & -123.17636861 \\
\hline D8 & -83.72229657 \\
\hline D9 & -49.69669308 \\
\hline D10 & 59.54977740 \\
\hline D11 & 54.60925713 \\
\hline D12 & -70.77187038 \\
\hline D13 & 49.39208897 \\
\hline D14 & 169.75182556 \\
\hline D15 & -68.04913608 \\
\hline D16 & -86.03575549 \\
\hline D17 & -36.76989291 \\
\hline D18 & -157.94346289 \\
\hline D19 & 64.92834302 \\
\hline D20 & -174.76444587 \\
\hline
\end{tabular}




$\begin{array}{lr}\text { D21 } & -54.97628096 \\ \text { D22 } & 75.81412038 \\ \text { D23 } & 75.87758265 \\ \text { D24 } & -112.35655170\end{array}$

Harmonic Vibrational Frequencies $\left(\mathbf{c m}^{-\mathbf{1}}\right)$ :

\begin{tabular}{lccccccccccc}
41.54 & 52.20 & 66.76 & 76.75 & 105.46 & 115.11 & 126.84 & 167.24 & 195.35 & 203.03 & 210.81 \\
219.95 & 225.44 & 278.17 & 307.79 & 325.31 & 355.86 & 363.10 & 411.34 & 469.87 & 473.96 \\
604.52 & 629.02 & 688.61 & 692.78 & 695.87 & 744.64 & 797.06 & 858.46 & 883.04 & 918.35 \\
941.62 & 977.80 & 997.95 & 1007.87 & 1033.74 & 1100.26 & 1127.16 & 1129.21 & 1148.76 \\
1149.72 & 1172.76 & 1184.31 & 1227.71 & 1357.87 & 1371.98 & 1379.03 & 1384.71 & 1420.28 \\
1422.21 & 1424.76 & 1438.88 & 1448.34 & 1475.82 & 1485.57 & 1487.55 & 1497.98 & 1501.67 \\
1528.55 & 1560.93 & 1689.25 & 3042.11 & 3056.43 & 3066.18 & 3076.12 & 3080.49 & 3108.10 \\
3152.40 & 3158.55 & 3173.82 & 3177.62 & 3178.01 & 3201.20 & 3474.26 & 3520.29 & \\
\multicolumn{7}{l}{ Rotational constants (B/GHZ): } & 1.0046496 & 0.6106915 & 0.4315555 &
\end{tabular}

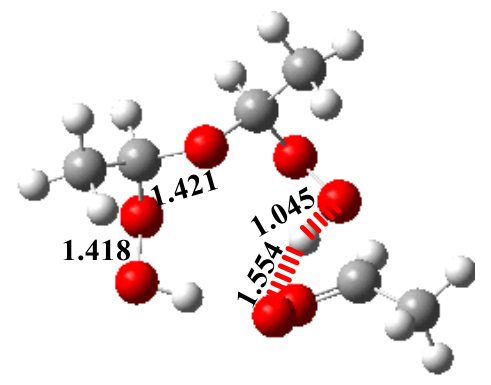

TS6g

C

$\begin{array}{lll}\mathrm{H} & 1 & \mathrm{~B} 1\end{array}$

$\begin{array}{lll}\text { O } & 1 & \text { B2 }\end{array}$

O 3

B2 2

A1

$\mathrm{H} \quad 3$

B3 1

B4 1

B5 1

A2 2

D1 0

A3 4

D2 0

C 3

A4 4

D3 0

C 6

B6 3

A5 1

D4 0

$\mathrm{H} \quad 7$

B7 6

A6 3

D5 0

H $\quad 7$

A7 3

D6 0

$\mathrm{H} \quad 7$

B8 6

A8 3

D7 0

O 6

B10 3

A9 1

D8 0

O 6

B11 3

A10 1

D9 0

H 6

B12 3

A11 1

D10 0

C 1

B13 4

A12 3

D11 0

H 14

B14 1

B15 1

A13 4

D12 0

A14 4

D13 0

$\mathrm{H} \quad 14$

B16 1

A15 4

D14 0

O 1

B17 4

A16 3

D15 0

C 18

B18 1

A17 4

D16 0

H 19

B19 18

A18 1

D17 0

C 19

B20 18

A19 1

D18 0

H 21

B21 19

A20 18

D19 0 


$\begin{array}{rrrrrrrr}\mathrm{H} & 21 & \text { B22 } & 19 & \text { A21 } & 18 & \text { D20 } & 0 \\ \mathrm{H} & 21 & \text { B23 } & 19 & \text { A22 } & 18 & \text { D21 } & 0 \\ \mathrm{O} & 19 & \text { B24 } & 18 & \text { A23 } & 1 & \text { D22 } & 0 \\ \mathrm{O} & 25 & \text { B25 } & 19 & \text { A24 } & 18 & \text { D23 } & 0 \\ \mathrm{H} & 26 & \text { B26 } & 25 & \text { A25 } & 19 & \text { D24 } & 0\end{array}$

Variables:

B1

B2

B3

B4

B5

B6

B7

B8

B9

B10

B11

B12

B13

B14

B15

B16

B17

B18

B19

B20

B21

B22

B23

B24

B25

B26

A1

A2

A3

A4

A5

A6

A7

A8

A9

A10

A11

A12
1.09558379

2.30796796

1.41624099

1.04545092

1.96121400

1.48480472

1.08615362

1.08368897

1.09358542

2.19697740

1.27454258

1.08825276

1.51099404

1.08695007

1.08799000

1.08772184

1.38680325

1.42092907

1.09567571

1.50948031

1.08799187

1.08769635

1.08577049

1.39307310

1.41760896

0.98796329

135.60381856

35.67478130

93.99629685

135.37052302

94.34337851

109.84282567

111.69150850

106.12902690

72.71747248

94.47102183

103.66268779

112.38231554 


\begin{tabular}{|c|c|}
\hline A13 & 110.08276496 \\
\hline A14 & 110.04809704 \\
\hline A 15 & 109.10279454 \\
\hline A16 & 112.09808856 \\
\hline A17 & 116.62889146 \\
\hline A18 & 108.35592337 \\
\hline A19 & 107.42426989 \\
\hline A20 & 109.41511823 \\
\hline A21 & 109.44260825 \\
\hline A22 & 109.97403183 \\
\hline A23 & 112.84578596 \\
\hline A24 & 110.16137251 \\
\hline A25 & 102.25998450 \\
\hline D1 & -9.47406200 \\
\hline D2 & -108.93240908 \\
\hline D3 & -29.16992006 \\
\hline D4 & -176.85975883 \\
\hline D5 & -63.05998865 \\
\hline D6 & 60.97143546 \\
\hline D7 & 179.73342166 \\
\hline D8 & -83.31089340 \\
\hline D9 & -51.92061995 \\
\hline D10 & 61.99639917 \\
\hline D11 & 54.31287876 \\
\hline D12 & -72.15875547 \\
\hline D13 & 48.48361470 \\
\hline D14 & 168.62095373 \\
\hline D15 & -68.74251223 \\
\hline D16 & -83.94308661 \\
\hline D17 & -35.01363359 \\
\hline D18 & -155.63381734 \\
\hline D19 & 66.18719670 \\
\hline D20 & -173.50716202 \\
\hline D21 & -53.98103850 \\
\hline D22 & 77.62520806 \\
\hline D23 & 81.02996891 \\
\hline D24 & -98.32368841 \\
\hline
\end{tabular}

Harmonic Vibrational Frequencies $\left(\mathbf{c m}^{-\mathbf{1}}\right)$ :

$\begin{array}{llllllllllll}404.04 i & 36.43 & 55.87 & 95.70 & 98.60 & 107.87 & 128.39 & 170.73 & 207.70 & 215.25 & 226.02 \\ 248.43 & 271.88 & 293.59 & 323.78 & 343.24 & 361.16 & 390.24 & 409.53 & 470.41 & 528.15 \\ 612.01 & 654.34 & 691.52 & 705.18 & 837.23 & 853.64 & 867.22 & 886.34 & 930.84 & 939.07 \\ 974.31 & 982.41 & 1009.47 & 1024.95 & 1101.98 & 1107.28 & 1137.13 & 1154.80 & 1166.03 \\ 1169.31 & 1189.09 & 1223.81 & 1235.29 & 1361.27 & 1363.44 & 1375.56 & 1385.34 & 1403.76 \\ 1419.71 & 1426.41 & 1440.41 & 1450.74 & 1480.56 & 1485.20 & 1492.34 & 1496.61 & 1500.10\end{array}$




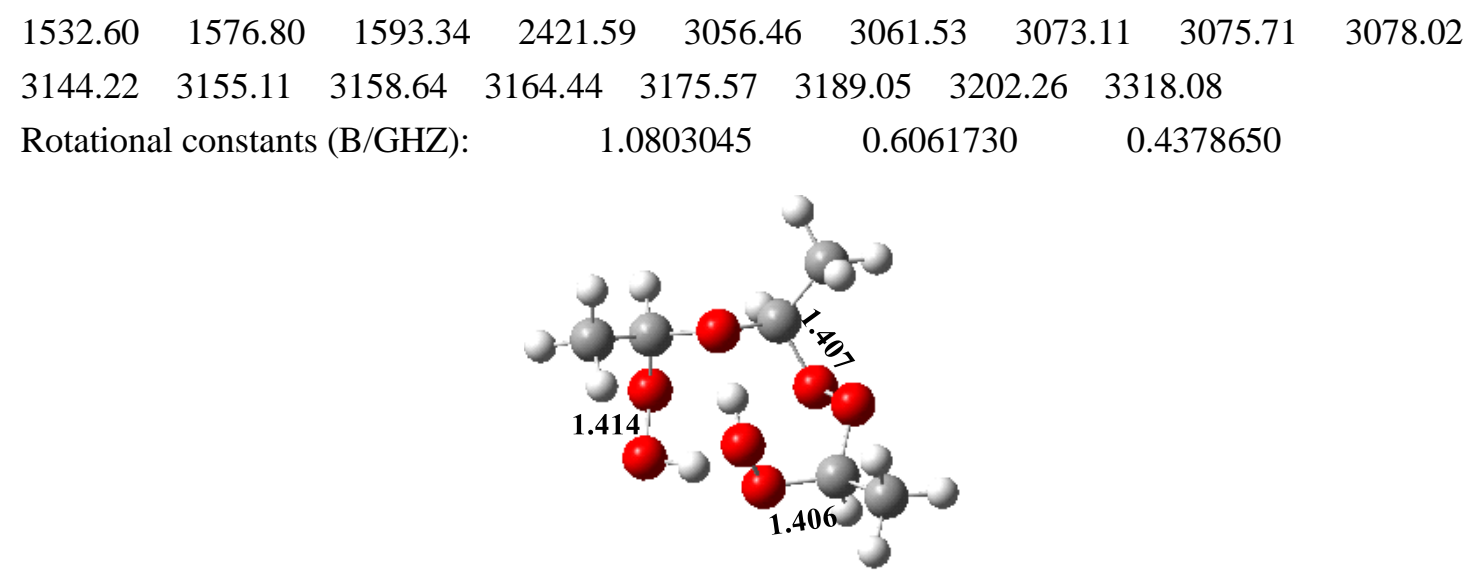

P6g

\begin{tabular}{|c|c|c|c|c|c|c|c|}
\hline C & & & & & & & \\
\hline $\mathrm{H}$ & 1 & B1 & & & & & \\
\hline $\mathrm{O}$ & 1 & B2 & 2 & A1 & & & \\
\hline $\mathrm{O}$ & 1 & B3 & 3 & A2 & 2 & D1 & 0 \\
\hline $\mathrm{H}$ & 3 & B4 & 1 & A3 & 4 & D2 & 0 \\
\hline $\mathrm{C}$ & 3 & B5 & 1 & A4 & 4 & D3 & 0 \\
\hline $\mathrm{C}$ & 6 & B6 & 3 & A5 & 1 & D4 & 0 \\
\hline $\mathrm{H}$ & 7 & B7 & 6 & A6 & 3 & D5 & 0 \\
\hline $\mathrm{H}$ & 7 & B8 & 6 & A7 & 3 & D6 & 0 \\
\hline $\mathrm{H}$ & 7 & B9 & 6 & A8 & 3 & D7 & 0 \\
\hline $\mathrm{O}$ & 6 & B10 & 3 & A9 & 1 & D8 & 0 \\
\hline $\mathrm{O}$ & 6 & B11 & 3 & A10 & 1 & D9 & 0 \\
\hline $\mathrm{H}$ & 6 & B12 & 3 & A11 & 1 & D10 & 0 \\
\hline $\mathrm{C}$ & 1 & B13 & 4 & A12 & 3 & D11 & 0 \\
\hline $\mathrm{H}$ & 14 & B14 & 1 & A13 & 4 & $\mathrm{D} 12$ & 0 \\
\hline $\mathrm{H}$ & 14 & B15 & 1 & A14 & 4 & D13 & 0 \\
\hline $\mathrm{H}$ & 14 & B16 & 1 & A15 & 4 & D14 & 0 \\
\hline $\mathrm{O}$ & 1 & B17 & 4 & A16 & 3 & D15 & 0 \\
\hline $\mathrm{C}$ & 18 & B18 & 1 & A17 & 4 & D16 & 0 \\
\hline $\mathrm{H}$ & 19 & B19 & 18 & A18 & 1 & D17 & 0 \\
\hline $\mathrm{C}$ & 19 & B20 & 18 & A19 & 1 & D18 & 0 \\
\hline $\mathrm{H}$ & 21 & B21 & 19 & A20 & 18 & D19 & 0 \\
\hline $\mathrm{H}$ & 21 & B22 & 19 & A21 & 18 & D20 & 0 \\
\hline $\mathrm{H}$ & 21 & B23 & 19 & A22 & 18 & D21 & 0 \\
\hline $\mathrm{O}$ & 19 & B24 & 18 & A23 & 1 & D22 & 0 \\
\hline $\mathrm{O}$ & 25 & B25 & 19 & A24 & 18 & D23 & 0 \\
\hline $\mathrm{H}$ & 26 & B26 & 25 & A25 & 19 & D24 & 0 \\
\hline \multicolumn{7}{|c|}{ Variables: } & \\
\hline B1 & & 1.09272989 & & & & & \\
\hline B2 & & 2.28738625 & & & & & \\
\hline B3 & & 1.40698772 & & & & & \\
\hline B4 & & 2.56868194 & & & & & \\
\hline
\end{tabular}




\begin{tabular}{|c|c|}
\hline B5 & 1.39920313 \\
\hline B6 & 1.50922735 \\
\hline B7 & 1.08729649 \\
\hline B8 & 1.08662671 \\
\hline B9 & 1.08742064 \\
\hline B10 & 2.30679945 \\
\hline B11 & 1.40625469 \\
\hline B12 & 1.09308523 \\
\hline B13 & 1.51066940 \\
\hline B14 & 1.08670114 \\
\hline B15 & 1.08794873 \\
\hline B16 & 1.08810724 \\
\hline B17 & 1.41032996 \\
\hline B18 & 1.42039824 \\
\hline B19 & 1.09502270 \\
\hline B20 & 1.50769118 \\
\hline B21 & 1.08784406 \\
\hline B22 & 1.08768902 \\
\hline $\mathrm{B} 23$ & 1.08721152 \\
\hline B24 & 1.39559400 \\
\hline $\mathrm{B} 25$ & 1.41447171 \\
\hline B26 & 0.97305510 \\
\hline $\mathrm{A} 1$ & 137.30409043 \\
\hline $\mathrm{A} 2$ & 36.42963001 \\
\hline $\mathrm{A} 3$ & 70.59886783 \\
\hline A4 & 128.01426630 \\
\hline A5 & 106.30743081 \\
\hline A6 & 109.76497702 \\
\hline A7 & 110.01312293 \\
\hline A8 & 108.85366188 \\
\hline A9 & 92.47243809 \\
\hline A10 & 111.35642264 \\
\hline A11 & 110.12693569 \\
\hline A 12 & 113.19243272 \\
\hline A13 & 110.12627757 \\
\hline A14 & 109.96080372 \\
\hline A15 & 109.12403297 \\
\hline A16 & 110.99214774 \\
\hline A17 & 115.07829102 \\
\hline A18 & 108.77957929 \\
\hline A19 & 107.84048318 \\
\hline A20 & 109.48365548 \\
\hline A 21 & 109.22706388 \\
\hline A22 & 110.34010596 \\
\hline
\end{tabular}




$\begin{array}{lr}\text { A23 } & 111.25560139 \\ \text { A24 } & 109.85096152 \\ \text { A25 } & 103.58561194 \\ \text { D1 } & 14.72094049 \\ \text { D2 } & -117.51504677 \\ \text { D3 } & -64.67283429 \\ \text { D4 } & -153.95826857 \\ \text { D5 } & -62.83847293 \\ \text { D6 } & 57.82885392 \\ \text { D7 } & 177.35162269 \\ \text { D8 } & -60.27776248 \\ \text { D9 } & -29.64754197 \\ \text { D10 } & 83.30881855 \\ \text { D11 } & 49.58616740 \\ \text { D12 } & -70.97191214 \\ \text { D13 } & 49.44352532 \\ \text { D14 } & 169.48585131 \\ \text { D15 } & -73.48106921 \\ \text { D16 } & -100.75914071 \\ \text { D17 } & -54.50400627 \\ \text { D18 } & -175.95925180 \\ \text { D19 } & 63.95504164 \\ \text { D20 } & -176.14085651 \\ \text { D21 } & -56.64373524 \\ \text { D22 } & 57.34956199 \\ \text { D23 } & 74.46974902 \\ \text { D24 } & -78.09749349 \\ \text { D19 }\end{array}$

Harmonic Vibrational Frequencies $\left(\mathbf{c m}^{-1}\right)$ :

$\begin{array}{lllllllllll}58.79 & 61.00 & 87.94 & 114.19 & 127.06 & 186.32 & 190.49 & 202.63 & 213.19 & 228.44 & 242.95\end{array}$ $\begin{array}{llllllllll}273.71 & 298.91 & 314.06 & 333.40 & 358.97 & 417.66 & 437.76 & 477.66 & 544.22 & 566.07\end{array}$ $\begin{array}{llllllllll}600.08 & 650.53 & 675.06 & 713.46 & 716.82 & 855.60 & 882.30 & 895.10 & 937.09 & 947.39\end{array}$ $\begin{array}{lllllllll}984.96 & 1006.66 & 1019.40 & 1030.68 & 1112.84 & 1137.19 & 1148.33 & 1151.44 & 1155.35\end{array}$ $\begin{array}{lllllllll}1170.86 & 1174.26 & 1207.09 & 1230.16 & 1358.25 & 1365.23 & 1377.82 & 1393.18 & 1397.86\end{array}$ $\begin{array}{lllllllll}1423.19 & 1426.42 & 1431.17 & 1439.11 & 1469.19 & 1486.57 & 1488.32 & 1489.63 & 1498.17\end{array}$ $\begin{array}{lllllllll}1500.52 & 1504.34 & 1525.22 & 3070.83 & 3074.95 & 3078.01 & 3085.72 & 3111.40 & 3113.12\end{array}$ $\begin{array}{llllllll}3151.75 & 3157.51 & 3163.49 & 3164.74 & 3166.85 & 3178.31 & 3638.68 & 3670.62\end{array}$

$\begin{array}{llll}\text { Rotational constants (B/GHZ): } & 1.0949408 & 0.6441656 & 0.5157917\end{array}$ 


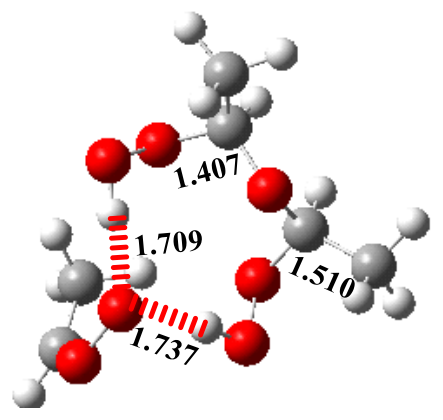

IM6h

C

C 1

H 2

O 2

O 2

H 4

C 4

$\begin{array}{ll}\text { C } & 7\end{array}$

H 8

H 8

$\mathrm{H} \quad 8$

$\begin{array}{ll}\mathrm{O} & 7\end{array}$

$\begin{array}{ll}\mathrm{O} & 7\end{array}$

H $\quad 7$

$\mathrm{H} \quad 1$

H 1

$\mathrm{H} \quad 1$

O 2

C 18

H 19

$\begin{array}{ll}\text { C } & 19\end{array}$

$\mathrm{H} \quad 21$

$\mathrm{H} \quad 21$

$\mathrm{H} \quad 21$

O 19

O 25

H 26

Variables:

B

B2

B3

B4

B5

B6

B7
B1

B2

B3 1

B4 1

B5 2

B6 2

B7 4

B8 7

B9 7

B10 7

B11 4

B12 4

B13 4

B14 2

B15 2

B16 2

B17 1

B18 2

B19 18

B20 18

B21 19

B22 19

B23 19

B24 18

B25 19

B26 25
A1

A2 $3 \quad$ D1 0

A3 $4 \quad$ D2 0

$\begin{array}{llll}\text { A4 } & 1 & \text { D3 } & 0\end{array}$

A5 $1 \quad$ D4 0

$\begin{array}{llll}\text { A6 } 2 & \text { D5 } & 0\end{array}$

A7 $4 \quad$ D6 0

$\begin{array}{llll}\text { A8 } & 4 & \text { D7 } & 0\end{array}$

A9 $4 \quad$ D8 0

$\begin{array}{llll}\mathrm{A} 10 & 2 & \mathrm{D} 9 & 0\end{array}$

A11 $2 \quad$ D10 0

A12 2 D11 0

A13 $5 \quad$ D12 0

A14 $5 \quad$ D13 0

A15 $5 \quad$ D14 0

A16 $5 \quad$ D15 0

A17 $1 \quad$ D16 0

A18 $2 \quad$ D17 0

$\begin{array}{llll}\mathrm{A} 19 & 2 & \mathrm{D} 18 & 0\end{array}$

A20 $18 \quad$ D19 0

A21 $18 \quad$ D20 0

$\begin{array}{llll}\text { A22 } & 18 & \text { D21 } & 0\end{array}$

$\begin{array}{llll}\text { A23 } 2 & \text { D22 } & 0\end{array}$

A24 $18 \quad$ D23 0

$\begin{array}{llll}\text { A25 } & 19 & \text { D24 } & 0\end{array}$

$\begin{array}{ll}1 & 1.50975000 \\ 2 & 1.09578514 \\ 3 & 2.31556026 \\ 4 & 1.40714344 \\ 5 & 0.98046850 \\ 6 & 3.84902584 \\ 7 & 1.46004358\end{array}$




\begin{tabular}{|c|c|}
\hline B8 & 1.09613712 \\
\hline B9 & 1.08441359 \\
\hline B 10 & 1.09536997 \\
\hline B11 & 2.24965885 \\
\hline B12 & 1.24497071 \\
\hline B13 & 1.08677102 \\
\hline B14 & 1.08784423 \\
\hline B 15 & 1.08785481 \\
\hline B16 & 1.08598230 \\
\hline B17 & 1.40372857 \\
\hline B18 & 1.40174794 \\
\hline B19 & 1.09569412 \\
\hline B20 & 1.51006130 \\
\hline B21 & 1.08601017 \\
\hline B22 & 1.08777135 \\
\hline B23 & 1.08798779 \\
\hline B24 & 1.40888036 \\
\hline B25 & 1.41666391 \\
\hline B26 & 0.98632258 \\
\hline $\mathrm{A} 1$ & 111.47914265 \\
\hline $\mathrm{A} 2$ & 87.20621932 \\
\hline A3 & 113.37602806 \\
\hline A4 & 101.76816920 \\
\hline A5 & 110.08448304 \\
\hline A6 & 54.09431372 \\
\hline A7 & 109.81958089 \\
\hline A8 & 110.62382869 \\
\hline A9 & 109.05808642 \\
\hline A10 & 43.84357246 \\
\hline A11 & 73.64872672 \\
\hline A12 & 160.46698622 \\
\hline A13 & 109.11806410 \\
\hline A14 & 109.79882643 \\
\hline A15 & 110.02064874 \\
\hline A16 & 108.18372035 \\
\hline A17 & 116.67332246 \\
\hline A18 & 109.72891903 \\
\hline A19 & 108.33921583 \\
\hline A20 & 110.01420189 \\
\hline A 21 & 109.86706400 \\
\hline $\mathrm{A} 22$ & 109.12058949 \\
\hline A23 & 112.39082847 \\
\hline $\mathrm{A} 24$ & 110.12173364 \\
\hline A25 & 102.83429012 \\
\hline
\end{tabular}




$\begin{array}{lr}\text { D1 } & -137.88357664 \\ \text { D2 } & 24.02516668 \\ \text { D3 } & -124.96201807 \\ \text { D4 } & -160.42948440 \\ \text { D5 } & -62.15702076 \\ \text { D6 } & 76.86485999 \\ \text { D7 } & -159.71094327 \\ \text { D8 } & -37.74059032 \\ \text { D9 } & 82.16844064 \\ \text { D10 } & 98.41233760 \\ \text { D11 } & -152.67672306 \\ \text { D12 } & -168.01053792 \\ \text { D13 } & -47.77990806 \\ \text { D14 } & 72.26079348 \\ \text { D15 } & -125.75078509 \\ \text { D16 } & -153.46155662 \\ \text { D17 } & 28.74718576 \\ \text { D18 } & 150.78401800 \\ \text { D19 } & 52.46523804 \\ \text { D20 } & 172.55061740 \\ \text { D21 } & -67.17284529 \\ \text { D22 } & -83.51553832 \\ \text { D23 } & -76.53769192 \\ \text { D24 } & 84.38735404\end{array}$

Harmonic Vibrational Frequencies $\left(\mathbf{c m}^{-1}\right)$ :

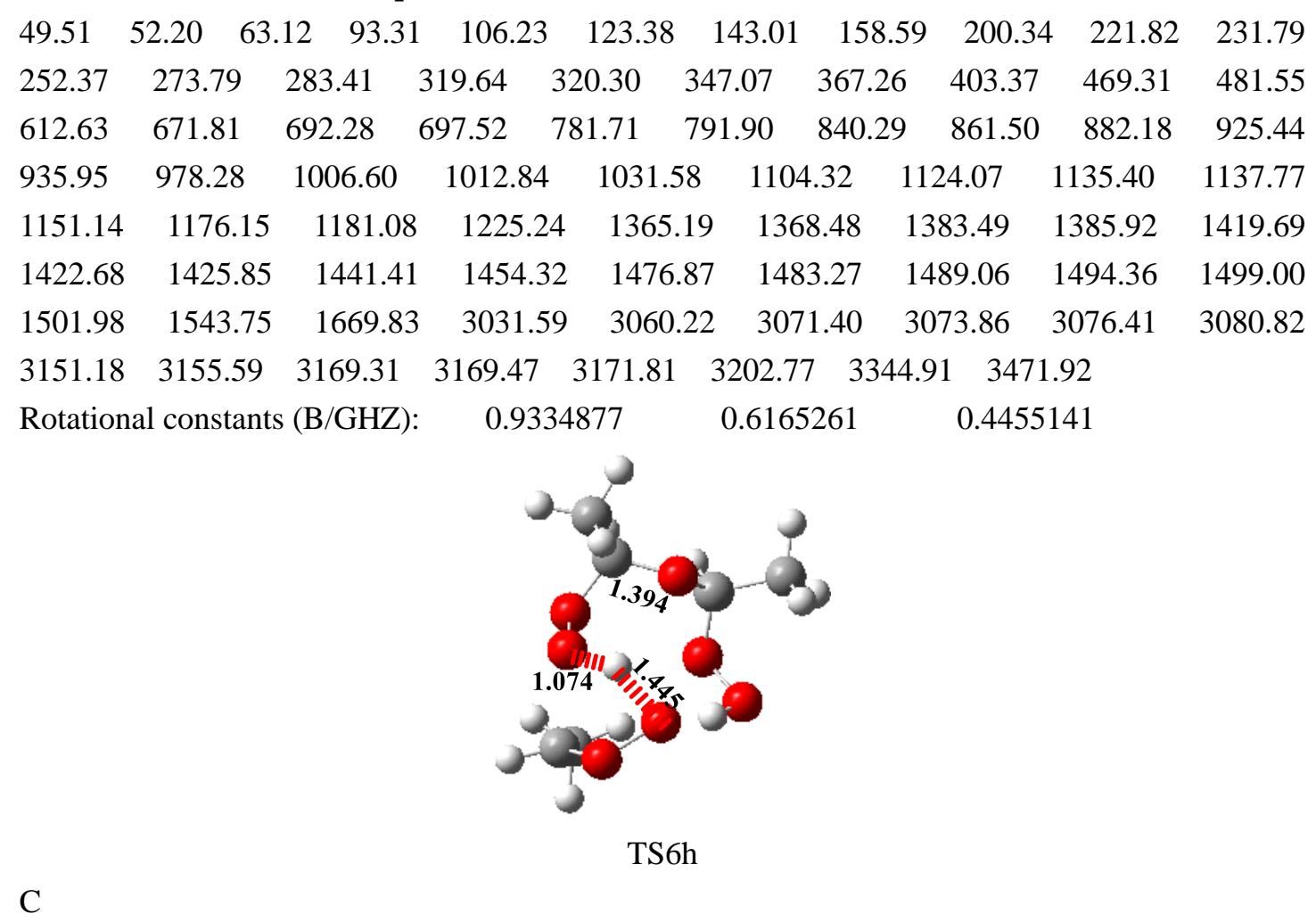




\begin{tabular}{|c|c|c|c|c|c|c|c|}
\hline $\mathrm{C}$ & 1 & B 1 & & & & & \\
\hline $\mathrm{H}$ & 2 & B2 & 1 & A1 & & & \\
\hline $\mathrm{O}$ & 2 & B3 & 1 & A2 & 3 & D1 & 0 \\
\hline $\mathrm{O}$ & 4 & B4 & 2 & A3 & 1 & D2 & 0 \\
\hline $\mathrm{H}$ & 4 & B5 & 2 & A4 & 1 & D3 & 0 \\
\hline $\mathrm{C}$ & 4 & B6 & 2 & A5 & 1 & D4 & 0 \\
\hline $\mathrm{C}$ & 7 & B7 & 4 & A6 & 2 & D5 & 0 \\
\hline $\mathrm{H}$ & 8 & B8 & 7 & A7 & 4 & D6 & 0 \\
\hline $\mathrm{H}$ & 8 & B9 & 7 & A8 & 4 & D7 & 0 \\
\hline $\mathrm{H}$ & 8 & B10 & 7 & A9 & 4 & D8 & 0 \\
\hline $\mathrm{O}$ & 7 & B11 & 4 & A10 & 2 & D9 & 0 \\
\hline $\mathrm{O}$ & 7 & B12 & 4 & A11 & 2 & D10 & 0 \\
\hline $\mathrm{H}$ & 7 & B13 & 4 & A12 & 2 & D11 & 0 \\
\hline $\mathrm{H}$ & 1 & B14 & 2 & A13 & 5 & $\mathrm{D} 12$ & 0 \\
\hline $\mathrm{H}$ & 1 & B15 & 2 & A14 & 5 & D13 & 0 \\
\hline $\mathrm{H}$ & 1 & B16 & 2 & A15 & 5 & D14 & 0 \\
\hline $\mathrm{O}$ & 2 & B17 & 1 & A16 & 5 & D15 & 0 \\
\hline $\mathrm{C}$ & 18 & B18 & 2 & A17 & 1 & D16 & 0 \\
\hline $\mathrm{H}$ & 19 & B19 & 18 & A18 & 2 & D17 & 0 \\
\hline $\mathrm{C}$ & 19 & B20 & 18 & A19 & 2 & D18 & 0 \\
\hline $\mathrm{H}$ & 21 & B21 & 19 & A 20 & 18 & D19 & 0 \\
\hline $\mathrm{H}$ & 21 & B22 & 19 & A21 & 18 & D20 & 0 \\
\hline $\mathrm{H}$ & 21 & B23 & 19 & A22 & 18 & D21 & 0 \\
\hline $\mathrm{O}$ & 19 & B24 & 18 & A23 & 2 & D22 & 0 \\
\hline $\mathrm{O}$ & 25 & B25 & 19 & A24 & 18 & $\mathrm{D} 23$ & 0 \\
\hline $\mathrm{H}$ & 26 & B26 & 25 & A25 & 19 & D24 & 0 \\
\hline \multicolumn{7}{|c|}{ Variables: } & \\
\hline B1 & \multicolumn{3}{|c|}{1.51034559} & & & & \\
\hline B2 & \multicolumn{3}{|c|}{1.09571987} & & & & \\
\hline B3 & \multicolumn{2}{|r|}{2.30216649} & & & & & \\
\hline B4 & \multicolumn{2}{|r|}{1.41423500} & & & & & \\
\hline B5 & \multicolumn{2}{|r|}{1.07363063} & & & & & \\
\hline B6 & \multicolumn{2}{|r|}{2.01050930} & & & & & \\
\hline B7 & \multicolumn{2}{|r|}{1.47655379} & & & & & \\
\hline B8 & \multicolumn{2}{|r|}{1.08274644} & & & & & \\
\hline B9 & \multicolumn{2}{|r|}{1.09528129} & & & & & \\
\hline B10 & \multicolumn{2}{|r|}{1.08694291} & & & & & \\
\hline B11 & \multicolumn{2}{|r|}{2.21912884} & & & & & \\
\hline B12 & \multicolumn{2}{|r|}{1.27771680} & & & & & \\
\hline B13 & \multicolumn{2}{|r|}{1.08854666} & & & & & \\
\hline B14 & \multicolumn{2}{|r|}{1.08769019} & & & & & \\
\hline B15 & \multicolumn{2}{|r|}{1.08789183} & & & & & \\
\hline B16 & \multicolumn{2}{|r|}{1.08685170} & & & & & \\
\hline B17 & \multicolumn{2}{|r|}{1.39354251} & & & & & \\
\hline
\end{tabular}




$\begin{array}{lr}\text { B18 } & 1.41275009 \\ \text { B19 } & 1.09530639 \\ \text { B20 } & 1.50905551 \\ \text { B21 } & 1.08599086 \\ \text { B22 } & 1.08755708 \\ \text { B23 } & 1.08785043 \\ \text { B24 } & 1.40230223 \\ \text { B25 } & 1.41779428 \\ \text { B26 } & 0.98293214 \\ \text { A1 } & 111.83499594 \\ \text { A2 } & 90.04794310 \\ \text { A3 } & 35.68923185 \\ \text { A4 } & 87.50780907 \\ \text { A5 } & 139.11483110 \\ \text { A6 } & 98.08424818 \\ \text { A7 } & 113.89605568 \\ \text { A8 } & 104.96896989 \\ \text { A9 } & 109.50771547 \\ \text { A10 } & 69.53201868 \\ \text { A11 } & 90.47267619 \\ \text { A12 } & 103.94417710 \\ \text { A13 } & 109.16640977 \\ \text { A14 } & 109.82711519 \\ \text { A15 } & 110.16764572 \\ \text { A16 } & 108.50816372 \\ \text { A17 } & 116.98750431 \\ \text { A18 } & 108.86956507 \\ \text { A19 } & 107.79568017 \\ \text { A20 } & 109.90009319 \\ \text { A21 } & 109.82971510 \\ \text { A22 } & 109.1332180606 \\ \text { A23 } & 112.70700612 \\ \text { A24 } & 110.93510190 \\ \text { A25 } & 102.70336293 \\ \text { D1 } & \\ \text { D2 } & -1377659565 \\ \text { D3 } & \end{array}$




$\begin{array}{lr}\text { D11 } & -140.99067049 \\ \text { D12 } & -169.40750779 \\ \text { D13 } & -49.23037514 \\ \text { D14 } & 71.18698642 \\ \text { D15 } & -124.55334350 \\ \text { D16 } & -156.25993115 \\ \text { D17 } & 30.19507669 \\ \text { D18 } & 151.55435296 \\ \text { D19 } & 53.23911619 \\ \text { D20 } & 173.14420229 \\ \text { D21 } & -66.49153684 \\ \text { D22 } & -82.16434402 \\ \text { D23 } & -81.85434054 \\ \text { D24 } & 94.31677646\end{array}$

Harmonic Vibrational Frequencies $\left(\mathbf{c m}^{-1}\right)$ :

$\begin{array}{llllllllllll}423.44 i & 48.52 & 89.87 & 103.52 & 127.85 & 140.36 & 173.14 & 192.53 & 202.53 & 220.15 & 223.59 \\ 252.89 & 281.91 & 311.41 & 328.67 & 359.16 & 378.05 & 402.72 & 425.73 & 458.58 & 480.07 \\ 610.03 & 640.66 & 688.28 & 710.89 & 791.92 & 858.23 & 867.39 & 883.83 & 932.32 & 937.52 \\ 960.78 & 984.04 & 1012.17 & 1028.15 & 1097.01 & 1112.67 & 1143.00 & 1149.36 & 1167.28 \\ 1176.96 & 1184.37 & 1224.15 & 1270.11 & 1361.04 & 1366.23 & 1377.76 & 1384.93 & 1411.29 \\ 1422.92 & 1425.97 & 1439.61 & 1444.02 & 1478.06 & 1487.02 & 1489.58 & 1494.93 & 1501.19 \\ 1514.53 & 1542.76 & 1643.10 & 2148.77 & 3039.48 & 3069.92 & 3076.68 & 3081.09 & 3081.93 \\ 3137.71 & 3157.61 & 3160.76 & 3167.47 & 3173.90 & 3187.96 & 3226.86 & 3427.04 & \\ \text { Rotational constants (B/GHZ): } & 0.9709020 & 0.6823635 & 0.4977185 & \end{array}$

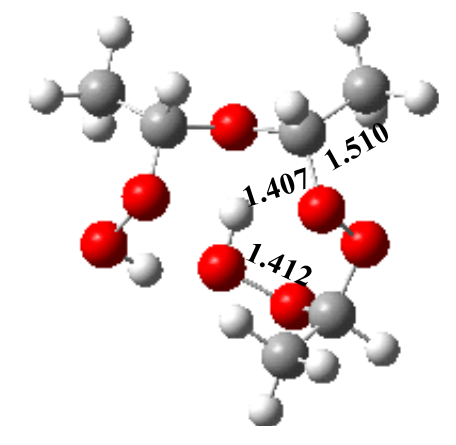

P6h

C

$\begin{array}{lll}\mathrm{C} & 1 & \mathrm{~B} 1\end{array}$

$\begin{array}{lll}\mathrm{H} & 2 & \mathrm{~B} 2\end{array}$

O 2

$\begin{array}{lll}\mathrm{B} 2 & 1 & \mathrm{~A} 1\end{array}$

O 2

B3

B4 1

B5 2

A2 3

D1 0

$\mathrm{H} \quad 4$

A3 4

D2 0

A4 $1 \quad$ D3 0

C 4

B6 2

A5

D4 0

C 7

B7 4

A6 2

D5 0

$\mathrm{H} \quad 8$

B8 7

A7 4

D6 0

$\mathrm{H} \quad 8$

$\begin{array}{llll}\text { B9 } & 7 & \text { A8 } & 4\end{array}$

D7 0

$\mathrm{H} \quad 8$

B10 7

A9 4

D8 0 


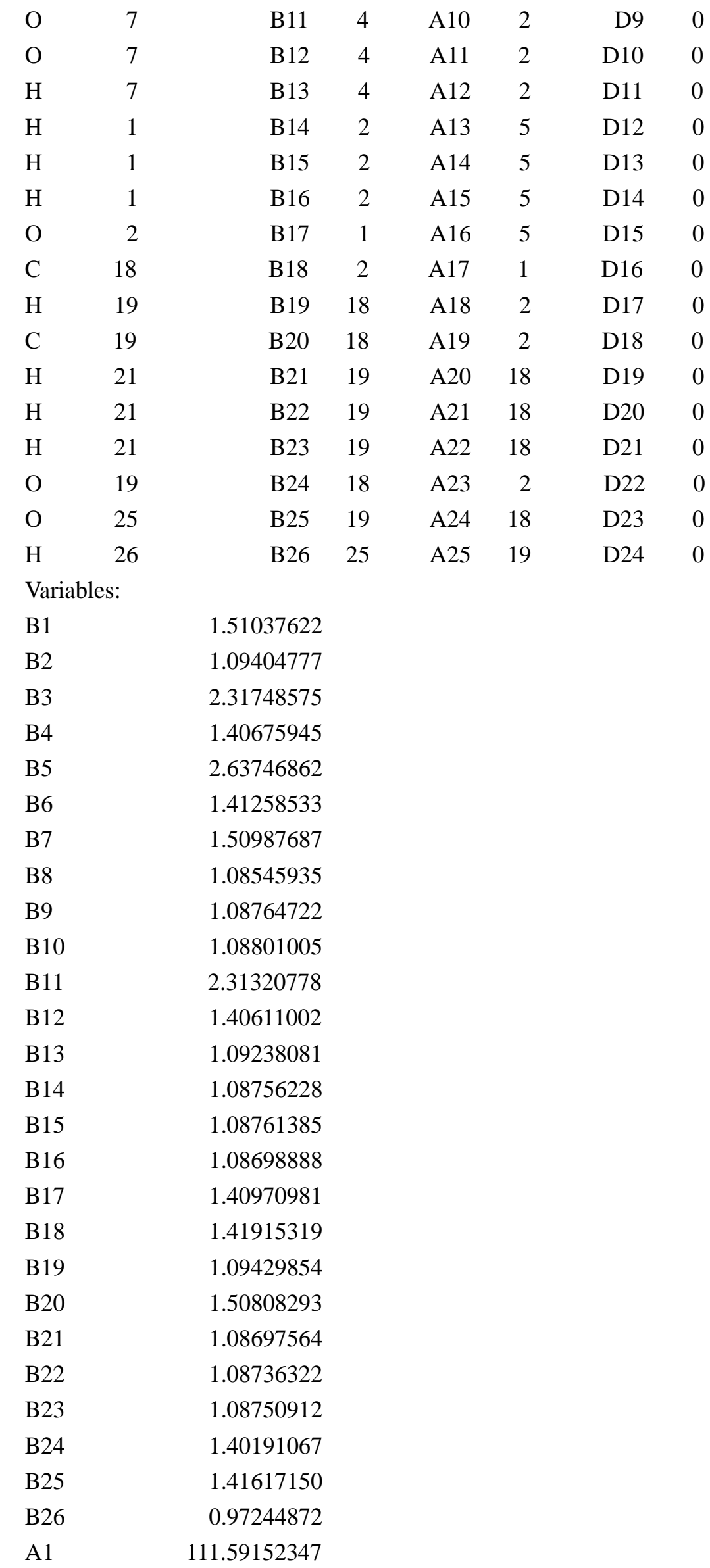




\begin{tabular}{|c|c|}
\hline A 2 & 85.22497751 \\
\hline $\mathrm{A} 3$ & 113.76621574 \\
\hline A4 & 66.95918870 \\
\hline A5 & 128.77634772 \\
\hline A6 & 114.44182151 \\
\hline A7 & 112.00341507 \\
\hline A8 & 108.38515873 \\
\hline A9 & 109.20726483 \\
\hline A10 & 98.96044606 \\
\hline A11 & 110.67018242 \\
\hline A12 & 103.37634136 \\
\hline A13 & 108.98410509 \\
\hline A14 & 109.99618754 \\
\hline A 15 & 110.22366049 \\
\hline A16 & 108.42767768 \\
\hline A17 & 115.73055313 \\
\hline A18 & 108.15772300 \\
\hline A19 & 107.85253448 \\
\hline A20 & 110.48660912 \\
\hline A 21 & 109.69575004 \\
\hline $\mathrm{A} 22$ & 109.04210883 \\
\hline A23 & 112.43513230 \\
\hline A24 & 111.08035088 \\
\hline A 25 & 103.13871838 \\
\hline D1 & -135.24045782 \\
\hline D2 & 21.31696023 \\
\hline D3 & -104.90770045 \\
\hline D4 & -143.68693540 \\
\hline D5 & -61.48950710 \\
\hline D6 & 61.57490877 \\
\hline D7 & -177.01158260 \\
\hline D8 & -57.86019380 \\
\hline D9 & 35.53293368 \\
\hline D10 & 69.65833247 \\
\hline D11 & 177.13167157 \\
\hline D12 & -167.66866202 \\
\hline D13 & -47.37266197 \\
\hline D14 & 72.75345507 \\
\hline D15 & -126.05987199 \\
\hline D16 & -147.58634571 \\
\hline D17 & 39.65488219 \\
\hline D18 & 160.49918005 \\
\hline D19 & 53.77394492 \\
\hline D20 & 173.57584731 \\
\hline
\end{tabular}




$\begin{array}{lr}\text { D21 } & -66.12868612 \\ \text { D22 } & -72.39186670 \\ \text { D23 } & -86.08939000 \\ \text { D24 } & 86.12215251\end{array}$

Harmonic Vibrational Frequencies $\left(\mathrm{cm}^{-\mathbf{1}}\right)$ :

$\begin{array}{lccccccccccc}33.43 & 52.70 & 72.95 & 92.59 & 131.80 & 173.69 & 211.02 & 218.51 & 228.24 & 244.20 & 264.34 \\ 294.49 & 305.04 & 322.59 & 336.56 & 355.57 & 395.98 & 458.12 & 484.58 & 511.82 & 554.57 \\ 600.67 & 639.88 & 667.66 & 697.40 & 731.32 & 845.88 & 863.05 & 887.74 & 926.86 & 939.92 \\ 969.32 & 1000.09 & 1021.09 & 1026.52 & 1109.83 & 1128.37 & 1142.55 & 1151.31 & 1163.36 \\ 1173.68 & 1178.29 & 1192.92 & 1223.93 & 1354.97 & 1370.34 & 1386.25 & 1391.50 & 1404.48 \\ 1424.33 & 1429.04 & 1434.45 & 1448.60 & 1464.61 & 1483.43 & 1487.59 & 1489.01 & 1496.14 \\ 1499.48 & 1506.33 & 1510.84 & 3077.10 & 3077.27 & 3079.04 & 3083.18 & 3097.99 & 3117.81 \\ 3155.90 & 3159.72 & 3160.47 & 3165.92 & 3166.25 & 3181.06 & 3682.68 & 3708.78 & \\ \text { Rotational constants (B/GHZ): } & 1.0039743 & 0.6955716 & 0.5293500 & \end{array}$


Figure S8. Optimized geometries, z-matrices and vibrational frequencies of all stationary points in the reaction of $\left(\mathrm{CH}_{3}\right)_{2} \mathrm{COO}$ with $\mathrm{HO}-\mathrm{C}\left(\mathrm{CH}_{3}\right)_{2} \mathrm{OO}-\mathrm{H}$ at the M06-2X/6-311+G(2df,2p) level of theory

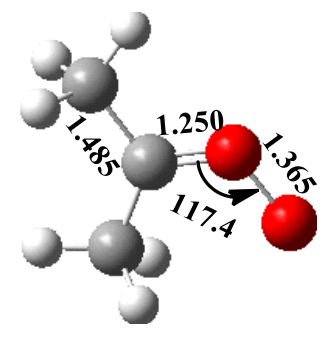

$\begin{array}{llllllll}\mathrm{C} & & & & & & & \\ \mathrm{O} & 1 & \text { B1 } & & & & & \\ \mathrm{O} & 2 & \text { B2 } & 1 & \text { A1 } & & & \\ \mathrm{C} & 1 & \text { B3 } & 2 & \text { A2 } & 3 & \text { D1 } & 0 \\ \mathrm{C} & 1 & \text { B4 } & 2 & \text { A3 } & 3 & \text { D2 } & 0 \\ \mathrm{H} & 4 & \text { B5 } & 1 & \text { A4 } & 2 & \text { D3 } & 0 \\ \mathrm{H} & 4 & \text { B6 } & 1 & \text { A5 } & 2 & \text { D4 } & 0 \\ \text { H } & 4 & \text { B7 } & 1 & \text { A6 } & 2 & \text { D5 } & 0 \\ \text { H } & 5 & \text { B8 } & 1 & \text { A7 } & 2 & \text { D6 } & 0 \\ \text { H } & 5 & \text { B9 } & 1 & \text { A8 } & 2 & \text { D7 } & 0 \\ \text { H } & 5 & \text { B10 } & 1 & \text { A9 } & 2 & \text { D8 } & 0\end{array}$

Variables:

$\begin{array}{lr}\text { B1 } & 1.24973026 \\ \text { B2 } & 1.36521073 \\ \text { B3 } & 1.47294272 \\ \text { B4 } & 1.48491383 \\ \text { B5 } & 1.08561662 \\ \text { B6 } & 1.09251273 \\ \text { B7 } & 1.09251273 \\ \text { B8 } & 1.09132373 \\ \text { B9 } & 1.09132373 \\ \text { B10 } & 1.08604848 \\ \text { A1 } & 117.44317761 \\ \text { A2 } & 119.44468526 \\ \text { A3 } & 116.56507938 \\ \text { A4 } & 111.53606452 \\ \text { A5 } & 108.40498430 \\ \text { A6 } & 108.40498430 \\ \text { A7 } & 109.64754076 \\ \text { A8 } & 109.64754076 \\ \text { A9 } & 110.67329046 \\ \text { D1 } & 0.00000000 \\ \text { D2 } & 180.00000000 \\ \text { D3 } & 180.00000000 \\ \text { D4 } & -56.61851850\end{array}$


D5

56.61851850

D6

121.30347974

D7

$-121.30347974$

D8

0.00000000

Harmonic Vibrational Frequencies $\left(\mathbf{c m}^{-\mathbf{1}}\right)$ :

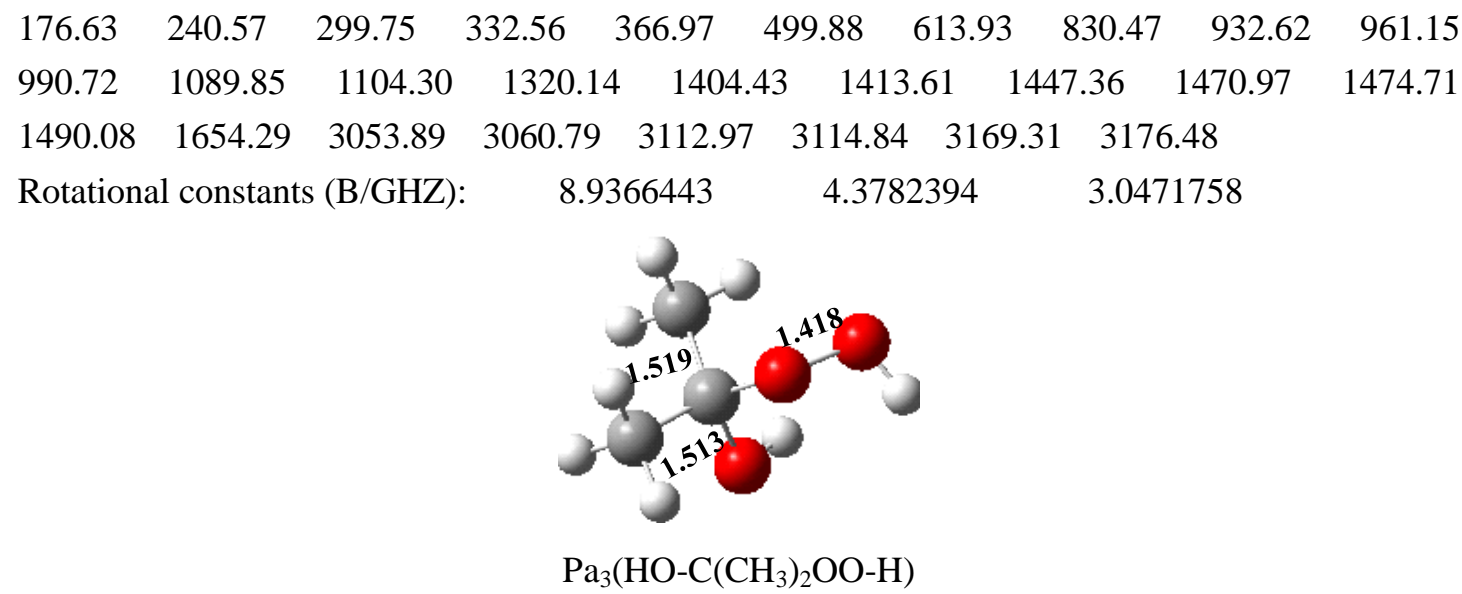

C

$\begin{array}{lll}\mathrm{O} & 1 & \mathrm{~B} 1\end{array}$

$\begin{array}{lll}\mathrm{O} & 2 & \mathrm{~B} 2\end{array}$

O 1

$\mathrm{H} \quad 3$

$\mathrm{H} \quad 4$

C 1

$\begin{array}{ll}\mathrm{H} & 7\end{array}$

$\mathrm{H} \quad 7$

$\begin{array}{ll}\mathrm{H} & 7\end{array}$

C 1

$\mathrm{H} \quad 11$

$\mathrm{H} \quad 11$

B2 1

B3 2

A1

B4 2

A2 $3 \quad$ D1 0

A3 $1 \quad$ D2 0

B5 1

A4 2 D3 0

B6 4

A5 2 D4 0

B7 1

A6 4 D5 0

B8 1

A7 4 D6 0

B9 1

A8

D7 0

$\begin{array}{llll}\text { A9 } 2 & \text { D8 } & 0\end{array}$

B10 4

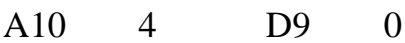

$\mathrm{H} \quad 11$

B12

A11 4

D10 0

Variables:

$\begin{array}{ll}\text { B1 } & 1.42803919 \\ \text { B2 } & 1.41800530 \\ \text { B3 } & 1.39759751 \\ \text { B4 } & 0.96418076 \\ \text { B5 } & 0.96115215 \\ \text { B6 } & 1.51302090 \\ \text { B7 } & 1.08771177 \\ \text { B8 } & 1.08772335 \\ \text { B9 } & 1.08778728 \\ \text { B10 } & 1.51907972 \\ \text { B11 } & 1.08858997 \\ \text { B12 } & 1.08832637 \\ \text { B13 } & 1.08934092\end{array}$




$\begin{array}{lr}\text { A1 } & 108.25990832 \\ \text { A2 } & 110.17159819 \\ \text { A3 } & 101.12148048 \\ \text { A4 } & 108.38858916 \\ \text { A5 } & 107.86333905 \\ \text { A6 } & 110.19455398 \\ \text { A7 } & 109.21211180 \\ \text { A8 } & 109.40787479 \\ \text { A9 } & 111.49446172 \\ \text { A10 } & 109.55770894 \\ \text { A11 } & 110.04067083 \\ \text { A12 } & 110.35289730 \\ \text { D1 } & 60.09421131 \\ \text { D2 } & -107.24435152 \\ \text { D3 } & -78.39442433 \\ \text { D4 } & -112.40333608 \\ \text { D5 } & 174.17307356 \\ \text { D6 } & -65.61309310 \\ \text { D7 } & 53.78620215 \\ \text { D8 } & 122.64462128 \\ \text { D9 } & 59.21801019 \\ \text { D10 } & 179.39905446 \\ \text { D11 } & -61.01471649 \\ \text { Har }\end{array}$

Harmonic Vibrational Frequencies $\left(\mathbf{c m}^{-1}\right)$ :

$\begin{array}{llllllllll}157.10 & 215.45 & 254.64 & 266.88 & 289.13 & 351.63 & 395.94 & 438.87 & 469.01 & 533.39\end{array}$ $\begin{array}{llllllllll}610.62 & 811.41 & 900.45 & 942.08 & 1009.93 & 1018.99 & 1052.21 & 1156.25 & 1222.47 & 1275.33\end{array}$ $\begin{array}{lllllllll}1379.33 & 1414.04 & 1419.69 & 1440.88 & 1488.01 & 1488.58 & 1504.38 & 1512.80 & 3065.61\end{array}$ $\begin{array}{lllllll}3073.57 & 3143.79 & 3154.79 & 3158.62 & 3161.33 & 3840.02 & 3882.61\end{array}$

$\begin{array}{llll}\text { Rotational constants (B/GHZ): } & 4.8013572 & 2.9558337 & 2.8668841\end{array}$

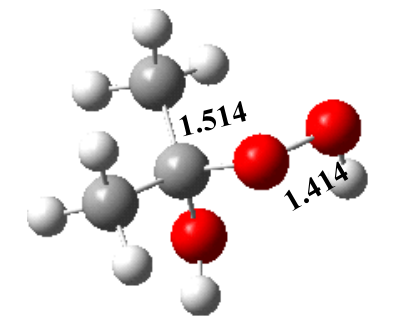

$\mathrm{Pb}_{3}\left(\mathrm{HO}-\mathrm{C}\left(\mathrm{CH}_{3}\right)_{2} \mathrm{OO}-\mathrm{H}\right)$

$\begin{array}{llllllll}\mathrm{C} & & & & & & & \\ \mathrm{O} & 1 & \mathrm{~B} 1 & & & & & \\ \mathrm{O} & 2 & \mathrm{~B} 2 & 1 & \mathrm{~A} 1 & & & \\ \mathrm{O} & 1 & \mathrm{~B} 3 & 2 & \mathrm{~A} 2 & 3 & \mathrm{D} 1 & 0 \\ \mathrm{H} & 3 & \mathrm{~B} 4 & 2 & \mathrm{~A} 3 & 1 & \mathrm{D} 2 & 0 \\ \mathrm{H} & 4 & \mathrm{~B} 5 & 1 & \mathrm{~A} 4 & 2 & \mathrm{D} 3 & 0 \\ \mathrm{C} & 1 & \mathrm{~B} 6 & 4 & \mathrm{~A} 5 & 2 & \mathrm{D} 4 & 0 \\ \mathrm{H} & 7 & \mathrm{~B} 7 & 1 & \text { A6 } & 4 & \text { D5 } & 0\end{array}$




$\begin{array}{rrrrrrrr}\mathrm{H} & 7 & \text { B8 } & 1 & \text { A7 } & 4 & \text { D6 } & 0 \\ \mathrm{H} & 7 & \text { B9 } & 1 & \text { A8 } & 4 & \text { D7 } & 0 \\ \mathrm{C} & 1 & \text { B10 } & 4 & \text { A9 } & 2 & \text { D8 } & 0 \\ \mathrm{H} & 11 & \text { B11 } & 1 & \text { A10 } & 4 & \text { D9 } & 0 \\ \mathrm{H} & 11 & \text { B12 } & 1 & \text { A11 } & 4 & \text { D10 } & 0 \\ \mathrm{H} & 11 & \text { B13 } & 1 & \text { A12 } & 4 & \text { D11 } & 0\end{array}$

Variables:

$\begin{array}{ll}\text { B1 } & 1.42036903 \\ \text { B2 } & 1.41353351 \\ \text { B3 } & 1.40771550 \\ \text { B4 } & 0.96704094 \\ \text { B5 } & 0.96174753 \\ \text { B6 } & 1.51724115 \\ \text { B7 } & 1.08774105 \\ \text { B8 } & 1.08800934 \\ \text { B9 } & 1.09065644 \\ \text { B10 } & 1.51409413 \\ \text { B11 } & 1.08817133 \\ \text { B12 } & 1.08811607 \\ \text { B13 } & 1.08592678\end{array}$

A1 $\quad 109.52808369$

A2 109.02539061

A3 $\quad 102.33339650$

A4 108.69209589

A5 111.87981629

A6 110.32651224

A7 $\quad 109.29683717$

A8 $\quad 110.15669170$

A9 $\quad 107.22797735$

A10 $\quad 109.38232930$

A11 $\quad 109.51457284$

A12 $\quad 110.32563548$

D1 $\quad 63.69901589$

D2 $\quad-70.42057341$

D3 71.96629503

D4 $\quad-113.82811305$

D5 $\quad-179.46084328$

D6 $\quad-59.25388260$

D7 $\quad 60.90401865$

D8 $\quad 121.63715387$

D9 $\quad 67.14427196$

D10 $\quad-172.83920079$

D11 $\quad-52.78580086$

Harmonic Vibrational Frequencies $\left(\mathrm{cm}^{-1}\right)$ : 


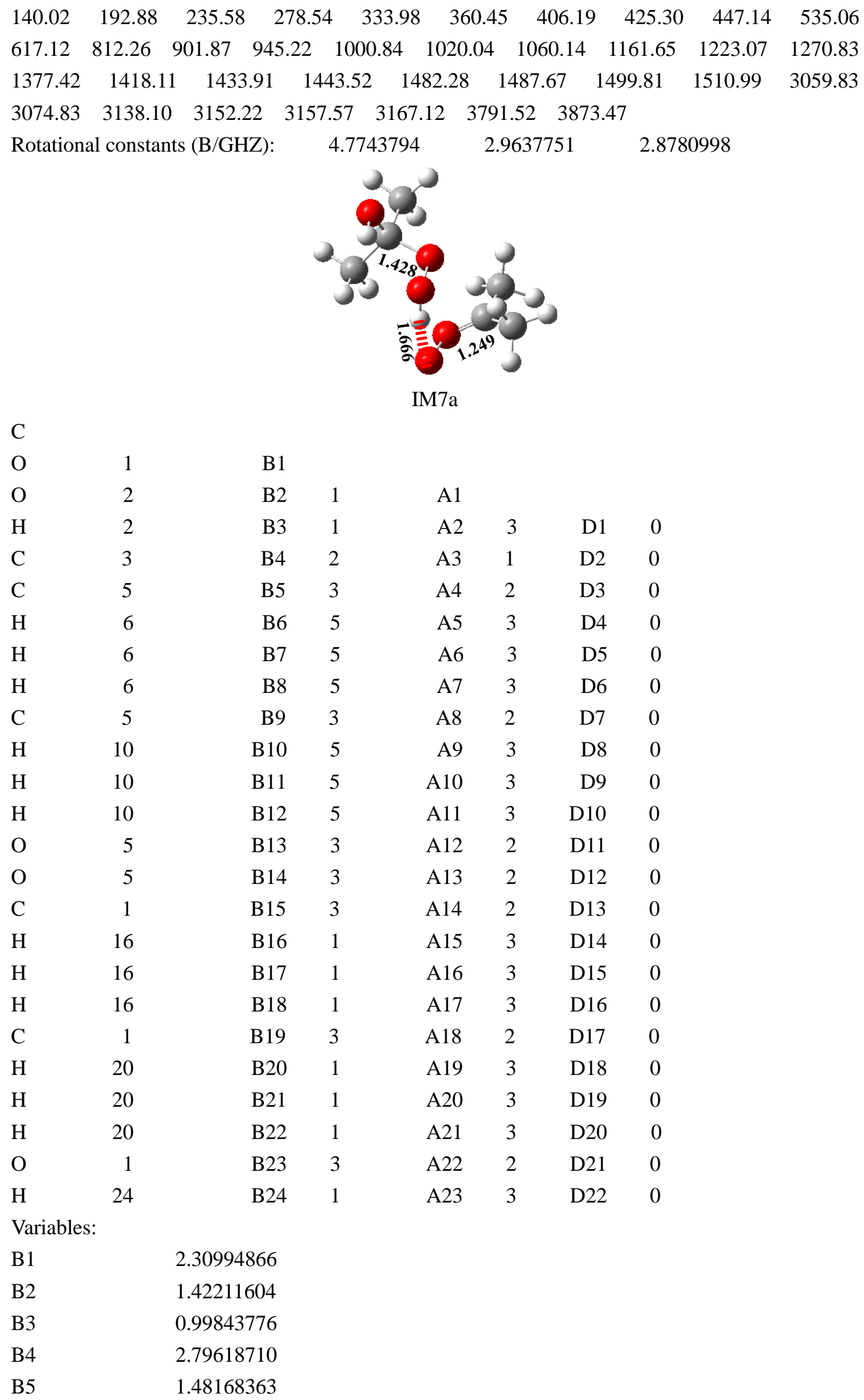




\begin{tabular}{|c|c|}
\hline B6 & 1.09006107 \\
\hline B7 & 1.08587289 \\
\hline B8 & 1.09265242 \\
\hline B9 & 1.47232181 \\
\hline B10 & 1.09380200 \\
\hline B11 & 1.09000982 \\
\hline B12 & 1.08579104 \\
\hline B13 & 2.25260185 \\
\hline B14 & 1.24854891 \\
\hline B15 & 1.51350945 \\
\hline B16 & 1.08788603 \\
\hline B17 & 1.08822470 \\
\hline B18 & 1.08903814 \\
\hline B19 & 1.52108723 \\
\hline B20 & 1.08823829 \\
\hline B21 & 1.08855579 \\
\hline $\mathrm{B} 22$ & 1.08915728 \\
\hline B23 & 1.39546628 \\
\hline B24 & 0.96344045 \\
\hline A1 & 35.93192591 \\
\hline $\mathrm{A} 2$ & 105.41595123 \\
\hline A3 & 95.30499225 \\
\hline A4 & 101.49527504 \\
\hline A5 & 110.05142669 \\
\hline A6 & 111.13924999 \\
\hline A7 & 107.45889653 \\
\hline A8 & 88.88193845 \\
\hline A9 & 108.13676696 \\
\hline A10 & 108.91371112 \\
\hline A11 & 110.64801682 \\
\hline A12 & 73.67567531 \\
\hline A13 & 78.71323115 \\
\hline A14 & 104.21642285 \\
\hline A15 & 109.09102198 \\
\hline A16 & 109.47103905 \\
\hline A17 & 110.41026475 \\
\hline A18 & 110.73296624 \\
\hline A19 & 110.61062393 \\
\hline $\mathrm{A} 20$ & 109.06225400 \\
\hline A21 & 110.73356189 \\
\hline $\mathrm{A} 22$ & 109.61652926 \\
\hline A23 & 106.96024250 \\
\hline D1 & -87.55734855 \\
\hline D2 & -134.98283473 \\
\hline
\end{tabular}




$\begin{array}{lr}\text { D3 } & -176.02956737 \\ \text { D4 } & 151.37311366 \\ \text { D5 } & -85.78033606 \\ \text { D6 } & 34.42173545 \\ \text { D7 } & -52.88037573 \\ \text { D8 } & 4.09999543 \\ \text { D9 } & 119.46283527 \\ \text { D10 } & -116.61175937 \\ \text { D11 } & 35.76249037 \\ \text { D12 } & 69.61050685 \\ \text { D13 } & 175.48984732 \\ \text { D14 } & -175.99046789 \\ \text { D15 } & -56.81423625 \\ \text { D16 } & 64.31193115 \\ \text { D17 } & -62.89038753 \\ \text { D18 } & 64.96293393 \\ \text { D19 } & -175.61793030 \\ \text { D20 } & -55.04328821 \\ \text { D21 } & 60.43622906 \\ \text { D22 } & -56.21232413\end{array}$

Harmonic Vibrational Frequencies $\left(\mathbf{c m}^{-1}\right)$ :

$\begin{array}{lccccccccccr}45.49 & 61.51 & 63.33 & 84.28 & 122.29 & 154.44 & 182.91 & 187.09 & 207.51 & 251.96 & 275.83 \\ 296.25 & 332.63 & 348.67 & 359.89 & 380.38 & 396.77 & 436.52 & 444.46 & 496.78 & 542.75 \\ 602.99 & 610.26 & 814.50 & 827.74 & 900.82 & 902.10 & 915.92 & 938.06 & 955.78 & 991.10 \\ 1008.56 & 1013.21 & 1053.06 & 1091.85 & 1110.40 & 1150.86 & 1238.28 & 1275.29 & 1319.24 \\ 1377.69 & 1394.47 & 1410.65 & 1413.78 & 1431.90 & 1452.69 & 1462.69 & 1474.66 & 1481.59 \\ 1487.90 & 1493.83 & 1502.65 & 1506.48 & 1618.90 & 1668.91 & 3047.49 & 3053.96 & 3063.14 \\ 3067.21 & 3108.93 & 3119.05 & 3124.64 & 3143.10 & 3148.30 & 3151.56 & 3154.49 & 3172.35 \\ 3177.49 & 3863.49 & & & & & & & & \end{array}$

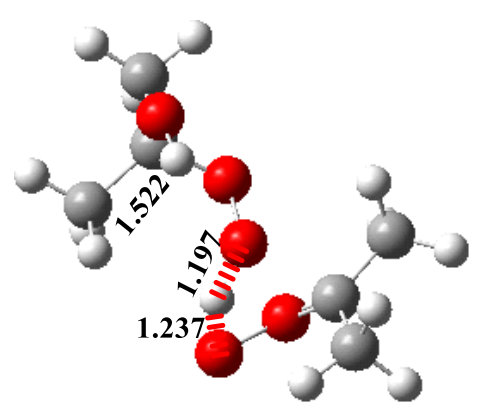

TS7a

$\mathrm{C}$

$\begin{array}{lll}\mathrm{O} & 1 & \mathrm{~B} 1\end{array}$

$\begin{array}{lllll}\mathrm{O} & 2 & \mathrm{~B} 2 & 1 & \mathrm{~A} 1\end{array}$

$\begin{array}{llllllll}\mathrm{H} & 2 & \mathrm{~B} 3 & 1 & \mathrm{~A} 2 & 3 & \mathrm{D} 1 & 0\end{array}$

$\begin{array}{llllllll}\mathrm{C} & 2 & \mathrm{~B} 4 & 1 & \mathrm{~A} 3 & 3 & \mathrm{D} 2 & 0\end{array}$ 


\begin{tabular}{|c|c|c|c|c|c|c|}
\hline $\mathrm{C}$ & 5 & B5 & 2 & A4 & 1 & D3 \\
\hline $\mathrm{H}$ & 6 & B6 & 5 & A5 & 2 & D4 \\
\hline $\mathrm{H}$ & 6 & B7 & 5 & A6 & 2 & D5 \\
\hline $\mathrm{H}$ & 6 & B8 & 5 & A7 & 2 & D6 \\
\hline $\mathrm{C}$ & 5 & B9 & 2 & A8 & 1 & D7 \\
\hline $\mathrm{H}$ & 10 & B10 & 5 & A9 & 2 & D8 \\
\hline $\mathrm{H}$ & 10 & B11 & 5 & $\mathrm{~A} 10$ & 2 & D9 \\
\hline $\mathrm{H}$ & 10 & B12 & 5 & A11 & 2 & D10 \\
\hline $\mathrm{O}$ & 5 & B13 & 2 & $\mathrm{~A} 12$ & 1 & D11 \\
\hline $\mathrm{O}$ & 5 & B14 & 2 & A13 & 1 & D12 \\
\hline $\mathrm{C}$ & 1 & B15 & 3 & A14 & 2 & D13 \\
\hline $\mathrm{H}$ & 16 & B16 & 1 & A15 & 3 & D14 \\
\hline $\mathrm{H}$ & 16 & B17 & 1 & A16 & 3 & D15 \\
\hline $\mathrm{H}$ & 16 & B18 & 1 & A17 & 3 & D16 \\
\hline $\mathrm{C}$ & 1 & B19 & 3 & A18 & 2 & D17 \\
\hline $\mathrm{H}$ & 20 & B20 & 1 & A19 & 3 & D18 \\
\hline $\mathrm{H}$ & 20 & B21 & 1 & A20 & 3 & D19 \\
\hline $\mathrm{H}$ & 20 & B22 & 1 & A21 & 3 & D20 \\
\hline $\mathrm{O}$ & 1 & B23 & 3 & $\mathrm{~A} 22$ & 2 & D21 \\
\hline $\mathrm{H}$ & 24 & B24 & 1 & A23 & 3 & D22 \\
\hline Varia & & & & & & \\
\hline B1 & \multicolumn{2}{|r|}{2.30587951} & & & & \\
\hline B2 & \multicolumn{2}{|r|}{1.42029801} & & & & \\
\hline B3 & \multicolumn{2}{|r|}{1.19661251} & & & & \\
\hline B4 & \multicolumn{2}{|r|}{2.12665176} & & & & \\
\hline B5 & \multicolumn{2}{|r|}{1.48139931} & & & & \\
\hline B6 & \multicolumn{2}{|r|}{1.08782503} & & & & \\
\hline B7 & \multicolumn{2}{|r|}{1.08628347} & & & & \\
\hline B8 & \multicolumn{2}{|r|}{1.09280403} & & & & \\
\hline B9 & \multicolumn{2}{|r|}{1.48602114} & & & & \\
\hline B 10 & \multicolumn{2}{|r|}{1.08658587} & & & & \\
\hline B11 & \multicolumn{2}{|r|}{1.08265787} & & & & \\
\hline B12 & \multicolumn{2}{|r|}{1.09435380} & & & & \\
\hline B13 & \multicolumn{2}{|r|}{2.21718167} & & & & \\
\hline B14 & \multicolumn{2}{|r|}{1.27984906} & & & & \\
\hline B15 & \multicolumn{2}{|r|}{1.51301310} & & & & \\
\hline B16 & \multicolumn{2}{|r|}{1.08794896} & & & & \\
\hline B17 & \multicolumn{2}{|r|}{1.08794379} & & & & \\
\hline B18 & \multicolumn{2}{|r|}{1.08803628} & & & & \\
\hline B19 & \multicolumn{2}{|r|}{1.52213984} & & & & \\
\hline B20 & \multicolumn{2}{|r|}{1.08926000} & & & & \\
\hline B 21 & \multicolumn{2}{|r|}{1.08847705} & & & & \\
\hline B22 & \multicolumn{2}{|r|}{1.08898851} & & & & \\
\hline B23 & \multicolumn{2}{|r|}{1.39930155} & & & & \\
\hline
\end{tabular}




\begin{tabular}{|c|c|}
\hline B24 & 0.96315731 \\
\hline A1 & 35.83803365 \\
\hline $\mathrm{A} 2$ & 102.50876962 \\
\hline A3 & 133.68778180 \\
\hline A4 & 107.71111012 \\
\hline A5 & 111.06572004 \\
\hline A6 & 110.62536248 \\
\hline A7 & 105.94723297 \\
\hline A8 & 89.71485017 \\
\hline A9 & 109.33822751 \\
\hline A10 & 112.18720316 \\
\hline A11 & 106.22855010 \\
\hline A12 & 66.10533091 \\
\hline A13 & 89.20506929 \\
\hline A14 & 104.41883384 \\
\hline A15 & 109.04959610 \\
\hline A16 & 109.40569395 \\
\hline A17 & 110.32367851 \\
\hline A18 & 110.59789523 \\
\hline A19 & 110.64868051 \\
\hline A 20 & 109.20452138 \\
\hline A 21 & 110.36947028 \\
\hline A22 & 109.79157084 \\
\hline A 23 & 106.78025961 \\
\hline D1 & -95.26408199 \\
\hline D2 & -18.41756165 \\
\hline D3 & 56.85862266 \\
\hline D4 & 129.39295618 \\
\hline D5 & -106.14255269 \\
\hline D6 & 11.10801703 \\
\hline D7 & 178.42483015 \\
\hline D8 & -63.55549936 \\
\hline D9 & 59.97693823 \\
\hline D10 & 179.64737076 \\
\hline D11 & -87.61971874 \\
\hline D12 & -58.22783812 \\
\hline D13 & 176.93526894 \\
\hline D14 & -176.17895241 \\
\hline D15 & -57.07171307 \\
\hline D16 & 63.67123527 \\
\hline D17 & -61.53339509 \\
\hline D18 & 67.88568348 \\
\hline D19 & -173.04361901 \\
\hline D20 & -52.45989794 \\
\hline
\end{tabular}


Harmonic Vibrational Frequencies $\left(\mathbf{c m}^{-1}\right)$ :

$\begin{array}{lccccccccccr}1021.22 i & 34.14 & 42.48 & 60.79 & 87.49 & 173.09 & 183.06 & 202.05 & 206.32 & 211.63 & 267.60 \\ 304.61 & 332.58 & 356.59 & 373.77 & 383.44 & 402.53 & 438.17 & 453.30 & 522.45 & 553.22 \\ 580.55 & 625.64 & 730.11 & 815.82 & 821.98 & 911.58 & 943.08 & 954.71 & 959.72 & 986.92 \\ 1006.92 & 1014.11 & 1066.57 & 1084.06 & 1105.74 & 1147.82 & 1242.80 & 1267.39 & 1316.37 \\ 1378.22 & 1401.27 & 1412.22 & 1423.78 & 1433.06 & 1438.91 & 1457.13 & 1477.36 & 1485.91 \\ 1487.14 & 1493.24 & 1503.17 & 1510.24 & 1543.59 & 1588.20 & 1830.56 & 3052.57 & 3057.51 \\ 3062.11 & 3075.41 & 3132.35 & 3142.72 & 3146.46 & 3146.64 & 3160.17 & 3163.32 & 3172.81 \\ 3217.82 & 3863.02 & & & & & & & & \end{array}$

Rotational constants (B/GHZ): $\quad 1.8611556 \quad 0.6571852 \quad 0.6168925$

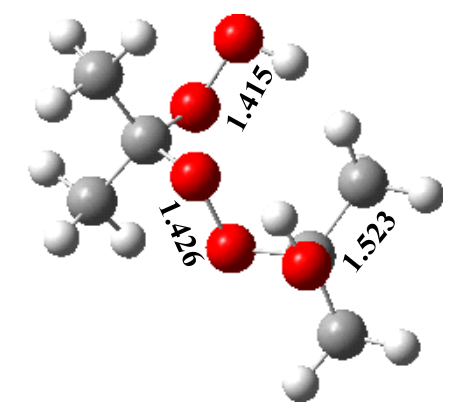

P7a

C

$\begin{array}{lll}\text { O } & 1 & \text { B1 }\end{array}$

O 1

$\mathrm{H} \quad 2$

C 2

C 5

$\mathrm{H} \quad 6$

$\mathrm{H} \quad 6$

$\mathrm{H} \quad 6$

C 5

$\mathrm{H} \quad 10$

$\mathrm{H} \quad 10$

$\mathrm{H} \quad 10$

O 5

$\mathrm{O} \quad 5$

C 1

$\mathrm{H} \quad 16$

$\mathrm{H} \quad 16$

$\mathrm{H} \quad 16$

C 1

$\mathrm{H} \quad 20$

B2 2

A1

B3 1

A2

B4 1

A3 3

D1 0

B5 2

A4 1

D2 0

B6 5

A5 2

D3 0

B7 5

A6 2

D4 0

B8 5

A7 2

D5 0

$\begin{array}{llll}\text { B9 } & 2 & \text { A8 } & 1\end{array}$

D6 0

B10 5

A9 2

D7 0

B11 5

A10 2

D8 0

$\begin{array}{llll}\mathrm{B} 12 & 5 & \mathrm{~A} 11 & 2\end{array}$

D9 0

B13 2

A12 1

D10 0

$\begin{array}{llll}\text { B14 } 2 & \text { A13 } & 1\end{array}$

D11 0

B15 3

A14 2

D12 0

B16 1

A15 3

D13 0

B17 1 A16 3

D14 0

$\begin{array}{llll}\text { B18 } 1 & \text { A17 } & 3\end{array}$

D15 0

$\begin{array}{llll}\text { B } 19 & 3 & \text { A18 } & 2\end{array}$

D16 0

$\begin{array}{llll}\text { B20 } & 1 & \text { A19 } & 3\end{array}$

D17 0

$\mathrm{H} \quad 20$

$\mathrm{H} \quad 20$

B21 1

B22 1

A20 3

D18 0

D19 0

A21 3

D20 0 


$\begin{array}{rrrrrrrr}\mathrm{O} & 1 & \text { B23 } & 3 & \text { A22 } & 2 & \text { D21 } & 0 \\ \mathrm{H} & 24 & \text { B24 } & 1 & \text { A23 } & 3 & \text { D22 } & 0\end{array}$

Variables:

$\begin{array}{ll}\text { B1 } & 2.29384480 \\ \text { B2 } & 1.41991671 \\ \text { B3 } & 2.77181151 \\ \text { B4 } & 1.41563488 \\ \text { B5 } & 1.51728812 \\ \text { B6 } & 1.08764905 \\ \text { B7 } & 1.08617650 \\ \text { B8 } & 1.08789207 \\ \text { B9 } & 1.51671525 \\ \text { B10 } & 1.08765668 \\ \text { B11 } & 1.08606173 \\ \text { B12 } & 1.08799280 \\ \text { B13 } & 2.31572911 \\ \text { B14 } & 1.41117263 \\ \text { B15 } & 1.51388283 \\ \text { B16 } & 1.08776132 \\ \text { B17 } & 1.08760462 \\ \text { B18 } & 1.08792566 \\ \text { B19 } & 1.52265333 \\ \text { B20 } & 1.09004253 \\ \text { B21 } & 1.08803858 \\ \text { B22 } & 1.09125397 \\ \text { B23 } & 1.39767133 \\ \text { B24 } & 0.96216003 \\ \text { A1 } & 36.381339\end{array}$

A1

36.38133949

A2 85.52290916

A3 $\quad 135.40767946$

A4 112.37419632

A5 109.19952253

A6 110.58992543

A7 109.03597300

A8 $\quad 104.14563950$

A9 109.33528602

A10 $\quad 110.80978867$

A11 $\quad 108.75616587$

A12 95.80308420

A13 $\quad 110.62684998$

A14 $\quad 103.81168838$

A15 108.90985249

A16 $\quad 109.29889992$

A17 $\quad 110.41727385$ 


$\begin{array}{lr}\text { A18 } & 111.04645212 \\ \text { A19 } & 110.19355451 \\ \text { A20 } & 109.35028182 \\ \text { A21 } & 109.83046578 \\ \text { A22 } & 110.45008760 \\ \text { A23 } & 108.29381451 \\ \text { D1 } & -111.10611994 \\ \text { D2 } & -51.78079758 \\ \text { D3 } & 75.42219827 \\ \text { D4 } & 168.02421496 \\ \text { D5 } & -71.68699072 \\ \text { D6 } & 48.26708841 \\ \text { D7 } & -161.86460697 \\ \text { D8 } & -70.99514665 \\ \text { D9 } & 49.79038951 \\ \text { D10 } & 169.45649836 \\ \text { D11 } & -73.13700182 \\ \text { D12 } & -40.39593049 \\ \text { D13 } & 177.90328299 \\ \text { D14 } & -176.30675413 \\ \text { D15 } & -57.25506156 \\ \text { D16 } & 63.47108447 \\ \text { D17 } & -60.86698402 \\ \text { D18 } & 71.34161754 \\ \text { D19 } & -170.08907487 \\ \text { D20 } & -50.46411455 \\ \text { D21 } & 62.87615714 \\ \text { D22 } & -59.93356923 \\ \text { Hart }\end{array}$

Harmonic Vibrational Frequencies $\left(\mathrm{cm}^{-1}\right)$ :

$\begin{array}{lllllllllll}44.60 & 81.92 & 119.11 & 159.04 & 198.21 & 208.50 & 232.54 & 236.86 & 244.12 & 266.78 & 284.27\end{array}$ $\begin{array}{llllllllll}328.52 & 359.33 & 370.67 & 375.64 & 390.26 & 410.17 & 452.89 & 463.93 & 537.98 & 590.50\end{array}$ $\begin{array}{llllllllll}607.60 & 619.05 & 812.34 & 846.62 & 907.81 & 927.49 & 946.56 & 960.44 & 974.63 & 1010.71\end{array}$ $\begin{array}{lllllllll}1026.22 & 1030.27 & 1054.70 & 1066.04 & 1155.43 & 1207.34 & 1246.33 & 1255.52 & 1269.86\end{array}$ $\begin{array}{lllllllll}1313.94 & 1377.50 & 1405.31 & 1414.35 & 1423.90 & 1429.68 & 1439.70 & 1481.87 & 1487.21\end{array}$ $\begin{array}{lllllllll}1487.95 & 1492.98 & 1497.10 & 1504.98 & 1510.39 & 1529.69 & 3049.53 & 3072.82 & 3083.14\end{array}$ $\begin{array}{lllllllll}3085.96 & 3128.87 & 3143.74 & 3159.15 & 3160.93 & 3162.20 & 3167.27 & 3176.21 & 3181.38\end{array}$ $3842.24 \quad 3877.61$

$\begin{array}{llll}\text { Rotational constants (B/GHZ): } & 1.7988145 & 0.7309428 & 0.6661869\end{array}$ 


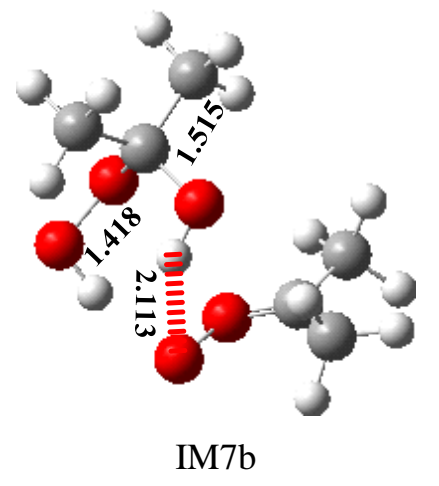

C

$\begin{array}{lll}\text { C } & 1 & \text { B1 }\end{array}$

$\mathrm{H} \quad 2$

$\mathrm{H} \quad 2$

$\mathrm{H} \quad 2$

C 1

$\mathrm{H} \quad 6$

$\mathrm{H} \quad 6$

$\mathrm{H} \quad 6$

O 1

$\mathrm{H} \quad 10$

O 1

C $\quad 10$

C 13

$\mathrm{H} \quad 14$

$\mathrm{H} \quad 14$

$\mathrm{H} \quad 14$

C 13

$\mathrm{H} \quad 18$

$\mathrm{H} \quad 18$

$\mathrm{H} \quad 18$

O 13

O 13

O 1

$\mathrm{H} \quad 12$

Variables:

B1

1.51912522

B2

1.08666541

B3

1.08870560

B4

1.08886031

B5

1.51467507

B6

1.08909095

B7

1.08754406

B8

1.08813772

B9

1.41146083

$\begin{array}{llllll}\text { B3 } & 1 & \text { A2 } & 3 & \text { D1 } & 0\end{array}$

$\begin{array}{llllll}\text { B4 } & 1 & \text { A3 } & 4 & \text { D2 } & 0\end{array}$

$\begin{array}{llllll}\text { B5 } & 2 & \text { A4 } & 3 & \text { D3 } & 0\end{array}$

$\begin{array}{llllll}\text { B6 } & 1 & \text { A5 } & 2 & \text { D4 } & 0\end{array}$

$\begin{array}{llllll}\text { B7 } & 1 & \text { A6 } & 2 & \text { D5 } & 0\end{array}$

$\begin{array}{llllll}\text { B8 } & 1 & \text { A7 } & 2 & \text { D6 } & 0\end{array}$

$\begin{array}{llllll}\text { B9 } & 6 & \text { A8 } & 2 & \text { D7 } & 0\end{array}$

$\begin{array}{llllll}\text { B10 } & 1 & \text { A9 } & 6 & \text { D8 } & 0\end{array}$

$\begin{array}{llllll}\mathrm{B} 11 & 10 & \mathrm{~A} 10 & 6 & \mathrm{D} 9 & 0\end{array}$

$\begin{array}{llllll}\mathrm{B} 12 & 1 & \mathrm{~A} 11 & 6 & \mathrm{D} 10 & 0\end{array}$

$\begin{array}{llllll}\text { B13 } & 10 & \text { A12 } & 1 & \text { D11 } & 0\end{array}$

$\begin{array}{llllll}\mathrm{B} 14 & 13 & \mathrm{~A} 13 & 10 & \mathrm{D} 12 & 0\end{array}$

$\begin{array}{llllll}\text { B15 } & 13 & \text { A14 } & 10 & \text { D13 } & 0\end{array}$

$\begin{array}{llllll}\text { B16 } & 13 & \text { A15 } & 10 & \text { D14 } & 0\end{array}$

$\begin{array}{llllll}\text { B17 } & 10 & \text { A16 } & 1 & \text { D15 } & 0\end{array}$

$\begin{array}{llllll}\text { B18 } & 13 & \text { A17 } & 10 & \text { D16 } & 0\end{array}$

$\begin{array}{llllll}\mathrm{B} 19 & 13 & \mathrm{~A} 18 & 10 & \mathrm{D} 17 & 0\end{array}$

$\begin{array}{llllll}\text { B20 } & 13 & \text { A19 } & 10 & \text { D18 } & 0\end{array}$

$\begin{array}{llllll}\text { B21 } & 10 & \text { A20 } & 1 & \text { D19 } & 0\end{array}$

$\begin{array}{llllll}\mathrm{B} 22 & 10 & \mathrm{~A} 21 & 1 & \mathrm{D} 20 & 0\end{array}$

$\begin{array}{llllll}\mathrm{B} 23 & 10 & \mathrm{~A} 22 & 6 & \mathrm{D} 21 & 0\end{array}$

$\begin{array}{llllll}\text { B24 } & 1 & \text { A23 } & 10 & \text { D22 } & 0\end{array}$ 


\begin{tabular}{|c|c|}
\hline B 10 & 0.96929819 \\
\hline B11 & 2.31632335 \\
\hline B12 & 2.96479402 \\
\hline B13 & 1.48190801 \\
\hline B14 & 1.09184188 \\
\hline B15 & 1.09080734 \\
\hline B16 & 1.08574494 \\
\hline B17 & 1.47302927 \\
\hline B18 & 1.09259673 \\
\hline B19 & 1.08958266 \\
\hline B20 & 1.08596796 \\
\hline B21 & 2.24756788 \\
\hline B22 & 1.24695040 \\
\hline $\mathrm{B} 23$ & 1.41527190 \\
\hline B24 & 0.98294912 \\
\hline $\mathrm{A} 1$ & 110.46521688 \\
\hline $\mathrm{A} 2$ & 109.13296872 \\
\hline A3 & 110.03360884 \\
\hline A4 & 112.70390304 \\
\hline A5 & 109.43502513 \\
\hline A6 & 110.37291320 \\
\hline A7 & 109.23982934 \\
\hline A8 & 107.11708914 \\
\hline A9 & 107.95300410 \\
\hline A 10 & 96.35845866 \\
\hline A11 & 117.42934070 \\
\hline A 12 & 112.80938637 \\
\hline A13 & 108.04598173 \\
\hline A14 & 109.75687501 \\
\hline A 15 & 110.95799654 \\
\hline A16 & 82.71749073 \\
\hline A 17 & 107.76469556 \\
\hline A18 & 109.07558139 \\
\hline A19 & 110.68169179 \\
\hline A20 & 64.97485761 \\
\hline A21 & 74.90335490 \\
\hline A22 & 110.13469824 \\
\hline $\mathrm{A} 23$ & 101.79388520 \\
\hline D1 & 119.88699709 \\
\hline D2 & 120.11734103 \\
\hline D3 & -176.44539623 \\
\hline D4 & -175.09092970 \\
\hline D5 & -55.02475699 \\
\hline D6 & 65.42921691 \\
\hline
\end{tabular}




$\begin{array}{lr}\text { D7 } & -122.13652958 \\ \text { D8 } & -161.86038955 \\ \text { D9 } & 146.06370822 \\ \text { D10 } & -78.92314007 \\ \text { D11 } & 56.45916003 \\ \text { D12 } & 38.78233848 \\ \text { D13 } & 155.55831450 \\ \text { D14 } & -81.89096438 \\ \text { D15 } & 179.26521920 \\ \text { D16 } & -4.83226908 \\ \text { D17 } & 110.93706419 \\ \text { D18 } & -125.05719537 \\ \text { D19 } & -89.43149150 \\ \text { D20 } & -55.53915016 \\ \text { D21 } & 112.64700173 \\ \text { D22 } & -21.32732074\end{array}$

Harmonic Vibrational Frequencies $\left(\mathbf{c m}^{-1}\right)$ :

$\begin{array}{lccccccccccr}28.00 & 54.96 & 74.22 & 98.79 & 128.92 & 131.86 & 156.07 & 176.53 & 194.91 & 228.09 & 268.45 \\ 294.48 & 325.16 & 356.74 & 361.33 & 379.17 & 400.56 & 444.30 & 489.45 & 525.04 & 551.30 \\ 606.64 & 616.86 & 738.89 & 810.41 & 830.64 & 907.80 & 910.64 & 943.81 & 965.27 & 993.66 \\ 1000.92 & 1015.21 & 1062.93 & 1096.05 & 1105.62 & 1181.33 & 1239.99 & 1267.99 & 1320.10 \\ 1383.66 & 1386.15 & 1411.94 & 1413.79 & 1434.89 & 1458.44 & 1468.25 & 1477.20 & 1482.13 \\ 1484.45 & 1492.04 & 1498.52 & 1510.82 & 1575.54 & 1679.24 & 3052.60 & 3056.50 & 3061.10 \\ 3066.30 & 3117.84 & 3120.74 & 3139.80 & 3141.69 & 3151.01 & 3161.59 & 3168.61 & 3182.01 \\ 3455.59 & 3739.52 & & & & & & & & \end{array}$

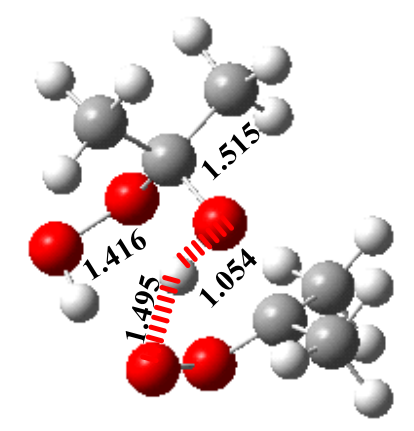

TS7b

$\mathrm{C}$

$\begin{array}{lll}\mathrm{C} & 1 & \mathrm{~B} 1\end{array}$

$\begin{array}{lllll}\mathrm{H} & 2 & \mathrm{~B} 2 & 1 & \mathrm{~A} 1\end{array}$

$\begin{array}{llllllll}\mathrm{H} & 2 & \mathrm{~B} 3 & 1 & \mathrm{~A} 2 & 3 & \mathrm{D} 1 & 0\end{array}$

$\begin{array}{lllllllll}\mathrm{H} & 2 & \mathrm{~B} 4 & 1 & \mathrm{~A} 3 & 4 & \mathrm{D} 2 & 0\end{array}$

$\begin{array}{llllllll}\text { C } & 1 & \text { B5 } & 2 & \text { A4 } & 3 & \text { D3 } & 0\end{array}$

$\begin{array}{llllllll}\mathrm{H} & 6 & \mathrm{~B} 6 & 1 & \mathrm{~A} 5 & 2 & \mathrm{D} 4 & 0\end{array}$

$\begin{array}{llllllll}\mathrm{H} & 6 & \mathrm{~B} 7 & 1 & \mathrm{~A} 6 & 2 & \mathrm{D} 5 & 0\end{array}$

$\begin{array}{llllllll}\mathrm{H} & 6 & \mathrm{~B} 8 & 1 & \mathrm{~A} 7 & 2 & \mathrm{D} 6 & 0\end{array}$ 


\begin{tabular}{|c|c|c|c|c|c|c|}
\hline $\mathrm{O}$ & 1 & B9 & 6 & A 8 & 2 & D7 \\
\hline $\mathrm{H}$ & 10 & B10 & 1 & A9 & 6 & D8 \\
\hline $\mathrm{O}$ & 1 & B11 & 10 & A10 & 2 & D9 \\
\hline $\mathrm{C}$ & 10 & B12 & 1 & A11 & 6 & D10 \\
\hline $\mathrm{C}$ & 13 & B13 & 10 & A12 & 1 & D11 \\
\hline $\mathrm{H}$ & 14 & B14 & 13 & A13 & 10 & D12 \\
\hline $\mathrm{H}$ & 14 & B15 & 13 & A14 & 10 & D13 \\
\hline $\mathrm{H}$ & 14 & B16 & 13 & A15 & 10 & D14 \\
\hline $\mathrm{C}$ & 13 & B17 & 10 & A16 & 1 & D15 \\
\hline $\mathrm{H}$ & 18 & B18 & 13 & A17 & 10 & D16 \\
\hline $\mathrm{H}$ & 18 & B19 & 13 & A18 & 10 & D17 \\
\hline $\mathrm{H}$ & 18 & B20 & 13 & A19 & 10 & D18 \\
\hline $\mathrm{O}$ & 13 & B21 & 10 & A20 & 1 & D19 \\
\hline $\mathrm{O}$ & 13 & B22 & 10 & A21 & 1 & D20 \\
\hline $\mathrm{O}$ & 1 & B23 & 10 & A22 & 13 & D21 \\
\hline $\mathrm{H}$ & 12 & B24 & 1 & A23 & 24 & D22 \\
\hline \multicolumn{7}{|c|}{ Variables: } \\
\hline B1 & \multicolumn{2}{|c|}{1.51793124} & & & & \\
\hline B2 & \multicolumn{2}{|c|}{1.08683102} & & & & \\
\hline B3 & \multicolumn{2}{|c|}{1.08826680} & & & & \\
\hline B4 & \multicolumn{2}{|c|}{1.08839803} & & & & \\
\hline B5 & \multicolumn{2}{|c|}{1.51542228} & & & & \\
\hline B6 & \multicolumn{2}{|c|}{1.08740288} & & & & \\
\hline B7 & \multicolumn{2}{|c|}{1.08756003} & & & & \\
\hline B8 & \multicolumn{2}{|c|}{1.08804320} & & & & \\
\hline B9 & \multicolumn{2}{|c|}{1.43103489} & & & & \\
\hline B10 & \multicolumn{2}{|c|}{1.05380252} & & & & \\
\hline B11 & \multicolumn{2}{|c|}{2.33143227} & & & & \\
\hline B12 & \multicolumn{2}{|c|}{1.92870523} & & & & \\
\hline B13 & \multicolumn{2}{|c|}{1.49058817} & & & & \\
\hline B14 & \multicolumn{2}{|c|}{1.08707406} & & & & \\
\hline B15 & \multicolumn{2}{|c|}{1.09194538} & & & & \\
\hline B16 & \multicolumn{2}{|c|}{1.08631594} & & & & \\
\hline B17 & \multicolumn{2}{|c|}{1.49488298} & & & & \\
\hline B18 & \multicolumn{2}{|c|}{1.08563631} & & & & \\
\hline B19 & \multicolumn{2}{|c|}{1.08380553} & & & & \\
\hline B20 & \multicolumn{2}{|c|}{1.09312044} & & & & \\
\hline B 21 & \multicolumn{2}{|c|}{2.23457018} & & & & \\
\hline B22 & \multicolumn{2}{|c|}{1.29274492} & & & & \\
\hline B23 & \multicolumn{2}{|c|}{1.40750391} & & & & \\
\hline B24 & & & & & & \\
\hline $\mathrm{A} 1$ & & & & & & \\
\hline $\mathrm{A} 2$ & & & & & & \\
\hline A3 & & 88 & & & & \\
\hline
\end{tabular}




\begin{tabular}{|c|c|}
\hline A4 & 112.43505581 \\
\hline A5 & 110.69396512 \\
\hline A6 & 109.59455493 \\
\hline A7 & 109.25702145 \\
\hline A8 & 108.40061155 \\
\hline A9 & 108.69906868 \\
\hline A 10 & 100.85572238 \\
\hline A11 & 122.96840554 \\
\hline A12 & 109.67233791 \\
\hline A13 & 110.13278609 \\
\hline A14 & 107.50459589 \\
\hline A15 & 110.37651183 \\
\hline A16 & 93.01875964 \\
\hline A17 & 110.37445754 \\
\hline A18 & 111.33002613 \\
\hline A19 & 106.15690301 \\
\hline A20 & 71.24528107 \\
\hline A21 & 95.84259798 \\
\hline A22 & 110.21663850 \\
\hline $\mathrm{A} 23$ & 94.28445951 \\
\hline D1 & 120.39119833 \\
\hline D2 & 119.98823918 \\
\hline D3 & -172.50452175 \\
\hline D4 & -171.92428843 \\
\hline D5 & -52.25939734 \\
\hline D6 & 67.60809322 \\
\hline D7 & -119.32008888 \\
\hline D8 & -173.75650334 \\
\hline D9 & -89.19244136 \\
\hline D10 & -84.16805698 \\
\hline D11 & 46.03441556 \\
\hline D12 & 56.57286927 \\
\hline D13 & 174.27846511 \\
\hline D14 & -66.54691392 \\
\hline D15 & 167.75586601 \\
\hline D16 & -61.89314937 \\
\hline D17 & 61.70496038 \\
\hline D18 & -179.76365708 \\
\hline D19 & -99.13876586 \\
\hline D20 & -71.07370870 \\
\hline D21 & 30.07421026 \\
\hline D22 & 108.47346682 \\
\hline
\end{tabular}

Harmonic Vibrational Frequencies $\left(\mathbf{c m}^{-1}\right)$ :

$\begin{array}{llllllllll}389.61 i & 62.31 & 120.67 & 145.61 & 162.48 & 195.53 & 208.44 & 225.07 & 235.09 & 245.37\end{array}$ 


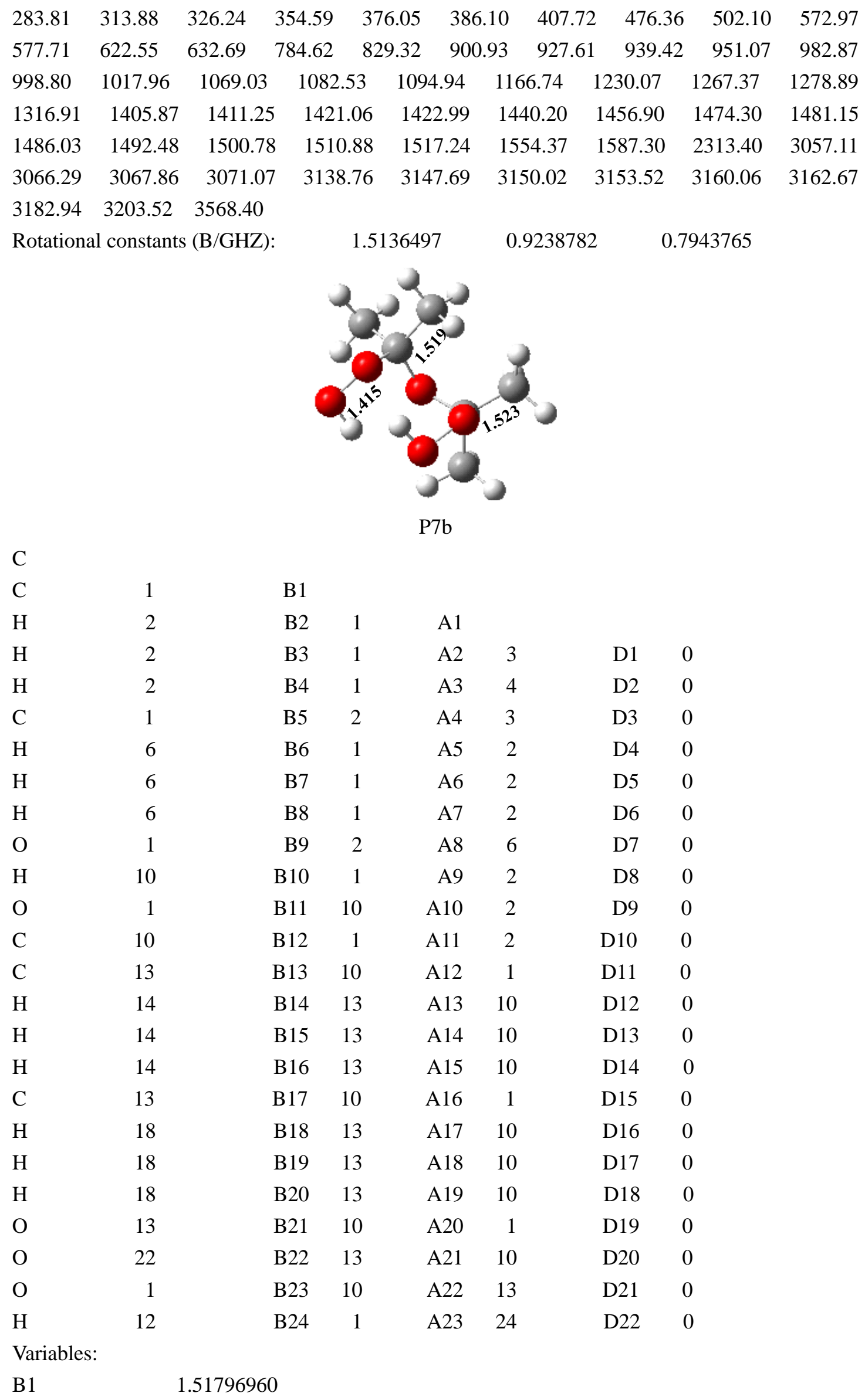




\begin{tabular}{|c|c|}
\hline B2 & 1.08631684 \\
\hline B3 & 1.08829144 \\
\hline B4 & 1.08808809 \\
\hline B5 & 1.52325025 \\
\hline B6 & 1.08565516 \\
\hline B7 & 1.08797718 \\
\hline B8 & 1.08811447 \\
\hline B9 & 1.42255353 \\
\hline B10 & 2.29473798 \\
\hline B11 & 2.32069363 \\
\hline B12 & 1.40884321 \\
\hline B13 & 1.51920615 \\
\hline B14 & 1.08785477 \\
\hline B15 & 1.08813714 \\
\hline B16 & 1.08646695 \\
\hline B 17 & 1.51720452 \\
\hline B18 & 1.08835907 \\
\hline B19 & 1.08581886 \\
\hline B20 & 1.08816468 \\
\hline B21 & 2.32495472 \\
\hline B22 & 1.41502654 \\
\hline B23 & 1.40940663 \\
\hline B24 & 0.96914405 \\
\hline $\mathrm{A} 1$ & 110.50374449 \\
\hline $\mathrm{A} 2$ & 109.28436959 \\
\hline $\mathrm{A} 3$ & 109.46802991 \\
\hline A4 & 111.11830414 \\
\hline A5 & 111.89257328 \\
\hline A6 & 109.40296879 \\
\hline A7 & 108.72989545 \\
\hline A8 & 104.83494213 \\
\hline A9 & 106.24586544 \\
\hline A 10 & 95.67208426 \\
\hline A11 & 124.32100140 \\
\hline A12 & 116.73269760 \\
\hline A13 & 109.55514170 \\
\hline A14 & 108.33357807 \\
\hline A 15 & 111.39150013 \\
\hline A16 & 105.45983152 \\
\hline A17 & 109.19833860 \\
\hline A18 & 110.35968385 \\
\hline A19 & 109.54484776 \\
\hline A20 & 89.02886257 \\
\hline A 21 & 35.38133353 \\
\hline
\end{tabular}




$\begin{array}{lr}\text { A22 } & 112.64923397 \\ \text { A23 } & 107.21663867 \\ \text { D1 } & 119.95094209 \\ \text { D2 } & 119.89265735 \\ \text { D3 } & -178.06251157 \\ \text { D4 } & -166.65239770 \\ \text { D5 } & -46.70314912 \\ \text { D6 } & 72.38135463 \\ \text { D7 } & 121.87549827 \\ \text { D8 } & 70.25016533 \\ \text { D9 } & -90.37857456 \\ \text { D10 } & 158.58911122 \\ \text { D11 } & 38.99692642 \\ \text { D12 } & 58.23453085 \\ \text { D13 } & 176.55327352 \\ \text { D14 } & -63.60747206 \\ \text { D15 } & 163.74389055 \\ \text { D16 } & -69.79780907 \\ \text { D17 } & 50.01722280 \\ \text { D18 } & 170.29621483 \\ \text { D19 } & -106.68644926 \\ \text { D20 } & 125.98673456 \\ \text { D21 } & 36.57631911 \\ \text { D22 } & 83.22823206\end{array}$

Harmonic Vibrational Frequencies $\left(\mathbf{c m}^{-1}\right)$ :

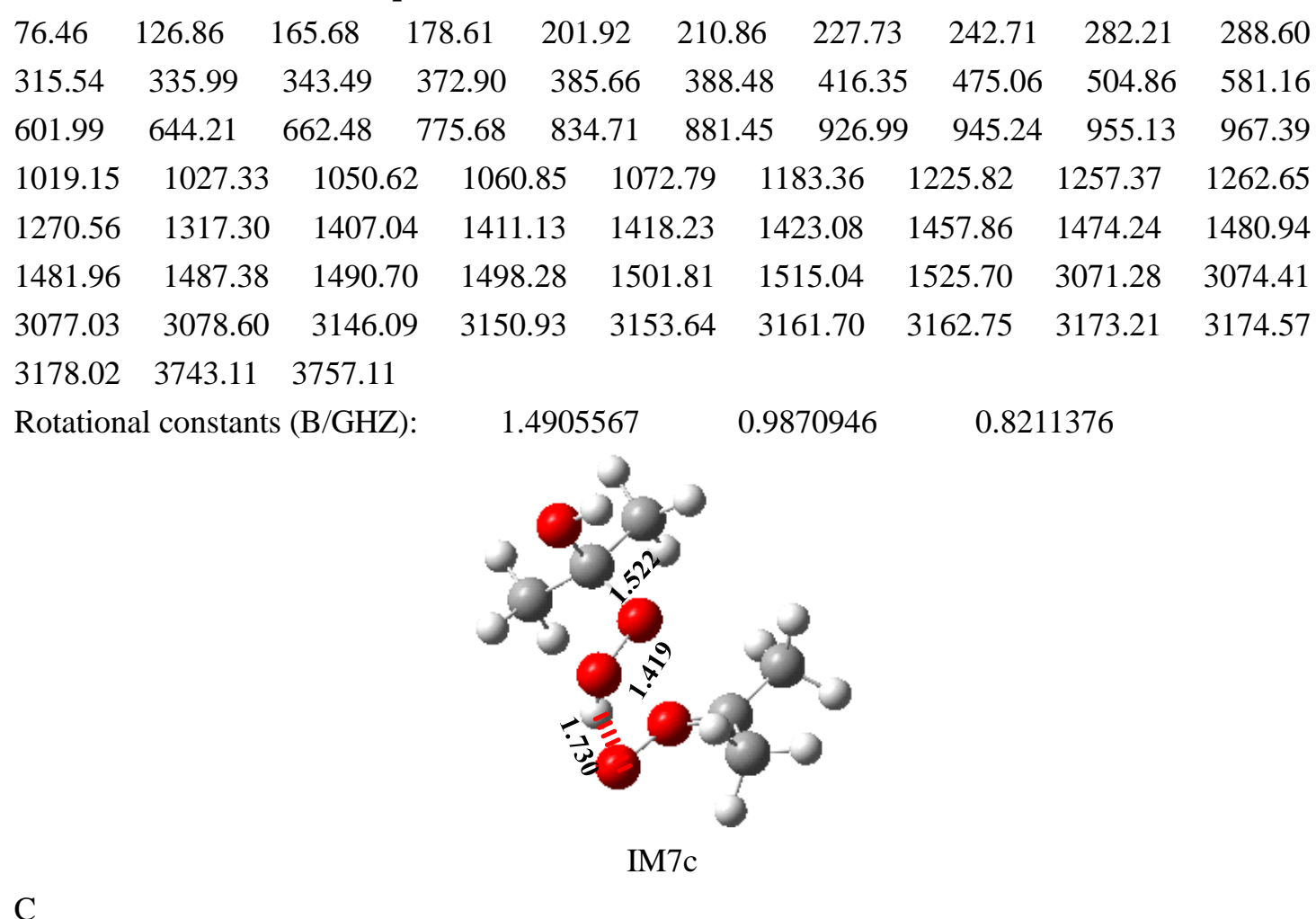




\begin{tabular}{|c|c|c|c|c|c|c|c|}
\hline $\mathrm{O}$ & 1 & B1 & & & & & \\
\hline $\mathrm{O}$ & 2 & B2 & 1 & A1 & & & \\
\hline $\mathrm{H}$ & 2 & B3 & 1 & $\mathrm{~A} 2$ & 3 & D1 & 0 \\
\hline $\mathrm{C}$ & 3 & B4 & 2 & A3 & 1 & D2 & 0 \\
\hline $\mathrm{C}$ & 5 & B5 & 3 & A4 & 2 & D3 & 0 \\
\hline $\mathrm{H}$ & 6 & B6 & 5 & A5 & 3 & D4 & 0 \\
\hline $\mathrm{H}$ & 6 & B7 & 5 & A6 & 3 & D5 & 0 \\
\hline $\mathrm{H}$ & 6 & B8 & 5 & A7 & 3 & D6 & 0 \\
\hline $\mathrm{C}$ & 5 & B9 & 3 & A8 & 2 & D7 & 0 \\
\hline $\mathrm{H}$ & 10 & B10 & 5 & A9 & 3 & D8 & 0 \\
\hline $\mathrm{H}$ & 10 & B11 & 5 & A10 & 3 & D9 & 0 \\
\hline $\mathrm{H}$ & 10 & B12 & 5 & A11 & 3 & D10 & 0 \\
\hline $\mathrm{O}$ & 5 & B13 & 3 & A12 & 2 & D11 & 0 \\
\hline $\mathrm{O}$ & 5 & B14 & 3 & A13 & 2 & D12 & 0 \\
\hline $\mathrm{C}$ & 1 & B15 & 3 & A14 & 2 & D13 & 0 \\
\hline $\mathrm{H}$ & 16 & B16 & 1 & A15 & 3 & D14 & 0 \\
\hline $\mathrm{H}$ & 16 & B17 & 1 & A16 & 3 & D15 & 0 \\
\hline $\mathrm{H}$ & 16 & B18 & 1 & A17 & 3 & D16 & 0 \\
\hline $\mathrm{C}$ & 1 & B19 & 3 & A18 & 2 & D17 & 0 \\
\hline $\mathrm{H}$ & 20 & B20 & 1 & A19 & 3 & D18 & 0 \\
\hline $\mathrm{H}$ & 20 & B21 & 1 & A20 & 3 & D19 & 0 \\
\hline $\mathrm{H}$ & 20 & B22 & 1 & A21 & 3 & D20 & 0 \\
\hline $\mathrm{O}$ & 1 & B23 & 3 & $\mathrm{~A} 22$ & 2 & D21 & 0 \\
\hline $\mathrm{H}$ & 24 & B24 & 1 & A23 & 3 & D22 & 0 \\
\hline
\end{tabular}

Variables:

$\begin{array}{ll}\text { B1 } & 2.33690441 \\ \text { B2 } & 1.41895120 \\ \text { B3 } & 0.99080142 \\ \text { B4 } & 2.81671118 \\ \text { B5 } & 1.48243491 \\ \text { B6 } & 1.08597064 \\ \text { B7 } & 1.09249738 \\ \text { B8 } & 1.09019034 \\ \text { B9 } & 1.47215910 \\ \text { B10 } & 1.09373609 \\ \text { B11 } & 1.09042399 \\ \text { B12 } & 1.08574587 \\ \text { B13 } & 2.25101099 \\ \text { B14 } & 1.24840694 \\ \text { B15 } & 1.52246351 \\ \text { B16 } & 1.08772020 \\ \text { B17 } & 1.09120934 \\ \text { B18 } & 1.08947578 \\ \text { B19 } & 1.51409828\end{array}$




\begin{tabular}{|c|c|}
\hline B20 & 1.08623343 \\
\hline B21 & 1.08850967 \\
\hline B22 & 1.08868638 \\
\hline B23 & 1.39822778 \\
\hline B24 & 0.96154362 \\
\hline A1 & 34.75486579 \\
\hline A2 & 102.83540669 \\
\hline A3 & 97.00073580 \\
\hline A4 & 103.49862069 \\
\hline A5 & 111.15704869 \\
\hline A6 & 107.83869880 \\
\hline A7 & 109.86181039 \\
\hline A8 & 88.19096701 \\
\hline A9 & 108.22559825 \\
\hline A10 & 108.76293200 \\
\hline A11 & 110.74827249 \\
\hline A12 & 72.79107041 \\
\hline A13 & 77.91856722 \\
\hline A14 & 102.79669386 \\
\hline A15 & 109.01471973 \\
\hline A16 & 110.11304635 \\
\hline A17 & 110.61291504 \\
\hline A18 & 112.40902089 \\
\hline A19 & 109.70608626 \\
\hline A20 & 109.18169219 \\
\hline A 21 & 110.14673269 \\
\hline A22 & 110.23639208 \\
\hline A23 & 108.38277272 \\
\hline D1 & -89.60573413 \\
\hline D2 & -132.60189661 \\
\hline D3 & -174.00933323 \\
\hline D4 & -86.31818671 \\
\hline D5 & 34.09321727 \\
\hline D6 & 151.10760556 \\
\hline D7 & -50.73300214 \\
\hline D8 & 4.45184323 \\
\hline D9 & 119.57782651 \\
\hline D10 & -116.63097843 \\
\hline D11 & 37.98335264 \\
\hline D12 & 71.89990250 \\
\hline D13 & -172.75310203 \\
\hline D14 & -172.96335224 \\
\hline D15 & -53.62400233 \\
\hline D16 & 67.08664701 \\
\hline
\end{tabular}




$\begin{array}{lr}\text { D17 } & -51.34205653 \\ \text { D18 } & 69.64992991 \\ \text { D19 } & -171.10239214 \\ \text { D20 } & -50.53017296 \\ \text { D21 } & 68.23188301 \\ \text { D22 } & 48.74910928\end{array}$

Harmonic Vibrational Frequencies $\left(\mathbf{c m}^{-1}\right)$ :

$\begin{array}{lccccccccccr}41.59 & 67.66 & 70.49 & 78.90 & 124.35 & 161.84 & 175.65 & 181.79 & 230.26 & 244.59 & 269.22 \\ 286.27 & 308.75 & 328.72 & 344.73 & 362.48 & 378.57 & 397.32 & 449.69 & 492.33 & 529.01 \\ 602.47 & 606.05 & 810.14 & 823.73 & 858.72 & 903.13 & 909.69 & 941.13 & 951.60 & 988.00 \\ 1004.73 & 1019.84 & 1061.68 & 1089.76 & 1106.84 & 1149.62 & 1228.14 & 1271.86 & 1316.06 \\ 1372.98 & 1392.38 & 1406.88 & 1409.22 & 1431.27 & 1450.43 & 1462.62 & 1474.73 & 1485.00 \\ 1488.48 & 1492.35 & 1502.89 & 1512.09 & 1602.24 & 1667.42 & 3044.98 & 3052.37 & 3058.02 \\ 3071.75 & 3112.40 & 3117.93 & 3134.70 & 3147.28 & 3153.52 & 3169.98 & 3175.81 & 3176.19 \\ 3279.23 & 3884.86 & & & & & & & & \end{array}$

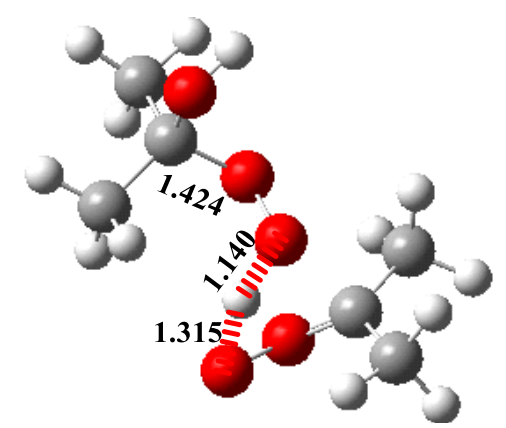

TS7c

C

$\begin{array}{lll}\mathrm{O} & 1 & \mathrm{~B} 1\end{array}$

$\begin{array}{lll}\mathrm{O} & 2 & \mathrm{~B} 2\end{array}$

$\mathrm{H} \quad 2$

C 2

C 5

$\mathrm{H} \quad 6$

$\mathrm{H} \quad 6$

$\mathrm{H} \quad 6$

C 5

$\mathrm{H} \quad 10$

$\mathrm{H} \quad 10$

$\mathrm{H} \quad 10$

O 5

O 5

C 1

$\mathrm{H} \quad 16$

$\mathrm{H} \quad 16$

$\mathrm{H} \quad 16$

$\begin{array}{rrrrrr}\text { B2 } & 1 & \text { A1 } & & & \\ \text { B3 } & 1 & \text { A2 } & 3 & \text { D1 } & 0 \\ \text { B4 } & 1 & \text { A3 } & 3 & \text { D2 } & 0 \\ \text { B5 } & 2 & \text { A4 } & 1 & \text { D3 } & 0 \\ \text { B6 } & 5 & \text { A5 } & 2 & \text { D4 } & 0 \\ \text { B7 } & 5 & \text { A6 } & 2 & \text { D5 } & 0 \\ \text { B8 } & 5 & \text { A7 } & 2 & \text { D6 } & 0 \\ \text { B9 } & 2 & \text { A8 } & 1 & \text { D7 } & 0 \\ \text { B10 } & 5 & \text { A9 } & 2 & \text { D8 } & 0 \\ \text { B11 } & 5 & \text { A10 } & 2 & \text { D9 } & 0 \\ \text { B12 } & 5 & \text { A11 } & 2 & \text { D10 } & 0 \\ \text { B13 } & 2 & \text { A12 } & 1 & \text { D11 } & 0 \\ \text { B14 } & 2 & \text { A13 } & 1 & \text { D12 } & 0 \\ \text { B15 } & 3 & \text { A14 } & 2 & \text { D13 } & 0 \\ \text { B16 } & 1 & \text { A15 } & 3 & \text { D14 } & 0 \\ \text { B17 } & 1 & \text { A16 } & 3 & \text { D15 } & 0 \\ \text { B18 } & 1 & \text { A17 } & 3 & \text { D16 } & 0\end{array}$




$\begin{array}{lrllllll}\mathrm{C} & 1 & \mathrm{~B} 19 & 3 & \mathrm{~A} 18 & 2 & \mathrm{D} 17 & 0 \\ \mathrm{H} & 20 & \mathrm{~B} 20 & 1 & \text { A19 } & 3 & \text { D18 } & 0 \\ \mathrm{H} & 20 & \text { B21 } & 1 & \text { A20 } & 3 & \text { D19 } & 0 \\ \mathrm{H} & 20 & \text { B22 } & 1 & \text { A21 } & 3 & \text { D20 } & 0 \\ \mathrm{O} & 1 & \text { B23 } & 3 & \text { A22 } & 2 & \text { D21 } & 0 \\ \mathrm{H} & 24 & \text { B24 } & 1 & \text { A23 } & 3 & \text { D22 } & 0\end{array}$

Variables:

$\begin{array}{ll}\text { B1 } & 2.33450667 \\ \text { B2 } & 1.41576394 \\ \text { B3 } & 1.14015447 \\ \text { B4 } & 2.06849195 \\ \text { B5 } & 1.48482479 \\ \text { B6 } & 1.08546220 \\ \text { B7 } & 1.09037854 \\ \text { B8 } & 1.08959154 \\ \text { B9 } & 1.48922545 \\ \text { B10 } & 1.08653314 \\ \text { B11 } & 1.08284678 \\ \text { B12 } & 1.09385049 \\ \text { B13 } & 2.21383823 \\ \text { B14 } & 1.28087117 \\ \text { B15 } & 1.52090371 \\ \text { B16 } & 1.08781970 \\ \text { B17 } & 1.09071678 \\ \text { B18 } & 1.08799670 \\ \text { B19 } & 1.51471941 \\ \text { B20 } & 1.08758826 \\ \text { B21 } & 1.08825803 \\ \text { B22 } & 1.08831732 \\ \text { B23 } & 1.39904341 \\ \text { B24 } & 0.96133824 \\ \text { A1 } & 34.8033571\end{array}$

A1 34.80333571

A2 97.22019966

A3 $\quad 132.60857670$

A4 $\quad 107.53436679$

A5 110.66645403

A6 107.19026734

A7 $\quad 110.17775288$

A8 90.76312854

A9 $\quad 109.55795648$

A10 $\quad 111.95840167$

A11 $\quad 106.40094650$

A12 $\quad 67.59274225$

A13 90.80709895 


$\begin{array}{lr}\text { A14 } & 102.76272050 \\ \text { A15 } & 108.91387081 \\ \text { A16 } & 110.06024341 \\ \text { A17 } & 110.63019972 \\ \text { A18 } & 112.19422569 \\ \text { A19 } & 109.94006364 \\ \text { A20 } & 109.16623950 \\ \text { A21 } & 110.06984658 \\ \text { A22 } & 110.47471355 \\ \text { A23 } & 108.44263541 \\ \text { D1 } & -103.00535301 \\ \text { D2 } & -28.71512577 \\ \text { D3 } & 63.71487937 \\ \text { D4 } & -93.49447900 \\ \text { D5 } & 25.53261919 \\ \text { D6 } & 143.24692415 \\ \text { D7 } & -175.11668455 \\ \text { D8 } & -64.54974324 \\ \text { D9 } & 58.90201580 \\ \text { D10 } & 178.19473478 \\ \text { D11 } & -81.74818894 \\ \text { D12 } & -52.21231230 \\ \text { D13 } & -174.33223843 \\ \text { D14 } & -174.96287212 \\ \text { D15 } & -55.54732773 \\ \text { D16 } & 64.65649235 \\ \text { D17 } & -52.93181149 \\ \text { D18 } & 69.49225863 \\ \text { D19 } & -171.68861691 \\ \text { D20 } & -51.15363952 \\ \text { D21 } & 66.33937695 \\ \text { D22 } & 54.94104970 \\ \text { Da } & \end{array}$

Harmonic Vibrational Frequencies $\left(\mathbf{c m}^{-1}\right)$ :

$\begin{array}{lllllllllll}774.33 i & 41.79 & 65.22 & 81.55 & 87.04 & 149.22 & 167.63 & 185.53 & 209.06 & 221.04 & 261.54\end{array}$ $\begin{array}{llllllllll}292.66 & 325.39 & 334.83 & 363.06 & 380.16 & 409.93 & 445.34 & 448.93 & 522.13 & 548.36\end{array}$ $\begin{array}{llllllllll}581.11 & 620.46 & 806.70 & 815.90 & 891.65 & 908.62 & 943.76 & 944.88 & 959.75 & 983.79\end{array}$ $\begin{array}{lllllllll}1004.65 & 1020.09 & 1079.26 & 1082.67 & 1126.36 & 1153.56 & 1230.31 & 1274.47 & 1314.55\end{array}$ $\begin{array}{lllllllll}1373.05 & 1402.46 & 1412.76 & 1422.27 & 1432.99 & 1447.55 & 1457.23 & 1470.00 & 1482.02\end{array}$ $\begin{array}{lllllllll}1485.86 & 1493.06 & 1502.62 & 1512.27 & 1588.04 & 1604.33 & 1786.55 & 3055.04 & 3058.06\end{array}$ $\begin{array}{llllllllll}3065.87 & 3072.04 & 3136.25 & 3136.38 & 3144.18 & 3152.25 & 3157.44 & 3160.19 & 3176.32\end{array}$ $3213.07 \quad 3875.44$

Rotational constants (B/GHZ): $\quad 1.8634429 \quad 0.6631601 \quad 0.6226989$ 


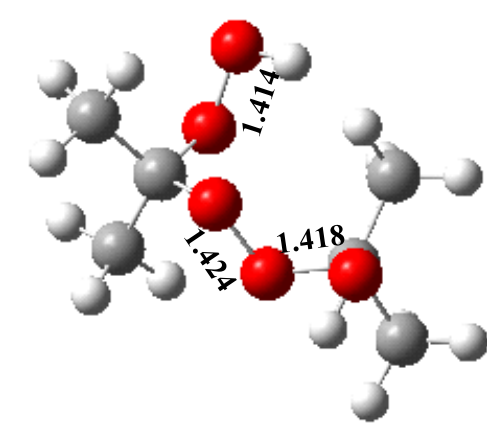

P7c

C

$\begin{array}{lll}\mathrm{O} & 1 & \mathrm{~B} 1\end{array}$

O 1

$\mathrm{H} \quad 2$

C 2

C 5

$\mathrm{H} \quad 6$

$\mathrm{H} \quad 6$

$\mathrm{H} \quad 6$

C 5

$\mathrm{H} \quad 10$

$\mathrm{H} \quad 10$

$\mathrm{H} \quad 10$

O 5

$\mathrm{O} \quad 5$

C 1

$\mathrm{H} \quad 16$

$\mathrm{H} \quad 16$

$\mathrm{H} \quad 16$

C 1

$\mathrm{H} \quad 20$

$\mathrm{H} \quad 20$

$\mathrm{H} \quad 20$

O 1

$\mathrm{H} \quad 24$

Variables:

B1

B2

B3

B4

B5

B6

B7

B8

B9

$\begin{array}{ll} & 2.32041754 \\ 2 & 1.41793950 \\ 3 & 2.69121487 \\ 4 & 1.41544267 \\ 5 & 1.51746648 \\ 6 & 1.08615272 \\ 7 & 1.08799285 \\ 8 & 1.08769628 \\ 9 & 1.51706057\end{array}$

B2 2

B3 1

B4 1

B5 2

B6 5

B7 5

B8 5

B9 2

B10 5

B11 5

B12 5

B13 2

B14 2

B15 3

B16 1

B17 1

B18 1

B19 3

B20 1

B21 1

B22 1

B23 3

B24 1
A1

A2 $3 \quad$ D1 0

A3 $3 \quad$ D2 0

A4 $1 \quad$ D3 0

A5 $2 \quad$ D4 0

A6 2 D5 0

A7 2 D6 0

A8 $1 \quad$ D7 0

A9 $2 \quad$ D8 0

$\begin{array}{llll}\mathrm{A} 10 & 2 & \mathrm{D} 9 & 0\end{array}$

A11 2 D10 0

A12 $1 \quad$ D11 0

A13 $1 \quad$ D12 0

A14 $2 \quad$ D13 0

A15 $3 \quad$ D14 0

A16 $3 \quad$ D15 0

A17 $3 \quad$ D16 0

A18 $2 \quad$ D17 0

A19 $3 \quad$ D18 0

A20 $3 \quad$ D19 0

A21 $3 \quad$ D20 0

A22 $2 \quad$ D21 0

A23 $3 \quad$ D22 0 


\begin{tabular}{|c|c|}
\hline B10 & 1.08766149 \\
\hline B11 & 1.08583801 \\
\hline B12 & 1.08790715 \\
\hline B13 & 2.31752023 \\
\hline B14 & 1.41310791 \\
\hline B15 & 1.52139292 \\
\hline B16 & 1.08775033 \\
\hline B17 & 1.09016254 \\
\hline B18 & 1.08817114 \\
\hline B19 & 1.51544068 \\
\hline B20 & 1.08890228 \\
\hline B21 & 1.08792015 \\
\hline B22 & 1.09018726 \\
\hline $\mathrm{B} 23$ & 1.39785127 \\
\hline B24 & 0.96173873 \\
\hline A1 & 35.35543874 \\
\hline $\mathrm{A} 2$ & 85.43264168 \\
\hline A3 & 132.03010964 \\
\hline A4 & 112.76374466 \\
\hline A5 & 110.66381344 \\
\hline A6 & 109.10055389 \\
\hline A7 & 109.14831159 \\
\hline A8 & 104.01980557 \\
\hline A9 & 109.20087335 \\
\hline A10 & 110.86240715 \\
\hline A11 & 108.84495993 \\
\hline A12 & 96.36497666 \\
\hline A13 & 110.46633638 \\
\hline A14 & 102.65815351 \\
\hline A15 & 108.86686466 \\
\hline A16 & 109.93582699 \\
\hline A17 & 110.76140721 \\
\hline A18 & 112.52158495 \\
\hline A19 & 109.18452195 \\
\hline A20 & 109.52817862 \\
\hline A21 & 109.55678851 \\
\hline A22 & 110.64316925 \\
\hline $\mathrm{A} 23$ & 108.58025354 \\
\hline D1 & -118.95618914 \\
\hline D2 & -56.93075538 \\
\hline D3 & 75.10730044 \\
\hline D4 & -72.54917223 \\
\hline D5 & 47.53042304 \\
\hline D6 & 167.20383946 \\
\hline
\end{tabular}




$\begin{array}{lr}\text { D7 } & -162.19588705 \\ \text { D8 } & -72.97587626 \\ \text { D9 } & 47.54122160 \\ \text { D10 } & 167.38697170 \\ \text { D11 } & -73.84091976 \\ \text { D12 } & -40.83006114 \\ \text { D13 } & -176.36573308 \\ \text { D14 } & -175.40650350 \\ \text { D15 } & -56.07286275 \\ \text { D16 } & 64.07024469 \\ \text { D17 } & -55.03507989 \\ \text { D18 } & 72.04070069 \\ \text { D19 } & -169.81285633 \\ \text { D20 } & -50.13815036 \\ \text { D21 } & 64.10535551 \\ \text { D22 } & 54.31641570\end{array}$

Harmonic Vibrational Frequencies $\left(\mathbf{c m}^{-1}\right)$ :

$\begin{array}{lllllllllll}53.26 & 87.59 & 118.96 & 155.72 & 192.87 & 219.14 & 231.90 & 237.98 & 254.76 & 268.65 & 283.51\end{array}$ $\begin{array}{llllllllll}310.11 & 333.45 & 363.74 & 375.24 & 380.00 & 411.11 & 451.05 & 464.43 & 526.29 & 596.36\end{array}$ $\begin{array}{llllllllll}607.78 & 620.39 & 812.44 & 842.52 & 903.23 & 925.88 & 947.45 & 958.60 & 971.97 & 1013.24\end{array}$ $\begin{array}{lllllllll}1019.63 & 1028.44 & 1055.70 & 1065.50 & 1155.49 & 1209.22 & 1230.79 & 1251.87 & 1281.03\end{array}$ $\begin{array}{lllllllll}1313.58 & 1368.04 & 1412.27 & 1415.55 & 1421.09 & 1424.81 & 1430.95 & 1478.90 & 1487.05\end{array}$ $\begin{array}{lllllllll}1488.15 & 1493.82 & 1494.93 & 1503.04 & 1508.43 & 1529.29 & 3057.81 & 3063.48 & 3076.47\end{array}$ $\begin{array}{llllllllll}3081.50 & 3141.85 & 3144.26 & 3149.42 & 3156.88 & 3159.79 & 3161.92 & 3169.96 & 3175.16\end{array}$ $3826.81 \quad 3883.11$

Rotational constants (B/GHZ): $\quad 1.7919387 \quad 0.7346323 \quad 0.6691134$

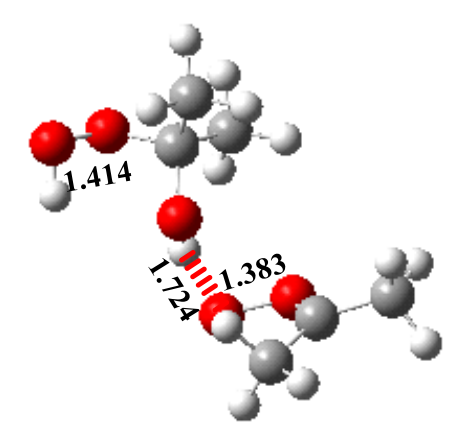

IM7d

$\mathrm{C}$ C 11

$\begin{array}{llllllll}\mathrm{H} & 2 & \text { B2 } & 1 & \text { A1 } & & & \\ \mathrm{H} & 2 & \text { B3 } & 1 & \text { A2 } & 3 & \text { D1 } & 0 \\ \mathrm{H} & 2 & \text { B4 } & 1 & \text { A3 } & 3 & \text { D2 } & 0 \\ \mathrm{C} & 1 & \text { B5 } & 2 & \text { A4 } & 5 & \text { D3 } & 0 \\ \mathrm{H} & 6 & \text { B6 } & 1 & \text { A5 } & 2 & \text { D4 } & 0 \\ \text { H } & 6 & \text { B7 } & 1 & \text { A6 } & 2 & \text { D5 } & 0 \\ \text { H } & 6 & \text { B8 } & 1 & \text { A7 } & 2 & \text { D6 } & 0\end{array}$




$\begin{array}{rrrrrrrr}\text { O } & 1 & \text { B9 } & 6 & \text { A8 } & 2 & \text { D7 } & 0 \\ \text { H } & 10 & \text { B10 } & 1 & \text { A9 } & 6 & \text { D8 } & 0 \\ \text { O } & 1 & \text { B11 } & 10 & \text { A10 } & 6 & \text { D9 } & 0 \\ \mathrm{C} & 10 & \text { B12 } & 1 & \text { A11 } & 6 & \text { D10 } & 0 \\ \mathrm{C} & 13 & \text { B13 } & 10 & \text { A12 } & 1 & \text { D11 } & 0 \\ \mathrm{H} & 14 & \text { B14 } & 13 & \text { A13 } & 10 & \text { D12 } & 0 \\ \mathrm{H} & 14 & \text { B15 } & 13 & \text { A14 } & 10 & \text { D13 } & 0 \\ \mathrm{H} & 14 & \text { B16 } & 13 & \text { A15 } & 10 & \text { D14 } & 0 \\ \mathrm{C} & 13 & \text { B17 } & 10 & \text { A16 } & 1 & \text { D15 } & 0 \\ \mathrm{H} & 18 & \text { B18 } & 13 & \text { A17 } & 10 & \text { D16 } & 0 \\ \text { H } & 18 & \text { B19 } & 13 & \text { A18 } & 10 & \text { D17 } & 0 \\ \text { H } & 18 & \text { B20 } & 13 & \text { A19 } & 10 & \text { D18 } & 0 \\ \text { O } & 13 & \text { B21 } & 10 & \text { A20 } & 1 & \text { D19 } & 0 \\ \text { O } & 13 & \text { B22 } & 10 & \text { A21 } & 1 & \text { D20 } & 0 \\ \text { O } & 12 & \text { B23 } & 1 & \text { A22 } & 10 & \text { D21 } & 0 \\ \text { H } & 12 & \text { B24 } & 1 & \text { A23 } & 10 & \text { D22 } & 0 \\ \text { Var } & & & & & & & \end{array}$

Variables:

$\begin{array}{lr}\text { B1 } & 1.51935236 \\ \text { B2 } & 1.08948458 \\ \text { B3 } & 1.08953774 \\ \text { B4 } & 1.08819841 \\ \text { B5 } & 1.51610767 \\ \text { B6 } & 1.08903208 \\ \text { B7 } & 1.08833762 \\ \text { B8 } & 1.08664537 \\ \text { B9 } & 1.39928720 \\ \text { B10 } & 0.98674675 \\ \text { B11 } & 2.31266600 \\ \text { B12 } & 3.12791732 \\ \text { B13 } & 1.48324666 \\ \text { B14 } & 1.09190625 \\ \text { B15 } & 1.09071787 \\ \text { B16 } & 1.08579597 \\ \text { B17 } & 1.47257889 \\ \text { B18 } & 1.09404573 \\ \text { B19 } & 1.09034209 \\ \text { B20 } & 1.08570871 \\ \text { B21 } & 2.24848582 \\ \text { B22 } & 1.24936484 \\ \text { B23 } & 1.41397679 \\ \text { B24 } & 0.96680923 \\ \text { A1 } & 109.18980975 \\ \text { A2 } & 109.97340333 \\ \text { A3 } & 110.29604306\end{array}$




\begin{tabular}{|c|c|}
\hline A4 & 113.08088007 \\
\hline A5 & 109.46342504 \\
\hline A6 & 109.88853742 \\
\hline A7 & 110.06523363 \\
\hline A8 & 107.94800798 \\
\hline A9 & 109.81889551 \\
\hline A 10 & 89.78275673 \\
\hline A11 & 122.73287256 \\
\hline A12 & 123.67155223 \\
\hline A13 & 108.53704990 \\
\hline A14 & 109.51959725 \\
\hline A15 & 111.11497556 \\
\hline A16 & 77.35542522 \\
\hline A17 & 107.84916989 \\
\hline A18 & 108.77009665 \\
\hline A19 & 110.86139864 \\
\hline A 20 & 56.74562939 \\
\hline A21 & 70.49563163 \\
\hline A22 & 35.58274494 \\
\hline $\mathrm{A} 23$ & 87.23848434 \\
\hline D1 & 119.47598533 \\
\hline D2 & -119.78129867 \\
\hline D3 & -62.04506226 \\
\hline D4 & -60.20981946 \\
\hline D5 & 59.80438117 \\
\hline D6 & 179.93933073 \\
\hline D7 & 124.37751295 \\
\hline D8 & -164.50633581 \\
\hline D9 & -91.20075804 \\
\hline D10 & -100.38428653 \\
\hline D11 & 48.09315491 \\
\hline D12 & 37.28149027 \\
\hline D13 & 154.06698654 \\
\hline D14 & -83.84732302 \\
\hline D15 & 169.54931514 \\
\hline D16 & -12.19185258 \\
\hline D17 & 102.84631150 \\
\hline D18 & -133.11348862 \\
\hline D19 & -94.52323292 \\
\hline D20 & -60.60163843 \\
\hline D21 & -125.37564924 \\
\hline D22 & -10.58183698 \\
\hline
\end{tabular}

Harmonic Vibrational Frequencies $\left(\mathbf{c m}^{-1}\right)$ :

$\begin{array}{lllllllllll}35.82 & 42.15 & 67.45 & 88.69 & 111.10 & 148.44 & 166.36 & 187.65 & 207.34 & 222.48 & 252.78\end{array}$ 


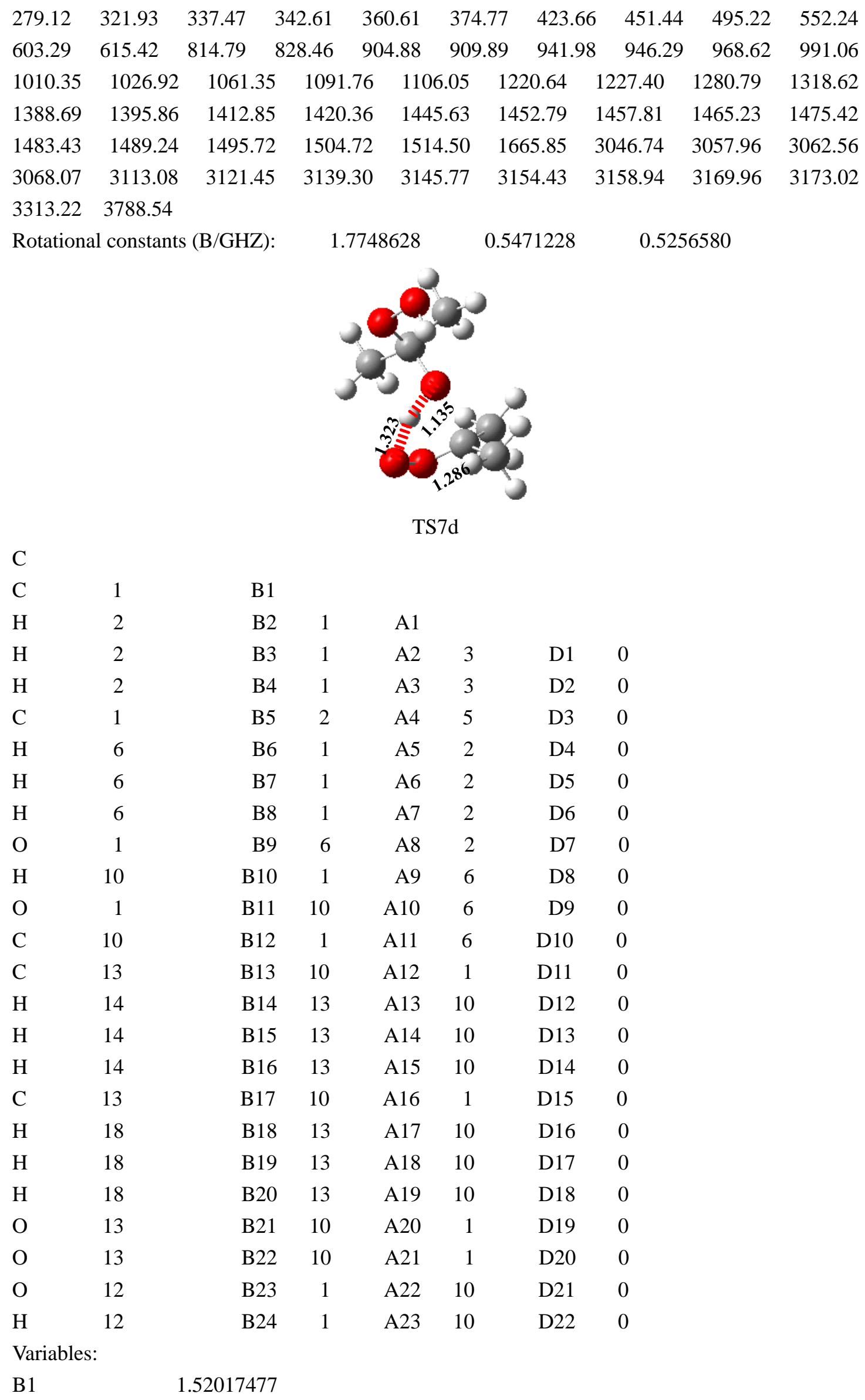




\begin{tabular}{|c|c|}
\hline B2 & 1.08822179 \\
\hline B3 & 1.08835353 \\
\hline B4 & 1.08810005 \\
\hline B5 & 1.51785486 \\
\hline B6 & 1.08886157 \\
\hline B7 & 1.08868961 \\
\hline B8 & 1.08688076 \\
\hline B9 & 1.40885578 \\
\hline B10 & 1.13514638 \\
\hline B11 & 2.31451369 \\
\hline B12 & 2.00971629 \\
\hline B13 & 1.48873615 \\
\hline B14 & 1.08737058 \\
\hline B15 & 1.09189561 \\
\hline B16 & 1.08593925 \\
\hline B17 & 1.49193143 \\
\hline B18 & 1.08557186 \\
\hline B19 & 1.08245384 \\
\hline B20 & 1.09386159 \\
\hline B21 & 2.21445415 \\
\hline B22 & 1.28551325 \\
\hline B23 & 1.41409901 \\
\hline B24 & 0.96599734 \\
\hline $\mathrm{A} 1$ & 109.99217325 \\
\hline $\mathrm{A} 2$ & 110.28310897 \\
\hline A3 & 109.49390842 \\
\hline A4 & 112.89320513 \\
\hline A5 & 110.05371781 \\
\hline A6 & 109.57320180 \\
\hline A7 & 110.26625911 \\
\hline A8 & 109.42525331 \\
\hline A9 & 110.72129389 \\
\hline A10 & 89.53758679 \\
\hline A11 & 125.88002182 \\
\hline A12 & 110.56062128 \\
\hline A 13 & 109.53072092 \\
\hline A14 & 108.09629381 \\
\hline A 15 & 111.23926397 \\
\hline A16 & 91.16096194 \\
\hline A17 & 110.00381547 \\
\hline A 18 & 111.58583342 \\
\hline A19 & 106.09893433 \\
\hline A20 & 68.38275298 \\
\hline A21 & 92.83893192 \\
\hline
\end{tabular}




$\begin{array}{lr}\text { A22 } & 35.35211810 \\ \text { A23 } & 90.94224359 \\ \text { D1 } & 119.63256267 \\ \text { D2 } & -119.73964054 \\ \text { D3 } & -59.85221532 \\ \text { D4 } & -55.04929014 \\ \text { D5 } & 64.30026564 \\ \text { D6 } & -175.81090149 \\ \text { D7 } & 126.66682366 \\ \text { D8 } & -164.57720631 \\ \text { D9 } & -89.63307280 \\ \text { D10 } & -78.01548674 \\ \text { D11 } & 43.36468269 \\ \text { D12 } & 49.91400549 \\ \text { D13 } & 167.41648044 \\ \text { D14 } & -72.72368277 \\ \text { D15 } & 164.05271536 \\ \text { D16 } & -62.58583517 \\ \text { D17 } & 60.92866682 \\ \text { D18 } & 179.91845969 \\ \text { D19 } & -102.63007406 \\ \text { D20 } & -73.86177324 \\ \text { D21 } & -121.62153940 \\ \text { D22 } & -12.14042462\end{array}$

Harmonic Vibrational Frequencies $\left(\mathbf{c m}^{-1}\right)$ :

$\begin{array}{llllllllllr}774.49 i & 49.74 & 114.29 & 118.06 & 144.31 & 163.33 & 179.41 & 202.61 & 213.46 & 233.49 \\ 257.60 & 295.97 & 317.57 & 338.66 & 360.16 & 364.54 & 394.90 & 423.08 & 443.47 & 529.89 \\ 567.61 & 583.78 & 637.49 & 755.33 & 833.85 & 895.62 & 912.85 & 926.12 & 942.53 & 953.25 \\ 994.27 & 1013.47 & 1032.20 & 1062.32 & 1082.00 & 1109.86 & 1228.52 & 1258.21 & 1270.52 \\ 1314.27 & 1404.26 & 1408.08 & 1422.25 & 1425.07 & 1443.30 & 1444.98 & 1455.75 & 1473.92 \\ 1476.73 & 1479.62 & 1495.70 & 1500.54 & 1501.58 & 1518.61 & 1576.11 & 1854.01 & 3055.10 \\ 3064.84 & 3067.68 & 3075.00 & 3137.77 & 3149.18 & 3151.88 & 3152.46 & 3154.66 & 3166.87 \\ 3186.36 & 3207.22 & 3809.33 & & & & & & & \end{array}$

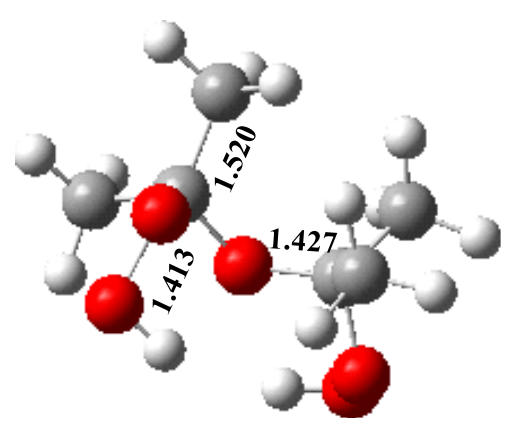

P7d

C 


\begin{tabular}{|c|c|c|c|c|c|c|}
\hline $\mathrm{C}$ & 1 & B1 & & & & \\
\hline $\mathrm{H}$ & 2 & B2 & 1 & A1 & & \\
\hline $\mathrm{H}$ & 2 & B3 & 1 & A2 & 3 & D1 \\
\hline $\mathrm{H}$ & 2 & B4 & 1 & A3 & 4 & D2 \\
\hline $\mathrm{C}$ & 1 & B5 & 2 & A4 & 3 & D3 \\
\hline $\mathrm{H}$ & 6 & B6 & 1 & A5 & 2 & D4 \\
\hline $\mathrm{H}$ & 6 & B7 & 1 & A6 & 2 & D5 \\
\hline $\mathrm{H}$ & 6 & B8 & 1 & A7 & 2 & D6 \\
\hline $\mathrm{O}$ & 1 & B9 & 2 & A8 & 6 & D7 \\
\hline $\mathrm{H}$ & 10 & B10 & 1 & A9 & 2 & D8 \\
\hline $\mathrm{O}$ & 1 & B11 & 10 & A 10 & 6 & D9 \\
\hline $\mathrm{C}$ & 10 & B12 & 1 & A11 & 2 & D10 \\
\hline $\mathrm{C}$ & 13 & B13 & 10 & A12 & 1 & D11 \\
\hline $\mathrm{H}$ & 14 & B14 & 13 & A13 & 10 & D12 \\
\hline $\mathrm{H}$ & 14 & B15 & 13 & A14 & 10 & D13 \\
\hline $\mathrm{H}$ & 14 & B16 & 13 & A15 & 10 & D14 \\
\hline $\mathrm{C}$ & 13 & B17 & 10 & A16 & 1 & D15 \\
\hline $\mathrm{H}$ & 18 & B18 & 13 & A17 & 10 & D16 \\
\hline $\mathrm{H}$ & 18 & B19 & 13 & A18 & 10 & D17 \\
\hline $\mathrm{H}$ & 18 & B20 & 13 & A19 & 10 & D18 \\
\hline $\mathrm{O}$ & 13 & B21 & 10 & $\mathrm{~A} 20$ & 1 & D19 \\
\hline $\mathrm{O}$ & 22 & B22 & 13 & A 21 & 10 & D20 \\
\hline $\mathrm{O}$ & 1 & B23 & 10 & A22 & 13 & D21 \\
\hline $\mathrm{H}$ & 12 & B24 & 1 & A23 & 24 & D22 \\
\hline
\end{tabular}

Variables:

$\begin{array}{ll}\text { B1 } & 1.51975844 \\ \text { B2 } & 1.08510851 \\ \text { B3 } & 1.08831892 \\ \text { B4 } & 1.08849776 \\ \text { B5 } & 1.52089814 \\ \text { B6 } & 1.08694474 \\ \text { B7 } & 1.08863574 \\ \text { B8 } & 1.08644301 \\ \text { B9 } & 1.42740681 \\ \text { B10 } & 2.35894884 \\ \text { B11 } & 2.31046110 \\ \text { B12 } & 1.40993266 \\ \text { B13 } & 1.52022979 \\ \text { B14 } & 1.08793957 \\ \text { B15 } & 1.08787123 \\ \text { B16 } & 1.08703109 \\ \text { B17 } & 1.51834295 \\ \text { B18 } & 1.08841555 \\ \text { B19 } & 1.08585966\end{array}$




\begin{tabular}{|c|c|}
\hline B20 & 1.08802660 \\
\hline B21 & 2.31356060 \\
\hline B22 & 1.41273241 \\
\hline B23 & 1.41290913 \\
\hline B24 & 0.96657963 \\
\hline A1 & 111.52128219 \\
\hline $\mathrm{A} 2$ & 110.85853846 \\
\hline A3 & 108.04806701 \\
\hline A4 & 112.84620699 \\
\hline A5 & 110.97598616 \\
\hline A6 & 108.65228146 \\
\hline A7 & 110.32134206 \\
\hline A 8 & 114.98573393 \\
\hline A9 & 101.16071446 \\
\hline A10 & 86.36020338 \\
\hline A11 & 124.86474696 \\
\hline A12 & 115.28817617 \\
\hline A13 & 109.43189990 \\
\hline A14 & 108.70713214 \\
\hline A15 & 112.04165269 \\
\hline A16 & 105.19988632 \\
\hline A17 & 109.33648987 \\
\hline A18 & 110.39085652 \\
\hline A19 & 109.42589555 \\
\hline A 20 & 90.99216125 \\
\hline A 21 & 35.23093048 \\
\hline A 22 & 103.28018597 \\
\hline A 23 & 91.26800033 \\
\hline D1 & 121.01719067 \\
\hline D2 & 118.88689337 \\
\hline D3 & 63.61090912 \\
\hline D4 & -51.37173866 \\
\hline D5 & 67.96401097 \\
\hline D6 & -172.83122748 \\
\hline D7 & -129.40868284 \\
\hline D8 & -36.67435283 \\
\hline D9 & -87.84312708 \\
\hline D10 & 46.27258105 \\
\hline D11 & 47.98797546 \\
\hline D12 & 53.50978221 \\
\hline D13 & 172.48843577 \\
\hline D14 & -68.04775717 \\
\hline D15 & 170.92537626 \\
\hline D16 & -68.71298204 \\
\hline
\end{tabular}




$\begin{array}{lr}\text { D17 } & 51.42836618 \\ \text { D18 } & 171.42415332 \\ \text { D19 } & -99.09829213 \\ \text { D20 } & 124.09315111 \\ \text { D21 } & 157.27375643 \\ \text { D22 } & 108.92634121\end{array}$

Harmonic Vibrational Frequencies $\left(\mathbf{c m}^{-1}\right)$ :

$\begin{array}{lcccccccccr}54.46 & 106.02 & 136.80 & 161.28 & 205.40 & 223.22 & 241.23 & 258.97 & 273.25 & 289.25 \\ 302.42 & 324.73 & 340.11 & 358.83 & 373.39 & 380.90 & 398.37 & 404.10 & 491.79 & 539.38 \\ 558.34 & 603.47 & 641.66 & 752.33 & 849.45 & 899.42 & 925.81 & 939.16 & 948.44 & 972.48 \\ 1017.99 & 1022.21 & 1052.37 & 1063.94 & 1074.19 & 1196.00 & 1231.87 & 1249.32 & 1261.80 \\ 1280.31 & 1307.65 & 1410.77 & 1412.68 & 1419.87 & 1429.97 & 1441.85 & 1450.05 & 1474.40 \\ 1483.25 & 1487.94 & 1494.65 & 1495.58 & 1504.63 & 1511.27 & 1520.74 & 3074.88 & 3075.59 \\ 3078.01 & 3081.30 & 3149.27 & 3152.36 & 3154.64 & 3160.43 & 3166.98 & 3168.92 & 3172.26 \\ 3193.58 & 3779.71 & 3809.13 & & & & & & & \\ \text { Rotational constants (B/GHZ): } & 1.5565329 & 0.8072245 & 0.7806714 & \end{array}$

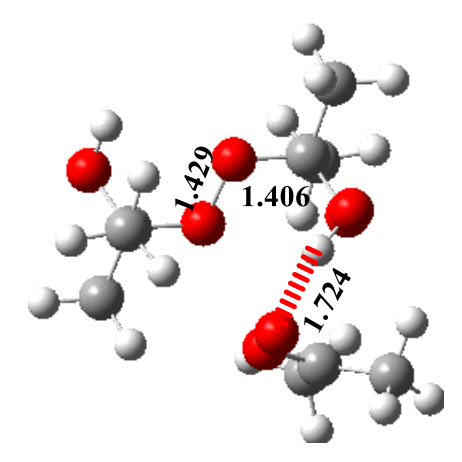

IM8a

C

\begin{tabular}{|c|c|c|c|c|c|c|c|}
\hline $\mathrm{O}$ & 1 & B1 & & & & & \\
\hline $\mathrm{O}$ & 1 & B2 & 2 & A1 & & & \\
\hline $\mathrm{H}$ & 2 & B3 & 1 & A2 & 3 & D1 & 0 \\
\hline $\mathrm{C}$ & 3 & B4 & 1 & A3 & 2 & D2 & 0 \\
\hline $\mathrm{O}$ & 5 & B5 & 3 & A4 & 1 & D3 & 0 \\
\hline $\mathrm{O}$ & 5 & B6 & 3 & A5 & 1 & D4 & 0 \\
\hline $\mathrm{C}$ & 1 & B7 & 3 & A6 & 2 & D5 & 0 \\
\hline $\mathrm{H}$ & 8 & B8 & 1 & A7 & 3 & D6 & 0 \\
\hline $\mathrm{H}$ & 8 & B9 & 1 & A8 & 3 & D7 & 0 \\
\hline $\mathrm{H}$ & 8 & B 10 & 1 & A9 & 3 & D8 & 0 \\
\hline $\mathrm{O}$ & 1 & B11 & 3 & A10 & 2 & D9 & 0 \\
\hline $\mathrm{C}$ & 12 & B12 & 1 & A11 & 3 & D10 & 0 \\
\hline $\mathrm{C}$ & 5 & B13 & 3 & A12 & 1 & D11 & 0 \\
\hline $\mathrm{H}$ & 14 & B14 & 5 & A13 & 3 & D12 & 0 \\
\hline $\mathrm{H}$ & 14 & B15 & 5 & A14 & 3 & D13 & 0 \\
\hline $\mathrm{H}$ & 14 & B 16 & 5 & A15 & 3 & D14 & 0 \\
\hline $\mathrm{C}$ & 5 & B17 & 3 & A16 & 1 & D15 & 0 \\
\hline
\end{tabular}




\begin{tabular}{|c|c|c|c|c|c|c|}
\hline $\mathrm{H}$ & 18 & B18 & 5 & A17 & 3 & D16 \\
\hline $\mathrm{H}$ & 18 & B19 & 5 & A18 & 3 & D17 \\
\hline $\mathrm{H}$ & 18 & B20 & 5 & A19 & 3 & D18 \\
\hline $\mathrm{C}$ & 1 & B21 & 12 & A20 & 13 & D19 \\
\hline $\mathrm{H}$ & 22 & B22 & 1 & A21 & 12 & D20 \\
\hline $\mathrm{H}$ & 22 & B23 & 1 & A22 & 12 & D21 \\
\hline $\mathrm{H}$ & 22 & B24 & 1 & A23 & 12 & $\mathrm{D} 22$ \\
\hline $\mathrm{O}$ & 13 & B25 & 12 & A24 & 1 & D23 \\
\hline $\mathrm{C}$ & 13 & B26 & 12 & A25 & 1 & D24 \\
\hline $\mathrm{H}$ & 27 & B27 & 13 & A26 & 12 & D25 \\
\hline $\mathrm{H}$ & 27 & B28 & 13 & A27 & 12 & D26 \\
\hline $\mathrm{H}$ & 27 & B29 & 13 & A28 & 12 & D27 \\
\hline $\mathrm{C}$ & 13 & B30 & 12 & A29 & 1 & D28 \\
\hline $\mathrm{H}$ & 31 & B31 & 13 & A30 & 12 & D29 \\
\hline $\mathrm{H}$ & 31 & B32 & 13 & A31 & 12 & D30 \\
\hline $\mathrm{H}$ & 31 & B33 & 13 & A32 & 12 & D31 \\
\hline $\mathrm{O}$ & 13 & B34 & 12 & A33 & 1 & D32 \\
\hline $\mathrm{H}$ & 35 & B35 & 13 & A34 & 12 & D33 \\
\hline
\end{tabular}

Variables:

$\begin{array}{ll}\text { B1 } & 2.31968155 \\ \text { B2 } & 1.41073015 \\ \text { B3 } & 0.99240356 \\ \text { B4 } & 2.79512694 \\ \text { B5 } & 2.24983357 \\ \text { B6 } & 1.24736331 \\ \text { B7 } & 1.51881329 \\ \text { B8 } & 1.08788767 \\ \text { B9 } & 1.08639059 \\ \text { B10 } & 1.08796568 \\ \text { B11 } & 1.40648711 \\ \text { B12 } & 2.28389634 \\ \text { B13 } & 1.48148482 \\ \text { B14 } & 1.08628662 \\ \text { B15 } & 1.09124945 \\ \text { B16 } & 1.09140505 \\ \text { B17 } & 1.47406083 \\ \text { B18 } & 1.09253058 \\ \text { B19 } & 1.09041646 \\ \text { B20 } & 1.08586134 \\ \text { B21 } & 1.51725893 \\ \text { B22 } & 1.08807250 \\ \text { B23 } & 1.08792238 \\ \text { B24 } & 1.08567861 \\ \text { B25 } & 1.42471061\end{array}$




\begin{tabular}{|c|c|}
\hline B26 & 1.51448664 \\
\hline B27 & 1.08904415 \\
\hline B28 & 1.08790724 \\
\hline B29 & 1.08766472 \\
\hline B30 & 1.51937191 \\
\hline B31 & 1.08940057 \\
\hline B32 & 1.08861881 \\
\hline B33 & 1.08884347 \\
\hline B34 & 1.39982303 \\
\hline B35 & 0.96141261 \\
\hline $\mathrm{A} 1$ & 35.13324428 \\
\hline $\mathrm{A} 2$ & 110.68768202 \\
\hline A3 & 131.14698969 \\
\hline A4 & 77.88597221 \\
\hline A5 & 83.67622646 \\
\hline A6 & 104.51901777 \\
\hline A7 & 109.58868384 \\
\hline A8 & 110.71945938 \\
\hline A9 & 108.90630313 \\
\hline A10 & 110.79722842 \\
\hline A11 & 144.70602573 \\
\hline A12 & 99.30508026 \\
\hline A13 & 110.84414679 \\
\hline A14 & 107.79940001 \\
\hline A 15 & 109.70512477 \\
\hline A16 & 87.24006640 \\
\hline A 17 & 108.56045476 \\
\hline A18 & 108.58629338 \\
\hline A19 & 110.65881243 \\
\hline A20 & 104.22536140 \\
\hline A21 & 108.77728410 \\
\hline $\mathrm{A} 22$ & 109.59125879 \\
\hline A23 & 110.30649333 \\
\hline A24 & 36.89425555 \\
\hline A25 & 140.48903187 \\
\hline A26 & 109.64216898 \\
\hline A 27 & 110.14081877 \\
\hline A28 & 108.74286797 \\
\hline A29 & 89.06939618 \\
\hline A 30 & 110.56054919 \\
\hline A31 & 110.59465807 \\
\hline A 32 & 108.77329832 \\
\hline A33 & 93.04697218 \\
\hline A34 & 108.05585123 \\
\hline
\end{tabular}




$\begin{array}{lr}\text { D1 } & -81.80759302 \\ \text { D2 } & 116.88058604 \\ \text { D3 } & -97.18246834 \\ \text { D4 } & -63.89471874 \\ \text { D5 } & 171.61575420 \\ \text { D6 } & -67.81797423 \\ \text { D7 } & 53.26297116 \\ \text { D8 } & 172.43428268 \\ \text { D9 } & -67.20895478 \\ \text { D10 } & -60.43575250 \\ \text { D11 } & 51.32686259 \\ \text { D12 } & -81.30555133 \\ \text { D13 } & 39.10657522 \\ \text { D14 } & 155.92395966 \\ \text { D15 } & 174.36824032 \\ \text { D16 } & 11.83670620 \\ \text { D17 } & 127.11344084 \\ \text { D18 } & -109.36652676 \\ \text { D19 } & 178.50669780 \\ \text { D20 } & 171.24055265 \\ \text { D21 } & -69.17048010 \\ \text { D22 } & 51.76156921 \\ \text { D23 } & -5.39950991 \\ \text { D24 } & -2.99886798 \\ \text { D25 } & -57.09044284 \\ \text { D26 } & 64.40066710 \\ \text { D27 } & -175.88202491 \\ \text { D28 } & 123.10310053 \\ \text { D29 } & -81.55632291 \\ \text { D30 } & 38.39650041 \\ \text { D31 } & -34.73845411 \\ \text { D32 }\end{array}$

Harmonic Vibrational Frequencies $\left(\mathbf{c m}^{-1}\right)$ :

$\begin{array}{lccccccccccc}35.22 & 56.04 & 73.25 & 84.73 & 88.63 & 95.84 & 114.42 & 132.82 & 139.72 & 164.50 & 175.98 \\ 191.50 & 200.12 & 204.42 & 222.69 & 246.40 & 265.90 & 274.30 & 287.79 & 323.20 & 335.04 \\ 348.28 & 361.79 & 371.07 & 380.65 & 395.91 & 406.62 & 442.91 & 470.67 & 504.58 & 538.70 \\ 581.33 & 596.45 & 603.73 & 615.02 & 771.50 & 815.11 & 831.90 & 855.56 & 912.31 & 918.86 \\ 930.42 & 947.33 & 953.54 & 960.68 & 972.19 & 993.84 & 1012.12 & 1023.74 & 1033.52 & 1057.62 \\ 1068.41 & 1094.07 & 1110.58 & 1156.18 & 1208.49 & 1237.22 & 1258.31 & 1271.93 & 1310.03 \\ 1319.19 & 1378.00 & 1397.67 & 1406.65 & 1410.78 & 1415.40 & 1432.60 & 1433.69 & 1457.07 \\ 1462.43 & 1474.87 & 1481.25 & 1486.14 & 1488.62 & 1490.98 & 1492.23 & 1496.63 & 1504.32 \\ 1509.73 & 1515.80 & 1625.41 & 1673.66 & 3057.05 & 3061.80 & 3062.33 & 3065.62 & 3071.79 \\ 3075.60 & 3125.16 & 3125.90 & 3143.01 & 3146.67 & 3150.56 & 3150.95 & 3155.90 & 3157.46\end{array}$


$\begin{array}{llllll}3163.76 & 3169.09 & 3170.74 & 3173.74 & 3231.19 & 3889.84\end{array}$

$\begin{array}{llll}\text { Rotational constants (B/GHZ): } & 0.5932267 & 0.4720037 & 0.3590494\end{array}$

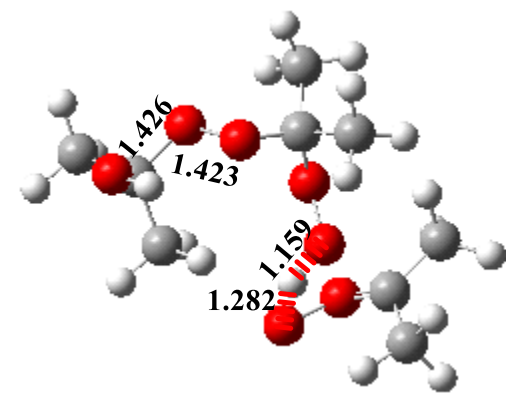

TS8a

$\mathrm{C}$

O 11 B1

O 1

$\mathrm{H} 2$

C 2

O 5

O $\quad 5$

C 1

$\mathrm{H} \quad 8$

H 8

$\mathrm{H} \quad 8$

O 1

C 12

C 5

$\mathrm{H} \quad 14$

$\mathrm{H} \quad 14$

H 14

C 5

H 18

H 18

$\mathrm{H} \quad 18$

C 1

H 22

H 22

$\mathrm{H} \quad 22$

O 12

C 13

H 27

H 27

H 27

C 13

$\mathrm{H} \quad 31$

$\mathrm{H} \quad 31$

$\begin{array}{rrrrrr}\text { B2 } & 2 & \text { A1 } & & & \\ \text { B3 } & 1 & \text { A2 } & 3 & \text { D1 } & 0 \\ \text { B4 } & 1 & \text { A3 } & 3 & \text { D2 } & 0 \\ \text { B5 } & 2 & \text { A4 } & 1 & \text { D3 } & 0 \\ \text { B6 } & 2 & \text { A5 } & 1 & \text { D4 } & 0 \\ \text { B7 } & 3 & \text { A6 } & 2 & \text { D5 } & 0 \\ \text { B8 } & 1 & \text { A7 } & 3 & \text { D6 } & 0 \\ \text { B9 } & 1 & \text { A8 } & 3 & \text { D7 } & 0 \\ \text { B10 } & 1 & \text { A9 } & 3 & \text { D8 } & 0 \\ \text { B11 } & 3 & \text { A10 } & 2 & \text { D9 } & 0 \\ \text { B12 } & 1 & \text { A11 } & 3 & \text { D10 } & 0 \\ \text { B13 } & 2 & \text { A12 } & 1 & \text { D11 } & 0 \\ \text { B14 } & 5 & \text { A13 } & 2 & \text { D12 } & 0 \\ \text { B15 } & 5 & \text { A14 } & 2 & \text { D13 } & 0 \\ \text { B16 } & 5 & \text { A15 } & 2 & \text { D14 } & 0 \\ \text { B17 } & 2 & \text { A16 } & 1 & \text { D15 } & 0 \\ \text { B18 } & 5 & \text { A17 } & 2 & \text { D16 } & 0 \\ \text { B19 } & 5 & \text { A18 } & 2 & \text { D17 } & 0 \\ \text { B20 } & 5 & \text { A19 } & 2 & \text { D18 } & 0 \\ \text { B21 } & 3 & \text { A20 } & 2 & \text { D19 } & 0 \\ \text { B22 } & 1 & \text { A21 } & 3 & \text { D20 } & 0 \\ \text { B23 } & 1 & \text { A22 } & 3 & \text { D21 } & 0 \\ \text { B24 } & 1 & \text { A23 } & 3 & \text { D22 } & 0 \\ \text { B25 } & 1 & \text { A24 } & 3 & \text { D23 } & 0 \\ \text { B26 } & 12 & \text { A25 } & 1 & \text { D24 } & 0 \\ \text { B27 } & 13 & \text { A26 } & 12 & \text { D25 } & 0 \\ \text { B28 } & 13 & \text { A27 } & 12 & \text { D26 } & 0 \\ \text { B29 } & 13 & \text { A28 } & 12 & \text { D27 } & 0 \\ \text { B30 } & 12 & \text { A29 } & 1 & \text { D28 } & 0 \\ \text { B31 } & 13 & \text { A30 } & 12 & \text { D29 } & 0 \\ \text { B32 } & 13 & \text { A31 } & 12 & \text { D30 } & 0 \\ & & & & & \end{array}$




$\begin{array}{rrrrrrrr}\mathrm{H} & 31 & \text { B33 } & 13 & \text { A32 } & 12 & \text { D31 } & 0 \\ \mathrm{O} & 13 & \text { B34 } & 12 & \text { A33 } & 1 & \text { D32 } & 0 \\ \mathrm{H} & 35 & \text { B35 } & 13 & \text { A34 } & 12 & \text { D33 } & 0\end{array}$

Variables:

\begin{tabular}{|c|c|}
\hline B1 & 2.31326192 \\
\hline B2 & 1.41023439 \\
\hline B3 & 1.15901384 \\
\hline B4 & 2.11704371 \\
\hline B5 & 2.21839799 \\
\hline B6 & 1.27982971 \\
\hline B7 & 1.51882049 \\
\hline B8 & 1.08852137 \\
\hline B9 & 1.08605009 \\
\hline B10 & 1.08787818 \\
\hline B11 & 1.41042674 \\
\hline B12 & 2.30071068 \\
\hline B13 & 1.48269256 \\
\hline B14 & 1.08736657 \\
\hline B15 & 1.09326133 \\
\hline B16 & 1.08671050 \\
\hline B17 & 1.48638690 \\
\hline B18 & 1.08262707 \\
\hline B19 & 1.09466325 \\
\hline B20 & 1.08668649 \\
\hline B21 & 1.51872724 \\
\hline B22 & 1.08896338 \\
\hline B 23 & 1.08772513 \\
\hline B24 & 1.08617550 \\
\hline B25 & 1.42279617 \\
\hline B26 & 1.51391167 \\
\hline B27 & 1.08782097 \\
\hline B28 & 1.08772480 \\
\hline B29 & 1.08775376 \\
\hline B30 & 1.51791713 \\
\hline B31 & 1.08899958 \\
\hline B32 & 1.08937375 \\
\hline B33 & 1.08859079 \\
\hline B34 & 1.39890900 \\
\hline B35 & 0.96176878 \\
\hline A1 & 35.18218477 \\
\hline A2 & 126.40637646 \\
\hline A3 & 130.14059860 \\
\hline A4 & 66.32413606 \\
\hline A5 & 89.67816031 \\
\hline
\end{tabular}




\begin{tabular}{|c|c|}
\hline A6 & 104.15087633 \\
\hline A7 & 109.47740940 \\
\hline A8 & 110.40664496 \\
\hline A9 & 109.04414490 \\
\hline A10 & 111.45723426 \\
\hline A11 & 131.68103828 \\
\hline A12 & 107.66684882 \\
\hline A13 & 110.24538513 \\
\hline A14 & 105.94350667 \\
\hline A 15 & 111.70297372 \\
\hline A16 & 89.63924695 \\
\hline A 17 & 112.07625955 \\
\hline A18 & 106.15884191 \\
\hline A19 & 109.55093716 \\
\hline A20 & 112.69868640 \\
\hline A21 & 109.28294600 \\
\hline A22 & 109.33904463 \\
\hline A23 & 110.54349600 \\
\hline A24 & 108.90195231 \\
\hline A 25 & 139.49127567 \\
\hline A26 & 109.43671501 \\
\hline A27 & 110.33105670 \\
\hline A28 & 108.81284181 \\
\hline A29 & 91.89463276 \\
\hline A30 & 110.55581070 \\
\hline A 31 & 110.46353133 \\
\hline A32 & 109.07902793 \\
\hline A33 & 90.91882098 \\
\hline A34 & 107.99806822 \\
\hline D1 & -53.81015742 \\
\hline D2 & 44.75654729 \\
\hline D3 & -121.86649866 \\
\hline D4 & -92.68738650 \\
\hline D5 & 166.99014646 \\
\hline D6 & -72.38574704 \\
\hline D7 & 48.09181886 \\
\hline D8 & 167.86151006 \\
\hline D9 & -71.89787126 \\
\hline D10 & -37.02595165 \\
\hline D11 & 22.12174168 \\
\hline D12 & -117.40348400 \\
\hline D13 & -1.07156017 \\
\hline D14 & 118.19682126 \\
\hline D15 & 144.16861735 \\
\hline
\end{tabular}




$\begin{array}{lr}\text { D16 } & 58.98332448 \\ \text { D17 } & 178.45551645 \\ \text { D18 } & -64.72677988 \\ \text { D19 } & 44.30949488 \\ \text { D20 } & 48.96155429 \\ \text { D21 } & 168.40310633 \\ \text { D22 } & -71.02860377 \\ \text { D23 } & -70.18177856 \\ \text { D24 } & -66.30914134 \\ \text { D25 } & -54.63541830 \\ \text { D26 } & 66.21813816 \\ \text { D27 } & -173.78449002 \\ \text { D28 } & 63.41139262 \\ \text { D29 } & -84.38205004 \\ \text { D30 } & 35.71117815 \\ \text { D31 } & 155.25180405 \\ \text { D32 } & 175.02972869 \\ \text { D33 } & -34.38888159\end{array}$

Harmonic Vibrational Frequencies $\left(\mathbf{c m}^{-1}\right)$ :

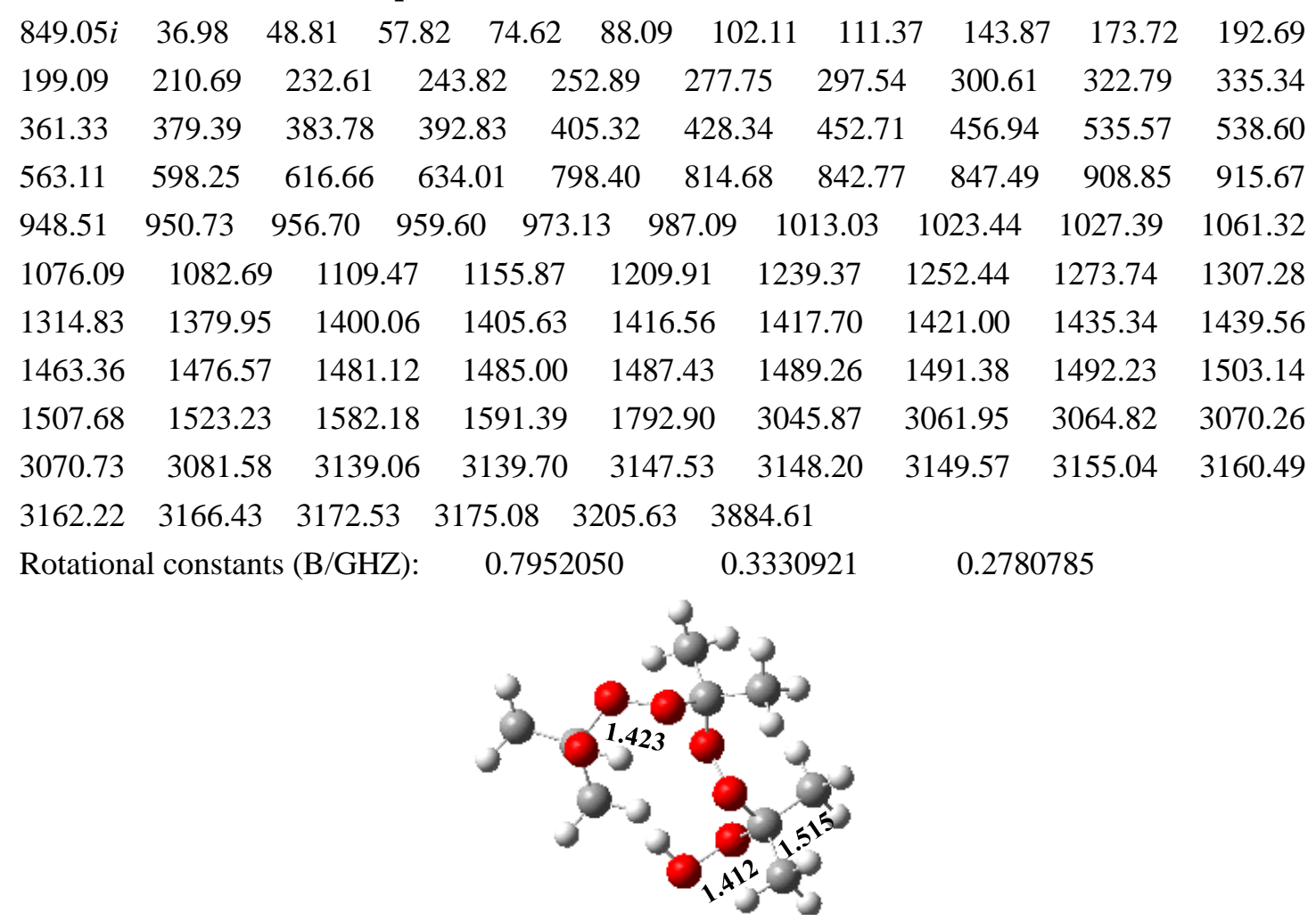

P8a

$\mathrm{C}$

O $\quad 1 \quad$ B1

$\begin{array}{lllll}\mathrm{O} & 1 & \mathrm{~B} 2 & 2 & \mathrm{~A} 1\end{array}$

$\begin{array}{llllllll}\mathrm{H} & 2 & \mathrm{~B} 3 & 1 & \mathrm{~A} 2 & 3 & \mathrm{D} 1 & 0\end{array}$

$\begin{array}{llllllll}\mathrm{C} & 2 & \mathrm{~B} 4 & 1 & \mathrm{~A} 3 & 3 & \mathrm{D} 2 & 0\end{array}$ 


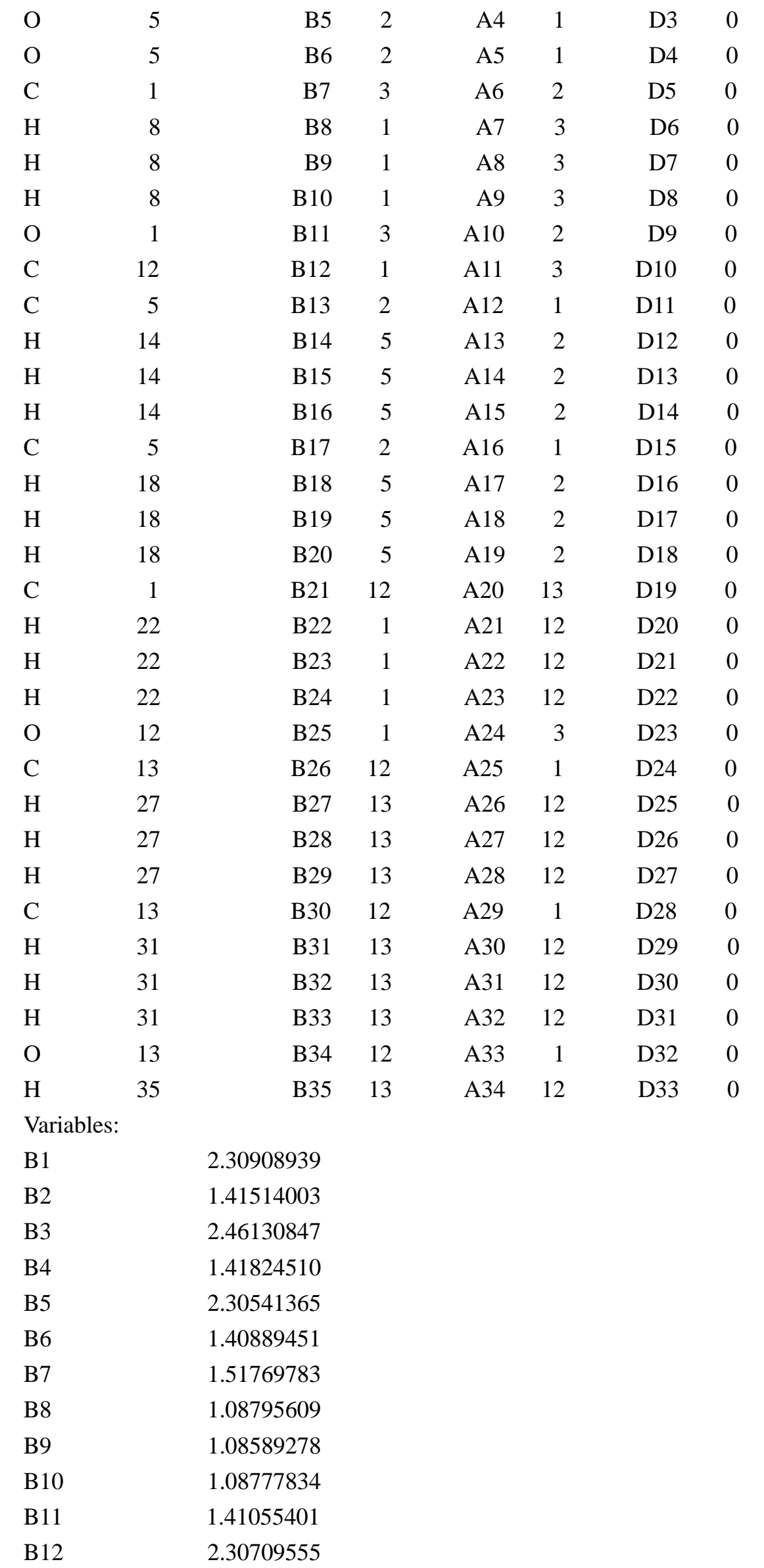




\begin{tabular}{|c|c|}
\hline B13 & 1.51541517 \\
\hline B14 & 1.08780055 \\
\hline B15 & 1.08718928 \\
\hline B16 & 1.08843281 \\
\hline B17 & 1.51636819 \\
\hline B18 & 1.08629214 \\
\hline B19 & 1.08816886 \\
\hline B20 & 1.08822181 \\
\hline B21 & 1.51800790 \\
\hline B22 & 1.08803484 \\
\hline B23 & 1.08755312 \\
\hline B24 & 1.08648794 \\
\hline B25 & 1.42255904 \\
\hline B26 & 1.51393200 \\
\hline B27 & 1.08743287 \\
\hline B28 & 1.08814905 \\
\hline B29 & 1.08787236 \\
\hline B30 & 1.51968437 \\
\hline B31 & 1.09118388 \\
\hline B32 & 1.08914846 \\
\hline B33 & 1.08840247 \\
\hline B34 & 1.39460138 \\
\hline B35 & 0.96219433 \\
\hline A1 & 35.74007698 \\
\hline $\mathrm{A} 2$ & 109.11323312 \\
\hline A3 & 133.66469209 \\
\hline A4 & 88.92188066 \\
\hline A5 & 109.81257454 \\
\hline A6 & 103.85823732 \\
\hline A7 & 109.19834436 \\
\hline A8 & 110.62851480 \\
\hline A9 & 108.97011961 \\
\hline A10 & 110.96440846 \\
\hline A11 & 130.87661854 \\
\hline A12 & 113.02854803 \\
\hline A 13 & 109.41028452 \\
\hline A14 & 110.21454072 \\
\hline A 15 & 109.22858423 \\
\hline A16 & 103.78420199 \\
\hline A17 & 110.27246445 \\
\hline A 18 & 108.63423337 \\
\hline A19 & 110.01285835 \\
\hline A20 & 103.95217064 \\
\hline A21 & 109.20321262 \\
\hline
\end{tabular}




\begin{tabular}{|c|c|}
\hline $\mathrm{A} 22$ & 108.97111217 \\
\hline A23 & 110.94289935 \\
\hline A24 & 109.94430385 \\
\hline A25 & 138.74273832 \\
\hline A26 & 109.32663130 \\
\hline $\mathrm{A} 27$ & 110.44390640 \\
\hline A28 & 108.89757599 \\
\hline A29 & 94.93898393 \\
\hline A 30 & 109.11499059 \\
\hline A31 & 110.75169860 \\
\hline A32 & 109.25016912 \\
\hline A33 & 88.20478830 \\
\hline A34 & 108.10074895 \\
\hline D1 & -37.44451449 \\
\hline D2 & 54.53773797 \\
\hline D3 & -114.96791900 \\
\hline D4 & -86.00771262 \\
\hline D5 & 171.22973549 \\
\hline D6 & -72.27191213 \\
\hline D7 & 48.26471804 \\
\hline D8 & 168.02416362 \\
\hline D9 & -67.33607946 \\
\hline D10 & -41.42945877 \\
\hline D11 & 29.96220233 \\
\hline D12 & -179.11136690 \\
\hline D13 & -59.21070008 \\
\hline D14 & 61.57625895 \\
\hline D15 & 153.34524441 \\
\hline D16 & 58.21697803 \\
\hline D17 & 177.78062874 \\
\hline D18 & -62.67782159 \\
\hline D19 & -163.03765445 \\
\hline D20 & 172.41195369 \\
\hline D21 & -68.38335850 \\
\hline D22 & 51.83685414 \\
\hline D23 & -76.05280088 \\
\hline D24 & -79.47633929 \\
\hline D25 & -51.62608081 \\
\hline D26 & 69.09725425 \\
\hline D27 & -170.85021729 \\
\hline D28 & 54.04164616 \\
\hline D29 & -91.65146504 \\
\hline D30 & 28.77598499 \\
\hline D31 & 149.10903180 \\
\hline
\end{tabular}


D32

165.53981495

D33

$-34.21983325$

Harmonic Vibrational Frequencies $\left(\mathrm{cm}^{-1}\right)$ :

$\begin{array}{lccccccccccc}35.19 & 52.52 & 67.69 & 82.09 & 106.38 & 120.22 & 141.20 & 174.84 & 187.80 & 217.51 & 223.81 \\ 232.67 & 250.58 & 262.79 & 270.39 & 287.64 & 297.40 & 305.39 & 327.61 & 350.23 & 358.14 \\ 373.32 & 380.66 & 389.51 & 407.48 & 424.38 & 439.91 & 460.67 & 479.14 & 534.41 & 574.94 \\ 591.73 & 602.52 & 614.80 & 631.03 & 812.69 & 831.22 & 866.23 & 904.55 & 912.93 & 917.10 \\ 944.54 & 951.53 & 955.12 & 962.19 & 982.34 & 1017.03 & 1023.89 & 1028.26 & 1034.90 & 1049.24 \\ 1062.07 & 1068.97 & 1154.05 & 1193.73 & 1225.50 & 1238.57 & 1250.82 & 1256.09 & 1277.48 \\ 1297.91 & 1323.02 & 1381.99 & 1407.67 & 1411.79 & 1413.28 & 1415.20 & 1420.52 & 1437.30 \\ 1448.62 & 1478.20 & 1483.22 & 1486.00 & 1486.67 & 1488.83 & 1495.03 & 1496.84 & 1499.46 \\ 1507.70 & 1510.76 & 1513.43 & 1525.90 & 3057.21 & 3074.19 & 3076.02 & 3077.42 & 3078.92 \\ 3081.03 & 3137.17 & 3153.42 & 3156.08 & 3158.14 & 3159.73 & 3161.30 & 3162.75 & 3167.21 \\ 3168.14 & 3169.99 & 3170.10 & 3170.91 & 3799.00 & 3878.96 & & & & \end{array}$

$\begin{array}{llll}\text { Rotational constants (B/GHZ): } & 0.7749678 & 0.3589879 & 0.2955461\end{array}$

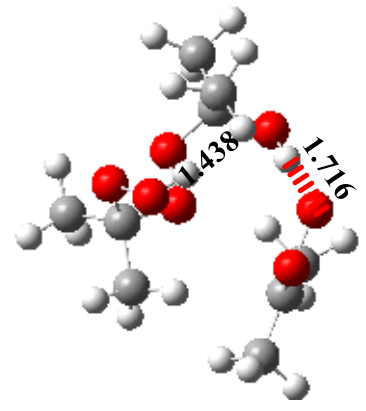

IM8b

C

$\begin{array}{lll}\mathrm{C} & 1 & \mathrm{~B} 1\end{array}$

$\begin{array}{lllll}\mathrm{H} & 2 & \mathrm{~B} 2 & 1 & \mathrm{~A} 1\end{array}$

$\begin{array}{lllll}\mathrm{H} & 2 & \mathrm{~B} 3 & 1 & \mathrm{~A} 2\end{array}$

$\mathrm{H} \quad 2$

C 1

$\mathrm{H} \quad 6$

B4 1

B5 2

B6 1

A3 $3 \quad$ D2 0

$\mathrm{H} \quad 6$

$\mathrm{H} \quad 6$

B7 1

A4 3

D3 0

A5 2 D4 0

A6 2 D5 0

O 1

B8 1

A7 2

D6 0

$\mathrm{H} \quad 10$

B9 2

A8 6

D7 0

B10 1

A9 2 D8 0

C 10

B11 1

A10 2

D9 0

C 12

B12 10

A11 1

D10 0

B13 12

A12 10

D11 0

B14 12

A13 10

D12 0

B15 12

A14 10

D13 0

C 12

B16 10

A15 1

D14 0

$\begin{array}{ll}\mathrm{H} & 17\end{array}$

B17 12

A16 10

D15 0

$\begin{array}{ll}\mathrm{H} & 17\end{array}$

B18 12

A17 10

D16 0

$\begin{array}{ll}\mathrm{H} & 17\end{array}$

B19 12

A18 10

D17 0 


$\begin{array}{rrrrrrrr}\mathrm{O} & 12 & \text { B20 } & 10 & \text { A19 } & 1 & \text { D18 } & 0 \\ \mathrm{O} & 12 & \text { B21 } & 10 & \text { A20 } & 1 & \text { D19 } & 0 \\ \mathrm{O} & 1 & \text { B22 } & 10 & \text { A21 } & 2 & \text { D20 } & 0 \\ \mathrm{O} & 23 & \text { B23 } & 1 & \text { A22 } & 10 & \text { D21 } & 0 \\ \mathrm{C} & 24 & \text { B24 } & 23 & \text { A23 } & 1 & \text { D22 } & 0 \\ \mathrm{C} & 25 & \text { B25 } & 24 & \text { A24 } & 23 & \text { D23 } & 0 \\ \mathrm{H} & 26 & \text { B26 } & 25 & \text { A25 } & 24 & \text { D24 } & 0 \\ \mathrm{H} & 26 & \text { B27 } & 25 & \text { A26 } & 24 & \text { D25 } & 0 \\ \mathrm{H} & 26 & \text { B28 } & 25 & \text { A27 } & 24 & \text { D26 } & 0 \\ \mathrm{C} & 25 & \text { B29 } & 24 & \text { A28 } & 23 & \text { D27 } & 0 \\ \mathrm{H} & 30 & \text { B30 } & 25 & \text { A29 } & 24 & \text { D28 } & 0 \\ \mathrm{H} & 30 & \text { B31 } & 25 & \text { A30 } & 24 & \text { D29 } & 0 \\ \mathrm{H} & 30 & \text { B32 } & 25 & \text { A31 } & 24 & \text { D30 } & 0 \\ \text { O } & 25 & \text { B33 } & 24 & \text { A32 } & 23 & \text { D31 } & 0 \\ \text { O } & 34 & \text { B34 } & 25 & \text { A33 } & 24 & \text { D32 } & 0 \\ \text { H } & 35 & \text { B35 } & 34 & \text { A34 } & 25 & \text { D33 } & 0 \\ \text { Var } & & & & & & & \end{array}$

Variables:

$\begin{array}{ll}\text { B1 } & 1.51730555 \\ \text { B2 } & 1.08753630 \\ \text { B3 } & 1.08821852 \\ \text { B4 } & 1.08825952 \\ \text { B5 } & 1.52213938 \\ \text { B6 } & 1.08865668 \\ \text { B7 } & 1.08914895 \\ \text { B8 } & 1.09109496 \\ \text { B9 } & 1.38278939 \\ \text { B10 } & 0.98397369 \\ \text { B11 } & 3.69130209 \\ \text { B12 } & 1.48428764 \\ \text { B13 } & 1.08552829 \\ \text { B14 } & 1.09041186 \\ \text { B15 } & 1.09187943 \\ \text { B16 } & 1.47094734 \\ \text { B17 } & 1.09510810 \\ \text { B18 } & 1.09051260 \\ \text { B19 } & 1.08595366 \\ \text { B20 } & 2.25855798 \\ \text { B21 } & 1.24905942 \\ \text { B22 } & 1.43778211 \\ \text { B23 } & 1.42421409 \\ \text { B24 } & 1.41913110 \\ \text { B25 } & 1.51768533 \\ \text { B26 } & 1.08655121 \\ \text { B27 } & 1.08799923\end{array}$




\begin{tabular}{|c|c|}
\hline B28 & 1.08841320 \\
\hline B29 & 1.51729775 \\
\hline B30 & 1.08565126 \\
\hline B31 & 1.08804648 \\
\hline B32 & 1.08786489 \\
\hline B33 & 1.40961621 \\
\hline B34 & 1.41339449 \\
\hline B35 & 0.96683142 \\
\hline $\mathrm{A} 1$ & 108.55230834 \\
\hline $\mathrm{A} 2$ & 109.51274678 \\
\hline A3 & 110.74233419 \\
\hline A4 & 112.64674933 \\
\hline A5 & 108.84609217 \\
\hline A6 & 110.95741928 \\
\hline A7 & 109.34473611 \\
\hline A 8 & 108.60548826 \\
\hline A9 & 108.84205278 \\
\hline A10 & 110.46461269 \\
\hline A11 & 153.28763450 \\
\hline A12 & 111.47098701 \\
\hline A 13 & 109.09653828 \\
\hline A14 & 108.60103925 \\
\hline A15 & 54.91445558 \\
\hline A16 & 108.38977903 \\
\hline A 17 & 109.36970677 \\
\hline A18 & 110.67011323 \\
\hline A19 & 46.68262382 \\
\hline $\mathrm{A} 20$ & 74.01286267 \\
\hline A21 & 110.57504946 \\
\hline $\mathrm{A} 22$ & 109.92105939 \\
\hline A 23 & 108.51280392 \\
\hline A24 & 104.52721952 \\
\hline A25 & 110.66731027 \\
\hline A26 & 108.83549142 \\
\hline A 27 & 109.32502593 \\
\hline A28 & 112.80752640 \\
\hline A29 & 110.54120271 \\
\hline A 30 & 109.32300830 \\
\hline A31 & 109.05347636 \\
\hline A 32 & 110.46673031 \\
\hline A33 & 109.93582435 \\
\hline A34 & 102.51828441 \\
\hline D1 & 118.81408731 \\
\hline D2 & -120.27556037 \\
\hline
\end{tabular}




$\begin{array}{lr}\text { D3 } & 64.01965328 \\ \text { D4 } & -49.13747622 \\ \text { D5 } & 71.13314538 \\ \text { D6 } & -165.72416520 \\ \text { D7 } & -123.56162073 \\ \text { D8 } & 168.41992115 \\ \text { D9 } & -151.64813968 \\ \text { D10 } & 42.01211735 \\ \text { D11 } & -102.13017764 \\ \text { D12 } & 19.93586893 \\ \text { D13 } & 136.53039638 \\ \text { D14 } & 135.11863361 \\ \text { D15 } & -38.56074792 \\ \text { D16 } & 76.51097647 \\ \text { D17 } & -159.53642966 \\ \text { D18 } & -94.65697155 \\ \text { D19 } & -74.11807770 \\ \text { D20 } & 111.11953106 \\ \text { D21 } & 65.26394781 \\ \text { D22 } & 132.82810384 \\ \text { D23 } & 164.24013634 \\ \text { D24 } & 47.83190920 \\ \text { D25 } & 167.12361888 \\ \text { D26 } & -73.63810326 \\ \text { D27 } & 41.45546951 \\ \text { D28 } & -72.96493746 \\ \text { D29 } & 46.95950645 \\ \text { D30 } & 166.79861019 \\ \text { D31 } & -74.87410950 \\ \text { D32 } & -70.85260150 \\ \text { D33 } & 82.57674467 \\ \text { Hart }\end{array}$

Harmonic Vibrational Frequencies $\left(\mathbf{c m}^{-1}\right)$ :

$\begin{array}{lccccccccccc}19.72 & 45.50 & 63.58 & 77.53 & 101.46 & 119.94 & 120.62 & 135.30 & 153.59 & 161.32 & 177.59 \\ 215.19 & 217.19 & 230.42 & 245.52 & 268.74 & 279.63 & 305.94 & 316.87 & 321.59 & 343.91 \\ 351.83 & 368.93 & 378.22 & 383.61 & 390.14 & 420.81 & 459.00 & 469.70 & 493.40 & 544.28 \\ 598.34 & 604.42 & 613.40 & 626.45 & 811.61 & 826.61 & 843.75 & 898.24 & 900.81 & 912.08 \\ 920.97 & 950.32 & 952.99 & 962.96 & 970.92 & 992.61 & 1018.34 & 1026.79 & 1036.42 & 1055.70 \\ 1067.65 & 1096.57 & 1104.16 & 1191.38 & 1210.39 & 1240.68 & 1252.47 & 1287.65 & 1311.21 \\ 1321.87 & 1386.91 & 1399.20 & 1407.79 & 1411.27 & 1413.10 & 1416.04 & 1428.06 & 1456.32 \\ 1466.07 & 1470.94 & 1473.39 & 1480.88 & 1490.19 & 1491.80 & 1496.44 & 1498.67 & 1499.64 \\ 1514.55 & 1525.87 & 1546.27 & 1665.24 & 3044.69 & 3051.35 & 3056.55 & 3073.32 & 3073.89 \\ 3077.57 & 3109.36 & 3123.00 & 3129.90 & 3149.12 & 3153.44 & 3159.14 & 3159.29 & 3160.07 \\ 3167.22 & 3170.50 & 3175.33 & 3176.69 & 3345.74 & 3803.56 & & & & \\ \text { Rotational constants (B/GHZ): } & 0.5626837 & 0.4730170 & 0.3462310 & \end{array}$




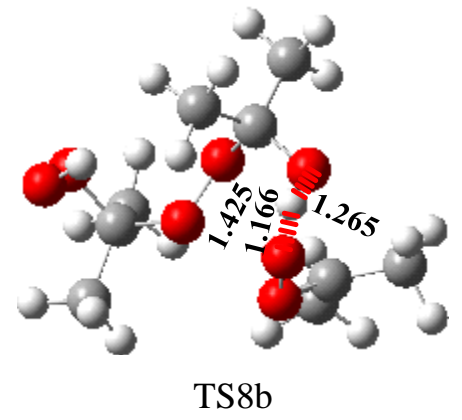

C

$\begin{array}{lll}\text { C } & 1 & \text { B1 }\end{array}$

$\mathrm{H} \quad 2$

$\mathrm{H} \quad 2$

$\mathrm{H} \quad 2$

C 1

$\mathrm{H} \quad 6$

$\mathrm{H} \quad 6$

$\mathrm{H} \quad 6$

O 1

$\mathrm{H} \quad 10$

C $\quad 10$

C 12

$\mathrm{H} \quad 13$

$\mathrm{H} \quad 13$

$\mathrm{H} \quad 13$

C 12

$\mathrm{H} \quad 17$

$\begin{array}{ll}\mathrm{H} & 17\end{array}$

$\mathrm{H} \quad 17$

O 12

O 12

O 1

O 23

C 24

C 25

$\mathrm{H} \quad 26$

$\mathrm{H} \quad 26$

H 26

C 25

$\mathrm{H} \quad 30$

$\mathrm{H} \quad 30$

$\mathrm{H} \quad 30$

O 25

O 34

$\mathrm{H} \quad 35$

B2 1

B3 1

A1

B4 1

$\begin{array}{llll}\text { A2 } & 3 & \text { D1 } & 0\end{array}$

B5 2

A3 3

D2 0

B6 1

A4 3

D3 0

B7 1

A5 2

D4 0

B8 1

A6 $2 \quad$ D5 0

B9 2

A7 2

D6 0

B10 1

B11 1

A8 6

D7 0

$\begin{array}{llll}\text { A9 } & 2 & \text { D8 } & 0\end{array}$

B12 10

$\begin{array}{llll}\mathrm{A} 10 & 2 & \mathrm{D} 9 & 0\end{array}$

B13 12

$\begin{array}{llll}\text { A11 } & 1 & \text { D10 } & 0\end{array}$

B14 12

A12 10

D11 0

B15 12

A13 10

D12 0

B16 10

A14 10

D13 0

B17 12

A15 1

D14 0

B18 12

A16 10

D15 0

A17 $10 \quad$ D16 0

B19 12

A18 10

D17 0

B20 10

A19 1

D18 0

B21 10

A20 1

D19 0

B22 10

A21 12

D20 0

B23 1

A22 10

D21 0

B24 23

A23 1

D22 0

B25 24

A24 23

D23 0

B26 25

A25 24

D24 0

B27 25

A26 24

D25 0

B28 25

A27 24

D26 0

B29 24

A28 23

D27 0

B30 25

A29 24

D28 0

B31 25

A30 24

D29 0

B32 25

A31 24

D30 0

B33 24

A32 23

D31 0

B34 25

A33 24

D32 0

B35 34

A34 25

D33 0 
Variables:

\begin{tabular}{|c|c|}
\hline B1 & 1.52311122 \\
\hline $\mathrm{B} 2$ & 1.08792015 \\
\hline B3 & 1.08890482 \\
\hline B4 & 1.08871281 \\
\hline B5 & 1.52443625 \\
\hline B6 & 1.08894247 \\
\hline B7 & 1.09114024 \\
\hline B8 & 1.09071869 \\
\hline B9 & 1.37844482 \\
\hline B10 & 1.26474017 \\
\hline B11 & 2.11126607 \\
\hline B12 & 1.48392616 \\
\hline B13 & 1.08537033 \\
\hline B14 & 1.09152395 \\
\hline B15 & 1.08883726 \\
\hline B16 & 1.48726939 \\
\hline B17 & 1.08569335 \\
\hline B18 & 1.08140113 \\
\hline B19 & 1.09494215 \\
\hline B20 & 2.21735200 \\
\hline B21 & 1.28387695 \\
\hline B22 & 1.43599161 \\
\hline B23 & 1.42466020 \\
\hline B24 & 1.41440086 \\
\hline B25 & 1.51811281 \\
\hline B26 & 1.08631163 \\
\hline B27 & 1.08790780 \\
\hline B28 & 1.08852627 \\
\hline B29 & 1.51768793 \\
\hline B30 & 1.08607332 \\
\hline B31 & 1.08871949 \\
\hline B32 & 1.08780449 \\
\hline B33 & 1.41106207 \\
\hline B34 & 1.41386529 \\
\hline B35 & 0.96576667 \\
\hline A1 & 108.53875156 \\
\hline A2 & 109.31928944 \\
\hline A3 & 111.20129083 \\
\hline A4 & 111.83431201 \\
\hline A5 & 109.10407124 \\
\hline A6 & 110.26755974 \\
\hline A7 & 109.78585561 \\
\hline A8 & 109.55982742 \\
\hline
\end{tabular}




\begin{tabular}{|c|c|}
\hline A9 & 116.07977239 \\
\hline A10 & 129.18316204 \\
\hline A11 & 112.46972470 \\
\hline A12 & 110.68715916 \\
\hline A 13 & 106.66476645 \\
\hline A14 & 110.05931375 \\
\hline A 15 & 87.24502451 \\
\hline A16 & 109.36259769 \\
\hline A17 & 112.55838544 \\
\hline A 18 & 105.57705711 \\
\hline A19 & 66.22840656 \\
\hline A20 & 91.29908197 \\
\hline A21 & 111.54922700 \\
\hline A22 & 108.86819868 \\
\hline A23 & 108.83353981 \\
\hline A24 & 103.94587119 \\
\hline A25 & 110.76087281 \\
\hline A26 & 109.00605355 \\
\hline A 27 & 109.37558585 \\
\hline A28 & 112.63837078 \\
\hline A29 & 110.56796248 \\
\hline A30 & 109.13748839 \\
\hline A31 & 109.26178416 \\
\hline A32 & 110.57757803 \\
\hline A33 & 109.95669524 \\
\hline A34 & 102.21718438 \\
\hline D1 & 118.44720802 \\
\hline D2 & -120.60821959 \\
\hline D3 & 65.31075318 \\
\hline D4 & -55.38804774 \\
\hline D5 & 64.07812490 \\
\hline D6 & -173.33939667 \\
\hline D7 & -123.91355848 \\
\hline D8 & 146.59506370 \\
\hline D9 & -126.44651589 \\
\hline D10 & 36.45982770 \\
\hline D11 & -104.56072470 \\
\hline D12 & 13.33428715 \\
\hline D13 & 131.44620027 \\
\hline D14 & 156.78344929 \\
\hline D15 & -67.42059270 \\
\hline D16 & 55.89507138 \\
\hline D17 & 175.68219676 \\
\hline D18 & -107.97262289 \\
\hline
\end{tabular}




$\begin{array}{lr}\text { D19 } & -80.45505311 \\ \text { D20 } & -14.54368603 \\ \text { D21 } & 68.08310616 \\ \text { D22 } & 140.38632200 \\ \text { D23 } & 166.73471982 \\ \text { D24 } & 45.57129973 \\ \text { D25 } & 165.50378954 \\ \text { D26 } & -74.97922056 \\ \text { D27 } & 44.02507780 \\ \text { D28 } & -71.99029807 \\ \text { D29 } & 47.99484361 \\ \text { D30 } & 167.56961025 \\ \text { D31 } & -71.99363305 \\ \text { D32 } & -70.40935930 \\ \text { D33 } & 86.32197881\end{array}$

Harmonic Vibrational Frequencies $\left(\mathbf{c m}^{-1}\right)$ :

\begin{tabular}{lccccccccccc}
$858.84 i$ & 29.25 & 37.32 & 59.11 & 98.11 & 119.17 & 134.06 & 156.53 & 180.63 & 196.45 & 213.34 \\
220.55 & 231.58 & 234.18 & 248.37 & 252.54 & 274.41 & 279.20 & 283.47 & 316.74 & 336.06 \\
352.19 & 365.32 & 372.55 & 391.36 & 394.97 & 418.61 & 460.51 & 491.85 & 503.18 & 544.83 \\
598.84 & 607.42 & 619.36 & 622.04 & 703.15 & 810.33 & 817.75 & 853.51 & 903.22 & 911.62 \\
938.90 & 957.46 & 957.72 & 966.40 & 971.01 & 995.53 & 1017.02 & 1024.19 & 1030.71 & 1053.53 \\
1067.01 & 1087.27 & 1098.67 & 1197.64 & 1219.09 & 1250.44 & 1252.88 & 1283.26 & 1310.38 \\
1323.85 & 1385.57 & 1393.79 & 1406.50 & 1410.25 & 1414.13 & 1419.80 & 1424.14 & 1430.79 \\
1452.03 & 1465.63 & 1473.08 & 1481.03 & 1485.60 & 1485.92 & 1491.15 & 1494.27 & 1495.36 \\
1500.31 & 1506.12 & 1534.21 & 1575.03 & 1921.67 & 3037.62 & 3054.54 & 3063.18 & 3068.37 \\
3070.25 & 3073.02 & 3120.20 & 3130.06 & 3134.82 & 3147.51 & 3149.51 & 3153.36 & 3156.27 \\
3162.19 & 3165.80 & 3166.68 & 3176.02 & 3214.90 & 3817.74 & & & & \\
Rotational constants (B/GHZ): & \multicolumn{8}{c}{0.7279719} & 0.3936212 & 0.3535498 &
\end{tabular}

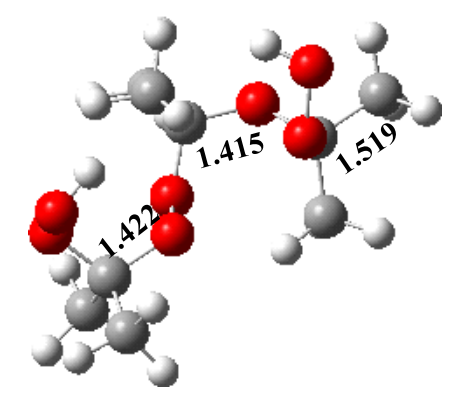

$\mathrm{P} 8 \mathrm{~b}$

$\mathrm{C}$

$\begin{array}{lll}\text { C } & 1 & \text { B1 }\end{array}$

$\begin{array}{lllll}\mathrm{H} & 2 & \mathrm{~B} 2 & 1 & \mathrm{~A} 1\end{array}$

$\begin{array}{llllllll}\mathrm{H} & 2 & \mathrm{~B} 3 & 1 & \mathrm{~A} 2 & 3 & \mathrm{D} 1 & 0\end{array}$

$\begin{array}{lllllllll}\mathrm{H} & 2 & \mathrm{~B} 4 & 1 & \mathrm{~A} 3 & 4 & \mathrm{D} 2 & 0\end{array}$

$\begin{array}{llllllll}\text { C } & 1 & \text { B5 } & 2 & \text { A4 } & 3 & \text { D3 } & 0\end{array}$

$\begin{array}{llllllll}\mathrm{H} & 6 & \mathrm{~B} 6 & 1 & \mathrm{~A} 5 & 2 & \mathrm{D} 4 & 0\end{array}$ 


\begin{tabular}{|c|c|c|c|c|c|c|}
\hline $\mathrm{H}$ & 6 & B7 & 1 & A6 & 2 & D5 \\
\hline $\mathrm{H}$ & 6 & B8 & 1 & A7 & 2 & D6 \\
\hline $\mathrm{O}$ & 1 & B9 & 6 & A8 & 2 & D7 \\
\hline $\mathrm{H}$ & 6 & B10 & 1 & A9 & 10 & D8 \\
\hline $\mathrm{C}$ & 10 & B11 & 1 & A 10 & 6 & D9 \\
\hline $\mathrm{C}$ & 12 & B12 & 10 & A11 & 1 & D10 \\
\hline $\mathrm{H}$ & 13 & B13 & 12 & A12 & 10 & D11 \\
\hline $\mathrm{H}$ & 13 & B14 & 12 & A 13 & 10 & D12 \\
\hline $\mathrm{H}$ & 13 & B15 & 12 & A14 & 10 & D13 \\
\hline $\mathrm{C}$ & 12 & B16 & 10 & A15 & 1 & D14 \\
\hline $\mathrm{H}$ & 17 & B17 & 12 & A16 & 10 & D15 \\
\hline $\mathrm{H}$ & 17 & B18 & 12 & A 17 & 10 & D16 \\
\hline $\mathrm{H}$ & 17 & B19 & 12 & A18 & 10 & D17 \\
\hline $\mathrm{O}$ & 12 & B20 & 10 & A19 & 1 & D18 \\
\hline $\mathrm{O}$ & 21 & B21 & 12 & A20 & 10 & D19 \\
\hline $\mathrm{O}$ & 1 & B22 & 10 & A21 & 12 & D20 \\
\hline $\mathrm{O}$ & 23 & B23 & 1 & A22 & 10 & D21 \\
\hline $\mathrm{C}$ & 24 & B24 & 23 & A23 & 1 & D22 \\
\hline $\mathrm{C}$ & 25 & B 25 & 24 & A24 & 23 & D23 \\
\hline $\mathrm{H}$ & 26 & B26 & 25 & A25 & 24 & D24 \\
\hline $\mathrm{H}$ & 26 & B27 & 25 & A26 & 24 & D25 \\
\hline $\mathrm{H}$ & 26 & B28 & 25 & A27 & 24 & D26 \\
\hline $\mathrm{C}$ & 25 & B29 & 24 & A28 & 23 & D27 \\
\hline $\mathrm{H}$ & 30 & B30 & 25 & A29 & 24 & D28 \\
\hline $\mathrm{H}$ & 30 & B31 & 25 & A30 & 24 & D29 \\
\hline $\mathrm{H}$ & 30 & B32 & 25 & A31 & 24 & D30 \\
\hline $\mathrm{O}$ & 25 & B33 & 24 & A32 & 23 & D31 \\
\hline $\mathrm{O}$ & 34 & B34 & 25 & A33 & 24 & D32 \\
\hline $\mathrm{H}$ & 35 & B35 & 34 & A34 & 25 & D33 \\
\hline
\end{tabular}

Variables:

$\begin{array}{ll}\text { B1 } & 1.52108106 \\ \text { B2 } & 1.08769472 \\ \text { B3 } & 1.08813701 \\ \text { B4 } & 1.08798034 \\ \text { B5 } & 1.52070302 \\ \text { B6 } & 1.09025078 \\ \text { B7 } & 1.09003681 \\ \text { B8 } & 1.08843790 \\ \text { B9 } & 1.41492568 \\ \text { B10 } & 2.47023965 \\ \text { B11 } & 1.41666094 \\ \text { B12 } & 1.52198207 \\ \text { B13 } & 1.08377634 \\ \text { B14 } & 1.08796481\end{array}$




\begin{tabular}{|c|c|}
\hline B15 & 1.08839260 \\
\hline B16 & 1.51904027 \\
\hline B17 & 1.08834640 \\
\hline B18 & 1.08534959 \\
\hline B19 & 1.08818908 \\
\hline B20 & 2.32315013 \\
\hline B21 & 1.41470646 \\
\hline B22 & 1.41689474 \\
\hline B23 & 1.42250691 \\
\hline B24 & 1.42151250 \\
\hline B25 & 1.51730254 \\
\hline B26 & 1.08618309 \\
\hline B27 & 1.08777258 \\
\hline B28 & 1.08781546 \\
\hline B29 & 1.51625901 \\
\hline B30 & 1.08603262 \\
\hline B31 & 1.08785495 \\
\hline B32 & 1.08765782 \\
\hline B33 & 1.41235739 \\
\hline B34 & 1.41366128 \\
\hline B35 & 0.96600246 \\
\hline A1 & 109.31070787 \\
\hline $\mathrm{A} 2$ & 109.19329888 \\
\hline $\mathrm{A} 3$ & 110.04835237 \\
\hline A4 & 111.20121293 \\
\hline A5 & 108.29445314 \\
\hline A6 & 109.15584829 \\
\hline A7 & 111.06388448 \\
\hline A8 & 112.00627143 \\
\hline A9 & 96.23289555 \\
\hline A 10 & 125.46241953 \\
\hline A11 & 114.35410162 \\
\hline A12 & 111.80073692 \\
\hline A13 & 108.73427053 \\
\hline A14 & 108.65061308 \\
\hline A 15 & 104.48113588 \\
\hline A16 & 108.86442221 \\
\hline A 17 & 110.89032831 \\
\hline A18 & 109.34041552 \\
\hline A19 & 96.31671829 \\
\hline A20 & 34.91082502 \\
\hline A21 & 113.15908449 \\
\hline A22 & 113.02560960 \\
\hline A23 & 108.92241075 \\
\hline
\end{tabular}




\begin{tabular}{|c|c|}
\hline A24 & 103.84240866 \\
\hline A 25 & 111.12419147 \\
\hline A26 & 108.93954855 \\
\hline A27 & 108.99134610 \\
\hline A28 & 113.06219167 \\
\hline A29 & 110.81806484 \\
\hline $\mathrm{A} 30$ & 109.32332160 \\
\hline A31 & 108.76555322 \\
\hline A 32 & 109.95160318 \\
\hline A33 & 110.12981216 \\
\hline A34 & 102.74891137 \\
\hline D1 & 118.91213093 \\
\hline D2 & 120.28062245 \\
\hline D3 & 66.06279510 \\
\hline D4 & -58.83208850 \\
\hline D5 & 58.33177204 \\
\hline D6 & -179.61039020 \\
\hline D7 & 116.19334067 \\
\hline D8 & -5.85427545 \\
\hline D9 & 78.00356633 \\
\hline $\mathrm{D} 10$ & 63.04843105 \\
\hline D11 & -76.47332812 \\
\hline D12 & 44.37250652 \\
\hline D13 & 163.16909214 \\
\hline D14 & -175.59711866 \\
\hline D15 & -70.65266744 \\
\hline D16 & 49.31558708 \\
\hline D17 & 169.88432471 \\
\hline D18 & -87.18300858 \\
\hline D19 & 118.25440408 \\
\hline D20 & -50.39515208 \\
\hline D21 & 92.89896189 \\
\hline $\mathrm{D} 22$ & 129.05692073 \\
\hline D23 & 161.17321314 \\
\hline D24 & 45.65789719 \\
\hline $\mathrm{D} 25$ & 165.62952361 \\
\hline D26 & -74.72846648 \\
\hline D27 & 38.41166245 \\
\hline D28 & -73.44328505 \\
\hline D29 & 46.80938158 \\
\hline D30 & 166.39464136 \\
\hline D31 & -77.73656278 \\
\hline D32 & -72.42633099 \\
\hline D33 & 81.07444508 \\
\hline
\end{tabular}


Harmonic Vibrational Frequencies $\left(\mathrm{cm}^{-1}\right)$ :

$\begin{array}{lccccccccccc}28.23 & 42.40 & 74.24 & 115.10 & 140.50 & 148.25 & 159.71 & 189.20 & 214.41 & 217.94 & 232.82 \\ 239.34 & 241.76 & 250.21 & 266.53 & 270.41 & 286.45 & 314.55 & 321.62 & 333.60 & 339.83 \\ 360.88 & 379.77 & 388.33 & 403.39 & 415.15 & 438.58 & 447.17 & 495.32 & 527.27 & 548.18 \\ 604.55 & 615.94 & 621.38 & 643.82 & 779.34 & 821.04 & 838.52 & 890.58 & 894.69 & 933.57 \\ 950.91 & 961.47 & 966.26 & 970.35 & 975.73 & 1024.39 & 1027.03 & 1032.71 & 1053.26 & 1056.32 \\ 1065.31 & 1079.79 & 1175.96 & 1210.05 & 1233.84 & 1248.24 & 1254.56 & 1258.29 & 1275.70 \\ 1304.74 & 1320.40 & 1394.68 & 1412.88 & 1415.77 & 1417.04 & 1424.67 & 1425.16 & 1426.73 \\ 1436.89 & 1481.32 & 1481.82 & 1486.27 & 1488.95 & 1491.14 & 1494.47 & 1496.78 & 1502.57 \\ 1508.79 & 1511.91 & 1521.02 & 1559.45 & 3057.83 & 3070.33 & 3077.56 & 3079.14 & 3081.37 \\ 3081.81 & 3132.45 & 3152.83 & 3152.93 & 3157.78 & 3158.19 & 3158.86 & 3162.39 & 3165.99 \\ 3172.71 & 3179.18 & 3179.39 & 3202.62 & 3807.51 & 3810.42 & & & & \end{array}$

Rotational constants (B/GHZ): $\quad 0.8346450 \quad 0.3441726 \quad 0.3375178$

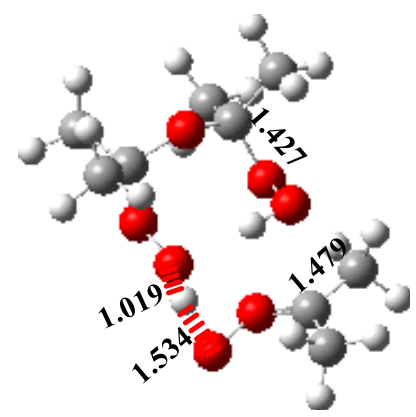

IM8c

$\mathrm{C}$

O 11

$\begin{array}{lll}\mathrm{O} & 2 & \mathrm{~B} 2\end{array}$

$\mathrm{H} \quad 2$

B3 1

A1

C 2

O 5

B4 1

B5 2

B6 2

B7 3

B8 1

B9 1

B10 1

B11 3

$\begin{array}{llll}\mathrm{A} 2 & 3 & \mathrm{D} 1 & 0\end{array}$

A3 $3 \quad$ D2 0

A4 $1 \quad$ D3 0

A5 $1 \quad$ D4 0

A6 2 D5 0

$\begin{array}{ll}\mathrm{H} & 8\end{array}$

A7 3

D6 0

H $\quad 8$

$\mathrm{H} \quad 8$

O 1

C 12

B12 1

B13 12

A8 3

D7 0

A9 $3 \quad$ D8 0

C 13

$\mathrm{H} \quad 14$

B14 13

A10 2

D9 0

A11 $3 \quad$ D10 0

A12 $1 \quad$ D11 0

$\mathrm{H} \quad 14$

B15 13

A13 12

D12 0

$\mathrm{H} \quad 14$

B16 13

A14 12

D13 0

O 13

O 18

$\mathrm{H} \quad 19$

C 5

$\mathrm{H} \quad 21$

B17 12

A15 12

D14 0

A16 $1 \quad$ D15 0

$\begin{array}{llllll}\text { B18 } & 13 & \text { A17 } & 12 & \text { D16 } & 0\end{array}$

$\begin{array}{llllll}\text { B19 } & 18 & \text { A18 } & 13 & \text { D17 } & 0\end{array}$

$\begin{array}{llllll}\mathrm{B} 20 & 2 & \mathrm{~A} 19 & 1 & \mathrm{D} 18 & 0\end{array}$

$\begin{array}{llllll}\mathrm{B} 21 & 5 & \mathrm{~A} 20 & 2 & \mathrm{D} 19 & 0\end{array}$ 


$\begin{array}{rrrrrrrr}\mathrm{H} & 21 & \text { B22 } & 5 & \text { A21 } & 2 & \text { D20 } & 0 \\ \mathrm{H} & 21 & \text { B23 } & 5 & \text { A22 } & 2 & \text { D21 } & 0 \\ \mathrm{C} & 5 & \text { B24 } & 2 & \text { A23 } & 1 & \text { D22 } & 0 \\ \mathrm{H} & 25 & \text { B25 } & 5 & \text { A24 } & 2 & \text { D23 } & 0 \\ \mathrm{H} & 25 & \text { B26 } & 5 & \text { A25 } & 2 & \text { D24 } & 0 \\ \mathrm{H} & 25 & \text { B27 } & 5 & \text { A26 } & 2 & \text { D25 } & 0 \\ \mathrm{C} & 1 & \text { B28 } & 12 & \text { A27 } & 13 & \text { D26 } & 0 \\ \mathrm{H} & 29 & \text { B29 } & 1 & \text { A28 } & 12 & \text { D27 } & 0 \\ \mathrm{H} & 29 & \text { B30 } & 1 & \text { A29 } & 12 & \text { D28 } & 0 \\ \mathrm{H} & 29 & \text { B31 } & 1 & \text { A30 } & 12 & \text { D29 } & 0 \\ \mathrm{C} & 13 & \text { B32 } & 12 & \text { A31 } & 1 & \text { D30 } & 0 \\ \mathrm{H} & 33 & \text { B33 } & 13 & \text { A32 } & 12 & \text { D31 } & 0 \\ \mathrm{H} & 33 & \text { B34 } & 13 & \text { A33 } & 12 & \text { D32 } & 0 \\ \mathrm{H} & 33 & \text { B35 } & 13 & \text { A34 } & 12 & \text { D33 } & 0\end{array}$

Variables:

$\begin{array}{ll}\text { B1 } & 2.32959602 \\ \text { B2 } & 1.41521699 \\ \text { B3 } & 0.97918966 \\ \text { B4 } & 2.83161492 \\ \text { B5 } & 2.24648820 \\ \text { B6 } & 1.24849208 \\ \text { B7 } & 1.52074551 \\ \text { B8 } & 1.08852128 \\ \text { B9 } & 1.08700022 \\ \text { B10 } & 1.08790118 \\ \text { B11 } & 1.40210153 \\ \text { B12 } & 1.42408078 \\ \text { B13 } & 1.52050410 \\ \text { B14 } & 1.08855169 \\ \text { B15 } & 1.08821171 \\ \text { B16 } & 1.08541224 \\ \text { B17 } & 1.39871734 \\ \text { B18 } & 1.42701766 \\ \text { B19 } & 1.01878173 \\ \text { B20 } & 1.47934639 \\ \text { B21 } & 1.08509235 \\ \text { B22 } & 1.09033112 \\ \text { B23 } & 1.09160887 \\ \text { B24 } & 1.47542341 \\ \text { B25 } & 1.08914942 \\ \text { B26 } & 1.09236565 \\ \text { B27 } & 1.08508648 \\ \text { B28 } & 1.51835671 \\ \text { B29 } & 1.08858772\end{array}$




$\begin{array}{lr}\text { B30 } & 1.08847610 \\ \text { B31 } & 1.08673219 \\ \text { B32 } & 1.52541985 \\ \text { B33 } & 1.08845739 \\ \text { B34 } & 1.08522394 \\ \text { B35 } & 1.08804755 \\ \text { A1 } & 35.12038183 \\ \text { A2 } & 92.64429255 \\ \text { A3 } & 114.33643778 \\ \text { A4 } & 96.75800277 \\ \text { A5 } & 98.39289513 \\ \text { A6 } & 102.12785376 \\ \text { A7 } & 108.73581122 \\ \text { A8 } & 110.64113542 \\ \text { A9 } & 109.94217777 \\ \text { A10 } & 111.12502653 \\ \text { A11 } & 124.75520708 \\ \text { A12 } & 104.14800820 \\ \text { A13 } & 109.16600840 \\ \text { A14 } & 109.31121697 \\ \text { A15 } & 110.44313103 \\ \text { A16 } & 112.25533008 \\ \text { A17 } & 108.75730292 \\ \text { A18 } & 100.64023145 \\ \text { A19 } & 86.97922862 \\ \text { A20 } & 110.89962272 \\ \text { A21 } & 107.75270351 \\ \text { A22 } & 108.96305891 \\ \text { A23 } & 87.01408433 \\ \text { A24 } & 109.15975057 \\ \text { A25 } & 108.15046917 \\ \text { A26 } & 110.75813024 \\ \text { A27 } & 104.74542722 \\ \text { A28 } & 109.77801424 \\ \text { A29 } & 109.59962925 \\ & 109.54931457 \\ \text { A30 } & 13.36892692 \\ \text { A3 } & \end{array}$




$\begin{array}{lr}\text { D5 } & -178.48203379 \\ \text { D6 } & -66.68401384 \\ \text { D7 } & 52.53795174 \\ \text { D8 } & 174.37025970 \\ \text { D9 } & -53.79149656 \\ \text { D10 } & -70.46274827 \\ \text { D11 } & 165.08507380 \\ \text { D12 } & 67.96901279 \\ \text { D13 } & -172.19531797 \\ \text { D14 } & -51.96852848 \\ \text { D15 } & 43.18470503 \\ \text { D16 } & 69.05323071 \\ \text { D17 } & -171.01351873 \\ \text { D18 } & 73.36305119 \\ \text { D19 } & -96.01937674 \\ \text { D20 } & 24.36911091 \\ \text { D21 } & 141.70988394 \\ \text { D22 } & -163.37316201 \\ \text { D23 } & 42.89642844 \\ \text { D24 } & 157.73219746 \\ \text { D25 } & -79.73404355 \\ \text { D26 } & 170.23807743 \\ \text { D27 } & 174.15987847 \\ \text { D28 } & -65.65525212 \\ \text { D29 } & 53.92295213 \\ \text { D30 } & -74.73515991 \\ \text { D31 } & -41.25640360 \\ \text { D32 } & 80.21041861 \\ \text { D33 } & -160.05861183 \\ \text { Dar }\end{array}$

Harmonic Vibrational Frequencies $\left(\mathrm{cm}^{-1}\right)$ :

$\begin{array}{lccccccccccc}32.34 & 42.10 & 60.68 & 93.21 & 103.23 & 127.24 & 147.04 & 175.73 & 184.94 & 197.03 & 215.97 \\ 230.55 & 239.86 & 246.66 & 266.32 & 271.71 & 282.08 & 302.77 & 311.46 & 331.79 & 341.77 \\ 350.69 & 364.49 & 379.98 & 386.96 & 410.98 & 428.08 & 471.94 & 491.51 & 507.00 & 585.94 \\ 603.30 & 609.06 & 649.25 & 736.61 & 779.44 & 826.97 & 837.17 & 887.92 & 919.13 & 923.16 \\ 942.15 & 948.28 & 960.14 & 964.61 & 994.64 & 1015.64 & 1025.04 & 1056.21 & 1065.03 & 1072.16 \\ 1087.69 & 1104.81 & 1116.46 & 1181.62 & 1226.69 & 1259.13 & 1263.17 & 1265.48 & 1312.39 \\ 1320.58 & 1396.06 & 1403.19 & 1409.26 & 1414.50 & 1415.25 & 1419.91 & 1452.01 & 1468.82 \\ 1471.57 & 1474.87 & 1479.82 & 1485.79 & 1489.35 & 1492.12 & 1495.00 & 1501.13 & 1511.39 \\ 1527.01 & 1532.55 & 1584.09 & 1671.59 & 2771.51 & 3058.53 & 3063.41 & 3068.00 & 3071.74 \\ 3072.94 & 3079.92 & 3134.59 & 3136.55 & 3143.21 & 3148.85 & 3149.89 & 3155.07 & 3161.78 \\ 3170.22 & 3174.60 & 3181.46 & 3182.48 & 3182.84 & 3555.21 & & & \\ \text { Rotational constants (B/GHZ): } & 0.7505620 & 0.3992725 & 0.3239009 & \end{array}$




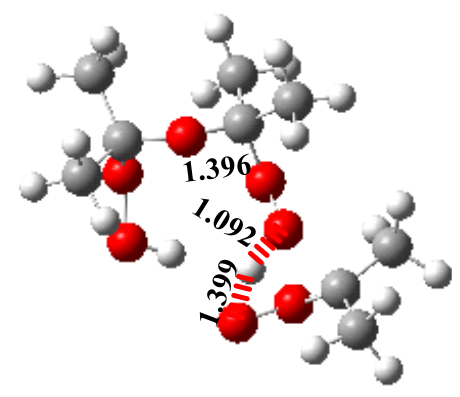

TS8c

C

O 1

O 2

H 2

C 2

O 5

O 5

C 1

$\begin{array}{ll}\mathrm{H} & 8\end{array}$

$\begin{array}{ll}\mathrm{H} & 8\end{array}$

$\begin{array}{ll}\mathrm{H} & 8\end{array}$

O 1

C 12

C 13

$\mathrm{H} \quad 14$

$\mathrm{H} \quad 14$

H 14

O 13

O 18

H 19

C 5

H 21

$\mathrm{H} \quad 21$

$\mathrm{H} \quad 21$

C 5

H 25

H 25

H 25

C 1

H 29

H 29

H 29

C 13

$\mathrm{H} \quad 33$

$\mathrm{H}$
B1

B2 1

B3 1

B4 1

B5 2

B6 2

B7 3

B8 1

B9 1

B10 1

B11 3

B12 1

B13 12

B14 13

B15 13

B16 13

B17 12

B18 13

B19 18

B20 2

B21 5

B22 5

B23 5

B24 2

B25 5

B26 5

B27 5

B28 12

B29 1

B30 1

B31 1

B32 12

B33 13

B34 13
A1

$\begin{array}{llll}\text { A2 } & 3 & \text { D1 } & 0\end{array}$

A3 $3 \quad$ D2 0

A4 $1 \quad$ D3 0

A5 $1 \quad$ D4 0

A6 2 D5 0

A7 $3 \quad$ D6 0

A8 $3 \quad$ D7 0

$\begin{array}{llll}\text { A9 } & 3 & \text { D8 } & 0\end{array}$

$\begin{array}{llll}\text { A10 } 2 & \text { D9 } & 0\end{array}$

$\begin{array}{llll}\text { A11 } & 3 & \text { D10 } & 0\end{array}$

A12 $1 \quad$ D11 0

$\begin{array}{llll}\text { A13 } & 12 & \text { D12 } & 0\end{array}$

A14 $12 \quad$ D13 0

$\begin{array}{llll}\text { A15 } 12 & \text { D14 }\end{array}$

A16 $1 \quad$ D15 0

A17 $12 \quad$ D16 0

A18 $13 \quad$ D17 0

A19 $1 \quad$ D18 0

$\begin{array}{llll}\text { A20 } 2 & \text { D19 } & 0\end{array}$

A21 $2 \quad$ D20 0

$\begin{array}{llll}\mathrm{A} 22 & 2 & \mathrm{D} 21 & 0\end{array}$

A23 $1 \quad$ D22 0

A24 2 D23 0

A25 2 D24 0

A26 2 D25 0

A27 $13 \quad$ D26 0

$\begin{array}{llll}\text { A28 } & 12 & \text { D27 } & 0\end{array}$

$\begin{array}{llll}\text { A29 } & 12 & \text { D28 } & 0\end{array}$

$\begin{array}{llll}\text { A30 } & 12 & \text { D29 } & 0\end{array}$

A31 $1 \quad$ D30 0

A32 $12 \quad$ D31 0

$\begin{array}{llll}\text { A33 } & 12 & \text { D32 } & 0\end{array}$ 


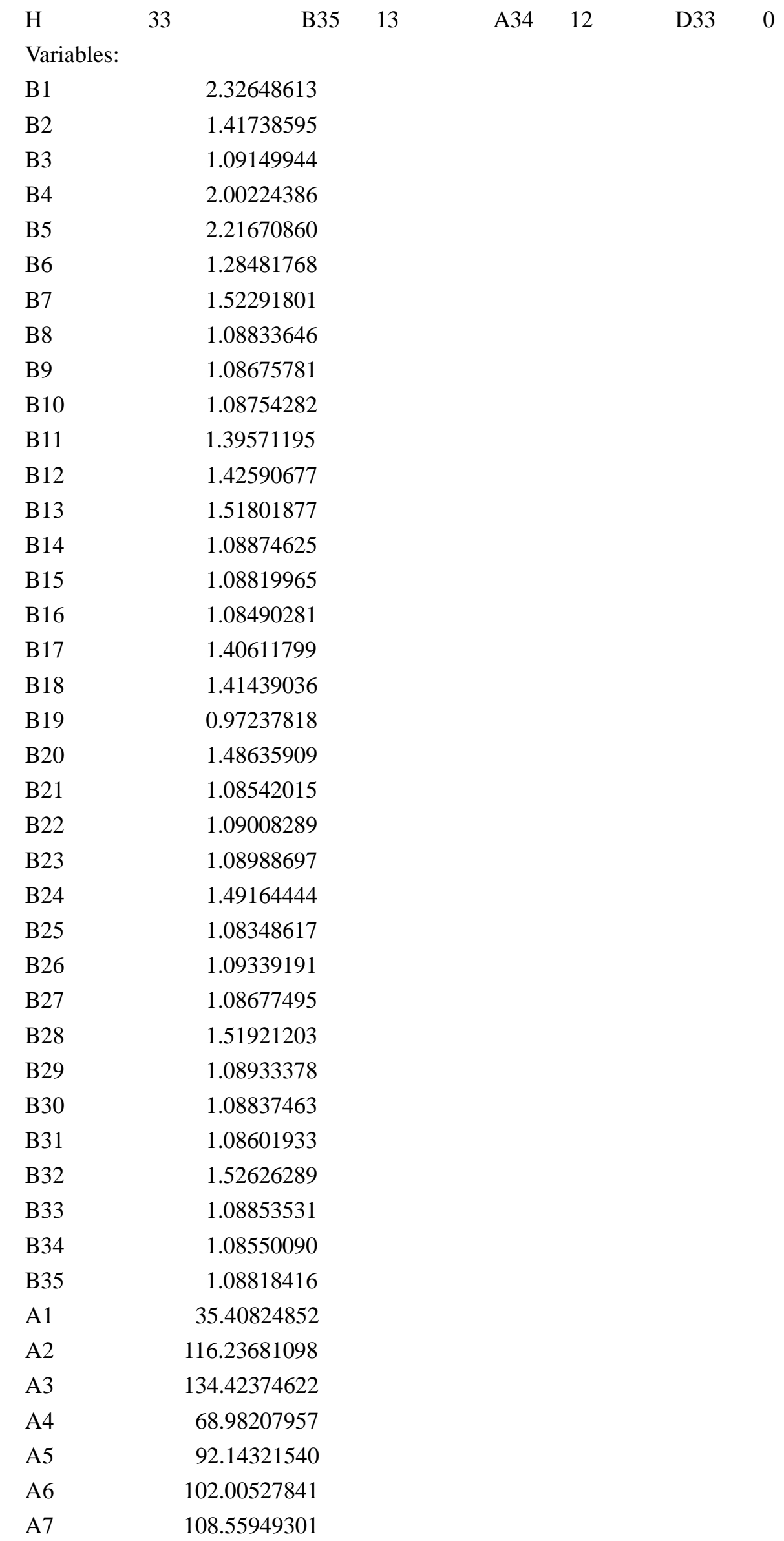




\begin{tabular}{|c|c|}
\hline A8 & 111.06909875 \\
\hline A9 & 109.61160946 \\
\hline A 10 & 110.60903860 \\
\hline A11 & 123.99810037 \\
\hline A12 & 104.31702037 \\
\hline A13 & 108.91997757 \\
\hline A14 & 109.46316248 \\
\hline A15 & 110.24283053 \\
\hline A16 & 112.81261124 \\
\hline A 17 & 110.49838473 \\
\hline A18 & 104.44915380 \\
\hline A19 & 106.79011332 \\
\hline A20 & 110.59972278 \\
\hline A 21 & 107.62521746 \\
\hline A22 & 109.89545465 \\
\hline $\mathrm{A} 23$ & 92.33306011 \\
\hline A24 & 111.96457236 \\
\hline A25 & 106.34782307 \\
\hline A26 & 109.78760126 \\
\hline A27 & 105.43061293 \\
\hline A28 & 110.03687375 \\
\hline A29 & 109.08892875 \\
\hline A 30 & 109.98932895 \\
\hline A31 & 112.54676471 \\
\hline A 32 & 109.24082564 \\
\hline A33 & 112.08555237 \\
\hline A34 & 108.93152600 \\
\hline D1 & -69.86389231 \\
\hline D2 & 27.31618746 \\
\hline D3 & -107.29352319 \\
\hline D4 & -77.64410753 \\
\hline D5 & 170.37493596 \\
\hline D6 & -63.75450038 \\
\hline D7 & 56.00408926 \\
\hline D8 & 177.72018241 \\
\hline D9 & -64.71343679 \\
\hline D10 & -78.89181326 \\
\hline D11 & 162.55443712 \\
\hline D12 & 67.61432415 \\
\hline D13 & -172.65661426 \\
\hline D14 & -52.39603285 \\
\hline D15 & 39.99070599 \\
\hline D16 & 77.51162135 \\
\hline D17 & -109.19827224 \\
\hline
\end{tabular}




$\begin{array}{lr}\text { D18 } & 38.48480249 \\ \text { D19 } & -90.82503641 \\ \text { D20 } & 28.70778614 \\ \text { D21 } & 146.41503014 \\ \text { D22 } & 159.96138145 \\ \text { D23 } & 57.73554553 \\ \text { D24 } & 176.61333723 \\ \text { D25 } & -66.03211678 \\ \text { D26 } & 161.47634526 \\ \text { D27 } & 171.49817215 \\ \text { D28 } & -68.83103721 \\ \text { D29 } & 50.59971499 \\ \text { D30 } & -77.45406524 \\ \text { D31 } & -40.28043677 \\ \text { D32 } & 81.37142968 \\ \text { D33 } & -159.29221066\end{array}$

Harmonic Vibrational Frequencies $\left(\mathbf{c m}^{-1}\right)$ :

$\begin{array}{lccccccccccc}478.38 i & 35.08 & 50.29 & 69.75 & 100.91 & 106.67 & 132.65 & 155.98 & 171.92 & 192.13 & 193.49 \\ 212.36 & 222.87 & 236.58 & 246.70 & 264.24 & 301.98 & 322.38 & 326.03 & 338.89 & 345.40 \\ 352.20 & 380.14 & 390.67 & 402.76 & 415.80 & 439.83 & 477.31 & 495.17 & 548.93 & 572.35 \\ 582.39 & 610.01 & 633.00 & 652.94 & 776.21 & 819.62 & 836.71 & 890.10 & 926.59 & 943.66 \\ 951.07 & 959.39 & 962.19 & 968.01 & 989.04 & 1021.84 & 1027.29 & 1039.86 & 1067.49 & 1078.19 \\ 1082.69 & 1088.41 & 1170.35 & 1218.55 & 1246.65 & 1254.81 & 1268.76 & 1272.09 & 1314.22 \\ 1317.46 & 1401.36 & 1405.22 & 1410.99 & 1414.02 & 1418.08 & 1422.57 & 1427.37 & 1453.42 \\ 1462.05 & 1471.43 & 1478.06 & 1480.59 & 1488.41 & 1492.88 & 1496.46 & 1499.31 & 1502.87 \\ 1516.46 & 1531.51 & 1583.27 & 1632.26 & 2013.29 & 3063.81 & 3066.39 & 3070.11 & 3071.67 \\ 3079.88 & 3082.92 & 3142.02 & 3142.08 & 3145.65 & 3155.26 & 3156.75 & 3159.64 & 3171.13 \\ 3176.75 & 3179.48 & 3182.41 & 3182.53 & 3213.22 & 3687.43 & & & & \\ \text { Rotational constants } & (\mathrm{B} / \mathrm{GHZ}) & 0.8590051 & 0.3646711 & 0.3079698 & \end{array}$

$\begin{array}{llll}\text { Rotational constants (B/GHZ): } & 0.8590051 & 0.3646711 & 0.3079698\end{array}$

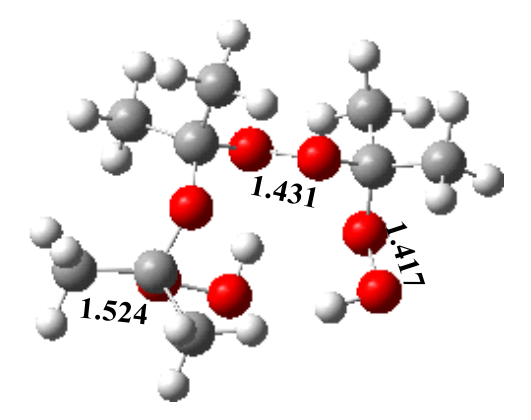

P8c

$\mathrm{C}$

$\begin{array}{lll}\mathrm{O} & 1 & \mathrm{~B} 1\end{array}$

$\begin{array}{lllll}\mathrm{O} & 1 & \mathrm{~B} 2 & 2 & \mathrm{~A} 1\end{array}$

$\begin{array}{llllllll}\mathrm{H} & 2 & \mathrm{~B} 3 & 1 & \mathrm{~A} 2 & 3 & \mathrm{D} 1 & 0\end{array}$

$\begin{array}{lllllllll}\mathrm{C} & 2 & \mathrm{~B} 4 & 1 & \mathrm{~A} 3 & 3 & \mathrm{D} 2 & 0\end{array}$

$\begin{array}{llllllll}\mathrm{O} & 5 & \mathrm{~B} 5 & 2 & \mathrm{~A} 4 & 1 & \mathrm{D} 3 & 0\end{array}$ 


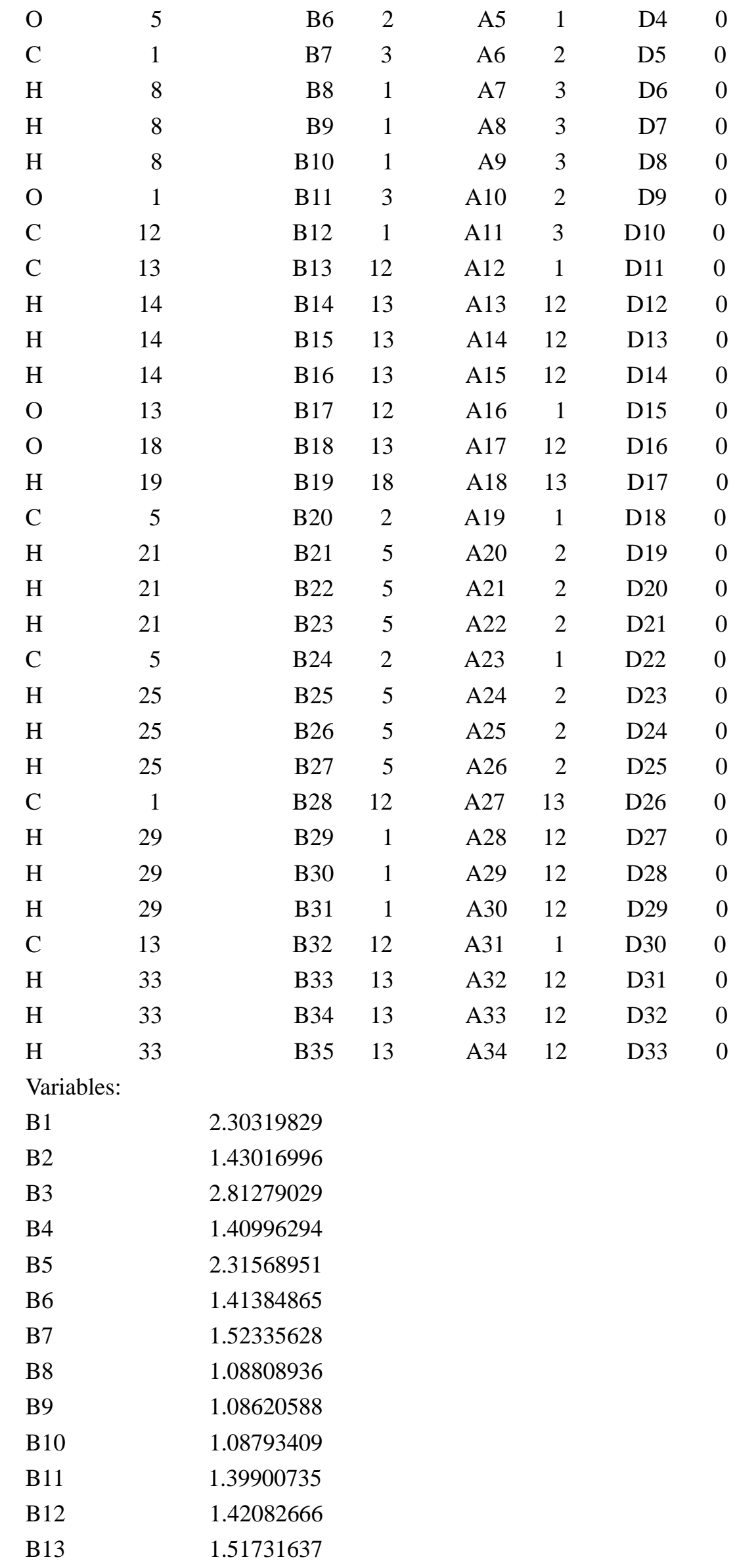




\begin{tabular}{|c|c|}
\hline B14 & 1.08830565 \\
\hline B15 & 1.08819527 \\
\hline B16 & 1.08628516 \\
\hline B17 & 1.41012666 \\
\hline B18 & 1.41206010 \\
\hline B19 & 0.97203683 \\
\hline B20 & 1.51757769 \\
\hline B21 & 1.08658657 \\
\hline B22 & 1.08813251 \\
\hline B23 & 1.08750954 \\
\hline B24 & 1.51682159 \\
\hline B 25 & 1.08590770 \\
\hline B26 & 1.08807909 \\
\hline $\mathrm{B} 27$ & 1.08773403 \\
\hline B28 & 1.51970178 \\
\hline B29 & 1.08865867 \\
\hline B30 & 1.08825301 \\
\hline B31 & 1.08538101 \\
\hline B32 & 1.52362465 \\
\hline B33 & 1.08797254 \\
\hline B34 & 1.08638996 \\
\hline B35 & 1.08815429 \\
\hline $\mathrm{A} 1$ & 36.41996293 \\
\hline $\mathrm{A} 2$ & 102.95257338 \\
\hline A3 & 144.84159203 \\
\hline A4 & 94.44126889 \\
\hline A5 & 110.32711463 \\
\hline A6 & 103.02870718 \\
\hline A7 & 108.56713690 \\
\hline A8 & 111.76633513 \\
\hline A9 & 109.03065010 \\
\hline A 10 & 110.28797298 \\
\hline A11 & 124.60580302 \\
\hline A 12 & 104.97669550 \\
\hline A13 & 109.13674842 \\
\hline A14 & 109.73420725 \\
\hline A15 & 110.36514113 \\
\hline A 16 & 113.32577684 \\
\hline A17 & 111.67793699 \\
\hline A 18 & 101.82424692 \\
\hline A19 & 112.99879674 \\
\hline A20 & 110.98836055 \\
\hline A21 & 108.94098911 \\
\hline A22 & 109.22400990 \\
\hline
\end{tabular}




\begin{tabular}{|c|c|}
\hline A23 & 103.91629696 \\
\hline A24 & 110.50348967 \\
\hline A25 & 108.69729456 \\
\hline A26 & 109.52359046 \\
\hline A27 & 105.40598437 \\
\hline A28 & 109.76714375 \\
\hline A29 & 108.90521843 \\
\hline A30 & 110.23752723 \\
\hline A31 & 111.77228323 \\
\hline A32 & 108.73407221 \\
\hline A33 & 111.45978398 \\
\hline A34 & 109.58031539 \\
\hline D1 & -80.78165207 \\
\hline D2 & -2.28290853 \\
\hline D3 & -99.03902231 \\
\hline D4 & -66.80732091 \\
\hline D5 & 170.02450629 \\
\hline D6 & -63.94056787 \\
\hline D7 & 56.55198250 \\
\hline D8 & 177.97645021 \\
\hline D9 & -64.94226943 \\
\hline D10 & -76.97954870 \\
\hline D11 & 153.66954162 \\
\hline D12 & 66.95481769 \\
\hline D13 & -173.11094312 \\
\hline D14 & -52.02354573 \\
\hline D15 & 30.77408800 \\
\hline D16 & 69.54687540 \\
\hline D17 & -96.47154429 \\
\hline D18 & 49.39978250 \\
\hline D19 & -70.97400319 \\
\hline D20 & 49.13337442 \\
\hline D21 & 168.67447484 \\
\hline D22 & 172.42559678 \\
\hline D23 & 48.27617800 \\
\hline D24 & 167.84055732 \\
\hline D25 & -72.51992023 \\
\hline D26 & 163.60416377 \\
\hline D27 & 168.43148043 \\
\hline D28 & -71.85417564 \\
\hline D29 & 47.75073479 \\
\hline D30 & -85.41415721 \\
\hline D31 & -46.41190321 \\
\hline D32 & 74.26939422 \\
\hline
\end{tabular}


D33

$-165.80946040$

Harmonic Vibrational Frequencies $\left(\mathrm{cm}^{-\mathbf{1}}\right)$ :

$\begin{array}{lllllllllll}38.59 & 54.32 & 101.03 & 130.44 & 139.83 & 169.26 & 179.48 & 198.40 & 210.10 & 213.68 & 219.31\end{array}$ $\begin{array}{llllllllll}227.22 & 239.93 & 256.20 & 277.59 & 285.90 & 293.36 & 321.93 & 326.99 & 347.61 & 358.65\end{array}$

$\begin{array}{lllllllllll}373.31 & 378.54 & 399.50 & 405.87 & 428.18 & 436.63 & 490.90 & 507.15 & 567.19 & 589.36\end{array}$

$\begin{array}{llllllllll}600.77 & 608.55 & 641.87 & 666.48 & 779.32 & 827.24 & 854.30 & 895.73 & 918.71 & 928.70\end{array}$

$\begin{array}{llllllllll}944.83 & 955.49 & 961.04 & 967.43 & 978.94 & 1020.25 & 1025.60 & 1034.67 & 1050.19 & 1065.25\end{array}$

$\begin{array}{lllllllll}1073.20 & 1079.78 & 1175.81 & 1219.47 & 1225.48 & 1251.45 & 1254.66 & 1266.45 & 1271.21\end{array}$

$\begin{array}{lllllllll}1304.14 & 1325.58 & 1408.07 & 1410.39 & 1415.26 & 1419.84 & 1421.79 & 1424.76 & 1463.65\end{array}$

$\begin{array}{llllllllll}1479.93 & 1481.54 & 1481.77 & 1485.45 & 1489.67 & 1493.99 & 1497.67 & 1501.30 & 1501.64\end{array}$

$\begin{array}{lllllllll}1511.45 & 1515.27 & 1522.05 & 1526.85 & 3072.98 & 3074.53 & 3077.98 & 3078.42 & 3080.28\end{array}$

$\begin{array}{lllllllll}3086.32 & 3148.32 & 3152.54 & 3154.58 & 3157.90 & 3159.15 & 3164.37 & 3170.69 & 3170.75\end{array}$

$\begin{array}{llllll}3170.91 & 3175.64 & 3181.59 & 3188.68 & 3710.21 & 3742.96\end{array}$

$\begin{array}{llll}\text { Rotational constants (B/GHZ): } & 0.8088231 & 0.4164583 & 0.3411174\end{array}$

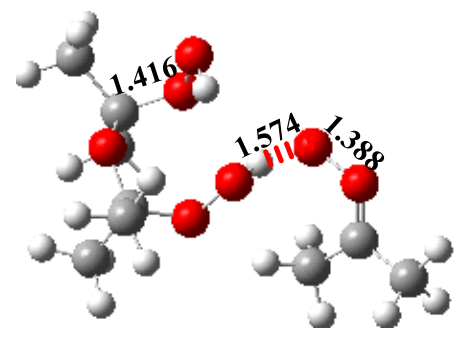

IM8d

$\mathrm{C}$

C $\quad 1 \quad$ B1

$\begin{array}{lllll}\mathrm{O} & 2 & \mathrm{~B} 2 & 1 & \mathrm{~A} 1\end{array}$

$\begin{array}{lllll}\mathrm{O} & 2 & \mathrm{~B} 3 & 1 & \mathrm{~A} 2\end{array}$

$\mathrm{H} \quad 3$

C 3

B4 2

A2 3

D1 0

B5 2

A3 1

D2 0

C 6

B6 3

A4 1

D3 0

$\begin{array}{ll}\mathrm{H} & 7\end{array}$

$\begin{array}{ll}\mathrm{H} & 7\end{array}$

B7 6

A5 2

D4 0

A6 3

D5 0

$\begin{array}{llll}\text { B8 } & 6 & \text { A7 } & 3\end{array}$

D6 0

$\begin{array}{llll}\text { B9 } & 6 & \text { A8 } & 3\end{array}$

D7 0

$\begin{array}{llllll}\mathrm{B} 10 & 3 & \mathrm{~A} 9 & 2 & \mathrm{D} 8 & 0\end{array}$

O 6

O 6

B11 3

A10 2

D9 0

B12 2 A11 4

D10 0

$\begin{array}{llll}\mathrm{B} 13 & 2 & \mathrm{~A} 12 & 4\end{array}$

D11 0

$\begin{array}{llll}\text { B14 } 2 & \text { A13 } 4\end{array}$

D12 0

B15 1

A14 4

D13 0

$\begin{array}{llll}\mathrm{B} 16 & 2 & \mathrm{~A} 15 & 1\end{array}$

D14 0

$\begin{array}{llllll}\text { B17 } & 16 & \text { A16 } & 2 & \text { D15 } & 0\end{array}$

C $\quad 17$

$\mathrm{H} \quad 18$

B18 17

A17 16

D16 0

B19 17

A18 16

D17 0

$\mathrm{H} \quad 18$

B20 17

A19 16

D18 0

O 17

$\begin{array}{llll}\mathrm{B} 21 & 16 & \mathrm{~A} 20 & 2\end{array}$

D19 0 


$\begin{array}{rrrrrrrr}\mathrm{O} & 22 & \text { B22 } & 17 & \text { A21 } & 16 & \text { D20 } & 0 \\ \mathrm{H} & 23 & \text { B23 } & 22 & \text { A22 } & 17 & \text { D21 } & 0 \\ \mathrm{C} & 6 & \text { B24 } & 3 & \text { A23 } & 2 & \text { D22 } & 0 \\ \mathrm{H} & 25 & \text { B25 } & 6 & \text { A24 } & 3 & \text { D23 } & 0 \\ \mathrm{H} & 25 & \text { B26 } & 6 & \text { A25 } & 3 & \text { D24 } & 0 \\ \mathrm{H} & 25 & \text { B27 } & 6 & \text { A26 } & 3 & \text { D25 } & 0 \\ \mathrm{C} & 2 & \text { B28 } & 1 & \text { A27 } & 16 & \text { D26 } & 0 \\ \mathrm{H} & 29 & \text { B29 } & 2 & \text { A28 } & 1 & \text { D27 } & 0 \\ \mathrm{H} & 29 & \text { B30 } & 2 & \text { A29 } & 1 & \text { D28 } & 0 \\ \mathrm{H} & 29 & \text { B31 } & 2 & \text { A30 } & 1 & \text { D29 } & 0 \\ \mathrm{C} & 17 & \text { B32 } & 16 & \text { A31 } & 2 & \text { D30 } & 0 \\ \mathrm{H} & 33 & \text { B33 } & 17 & \text { A32 } & 16 & \text { D31 } & 0 \\ \mathrm{H} & 33 & \text { B34 } & 17 & \text { A33 } & 16 & \text { D32 } & 0 \\ \text { H } & 33 & \text { B35 } & 17 & \text { A34 } & 16 & \text { D33 } & 0\end{array}$

Variables:

$\begin{array}{ll}\text { B1 } & 1.51984898 \\ \text { B2 } & 2.32251789 \\ \text { B3 } & 1.40885104 \\ \text { B4 } & 1.01291570 \\ \text { B5 } & 3.30687211 \\ \text { B6 } & 1.47169044 \\ \text { B7 } & 1.09491584 \\ \text { B8 } & 1.09120846 \\ \text { B9 } & 1.08568259 \\ \text { B10 } & 2.24680336 \\ \text { B11 } & 1.24878598 \\ \text { B12 } & 1.08849419 \\ \text { B13 } & 1.08852340 \\ \text { B14 } & 1.08584610 \\ \text { B15 } & 1.41190883 \\ \text { B16 } & 1.41186982 \\ \text { B17 } & 1.51833228 \\ \text { B18 } & 1.08619233 \\ \text { B19 } & 1.08830398 \\ \text { B20 } & 1.08866098 \\ \text { B21 } & 1.41657481 \\ \text { B22 } & 1.41201179 \\ \text { B23 } & 0.97004727 \\ \text { B24 } & 1.48313122 \\ \text { B25 } & 1.08557117 \\ \text { B26 } & 1.09118164 \\ \text { B27 } & 1.09101968 \\ \text { B28 } & 1.52605369 \\ \text { B29 } & 1.08842258\end{array}$




\begin{tabular}{|c|c|}
\hline B30 & 1.08829181 \\
\hline B31 & 1.08597430 \\
\hline B32 & 1.52066603 \\
\hline B33 & 1.08722113 \\
\hline B34 & 1.08824112 \\
\hline B35 & 1.08817121 \\
\hline A1 & 87.90666797 \\
\hline A2 & 111.63128225 \\
\hline A3 & 122.76156981 \\
\hline A4 & 129.62392146 \\
\hline A5 & 81.55254121 \\
\hline A6 & 108.60735459 \\
\hline A7 & 108.43911686 \\
\hline A8 & 110.94104942 \\
\hline A9 & 50.76741577 \\
\hline A10 & 63.25357400 \\
\hline A11 & 109.18757296 \\
\hline A12 & 109.76137003 \\
\hline A13 & 110.08198036 \\
\hline A14 & 104.74261964 \\
\hline A15 & 124.56977020 \\
\hline A16 & 104.65042700 \\
\hline A17 & 109.66251693 \\
\hline A18 & 109.53930892 \\
\hline A19 & 109.60472886 \\
\hline A20 & 110.61033599 \\
\hline A21 & 109.55052958 \\
\hline A22 & 100.54290519 \\
\hline A23 & 126.63403186 \\
\hline A24 & 111.05580507 \\
\hline A25 & 108.73504170 \\
\hline A26 & 109.27656864 \\
\hline A27 & 110.87977700 \\
\hline A28 & 109.63755822 \\
\hline A29 & 108.65144535 \\
\hline $\mathrm{A} 30$ & 111.80642836 \\
\hline A31 & 115.89066989 \\
\hline A32 & 110.74533153 \\
\hline A33 & 109.91572777 \\
\hline A34 & 108.46749000 \\
\hline D1 & 26.42195236 \\
\hline D2 & -168.65283426 \\
\hline D3 & 126.91787508 \\
\hline D4 & 13.97752610 \\
\hline
\end{tabular}




$\begin{array}{lr}\text { D5 } & -14.78421350 \\ \text { D6 } & 99.50616049 \\ \text { D7 } & -136.72366914 \\ \text { D8 } & 107.54944821 \\ \text { D9 } & 144.95650423 \\ \text { D10 } & -172.65092240 \\ \text { D11 } & -52.67438920 \\ \text { D12 } & 67.77541991 \\ \text { D13 } & -122.87495284 \\ \text { D14 } & 158.79632276 \\ \text { D15 } & 167.39626377 \\ \text { D16 } & 53.61873783 \\ \text { D17 } & 173.53388299 \\ \text { D18 } & -66.25353307 \\ \text { D19 } & -72.84152723 \\ \text { D20 } & -57.29042661 \\ \text { D21 } & 86.63242941 \\ \text { D22 } & -111.38402857 \\ \text { D23 } & -73.77387395 \\ \text { D24 } & 47.54824886 \\ \text { D25 } & 164.34880135 \\ \text { D26 } & -121.88457859 \\ \text { D27 } & -46.74695441 \\ \text { D28 } & 72.28374133 \\ \text { D29 } & -167.18240153 \\ \text { D30 } & 43.72757243 \\ \text { D31 } & -66.47698646 \\ \text { D32 } & 55.55772208 \\ \text { D33 } & 174.51004891\end{array}$

Harmonic Vibrational Frequencies $\left(\mathrm{cm}^{-1}\right)$ :

\begin{tabular}{lccccccccccc}
22.20 & 35.66 & 47.02 & 58.15 & 91.07 & 105.46 & 131.96 & 141.53 & 172.94 & 182.26 & 190.41 \\
205.43 & 218.26 & 234.77 & 244.19 & 258.06 & 290.70 & 297.74 & 317.10 & 336.12 & 338.69 \\
345.91 & 372.46 & 380.02 & 385.73 & 394.05 & 415.54 & 459.88 & 490.90 & 503.29 & 524.01 \\
586.53 & 603.57 & 610.93 & 652.31 & 779.90 & 828.38 & 840.67 & 896.22 & 913.98 & 928.07 \\
945.16 & 946.28 & 947.68 & 964.55 & 991.52 & 1020.52 & 1028.25 & 1042.11 & 1061.11 & 1073.53 \\
1082.89 & 1093.44 & 1109.30 & 1189.91 & 1237.96 & 1258.43 & 1259.13 & 1267.58 & 1311.73 \\
1318.50 & 1394.76 & 1404.07 & 1407.98 & 1411.27 & 1416.30 & 1418.64 & 1448.82 & 1466.67 \\
1477.68 & 1478.75 & 1480.85 & 1485.08 & 1486.43 & 1490.19 & 1492.26 & 1500.78 & 1502.69 \\
1514.16 & 1526.18 & 1588.02 & 1668.23 & 2895.84 & 3045.29 & 3058.81 & 3066.24 & 3068.28 \\
3073.35 & 3080.84 & 3111.66 & 3123.18 & 3142.34 & 3145.84 & 3151.82 & 3159.72 & 3168.34 \\
3169.22 & 3170.04 & 3170.82 & 3170.95 & 3174.11 & 3739.95 & & & \\
Rotational constants (B/GHZ): & \multicolumn{8}{c}{0.7879742} & 0.3169244 & 0.2789207 &
\end{tabular}




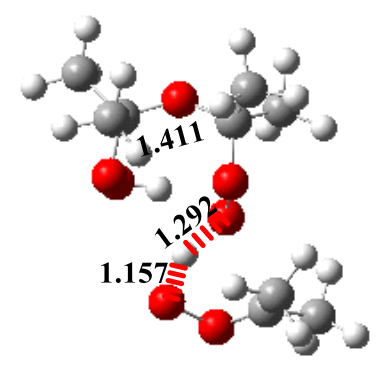

TS8d

C

$\begin{array}{lll}\text { C } & 1 & \text { B1 }\end{array}$

O 2

$\mathrm{O} 2$

$\mathrm{H} \quad 3$

C 3

C 6

$\begin{array}{ll}\mathrm{H} & 7\end{array}$

$\begin{array}{ll}\mathrm{H} & 7\end{array}$

$\mathrm{H} \quad 7$

O 6

$\mathrm{O}$

$\mathrm{H}$

$\mathrm{H}$

$\mathrm{H}$

O

$\mathrm{C}$

$\mathrm{C}$

$\mathrm{H} \quad 18$

$\mathrm{H} \quad 18$

$\mathrm{H} \quad 18$

O 17

O 22

$\mathrm{H} \quad 23$

C 6

$\mathrm{H} \quad 25$

$\mathrm{H} \quad 25$

H 25

C 2

H 29

H 29

H 29

C 17

$\mathrm{H} \quad 33$

$\mathrm{H} \quad 33$

$\mathrm{H} \quad 33$

B

B3 1

A1

B4 2

A2

A3 1

D1 0

B5 2

A4 1

D2 0

B6 3

A5 2

D3 0

B7 6

A6 3

D4 0

B8 6

A7 3

D5 0

B9 6

A8 3

D6 0

B10 3

A9 2

D7 0

$\begin{array}{llll}\mathrm{B} 11 & 3 & \mathrm{~A} 10 & 2\end{array}$

D8 0

$\begin{array}{llll}\mathrm{B} 12 & 2 & \mathrm{~A} 11 & 4\end{array}$

D9 0

B13 $2 \quad \mathrm{~A} 12 \quad 4$

D10 0

D11 0

$\begin{array}{llll}\text { B } 14 & 2 & \text { A13 } & 4\end{array}$

D12 0

$\begin{array}{llll}\text { B } 15 & 1 & \text { A14 } & 4\end{array}$

D13 0

$\begin{array}{llll}\mathrm{B} 16 & 2 & \mathrm{~A} 15 & 1\end{array}$

D14 0

B17 $16 \quad$ A $16 \quad 2$

D15 0

$\begin{array}{llll}\text { B } 18 & 17 & \text { A17 } & 16\end{array}$

D16 0

$\begin{array}{llll}\text { B } 19 & 17 & \text { A18 } & 16\end{array}$

D17 0

$\begin{array}{llll}\text { B20 } & 17 & \text { A19 } & 16\end{array}$

D18 0

B21 $16 \quad$ A20 2

D19 0

$\begin{array}{llll}\text { B22 } & 17 & \text { A21 } & 16\end{array}$

D20 0

$\begin{array}{llll}\text { B23 } & 22 & \text { A22 } & 17\end{array}$

D21 0

B24 $3 \quad$ A23 2

D22 0

$\begin{array}{llllll}\text { B25 } & 6 & \text { A24 } & 3 & \text { D23 } & 0\end{array}$

$\begin{array}{llllll}\text { B26 } & 6 & \text { A25 } & 3 & \text { D24 } & 0\end{array}$

$\begin{array}{llllll}\text { B27 } & 6 & \text { A26 } & 3 & \text { D25 } & 0\end{array}$

$\begin{array}{llllll}\text { B28 } & 1 & \text { A27 } & 16 & \text { D26 } & 0\end{array}$

$\begin{array}{llllll}\text { B29 } & 2 & \text { A28 } & 1 & \text { D27 } & 0\end{array}$

$\begin{array}{llllll}\text { B30 } & 2 & \text { A29 } & 1 & \text { D28 } & 0\end{array}$

$\begin{array}{llllll}\text { B31 } & 2 & \text { A30 } & 1 & \text { D29 } & 0\end{array}$

$\begin{array}{llllll}\text { B32 } & 16 & \text { A31 } & 2 & \text { D30 } & 0\end{array}$

$\begin{array}{llllll}\text { B33 } & 17 & \text { A32 } & 16 & \text { D31 } & 0\end{array}$

$\begin{array}{llllll}\text { B34 } & 17 & \text { A33 } & 16 & \text { D32 } & 0\end{array}$

$\begin{array}{llllll}\text { B35 } & 17 & \text { A34 } & 16 & \text { D33 } & 0\end{array}$ 
Variables:

\begin{tabular}{|c|c|}
\hline B1 & 1.52078622 \\
\hline B2 & 2.31936148 \\
\hline B3 & 1.40443606 \\
\hline B4 & 1.29168429 \\
\hline B5 & 2.27435536 \\
\hline B6 & 1.47731291 \\
\hline B7 & 1.08797363 \\
\hline B8 & 1.08173973 \\
\hline B9 & 1.09460900 \\
\hline B10 & 2.23010100 \\
\hline B11 & 1.27202304 \\
\hline B12 & 1.08859755 \\
\hline B13 & 1.08953608 \\
\hline B14 & 1.08567855 \\
\hline B15 & 1.41585983 \\
\hline B16 & 1.41079385 \\
\hline B17 & 1.51846886 \\
\hline B18 & 1.08616790 \\
\hline B19 & 1.08837525 \\
\hline B20 & 1.08872053 \\
\hline B21 & 1.41847576 \\
\hline B22 & 1.41213963 \\
\hline B23 & 0.97170844 \\
\hline B24 & 1.48150343 \\
\hline B25 & 1.08613104 \\
\hline B26 & 1.09295231 \\
\hline B27 & 1.08782472 \\
\hline B28 & 1.52610070 \\
\hline B29 & 1.08882788 \\
\hline B30 & 1.08837309 \\
\hline B31 & 1.08554390 \\
\hline B32 & 1.52093295 \\
\hline B33 & 1.08702565 \\
\hline B34 & 1.08818605 \\
\hline B35 & 1.08818700 \\
\hline A1 & 88.09868936 \\
\hline $\mathrm{A} 2$ & 111.98500603 \\
\hline A3 & 124.22005603 \\
\hline A4 & 140.22993995 \\
\hline A5 & 90.06403067 \\
\hline A6 & 109.24759674 \\
\hline A7 & 112.21480461 \\
\hline A8 & 106.03005575 \\
\hline
\end{tabular}




\begin{tabular}{|c|c|}
\hline A9 & 63.76095663 \\
\hline A10 & 83.53954898 \\
\hline A11 & 109.29403032 \\
\hline A12 & 109.57359001 \\
\hline A13 & 110.11073676 \\
\hline A14 & 104.55321826 \\
\hline A15 & 124.10686459 \\
\hline A16 & 104.64573302 \\
\hline A17 & 109.66719888 \\
\hline A 18 & 109.55255794 \\
\hline A19 & 109.59166181 \\
\hline A20 & 110.86145766 \\
\hline A21 & 109.50553961 \\
\hline A22 & 99.88667073 \\
\hline A23 & 108.42886967 \\
\hline A24 & 111.12146511 \\
\hline A25 & 105.63256434 \\
\hline A26 & 111.03402951 \\
\hline A 27 & 110.65350871 \\
\hline A28 & 109.52789102 \\
\hline A29 & 108.83354855 \\
\hline A30 & 111.82612337 \\
\hline A31 & 115.89569781 \\
\hline A32 & 110.69272391 \\
\hline A33 & 109.93125881 \\
\hline A34 & 108.50638261 \\
\hline D1 & 26.29324589 \\
\hline D2 & -169.85278926 \\
\hline D3 & 90.84906031 \\
\hline D4 & 30.12556954 \\
\hline D5 & -58.85048819 \\
\hline D6 & 63.39215248 \\
\hline D7 & -175.55409678 \\
\hline D8 & 123.14952010 \\
\hline D9 & 154.69185946 \\
\hline D10 & -171.99566928 \\
\hline D11 & -52.36195718 \\
\hline D12 & 68.20036771 \\
\hline D13 & -122.41576785 \\
\hline D14 & 159.73081017 \\
\hline D15 & 168.55656568 \\
\hline D16 & 53.82355269 \\
\hline D17 & 173.74227414 \\
\hline D18 & -66.05222708 \\
\hline
\end{tabular}




$\begin{array}{lr}\text { D19 } & -71.76747612 \\ \text { D20 } & -56.70506992 \\ \text { D21 } & 85.57853900 \\ \text { D22 } & -92.01698470 \\ \text { D23 } & -100.64755351 \\ \text { D24 } & 17.38547278 \\ \text { D25 } & 134.70927735 \\ \text { D26 } & -121.54777660 \\ \text { D27 } & -46.33577180 \\ \text { D28 } & 72.66262616 \\ \text { D29 } & -166.55442744 \\ \text { D30 } & 44.88261137 \\ \text { D31 } & -67.04801970 \\ \text { D32 } & 54.89559204 \\ \text { D33 } & 173.84973867\end{array}$

Harmonic Vibrational Frequencies $\left(\mathbf{c m}^{-1}\right)$ :

\begin{tabular}{lccccccccccc}
$784.25 i$ & 25.65 & 45.64 & 65.61 & 91.36 & 112.83 & 123.21 & 140.72 & 173.84 & 176.51 & 197.78 \\
202.32 & 212.27 & 229.60 & 255.33 & 259.72 & 288.10 & 293.24 & 316.03 & 326.90 & 340.98 \\
349.06 & 371.36 & 385.36 & 388.22 & 407.10 & 444.80 & 471.67 & 489.05 & 495.82 & 564.74 \\
594.57 & 603.96 & 613.53 & 655.27 & 756.30 & 785.57 & 818.37 & 843.02 & 895.76 & 928.70 \\
932.19 & 942.52 & 946.09 & 955.95 & 965.78 & 987.41 & 1017.46 & 1027.46 & 1058.96 & 1069.51 \\
1076.61 & 1090.08 & 1103.72 & 1190.63 & 1234.90 & 1253.86 & 1260.39 & 1265.73 & 1309.24 \\
1319.52 & 1362.72 & 1397.26 & 1402.29 & 1407.39 & 1415.81 & 1418.57 & 1424.65 & 1441.58 \\
1457.23 & 1470.55 & 1476.65 & 1480.20 & 1487.73 & 1490.10 & 1490.70 & 1495.26 & 1496.71 \\
1502.60 & 1516.10 & 1526.99 & 1610.80 & 1927.26 & 3053.70 & 3059.94 & 3061.13 & 3070.93 \\
3073.89 & 3077.02 & 3131.95 & 3135.51 & 3136.58 & 3148.43 & 3148.97 & 3156.38 & 3160.58 \\
3160.97 & 3172.53 & 3174.27 & 3183.67 & 3209.90 & 3687.40 & & & \\
Rotational constants (B/GHZ): & \multicolumn{8}{c}{0.7989229} & 0.3705004 & 0.3140995 &
\end{tabular}

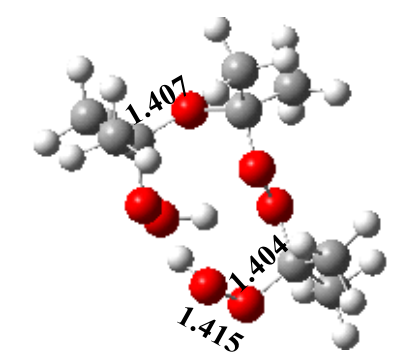

P8d

$\begin{array}{llllllll}\mathrm{C} & & & & & & & \\ \mathrm{C} & 1 & \mathrm{~B} 1 & & & & & \\ \mathrm{O} & 2 & \mathrm{~B} 2 & 1 & \text { A1 } & & & \\ \mathrm{O} & 2 & \mathrm{~B} 3 & 1 & \mathrm{~A} 2 & 3 & \mathrm{D} 1 & 0 \\ \mathrm{H} & 4 & \mathrm{~B} 4 & 2 & \mathrm{~A} 3 & 1 & \text { D2 } & 0 \\ \mathrm{C} & 3 & \text { B5 } & 2 & \text { A4 } & 1 & \text { D3 } & 0 \\ \mathrm{C} & 6 & \text { B6 } & 3 & \text { A5 } & 2 & \text { D4 } & 0 \\ \mathrm{H} & 7 & \text { B7 } & 6 & \text { A6 } & 3 & \text { D5 } & 0\end{array}$




\begin{tabular}{|c|c|c|c|c|c|c|}
\hline $\mathrm{H}$ & 7 & B8 & 6 & A7 & 3 & D6 \\
\hline $\mathrm{H}$ & 7 & B9 & 6 & A8 & 3 & D7 \\
\hline $\mathrm{O}$ & 6 & B10 & 3 & A9 & 2 & D8 \\
\hline $\mathrm{O}$ & 6 & B11 & 3 & A10 & 2 & D9 \\
\hline $\mathrm{H}$ & 1 & B12 & 2 & A11 & 4 & D10 \\
\hline $\mathrm{H}$ & 1 & B13 & 2 & $\mathrm{~A} 12$ & 4 & D11 \\
\hline $\mathrm{H}$ & 1 & B14 & 2 & A13 & 4 & D12 \\
\hline $\mathrm{O}$ & 2 & B15 & 1 & A14 & 4 & D13 \\
\hline $\mathrm{C}$ & 16 & B16 & 2 & A15 & 1 & D14 \\
\hline $\mathrm{C}$ & 17 & B17 & 16 & A16 & 2 & D15 \\
\hline $\mathrm{H}$ & 18 & B18 & 17 & A17 & 16 & D16 \\
\hline $\mathrm{H}$ & 18 & B19 & 17 & A18 & 16 & D17 \\
\hline $\mathrm{H}$ & 18 & B20 & 17 & A19 & 16 & D18 \\
\hline $\mathrm{O}$ & 17 & B21 & 16 & A20 & 2 & D19 \\
\hline $\mathrm{O}$ & 22 & B22 & 17 & A21 & 16 & D20 \\
\hline $\mathrm{H}$ & 23 & B23 & 22 & A22 & 17 & D21 \\
\hline $\mathrm{C}$ & 6 & B24 & 3 & A23 & 2 & D22 \\
\hline $\mathrm{H}$ & 25 & B25 & 6 & A24 & 3 & D23 \\
\hline $\mathrm{H}$ & 25 & B26 & 6 & A25 & 3 & D24 \\
\hline $\mathrm{H}$ & 25 & B27 & 6 & A26 & 3 & D25 \\
\hline $\mathrm{C}$ & 2 & B28 & 1 & A27 & 16 & D26 \\
\hline $\mathrm{H}$ & 29 & B29 & 2 & A28 & 1 & D27 \\
\hline $\mathrm{H}$ & 29 & B30 & 2 & A29 & 1 & D28 \\
\hline $\mathrm{H}$ & 29 & B31 & 2 & A30 & 1 & D29 \\
\hline $\mathrm{C}$ & 17 & B32 & 16 & A31 & 2 & D30 \\
\hline $\mathrm{H}$ & 33 & B33 & 17 & A32 & 16 & D31 \\
\hline $\mathrm{H}$ & 33 & B34 & 17 & A33 & 16 & D32 \\
\hline $\mathrm{H}$ & 33 & B35 & 17 & A34 & 16 & D33 \\
\hline \multicolumn{7}{|c|}{ Variables: } \\
\hline B1 & & 1.52016370 & & & & \\
\hline B2 & & 2.29963054 & & & & \\
\hline B3 & & 1.41059122 & & & & \\
\hline B4 & & 2.60713139 & & & & \\
\hline B5 & & 1.42779127 & & & & \\
\hline B6 & & 1.51436369 & & & & \\
\hline B7 & & 1.08851039 & & & & \\
\hline B8 & & 1.08416930 & & & & \\
\hline B9 & & 1.08823600 & & & & \\
\hline B10 & & 2.31570622 & & & & \\
\hline B11 & & 1.40392251 & & & & \\
\hline $\mathrm{B} 12$ & & 1.08842625 & & & & \\
\hline B13 & & 1.08853775 & & & & \\
\hline B14 & & 1.08543077 & & & & \\
\hline B15 & & 1.41355338 & & & & \\
\hline
\end{tabular}




\begin{tabular}{|c|c|}
\hline B16 & 1.40664548 \\
\hline B17 & 1.51648534 \\
\hline B18 & 1.08614036 \\
\hline B19 & 1.08806768 \\
\hline B20 & 1.08844939 \\
\hline B21 & 1.42641054 \\
\hline B22 & 1.40993238 \\
\hline B23 & 0.97037467 \\
\hline B24 & 1.51826934 \\
\hline B25 & 1.08894773 \\
\hline B26 & 1.08730640 \\
\hline B27 & 1.08719607 \\
\hline B28 & 1.52705524 \\
\hline B29 & 1.08808127 \\
\hline B30 & 1.08840824 \\
\hline B31 & 1.08525381 \\
\hline B32 & 1.52060750 \\
\hline B33 & 1.08793761 \\
\hline B34 & 1.08782299 \\
\hline B35 & 1.08800943 \\
\hline $\mathrm{A} 1$ & 86.30175837 \\
\hline $\mathrm{A} 2$ & 112.45194201 \\
\hline A3 & 125.20821276 \\
\hline A4 & 145.10265987 \\
\hline A5 & 112.29094243 \\
\hline A6 & 109.70717096 \\
\hline A7 & 110.94874871 \\
\hline A8 & 108.24636255 \\
\hline A9 & 95.99401266 \\
\hline A10 & 110.15522544 \\
\hline A11 & 108.84546011 \\
\hline A12 & 109.53161303 \\
\hline A13 & 110.69538535 \\
\hline A14 & 104.76041354 \\
\hline A15 & 124.44280307 \\
\hline A16 & 104.92839659 \\
\hline A17 & 109.70431919 \\
\hline A 18 & 109.62465302 \\
\hline A19 & 109.39579987 \\
\hline A20 & 110.78209748 \\
\hline A21 & 110.50891683 \\
\hline A 22 & 100.42519573 \\
\hline A 23 & 103.63558559 \\
\hline A24 & 110.02610851 \\
\hline
\end{tabular}




\begin{tabular}{|c|c|}
\hline A25 & 109.68937408 \\
\hline A26 & 109.15050415 \\
\hline $\mathrm{A} 27$ & 110.48069552 \\
\hline A28 & 109.11899556 \\
\hline A29 & 108.70110812 \\
\hline A30 & 112.28987592 \\
\hline A31 & 116.17739162 \\
\hline A32 & 111.13520361 \\
\hline A33 & 109.85504328 \\
\hline A34 & 108.64687064 \\
\hline D1 & 25.68184359 \\
\hline D2 & -134.09282706 \\
\hline D3 & 126.78519519 \\
\hline D4 & -32.65265239 \\
\hline D5 & -50.52424036 \\
\hline D6 & 70.50681839 \\
\hline D7 & -169.78745661 \\
\hline D8 & 61.09225082 \\
\hline D9 & 94.00240574 \\
\hline D10 & -168.26508807 \\
\hline D11 & -48.78722418 \\
\hline D12 & 72.15535667 \\
\hline D13 & -121.91487072 \\
\hline D14 & 163.83047261 \\
\hline D15 & 168.67748988 \\
\hline D16 & 52.99597908 \\
\hline D17 & 173.12540667 \\
\hline D18 & -66.72026001 \\
\hline D19 & -72.11545705 \\
\hline D20 & -57.15472125 \\
\hline D21 & 90.29220756 \\
\hline D22 & -155.38582894 \\
\hline D23 & -54.70033639 \\
\hline D24 & 65.84416352 \\
\hline D25 & -173.94062946 \\
\hline D26 & -122.91923998 \\
\hline D27 & -42.18896324 \\
\hline D28 & 76.53316302 \\
\hline D29 & -162.25697674 \\
\hline D30 & 44.24395768 \\
\hline D31 & -67.77125422 \\
\hline D32 & 53.91318818 \\
\hline D33 & 172.80687010 \\
\hline
\end{tabular}

Harmonic Vibrational Frequencies $\left(\mathrm{cm}^{-1}\right)$ : 


\begin{tabular}{lccccccccccc}
54.28 & 61.11 & 77.38 & 121.11 & 131.16 & 161.20 & 172.57 & 186.99 & 198.51 & 206.38 & 218.46 \\
245.86 & 250.75 & 259.61 & 279.06 & 290.81 & 297.27 & 301.70 & 330.20 & 340.28 & 356.85 \\
366.08 & 375.70 & 397.08 & 431.66 & 440.72 & 456.40 & 499.92 & 503.36 & 533.60 & 541.69 \\
586.01 & 610.49 & 619.43 & 651.50 & 783.00 & 825.76 & 856.75 & 895.66 & 906.80 & 936.68 \\
947.02 & 960.20 & 961.00 & 970.22 & 974.11 & 1021.12 & 1022.04 & 1031.36 & 1057.68 & 1067.81 \\
1074.46 & 1077.78 & 1178.13 & 1213.64 & 1231.79 & 1252.32 & 1256.13 & 1262.96 & 1269.28 \\
1307.54 & 1327.34 & 1407.53 & 1412.05 & 1415.28 & 1417.81 & 1421.31 & 1423.62 & 1477.38 \\
1478.24 & 1482.37 & 1484.04 & 1487.33 & 1488.40 & 1492.53 & 1495.14 & 1502.85 & 1505.01 \\
1511.46 & 1514.08 & 1526.53 & 1539.46 & 3067.81 & 3067.85 & 3076.91 & 3078.08 & 3080.04 \\
3082.49 & 3145.08 & 3148.93 & 3150.95 & 3152.90 & 3158.01 & 3162.18 & 3165.02 & 3167.29 \\
3170.15 & 3178.99 & 3185.48 & 3188.83 & 3695.05 & 3736.56 & & & \\
Rotational constants (B/GHZ): & \multicolumn{8}{c}{0.7550587} & 0.4454084 & 0.3758664 &
\end{tabular}

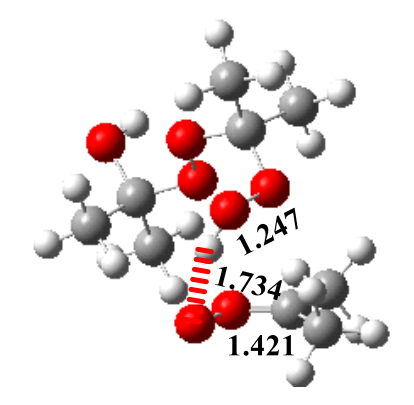

IM8e

C

\begin{tabular}{|c|c|c|c|c|c|c|c|}
\hline $\mathrm{O}$ & 1 & B1 & & & & & \\
\hline $\mathrm{O}$ & 1 & B2 & 2 & A1 & & & \\
\hline $\mathrm{H}$ & 2 & B3 & 1 & A2 & 3 & D1 & 0 \\
\hline $\mathrm{C}$ & 3 & B4 & 1 & A3 & 2 & D2 & 0 \\
\hline $\mathrm{C}$ & 5 & B5 & 3 & A4 & 1 & D3 & 0 \\
\hline $\mathrm{H}$ & 6 & B6 & 5 & A5 & 3 & D4 & 0 \\
\hline $\mathrm{H}$ & 6 & B7 & 5 & A6 & 3 & D5 & 0 \\
\hline $\mathrm{H}$ & 6 & B8 & 5 & A7 & 3 & D6 & 0 \\
\hline $\mathrm{C}$ & 5 & B9 & 3 & A8 & 1 & D7 & 0 \\
\hline $\mathrm{H}$ & 10 & B 10 & 5 & A9 & 3 & D8 & 0 \\
\hline $\mathrm{H}$ & 10 & B11 & 5 & A10 & 3 & D9 & 0 \\
\hline $\mathrm{H}$ & 10 & B12 & 5 & A11 & 3 & D10 & 0 \\
\hline $\mathrm{O}$ & 5 & B13 & 3 & A12 & 1 & D11 & 0 \\
\hline $\mathrm{O}$ & 5 & B14 & 3 & A13 & 1 & $\mathrm{D} 12$ & 0 \\
\hline $\mathrm{C}$ & 1 & B15 & 3 & A14 & 2 & D13 & 0 \\
\hline $\mathrm{H}$ & 16 & B 16 & 1 & A15 & 3 & D14 & 0 \\
\hline $\mathrm{H}$ & 16 & B17 & 1 & A16 & 3 & D15 & 0 \\
\hline $\mathrm{H}$ & 16 & B 18 & 1 & A17 & 3 & D16 & 0 \\
\hline $\mathrm{C}$ & 1 & B19 & 3 & A18 & 2 & D17 & 0 \\
\hline $\mathrm{H}$ & 20 & B20 & 1 & A19 & 3 & D18 & 0 \\
\hline $\mathrm{H}$ & 20 & B21 & 1 & A20 & 3 & D19 & 0 \\
\hline $\mathrm{H}$ & 20 & B22 & 1 & A21 & 3 & $\mathrm{D} 20$ & 0 \\
\hline
\end{tabular}




$\begin{array}{rrrrrrrr}\text { O } & 1 & \text { B23 } & 3 & \text { A22 } & 2 & \text { D21 } & 0 \\ \text { O } & 24 & \text { B24 } & 1 & \text { A23 } & 3 & \text { D22 } & 0 \\ \text { C } & 25 & \text { B25 } & 24 & \text { A24 } & 1 & \text { D23 } & 0 \\ \text { C } & 26 & \text { B26 } & 25 & \text { A25 } & 24 & \text { D24 } & 0 \\ \text { H } & 27 & \text { B27 } & 26 & \text { A26 } & 25 & \text { D25 } & 0 \\ \text { H } & 27 & \text { B28 } & 26 & \text { A27 } & 25 & \text { D26 } & 0 \\ \text { H } & 27 & \text { B29 } & 26 & \text { A28 } & 25 & \text { D27 } & 0 \\ \text { C } & 26 & \text { B30 } & 25 & \text { A29 } & 24 & \text { D28 } & 0 \\ \text { H } & 31 & \text { B31 } & 26 & \text { A30 } & 25 & \text { D29 } & 0 \\ \text { H } & 31 & \text { B32 } & 26 & \text { A31 } & 25 & \text { D30 } & 0 \\ \text { H } & 31 & \text { B33 } & 26 & \text { A32 } & 25 & \text { D31 } & 0 \\ \text { O } & 26 & \text { B34 } & 25 & \text { A33 } & 24 & \text { D32 } & 0 \\ \text { H } & 35 & \text { B35 } & 26 & \text { A34 } & 25 & \text { D33 } & 0\end{array}$

Variables:

$\begin{array}{ll}\text { B1 } & 2.32125941 \\ \text { B2 } & 1.41278768 \\ \text { B3 } & 0.99102410 \\ \text { B4 } & 2.76613958 \\ \text { B5 } & 1.48149396 \\ \text { B6 } & 1.09143067 \\ \text { B7 } & 1.08643016 \\ \text { B8 } & 1.09115544 \\ \text { B9 } & 1.47373363 \\ \text { B10 } & 1.08585998 \\ \text { B11 } & 1.09268948 \\ \text { B12 } & 1.09045898 \\ \text { B13 } & 2.24978577 \\ \text { B14 } & 1.24745543 \\ \text { B15 } & 1.51963346 \\ \text { B16 } & 1.08793249 \\ \text { B17 } & 1.08667844 \\ \text { B18 } & 1.08804845 \\ \text { B19 } & 1.51757303 \\ \text { B20 } & 1.08817704 \\ \text { B21 } & 1.08767342 \\ \text { B22 } & 1.08556727 \\ \text { B23 } & 1.40346740 \\ \text { B24 } & 1.42810111 \\ \text { B25 } & 1.42325418 \\ \text { B26 } & 1.51333629 \\ \text { B27 } & 1.08613898 \\ \text { B28 } & 1.08879280 \\ \text { B29 } & 1.08912202 \\ & 1.52175184\end{array}$




\begin{tabular}{|c|c|}
\hline B31 & 1.08785587 \\
\hline B32 & 1.09172365 \\
\hline B33 & 1.08786117 \\
\hline B34 & 1.40070056 \\
\hline B35 & 0.96141230 \\
\hline A1 & 35.10558118 \\
\hline $\mathrm{A} 2$ & 111.36495150 \\
\hline A3 & 132.88766006 \\
\hline A4 & 98.56362877 \\
\hline A5 & 109.69652263 \\
\hline A6 & 110.80798023 \\
\hline A7 & 107.85141376 \\
\hline A8 & 88.13734041 \\
\hline A9 & 110.71478426 \\
\hline A10 & 108.43276856 \\
\hline A11 & 108.62970227 \\
\hline A12 & 78.33986778 \\
\hline A13 & 83.46516013 \\
\hline A14 & 104.27601160 \\
\hline A15 & 109.59276944 \\
\hline A16 & 110.76550406 \\
\hline A17 & 108.88224707 \\
\hline A 18 & 112.24738176 \\
\hline A19 & 108.81852872 \\
\hline A20 & 109.53723895 \\
\hline A 21 & 110.24310944 \\
\hline A22 & 111.29614017 \\
\hline A23 & 108.12443459 \\
\hline A24 & 107.58918944 \\
\hline A25 & 112.48718503 \\
\hline A26 & 109.87403342 \\
\hline A27 & 108.78508156 \\
\hline A28 & 110.15309207 \\
\hline A29 & 102.80620634 \\
\hline A30 & 108.56964614 \\
\hline A 31 & 110.27187085 \\
\hline A 32 & 110.57358090 \\
\hline A33 & 110.04422314 \\
\hline A34 & 108.53299505 \\
\hline D1 & -80.79251249 \\
\hline D2 & 118.97687172 \\
\hline D3 & 50.61375542 \\
\hline D4 & 156.75507809 \\
\hline D5 & -80.59815266 \\
\hline
\end{tabular}




\begin{tabular}{lr} 
D6 & 39.86634069 \\
D7 & 173.76168173 \\
D8 & -108.75834289 \\
D9 & 12.46005882 \\
D10 & 127.66046195 \\
D11 & -98.03279599 \\
D12 & -64.63707003 \\
D13 & 170.83099596 \\
D14 & -67.03364479 \\
D15 & 53.97122567 \\
D16 & 173.39151375 \\
D17 & 48.51878497 \\
D18 & 50.74390249 \\
D19 & 170.52852765 \\
D20 & -68.83770429 \\
D21 & -67.63507712 \\
D22 & -64.70193429 \\
D23 & 171.71351352 \\
D24 & -52.26454282 \\
D25 & 69.51571159 \\
D26 & -171.46346268 \\
D27 & -51.17582162 \\
D28 & -173.76234999 \\
D29 & -173.10868338 \\
D30 & -54.05789206 \\
D31 & 67.05078839 \\
D32 & 67.45134010 \\
D33 & 55.45084733 \\
\hline
\end{tabular}

Harmonic Vibrational Frequencies $\left(\mathbf{c m}^{-1}\right)$ :

\begin{tabular}{lccccccccccc}
29.06 & 57.59 & 67.98 & 85.27 & 91.32 & 97.27 & 110.24 & 134.84 & 139.11 & 163.24 & 170.45 \\
176.01 & 189.71 & 215.96 & 223.66 & 247.46 & 260.46 & 273.41 & 288.10 & 305.04 & 325.84 \\
337.81 & 350.98 & 368.15 & 372.56 & 380.40 & 404.25 & 443.23 & 468.62 & 503.26 & 524.62 \\
583.52 & 597.13 & 603.51 & 611.60 & 757.50 & 814.09 & 830.69 & 852.48 & 910.95 & 918.21 \\
928.48 & 948.39 & 951.24 & 959.95 & 972.98 & 993.61 & 1010.90 & 1018.29 & 1029.72 & 1056.18 \\
1067.98 & 1092.61 & 1111.35 & 1156.13 & 1212.25 & 1230.41 & 1256.08 & 1271.87 & 1309.30 \\
1319.19 & 1380.02 & 1399.14 & 1403.36 & 1407.73 & 1411.18 & 1413.98 & 1442.32 & 1456.09 \\
1464.31 & 1475.07 & 1479.76 & 1483.06 & 1488.95 & 1490.22 & 1494.35 & 1496.08 & 1505.47 \\
1509.72 & 1516.83 & 1612.56 & 1672.13 & 3048.70 & 3054.48 & 3059.38 & 3069.37 & 3071.13 \\
3072.67 & 3121.97 & 3122.37 & 3128.42 & 3147.57 & 3149.55 & 3152.82 & 3153.34 & 3162.55 \\
3168.42 & 3171.05 & 3173.63 & 3174.76 & 3258.75 & 3882.03 & & & \\
Rotational constants (B/GHZ): & \multicolumn{8}{c}{0.5942399} & 0.4682707 & 0.3576222 &
\end{tabular}




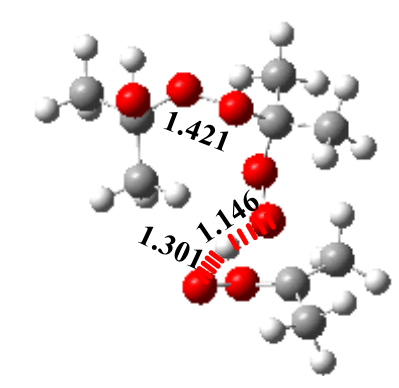

TS8e

C

$\begin{array}{lll}\mathrm{O} & 1 & \mathrm{~B} 1\end{array}$

$\begin{array}{lll}\mathrm{O} & 1 & \mathrm{~B}\end{array}$

$\mathrm{H} 2$

C 2

C 5

$\mathrm{H} \quad 6$

$\mathrm{H} \quad 6$

$\mathrm{H} \quad 6$

C 5

$\mathrm{H} \quad 10$

$\mathrm{H} \quad 10$

$\mathrm{H} \quad 10$

O 5

$\mathrm{O} \quad 5$

C 1

$\mathrm{H} \quad 16$

$\mathrm{H} \quad 16$

$\mathrm{H} \quad 16$

C 1

$\mathrm{H} \quad 20$

$\mathrm{H} \quad 20$

$\mathrm{H} \quad 20$

O 1

O 24

C $\quad 25$

C 26

$\mathrm{H} \quad 27$

$\mathrm{H} \quad 27$

$\begin{array}{ll}\mathrm{H} & 27\end{array}$

C 26

$\mathrm{H} \quad 31$

$\mathrm{H} \quad 31$

$\mathrm{H} \quad 31$

O 26

H $\quad 35$

B2 2

A1

B3 1

A2 3

D1 0

B4 1

A3 3

D2 0

B5 2

A4 1

D3 0

B6 $5 \quad$ A5 2

D4 0

B7 5

A6 2

D5 0

B8 5

A7 2

D6 0

$\begin{array}{llll}\text { B9 } & 2 & \text { A8 } & 1\end{array}$

D7 0

$\begin{array}{llllll}\text { B10 } & 5 & \text { A9 } & 2 & \text { D8 } & 0\end{array}$

$\begin{array}{llllll}\mathrm{B} 11 & 5 & \mathrm{~A} 10 & 2 & \mathrm{D} 9 & 0\end{array}$

$\begin{array}{llllll}\mathrm{B} 12 & 5 & \mathrm{~A} 11 & 2 & \mathrm{D} 10 & 0\end{array}$

$\begin{array}{llllll}\mathrm{B} 13 & 2 & \mathrm{~A} 12 & 1 & \mathrm{D} 11 & 0\end{array}$

$\begin{array}{llllll}\mathrm{B} 14 & 2 & \mathrm{~A} 13 & 1 & \mathrm{D} 12 & 0\end{array}$

$\begin{array}{llllll}\mathrm{B} 15 & 3 & \mathrm{~A} 14 & 2 & \mathrm{D} 13 & 0\end{array}$

$\begin{array}{llllll}\text { B } 16 & 1 & \text { A15 } & 3 & \text { D14 } & 0\end{array}$

$\begin{array}{llllll}\text { B17 } & 1 & \text { A16 } & 3 & \text { D15 } & 0\end{array}$

$\begin{array}{llllll}\text { B18 } & 1 & \text { A17 } & 3 & \text { D16 } & 0\end{array}$

$\begin{array}{llllll}\text { B } 19 & 3 & \text { A18 } & 2 & \text { D17 } & 0\end{array}$

$\begin{array}{llllll}\text { B20 } & 1 & \text { A19 } & 3 & \text { D18 } & 0\end{array}$

$\begin{array}{llllll}\text { B21 } 1 & \text { A20 } & 3 & \text { D19 } & 0\end{array}$

$\begin{array}{llllll}\text { B22 } & 1 & \text { A21 } & 3 & \text { D20 } & 0\end{array}$

$\begin{array}{llllll}\mathrm{B} 23 & 3 & \mathrm{~A} 22 & 2 & \mathrm{D} 21 & 0\end{array}$

$\begin{array}{llllll}\text { B24 } & 1 & \text { A23 } & 3 & \text { D22 } & 0\end{array}$

$\begin{array}{llllll}\text { B25 } & 24 & \text { A24 } & 1 & \text { D23 } & 0\end{array}$

$\begin{array}{llllll}\text { B26 } & 25 & \text { A25 } & 24 & \text { D24 } & 0\end{array}$

$\begin{array}{llllll}\text { B27 } & 26 & \text { A26 } & 25 & \text { D25 } & 0\end{array}$

$\begin{array}{llllll}\text { B28 } & 26 & \text { A27 } & 25 & \text { D26 } & 0\end{array}$

$\begin{array}{llllll}\text { B29 } & 26 & \text { A28 } & 25 & \text { D27 } & 0\end{array}$

$\begin{array}{llllll}\text { B30 } & 25 & \text { A29 } & 24 & \text { D28 } & 0\end{array}$

$\begin{array}{llllll}\text { B31 } & 26 & \text { A30 } & 25 & \text { D29 } & 0\end{array}$

$\begin{array}{llllll}\text { B } 32 & 26 & \text { A31 } & 25 & \text { D30 } & 0\end{array}$

$\begin{array}{llllll}\text { B } 33 & 26 & \text { A32 } & 25 & \text { D31 } & 0\end{array}$

$\begin{array}{llllll}\text { B34 } & 25 & \text { A33 } & 24 & \text { D32 } & 0\end{array}$

$\begin{array}{llllll}\text { B35 } & 26 & \text { A34 } & 25 & \text { D33 } & 0\end{array}$ 
Variables:

\begin{tabular}{|c|c|}
\hline B1 & 2.31261739 \\
\hline B2 & 1.41239530 \\
\hline B3 & 1.14627856 \\
\hline B4 & 2.10128450 \\
\hline B5 & 1.48348300 \\
\hline B6 & 1.08670903 \\
\hline B7 & 1.08743630 \\
\hline B8 & 1.09300807 \\
\hline B9 & 1.48703633 \\
\hline B10 & 1.08671245 \\
\hline B11 & 1.08272823 \\
\hline B12 & 1.09456675 \\
\hline B13 & 2.21820822 \\
\hline B14 & 1.28042305 \\
\hline B15 & 1.51925059 \\
\hline B16 & 1.08854047 \\
\hline B17 & 1.08613196 \\
\hline B18 & 1.08795361 \\
\hline B19 & 1.51892401 \\
\hline B20 & 1.08901812 \\
\hline B21 & 1.08775927 \\
\hline B22 & 1.08609250 \\
\hline B23 & 1.40833975 \\
\hline B24 & 1.42145937 \\
\hline B25 & 1.42382229 \\
\hline B26 & 1.51157285 \\
\hline B27 & 1.08706850 \\
\hline B28 & 1.08843679 \\
\hline B29 & 1.08848431 \\
\hline B30 & 1.52149428 \\
\hline B31 & 1.08781507 \\
\hline B32 & 1.09045389 \\
\hline B33 & 1.08803864 \\
\hline B34 & 1.39958354 \\
\hline B35 & 0.96153530 \\
\hline A1 & 35.20320370 \\
\hline $\mathrm{A} 2$ & 126.39216061 \\
\hline A3 & 130.57471912 \\
\hline A4 & 107.60014686 \\
\hline A5 & 111.68623732 \\
\hline A6 & 110.16732152 \\
\hline A7 & 106.10860528 \\
\hline A8 & 89.85405611 \\
\hline
\end{tabular}




\begin{tabular}{|c|c|}
\hline A9 & 109.58112011 \\
\hline A10 & 112.02397141 \\
\hline A11 & 106.18552042 \\
\hline A12 & 66.67191498 \\
\hline A 13 & 90.07226943 \\
\hline A14 & 103.95806638 \\
\hline A15 & 109.50141564 \\
\hline A16 & 110.46441840 \\
\hline A17 & 109.03664898 \\
\hline A18 & 112.61653682 \\
\hline A19 & 109.34666829 \\
\hline A20 & 109.33623065 \\
\hline A 21 & 110.42043416 \\
\hline A22 & 111.51826315 \\
\hline A23 & 108.62699959 \\
\hline A24 & 109.35930118 \\
\hline A25 & 112.61668619 \\
\hline A26 & 109.71808281 \\
\hline A27 & 109.06229601 \\
\hline A28 & 110.13119288 \\
\hline A29 & 102.42635171 \\
\hline A30 & 108.75147312 \\
\hline A31 & 110.12875299 \\
\hline A 32 & 110.67796095 \\
\hline A33 & 110.06898385 \\
\hline A34 & 108.34921473 \\
\hline D1 & -54.37354883 \\
\hline D2 & 45.48953420 \\
\hline D3 & 21.80047562 \\
\hline D4 & 117.57122642 \\
\hline D5 & -118.19307601 \\
\hline D6 & -1.80462590 \\
\hline D7 & 143.85730464 \\
\hline D8 & -64.59630932 \\
\hline D9 & 59.06955695 \\
\hline D10 & 178.51904205 \\
\hline D11 & -122.28741270 \\
\hline D12 & -93.11195286 \\
\hline D13 & 166.87696431 \\
\hline D14 & -72.32920171 \\
\hline D15 & 48.14654239 \\
\hline D16 & 168.00540633 \\
\hline D17 & 44.36543703 \\
\hline D18 & 48.25912482 \\
\hline
\end{tabular}




$\begin{array}{lc}\text { D19 } & 167.73889652 \\ \text { D20 } & -71.89020133 \\ \text { D21 } & -71.62536422 \\ \text { D22 } & -70.18025977 \\ \text { D23 } & 134.46054441 \\ \text { D24 } & -56.34684711 \\ \text { D25 } & 64.91669016 \\ \text { D26 } & -175.76178873 \\ \text { D27 } & -55.75160361 \\ \text { D28 } & -177.80312064 \\ \text { D29 } & -176.41915158 \\ \text { D30 } & -57.00459470 \\ \text { D31 } & 63.40013112 \\ \text { D32 } & 63.34338790 \\ \text { D33 } & 54.11233444\end{array}$

Harmonic Vibrational Frequencies $\left(\mathbf{c m}^{-\mathbf{1}}\right)$ :

$\begin{array}{lccccccccccc}784.16 i & 17.28 & 43.40 & 51.83 & 62.74 & 86.03 & 97.32 & 104.53 & 145.39 & 172.77 & 201.87 \\ 209.95 & 213.33 & 232.56 & 239.40 & 253.46 & 275.97 & 293.49 & 300.92 & 326.01 & 335.03 \\ 346.20 & 377.25 & 385.12 & 394.56 & 403.95 & 426.01 & 447.77 & 455.91 & 524.73 & 539.72 \\ 564.09 & 601.42 & 615.18 & 635.51 & 805.89 & 814.79 & 842.46 & 880.89 & 907.91 & 915.52 \\ 943.05 & 949.15 & 956.42 & 959.80 & 972.84 & 987.29 & 1003.10 & 1018.60 & 1027.55 & 1062.31 \\ 1076.67 & 1081.82 & 1115.97 & 1156.26 & 1214.94 & 1229.90 & 1249.74 & 1275.33 & 1307.08 \\ 1314.27 & 1374.20 & 1400.67 & 1405.13 & 1409.47 & 1416.07 & 1420.45 & 1430.51 & 1440.01 \\ 1464.25 & 1476.42 & 1481.96 & 1483.65 & 1487.76 & 1490.99 & 1492.51 & 1494.25 & 1499.00 \\ 1507.45 & 1516.20 & 1582.51 & 1597.24 & 1788.59 & 3046.38 & 3054.29 & 3064.34 & 3067.10 \\ 3070.44 & 3079.62 & 3133.06 & 3139.19 & 3145.42 & 3146.47 & 3148.51 & 3151.14 & 3158.68 \\ 3162.18 & 3168.04 & 3171.67 & 3172.26 & 3205.37 & 3881.20 & & & \\ \text { Rotational constants (B/GHZ): } & 0.7900059 & 0.3350680 & 0.2791479 & \end{array}$

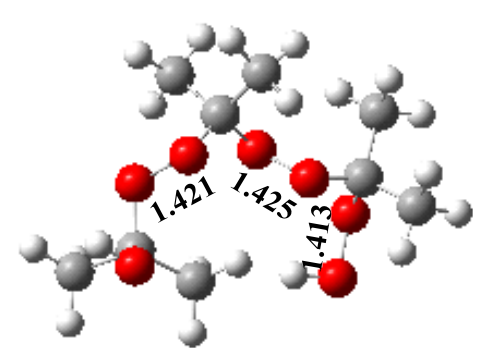

P8e

C

$\begin{array}{llllllll}\mathrm{O} & 1 & \mathrm{~B} 1 & & & & & \\ \mathrm{O} & 1 & \mathrm{~B} 2 & 2 & \mathrm{~A} 1 & & & \\ \mathrm{H} & 2 & \mathrm{~B} 3 & 1 & \mathrm{~A} 2 & 3 & \mathrm{D} 1 & 0 \\ \mathrm{C} & 2 & \mathrm{~B} 4 & 1 & \mathrm{~A} 3 & 3 & \mathrm{D} 2 & 0 \\ \mathrm{C} & 5 & \mathrm{~B} 5 & 2 & \mathrm{~A} 4 & 1 & \mathrm{D} 3 & 0 \\ \mathrm{H} & 6 & \mathrm{~B} 6 & 5 & \text { A5 } & 2 & \text { D4 } & 0 \\ \mathrm{H} & 6 & \text { B7 } & 5 & \text { A6 } & 2 & \text { D5 } & 0\end{array}$




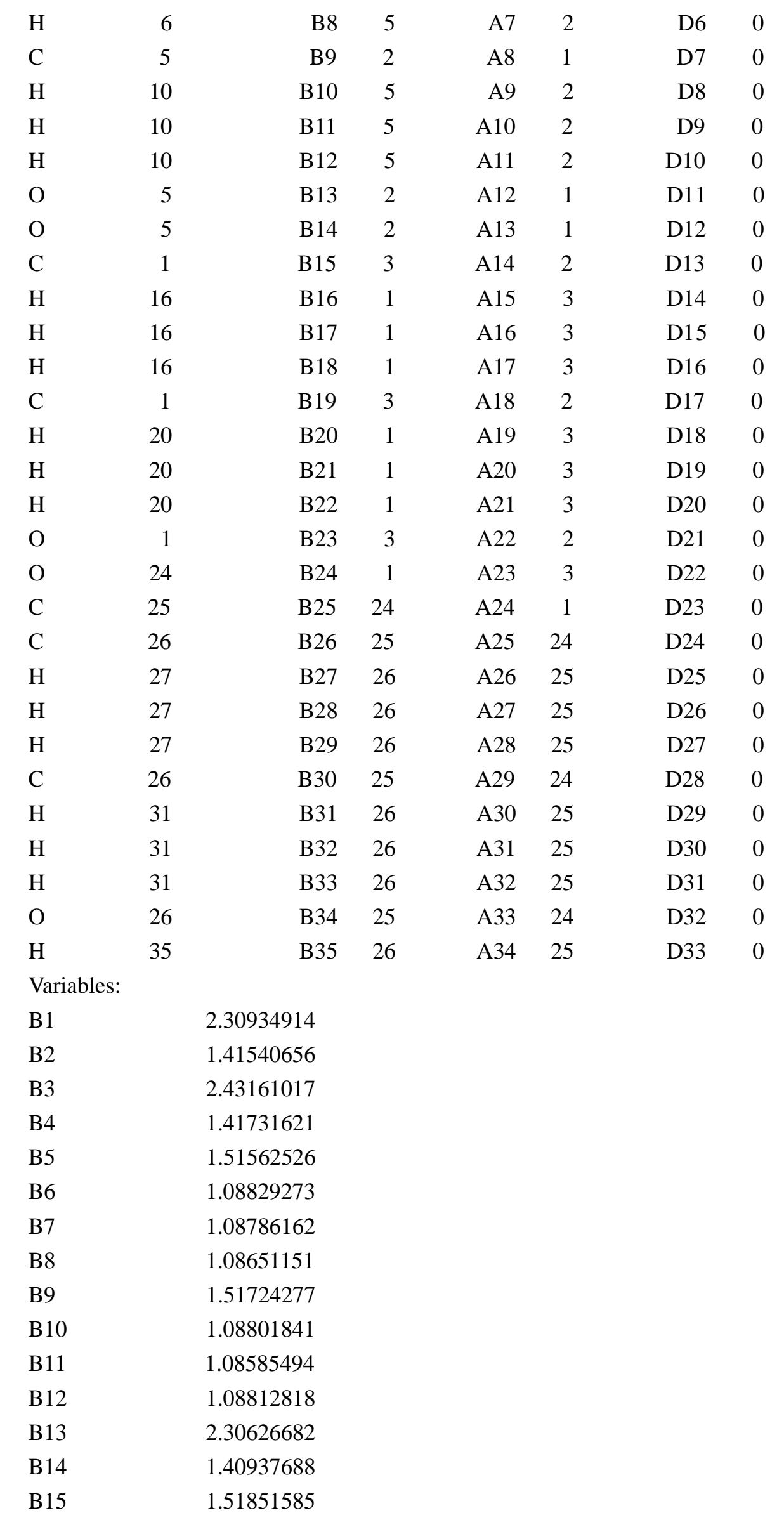




\begin{tabular}{|c|c|}
\hline B16 & 1.08806195 \\
\hline B17 & 1.08604131 \\
\hline B18 & 1.08788564 \\
\hline B19 & 1.51796499 \\
\hline B20 & 1.08809936 \\
\hline B21 & 1.08766784 \\
\hline B22 & 1.08622324 \\
\hline B23 & 1.41051625 \\
\hline B24 & 1.42114561 \\
\hline B25 & 1.42368513 \\
\hline B26 & 1.51364179 \\
\hline B27 & 1.08772811 \\
\hline B28 & 1.08835318 \\
\hline B29 & 1.09064688 \\
\hline B30 & 1.52155608 \\
\hline B31 & 1.08790418 \\
\hline B32 & 1.09000140 \\
\hline B33 & 1.08819587 \\
\hline B34 & 1.39588862 \\
\hline B35 & 0.96175774 \\
\hline $\mathrm{A} 1$ & 35.75412821 \\
\hline $\mathrm{A} 2$ & 115.99279212 \\
\hline A3 & 134.53395820 \\
\hline A4 & 113.38178498 \\
\hline A5 & 109.26758619 \\
\hline A6 & 109.13621944 \\
\hline A7 & 110.62355187 \\
\hline A8 & 103.82660210 \\
\hline A9 & 109.84193324 \\
\hline A10 & 110.52720649 \\
\hline A11 & 108.58937793 \\
\hline A 12 & 90.52380610 \\
\hline A13 & 109.58571940 \\
\hline A14 & 103.71758915 \\
\hline A15 & 109.17575202 \\
\hline A16 & 110.69172156 \\
\hline A17 & 109.04082000 \\
\hline A18 & 112.97823819 \\
\hline A19 & 109.18229857 \\
\hline A20 & 108.96082593 \\
\hline A21 & 110.83939664 \\
\hline A22 & 110.98664960 \\
\hline A23 & 109.41043541 \\
\hline A24 & 109.93392349 \\
\hline
\end{tabular}




\begin{tabular}{|c|c|}
\hline A25 & 112.27080332 \\
\hline A26 & 109.71755219 \\
\hline $\mathrm{A} 27$ & 109.30964009 \\
\hline A28 & 108.61323328 \\
\hline A29 & 102.41575972 \\
\hline A30 & 108.81837651 \\
\hline A31 & 110.04165875 \\
\hline A32 & 110.81845327 \\
\hline A33 & 110.53146750 \\
\hline A34 & 108.54058945 \\
\hline D1 & -45.66705532 \\
\hline D2 & 52.89145003 \\
\hline D3 & 24.92354447 \\
\hline D4 & 59.58759073 \\
\hline D5 & 178.56365993 \\
\hline D6 & -61.38704589 \\
\hline D7 & 148.16768839 \\
\hline D8 & -67.77572351 \\
\hline D9 & 53.09217098 \\
\hline D10 & 172.62097083 \\
\hline D11 & -121.44939058 \\
\hline D12 & -91.16742125 \\
\hline D13 & 168.36500341 \\
\hline D14 & -72.90227587 \\
\hline D15 & 47.56780143 \\
\hline D16 & 167.44293091 \\
\hline D17 & 46.09789913 \\
\hline D18 & 50.51746432 \\
\hline D19 & 169.70326869 \\
\hline D20 & -70.13864507 \\
\hline D21 & -70.01166209 \\
\hline D22 & -75.52853355 \\
\hline D23 & 129.41980306 \\
\hline D24 & -60.95661745 \\
\hline D25 & 62.53351228 \\
\hline D26 & -177.41314489 \\
\hline D27 & -57.79746737 \\
\hline D28 & 177.90228910 \\
\hline D29 & -178.01619978 \\
\hline D30 & -58.60279062 \\
\hline D31 & 61.68309483 \\
\hline D32 & 58.58696010 \\
\hline D33 & 53.74109330 \\
\hline
\end{tabular}

Harmonic Vibrational Frequencies $\left(\mathrm{cm}^{-1}\right)$ : 


$\begin{array}{lccccccccccc}27.07 & 52.93 & 71.04 & 83.15 & 99.17 & 119.75 & 137.52 & 173.41 & 187.93 & 204.85 & 226.47 \\ 230.47 & 242.85 & 260.29 & 268.22 & 285.17 & 292.78 & 300.12 & 309.85 & 331.23 & 345.76 \\ 359.12 & 373.24 & 388.45 & 390.01 & 423.85 & 439.96 & 461.64 & 477.33 & 528.51 & 582.91 \\ 588.88 & 601.87 & 619.99 & 633.03 & 812.78 & 830.88 & 861.96 & 902.33 & 912.58 & 916.75 \\ 948.29 & 949.89 & 956.48 & 963.63 & 981.13 & 1021.97 & 1026.39 & 1028.42 & 1033.48 & 1049.32 \\ 1060.90 & 1068.54 & 1152.01 & 1197.25 & 1225.35 & 1230.35 & 1247.58 & 1258.05 & 1286.50 \\ 1299.87 & 1324.42 & 1375.93 & 1407.50 & 1409.40 & 1412.44 & 1415.78 & 1420.25 & 1429.53 \\ 1448.94 & 1476.59 & 1483.98 & 1485.30 & 1487.48 & 1487.63 & 1492.04 & 1495.31 & 1499.41 \\ 1503.50 & 1509.15 & 1512.96 & 1532.73 & 3054.41 & 3059.09 & 3073.24 & 3074.07 & 3077.82 \\ 3080.46 & 3137.50 & 3138.12 & 3152.45 & 3152.55 & 3153.35 & 3159.69 & 3161.44 & 3163.30 \\ 3164.02 & 3168.24 & 3170.37 & 3171.75 & 3804.46 & 3881.05 & & & \end{array}$

Rotational constants (B/GHZ): $\quad 0.7660137 \quad 0.3617709 \quad 0.2938404$

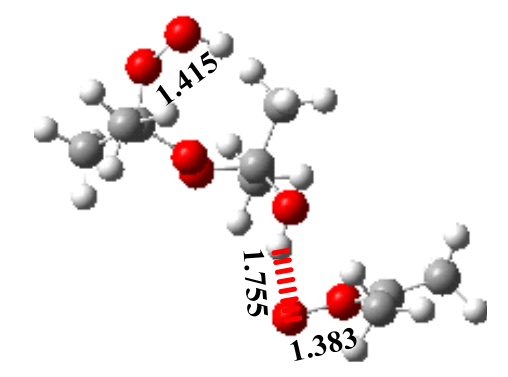

IM8f

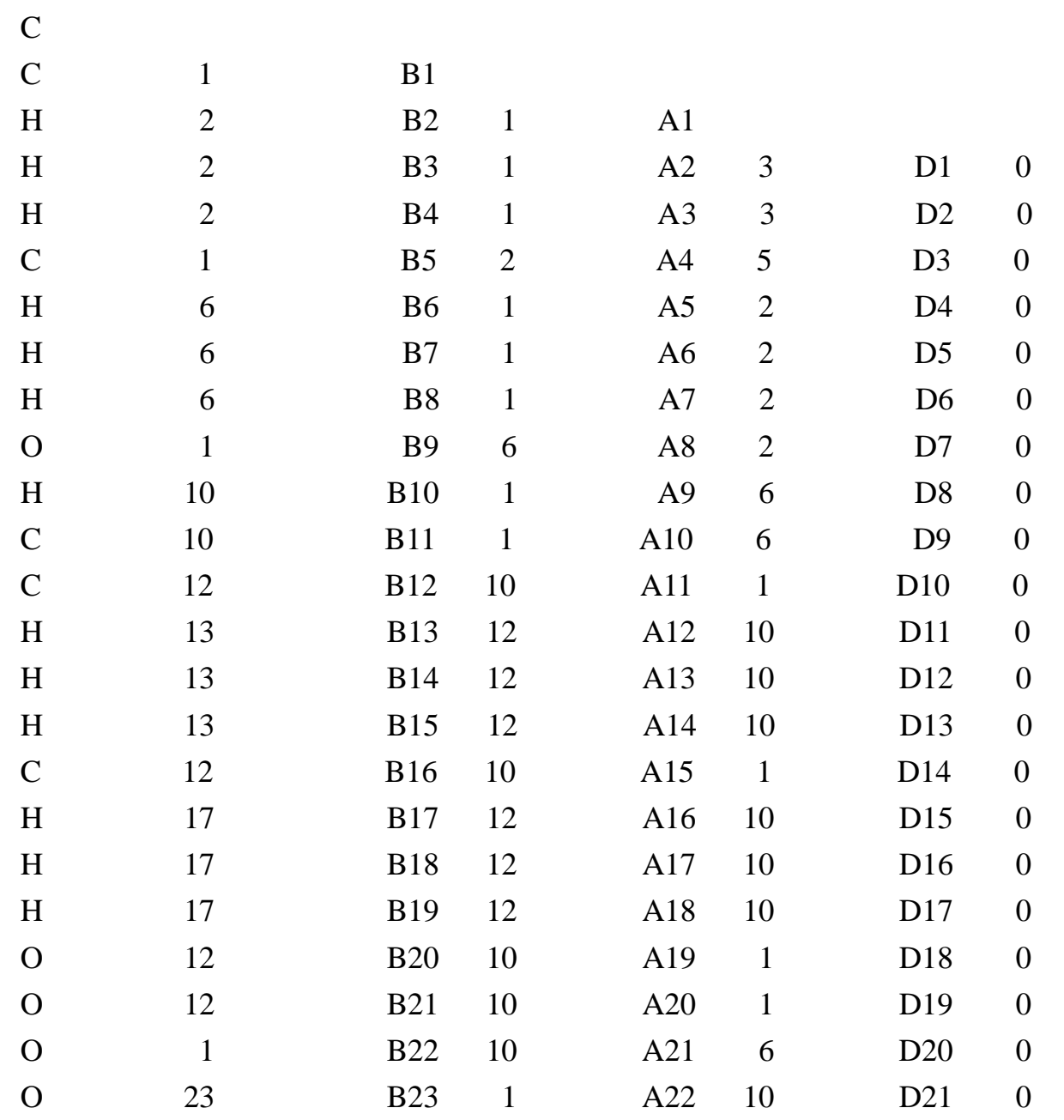




$\begin{array}{llllllll}\mathrm{C} & 24 & \text { B24 } & 23 & \text { A23 } & 1 & \text { D22 } & 0 \\ \mathrm{C} & 25 & \text { B25 } & 24 & \text { A24 } & 23 & \text { D23 } & 0 \\ \text { H } & 26 & \text { B26 } & 25 & \text { A25 } & 24 & \text { D24 } & 0 \\ \text { H } & 26 & \text { B27 } & 25 & \text { A26 } & 24 & \text { D25 } & 0 \\ \text { H } & 26 & \text { B28 } & 25 & \text { A27 } & 24 & \text { D26 } & 0 \\ \text { C } & 25 & \text { B29 } & 24 & \text { A28 } & 23 & \text { D27 } & 0 \\ \text { H } & 30 & \text { B30 } & 25 & \text { A29 } & 24 & \text { D28 } & 0 \\ \text { H } & 30 & \text { B31 } & 25 & \text { A30 } & 24 & \text { D29 } & 0 \\ \text { H } & 30 & \text { B32 } & 25 & \text { A31 } & 24 & \text { D30 } & 0 \\ \text { O } & 25 & \text { B33 } & 24 & \text { A32 } & 23 & \text { D31 } & 0 \\ \text { O } & 34 & \text { B34 } & 25 & \text { A33 } & 24 & \text { D32 } & 0 \\ \text { H } & 35 & \text { B35 } & 34 & \text { A34 } & 25 & \text { D33 } & 0\end{array}$

Variables:

$\begin{array}{ll}\text { B1 } & 1.52273924 \\ \text { B2 } & 1.08892690 \\ \text { B3 } & 1.08953765 \\ \text { B4 } & 1.08833210 \\ \text { B5 } & 1.51741728 \\ \text { B6 } & 1.08840973 \\ \text { B7 } & 1.09067946 \\ \text { B8 } & 1.08940207 \\ \text { B9 } & 1.38980252 \\ \text { B10 } & 0.98411234 \\ \text { B11 } & 3.06958751 \\ \text { B12 } & 1.48312208 \\ \text { B13 } & 1.09148356 \\ \text { B14 } & 1.09097335 \\ \text { B15 } & 1.08576888 \\ \text { B16 } & 1.47296373 \\ \text { B17 } & 1.09388032 \\ \text { B18 } & 1.09026640 \\ \text { B19 } & 1.08593259 \\ \text { B20 } & 2.24727700 \\ \text { B21 } & 1.24924983 \\ \text { B22 } & 1.42267467 \\ \text { B23 } & 1.42452070 \\ \text { B24 } & 1.41190134 \\ \text { B25 } & 1.51765364 \\ \text { B26 } & 1.08606270 \\ \text { B27 } & 1.08802211 \\ \text { B28 } & 1.08778151 \\ \text { B29 } & 1.51757123 \\ \text { B30 } & 1.08782588 \\ \text { B31 } & 1.08609357\end{array}$




\begin{tabular}{|c|c|}
\hline B32 & 1.08789906 \\
\hline B33 & 1.41520018 \\
\hline B34 & 1.41454686 \\
\hline B35 & 0.96524603 \\
\hline A1 & 108.75446983 \\
\hline $\mathrm{A} 2$ & 109.58876811 \\
\hline A3 & 110.86394040 \\
\hline A4 & 112.75067188 \\
\hline A5 & 109.64707462 \\
\hline A6 & 109.77276708 \\
\hline A7 & 109.21666106 \\
\hline A8 & 107.64602743 \\
\hline A9 & 108.88215335 \\
\hline A 10 & 134.49147623 \\
\hline A11 & 121.99119623 \\
\hline A12 & 108.63716713 \\
\hline A13 & 109.43529171 \\
\hline A14 & 111.13428815 \\
\hline A15 & 78.39352075 \\
\hline A16 & 108.11531518 \\
\hline A 17 & 108.75699048 \\
\hline A18 & 110.82229869 \\
\hline A19 & 58.30923998 \\
\hline A20 & 71.18809167 \\
\hline $\mathrm{A} 21$ & 110.92889986 \\
\hline A22 & 109.14084912 \\
\hline A23 & 108.23959772 \\
\hline A24 & 104.15385882 \\
\hline A25 & 110.87716472 \\
\hline A26 & 108.88549462 \\
\hline $\mathrm{A} 27$ & 109.21436365 \\
\hline A28 & 112.92377168 \\
\hline A29 & 109.17737088 \\
\hline A 30 & 110.60768188 \\
\hline A31 & 109.00983418 \\
\hline A 32 & 110.48691813 \\
\hline A33 & 109.95013923 \\
\hline A34 & 102.12326460 \\
\hline D1 & 118.67779047 \\
\hline D2 & -120.20832908 \\
\hline D3 & -59.20768823 \\
\hline D4 & -53.54669227 \\
\hline D5 & 66.10555658 \\
\hline D6 & -171.80991086 \\
\hline
\end{tabular}




$\begin{array}{lr}\text { D7 } & 123.53661697 \\ \text { D8 } & -171.35414114 \\ \text { D9 } & -105.43751914 \\ \text { D10 } & 60.23688442 \\ \text { D11 } & 39.10323737 \\ \text { D12 } & 155.86559376 \\ \text { D13 } & -82.18127696 \\ \text { D14 } & -177.99674119 \\ \text { D15 } & -10.88169015 \\ \text { D16 } & 104.27739444 \\ \text { D17 } & -131.77039215 \\ \text { D18 } & -83.25790190 \\ \text { D19 } & -49.20812591 \\ \text { D20 } & -122.23756293 \\ \text { D21 } & 63.80217318 \\ \text { D22 } & 141.47682763 \\ \text { D23 } & 166.79850843 \\ \text { D24 } & 46.88547658 \\ \text { D25 } & 166.75360974 \\ \text { D26 } & -73.55855581 \\ \text { D27 } & 44.00654089 \\ \text { D28 } & 166.90186655 \\ \text { D29 } & -72.70423306 \\ \text { D30 } & 47.15757891 \\ \text { D31 } & -72.04043939 \\ \text { D32 } & -69.14704749 \\ \text { D33 } & 84.89837234 \\ & \end{array}$

Harmonic Vibrational Frequencies $\left(\mathbf{c m}^{-1}\right)$ :

\begin{tabular}{lccccccccccc}
11.91 & 21.26 & 36.54 & 44.46 & 75.84 & 93.07 & 100.30 & 112.86 & 145.53 & 158.31 & 182.15 \\
195.30 & 213.81 & 217.36 & 227.87 & 237.90 & 257.47 & 278.09 & 287.54 & 324.86 & 332.74 \\
341.54 & 361.43 & 367.50 & 377.42 & 380.82 & 416.23 & 457.46 & 464.78 & 497.86 & 544.83 \\
594.04 & 605.50 & 611.53 & 619.89 & 813.67 & 827.31 & 844.73 & 870.47 & 904.52 & 918.75 \\
922.34 & 941.58 & 958.23 & 959.02 & 974.76 & 990.99 & 1019.06 & 1020.93 & 1031.52 & 1053.95 \\
1065.10 & 1092.43 & 1108.81 & 1203.80 & 1224.66 & 1232.75 & 1251.41 & 1284.77 & 1316.79 \\
1319.33 & 1384.29 & 1391.88 & 1412.40 & 1412.64 & 1415.84 & 1422.30 & 1427.76 & 1454.80 \\
1467.06 & 1468.90 & 1480.34 & 1481.21 & 1486.66 & 1488.12 & 1492.46 & 1495.69 & 1497.16 \\
1510.18 & 1521.19 & 1529.03 & 1663.67 & 3046.57 & 3051.24 & 3055.90 & 3062.64 & 3077.08 \\
3080.34 & 3116.11 & 3116.71 & 3128.66 & 3139.31 & 3142.76 & 3155.04 & 3156.62 & 3161.28 \\
3168.17 & 3177.25 & 3177.75 & 3183.34 & 3386.40 & 3824.30 & & & & \\
Rotational constants (B/GHZ): & \multicolumn{7}{c}{1.0443521} & 0.2161672 & 0.2111498 &
\end{tabular}




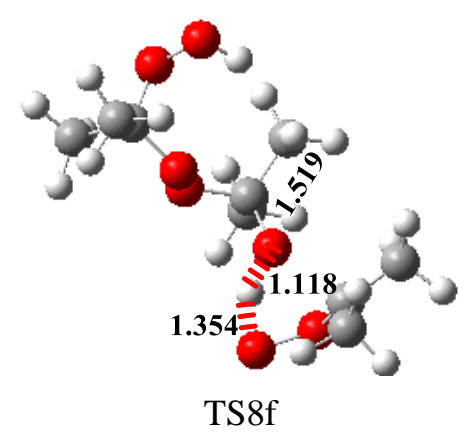

C

$\begin{array}{lll}\text { C } & 1 & \text { B1 }\end{array}$

$\mathrm{H} 2$

$\mathrm{H} \quad 2$

$\mathrm{H} \quad 2$

C 1

$\mathrm{H} \quad 6$

$\mathrm{H} \quad 6$

$\mathrm{H} \quad 6$

O 1

$\mathrm{H} \quad 10$

C 10

C 12

$\mathrm{H} \quad 13$

$\mathrm{H} \quad 13$

$\mathrm{H} \quad 13$

C 12

$\begin{array}{ll}\mathrm{H} & 17\end{array}$

$\begin{array}{ll}\mathrm{H} & 17\end{array}$

$\mathrm{H} \quad 17$

O 12

O 12

O 1

O 23

C 24

C 25

$\mathrm{H} \quad 26$

$\mathrm{H} \quad 26$

$\mathrm{H} \quad 26$

C 25

$\mathrm{H} \quad 30$

$\mathrm{H} \quad 30$

$\mathrm{H} \quad 30$

O 25

O 34

H $\quad 35$

B2 1

B3 1

A1

B4 1

A2 3

D1 0

B5 2

A3 4

D2 0

A4 3

D3 0

B6 1

A5 2

D4 0

B7 1

A6 2

D5 0

B8 1

A7 2

D6 0

B9 6

A8 2

D7 0

B10 1

A9 6

D8 0

B11 1

A10 6

D9 0

B12 10

A11 1

D10 0

B13 12

A12 10

D11 0

B14 12

A13 10

D12 0

B15 12

A14 10

D13 0

B16 10

A15 1

D14 0

B17 12

A16 10

D15 0

B18 12

A17 10

D16 0

B19 12

A18 10

D17 0

B20 10

A19 1

D18 0

B21 10

A20 1

D19 0

B22 10

A21 12

D20 0

B23 1

A22 10

D21 0

B24 23

A23 1

D22 0

B25 24

A24 23

D23 0

B26 25

A25 24

D24 0

B27 25

A26 24

D25 0

B28 25

A27 24

D26 0

B29 24

A28 23

D27 0

B30 25

A29 24

D28 0

B31 25

A30 24

D29 0

B32 25

A31 24

D30 0

B33 24

A32 23

D31 0

B34 25

A33 24

D32 0

B35 34

A34 25

D33 0 
Variables:

\begin{tabular}{|c|c|}
\hline B1 & 1.52292891 \\
\hline B2 & 1.08774116 \\
\hline B3 & 1.08850336 \\
\hline B4 & 1.08823772 \\
\hline B5 & 1.51881122 \\
\hline B6 & 1.08865399 \\
\hline B7 & 1.09087755 \\
\hline B8 & 1.08958795 \\
\hline B9 & 1.40296657 \\
\hline B10 & 1.11823038 \\
\hline B11 & 1.98463547 \\
\hline B12 & 1.48997617 \\
\hline B13 & 1.08754584 \\
\hline B14 & 1.09169300 \\
\hline B15 & 1.08595709 \\
\hline B16 & 1.49309639 \\
\hline B17 & 1.08560001 \\
\hline B18 & 1.08242306 \\
\hline B19 & 1.09365751 \\
\hline B20 & 2.21460198 \\
\hline B21 & 1.28634824 \\
\hline B22 & 1.41604706 \\
\hline $\mathrm{B} 23$ & 1.42456963 \\
\hline B24 & 1.41430839 \\
\hline B25 & 1.51724101 \\
\hline B26 & 1.08601902 \\
\hline B27 & 1.08794484 \\
\hline B28 & 1.08773845 \\
\hline B29 & 1.51732738 \\
\hline B30 & 1.08768798 \\
\hline B31 & 1.08598038 \\
\hline B32 & 1.08782364 \\
\hline B33 & 1.41464185 \\
\hline B34 & 1.41450970 \\
\hline B35 & 0.96473408 \\
\hline A1 & 110.01225807 \\
\hline $\mathrm{A} 2$ & 109.93129696 \\
\hline A3 & 109.69695735 \\
\hline A4 & 112.64478361 \\
\hline A5 & 110.03235749 \\
\hline A6 & 109.44286386 \\
\hline A7 & 109.36894348 \\
\hline A8 & 108.80518114 \\
\hline
\end{tabular}




\begin{tabular}{|c|c|}
\hline A9 & 109.94489217 \\
\hline A10 & 126.58062816 \\
\hline A11 & 110.27152375 \\
\hline A12 & 109.39542719 \\
\hline A13 & 108.26521946 \\
\hline A14 & 111.37860712 \\
\hline A15 & 91.41440307 \\
\hline A16 & 110.09189151 \\
\hline A17 & 111.57211418 \\
\hline A18 & 106.08417925 \\
\hline A19 & 69.03750650 \\
\hline A20 & 93.52167679 \\
\hline A21 & 108.39359476 \\
\hline A22 & 109.40623556 \\
\hline A23 & 108.24518331 \\
\hline A24 & 104.10401668 \\
\hline A25 & 110.96564270 \\
\hline A26 & 108.82564669 \\
\hline A 27 & 109.22609893 \\
\hline A28 & 112.89171662 \\
\hline A29 & 109.12456871 \\
\hline A30 & 110.65795043 \\
\hline A31 & 109.05598258 \\
\hline A32 & 110.44056820 \\
\hline A33 & 110.01330653 \\
\hline A34 & 102.19987485 \\
\hline D1 & 119.23860208 \\
\hline D2 & 120.82171425 \\
\hline D3 & 60.22757490 \\
\hline D4 & -51.69084916 \\
\hline D5 & 67.32620798 \\
\hline D6 & -170.79679304 \\
\hline D7 & 126.05873743 \\
\hline D8 & -169.66488206 \\
\hline D9 & -82.20148917 \\
\hline D10 & 45.02347720 \\
\hline D11 & 47.69495530 \\
\hline D12 & 165.10662643 \\
\hline D13 & -74.92638000 \\
\hline D14 & 165.50907104 \\
\hline D15 & -63.87812597 \\
\hline D16 & 59.44332394 \\
\hline D17 & 178.41398401 \\
\hline D18 & -101.38322835 \\
\hline
\end{tabular}




$\begin{array}{lr}\text { D19 } & -72.56013583 \\ \text { D20 } & 156.31976267 \\ \text { D21 } & 64.16378371 \\ \text { D22 } & 139.92163431 \\ \text { D23 } & 166.19139878 \\ \text { D24 } & 47.17617989 \\ \text { D25 } & 167.01288101 \\ \text { D26 } & -73.34276878 \\ \text { D27 } & 43.40949485 \\ \text { D28 } & 167.41114105 \\ \text { D29 } & -72.21392791 \\ \text { D30 } & 47.74044689 \\ \text { D31 } & -72.61888122 \\ \text { D32 } & -70.02219485 \\ \text { D33 } & 87.35763461\end{array}$

Harmonic Vibrational Frequencies $\left(\mathbf{c m}^{-\mathbf{1}}\right)$ :

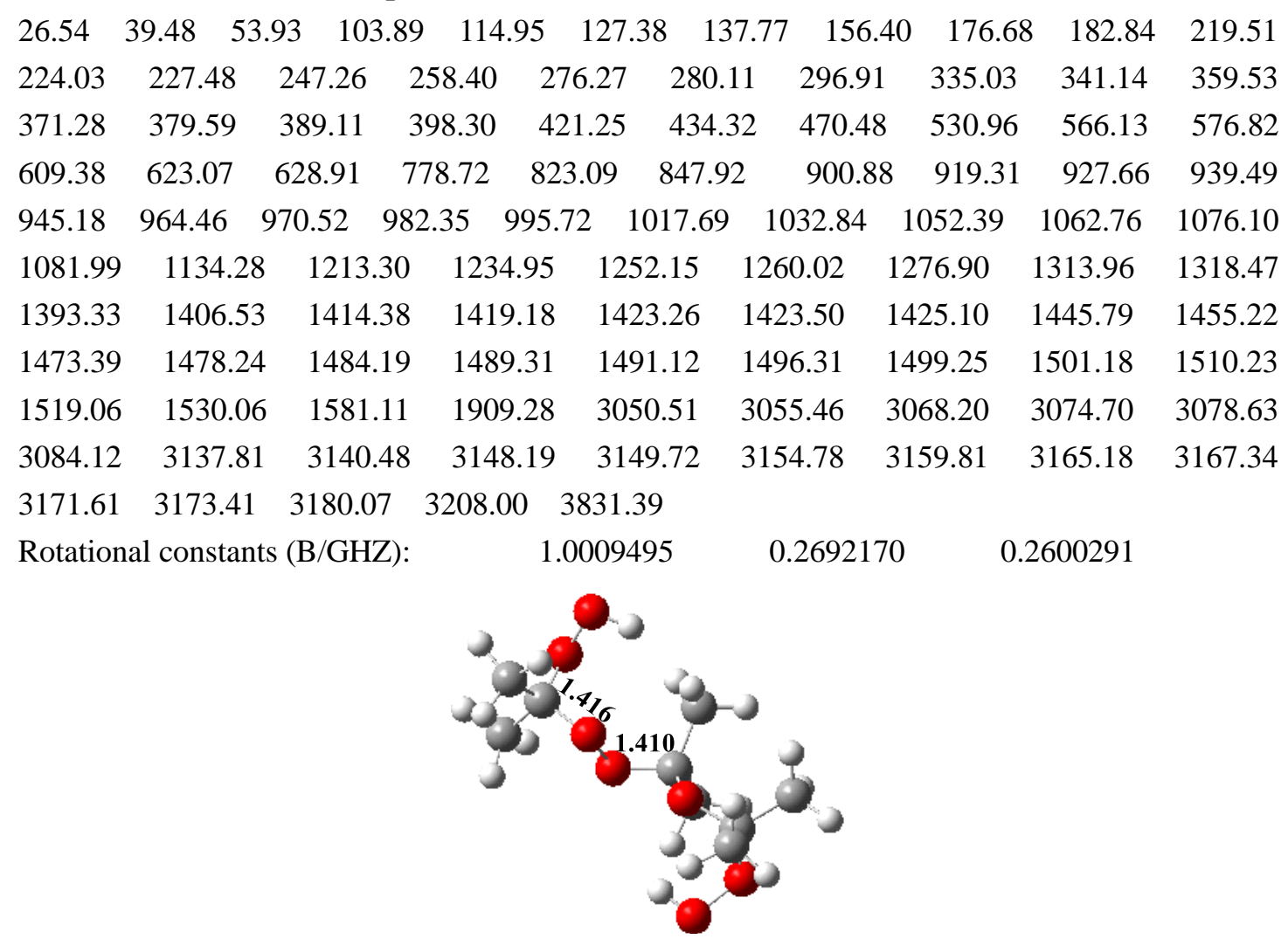

P8f

C

$\begin{array}{lll}\text { C } & 1 & \text { B1 }\end{array}$

$\begin{array}{lllll}\mathrm{H} & 2 & \mathrm{~B} 2 & 1 & \mathrm{~A} 1\end{array}$

$\begin{array}{llllllll}\mathrm{H} & 2 & \text { B3 } & 1 & \text { A2 } & 3 & \text { D1 } & 0 \\ \mathrm{H} & 2 & \text { B4 } & 1 & \text { A3 } & 4 & \text { D2 } & 0 \\ \mathrm{C} & 1 & \text { B5 } & 2 & \text { A4 } & 3 & \text { D3 } & 0 \\ \mathrm{H} & 6 & \text { B6 } & 1 & \text { A5 } & 2 & \text { D4 } & 0\end{array}$




\begin{tabular}{|c|c|c|c|c|c|c|}
\hline $\mathrm{H}$ & 6 & B7 & 1 & A6 & 2 & D5 \\
\hline $\mathrm{H}$ & 6 & B8 & 1 & A7 & 2 & D6 \\
\hline $\mathrm{O}$ & 1 & B9 & 6 & A8 & 2 & D7 \\
\hline $\mathrm{H}$ & 10 & B10 & 1 & A9 & 6 & D8 \\
\hline $\mathrm{C}$ & 10 & B11 & 1 & A10 & 6 & D9 \\
\hline $\mathrm{C}$ & 12 & B12 & 10 & A11 & 1 & D10 \\
\hline $\mathrm{H}$ & 13 & B13 & 12 & A12 & 10 & D11 \\
\hline $\mathrm{H}$ & 13 & B14 & 12 & A13 & 10 & D12 \\
\hline $\mathrm{H}$ & 13 & B15 & 12 & A14 & 10 & D13 \\
\hline $\mathrm{C}$ & 12 & B16 & 10 & A15 & 1 & D14 \\
\hline $\mathrm{H}$ & 17 & B17 & 12 & A16 & 10 & D15 \\
\hline $\mathrm{H}$ & 17 & B18 & 12 & A17 & 10 & D16 \\
\hline $\mathrm{H}$ & 17 & B19 & 12 & A18 & 10 & D17 \\
\hline $\mathrm{O}$ & 12 & B20 & 10 & A19 & 1 & D18 \\
\hline $\mathrm{O}$ & 21 & B21 & 12 & A20 & 10 & D19 \\
\hline $\mathrm{O}$ & 1 & B22 & 10 & A21 & 12 & D20 \\
\hline $\mathrm{O}$ & 23 & B23 & 1 & A 22 & 10 & D21 \\
\hline $\mathrm{C}$ & 24 & B24 & 23 & A23 & 1 & D22 \\
\hline $\mathrm{C}$ & 25 & B25 & 24 & A24 & 23 & D23 \\
\hline $\mathrm{H}$ & 26 & B26 & 25 & A25 & 24 & D24 \\
\hline $\mathrm{H}$ & 26 & B27 & 25 & A26 & 24 & D25 \\
\hline $\mathrm{H}$ & 26 & B28 & 25 & A27 & 24 & D26 \\
\hline $\mathrm{C}$ & 25 & B29 & 24 & A28 & 23 & D27 \\
\hline $\mathrm{H}$ & 30 & B30 & 25 & A29 & 24 & D28 \\
\hline $\mathrm{H}$ & 30 & B31 & 25 & $\mathrm{~A} 30$ & 24 & D29 \\
\hline $\mathrm{H}$ & 30 & B32 & 25 & A31 & 24 & D30 \\
\hline $\mathrm{O}$ & 25 & B33 & 24 & A32 & 23 & D31 \\
\hline $\mathrm{O}$ & 34 & B34 & 25 & A33 & 24 & D32 \\
\hline $\mathrm{H}$ & 35 & B35 & 34 & A34 & 25 & D33 \\
\hline
\end{tabular}

Variables:

$\begin{array}{ll}\text { B1 } & 1.52323570 \\ \text { B2 } & 1.08462549 \\ \text { B3 } & 1.08821815 \\ \text { B4 } & 1.08863244 \\ \text { B5 } & 1.52252559 \\ \text { B6 } & 1.08690584 \\ \text { B7 } & 1.09100579 \\ \text { B8 } & 1.08935748 \\ \text { B9 } & 1.41972855 \\ \text { B10 } & 2.26696523 \\ \text { B11 } & 1.40982482 \\ \text { B12 } & 1.52080138 \\ \text { B13 } & 1.08825513 \\ \text { B14 } & 1.08777496\end{array}$




\begin{tabular}{|c|c|}
\hline B15 & 1.08694670 \\
\hline B16 & 1.51846699 \\
\hline B17 & 1.08826994 \\
\hline B18 & 1.08553843 \\
\hline B19 & 1.08821535 \\
\hline B20 & 2.31265431 \\
\hline B21 & 1.41410261 \\
\hline B22 & 1.41022553 \\
\hline B23 & 1.42399169 \\
\hline B24 & 1.41604005 \\
\hline B25 & 1.51737491 \\
\hline B26 & 1.08604708 \\
\hline B27 & 1.08790671 \\
\hline B28 & 1.08763825 \\
\hline B29 & 1.51708388 \\
\hline B30 & 1.08761042 \\
\hline B31 & 1.08607505 \\
\hline B32 & 1.08782948 \\
\hline B33 & 1.41363539 \\
\hline B34 & 1.41415447 \\
\hline B35 & 0.96508446 \\
\hline A1 & 111.55367028 \\
\hline $\mathrm{A} 2$ & 110.66116666 \\
\hline $\mathrm{A} 3$ & 108.14972104 \\
\hline A4 & 112.32907726 \\
\hline A5 & 110.89932161 \\
\hline A6 & 108.60203229 \\
\hline A7 & 109.47687648 \\
\hline A8 & 110.99120760 \\
\hline A9 & 101.56890624 \\
\hline A 10 & 124.54656174 \\
\hline A11 & 115.40338212 \\
\hline A12 & 109.22186334 \\
\hline A13 & 108.85536360 \\
\hline A14 & 112.21242925 \\
\hline A 15 & 105.05282363 \\
\hline A16 & 109.42384384 \\
\hline A 17 & 110.34185917 \\
\hline A18 & 109.34421582 \\
\hline A19 & 90.20219656 \\
\hline A20 & 35.28236338 \\
\hline $\mathrm{A} 21$ & 104.57376450 \\
\hline A22 & 109.59625653 \\
\hline A23 & 108.36075782 \\
\hline
\end{tabular}




\begin{tabular}{|c|c|}
\hline $\mathrm{A} 24$ & 103.97156694 \\
\hline A25 & 110.96793453 \\
\hline A26 & 108.84490399 \\
\hline A 27 & 109.24775055 \\
\hline A28 & 112.89310615 \\
\hline A29 & 109.01917383 \\
\hline A30 & 110.74594179 \\
\hline A31 & 109.11393009 \\
\hline A 32 & 110.41064844 \\
\hline A33 & 110.08050208 \\
\hline A34 & 102.33085284 \\
\hline D1 & 120.57574930 \\
\hline D2 & 119.20701844 \\
\hline D3 & 63.45308665 \\
\hline D4 & -49.94534562 \\
\hline D5 & 69.01527944 \\
\hline D6 & -169.71740223 \\
\hline D7 & 130.77809170 \\
\hline D8 & -169.12418787 \\
\hline D9 & -85.30612839 \\
\hline D10 & 49.32201141 \\
\hline D11 & 53.35693515 \\
\hline D12 & 172.21135225 \\
\hline D13 & -68.20450785 \\
\hline D14 & 172.13098980 \\
\hline D15 & -69.04283053 \\
\hline D16 & 50.81040881 \\
\hline D17 & 170.96261062 \\
\hline D18 & -97.72722464 \\
\hline D19 & 125.03390516 \\
\hline D20 & 154.90226086 \\
\hline D21 & 64.34663352 \\
\hline D22 & 139.09039004 \\
\hline D23 & 165.80616169 \\
\hline D24 & 46.06274937 \\
\hline D25 & 165.89127130 \\
\hline D26 & -74.41057639 \\
\hline D27 & 43.10664219 \\
\hline D28 & 167.29556928 \\
\hline D29 & -72.44074115 \\
\hline D30 & 47.72235712 \\
\hline D31 & -72.96806768 \\
\hline D32 & -71.06835049 \\
\hline D33 & 87.04093739 \\
\hline
\end{tabular}


Harmonic Vibrational Frequencies $\left(\mathbf{c m}^{-1}\right)$ :

$\begin{array}{lccccccccccc}35.47 & 50.66 & 59.60 & 97.09 & 123.89 & 155.80 & 173.47 & 187.11 & 201.54 & 214.32 & 217.52 \\ 234.65 & 242.67 & 260.76 & 267.56 & 279.87 & 289.69 & 291.18 & 319.90 & 345.63 & 354.00 \\ 372.13 & 376.80 & 379.05 & 390.74 & 398.17 & 425.93 & 436.68 & 496.83 & 533.62 & 550.47 \\ 594.88 & 614.02 & 624.51 & 643.70 & 754.66 & 825.24 & 852.65 & 898.33 & 919.68 & 925.12 \\ 939.42 & 947.80 & 963.56 & 969.41 & 980.40 & 1018.73 & 1021.83 & 1030.82 & 1052.43 & 1057.83 \\ 1065.24 & 1073.37 & 1192.74 & 1214.04 & 1235.74 & 1246.30 & 1252.58 & 1262.57 & 1274.03 \\ 1305.90 & 1320.98 & 1402.53 & 1409.09 & 1414.83 & 1418.57 & 1422.21 & 1423.27 & 1428.65 \\ 1458.31 & 1474.49 & 1482.57 & 1483.23 & 1486.44 & 1487.08 & 1494.25 & 1497.55 & 1499.73 \\ 1507.11 & 1507.55 & 1514.32 & 1531.37 & 3054.27 & 3073.41 & 3074.80 & 3077.38 & 3079.38 \\ 3082.47 & 3138.82 & 3151.43 & 3151.86 & 3156.02 & 3156.53 & 3159.49 & 3163.15 & 3163.56 \\ 3167.95 & 3173.02 & 3177.96 & 3180.86 & 3757.38 & 3823.75 & & & & \end{array}$

$\begin{array}{llll}\text { Rotational constants (B/GHZ): } & 0.9827454 & 0.2863241 & 0.2751025\end{array}$

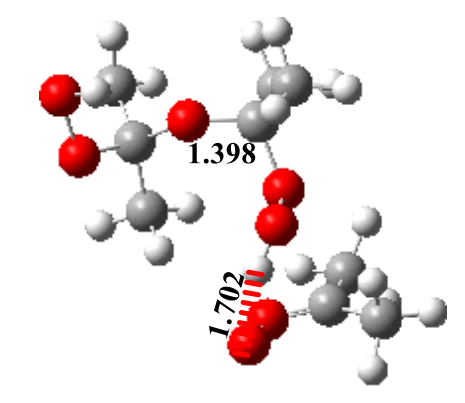

IM8g

$\mathrm{C}$

$\begin{array}{lll}\mathrm{O} & 1 & \mathrm{~B} 1\end{array}$

$\begin{array}{lll}\mathrm{O} & 1 & \mathrm{~B} 2\end{array}$

$\begin{array}{llll}\mathrm{H} & 2 & \mathrm{~B} 3 & 1\end{array}$

C 3

C $\quad 5$

$\mathrm{H} \quad 6$

$\mathrm{H} \quad 6$

$\mathrm{H} \quad 6$

C 5

$\mathrm{H} \quad 10$

$\mathrm{H} \quad 10$

B4 1

B5 3

B6 5

B7 5

B8 5

B9 3

B10 5

B11 5

B12 5

B13 3

B14 3

B15 3

B16 1

B17 1

B18 1

B19 3

B20 1

B21 1

A1

A2 $3 \quad$ D1 0

A3 $2 \quad$ D2 0

A4 $1 \quad$ D3 0

A5 $3 \quad$ D4 0

A6 $3 \quad$ D5 0

A7 $3 \quad$ D6 0

$\begin{array}{llll}\text { A8 } & 1 & \text { D7 } & 0\end{array}$

$\begin{array}{llll}\text { A9 } & 3 & \text { D8 } & 0\end{array}$

$\begin{array}{llll}\mathrm{A} 10 & 3 & \mathrm{D} 9 & 0\end{array}$

A11 $3 \quad$ D10 0

A12 $1 \quad$ D11 0

A13 $1 \quad$ D12 0

A14 2 D13 0

A15 $3 \quad$ D14 0

A16 $3 \quad$ D15 0

A17 $3 \quad$ D16 0

$\begin{array}{llll}\text { A18 } 2 & \text { D17 } & 0\end{array}$

A19 $3 \quad$ D18 0

$\mathrm{H} \quad 20$

A20 3

D19 0 


\begin{tabular}{|c|c|c|c|c|c|c|}
\hline $\mathrm{H}$ & 20 & B22 & 1 & $\mathrm{~A} 21$ & 3 & $\mathrm{D} 20$ \\
\hline $\mathrm{O}$ & 1 & B23 & 3 & A22 & 2 & D21 \\
\hline $\mathrm{C}$ & 24 & B24 & 1 & A23 & 3 & D22 \\
\hline $\mathrm{C}$ & 25 & B25 & 24 & A24 & 1 & D23 \\
\hline $\mathrm{H}$ & 26 & B26 & 25 & A25 & 24 & D24 \\
\hline $\mathrm{H}$ & 26 & B27 & 25 & A26 & 24 & D25 \\
\hline $\mathrm{H}$ & 26 & B28 & 25 & A 27 & 24 & D26 \\
\hline $\mathrm{C}$ & 25 & B29 & 24 & A28 & 1 & D27 \\
\hline $\mathrm{H}$ & 30 & B30 & 25 & A29 & 24 & D28 \\
\hline $\mathrm{H}$ & 30 & B31 & 25 & A30 & 24 & D29 \\
\hline $\mathrm{H}$ & 30 & B32 & 25 & A31 & 24 & D30 \\
\hline $\mathrm{O}$ & 25 & B33 & 24 & A32 & 1 & D31 \\
\hline $\mathrm{O}$ & 34 & B34 & 25 & A33 & 24 & D32 \\
\hline $\mathrm{H}$ & 35 & B35 & 34 & A34 & 25 & D33 \\
\hline
\end{tabular}

Variables:

\begin{tabular}{ll} 
B1 & 2.31853844 \\
B2 & 1.41828594 \\
B3 & 0.99408782 \\
B4 & 2.78739122 \\
B5 & 1.48250182 \\
B6 & 1.08606723 \\
B7 & 1.09246637 \\
B8 & 1.09018543 \\
B9 & 1.47262944 \\
B10 & 1.09393184 \\
B11 & 1.09045301 \\
B12 & 1.08571693 \\
B13 & 2.25215948 \\
B14 & 1.24795013 \\
B15 & 1.51928968 \\
B16 & 1.08845269 \\
B17 & 1.08843900 \\
B18 & 1.08604480 \\
B19 & 1.52389330 \\
B20 & 1.08857806 \\
B21 & 1.08847642 \\
B22 & 1.08785285 \\
B23 & 1.39828603 \\
B24 & 1.42439799 \\
B25 & 1.52310456 \\
B26 & 1.08885652 \\
B27 & 1.08682775 \\
B28 & 1.08704711 \\
B29 & 1.51668841 \\
& \\
\hline
\end{tabular}




$\begin{array}{lr}\text { B30 } & 1.08700260 \\ \text { B31 } & 1.08787657 \\ \text { B32 } & 1.08898064 \\ \text { B33 } & 1.41270178 \\ \text { B34 } & 1.41411684 \\ \text { B35 } & 0.96632749 \\ \text { A1 } & 35.39701443 \\ \text { A2 } & 123.39208814 \\ \text { A3 } & 155.63149642 \\ \text { A4 } & 100.17279658 \\ \text { A5 } & 111.00168193 \\ \text { A6 } & 107.63720103 \\ \text { A7 } & 110.09414549 \\ \text { A8 } & 90.36040851 \\ \text { A9 } & 108.11610179 \\ \text { A10 } & 108.67641230 \\ \text { A11 } & 110.80685276 \\ \text { A12 } & 74.48041482 \\ \text { A13 } & 78.49651031 \\ \text { A14 } & 110.80310733 \\ \text { A15 } & 109.87803335 \\ \text { A16 } & 109.41046141 \\ \text { A17 } & 109.54937119 \\ \text { A18 } & 102.86686799 \\ \text { D19 } & 109.15550229 \\ \text { A20 } & 111.39677123 \\ \text { A21 } & 109.56158283 \\ \text { A22 } & 112.32176818 \\ \text { A23 } & 125.36751287 \\ \text { A24 } & 111.53900694 \\ \text { A25 } & 108.56790299 \\ \text { A26 } & 110.29429350 \\ \text { A27 } & 111.28177260 \\ \text { A28 } & 114.64191989 \\ \text { A29 } & 110.99997089 \\ \text { A30 } & 110.74060395 \\ \text { A3 } & \end{array}$




$\begin{array}{lr}\text { D5 } & 35.49337289 \\ \text { D6 } & 152.58071343 \\ \text { D7 } & 142.86109003 \\ \text { D8 } & 6.64142460 \\ \text { D9 } & 121.60018803 \\ \text { D10 } & -114.54392638 \\ \text { D11 } & -128.99304855 \\ \text { D12 } & -95.15201306 \\ \text { D13 } & 53.53964410 \\ \text { D14 } & 52.04904216 \\ \text { D15 } & 172.20518153 \\ \text { D16 } & -67.98070988 \\ \text { D17 } & 172.70364417 \\ \text { D18 } & -66.73981256 \\ \text { D19 } & 53.18896654 \\ \text { D20 } & 174.04251048 \\ \text { D21 } & -63.36827522 \\ \text { D22 } & -66.54062301 \\ \text { D23 } & -83.06597635 \\ \text { D24 } & -161.42384972 \\ \text { D25 } & -42.35124530 \\ \text { D26 } & 79.25164638 \\ \text { D27 } & 47.25467762 \\ \text { D28 } & -68.61741084 \\ \text { D29 } & 52.36682363 \\ \text { D30 } & 172.05643725 \\ \text { D31 } & 158.52462700 \\ \text { D32 } & 61.42543371 \\ \text { D33 } & -76.68264449 \\ \text { Dar } & \end{array}$

Harmonic Vibrational Frequencies $\left(\mathbf{c m}^{-1}\right)$ :

\begin{tabular}{lccccccccccc}
39.46 & 40.92 & 66.54 & 75.64 & 94.93 & 111.47 & 128.11 & 133.60 & 152.44 & 179.44 & 194.76 \\
206.61 & 209.89 & 222.88 & 241.77 & 274.71 & 298.65 & 324.71 & 328.15 & 328.88 & 335.53 \\
338.39 & 360.21 & 373.35 & 378.54 & 390.91 & 407.13 & 414.46 & 485.07 & 501.60 & 536.55 \\
557.60 & 598.89 & 602.09 & 637.28 & 754.66 & 785.13 & 831.81 & 849.79 & 902.61 & 915.90 \\
924.30 & 940.25 & 946.28 & 956.00 & 973.17 & 990.14 & 1020.82 & 1023.84 & 1056.88 & 1066.23 \\
1077.33 & 1091.50 & 1110.38 & 1201.45 & 1232.76 & 1249.27 & 1263.11 & 1280.17 & 1303.03 \\
1318.02 & 1395.52 & 1405.59 & 1410.24 & 1410.77 & 1417.89 & 1427.07 & 1443.41 & 1450.91 \\
1468.08 & 1477.98 & 1480.72 & 1482.65 & 1490.82 & 1491.13 & 1494.74 & 1499.61 & 1504.56 \\
1517.38 & 1521.01 & 1620.56 & 1673.43 & 3048.56 & 3060.79 & 3066.12 & 3075.49 & 3079.30 \\
3084.41 & 3114.73 & 3127.24 & 3143.47 & 3148.73 & 3155.47 & 3162.60 & 3164.79 & 3168.52 \\
3170.76 & 3172.61 & 3174.38 & 3176.72 & 3203.82 & 3803.72 & & & & \\
Rotational constants (B/GHZ): & \multicolumn{8}{c}{0.7920870} & 0.3283419 & 0.3053114 &
\end{tabular}




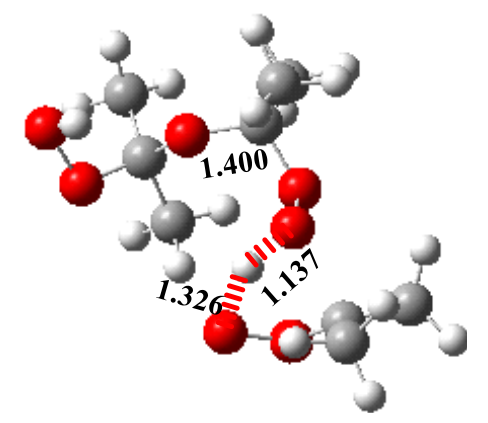

TS8g

C

$\begin{array}{lll}\mathrm{O} & 1 & \mathrm{~B} 1\end{array}$

O 2

B2

B3 1

B4 1

B5 2

B6 5

B7 5

B8 5

B9 2

B10 5

B11 5

B12 5

B13 2

B14 2

B15 3

B16 1

B17

B18 1

B19 3

B20 1

B21 1

B22 1

B23

B24 1

B25 24

B26 25

B27 25

B28 25

B29 24

B30 25

B31 25

B32 25

B33 24

A1

A2 3

D1 0

A3 3

D2 0

A4 1

D3 0

A5 2

D4 0

A6 2

D5 0

A7 2

D6 0

A8 1

D7 0

$\begin{array}{llll}\text { A9 } & 2 & \text { D8 } & 0\end{array}$

$\begin{array}{llll}\text { A10 } 2 & \text { D9 } & 0\end{array}$

$\begin{array}{llll}\text { A11 } 2 & \text { D10 } & 0\end{array}$

A12 1

D11 0

A13 1

D12 0

A14 2

D13 0

A15 3

D14 0

A16 3

D15 0

A17 3

D16 0

A18 2

D17 0

A19 3

D18 0

A20 3

D19 0

A21 3

D20 0

A22 2

D21 0

$\begin{array}{llll}\mathrm{A} 23 & 3 & \mathrm{D} 22 & 0\end{array}$

A24 11 D23 0

A25 $24 \quad$ D24 0

A26 $24 \quad$ D25 0

A27 $24 \quad$ D26 0

$\begin{array}{llll}\text { A28 } & 1 & \text { D27 } & 0\end{array}$

$\begin{array}{llll}\text { A29 } 24 & \text { D28 } & 0\end{array}$

$\begin{array}{llll}\text { A30 } & 24 & \text { D29 } & 0\end{array}$

$\begin{array}{llll}\text { A31 } & 24 & \text { D30 } & 0\end{array}$

A32 $1 \quad$ D31 0

$\begin{array}{llllll}\text { B34 } & 25 & \text { A33 } & 24 & \text { D32 } & 0\end{array}$ 


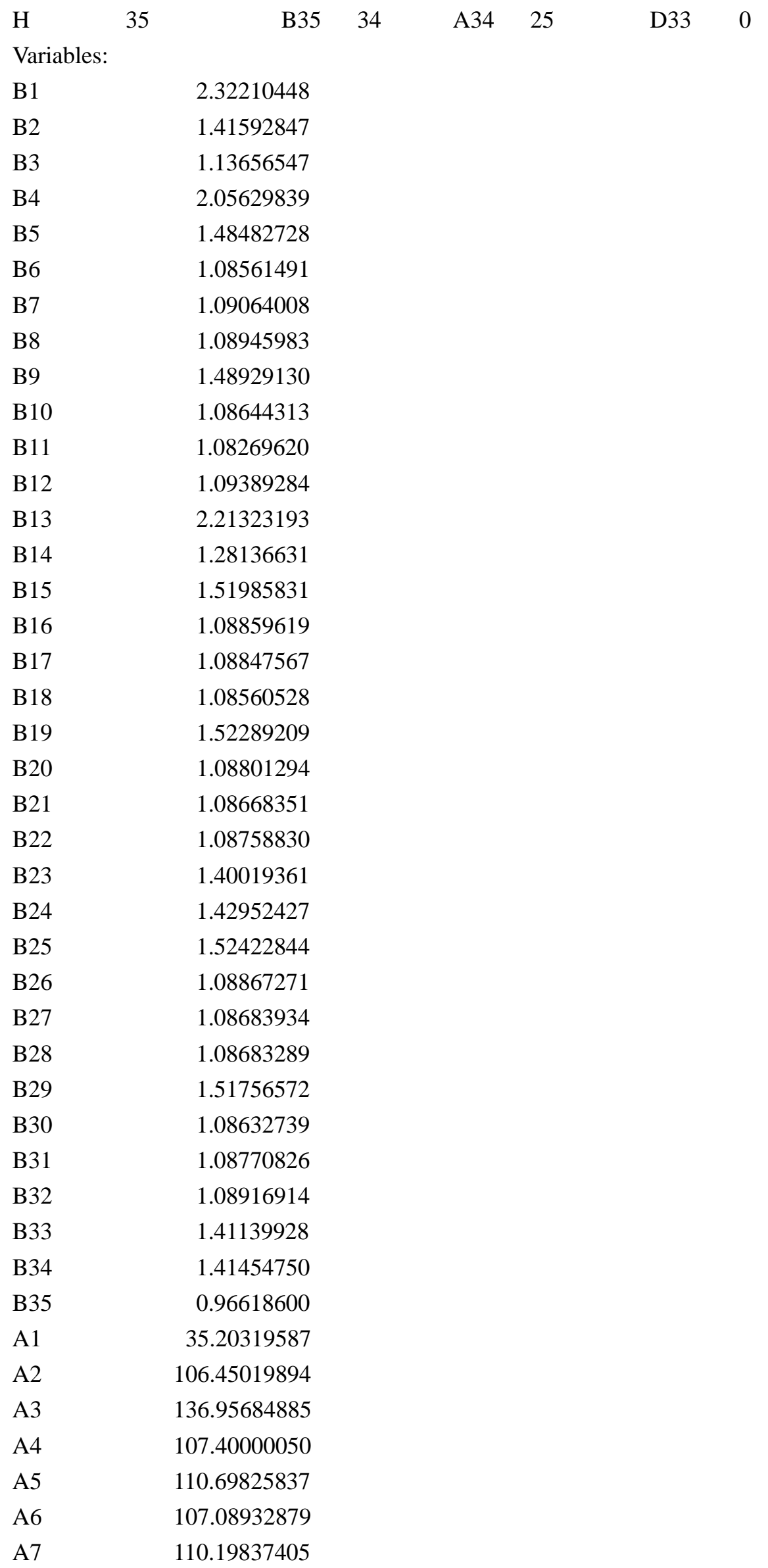




\begin{tabular}{|c|c|}
\hline A 8 & 90.98183625 \\
\hline A9 & 109.61461942 \\
\hline A 10 & 111.88785221 \\
\hline A11 & 106.30250244 \\
\hline A12 & 67.71480690 \\
\hline A13 & 90.97640390 \\
\hline A14 & 111.22881191 \\
\hline A15 & 109.78299764 \\
\hline A16 & 109.10323845 \\
\hline A 17 & 110.12483942 \\
\hline A18 & 103.18592891 \\
\hline A19 & 108.69572466 \\
\hline A20 & 111.87768147 \\
\hline A21 & 109.51047365 \\
\hline A22 & 111.07844750 \\
\hline $\mathrm{A} 23$ & 124.94060005 \\
\hline A24 & 111.69789769 \\
\hline A25 & 108.27906484 \\
\hline A26 & 110.59975367 \\
\hline A 27 & 111.18836855 \\
\hline A28 & 114.64747914 \\
\hline A29 & 111.09753033 \\
\hline A 30 & 110.26150076 \\
\hline A31 & 108.08616627 \\
\hline A32 & 103.29184706 \\
\hline A33 & 109.50353012 \\
\hline A34 & 101.51795860 \\
\hline D1 & -93.24753495 \\
\hline D2 & -7.60476205 \\
\hline D3 & 48.53986448 \\
\hline D4 & -95.04524278 \\
\hline D5 & 23.78551328 \\
\hline D6 & 141.59935625 \\
\hline D7 & 169.80344290 \\
\hline D8 & -67.12587435 \\
\hline D9 & 56.46320559 \\
\hline D10 & 175.56604397 \\
\hline D11 & -97.17945785 \\
\hline D12 & -67.52221967 \\
\hline D13 & 48.14996969 \\
\hline D14 & 49.72754848 \\
\hline D15 & 169.56973472 \\
\hline D16 & -70.51500502 \\
\hline D17 & 167.00506191 \\
\hline
\end{tabular}




$\begin{array}{lr}\text { D18 } & -66.52233343 \\ \text { D19 } & 53.15991507 \\ \text { D20 } & 174.54391476 \\ \text { D21 } & -68.67370834 \\ \text { D22 } & -73.07142732 \\ \text { D23 } & -79.81703988 \\ \text { D24 } & -157.52244561 \\ \text { D25 } & -38.54249103 \\ \text { D26 } & 83.42478458 \\ \text { D27 } & 50.55552095 \\ \text { D28 } & -64.58098663 \\ \text { D29 } & 56.36092132 \\ \text { D30 } & 176.06185134 \\ \text { D31 } & 161.80059164 \\ \text { D32 } & 63.24565856 \\ \text { D33 } & -76.83444381\end{array}$

Harmonic Vibrational Frequencies $\left(\mathbf{c m}^{-1}\right)$ :

$\begin{array}{llllllllllll}764.48 i & 30.41 & 45.26 & 83.37 & 88.13 & 116.58 & 119.94 & 134.91 & 154.44 & 179.36 & 194.44 \\ 214.08 & 226.50 & 228.49 & 255.67 & 265.53 & 280.87 & 293.57 & 311.91 & 322.98 & 343.85 \\ 356.90 & 367.78 & 374.63 & 383.64 & 391.11 & 414.19 & 427.89 & 482.96 & 533.45 & 545.56 \\ 554.32 & 575.87 & 624.66 & 637.38 & 752.89 & 808.23 & 848.80 & 879.67 & 902.09 & 922.09 \\ 943.62 & 945.06 & 948.99 & 961.03 & 973.21 & 982.53 & 1019.96 & 1023.32 & 1054.68 & 1069.06 \\ 1081.78 & 1082.71 & 1120.45 & 1205.52 & 1227.88 & 1247.91 & 1259.95 & 1280.15 & 1303.90 \\ 1313.61 & 1402.33 & 1407.58 & 1408.78 & 1418.75 & 1421.21 & 1429.94 & 1441.62 & 1448.56 \\ 1458.61 & 1472.61 & 1477.04 & 1484.70 & 1489.48 & 1493.18 & 1496.87 & 1497.43 & 1501.80 \\ 1512.33 & 1516.81 & 1582.38 & 1612.79 & 1784.91 & 3051.91 & 3072.68 & 3073.11 & 3075.43 \\ 3076.23 & 3080.81 & 3144.78 & 3147.92 & 3149.71 & 3151.04 & 3154.73 & 3159.15 & 3165.67 \\ 3171.62 & 3172.73 & 3179.56 & 3184.04 & 3209.60 & 3805.94 & & & & \end{array}$

$\begin{array}{llll}\text { Rotational constants (B/GHZ): } & 0.8198346 & 0.3409872 & 0.3016902\end{array}$

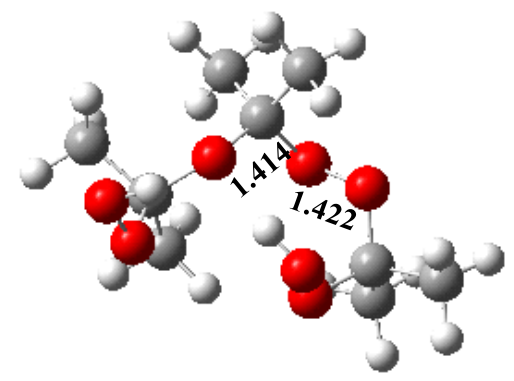

P8g

$\mathrm{C}$

$\begin{array}{lll}\mathrm{O} & 1 & \mathrm{~B} 1\end{array}$

$\begin{array}{lllll}\mathrm{O} & 1 & \mathrm{~B} 2 & 2 & \mathrm{~A} 1\end{array}$

$\begin{array}{llllllll}\mathrm{H} & 2 & \mathrm{~B} 3 & 1 & \mathrm{~A} 2 & 3 & \mathrm{D} 1 & 0\end{array}$

$\begin{array}{llllllll}\mathrm{C} & 2 & \mathrm{~B} 4 & 1 & \mathrm{~A} 3 & 3 & \mathrm{D} 2 & 0\end{array}$

$\begin{array}{llllllll}\mathrm{C} & 5 & \mathrm{~B} 5 & 2 & \mathrm{~A} 4 & 1 & \text { D3 } & 0\end{array}$

$\begin{array}{llllllll}\mathrm{H} & 6 & \mathrm{~B} 6 & 5 & \mathrm{~A} 5 & 2 & \mathrm{D} 4 & 0\end{array}$ 


\begin{tabular}{|c|c|c|c|c|c|c|}
\hline $\mathrm{H}$ & 6 & B7 & 5 & A6 & 2 & D5 \\
\hline $\mathrm{H}$ & 6 & B8 & 5 & A7 & 2 & D6 \\
\hline $\mathrm{C}$ & 5 & B9 & 2 & A8 & 1 & D7 \\
\hline $\mathrm{H}$ & 10 & B10 & 5 & A9 & 2 & D8 \\
\hline $\mathrm{H}$ & 10 & B11 & 5 & A10 & 2 & D9 \\
\hline $\mathrm{H}$ & 10 & B12 & 5 & A11 & 2 & D10 \\
\hline $\mathrm{O}$ & 5 & B13 & 2 & A12 & 1 & D11 \\
\hline $\mathrm{O}$ & 5 & B14 & 2 & A13 & 1 & D12 \\
\hline $\mathrm{C}$ & 1 & B15 & 3 & A14 & 2 & D13 \\
\hline $\mathrm{H}$ & 16 & B16 & 1 & A15 & 3 & D14 \\
\hline $\mathrm{H}$ & 16 & B 17 & 1 & A16 & 3 & D15 \\
\hline $\mathrm{H}$ & 16 & B18 & 1 & A17 & 3 & D16 \\
\hline $\mathrm{C}$ & 1 & B19 & 3 & A18 & 2 & D17 \\
\hline $\mathrm{H}$ & 20 & B20 & 1 & A19 & 3 & D18 \\
\hline $\mathrm{H}$ & 20 & B21 & 1 & A20 & 3 & D19 \\
\hline $\mathrm{H}$ & 20 & B22 & 1 & A21 & 3 & D20 \\
\hline $\mathrm{O}$ & 1 & B23 & 3 & A22 & 2 & D21 \\
\hline $\mathrm{C}$ & 24 & B24 & 1 & $\mathrm{~A} 23$ & 3 & D22 \\
\hline $\mathrm{C}$ & 25 & B25 & 24 & A24 & 1 & D23 \\
\hline $\mathrm{H}$ & 26 & B26 & 25 & A25 & 24 & D24 \\
\hline $\mathrm{H}$ & 26 & B27 & 25 & A26 & 24 & D25 \\
\hline $\mathrm{H}$ & 26 & B28 & 25 & A27 & 24 & D26 \\
\hline $\mathrm{C}$ & 25 & B29 & 24 & A28 & 1 & D27 \\
\hline $\mathrm{H}$ & 30 & B30 & 25 & A29 & 24 & D28 \\
\hline $\mathrm{H}$ & 30 & B31 & 25 & A30 & 24 & D29 \\
\hline $\mathrm{H}$ & 30 & B32 & 25 & $\mathrm{~A} 31$ & 24 & D30 \\
\hline $\mathrm{O}$ & 25 & B33 & 24 & A32 & 1 & D31 \\
\hline $\mathrm{O}$ & 34 & B34 & 25 & A33 & 24 & D32 \\
\hline $\mathrm{H}$ & 35 & B35 & 34 & A34 & 25 & D33 \\
\hline
\end{tabular}

Variables:

$\begin{array}{ll}\text { B1 } & 2.32158435 \\ \text { B2 } & 1.42022542 \\ \text { B3 } & 2.64911617 \\ \text { B4 } & 1.41310944 \\ \text { B5 } & 1.5163117 \\ \text { B6 } & 1.08685373 \\ \text { B7 } & 1.08802861 \\ \text { B8 } & 1.08783284 \\ \text { B9 } & 1.51793281 \\ \text { B10 } & 1.08769932 \\ \text { B11 } & 1.08644205 \\ \text { B12 } & 1.08802661 \\ \text { B13 } & 2.30820016 \\ \text { B14 } & 1.40780521\end{array}$




\begin{tabular}{|c|c|}
\hline B15 & 1.51981948 \\
\hline B16 & 1.08788338 \\
\hline B17 & 1.08829814 \\
\hline B18 & 1.08636329 \\
\hline B19 & 1.52252668 \\
\hline B20 & 1.08752177 \\
\hline B21 & 1.08670405 \\
\hline B22 & 1.08715216 \\
\hline B23 & 1.41426041 \\
\hline B24 & 1.43578929 \\
\hline B25 & 1.52346863 \\
\hline B26 & 1.08856740 \\
\hline B27 & 1.08656004 \\
\hline B28 & 1.08620012 \\
\hline B29 & 1.51805972 \\
\hline B30 & 1.08671291 \\
\hline B31 & 1.08617830 \\
\hline B32 & 1.08874064 \\
\hline B33 & 1.41052186 \\
\hline B34 & 1.41504141 \\
\hline B35 & 0.96508612 \\
\hline A1 & 35.26321706 \\
\hline $\mathrm{A} 2$ & 68.08487571 \\
\hline A3 & 127.65520861 \\
\hline A4 & 112.69575386 \\
\hline A5 & 110.39109228 \\
\hline A6 & 109.07805534 \\
\hline A7 & 109.49573294 \\
\hline A8 & 103.38811872 \\
\hline A9 & 109.99500913 \\
\hline A10 & 110.19505770 \\
\hline A11 & 108.52677048 \\
\hline A12 & 90.36764045 \\
\hline A13 & 111.07515571 \\
\hline A14 & 112.04002922 \\
\hline A15 & 109.91841814 \\
\hline A16 & 108.73127151 \\
\hline A17 & 110.46372841 \\
\hline A18 & 103.29026199 \\
\hline A19 & 108.05507898 \\
\hline A 20 & 112.24055157 \\
\hline A 21 & 109.88623948 \\
\hline A22 & 111.10050208 \\
\hline A 23 & 124.81501055 \\
\hline
\end{tabular}




\begin{tabular}{|c|c|}
\hline A24 & 112.20939126 \\
\hline A25 & 108.06581592 \\
\hline A26 & 110.95904285 \\
\hline A27 & 111.13399794 \\
\hline A28 & 114.09979369 \\
\hline A29 & 110.92784598 \\
\hline A30 & 109.78191471 \\
\hline A31 & 108.29743922 \\
\hline A 32 & 103.15395489 \\
\hline A33 & 109.84998723 \\
\hline A34 & 102.38554809 \\
\hline D1 & -121.15436441 \\
\hline D2 & -71.85217268 \\
\hline D3 & 86.46636630 \\
\hline D4 & -65.19704925 \\
\hline D5 & 54.77521231 \\
\hline D6 & 174.45547251 \\
\hline D7 & -150.74901670 \\
\hline D8 & -65.88193068 \\
\hline D9 & 55.00452859 \\
\hline D10 & 174.36852136 \\
\hline D11 & -59.42185252 \\
\hline D12 & -30.26898840 \\
\hline D13 & 36.82466006 \\
\hline D14 & 48.45958330 \\
\hline D15 & 168.38205868 \\
\hline D16 & -71.71979843 \\
\hline D17 & 155.79598382 \\
\hline D18 & -66.64491941 \\
\hline D19 & 52.43441143 \\
\hline D20 & 174.49264228 \\
\hline D21 & -80.19009417 \\
\hline D22 & -76.78326004 \\
\hline D23 & -76.56902352 \\
\hline D24 & -154.02023340 \\
\hline D25 & -35.08671776 \\
\hline D26 & 87.15965277 \\
\hline D27 & 53.60682935 \\
\hline D28 & -62.40320359 \\
\hline D29 & 58.20166096 \\
\hline D30 & 178.08389873 \\
\hline D31 & 164.64656410 \\
\hline D32 & 70.01985496 \\
\hline D33 & -88.18945459 \\
\hline
\end{tabular}


Harmonic Vibrational Frequencies $\left(\mathrm{cm}^{-1}\right)$ :

\begin{tabular}{lccccccccccc}
23.87 & 61.52 & 90.32 & 117.11 & 130.85 & 133.62 & 148.00 & 174.33 & 180.32 & 202.26 & 219.87 \\
228.57 & 232.74 & 254.29 & 273.52 & 276.02 & 287.07 & 298.26 & 307.72 & 322.82 & 340.87 \\
356.25 & 377.32 & 380.19 & 383.38 & 427.70 & 446.06 & 501.10 & 504.26 & 547.58 & 554.47 \\
589.95 & 597.63 & 631.85 & 640.56 & 750.53 & 831.09 & 853.23 & 895.23 & 913.31 & 928.43 \\
945.59 & 948.34 & 951.43 & 965.43 & 975.31 & 1019.67 & 1021.40 & 1030.53 & 1042.03 & 1057.02 \\
1066.07 & 1072.59 & 1174.89 & 1217.48 & 1230.64 & 1247.96 & 1249.10 & 1259.18 & 1283.94 \\
1297.79 & 1317.47 & 1409.15 & 1410.12 & 1412.96 & 1416.74 & 1419.35 & 1424.44 & 1427.12 \\
1471.60 & 1477.90 & 1479.99 & 1485.43 & 1487.45 & 1489.99 & 1493.45 & 1497.80 & 1503.50 \\
1504.82 & 1514.74 & 1517.27 & 1519.46 & 3069.10 & 3071.26 & 3072.55 & 3075.58 & 3081.78 \\
3085.94 & 3149.66 & 3151.50 & 3151.59 & 3156.22 & 3157.00 & 3158.10 & 3162.58 & 3168.45 \\
3169.81 & 3173.10 & 3176.29 & 3177.93 & 3710.63 & 3832.86 & & & & \\
Rotational constants (B/GHZ): & \multicolumn{8}{c}{0.7707336} & 0.4206507 & 0.3623053 &
\end{tabular}

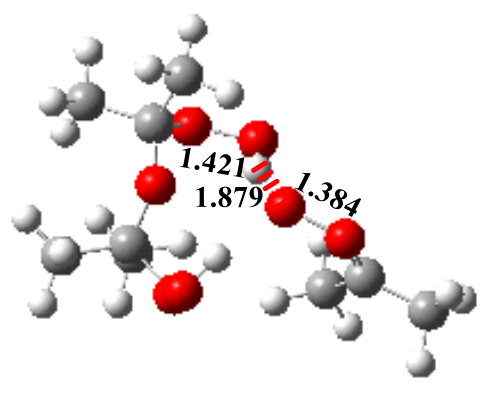

IM8h

C

$\begin{array}{lll}\text { C } & 1 & \text { B1 }\end{array}$

$\begin{array}{lll}\mathrm{O} & 2 & \mathrm{~B} 2\end{array}$

$\mathrm{O} \quad 3$

$\mathrm{H} \quad 3$

C 3

C 6

$\mathrm{H} \quad 7$

$\begin{array}{ll}\mathrm{H} & 7\end{array}$

$\mathrm{H} \quad 7$

O 6

O 6

$\mathrm{H} \quad 1$

$\mathrm{H} \quad 1$

$\mathrm{H} \quad 1$

O 2

C $\quad 16$

C $\quad 17$

$\mathrm{H} \quad 18$

$\mathrm{H} \quad 18$

$\begin{array}{ll}\mathrm{H} & 18\end{array}$

$\begin{array}{ll}\mathrm{O} & 17\end{array}$

$\begin{array}{rrrrrr}\text { B2 } & 1 & \text { A1 } & & & \\ \text { B3 } & 2 & \text { A2 } & 1 & \text { D1 } & 0 \\ \text { B4 } & 2 & \text { A3 } & 1 & \text { D2 } & 0 \\ \text { B5 } & 2 & \text { A4 } & 1 & \text { D3 } & 0 \\ \text { B6 } & 3 & \text { A5 } & 2 & \text { D4 } & 0 \\ \text { B7 } & 6 & \text { A6 } & 3 & \text { D5 } & 0 \\ \text { B8 } & 6 & \text { A7 } & 3 & \text { D6 } & 0 \\ \text { B9 } & 6 & \text { A8 } & 3 & \text { D7 } & 0 \\ \text { B10 } & 3 & \text { A9 } & 2 & \text { D8 } & 0 \\ \text { B11 } & 3 & \text { A10 } & 2 & \text { D9 } & 0 \\ \text { B12 } & 2 & \text { A11 } & 4 & \text { D10 } & 0 \\ \text { B13 } & 2 & \text { A12 } & 4 & \text { D11 } & 0 \\ \text { B14 } & 2 & \text { A13 } & 4 & \text { D12 } & 0 \\ \text { B15 } & 1 & \text { A14 } & 4 & \text { D13 } & 0 \\ \text { B16 } & 2 & \text { A15 } & 1 & \text { D14 } & 0 \\ \text { B17 } & 16 & \text { A16 } & 2 & \text { D15 } & 0 \\ \text { B18 } & 17 & \text { A17 } & 16 & \text { D16 } & 0 \\ \text { B19 } & 17 & \text { A18 } & 16 & \text { D17 } & 0 \\ \text { B20 } & 17 & \text { A19 } & 16 & \text { D18 } & 0 \\ \text { B21 } & 16 & \text { A20 } & 2 & \text { D19 } & 0\end{array}$




$\begin{array}{rrrrrrrr}\mathrm{O} & 22 & \text { B22 } & 17 & \text { A21 } & 16 & \text { D20 } & 0 \\ \mathrm{H} & 23 & \text { B23 } & 22 & \text { A22 } & 17 & \text { D21 } & 0 \\ \mathrm{C} & 6 & \text { B24 } & 3 & \text { A23 } & 2 & \text { D22 } & 0 \\ \mathrm{H} & 25 & \text { B25 } & 6 & \text { A24 } & 3 & \text { D23 } & 0 \\ \mathrm{H} & 25 & \text { B26 } & 6 & \text { A25 } & 3 & \text { D24 } & 0 \\ \mathrm{H} & 25 & \text { B27 } & 6 & \text { A26 } & 3 & \text { D25 } & 0 \\ \mathrm{C} & 2 & \text { B28 } & 1 & \text { A27 } & 16 & \text { D26 } & 0 \\ \mathrm{H} & 29 & \text { B29 } & 2 & \text { A28 } & 1 & \text { D27 } & 0 \\ \mathrm{H} & 29 & \text { B30 } & 2 & \text { A29 } & 1 & \text { D28 } & 0 \\ \mathrm{H} & 29 & \text { B31 } & 2 & \text { A30 } & 1 & \text { D29 } & 0 \\ \mathrm{C} & 17 & \text { B32 } & 16 & \text { A31 } & 2 & \text { D30 } & 0 \\ \mathrm{H} & 33 & \text { B33 } & 17 & \text { A32 } & 16 & \text { D31 } & 0 \\ \mathrm{H} & 33 & \text { B34 } & 17 & \text { A33 } & 16 & \text { D32 } & 0 \\ \text { H } & 33 & \text { B35 } & 17 & \text { A34 } & 16 & \text { D33 } & 0\end{array}$

Variables:

$\begin{array}{ll}\text { B1 } & 1.51757346 \\ \text { B2 } & 2.33124472 \\ \text { B3 } & 1.41232900 \\ \text { B4 } & 0.98229453 \\ \text { B5 } & 3.87534454 \\ \text { B6 } & 1.46983004 \\ \text { B7 } & 1.09561361 \\ \text { B8 } & 1.09504700 \\ \text { B9 } & 1.08551692 \\ \text { B10 } & 2.25308028 \\ \text { B11 } & 1.25008227 \\ \text { B12 } & 1.08884807 \\ \text { B13 } & 1.08892004 \\ \text { B14 } & 1.08722879 \\ \text { B15 } & 1.41881719 \\ \text { B16 } & 1.40995222 \\ \text { B17 } & 1.52134576 \\ \text { B18 } & 1.08729468 \\ \text { B19 } & 1.08828145 \\ \text { B20 } & 1.08831320 \\ \text { B21 } & 1.41103678 \\ \text { B22 } & 1.42113531 \\ \text { B23 } & 0.98078727 \\ \text { B24 } & 1.48352230 \\ \text { B25 } & 1.09119925 \\ \text { B26 } & 1.09103232 \\ \text { B27 } & 1.08559750 \\ \text { B28 } & 1.52245523 \\ \text { B29 } & 1.08902441\end{array}$




$\begin{array}{lr}\text { B30 } & 1.08489540 \\ \text { B31 } & 1.08712097 \\ \text { B32 } & 1.52097894 \\ \text { B33 } & 1.08612564 \\ \text { B34 } & 1.08818976 \\ \text { B35 } & 1.08813734 \\ \text { A1 } & 93.41099525 \\ \text { A2 } & 34.74323489 \\ \text { A3 } & 94.86131564 \\ \text { A4 } & 100.03218726 \\ \text { A5 } & 74.16746429 \\ \text { A6 } & 109.07054232 \\ \text { A7 } & 108.93647042 \\ \text { A8 } & 110.78946552 \\ \text { A9 } & 43.96847222 \\ \text { A10 } & 62.36383670 \\ \text { A11 } & 110.27931618 \\ \text { A12 } & 109.81402251 \\ \text { A13 } & 109.13845780 \\ \text { A14 } & 109.27090144 \\ \text { A15 } & 125.38965377 \\ \text { A16 } & 104.67252562 \\ \text { A17 } & 110.58982293 \\ \text { A18 } & 109.26491566 \\ \text { A19 } & 109.21800086 \\ \text { A20 } & 109.89564676 \\ \text { A21 } & 110.22178497 \\ \text { A22 } & 105.50092919 \\ \text { A23 } & 138.14031110 \\ \text { A24 } & 108.85921965 \\ \text { A25 } & 109.00226767 \\ \text { A26 } & 111.21570886 \\ \text { A27 } & 113.42667137 \\ \text { A28 } & 107.51911212 \\ \text { A29 } & 112.10750224 \\ \text { A30 } & 110.45023751 \\ \text { A3 } & \\ \text { D3 } & 112.3131847421786 \\ \text { A } & \end{array}$




$\begin{array}{lr}\text { D5 } & -9.07370884 \\ \text { D6 } & 105.49957591 \\ \text { D7 } & -132.91647803 \\ \text { D8 } & 70.95554472 \\ \text { D9 } & 105.79596058 \\ \text { D10 } & -177.70479024 \\ \text { D11 } & -57.56257245 \\ \text { D12 } & 62.24906627 \\ \text { D13 } & -115.35959741 \\ \text { D14 } & -97.13033404 \\ \text { D15 } & 176.11726054 \\ \text { D16 } & 48.97727829 \\ \text { D17 } & 169.64275749 \\ \text { D18 } & -70.46332555 \\ \text { D19 } & -63.42705496 \\ \text { D20 } & -61.95462750 \\ \text { D21 } & 33.59558227 \\ \text { D22 } & -155.86585644 \\ \text { D23 } & 44.37192781 \\ \text { D24 } & 160.93710725 \\ \text { D25 } & -77.25460418 \\ \text { D26 } & -131.72966213 \\ \text { D27 } & -60.78390532 \\ \text { D28 } & 58.55573637 \\ \text { D29 } & 179.71344320 \\ \text { D30 } & 54.19287541 \\ \text { D31 } & -66.25256140 \\ \text { D32 } & 54.88780737 \\ \text { D33 } & 173.61597745 \\ \text { Dar } & \end{array}$

Harmonic Vibrational Frequencies $\left(\mathbf{c m}^{-\mathbf{1}}\right)$ :

$\begin{array}{lllllllllll}25.10 & 43.77 & 67.81 & 87.71 & 94.80 & 112.07 & 132.92 & 136.58 & 154.59 & 171.32 & 193.69\end{array}$ $\begin{array}{llllllllll}211.31 & 228.78 & 242.75 & 257.30 & 267.25 & 272.43 & 291.80 & 303.29 & 332.85 & 338.95\end{array}$ $\begin{array}{llllllllll}349.99 & 358.25 & 376.55 & 385.62 & 387.66 & 407.58 & 475.76 & 506.95 & 522.65 & 565.57\end{array}$ $\begin{array}{llllllllll}597.79 & 608.02 & 636.52 & 662.01 & 752.33 & 773.09 & 832.25 & 847.80 & 893.53 & 914.40\end{array}$ $\begin{array}{llllllllll}925.77 & 938.27 & 951.71 & 958.90 & 973.09 & 995.00 & 1019.67 & 1024.46 & 1058.25 & 1070.18\end{array}$ $\begin{array}{lllllllll}1078.10 & 1096.48 & 1106.07 & 1194.96 & 1228.46 & 1248.08 & 1261.41 & 1270.76 & 1305.89\end{array}$ $\begin{array}{lllllllll}1320.42 & 1397.15 & 1405.55 & 1411.24 & 1413.01 & 1423.59 & 1428.19 & 1452.13 & 1466.16\end{array}$ $\begin{array}{lllllllll}1472.64 & 1477.84 & 1484.33 & 1487.50 & 1489.02 & 1491.70 & 1501.38 & 1503.26 & 1509.00\end{array}$ $\begin{array}{llllllllll}1516.15 & 1518.04 & 1548.57 & 1663.14 & 3027.98 & 3056.04 & 3068.90 & 3069.71 & 3081.23\end{array}$ $\begin{array}{lllllllll}3082.98 & 3088.85 & 3117.08 & 3147.86 & 3149.39 & 3159.68 & 3162.07 & 3162.81 & 3163.44\end{array}$ $\begin{array}{llllll}3166.36 & 3171.44 & 3182.07 & 3191.66 & 3476.22 & 3561.38\end{array}$

Rotational constants (B/GHZ): $\quad 0.7665196 \quad 0.3543521 \quad 0.3034123$ 


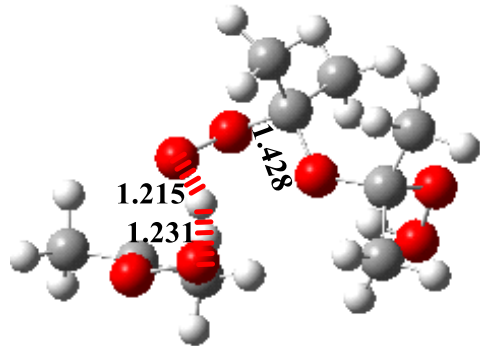

TS8h

C

$\begin{array}{lll}\mathrm{C} & 1 & \text { B1 }\end{array}$

O 2

O 2

B2 1

A1

B3 1

A2 $3 \quad$ D1 0

B4 2

A3 $1 \quad$ D2 0

C 3

B5 2

A4 $1 \quad$ D3 0

C 6

B6 3

A5 $2 \quad$ D4 0

B7 6

A6 $3 \quad$ D5 0

B8 6

A7 $3 \quad$ D6 0

B9 6

A8 $3 \quad$ D7 0

B10 3

A9 2 D8 0

B11 3

$\begin{array}{llll}\mathrm{A} 10 & 2 & \mathrm{D} 9 & 0\end{array}$

B12 2

A11 4 D10 0

B13 2

A12 4 D11 0

B14 2

A13 4

D12 0

B15 1

A14 4

D13 0

B16 2

A15 1 D14 0

B17 16

A16 2

D15 0

B18 17

A17 $16 \quad$ D16 0

B19 17

A18 $16 \quad$ D17 0

B20 17

A19 $16 \quad$ D18 0

B21 16

$\begin{array}{llll}\text { A20 } 2 & \text { D19 } & 0\end{array}$

B22 17

A21 16

D20 0

B23 22

A22 17

D21 0

B24 3

$\begin{array}{llll}\text { A23 } 2 & \text { D22 } & 0\end{array}$

B25 6

A24 3

D23 0

B26 6

A25 3

D24 0

B27 6

A26 $3 \quad$ D25 0

B28 1

A27 4

D26 0

B29 2

A28 1

D27 0

B30 2

A29

D28 0

B31 2

A30 1

D29 0

B32 16

$\begin{array}{llll}\mathrm{A} 31 & 2 & \mathrm{D} 30 & 0\end{array}$

B33 17

A32 16

D31 0

B34 17

A33 16

D32 0

B35 17

A34 $16 \quad$ D33 0 
Variables:

\begin{tabular}{|c|c|}
\hline B1 & 1.52214374 \\
\hline B2 & 2.31717819 \\
\hline B3 & 1.40702772 \\
\hline B4 & 1.21538070 \\
\hline B5 & 2.18173445 \\
\hline B6 & 1.48202667 \\
\hline B7 & 1.08745813 \\
\hline B8 & 1.08378641 \\
\hline B9 & 1.09474741 \\
\hline B10 & 2.21506623 \\
\hline B11 & 1.27670481 \\
\hline B12 & 1.08704920 \\
\hline B13 & 1.08856099 \\
\hline B14 & 1.08698123 \\
\hline B15 & 1.42802621 \\
\hline B16 & 1.40557612 \\
\hline B17 & 1.51785276 \\
\hline B18 & 1.08611283 \\
\hline B19 & 1.08839303 \\
\hline B20 & 1.08836856 \\
\hline B21 & 1.42245047 \\
\hline B22 & 1.41341199 \\
\hline B23 & 0.96789261 \\
\hline B24 & 1.48263146 \\
\hline B25 & 1.09155715 \\
\hline B26 & 1.08877019 \\
\hline B27 & 1.08591517 \\
\hline B28 & 1.51969064 \\
\hline B29 & 1.08851704 \\
\hline B30 & 1.08550282 \\
\hline B31 & 1.08950605 \\
\hline B32 & 1.51995501 \\
\hline B33 & 1.08721488 \\
\hline B34 & 1.08799453 \\
\hline B35 & 1.08790100 \\
\hline A1 & 88.25195254 \\
\hline $\mathrm{A} 2$ & 110.76724924 \\
\hline A3 & 87.76799474 \\
\hline A4 & 130.69427858 \\
\hline A5 & 92.26585079 \\
\hline A6 & 109.41622023 \\
\hline A7 & 111.85823211 \\
\hline A8 & 106.02153260 \\
\hline
\end{tabular}




\begin{tabular}{|c|c|}
\hline A9 & 65.27424690 \\
\hline A10 & 85.47738294 \\
\hline A11 & 111.02255228 \\
\hline A12 & 108.54691651 \\
\hline A13 & 110.35363259 \\
\hline A 14 & 111.54551172 \\
\hline A15 & 125.87645966 \\
\hline A 16 & 104.90071180 \\
\hline A17 & 110.23895157 \\
\hline A18 & 109.62295746 \\
\hline A19 & 109.31670739 \\
\hline A20 & 109.46466357 \\
\hline A 21 & 109.59429181 \\
\hline $\mathrm{A} 22$ & 102.24809777 \\
\hline A23 & 107.25813143 \\
\hline A24 & 106.27063379 \\
\hline A25 & 110.62829646 \\
\hline A26 & 110.95548113 \\
\hline A 27 & 112.79268129 \\
\hline A28 & 108.52721685 \\
\hline A29 & 111.40250144 \\
\hline A 30 & 110.58192226 \\
\hline A31 & 115.70649291 \\
\hline A 32 & 111.89947362 \\
\hline A33 & 109.40072628 \\
\hline A34 & 108.69873420 \\
\hline D1 & 27.16715611 \\
\hline D2 & -96.21172856 \\
\hline D3 & -158.36588189 \\
\hline D4 & -14.99733485 \\
\hline D5 & -64.02825920 \\
\hline D6 & 58.70514099 \\
\hline D7 & 179.07880507 \\
\hline D8 & 76.43731584 \\
\hline D9 & 108.43609377 \\
\hline D10 & -163.89656671 \\
\hline D11 & -44.40046395 \\
\hline D12 & 74.61495498 \\
\hline D13 & -114.39805188 \\
\hline D14 & -80.22560658 \\
\hline D15 & 169.43959361 \\
\hline D16 & 51.79226484 \\
\hline D17 & 172.25535975 \\
\hline D18 & -67.25728397 \\
\hline
\end{tabular}




$\begin{array}{lr}\text { D19 } & -70.42212057 \\ \text { D20 } & -58.95171516 \\ \text { D21 } & 66.45836935 \\ \text { D22 } & -137.34011218 \\ \text { D23 } & 23.38759829 \\ \text { D24 } & 140.82975446 \\ \text { D25 } & -95.26654255 \\ \text { D26 } & 115.54500795 \\ \text { D27 } & -55.30191837 \\ \text { D28 } & 65.16543147 \\ \text { D29 } & -174.24454646 \\ \text { D30 } & 46.03137277 \\ \text { D31 } & -67.21817865 \\ \text { D32 } & 54.29780671 \\ \text { D33 } & 173.26256522\end{array}$

Harmonic Vibrational Frequencies $\left(\mathbf{c m}^{-1}\right)$ :

$\begin{array}{lccccccccccc}1128.63 i & 39.70 & 45.29 & 62.24 & 82.79 & 87.04 & 108.54 & 133.22 & 172.25 & 179.08 & 196.24 \\ 219.59 & 236.88 & 243.35 & 255.02 & 264.38 & 281.23 & 286.69 & 311.31 & 314.57 & 321.12 \\ 351.14 & 356.61 & 371.17 & 382.74 & 390.16 & 400.75 & 407.55 & 481.68 & 497.67 & 539.63 \\ 563.85 & 579.17 & 605.31 & 641.80 & 690.65 & 754.71 & 821.67 & 848.37 & 901.16 & 922.16 \\ 939.51 & 945.11 & 949.00 & 954.46 & 971.16 & 989.39 & 1019.70 & 1025.24 & 1055.31 & 1068.43 \\ 1085.53 & 1090.84 & 1100.98 & 1195.79 & 1228.46 & 1250.58 & 1262.97 & 1276.21 & 1306.26 \\ 1318.44 & 1396.04 & 1404.40 & 1407.87 & 1413.28 & 1421.35 & 1425.74 & 1426.89 & 1447.30 \\ 1452.11 & 1460.75 & 1472.85 & 1473.77 & 1481.92 & 1484.31 & 1491.74 & 1493.95 & 1494.34 \\ 1503.09 & 1511.91 & 1517.54 & 1598.95 & 1917.55 & 3051.54 & 3068.76 & 3070.91 & 3072.63 \\ 3080.21 & 3081.03 & 3141.51 & 3143.67 & 3146.21 & 3146.49 & 3157.16 & 3157.61 & 3163.40 \\ 3168.69 & 3173.36 & 3178.81 & 3179.44 & 3203.44 & 3763.71 & & & \\ \text { Rotational constants (B/GHZ): } & 0.8060942 & 0.3540877 & 0.3256465 & \end{array}$

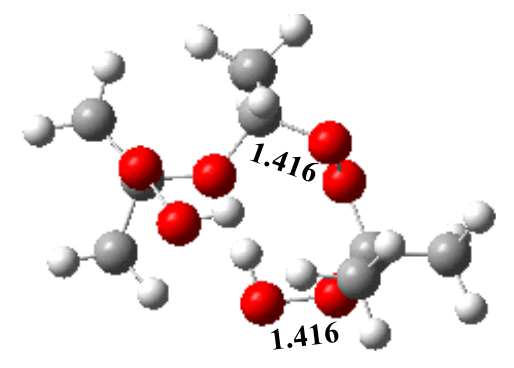

P8h

$\begin{array}{llllllll}\mathrm{C} & & & & & & & \\ \mathrm{C} & 1 & \mathrm{~B} 1 & & & & & \\ \mathrm{O} & 2 & \mathrm{~B} 2 & 1 & \mathrm{~A} 1 & & & \\ \mathrm{O} & 2 & \mathrm{~B} 3 & 1 & \mathrm{~A} 2 & 3 & \mathrm{D} 1 & 0 \\ \mathrm{H} & 3 & \mathrm{~B} 4 & 2 & \mathrm{~A} 3 & 1 & \mathrm{D} 2 & 0 \\ \mathrm{C} & 3 & \mathrm{~B} 5 & 2 & \mathrm{~A} 4 & 1 & \text { D3 } & 0 \\ \mathrm{C} & 6 & \text { B6 } & 3 & \text { A5 } & 2 & \text { D4 } & 0 \\ \mathrm{H} & 7 & \text { B7 } & 6 & \text { A6 } & 3 & \text { D5 } & 0\end{array}$




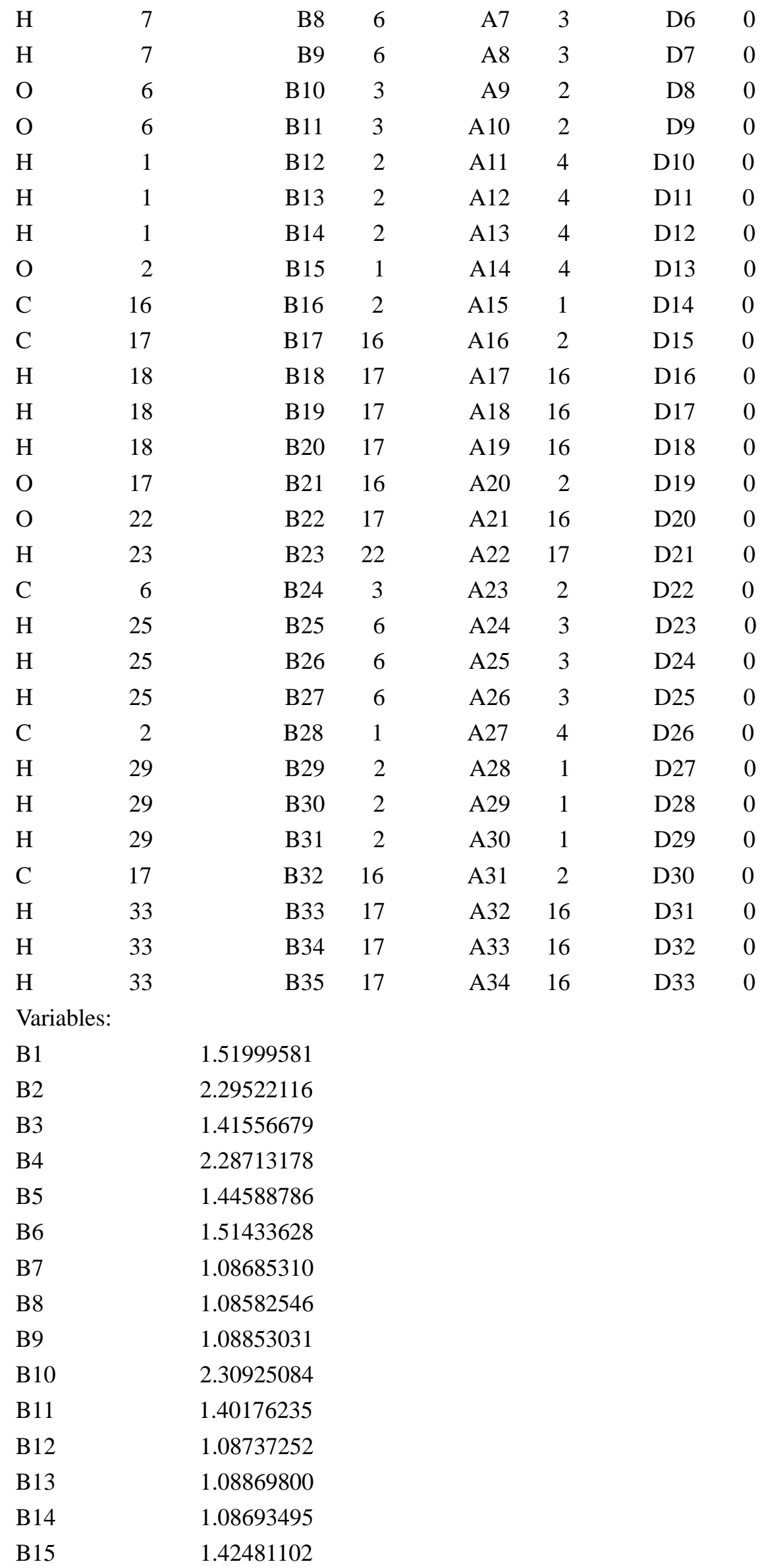




\begin{tabular}{|c|c|}
\hline B16 & 1.41522728 \\
\hline B17 & 1.51828429 \\
\hline B18 & 1.08662810 \\
\hline B19 & 1.08797701 \\
\hline B20 & 1.08935088 \\
\hline B21 & 1.41521147 \\
\hline B22 & 1.41253459 \\
\hline B23 & 0.96812056 \\
\hline B24 & 1.51524259 \\
\hline B25 & 1.08826708 \\
\hline B26 & 1.08798102 \\
\hline B27 & 1.08799065 \\
\hline B28 & 1.52078442 \\
\hline B29 & 1.08833138 \\
\hline B30 & 1.08480836 \\
\hline B31 & 1.08808921 \\
\hline B32 & 1.51978639 \\
\hline B33 & 1.08697238 \\
\hline B34 & 1.08811677 \\
\hline B35 & 1.08788261 \\
\hline $\mathrm{A} 1$ & 88.56171736 \\
\hline $\mathrm{A} 2$ & 110.79604196 \\
\hline A3 & 80.49879843 \\
\hline A4 & 130.70650365 \\
\hline A5 & 113.70270571 \\
\hline A6 & 110.45495516 \\
\hline A7 & 111.01977024 \\
\hline A8 & 107.33182437 \\
\hline A9 & 91.40603833 \\
\hline A10 & 103.75099985 \\
\hline A11 & 110.44016013 \\
\hline A 12 & 109.29193947 \\
\hline A13 & 110.09684267 \\
\hline A14 & 110.52691367 \\
\hline A15 & 125.40396453 \\
\hline A16 & 104.81368279 \\
\hline A17 & 110.50343310 \\
\hline A18 & 109.30832305 \\
\hline A19 & 109.56453143 \\
\hline A20 & 109.79837054 \\
\hline A21 & 109.76400100 \\
\hline A22 & 102.78518548 \\
\hline A23 & 107.72689666 \\
\hline A24 & 108.82358199 \\
\hline
\end{tabular}




\begin{tabular}{|c|c|}
\hline A25 & 110.38882289 \\
\hline A26 & 109.55844724 \\
\hline $\mathrm{A} 27$ & 112.66646058 \\
\hline A28 & 107.83215332 \\
\hline A29 & 111.53210941 \\
\hline A30 & 111.23109769 \\
\hline A31 & 114.82670349 \\
\hline A32 & 112.09336152 \\
\hline A33 & 109.29534534 \\
\hline A34 & 108.81021247 \\
\hline D1 & 28.76269698 \\
\hline D2 & -97.71851434 \\
\hline D3 & -159.49353719 \\
\hline D4 & -20.11371846 \\
\hline D5 & -67.05643783 \\
\hline D6 & 54.99859512 \\
\hline D7 & 173.69444424 \\
\hline D8 & 69.56426844 \\
\hline D9 & 102.86218371 \\
\hline D10 & -170.31182382 \\
\hline D11 & -50.68645443 \\
\hline D12 & 68.94319471 \\
\hline D13 & -115.31728114 \\
\hline D14 & -88.59573692 \\
\hline D15 & 174.41321122 \\
\hline D16 & 50.87068470 \\
\hline D17 & 171.19641539 \\
\hline D18 & -69.12402057 \\
\hline D19 & -65.19706597 \\
\hline D20 & -61.54204653 \\
\hline D21 & 71.47897466 \\
\hline D22 & -146.45114451 \\
\hline D23 & 62.95557980 \\
\hline D24 & -176.79626185 \\
\hline D25 & -56.22728661 \\
\hline D26 & 113.88389951 \\
\hline D27 & -58.42611579 \\
\hline D28 & 61.33383115 \\
\hline D29 & -177.48525925 \\
\hline D30 & 51.60507161 \\
\hline D31 & -67.60680992 \\
\hline D32 & 53.83922160 \\
\hline D33 & 172.79408537 \\
\hline
\end{tabular}

Harmonic Vibrational Frequencies $\left(\mathrm{cm}^{-1}\right)$ : 


$\begin{array}{lccccccccccc}16.99 & 54.47 & 66.92 & 81.98 & 108.22 & 158.33 & 174.37 & 185.84 & 202.66 & 217.77 & 222.99 \\ 237.82 & 255.89 & 264.67 & 274.20 & 285.72 & 296.95 & 301.70 & 319.79 & 328.96 & 354.34 \\ 362.15 & 364.64 & 377.67 & 394.57 & 402.89 & 457.47 & 471.50 & 486.44 & 529.52 & 535.50 \\ 582.90 & 600.14 & 611.41 & 640.29 & 757.56 & 797.53 & 851.15 & 892.58 & 909.10 & 925.83 \\ 940.47 & 946.61 & 948.26 & 973.15 & 981.43 & 1017.99 & 1021.41 & 1027.70 & 1055.08 & 1065.38 \\ 1070.09 & 1084.81 & 1179.05 & 1205.81 & 1231.63 & 1248.42 & 1258.38 & 1265.49 & 1278.32 \\ 1305.56 & 1310.53 & 1409.65 & 1411.14 & 1415.24 & 1417.71 & 1420.29 & 1428.67 & 1439.63 \\ 1466.36 & 1471.05 & 1480.92 & 1483.37 & 1486.40 & 1488.26 & 1493.88 & 1500.25 & 1501.20 \\ 1506.86 & 1508.76 & 1510.83 & 1521.06 & 3068.51 & 3070.02 & 3076.16 & 3079.27 & 3079.83 \\ 3083.36 & 3148.42 & 3149.24 & 3154.02 & 3157.16 & 3159.16 & 3160.93 & 3162.16 & 3163.41 \\ 3164.57 & 3167.57 & 3171.65 & 3182.81 & 3700.88 & 3760.83 & & & \\ \text { Rotational constants (B/GHZ): } & 0.8003076 & 0.3916650 & 0.3507378 & \end{array}$

